\title{
QUALITY AND RELIABILITY OF TECHNICAL SYSTEMS: THEORY AND PRACTICE
}

ANDRII GOROSHKO VILEN ROYZMAN MARYNA ZEMBYTSKA

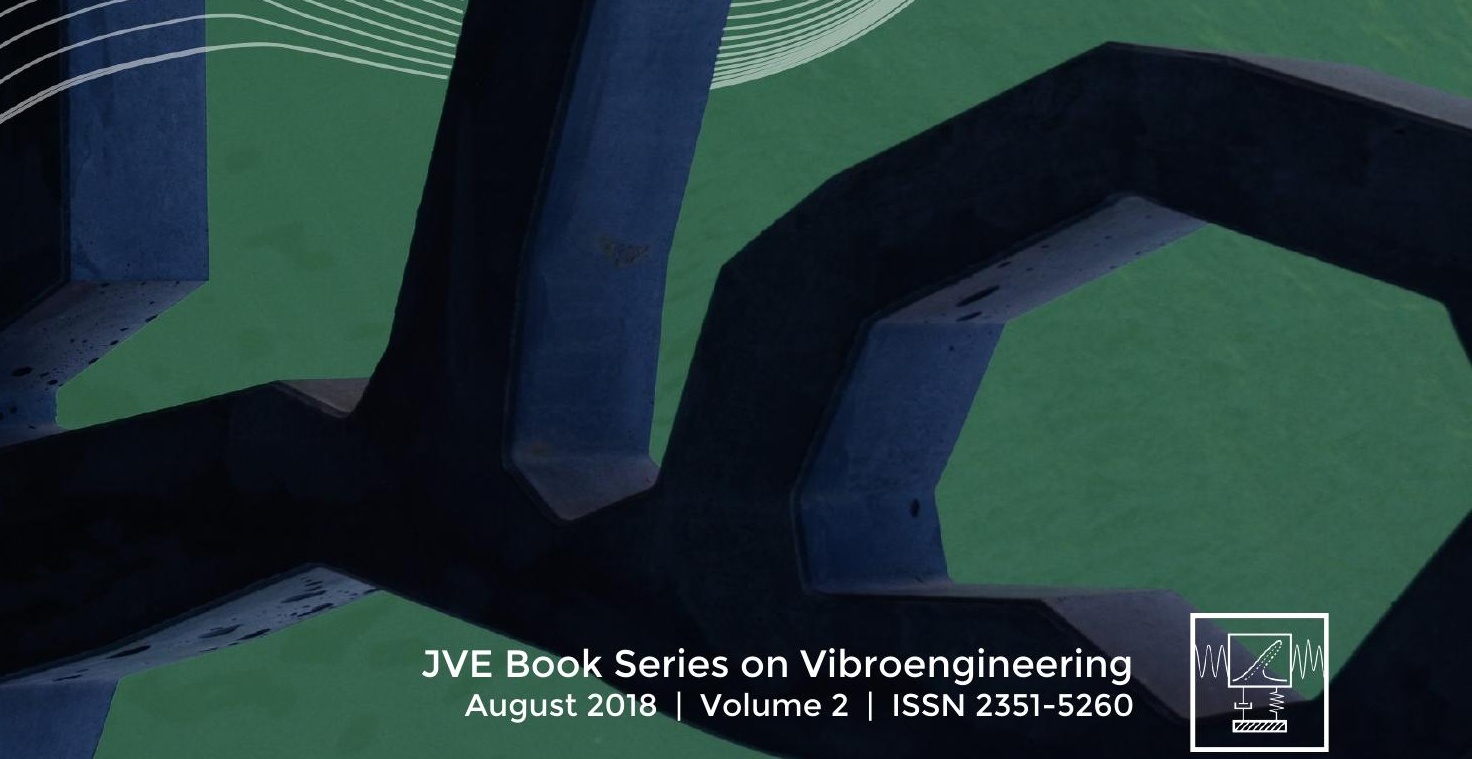




\section{Quality and Reliability of Technical Systems: Theory and Practice}




\title{
JVE Book Series on Vibroengineering
}

\author{
Aims and Scope \\ JVE Book Series on Vibroengineering covers a wide area of topics describing modern developments in \\ vibroengineering of dynamical systems (macro-, micro-, nano- mechanical, mechatronic and biomechanical \\ systems). \\ The following subjects are principal topics: \\ Vibration and wave processes; Vibration and wave technologies; \\ Nonlinear vibrations; Vibroshock systems; Generation of vibrations and waves; \\ Vibrostabilization; Transformation of motion by vibrations and waves; \\ Dynamics of intelligent mechanical systems; \\ Vibration control, identification, diagnostics and monitoring.
}

\begin{abstract}
About this Book
The monograph provides original approaches to the quality and reliability improvement in mechanical engineering, taking into account the functionally oriented management. The outlined studies validate the methods facilitating the increased reliability and durability of rotor systems and vibration processes. Methods for increasing the reliability and wear resistance of machine parts and units under dynamic and shock loads are considered and proposed. The issues of creation of new materials in mechanical engineering, in particular, nanostructures are studied and investigated. New quality and reliability improving methods for electronic equipment and energy systems are offered. The research also considers the issues of reliability and durability of construction materials.

The monograph is intended for scientists, graduate students and professionals in the field. It may also be useful to students of technology.
\end{abstract}




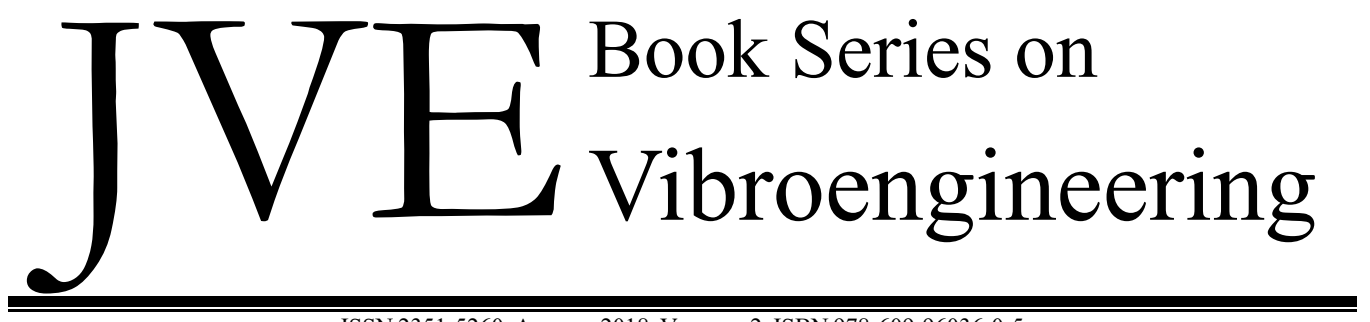

ISSN 2351-5260. AUGUST 2018. VOLUME 2, ISBN 978-609-96036-0-5

\title{
Quality and Reliability of Technical Systems: Theory and Practice
}

\author{
Andrii Goroshko
}

Khmelnytskyi National University, Ukraine

\section{Vilen Royzman}

Khmelnytskyi National University, Ukraine

\section{Maryna Zembytska}

Khmelnytskyi National University, Ukraine

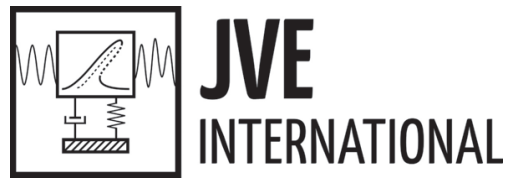




\section{Published by}

JVE International Ltd.

Naugarduko 41-906, Vilnius, LT-03227, Lithuania

https://www.jvejournals.com

JVE Book Series on Vibroengineering

QuAlity AND Reliability OF TeChNiCAl Systems: TheORY AND PRACTICE - Vol. 2

Copyright (C) 2018 by JVE International Ltd.

All rights reserved. This book, or parts thereof, may not be reproduced in any form or by any means, electronic or mechanical, including photocopying, recording or any information storage and retrieval system now know or to be invented, without written permission from the Publisher.

The bibliographic information about the publication is available in the National Bibliographic Data Bank (NBDB) of the Martynas Mažvydas National Library of Lithuania

ISSN 2351-5260

ISBN 978-609-96036-0-5

Printed in Lithuania by UAB "BMK Leidykla" 


\section{Introduction}

Creation of new technical systems necessitates the search for solutions aimed at improving their quality, reliability and durability. In this regard, the most important task in their design, manufacture and operation consists perhaps in ensuring the reliability of technical systems.

When manufacturing the machine, the necessary quality is ensured both by the quality of the parts, and by the quality of the assembly of the machine and its units, by finishing methods and other process indicators. The modern level of development of science and technology makes it possible to achieve any indicators of machine reliability. The decision on the need to improve the quality of products should be based on an economic analysis.

Modern development of technology is characterized by the intensification of its operating modes, greater construction complexity, unification of machines and devices in process lines, and process automation. These factors increase the likelihood of failures in operation and cause multiple problems with machinery hampering its stability and smooth running. The reliability is significantly reduced during the operation due to aging of the equipment as a result of wear, corrosion, fatigue, etc.

In order to be considered as reliable, a technical system shall perform all its functions for a certain specified operating time and under the required operating conditions while maintaining all the necessary performance characteristics of machinery and products within the specified limits.

This monograph outlines research findings, primarily the methods of increasing the reliability of various technical systems: machines and electronic equipment, power engineering facilities and building structures at the main stages of their life cycle.

The ISO 9000 standards contain recommendations and tools for companies and organizations (regardless of the scope of their activities) which want their products and services to meet constantly the customer's requirements, and their quality is constantly improved. The suggested monograph analyzes the existing approaches to process and functional management, as well as the formation of a process model of modern machine building organizations (based on the example of an enterprise for manufacturing modern compressor equipment), taking into account the requirements of ISO 9001:2015. The reorientation of organizations to process management will allow producers of compressor equipment to survive and develop under contemporary conditions.

The monograph primarily focuses on the ways of increasing the reliability of rotor systems and vibration technologies. The basic problem of these machines is the necessity to decrease their vibroactivity. The results of an experimental and theoretical research of fluid behavior in passive self-balancing devices, which are installed in rotors with vertical and horizontal rotation axes when passing the resonance, are given. Received relations show that automatic balancing by fluid is effective for elastically deformed rotors or (and) a rotor on elastic supports, where there is a difference in phases between the direction of centrifugal force and flexure (or movement) of the rotor. According to the experimental findings, this difference in phases occurs when the rotor reaches the resonance speed and increases up to $180^{\circ}$ while passing the resonance. In this case, the tangential force occurs, which influences the fluid to move to the side of flexure, opposite to imbalance, and further equilibration of the rotor takes place at the resonance rotation frequency.

The research introduces a method of indirect measurement of spindle vibrations based on the results of the vibration measurements of the spindle unit housing and theoretical compliance function. The method is based on the carried out experimental and theoretical studies of the spindle unit dynamic behavior, mounted on a special test rig with a low natural frequency.

The studies within this monograph, in particular, cover the creation of blood flow pulsations and the provision of the stable controlled behavior of the rotor in pulsating modes of functioning of the axial LVAD with magnetic bearings. The developed methods for ensuring the required rotor dynamics are accurate, fast, economical in the sense of the values of control currents, and most importantly reliability, which in general ensures the safety enhancement for the life support system - left ventricular assist device.

The studies are also aimed at solving the issues of increasing the productivity of turning parts 
at a rotational speed up to $10,000 \mathrm{rpm}$. The methods of studying the dynamic behavior of the lathe are reviewed, and the way of changing system properties to improve stability in the increased cutting conditions and productivity are reviewed too.

The authors also suggest methods for increasing the reliability and wear resistance of cylindrical blocks with surface plastic deformation. The findings, which were obtained by studying the efficiency of hardening the parts working in spalling conditions through reeling with rollers with the help of physical simulation, show a major effect of cast steels hardening. The process of the contact friction surface wear during reeling with consideration of slippage is investigated. A procedure for determining conditions of reeling with a wedge roller is developed.

The current series provides the analysis of high-rate elastic-plastic deformation of the elements of modern technology, taking into account the change of the material properties in the process of deformation under the impact and impulse loads. It is shown that multilayered protective elements more effectively withstand impact loads. A parametric analysis of technological processes of connecting and separating the elements due to the explosion energy is carried out.

A greater part of the work is devoted to the study of new materials, particularly the study of energy ions, their varieties and charge temperature, rate of temperature rise, thermal stresses, and also to the size, volume, number of particles in nanostructures and effective current density for nanostructures. The study is also concerned with the effect of technological factors on the electromagnetic wave absorption properties of polymer composites as well as with the investigation of the superhydrophobic surface properties.

The monograph covers the problems of the quality and reliability improvement of electronic equipment and power systems and introduces a modified method of spectral analysis of a reflection signal for damage detection systems in power lines, suggesting an algorithm for spectral analysis based on a change in the width of the analysis window.

The authors attempt to solve the current issues of rank analysis in the problems of optimization of local power engineering. The issue of application of the techno-enological approach for the optimal management of energy consumption with the use of rank analysis, which includes the procedures of interval estimation, parametric rationing, forecasting and standardization of consumption of resources is considered.

The paper represents the development principles invariant to disturbing factors of piezoresonance units with controlled dynamics, which are assumed to be an adaptive control system with predictive reference model.

The final part of the book is devoted to the problem of increasing the reliability and durability of building materials. The condition of the building industry and the building materials industry in Ukraine is considered, the production and sale of building materials is shown in dynamics. A new study of sound propagation in an impedance tube is presented, which is the basis of the sound absorption coefficient determination with greater simplicity.

The research findings presented in this book, which are aimed at ensuring the reliability of various technical systems, may be useful in the design, manufacture and operation of new machine-building products.

The monograph is primarily intended for specialists engaged in ensuring the reliability of machines, tools and equipment, as well as for researchers and engineers, students of technology interested in the issues discussed. 


\section{Contents}

INTRODUCTION

CHAPTER 1.

NEW METHODS FOR IMPROVING QUALITY AND RELIABILITY IN MECHANICAL ENGINEERING

1.1. APPLICATION OF PROCESS AND FUNCTIONAL APPROACHES TO QUALITY MANAGEMENT IN THE MACHINE-BUILDING ORGANIZATION WILIAM ZALOGA, KOSTIANTYN DYADYURA, IRYNA RYBALKA

1.2. CURRENT TRENDS IN MECHANICAL ENGINEERING

STEPAN D. POSTIL

DYNAMICS OF ROTOR SYSTEMS AND VIBRATION PROCESSES

ROTORS WITH LIQUID WORKING BODIES ON THE EXAMPLE OF DRUM TYPE WASHING MACHINES

ILONA DRACH, VILEN ROYZMAN, VitALII TKACHUK, ANDRII GOROSHKO

2.2. SPINDLE UNITS ON ROLLING BEARINGS DIAGNOSTICS BY INDIRECT MEASUREMENT OF SPINDLE VIBRATION

YURIY DANYLCHENKO, ANDRIY PETRYSHYN

2.3. METHODS OF ENSURING THE REQUIRED DYNAMICS OF AN AMB SUPPORTED AXIAL LVAD ROTOR

YULIA BOGDANOVA, ALEXANDER GOUSKOV

2.4. INCREASING OF EFFICIENCY OF PARTS TURNING AT SPINDLE SPEED FROM 5,000 TO 10,000 RPM BY CONTROLLING THE DYNAMICS OF MACHINING

WiLIAM ZALOGA, YURII SHAPOVAL, ViTALII KOLESNYK

CHAPTER 3.

INCREASING THE STRENGTH OF MACHINES UNDER STATIC, CYCLIC AND DYNAMIC LOADS

3.1. INCREASE OF RELIABILITY AND WEAR RESISTANCE OF CYLINDRICAL BLOCKS WITH SURFACE PLASTIC DEFORMATION

ALEKSANDR DYKHA, DMITRY MARCHENKO

3.2. PROBLEMS OF HIGH-RATE DEFORMATION OF ELEMENTS OF MODERN TECHNOLOGY UNDER THE INFLUENCE OF IMPACT AND IMPULSE LOADS YURII VOROBIOV, NATALIIA OVCHAROVA 
4.1. STUDY OF ENERGY IONS, THEIR VARIETIES AND CHARGE ON TEMPERATURE, RATE OF TEMPERATURE RISE, THERMAL STRESSES, AND ALSO ON THE SIZE, AMOUNT OF VOLUME, NUMBER OF PARTICLES IN NANOSTRUCTURES AND EFFECTIVE CURRENT DENSITY FOR NANOSTRUCTURES GENNADIY KOSTYUK

4.2. PREDICTION OF THE PHYSICAL AND MEChaNICAL CHARACTERISTICS, THE REMOVABLE MATERIAL VOLUME FOR THE DURABILITY PERIOD, CUTTING TOOLS DURABILITY AND PROCESSING PRODUCTIVITY DEPENDING ON THE GRAIN SIZE OF THE COATING OR CUTTING TOOL BASE MATERIAL GENNADIY KOSTYUK

4.3. EFFECT OF TECHNOLOGICAL FACTORS ON ELECTROMAGNETIC WAVE ABSORPTION PROPERTIES OF THE POLYMER COMPOSITES TAMAZ NATRIASHVILI, JIMSHER ANELI, LANA SHAMANAURI

4.4. STUDYING OF THE SEAWATER DROPS PROPERTIES ON SUPERHYDROPHOBIC SURFACE

TATIANA POPOVA, ALEKSEI UKOLOV

CHAPTER 5.

IMPROVING THE QUALITY AND RELIABILITY OF ELECTRONIC EQUIPMENT AND POWER SYSTEMS

5.1. ACOUSTIC EMISSION DIAGNOSTICS OF SOLDER JOINTS ON PRINTED CIRCUIT BOARDS IGOR KOVTUN, VILEN ROYZMAN, ANDRIY VOZNYAK

5.2. MODIFIED METHOD OF SPECTRAL ANALYSIS OF THE REFLECTION SIGNAL FOR DAMAGE DETECTION SYSTEMS IN POWER LINES KOSTYANTYN HORIASCHENKO, VICTOR STETCUK

5.3. RANK ANALYSIS IN THE PROBLEMS OF OPTIMIZATION OF LOCAL POWER ENGINEERING SERHII DENYSIUK, VASYLENKO VIRA

5.4. PRINCIPLES OF CONSTRUCTING INVARIANT PIEZORESONANCE DEVICES BASED ON ADAPTIVE MULTIFREQUENCY SYSTEMS WITH A PREDICTIVE STANDARD SERGEY PIDCHENKO, ALLA TARANCHUK

CHAPTER 6.

INCREASING THE RELIABILITY AND DURABILITY OF BUILDING MATERIALS

6.1. THE INDUSTRY OF BUILDING MATERIALS OF UKRAINE IN THE CONTEXT OF GLOBALIZATION

PETRO V. ZAKHARCHENKO, OLEKSANDR M. GAVRYSH, ROMAN D. ZAKHARIENKOV

6.2. A NEW METHOD FOR DETERMINING AN ACOUSTICAL CHARACTERISTIC OF BUILDING MATERIALS

Tamaz NatriashVili, Merab ChelidZe, DaVid NizHaRADZE, JEMAL JAVAKHISHVILI 


\section{Chapter 1}

New methods for improving quality and reliability in mechanical engineering 


\title{
1.1. Application of process and functional approaches to quality management in the machine-building organization
}

\author{
Wiliam Zaloga ${ }^{1}$, Kostiantyn Dyadyura ${ }^{2}$, Iryna Rybalka ${ }^{3}$ \\ Sumy State University, Sumy, Ukraine \\ E-mail: ${ }^{1}$ zalogav@gmail.com, ${ }^{2}$ dyadyura@pmtkm.sumdu.edu.ua, ${ }^{3}$ rybalka.ira@gmail.com
}

\begin{abstract}
The analysis of existing approaches to the construction of process models taking into account the functionally oriented management to ensure the appropriate quality of the products is carried out. The basic principles of process modeling are determined. The method of decision-making in the hierarchical structures of the system of enterprise processes is proposed. The results of the theoretical research can be used in the implementation of quality management systems based on international ISO standards and the description of processes by organizations.
\end{abstract}

Keywords: process, business process, process model, quality management system, functional approach, process approach.

\section{Introduction}

The ISO 9000 standards contain recommendations and tools for companies and organizations (regardless of the scope of their activities) who want their products and services to constantly meet the customer's requirements, and the quality is constantly improved. These standards are based on a number of principles for quality management such as customer orientation, motivation and involvement of senior management, process approach and continuous improvement [1]. The process approach is used as the base one [2]. According to the requirements of ISO 9001: 2015, the organization must determine the processes necessary for building a quality management system [3-5].

The problem is that the quality management system at each enterprise is, as a rule, unique and characterized by the content and importance of the hierarchical structures of the processes entering it, their input and output information, energy and material flows, quality indicators and their boundary values. The complex of interrelations between processes in each organization is complex and multifaceted [6-8]. Clients, competition, economic and political changes create new conditions for the existence of organizations and require flexibility and quick reaction in their management [9]. Until now, the methods of quality assurance applied at most machine-building enterprises were based, as a rule, on rigid multistage control of the product quality (functional approach) and services, and very insignificantly concerned improving the quality of production management systems based on the process approach (Fig. 1), which is the most important sign of perfect quality management and is used as a base in the International Standards ISO series 9000 [10-13]. For practical implementation in accordance with the requirements of ISO 9001: 2015, the organization must:

a) determine the required input data and the expected output of these processes;

b) determine the sequence and interaction of these processes;

c) identify and apply criteria and methods (including monitoring, measurements and relevant performance indicators) necessary to ensure the effective implementation and management of these processes;

d) identify the resources required for such processes and ensure their availability;

e) designate responsibilities and authority for the processes [14].

Under conditions of uncertainty in the external and internal environments characteristic of most modern enterprises, the formation of an optimal hierarchical structure of business processes that allow transforming "entrances" into "exits" and often al-most uncontrollable $[15,16]$, for the adoption of rational (optimal) management solutions to continuously improve quality is a complex 
scientific and practical task. This is especially relevant for enterprises, for example, machine building, which are involved in the life cycle (LC) - designing, manufacturing, servicing and recycling - of complex technical products such as compressor equipment. For them, a common scientific methodology is necessary both for the analysis and for synthesis of the system of processes at the stages of the LC of complex technical products, and for analysis and synthesis of systems for integrated process control, taking into account the corresponding dynamic, parametric and energy laws.

The purpose of this work is to analyze the existing approaches to process and functional management, as well as the formation of a process model of modern ma-chine building organizations (based on the example of an enterprise for manufacturing modern compressor equipment) based on the requirements of ISO 9001: 2015.

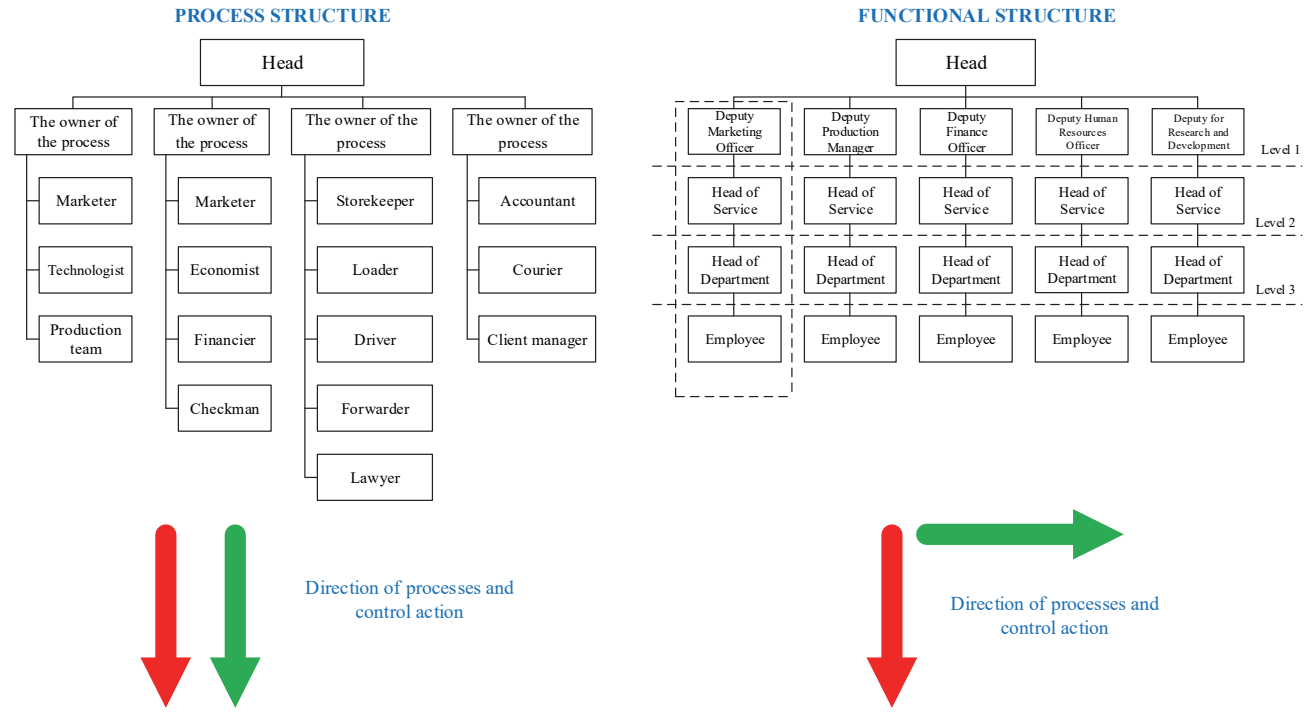

Fig. 1. Process and functional structures

\section{General: major recent advances}

As a part of a complex technical system (STS), in general, three different components can be distinguished [17] - a complex of technical means (CTM), software (SW) and operational personnel (OP) as shown in Fig. 2.

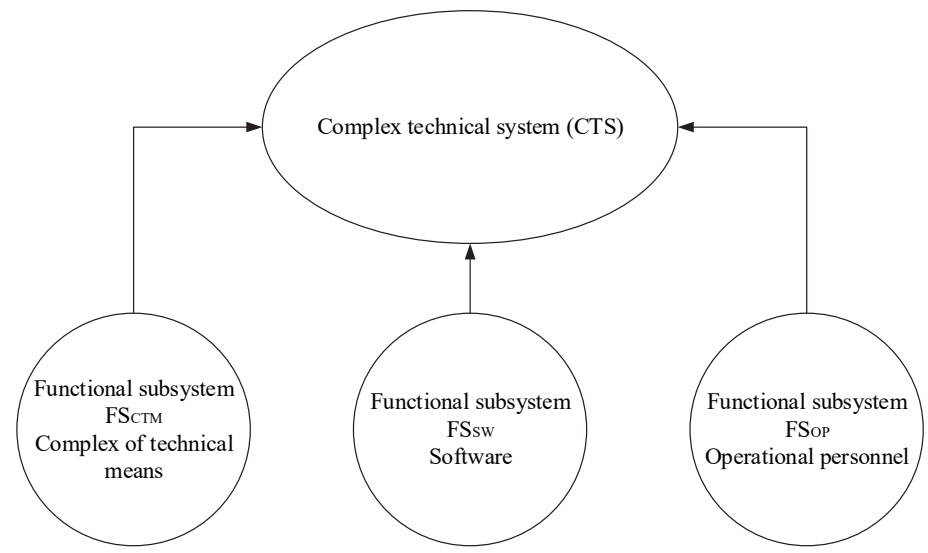

Fig. 2. Functional subsystems 
Designing, manufacturing and operation of compressor equipment, predetermines a community, which includes documented information, hardware and software, as well as personnel that are interconnected within a single system through information, energy and material flows. The components of the CTS compressor equipment are shown in Fig. 3.

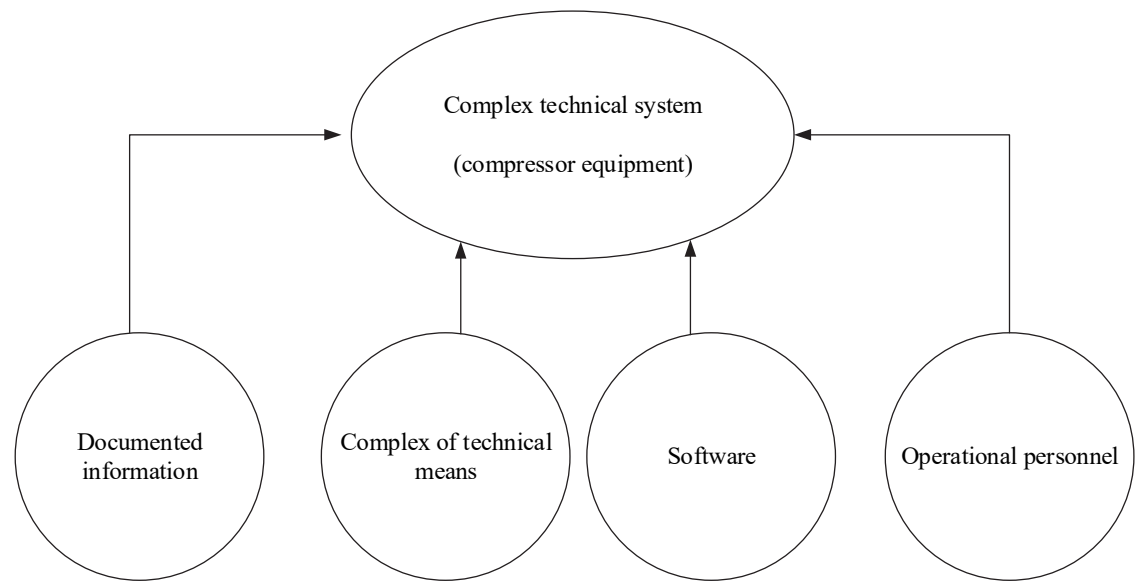

Fig. 3. Components of CTM compressor equipment

To ensure the quality of CTS (compressor equipment), it is necessary to ensure the appropriate quality of output data for each phase of the LC STS, which is realized through the identification and monitoring of KPI (Key Performance Indicator) key performance indicators of the LC processes. Highlighting indicators is most convenient for the process depicted in IDEF0 notation (Fig. 4), when the figure shows the inputs, outputs, control (process execution rules) and mechanisms (equipment, personnel).

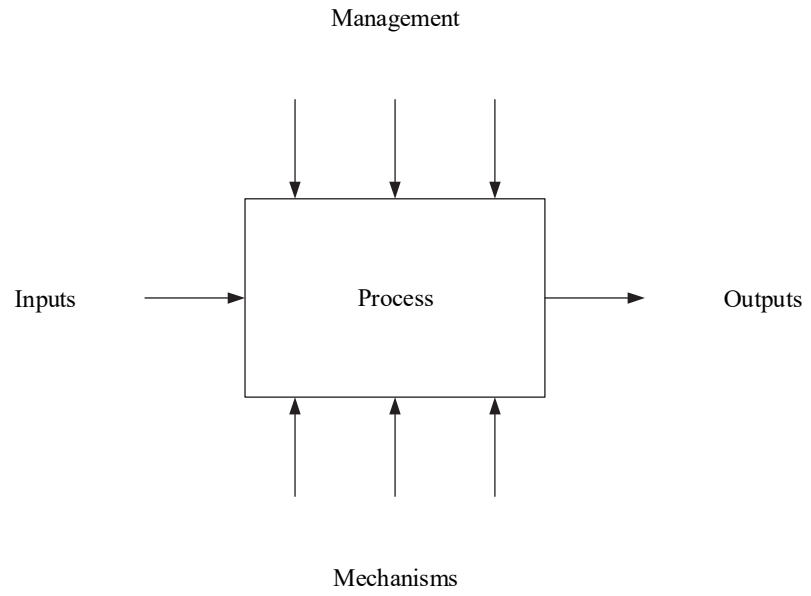

Fig. 4. Process in notation IDEF0

Key performance indicators and performance indicators, being derived, when using such a scheme characterize the overall process (Fig. 5).

The algorithm for developing the process indicators is as follows:

1. Identify the process and its result.

2. Identify inputs-resources (resources processed in one process cycle) and inputs-mechanisms (resources ensuring multiple execution of the process-equipment, personnel).

3. Identify the inputs and controls (rules and requirements for the process). 
4. Knowing the result that must be obtained, it is necessary to evaluate it quantitatively - to generate the outcome indicators. They can be either simple or calculated (by formula or otherwise).

5. Based on the inputs of the process, you can generate cost indicators.

6. Based on the mechanisms of the process, you can generate additional cost indicators.

7. The correctness of the process, in addition to the cost of performing activities, also reflects the performance indicators.

8. Performance indicators are calculated as the ratio of the result obtained to the time.

9. Calculation of key performance indicators is carried out on the basis of pre-selected KPI performance and KPI costs. Performance indicators, thus, are integral characteristics of activity.

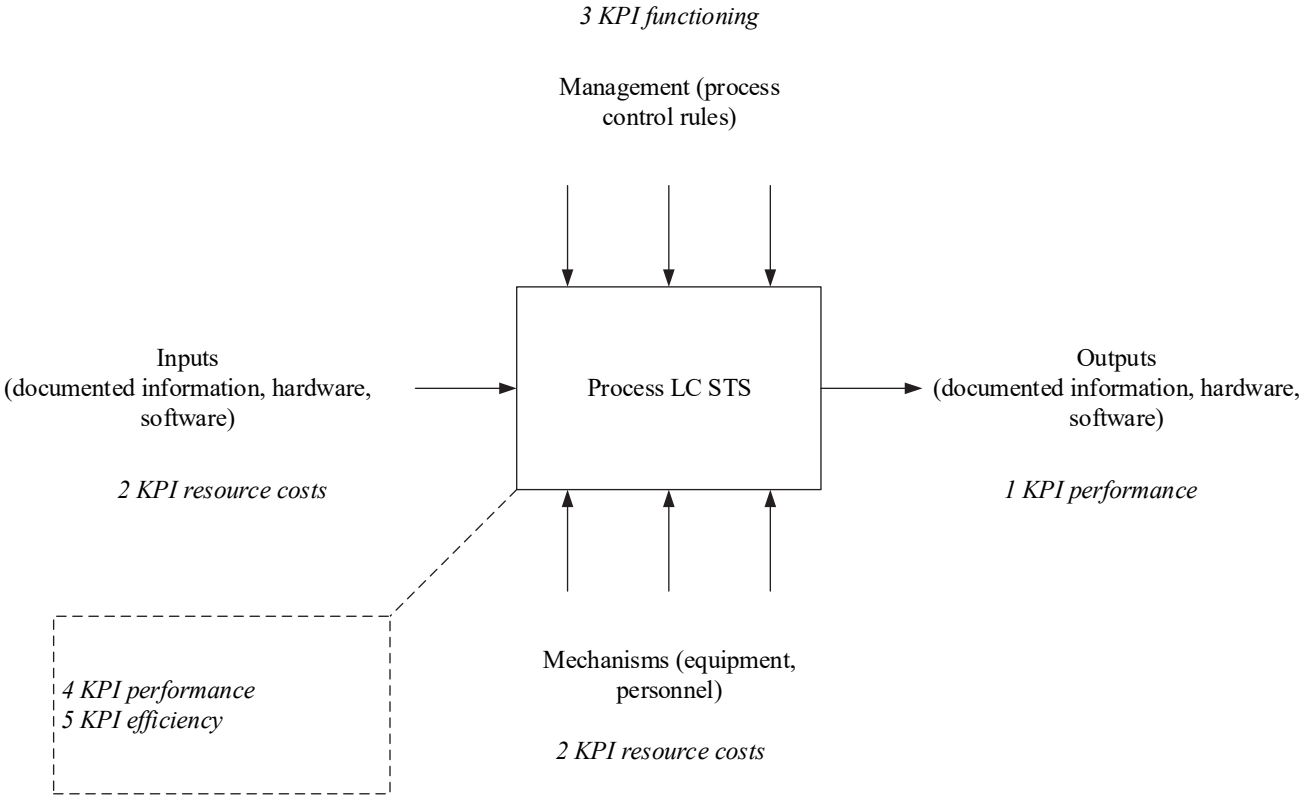

Fig. 5. KPI indicators

These components (Fig. 3) affect the effectiveness of the implementation of the LC is not isolated, but in close relationship with each other, which is expressed in the ability of one to correct the other two by the effectiveness of the implementation of its functions. Between the components, the space-time relations act.

The organization's processes are divided into main, providing, management processes and development processes, the definitions of which are presented in Table 1.

The main factors of the complexity of these processes are:

- the dynamic nature of the functioning of processes;

- the processes that take place both within the organization and when it interacts with the external environment, are non-linear, synergistic (dissipative);

- the natural evolutionary processes occurring in the organization are degradative, have a latent stage of development and are aimed at the destruction of the system;

- the time of the course of degradation processes considerably exceeds the time of the course of specific processes.

Every community of objects, processes and phenomena is characterized by the structure and interconnection of its components and can be represented in the form of a graph in which nodes correspond to components, and arcs to their connections.

Basic notations in modeling business processes (notation is a stable set of rules that describes the business process as part of the methodology): 
- structural models (control) - IDEF0 notation, VAD notation (ARIS, MS Visio);

- Workflow models - "process" and "procedure" notations, notation of eEPC (ARIS, MS Visio);

- models of executable processes - notations BPMN 2.0, notations S-BM, CFFC;

- data flow models - DFD notation $[18,19]$

Table 1. Table classification of processes

\begin{tabular}{|c|c|c|}
\hline Types of processes & Definitions & Organization processes \\
\hline $\begin{array}{c}\text { Basic } \\
\text { processes }\end{array}$ & $\begin{array}{l}\text { - Processes that create a product that is valuable to } \\
\text { the external consumer. } \\
\text { - Processes that create added value to a product or } \\
\text { service. }\end{array}$ & $\begin{array}{c}\text { Sales } \\
\text { Design and development } \\
\text { Service maintenance } \\
\text { Supply } \\
\text { Production } \\
\end{array}$ \\
\hline $\begin{array}{l}\text { Providing } \\
\text { processes }\end{array}$ & $\begin{array}{l}\text { - Processes whose consumers are the main } \\
\text { processes. } \\
\text { - Processes that create and maintain an } \\
\text { organization's infrastructure. }\end{array}$ & $\begin{array}{c}\text { Financial and Economic } \\
\text { Management } \\
\text { Human Resource } \\
\text { Management } \\
\text { Maintenance } \\
\text { management } \\
\text { Legal support } \\
\text { Business Security } \\
\text { Management } \\
\text { Managing documented } \\
\text { information } \\
\text { Knowledge Management } \\
\text { Communications } \\
\text { Management } \\
\text { Logistics Management } \\
\text { IT security }\end{array}$ \\
\hline $\begin{array}{l}\text { Processes } \\
\text { management }\end{array}$ & $\begin{array}{l}\text { - Processes, the main purpose of which is to } \\
\text { manage the activities of the organization. } \\
\text { - The processes that ensure the survival and } \\
\text { development of the organization regulate its } \\
\text { current activities. }\end{array}$ & $\begin{array}{l}\text { Strategic management } \\
\text { Business Process Quality } \\
\text { Management } \\
\text { Management of risks }\end{array}$ \\
\hline $\begin{array}{l}\text { Processes (projects } \\
\text { and programs) } \\
\text { development of }\end{array}$ & $\begin{array}{l}\text { - Irregular and innovative activities to improve and } \\
\text { develop the organization. } \\
\text { - Activities aimed at the long-term perspective. }\end{array}$ & Project management \\
\hline
\end{tabular}

Consider the IDEF0 notation. The IDEF0 submission form provides an opportunity to see and understand the interconnection of processes without going into details. Processes can be represented in the form of a hierarchical, tree structure with characteristics of links (branches), reflecting the utility, the probability of obtaining a result and the connection with previous and subsequent processes.

With the help of the graphical language IDEF0, the studied system appears as a set of interrelated functional blocks. Modeling of business processes using IDEF0 tools, as a rule, is the first stage of studying the system. Today, this technique of describing business processes has become the most widespread in the world and adopted as a standard in many countries [20-24]. The decomposition of processes in the form of IDEF0 is shown in Fig. 6.

The decomposition of variants is carried out by a set of characteristics. Criteria for choosing options are economic indicators (minimum cost, costs) or technical solutions that form a benefit function (utility). Description of business processes involves:

1) the definition of the owner of the business process, the boundaries of the business process, clients and outputs of the business process, suppliers and inputs of the business process, resources;

2) a description of the technology of the business process; 
3) development of indicators of the business process, the results of the business process, customer satisfaction of the business process;

4) a description of the work of the owner of the business process for analyzing, improving the business process, reporting to a superior manager.

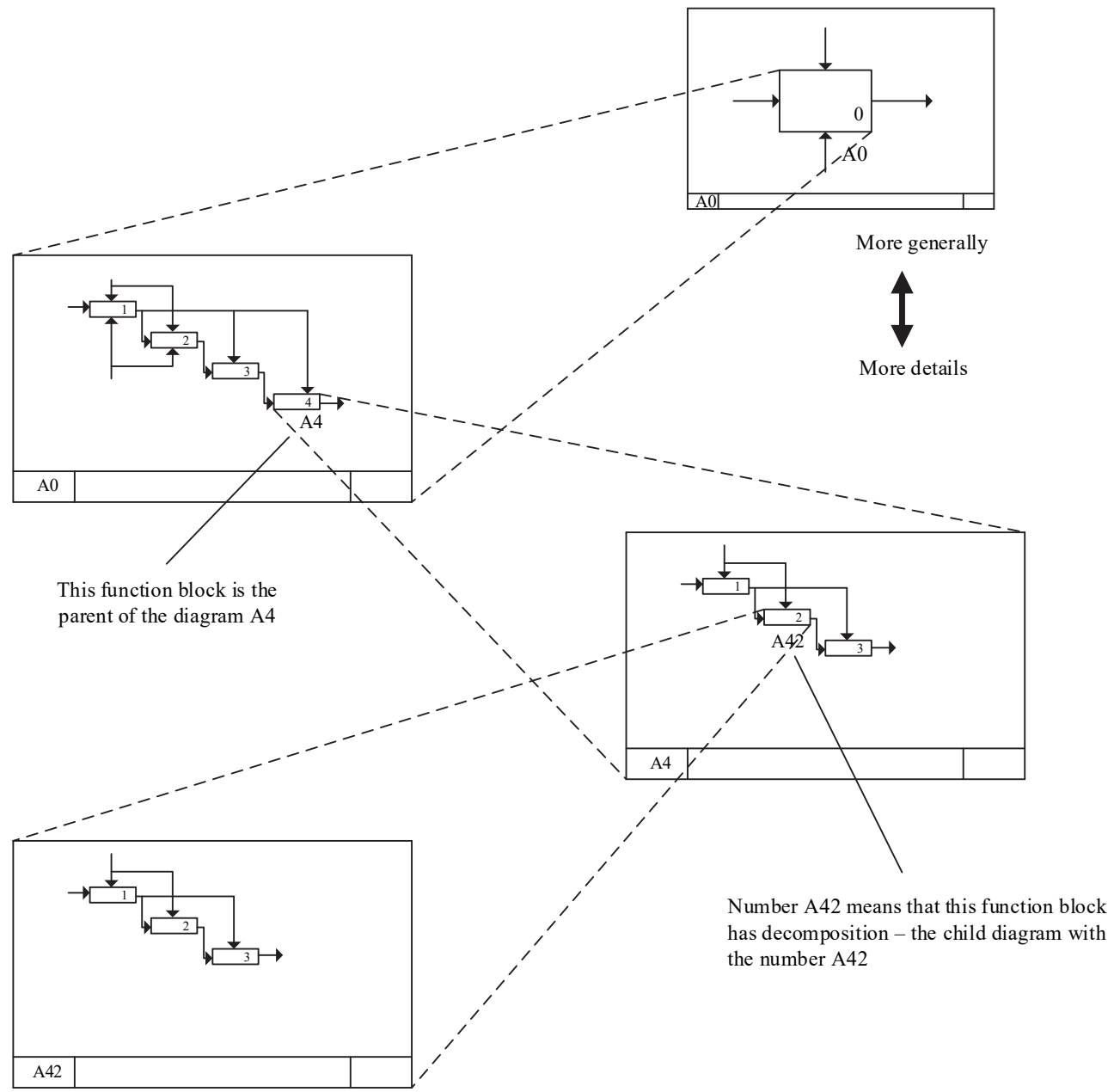

Fig. 6. The principle of the decomposition of processes

In describing and optimizing the organization's processes, two possible tasks can be solved:

- increase transparency and effectiveness of the organization;

- automation of business processes.

As criteria for evaluating the results of the description of processes, the following can be adopted:

- availability of verified descriptions of business processes;

- solution of problems identified during the implementation of business processes;

- the degree of coordination of units;

- the degree of minimization of possible variations as a result of the implementation of the corresponding business process, i.e. The business process is executed stably without failures.

Let us consider the choice of the best variant of describing processes under uncertainty conditions using vector optimization [25]. Among the various ways to optimize complex systems, which include the systems of business processes in enterprises, the formation of many possible 
solutions is paramount [26].

The problem of step-by-step vector optimization is formulated as follows. Let there be $N$ variants of processes with numbers $n=1, N$. Each variant of the process is characterized by $M$ number of indicators qnm, where $m=\overline{1, M}$. We assume that the exponents are normalized in such a way that $q_{n m} \in[0 ; 1]$, and the maximal quality of the process corresponds to $\max q_{n m}$. Then to each variant of the process there corresponds a quality vector $\bar{Q}\left[q_{n 1}, q_{n 2}, q_{n 3}, \cdots, q_{n m}\right]$.

Due to the fact that the systems of processes in the enterprise are multifunctional and the functions performed by them can differ significantly, when considering many issues, we use a functional approach. At the same time, a group of technical, program and ergatical (personnel) elements participating in the performance of this function are distinguished from the composition of all elements for considering the processes by some $(j$-th) function [27].

This group of elements forms the $j$-th functional subsystem ( $j$-th $F S$ or $F S_{j}$ ) of the system under consideration. It is this $F S_{j}$ that is to be analyzed when considering the characteristics of the system with respect to the $j$-th function it realizes.

The composition of the $F S_{j}$ (as well as the composition of the system as a whole) generally consists of three components:

- group of $j$-th functions of technical means participating in the implementation $(j$-th functional subsystem CTM $-F S_{\text {CTM } j}$ );

- group of software tools participating in the $j$-th function realization ( $j$-th functional subsystem $\left.\mathrm{SW}-F S_{S W j}\right)$;

- group of ergatic means participating in the $j$-th function realization ( $j$-th functional subsystem OP $-F S_{O P j}$ ).

On the basis of participation in the performance of a function, functional subsystems are distinguished, constructive features can be allocated to constructive subsystems, information subsystems, information subsystems, etc. Analysis of the functioning of the system is greatly simplified if its structure is constructed in such a way that the subsystems distinguished by functional and constructive characteristics coincide.

The system of functions forms the characteristics of the functional subsystems of the $X_{F P S}$. In this case, the functions can be divided into main and auxiliary. The main ones are those that directly form other functions, the auxiliary ones are only involved (help to form).

Between the hierarchy of functions (transformations) and the hierarchy of functional subsystems, there is a correspondence [28]. The interrelationship between the set of functions $(F)$ and the set of groups of means $\left(F S_{C T M}, F S_{S W}, F S_{O P}\right)$ is determined by the levels of the hierarchy. At each decomposition level, the $\mu$ function $F_{l j}^{\mu} \in F^{0}$ corresponds to a number $\left\{F S_{l j}^{\mu}\right\}_{Y}$ of types of means $(j$ is the index of the function at the level of the hierarchy; $l$ is the number of functions at the hierarchy level). The type of the facility provides $F S_{j} \in\left\{F S_{l j}^{\mu}\right\}_{Y}$ the principle possibility of performing the function $F_{l j}^{\mu} \in F^{0}$. Thus, we are given a multiple map of the system $F$ on the subsystem of the $F S$.

The system of funds based on the principle interconnection levels: forming a plurality of elementary means $F S=\left\{F S^{0}, F S^{1}, \ldots, F S^{\mu-1}\right\}$, each of which ensures the performance of a single elementary function of the set $F=\left\{F^{0}, F^{1}, \ldots, F^{\mu-1}\right\}$.

\section{Results}

The possibility of forming various variants of the characteristics of the $X_{F P S i}$ of the functional subsystem of processes is provided by a directed selection from the set $\left\{F S^{0}\right\}$ of a set of elements $F S_{i} \subset F S^{0}$, which ensures the fulfillment of a set of functions $\left\{F^{0}\right\}$ :

$X_{F S_{i}}:\left[\left\{F_{i}^{0}\right\} \rightarrow\left\{F S_{i}^{0}\right\} ;\left\{F_{i}^{1}\right\} \rightarrow\left\{F S_{i}^{1}\right\} ; \ldots ;\left\{F_{i}^{N}\right\} \rightarrow\left\{F S_{i}^{N}\right\}\right]$, 
with parameter constraints:

$$
\begin{array}{ll}
X_{F S_{\min }}^{1} \leq X_{F S}^{1} \leq X_{F S_{\text {max }}}^{1}, & X_{F S}^{1}=\left\{X_{F S_{1}}^{1}, X_{F S_{2}}^{1}, \ldots, X_{F S_{k 1}}^{1}\right\}, \\
X_{F S_{\min }}^{2} \leq X_{F S}^{2} \leq X_{F S_{\text {max }}}^{2}, & X_{F S}^{2}=\left\{X_{F S_{1}}^{2}, X_{F S_{2}}^{2}, \ldots, X_{F S_{k 2}}^{2}\right\}, \\
\vdots & \\
X_{F S_{\min }}^{N} \leq X_{F S}^{N} \leq X_{F S_{\max }}^{N}, & X_{F S}^{N}=\left\{X_{F S_{1}}^{N}, X_{F S_{2}}^{N}, \ldots, X_{F S_{k \mu}}^{N}\right\}
\end{array}
$$

for given connections of the set $F$ and $F S$ :

$$
\begin{aligned}
& \left\{F^{0}\right\} \times\left\{F S^{0}\right\} \rightarrow\left\{\Theta^{0}\right\}, \\
& \left\{F^{1}\right\} \times\left\{F S^{1}\right\} \rightarrow\left\{\Theta^{1}\right\}, \\
& \vdots \\
& \left\{F^{j}\right\} \times\left\{F S^{j}\right\} \rightarrow\left\{\Theta^{\mu}\right\}, \\
& \vdots \\
& \left\{F^{N}\right\} \times\left\{F S^{N}\right\} \rightarrow\left\{\Theta^{N}\right\},
\end{aligned}
$$

and the connections inside the set $F$ and $F S$ :

$$
\begin{aligned}
& \left\{F^{1}\right\} \times\left\{F^{2}\right\} \rightarrow\left\{f^{2}, v^{1-2}\right\}, \\
& \left\{F^{2}\right\} \times\left\{F^{3}\right\} \rightarrow\left\{f^{3}, v^{2-3}\right\}, \\
& \vdots \\
& \left\{F^{\mu}\right\} \times\left\{F^{\mu+1}\right\} \rightarrow\left\{f^{\mu+1}, v^{\mu-(\mu+1)}\right\}, \\
& \vdots \\
& \left\{F^{N-1}\right\} \times\left\{F^{N}\right\} \rightarrow\left\{f^{N}, v^{(N-1)-N}\right\}, \\
& \left\{F S^{1}\right\} \times\left\{F S^{2}\right\} \rightarrow\left\{\varepsilon^{1-2}\right\} \\
& \left\{F S^{2}\right\} \times\left\{F S^{3}\right\} \rightarrow\left\{\varepsilon^{2-3}\right\}, \\
& \vdots \\
& \left\{F S^{\mu}\right\} \times\left\{F S^{\mu+1}\right\} \rightarrow\left\{\varepsilon^{\mu-(\mu+1)}\right\}, \\
& \vdots \\
& \left\{F S^{N-1}\right\} \times\left\{F S^{N}\right\} \rightarrow\left\{\varepsilon^{(N-1)-N}\right\},
\end{aligned}
$$

where $i$ is the index of the characteristic of the functional subsystem of the $X_{F S i} 0 \leq i \leq M$; $M$ is the number of characteristics of $X_{F S i} ; \mu$ - hierarchy level index; $N$ - number of hierarchy levels; $X_{F S_{\text {min }}}, X_{F S_{\text {max }}}$ - ranges of parameters of functional efficiency relative to the $j$-th level of the hierarchy; $k_{j}$ - the number of parameters of functional efficiency at the $j$-th level of the hierarchy; $\Theta$ - connection of $F$ and $F S ; f$ - connection of levels of the hierarchy of the system $F ; v-$ connection within the levels of the system $\Phi ; \varepsilon$ - communication within the levels of the $F S$ system.

Thus, the functional subsystem of processes can be represented in the form of a set of hierarchically organized sets: elementary functions $F^{N}$, groups of means $\left(F S_{C T M}, F S_{S W}, F S_{O P}\right)$ participating in the realization of the function at each level and the corresponding characteristics of the $X_{F S}$ (Fig. 7). The set of elementary functions $F^{N}$ with respect to $\Theta^{N}$ bonds defines a set of groups $F S_{i N}^{N}$ and elementary means $\left(F S_{C T M_{i N}}, F S_{S W_{i N}}, F S_{O P_{i N}}\right)$. Inside the set $F S^{N}$ over the links $\Theta^{N}$, many characteristics $X_{F S}^{N}\left(X_{F S_{i}}^{N} \in X_{F S}^{N}\right)$ are formed, which ensure the efficiency of performance of functions in conditions of constraints on parameters [29, 30].

Each element of the system of means $F S_{i} \in F S^{0}$ can be described in addition to the links $\Theta$ and $\varepsilon$ by the readiness characteristics $r$ and the containment $C$ : 
$F S^{0}=\bigcup_{\mu=1}^{N} F S^{\mu}\left(\Theta^{\mu}, \varepsilon^{\mu}, r^{\mu}, C^{\mu}\right)$.

Many characteristics $X_{F S}^{N}$ can be represented by two subsets: $X_{F S_{i}}^{r}$ that includes the types of subsystems of elements ready for operational use, and a subset $X_{F S_{i}}^{r^{*}}$ that includes the types of subsystems and elements not ready for immediate use at the time $t$ :

$X_{F S_{i}}=X_{F S_{i}}^{r} \cup X_{F S_{i}}^{r^{*}}$

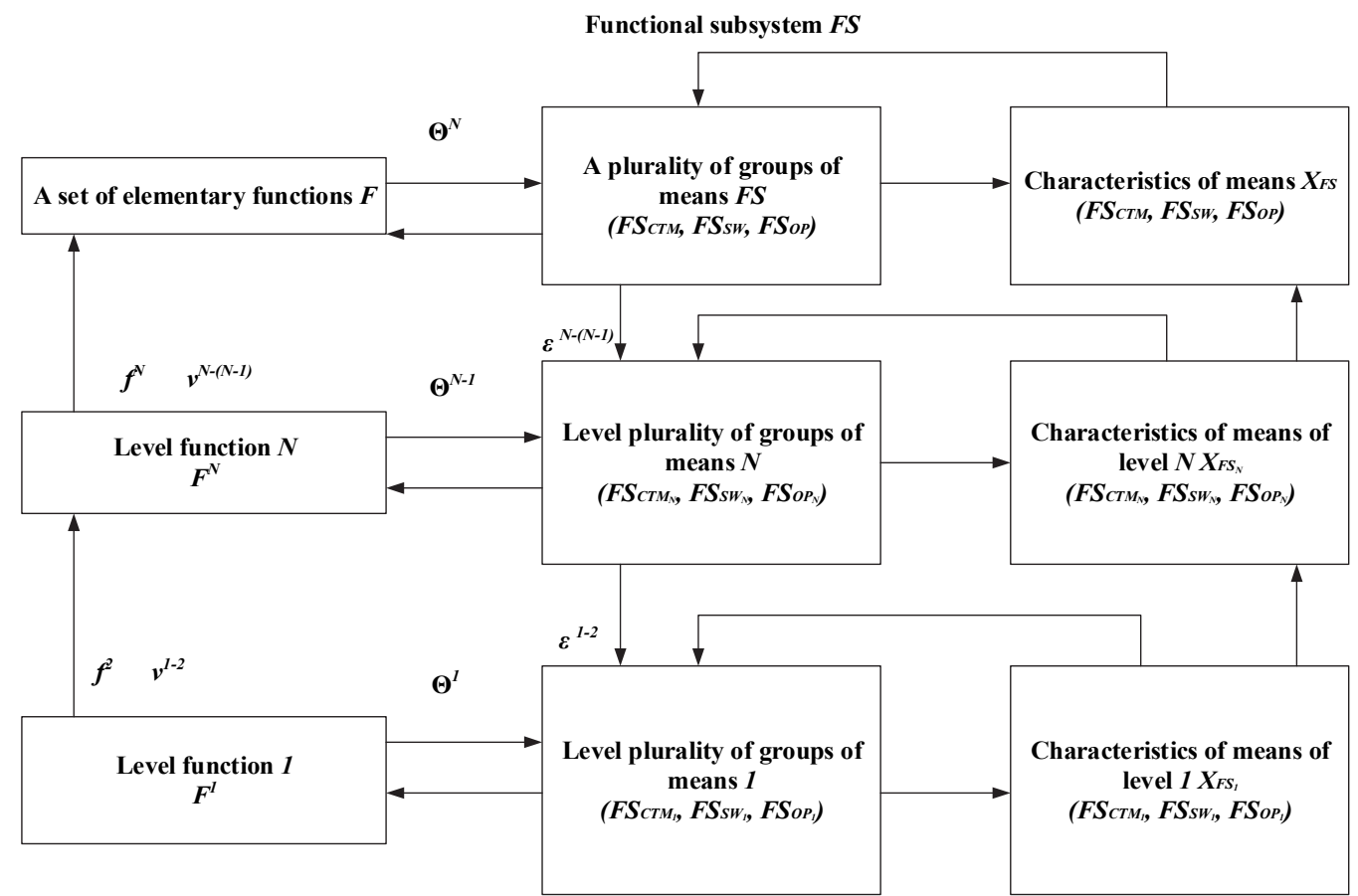

Fig. 7. Structure of the functional subsystem

\section{Discussion}

In this case, two types of business process models are considered: process and functional structures (see Fig. 1).

Differences and advantages of functional and process approaches:

1) the process approach shows:

- orientation to the client (on the result), i.e. CRM-approach;

- submission to the owner of the process;

- priority - performance of functions important for other participants in the process, active cooperation with participants in the process;

- higher management flexibility;

- focus on costs (cost of the process), its duration, quality;

- transparency of operations. It is easy to track 'failures';

2) with a functional approach:

- orientation to the manager;

- submission to the head of the unit;

- priority - the performance of functions in which the unit itself is interested; 
- propensity to "bureaucracy", as a consequence - loss of managerial flexibility, low speed of decision-making, loss of information;

- the contradiction between the objectives of the functional units;

- opacity of activity. The shifting of responsibility.

In our opinion, it is not only impossible to contrast the process and functional approaches to the management of an organization, but, conversely, the quality management system should be viewed as a system of interaction between process-oriented and functionally-oriented management.

In the process of implementation of processes under the influence of internal and external factors, a gradual and continuous (evolutionary) change in the structure of processes takes place.

In this case, the system of processes is proposed to be investigated in several directions:

- representation of complex systems of processes as an interconnected set of sub-systems of different hierarchical level, constructing a hierarchy of optimization tasks with appropriate information exchange in time and levels, i.e., the so-called hierarchical approach;

- development of special approaches and methods for accounting for information uncertainty, analysis and decision making;

- the development of gaming approaches, including games with non-conflicting interests and the adoption of collective decisions;

- development of methods for multicriteria optimization, methods of peer review, etc.

\section{Conclusions}

It is shown that due to the application of the process approach to the management of the organization it is possible to improve its activity to a considerable extent. Practical implementation of the process approach in the quality management system allows:

- make a list of the main business processes and draw conclusions on the rational use of resources, the number and load of personnel, etc., on the basis of the data obtained;

- identify missing and duplicated business processes and make appropriate adjustments;

- determine the list of functions of each division of the organization;

- establish interrelationships between departments and the functions performed in them.

As a result of the introduction of the process approach and the description of the processes, the order and responsibility for performing the work will be fixed, the failures in the implementation of processes will be eliminated, and the quality of the process performance will be improved.

The reorientation of organizations to process management will allow producers compressor equipment to survive and develop in the conditions of the modern world.

\section{References}

[1] Asensi S. S., Soler V. G., Bernabeu E. P. Seven principles of quality management in ISO 9001. Empresa, 2017, p. 10-18.

[2] Benner M. J. Dynamic or static capabilities? Process management practice-sand response to technological change. Journal of Product Innovation Management, Vol. 26, Issue 5, 2009, p. 473-486.

[3] Manders B., De Vries H. J., Blind K. ISO 9001 and product innovation: a literature review and research framework. Technovation, Vols. 48-49, 2016, p. 41-55.

[4] Enders C., Lang G. E., Lang G. K., Werner J. U. ISO 9001:2015 certification in quality management. Klinische Monatsblatter Fur Augenheilkund, Vol. 234, Issue 7, 2017, p. 886-890, (in German).

[5] Kontogiannis T., Leva M. C., Balfe N. Total safety management: principles, processes and methods. Safety Science, Vol. 100, 2017, p. 128-142.

[6] Aravind D., Christmann P. Decoupling of standard implementation from certification: does quality of ISO 14001 implementation affect facilities environmental performance. Business Ethics Quarterly, Vol. 21, Issue 1, 2011, p. 73-102. 
[7] Boiral O. Managing with ISO systems: lessons from practice. Long Range Plan, Vol. 44, Issue 3, 2011, p. 197-220.

[8] Cole R. E., Matsumiya T. Too much of a good thing? Quality as an impediment to innovation. California Management Review, Vol. 50, Issue 1, 2007, p. 77-93.

[9] Hammer M., Champy J. Reengineering the Corporation: A Manifesto for Business Revolution. Mann, Ivanov and Ferber, 2011, (in Russian).

[10] Arauz R., Suzuki H. ISO 9000 performance in Japanese industries. Total Quality Management and Business Excellence, Vol. 15, Issue 1, 2004, p. 3-33.

[11] Bayo Moriones A., Merino Díaz De Cerio J., Escamilla De León, S. A., Selvam R. M. The impact of ISO 9000 and EFQM on the use of flexible work practices. International Journal of Production Economics, Vol. 130, Issue 1, 2011, p. 33-42.

[12] Briscoe J. A., Fawcett S. E., Todd R. H. The implementation and impact of ISO 9000 among small manufacturing enterprises. Journal of Small Business Management, Vol. 43, Issue 3, 2005, p. 309-330.

[13] Chandrasekaran R., Karthikeyaan S. Measure of operational performance before and after implementation of ISO 9000 certification in pumpsets manufacturing industries in Coimbatore, India. Journal of Contemporary Research in Management, Vol. 4, Issue 2, 2013, p. 83-92.

[14] Quality Management Systems - Requirements. ISO 9001:2015, 2015.

[15] Abdikeev N. M., Danko P. P., et al. Reengineering of Business Processes. Eksmo-Press, 2006, (in Russian).

[16] Andersen B. Business Processes. Tools for Improvement. Standards and Quality, 2007, (in Russian).

[17] DSTU 3524-97 Reliability of Technology. Project Assessment of the Reliability of Complex Systems, Taking into Account Technical and Software and Operational Personnel. Substantive Provisions.

[18] Rother Shook Learn to See the Business Processes. the Practice of Constructing Maps of Value Streams. Alpina Business Books, 2008, (in Russian).

[19] Kalyanov G. N. Modeling, Analysis, Reorganization and Automation of Business Processes. Finance and Statistics, 2007, (in Russian).

[20] Kiselev A. D., Ildemenov S. V., Danko T. P., Abdikeev N. M. Reengineering of Business Processes. EKSMO, 2007, (in Russian).

[21] Renadive V. Foresight: a New Evolutionary Proactive Business Model. Eksmo, 2008, (in Russian).

[22] Repin V. V. Business Processes of the Company. Construction, Analysis, Regulation. Business Excellence, 2007, (in Russian).

[23] Repin V. V., Eliferov V. G. Process Approach to Management. Modeling of Business Processes. Mann, Ivanov and Ferber, 2013, (in Russian).

[24] Dzheston Nelis. Management of Business Processes. a Practical Guide to the Successful Implementation of Projects. Symbol, Plus, 2008, (in Russian).

[25] Zaloga V. O., Dyadyura K. O., Nagornyi V. V. The Hereditary Principles of Forming the Quality of Complex Machine-Building Products. SSU, 2012, Sumy, (in Ukrainian).

[26] Zaloga V. A., Dyadyura K. A., Prokopenko A. V. Methodology of increasing the effectiveness of interdependent processes of design, manufacture and operation in engineering projects. Cutting and the Tool in Technological Systems. Collection Kharkov: NTU "KhPI", Vol. 86, 2010, p. 34-42, (in Russian).

[27] Dmitrievsky B. S. Automated Information Systems for the Management of an Innovative Science-Intensive Enterprise. Mashinostroenie-1, Moscow, 2006, (in Russian).

[28] Lutsky S. V. Teoretiko-information approach to the development of technical systems. Herald of engine building. Motor Sich JSC, Vol. 2, 2007, p. 28-33, (in Russian).

[29] Buryak Yu I., Insarov V. V. Management in multi-site organizational systems. III: Parametric optimization in a system with several objective functions. System Analysis and Operations Research. Izvestiya RAN. Theory and Control Systems, Vol. 5, 2007, p. 89-99, (in Russian).

[30] Mykiychuk M. M. Prospects for the application of cluster analysis at the quality control of products. New Solutions in Modern Technologies, Herald of NTU KhPI, Vol. 23, 2011, p. 59-64. 


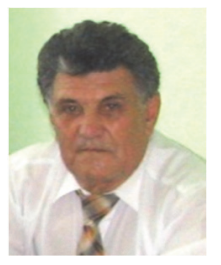

Viliyam Zaloga received Ph.D. degree in Information Science and Engineering Institute from University, City, Country, in 2014. Now he works at Company. His current research interests include control, dynamics and fault diagnosis. The scientific field is mechanical processing of materials, modeling of working processes of high technologies, standardization and certification of industrial winery

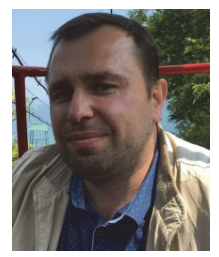

Kostiantyn Dyadyura is a Doctor of technical sciences, Professor, head of the Department of Applied Material Science of Sumy State University. The scientific field of machine elements includes both individual machine elements and machine elements in a product technology context. This relates specifically to machine elements with a product development and design application, focusing on condition monitoring. It is to unite theoretical and practical results of research, developmental and process work to form emergence properties of goods (of different industry branches) on the stages of their life circle taking into consideration self-consistent interaction of processes while designing, manufacturing and operating as an open system, self-organization of which influences the results of satisfaction of requirements.

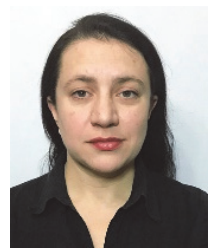

Iryna Rybalka is a postgraduate student of the Department of Manufacturing Engineering, Machines and Tools from Sumy State University, Sumy, Ukraine. She current research interests include integrated management systems, implementation of international standards. 


\title{
1.2. Current trends in mechanical engineering
}

\author{
Stepan D. Postil \\ University of the State Fiscal Service of Ukraine, Ukraine \\ E-mail: sdp_irp@ukr.net
}

\begin{abstract}
World science and industry face challenging problems that can be solved on the basis of the following: a) integration of specific scientific disciplines in inter-, multi- and transdisciplinary scientific areas; b) development of specific technologies into technological chains of new generation; c) integration of specific modules and components into hierarchical systems of higher level and development of megasystems. Innovative knowledge-based economy serves as critical characteristic of modern scientific and technical, industrial, socio-economic and all social processes. High-Tech Computer-Aided Engineering, "Simulation Based Design" (in development to "Digital Manufacturing"), integrated comprehensive systems and technologies to supporting life cycle of products act as the basis of engineering. The abstract presents science-based computer engineering of bimetallic casting which is based on the methods and algorithms of numerical problem solving with respect to heat exchange, nonlinear problem of thermoelasticity and inverse problems of heat conduction, that make it possible, based on the results of a natural experiment, to adapt a mathematical model to the conditions of the process under study. The experience of economically developed countries shows that one of the main conditions for economic growth and innovative development of the economy is development of the market engineering. The role of Ukraine in international engineering is insignificant, but the positive trend of our country is development of domestic engineering companies that have competitive advantages.
\end{abstract}

Keywords: engineering of products, innovation, computer-aided engineering, systems and technologies of computer design, integrated comprehensive systems and technologies, life cycle of products, bimetallic casting.

As of today the key point of engineering is its creative nature, presence of a common fund of technologies and inventions; the leading role of science (IT is in the first instance) in creation of new products and technics in general; system-based nature of activity. The abstract gives better outline of new integration trends that are related to deepening of design process understanding and change in technology of engineering [1].

The first key in this process is simulation of all aspects of human activity, i.e. social, organizational, technical, educational, recreational, etc. That is, the subjects of the activity move on to detailed forecasting of their future and to their most rapid implementation. In the process of such implementation, in materialization of ideas the role of engineering activity is significant, that organizes this process and implements the specific project based on innovative technologies. At the same time, the place and welfare of countries as well as the individuals depend ultimately on mastering and development of new.

Engineering, in the first instance, serves as the information process, the process of new information generation. This process in terms of quantity has an avalanche-type character since with transition to each new information level the number of possible combinations increases and, accordingly, the power of new sets of objects or their information substitutions. There is a need for proactive development of simulation and communication tools as compared to the means of material implementation of the project in the article. The main mean of systematic representation of new developments and forecasting of possible consequences is mathematical simulation.

Globalization of markets, competition, educational and industrial standards, financial capital and high-tech innovations requires faster growth rate, short cycles, low prices and high quality than ever before. Rapid, intense development of information and communication technologies and high-tech computer technologies has been observed.

Global science and industry face more complicated comprehensive challenges that cannot be 
solved using traditional ("highly targeted") approaches. This leads to integration of the separate scientific disciplines in inter-, multi- and transdisciplinary scientific areas; to development of separate technologies in technological chains of new generation; to integration of individual modules and components in hierarchical system of higher level, and to development of megasystems, that is, multiscale comprehensive scientific and technological systems that maintain the level of functionality that can be achieved through their individual components [2].

Intense blurring of sectoral boundaries, convergence of sectors and branches of economy, blurring of the boundaries between fundamental and applied science take place through the need to solve comprehensive scientific and technical problems, emergence of megaproblems and mega systems, diversification and intensification of activity. The most frequently this is done on the bases of outsourcing and outstaffing, as well as with effective cooperation of companies and institutions, both within the sector of economy and and from different sectors. For example, generation of high-tech clusters from scientific and educational organizations and industrial companies, from large state-owned companies to small innovative companies. A distinctive feature of the current stage of development is producing through modern nanotechnology of new functional materials and smart-materials, the materials with given physical and mechanical and controlled properties, alloys, polymers, ceramics, composites and composite structures, that on the one hand, are "materials-structures" and on the other hand, they are the integral part or the component of a macrostructure (a car, an aircraft, etc.).

Innovation is a critical characteristic of modern scientific, technical, industrial, socio-economic and social processes. The consistent pattern of transition from predominantly reproductive to inoovative type of development has been observed in the world. The future of Ukraine to the certain extent depends on mastering of innovative mechanisms of development: whether it moves towards accession to the number of developed countries or it will remain a stagnating country on the fringes of scientific-and-technological and social progress [3].

The basic principles of establishment of modern organizations, companies and institutions with innovative knowledge-based economy should be pointed out: 1) optimization of interactions between different areas of innovation process (education, science and industry); 2) priority ranking of long-term objectives; 3) a model of continuous improvement of processes after Shewhart-Deming; 4) kaizen principles; 5) "war for talents" from McKinsey international consulting company; 6) the knowledge creating company); 7) learning organization; 8) "firing rate" from Toyota; 9) learning through problem solving; 10) "education throughout the life"; 11) inter-, multi-, transdiscipline; 12) capitalization of "know-how" and key competencies; 13) "invariance" of multidisciplinary suprasectoral computer technologies [4, 5].

The principle of optimization of interactions between different actors of innovation process (education, science and industry) can be implemented under the corresponding state policy as an intermediary. As the analysis shows, in Ukraine innovative transfer of technology has always been a weak point in the National innovation system, which insufficiently ensures commercialization of scientific achievements and technological developments. The reason for this is administrativecommand tradition of managing Ukrainian educational and scientific areas, as well as maintaining of managerial organizational forms that serve the "linear" model of innovation cycle [6, 7].

Determination of the goals is a rather labor-intensive and responsible process, which consists of the following main stages: identification and analysis of trends within the environment; selection of the goals of the organization in general; building up of the hierarchy of the goals; identification of the individual goals. The basic rule when defining the goals, especially general ones, is involvement of the employees of the organization in their development.

Continuous Process Improvement Model or the Shewhart-Deming cycle PDCA (Deming Cycle is often used - Plan, Do, Check, Act) allows to manage effectively the activities in various areas on a system-wide basis. This is achieved through the following principles: a) sustainability of the goal through allocation of resources in such a manner as to maintain the long-term goals and high competitiveness; b) strict improvement of all processes; c) practice of leadership; 
d) promotion of effective bilateral ties within the organization and elimination of the barriers between units, services and divisions; e) practice of personnel training and retraining; e) stalwart devotion of senior management to continuous improvement of quality and effectiveness; e) implementation of educational programs and support of self-improvement of the employees under the "knowledge is a source of successful advancement in achievement of competitiveness" slogan [3].

Kaizen principles of Continuous Process Improvement of the Company Activity constitute central concept of Japanese management. The main components of Kaizen technologies are as follows: a) total quality control (TQC); b) process-oriented management; c) "standardized work" concept as the optimal combination of the employees and the resources; d) "just-in-time" concept; e) PDCA-cycle; $\quad$ f) 5-W / 1-H (Who-What-Where-When-Why / How) and 4-M (Man-Machine-Material-Method) concepts. It is critically important that everybody should be involved in Kaizen, from top management to ordinary employees, that is, "Kaizen is the cause of each and everyone" [8].

Thanks to implementation of the principles of McKinsey in the modern world, those organizations have revenues that are the most attractive in the labor market and do everything to attract, to assist development and to retain the most talented employees. The basis of success is appointment of the competent employees to the key positions within the organization. The shortage of the staff will force the companies to start creating their own sources of engagement and education of talents: partnership with the universities, the internship programs, career enhancement system and continuous education.

In the management model based on the "the company is the creator of knowledge" principle the main role is the "knowledge" The main provisions of this approach as follows: a) knowledge is the main competitive resource; b) organizational training; c) the theory of the knowledge creation by the organization is based on the methods of interaction and transformation of formalized and unformalized knowledge; d) the spiral of creation of knowledge that extends "up and in breadth" e) the knowledge creation team as a rule is composed of "knowledge officers", "knowledge engineers" and "knowledge practitioners" [9].

In the present-day conditions "stiff competition" of the organization becomes an obstacle for rapid respond to external changes and effective use of limited internal resources, therefore the organization must have such internal structure to allow it to adopt continuously to constant changes of the external environment. The main components of the learning organization are as follows: general vision, system-based thinking, skills of personal development, intellectual models, group learning based on regular dialogues and discussions.

Universal principles of Toyota can be used in any industry to form a perfect organizational structure which is capable of doing everything necessary to shorten the time period from the moment of the Customer's request and up to payment for the work performed. The following practical ideas are relevant for every business, which is focused on strict improvement and enhancement: a) making management decisions based on a long-term concept; b) standardized tasks are the basis for continuous improvement and empowerment of the employees; c) education of the leaders who have profound knowledge of their business, live with the company's philosophy and can share the knowledge about it to the others; d) it is mandatory to respect all partners and suppliers, to set them difficult tasks and help improve; e) making decisions slowly, jointly and carefully considering all options, but implement them quickly and immediately [10].

The principle of "learning through problem solving" provides for development of the system of regular participation of students and employees in joint implementation of real projects customized of the companies of domestic and world industry. This is achieved within the framework of the activities of virtual project-oriented teams based on proactive acquisition and application of modern key competencies, in the first instance, technologies of computer engineering [11].

The principle of "lifelong learning" facilitates development of comprehensive and 
interdisciplinary training or professional retraining of skilled and competent world-class experts in the area of computer engineering based on the corresponding advanced technologies.

The principle of inter-, multi-, transdiscipline means transition from field-specific sectoral qualifications to a set of key competencies, that is, capabilities and preparedness to conduct the certain activity (scientific, engineering, design, accounting, technological, etc.) that meets high requirements of the world market.

Implementation of the principle of "know-how" and the key competencies within the conditions of globalization and hypercompetition will allow confirming constantly high level of performed research work, to create new scientific and technological results through systematic capitalization and multiple replication in practice of both sectoral and inter-, multi-, transdisciplinary "know-how". This approach is fundamental for creation and dissemination of key competencies and advanced technology within the organization.

The principle of "invariance" of multidisciplinary suprasectoral computer technologies allows to create significant and unique scientific and educational projects by systematic capitalization and multiple application in practice of numerous inter-, multi, transdisciplinary "know-how", to establish rational effective schemes and algorithms of engineering (polytechnic) system of transfer, which is critically important for setting up of innovative infrastructure of the future.

It should be noted that at the present stage of civilization development any available resources, including information, cannot provide long-lasting competitive advantages. Today, success in global competition between the separate companies, regions and countries depends primarily on ability to create new knowledge and, on the basis of them, to develop new technologies, new products, identify continuously new problems and invent innovative ways for their solving. The leading role in creation of a "knowledge triangle" (education-research-innovation) belongs to the universities, that must act as the catalysts of innovation processes and, ultimately, the generators of welfare and well-being. The University as an organization is intended not just to transfer knowledge to students, but also to produce new knowledge and through involvement of students to permanent scientific research it must teach them to analyze critically, to think and act independently, freely, creatively, to formulate and solve new problems on their own, to develop and study throughout the life $[4,6]$.

The cornerstones of modern engineering are, first of all, high-tech computer engineering technologies: MultiDisciplinary and MultiScale and MultiStage Research and Engineering multidisciplinary, multi-scale (multi-level) and multistage research based on inter-, multi-, transdisciplinary, sometimes so-called "Multiphysical" ("MultiPhysics"), computer technologies [1]:

1. MultiDisciplinary Concept means transition from the separate disciplines, for example, thermal conductivity and mechanics, based on thermomechanics, electromagnetism and computational mathematics to multidisciplinary computational thermo-, electro-, magnetomechanics.

2. MultiScale Concept means transition from single-scale models to multi-scale hierarchical nano-, micro-, meso-, macromodels.

3. MultiStage Concept means application of MultiDisciplinary and MultiScale concepts when creating new materials with special properties, development of competitive systems, structures and products of new generation at all technological stages of engineering (for example, casting stamping / forging / ... / deformation - welding, etc.).

The second base of modern engineering is "Simulation Based Design" which means computer design of competitive products (products), based on effective and comprehensive application of the methods of numerical simulation and advanced computer technologies with the use of modern visualization tools [12]:

1. CAD, Computer-Aided Design. Currently three main CAD subgroups are distinguished:

- Mechanical CAD (MCAD - Mechanical CAD), being the most advanced technologies with the corresponding segment of the market; 


\begin{abstract}
- Printed circuit boards CAD (ECAD - Electronic CAD/EDA - Electronic Design Automation);

- Architectural and construction CAD (CAD/AEC - Architectural, Engineering and Construction).

2. FEA, Finite Element Analysis - numerical analysis, in the first instance, of the problems of mechanics of deformable solids, statics, oscillations, stability of dynamics and strength of machines, structures, devices, equipment, installations and constructions, that is, the entire spectrum of products and products of various sectors of industry. With the aid of various methods of numerical simulation, the problems of heat exchange, electromagnetism and acoustics, construction mechanics, technological problems (in the first instance, the problems of plastic processing of metals), the problems of fracture mechanics, mechanics of composites and composite structures are solved effectively.
\end{abstract}

3. CAE, Computer-Aided Engineering means a high-tech computer-aided engineering based on effective use of multidisciplinary suprasectoral CAE systems based on modern computing methods. When implementing CAE-systems, rational mathematical models are developed and applied that have high level of adequacy to real objects and real physical and mechanical processes, effective solving of multidimensional research and industrial problems are solved that are described by non-stationary nonlinear differential equations in private derivatives.

In XXI century, the main concept of computer-aided design of competitive products ("Simulation Based Design") is developed intensively by forces of the leading vendor companies of CAE-systems and industrial companies [5].

Evolution of the main approaches, trends, concepts and paradigms from "Simulation Based Design" to "Digital Manufacturing" can be represented as follows:

1. Simulation Based Design / Engineering (design and technical advisory services on development and preparation of production process, ensuring the normal course of the process and sales of products).

2. Multi-Disciplinary Simulation Based Design/Engineering (multidisciplinarity, the tasks become complex, they require knowledge of related disciplines to solve them).

3. Super Computer Simulation Based Design (widespread use of HPC technologies (High Performance Computing), supercomputers, high-performance computer systems and clusters within hierarchical cyber infrastructures for solving complex multidisciplinary tasks, multimodal and multiple-option calculations);

4. Super Computer (Multi Scale/Multi Stage and Multi-Disciplinary and Multi Technology) Simulation Based Design/Engineering (application of concepts: multi-scale/multi-stage and multidisciplinary and multi-technology).

5. Super Computer (Material Science and Mechanics) (Multi ** 3) Simulation Based Design/Engineering (simultaneous computer-aided design and engineering of materials and structural elements, among them: harmonious combination of mechanics of materials and structures);

6. Super Computer (Smart Mat and Mech) and (Multi ** 3) Simulation and Optimization Based Design/Engineering (application of Smart-materials/“intelligent" materials, application of various types of optimization (parametric, multidimensional, structural, topological, multicriterion, etc.), rational optimization of technological processes, etc.)

7. SuperComputer (SmartMat and Mech) and (Multi**3) Simulation and Optimization Based Product Development (design, engineering and optimization extends to manufacture of products and transition to Virtual Products/Products Development.

8. Digital Mock-Up/Digital Manufacturing ("digital prototype" means a virtual, digital 3-D model of the product and all its components, which allows to exclude from the product development process creation of expensive prototype models, it allows to "measure" and simulate any characteristics of the object in any conditions of operation/"digital production" as the main components of "smart" plants and factories). 
Integrated comprehensive systems and technologies to support the life cycle of products are also being developed, the evolution of which can be represented by the following stages [12]:

1. CAD/CAM-technologies (Computer-Aided Design/Manufacturing) that integrate CAD and CAM-systems and provide integrated solution of the tasks of computer aided design and technological design, including the means of 3-D parametric modeling, release of drawings, as well as technological production preparation aids, in the instance, with the aid of computer numerical control (CNC) or, recently, with Rapid Prototyping, RP or Additive Technologies (AD).

2. Concurrent Engineering (CE) means "competitive" design/parallel design/joint design) which is joint work of experts from different functional units of the company at the earliest stage of the product development to achieve high quality, functionality and processability in the shortest possible time with minimum costs. $\mathrm{CE}$ is basically expression of the desire to increase competitiveness of products through reduction of the life cycle of the article, as well as improvement of quality and price lowering.

3. Product Data Management Systems (PDM) are sometimes referred to as systems for collaborative work with engineering data.

4. In addition to application of CAD/CAM/CAE/PDM systems, starting from 1990s ERP (Enterprise Resources Planning) systems are used in the industry, they are enterprise resource planning and management systems. At the beginning of the century, the most serious attention was paid to Manufacturing Enterprise Solutions (MES) system, they are enterprise production planning and management systems at the level of production unit, SCM systems (Supply Chain Management, SCM), CRM systems (Customer Relationship Management, CRM).

5. The problems as to organization of teamwork on the projects and effective product information management throughout its life cycle attracted attention from the 1980s. Different approaches were proposed to address those issues, for example, the Unites States Department of Defense proposed the methodology of computer-aided acquisition and logistics support (CALS) and IBM Corporation introduced the concept of Computer Integrated Manufacturing (CIM). Perhaps those initiatives were ahead of time, but due to a number of reasons, they did not become widespread and did not cause stimulate enthusiasm among users.

6. At the end of the last millennium, IBM developed the new concept - Product Lifecycle Management (PLM). The demand for PLM products began to grow, despite the recession and global economic crisis. The main purpose of PLM-technologies is unification and effective interaction of isolated automation areas that were formed as a result of implementation of various systems (CAD, CAM, CAE, PDM, ERP, MES, SCM and CRM) within the single information space, as well as for implementation of end-to-end engineering, technological and commercial production cycles, from inception of an idea, creation of product, its operational use, and, finally, its disposal".

It is critically important that the basis of PLM-technologies form CAD-, CAM-, CAE- and PDM-technologies, due to the joint use of which traditional and consistent approach to development of new products is replaced by a modern integrated approach. This approach ensures simultaneous computer-aided design of the product with the aid of CAD system, execution of various engineering CAE calculations (computer engineering) and technological preparation of production with the help of a CAM system based on joint use of the project data from the earliest stages of design and engineering analysis, simultaneously by different groups of experts using the PDM-system.

The following presents is a high-tech computer-aided engineering of bimetallic casting (SAE system) that is based on reliable methods and algorithms of numerical solution of heat transfer problems, nonlinear thermoelasticity problem and inverse heat conduction problems, which make it possible, based on the results of a natural experiment, to adapt a mathematical model to the conditions of the process under study [13].

Using these algorithms, a multipart analysis of casting solidification process was performed, in the course of which thermal and geometric parameters of the system varied and the basic 
parameters of optimal casting regime were determined. Developed software tools release the researcher from conducting expensive and labor-intensive experiments on liquid metal and allow analyzing new technologies and new processes of casting.

The significant reserve for increase of metal use efficiency in machine structures is application of cast products and multilayered products within the structures of castings, including those obtained on the basis of worn parts.

Reconditioning of parts by filling of liquid metal into a solid base in diecasting die is carried out if the following processes related to heat exchange are in place: preliminary heating of the worn part (metal-base) using an induction heater; solidification of filled liquid metal; cooling of obtained bimetallic casting and diecasting die.

An important condition to form a high-quality casting is obtaining of metallic linkage between connecting parts. In the course of interaction of filled metal with the metal base of diffusion melting process the process of diffusion saturation of the surface layer is always preceded. After the certain concentration the alloying element is reached, the surface layer of the metal base is melting. The depth of diffusion layer for this temperature can be determined approximately by saturation time and diffusion coefficient of alloying element.

In the course of worn parts in diecasting die reconditioning in castings there is significant nonuniformity of temperature fields. The degree of nonuniformity of the temperature is determined by thermal interaction of build-up layer with metalize, conditions of heat transfer of casting with the environment, initial thermal state of the system, etc. The thermal stresses resulting from this nonuniformity can lead to the formation of various types of cracks.

An additional point is that insufficient metal junction may appear between built-up layer and metal base. Thus, the reconditioning of parts should be carried out under such values of technological parameters that ensure welding of bimetallic layer (built-up layer-metal base) and permissible level of emerging thermal stresses in the casting.

In connection with this, there was a need for mathematical simulation of heat transfer processes within the system of interacting bodies in the course of bimetallic casting formation.

The process of worn parts reconditioning is accompanied by the displacement of boundary of transformation phase as a result of metal crystallization and during mathematical description, it belongs to the class of Stefan's task.

The mathematical model of the task of casting hardening includes the parabolic equation of heat conductivity:

$c \rho \frac{\partial t}{\partial \tau}=\operatorname{div}(\lambda \operatorname{grad} t)+\Pi, \quad P=\left\{G \times\left[0 \times \tau_{\mathrm{k}}\right]\right\}$,

initial conditions:

$t_{\mid G}=\varphi$,

conditions of heat exchange on full external border $G$ :

$L\left(t_{r}\right)=\psi$,

on the $S$ surfaces of field links objects $G$ :

$\lambda \vec{\Delta} t_{\mid S^{-}}=\frac{\lambda_{S}}{\delta_{S}} \Delta t_{\mid S}=\lambda \vec{\Delta} t_{\mid S^{+}}$

and on the interphase border Stefan's approach is used in classical formulation: 
$\lambda \vec{\Delta} t_{\mid z^{-}}-\alpha \Delta t_{\mid z^{+}}=\rho \chi \frac{d z}{d \tau^{\prime}}$

where $G$ is the area in some spatial coordinates; $\tau$ is the time; $\lambda_{S}$ - effective heat transfer coefficient through gas gap with $\delta_{S}$ value; $L$ operator can correspond to the border-line conditions of type I, II or III. In this case, the inputs in the system of Eqs. (1)-(5) functions:

$\Phi=\left\{c, \rho, \lambda, \Pi, \chi, \varphi, \psi, \delta_{S}, \lambda_{S}\right\}$,

are considered as set for entire field $P$, in which the temperature and the problem (1)-(5) are determined which is considered to be a direct heat conduction problem if phase transformations are in place.

Given the known difficulties (nontranparancy of molten metal, its high temperature, aggressiveness, etc.) it is not always possible to accurately determine physical or geometric characteristics of the system. The situation might arise when at least one of the functions (6) is not determined or it is determined not in all field of the temperature definition and it is necessary to find this function on the basis of other known functions and some additional information about the temperature or heat flow in the cylinder $P$ :

$L_{\partial}(\mathrm{P})=g$.

Such a heat conduction task (1)-(7) is considered to be inverse. Since the additional information is taken from the experiment, the methods of solving the inverse problem of heat conduction allow us to adapt the mathematical model to the conditions of the process.

On the basis of the mathematical model of the heat conduction problem in the system of linked objects ("Surfacing", "Thermal insulation", "Diecasting die", "Environment") an algorithm and a software complex with the use of the difference method [14] have been developed.

Simulation experiments were carried out on the research of heat transfer processes on reconditioning of parts by filling of liquid metal on the worn surface of the part (metalbase).

In implemented technological line on reconditioning of parts (running roller) of a track type tractor, heat transfer processes run under the following conditions: 1) inductive heating of the metal base is carried out outside the diecasting die; 2) solidification of molten mass takes place without shielding; 3 ) cooling of the die-casting die and casting is carried out by air.

It is assumed that with a view to obtain high quality casting, the process of reconditioning of the running roller in the diecasting die, the inner surface of which is covered with thermal insulation layer, is carried out by liquid metal with overheating temperature of about 100 to $200 \mathrm{~K}$. At the same time, it is accepted that liquid metal at the time of metal base preheating completion is filled quickly enough and it hardens at the constant temperature $T_{t}$.

The defining parameters of the technological process can include the temperature of preheated surface of metal base $T_{m}$, the temperature of liquid metal $T_{j}$ and the amount offilled metal $D$, which is spent on reconditioning the unit surface of the worn part. In order to develop optimal modes of reconditioning of worn surface of the running roller of the track type tractor, computational study of the process was performed, depending on its determining parameters.

The following was determined in the course of computational study of reconditioning process of the running roller of the track type tractor: temperature distribution within the system of objects and the coordinates of phase transformation borders, the gap and the heat transfer coefficient between the casting and diecasting die as well as the temperature of the free surface of the thermal insulation.

A good match of design and of the calculated and experimental temperatures on external surface of metal base and inside building-up where the temperature is maximum shows sufficient accuracy of the mathematical model which is used.

For quality eating of metallic compounds of bimetallic pair, kinetics of solidification of liquid 
metal on the metal base was studied, depending on the key parameters of the technological process.

The results of calculating examination of the parts reconditioning process showed that molten metal was located near external surface of the metal base for some time in a liquid state. This time will be called as welding time of bimetallic pair. In the course of this time diffusion meltdown of metal base is carried out.

Based of the finding of experimental research the depth of qualitative diffused layer makes the order of $b=0.2-0.4 \mathrm{~mm}$.

Apparently, the optimal level of diffusion meltdown of metalbase corresponds to the depth of the surface irregularities and microcracks. Having in our dispodal $b$ value, we can determine the minimum time (saturation time) required to create the required connection.

As the optimal criteria of the parts reconditioning, stress intensity is used which determines permissible level of the emerging thermal stresses and $S$ ratio, which characterizes welding of bimetallic pair (meltdown - metalbase).

Correlation $S$ is obtained on the basis of comparison of calculated and experimental data:

$S=\frac{N * T * M}{1-M}$,

where dimensionless quantities are $N=\frac{T_{j}-T_{t}}{T_{t}}, T=\frac{T_{m}}{T_{t}}$ and $M=\frac{D}{D_{j}} ; D_{j}-$ input of liquid metal at full wear of a rim part.

$S$ value, obtained on the basis of relation (8) was used for the a-priori estimation of the parameters of the process of obtaining bimetallic casting by filling with cast iron under composition (on the mass): $2.8-3.2{ }^{\circ} \mathrm{C}$; $1.6-2.2 \mathrm{Si}$; 0.7-0.9 Mn; 0.2-0.4 Cg; 0.2-0.4 Cu; less than $0.05 \mathrm{~S}$; less than $0.1 \mathrm{R}$ on a solid metal base, made of steel grade 45 .

When input of liquid metal equals to $M=0.22-0.57$, its overheating equals to $N=0.1-0.25$ and preliminary heating of external surface of metal base reaches the value of $T=0.74-0.97$, then the qualitative weldability of bimetallic pair will be achieved if the following condition is fulfilled: $S \geq 0.04$.

Stressed state of bimetallic casting was determined by the solution of unlinked nonlinear thermoelasticity problem. The solution of the problem was carried out in movements by the method of installation [14]. In the course of numerous calculations, distribution of strain, stress and intensity of stresses were calculated. The value of permissible stress has been determined under the literature sources at the maximum temperature in each of the layers of the bimetallic casting, in which it takes minimum value and determines critical state of metal.

Calculations have shown that under the influence of substantially irregular temperature field in bimetallic casting the significant temperature stresses arise that depend on the temperature value difference along interconnection of the article. Based on the results of numerous experiments it can be concluded that in order to reduce the level of emerging thermal stresses in bimetallic casting, it is necessary to select metals as close to mechanical characteristics as possible and avoid high temperature differentials through the thickness of the article.

Studied problem with respect to formation of qualitative diffusion layer in bimetallic castings is also relevant in the processes of obtaining multilayer castings by filling of the next portion of liquid metal onto solidified metal after pre-filling.

Developed methodology allowed to carry out computational studies of thermal state of multilayer casting, obtained by layered filling of liquid metal on a pre-filled and solidified basis, and to obtain practical recommendations related to optimal modes of their formation.

The most active participants of the engineering projects are the countries of Europe and North America, but all these countries give ground to the United States. The trends in development of international engineering can be include the fact that among the exporters of engineering services the small companies dominate and the services themselves have national or regional nature [2].

Europe possesses a well-developed engineering consultancy service. There are all categories 
of companies that provide engineering services of all kinds in the region. The main lines of engineering services export from the EU are the EU countries themselves, the USA, Switzerland, Japan, that is, the most highly-developed countries. A small share of export falls to Russia, China, India, Turkey, Africa. Import of the EU is almost the same as export. For developed countries there is a widespread implementation of works and provision of services in the form of comprehensive engineering, that is, full range of services and supplies required for new production.

The market of engineering in Ukraine is in fact only at an early stage. More than $80 \%$ of domestic engineering companies have a market share of less than $0.3 \%$. This low indicator can be explained. First, in Ukraine, the demand for comprehensive engineering services is still insufficient. Second, lack of experience causes rise to a large number of errors in implementation of projects. The current problems of Ukraine also include shortage of skilled personnel, poor work performance, obsolete legislative framework, imperfect system for determination of the costs of the project design work, corruption phenomena at the stage of approval and examination of documents, low level of project design works automation and many others [1].

To ensure further development of engineering and advisory activities in Ukraine, the following main organizational and economic measures should be implemented: 1) establishment of worldclass competence engineering centers to permit Ukraine to reduce its dependence on foreign experts; 2) holding of professional international level forums; 3 ) adaptation of know-how to the specific conditions of Ukraine by local engineering companies; 4) setting up of the system of professional education and training of specialists in the area of engineering and ensuring of free access to professional literature; 5) regulation of legislative framework; 6) improvement of quality of engineering services by attracting the specialists from the certain sectors of science and industry.

The priority components should be as follows: a) building up of the National system of "digital" and STEM-education (focused on the scientific component of education and innovative technologies, and it actively develops creative component of personality and critical thinking); b) introduction of special training for cyberphysical productions and their continuous professional development; c) development of new approaches to acquisition of knowledge and skills at workplaces, including use of digital education methods.

\section{Conclusions}

In the context of international development, the engineering services become more widespread since they increase significantly the efficiency of invested capital. The experience of economically developed countries shows that one of the main prerequisites for economic growth and innovative development of the economy is precisely development of engineering market.

The role of Ukraine in international engineering at this stage is insignificant, but the positive trend is development of domestic engineering companies having competitive advantages in our country.

\section{References}

[1] Yaroschuk A. O. The World Market Environment of Engineering and Technical Services and Prospects for Development of Engineering Centers in Ukraine. Management of Economic Processes in World and National Economy: Collection of Abstracts of Scientific Papers. "New Economy" Analytical Center, 2015, p. 144.

[2] Kondratiuk A. A., Manaienko I. M. Development of International Engineering: World Trends and Domestic Realities. Current Problems of Economics and Management: Collection of Scientific Papers of Young Scientists. National Technical University of Ukraine "Igor Sikorsky Kyiv Polytechnic Institute", 2017, p. 7.

[3] Geyets V. M., et al. Innovative Ukraine 2020: National Report. National Academy of Sciences of Ukraine, 2015, p. 336, http://ief.org.ua/wp-content/uploads/2015/07/Innovation-Ukraine 2020++.pdf. 
[4] Pavlenko A. F., Antoniuk L. L., Vasilkova N. V., Ilnitsky D. O., et al. Research Universities: World Experience and Development Prospects in Ukraine: Monograph. Kyiv National Economic University, 2014, p. 350.

[5] Vyshnevsky V. P., Viyetska O. V., Garkushenko O. M., et al. Smart Industry in the Age of Digital Economy: Perspectives, Directions and Development Mechanisms. Institute of Industrial Economic of the National Academy of Sciences of Ukraine, 2018, p. 192.

[6] Bazhal Y. M. Development of innovation activity in the knowledge triangle "state-universities-industry". Economy and forecasting, No. 1, 2015, p. 76-88.

[7] Postil S. D., Kozak N. S. Integrity of system-based approach in the educational process. Physico Mathematical Education: Scientific Journal, Vol. 1, Issue 11, 2017, p. 84-88.

[8] Levytsky Y. K., Tsurkan B. Y. Modern Quality Management. Chernivtsi Institute of Trade and Economics of Kyiv National University of Trade and Economics, 2012, p. 4.

[9] Nonaka I., Takeuchi H. The Company is the Creator of Knowledge. Origin and Development of Innovations in Japanese Companies. "Olimp-Business" Closed Joint Stock Company, 2011, p. 384.

[10] Liker Jeffrey K. The Toyota Way. 14 Principles of Work by Coordinated Team. 2017, p. 424, https://biz.censor.net.ua/m3030359

[11] Postil S. D. CASE-technologies. Interdisciplinary Information Modeling: Teaching. UDFSU, 2018, p. 305.

[12] Ramazanov S. K., Veligura A. V., Ivanovska M. V. Information support of the lifecycle of technical objects of railway transport. Bulletin of Volodymyr Dahl East Ukrainian National University, Vol. 21, Issue 218, 2015, p. 194-199.

[13] Postil S. D. Heat Exchange during Solidification of Ingots and Bimetallic Castings. Extended Abstract of Ph.D. in Technical Sciences. Institute of Technical Thermophysics, Academy of Sciences of the Ukrainian SSR. 1989, p. 20.

[14] Nikitenko N. I. Conjugate and Inverse Problems of Heat and Mass Transfer. Scientific Thought (Naukova Dumka - in Ukrainian), 1988, p. 236.

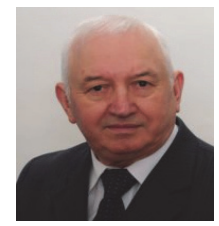

Stepan Postil obtained Ph.D. in technical sciences degree at the Institute of Technical Thermophysics of the National Academy of Sciences of Ukraine in 1989. At present he works at the University of the State Fiscal Service of Ukraine, he is the Associate Professor of the Department of Intellectual Management and Computer Systems. He is the author of more than 90 scientific and scientific-pedagogical papers. Current scientific interests include: numerical solution of tasks of mathematical physics, databases, interdisciplinary information modeling using CASE-technologies. 
Chapter 2

Dynamics of rotor systems and vibration processes 


\title{
2.1. System analysis of automatic balancing (self-balancing) machine rotors with liquid working bodies on the example of drum type washing machines
}

\author{
Ilona Drach ${ }^{1}$, Vilen Royzman², Vitalii Tkachuk ${ }^{3}$, Andrii Goroshko ${ }^{4}$ \\ Khmelnitsky National University, Institutska 11, Khmelnytskyi, 280016, Ukraine \\ E-mail: ${ }^{1}$ cogitare410@gmail.com, ${ }^{2}$ tkachukv.p@gmail.com, ${ }^{3}$ royzman@ukr.net, ${ }^{4}$ iftomm@ukr.net
}

\begin{abstract}
The main objective of the research is a solution of problems, connected with increasing reliability, efficiency and safety of modern rotor machines.

The class of rotary machines with varying imbalance while operating is rather diverse. These are separators, centrifuges used in chemical, food manufacturing, sugar, mining, medical industries, as well as sandblasters, washing and wringing machines and many others.

On the example of washing machines within the framework of the linear theory of oscillations, the dynamics of rotor machines is investigated, and the main causes of their vibrations and noise are revealed by experimental methods.

The basic problem of these machines is decreasing of their vibroactivity. We will ground the effective method of balancing rotor machines without stop during exploitation conditions passive automatic balancing with free movement of fluid.

The current work is dedicated to system research of the mentioned method, particularly for studies of fundamental natural phenomenon - self-balancing rotor with the help of passive auto-balancing units, which look like cavity chambers, partly filled with working media (fluid) being passive regulators of direct action that don't need energy supply and control system for movement of correction counterweights.
\end{abstract}

All theoretical materials are corroborated by results of experimental researches.

Keywords: rotor, vibration, automatic balancing (self-balancing), auto-balancing units (ABU), balancing effectiveness, vibroactivity, causes of vibration, washing-machine.

\section{Introduction}

Rotor mechanisms are used in a lot of areas of modern production, from mechanical engineering to computer and household appliances. Since these mechanisms have to operate under conditions of high velocities, strong vibrations, caused by shifting of rotor's center of gravity can become a serious problem and even lead to breakage of mechanism.

Problems of vibrations decreasing, stimulated by rotating rotors and having frequency of first rotor harmonic (also known as rotor vibration) are one of the most significant problems of construction, production and operation of almost all types of modern rotor machines [1]. Rotor vibration substantially defines reliability of these machines, for which high rotation frequencies, relatively small rigidity of construction are inherent, and critical operation modes are located within the limits of operation ranges of angular velocities [2].

Solution of tasks, concerning problems of decreasing rotor vibration is founded on the basis of linear theory of mechanical oscillations. The theory also indicates main directions of vibration control: vibroinsulation [3]; damping [4]; dynamic oscillation suppression [5]; perfecting methods of rotor balancing $[6,7]$, including those that take into consideration their flexibility [8].

Researches of rotor dynamics count more than 140 years, and the article of famous Scottish scientist W. Rankin [9] about rotational movement of rotor, written in 1869 is an evidence of this fact.

Practical value of this article is in the fact, that it for the first time adduced description of the effect of elastic and centrifugal forces on the rotation of flexible shaft. Other than this article showed how Poisson's theory about transverse vibrations of rods can be applied to dynamics of rotors. 
Discoveries made in the research of rotors dynamics at the beginning of 20th century are described in details in A. Stodol's monograph [10]. Expect the rest it describes the dynamics of flexible shafts with disk, the dynamics of distributed rotors without reference regard gyroscopic moment, static balancing of critical velocities.

With increasing rating and rapidity of machines increases the range of reverse effect of working environment on machine's rotor. It leads to the fact that, on one hand, rotor experiences significant load-bearing disturbance and under these conditions performs forced oscillations, and on the other hand - becomes less resistant and, under certain circumstances, enters the mode, in which besides forced oscillations there are also self-oscillating components [11]. For the first time the problem about self-oscillations of rotors were brought up in M. Ya Kushul's research [12], where stationary almost periodical oscillations of imbalanced rotor were examined, in which the reason of the origin of self-oscillating component is internal friction in the material of rotor. Further this subject was developed by G. I. Anikeev in his work [13], where lubricating film in supports and existence of dry friction were considered as reasons of the origin of self-oscillations. In the research of K. M. Ragulskis [14] except theoretical and experimental research of rotors' vibrations, the objectives of evaluation of out-of-balance rotor systems by vibration parameters are examined, and also the use of static approach to research of vibrations is described. Questions of the steadiness of rotor frequencies research are described by V. I. Siminivskiy in work [15]. The influence on constructive and mode parameters is analyzed, methods of calculation of multimass rotors steadiness are proposed in this work.

Majority of authors, researching dynamics of rotor systems, posed a problem of selection optimal parameters of rotor in respect to decrease vibrations. Among works, in which these type of problems are solved, one can mention work of B. Ya Kalmens [16], in which providing vibration reliability of machines by treating constructions of elements on the design stage is described, and also creating criteria of vibration reliability that corresponds to safe exploitation conditions. Similar approach to decreasing amplitudes of vibration movement of rotor by selecting characteristics of supports or form of the rotor is described in work of A. S. Kelzone and $\mathrm{Yu} \mathrm{M}$. Malinin [17]. This work adduces solutions of optimization tasks both for transitional and stationary operating modes.

Drum washing machines have become more widespread due to the simplicity of automation, more gentle washing, saving of water and detergent compared to the activator ones; but they are more complex and less reliable. Automatic washing machines are mainly drum type.

A washing machine as a object of research of the dynamics and vibration and noise decreasing is a particular interest. The reason is permanent presence of accidental imbalance because of bedclothes and small parts of machines and equipment in the drum and also as a result of low requirements for manufacturing and assembly accuracy of its parts and units in order to steer clear of possible increasing in value of manufacture.

Let's consider a rotary system using the example of a washing machine with a horizontal axis of rotation and implement theoretical studies using the theory of linear oscillations.

We will analyze the results and consider passive liquid auto-balancing as a means of reducing the vibration of rotary machines with variable imbalance.

The class of rotary machines with varying disbalance while operating is rather divers. These are separators, centrifuges used in chemical, food manufacturing, sugar, mining, medical industries, as well as sandblasters, washing and wringing machines and many others. For these machines traditional methods of reducing vibrations are of low efficiency, and the most effectual way is autobalancing by means of hollow chamber partly filled with working bodies.

To eliminate the imbalance of the rotating body, liquid auto-balancing devices (ABD) are used in the form of a hollow chamber with liquid working bodies. They are a standard feature in most household washing machines [18-21], but are also used in heavy industrial rotary machines. As an example, for washing machines, liquid stabilizers are used, which consist of a hollow ring similar to a rubber hoop but usually with a rectangular section containing a small amount of liquid. 
The ring, as a rule, attaches to the drum. The fluid moves freely and seeks to combine the main central axis of inertia of the rotor with its axis of rotation.

The main idea appeared already in 1912, and the US patent was granted in 1916 (Leblanc, 1916 [22]), where the working principle of the liquid balancer was outlined. Namely, when the machine rotates at a high angular velocity $\Omega$, the liquid forms a thin layer on the inner surface of the outer wall, depicted in Fig. 1.

A situation is considered when there is an unbalanced mass $\mathrm{m}$, for example, due to the uneven distribution of clothes in the washing machine. The rotor has a critical angular velocity $\Omega_{c r}$, where the centrifugal forces are balanced by the forces due to regenerative springs. Below this velocity $\left(\Omega<\Omega_{c r}\right)$, the center of mass of the fluid will be located "on the same side" as the unbalanced mass, as shown on the left side of Fig. 1 (here $M$ denotes the mass of the empty rotor, and $M$ is the mass of the fluid contained in camera) At a certain supercritical angular velocity $\Omega>\Omega_{c r}$ (say, during the drying process), the center of mass of the fluid moves to the "opposite side" relative to the unbalanced mass, as shown in the right side of Fig. 1, resulting in a "mass imbalance" and, thus, decreasing Thus, the centrifugal forces will decrease and the amplitude of the rotor fluctuations will decrease.

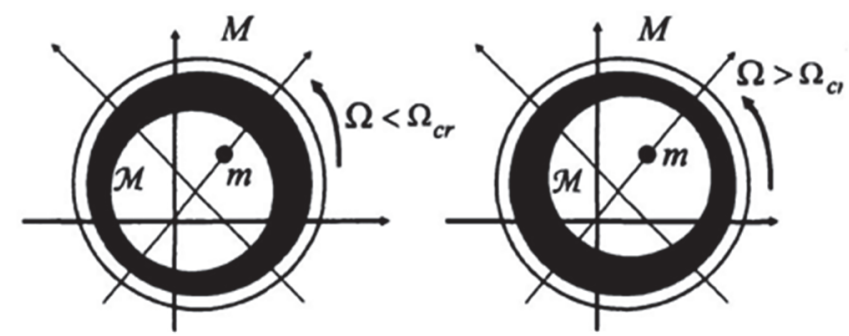

Fig. 1. Principle of operation of the liquid stabilizer for Leblanc

The original model consisted of one or more very narrow concentric channels (narrow in the radial direction, but wide in the axial direction, that is perpendicular to the plane in Fig. 1) partially filled with "liquid or very small steel balls or metal shreds" [22]. Thearle (1932) [23] considered and criticized the fluid balancer of Leblanc; and later Dan Hartog (1985) [24] supported Thearle's views. Here it is said that the Leblanc's balancer cannot work with the liquid, but only with the steel balls, and thus the invention was not recognized. This is probably due to very narrow channels, which mainly prevent the formation of surface waves.

Nevertheless, an automatic washing machine, equipped with a liquid balance, was introduced in 1940 and patented in 1945 (Dyer, 1945) [25]. The model of the liquid balancer was very similar to modern models, with a wide concentric camera, wide enough to allow the formation of a surface wave with large amplitudes.

The idea, therefore, is not new; but recently there has been a renewed interest in it both in industry and in scientific fields.

Experimental studies of liquid dampers were carried out by Kashara and others [26] and Nakamura [27]. As for mathematical models, simple models with lumped masses were considered by Bai in [28], Jun in [6], Maiewskyi in [29], Chen in [3], Urbiola-Soto and Lopez-Parra in [30]. The first and last two of these articles also include experimental studies. Jung's article [6] contains several numerical simulation results based on the computational fluid dynamics.

It should be emphasized that for the first time E. L. Thearle made an attempt to substantiate theoretically the principle of the action of Leblanc's ABD, ring, swing, and ball ABDs in the cycles of his articles [23]. He proposed a flat model of the rotor and the ABD. Within its framework, there is a single critical speed in the rotor, the excess of which the rotor begins to rotate light side outward and begins to manifest self-centered rotor phenomenon, which was laid by Thearle in the basis of the principle of the operation of all passive ABDs. In fact, here the phenomenon of self-centeredness appears as a phenomenon of self-balancing. The approaches and 
results of the work of $\mathrm{E}$. L. Thearle formed the basis of subsequent studies in the field of balancing the rotors of machines with passive ABDs. And the result obtained without taking into account the specific properties of the relatives, the forces of resistance, and thus the theoretically unreasonable and experimentally unconfirmed conclusion about the limitations of the range of performance of such auto-balancing devices by the supercritical (or retrograde) rotor rotation area is unexplored to this day. Therefore, a more detailed explanation of this phenomenon remains necessary.

Consequently, a more detailed explanation of the fluid balance dynamics remains necessary.

The approaches and the results of E. L. Thearle's work formed the basis of the following studies: J. Larry, O. Gusarov [31], V. P. Nesterenko [32], H. S. Hoon, L. J. Young, S. Suzuki, Ye M. Pashkov [33], Chung-Hyo Jung [6], M. A. Langthjem, T. Nakamura [34], Cunico M. W. M. [35] and other researchers in the field of balancing rotors of machines with passive liquid SBD. And the result obtained without taking into account the specific properties of correcting the masses, the forces of resistance, and thus, the theoretically unreasonable and experimentally unconfirmed conclusion about the limited range of performance of such automobile balancing devices with the supercritical (after resonance) rotor rotation zone is unexplored even nowadays.

According to the results of the review of Internet sources by the main manufacturers and industrial users of passive SBDs (in particular ball and liquid devices), there are such well-known companies as LG Electronics Inc. [18], Whirlpool Corporation [19], SKF AutoBalancer Systems [20], Samsung Electronics Co. Ltd [21]. However, information on the practical implementation of the above devices for automatic balancing of rotors of machines was not found. Such developments exist only as ideas in patents. Known companies use the idea of passive auto-balancing in advertising their products, which indicates the relevance of such SBDs, but their widespread use on serial machines is hampered by the unresolved many problems in the theory and practice of balancing rotors passive SBD, the presence of a number of phenomena and contradictions that have not yet found the proper theoretical justification.

Traditional methods are ineffective for machines with variable rotor imbalance and for balancing machine rotors without a halt in maintenance conditions. Automatic balancing by means of devices with free correcting masses movement is the most reliable, perspective and often the only possible method for reduction of vibration in such machines.

The current work is devoted to system research of the method mentioned, particularly to studying the fundamental natural phenomenon - rotor self-balancing with the help of passive auto-balancing units (ABD) which look like cavity chambers, partly filled with working media (liquid, granular materials) being passive regulators of direct action which don't need supply of energy and control system for correcting masses movement.

\section{Mathematical model of washing machines and the results of its investigation}

Matrix form was used to acquire mathematical equations that represent the oscillation of multiply connected tank-drum system fastened to the elastic suspensions for main types of machines and ring type centrifuges with the horizontal and vertical rotational axes. The problems have been solved in linear approach by using II type Lagrange equations.

The research is based on the theoretical analyzing concepts of rotor systems described in $[36,37]$.

Let us examine osciliations of elastic - suspended tank, containing console - fastened rotating imbalanced drum. Such sketch is typical for machines with the horizontally - stated tank for linen, for instance as in "Viatka", "LG F1222ND" and etc.

Assumptions used for schematization of the object of research are such that real typical construction was replaced by the design diagram (dynamic model), in which the absolutely solid body (tank with mass $m_{1}$ ) is elastically attached to the block by random number of bearings and is it capable of shifting in the space, having 6 degrees of freedom (Fig. 2). There is a cavity inside 
this body, in which the rotor rotates with the angular velocity $\omega$, having mass $m_{2}$, leaning on the absolutely rigid bearings, contained in the same body.

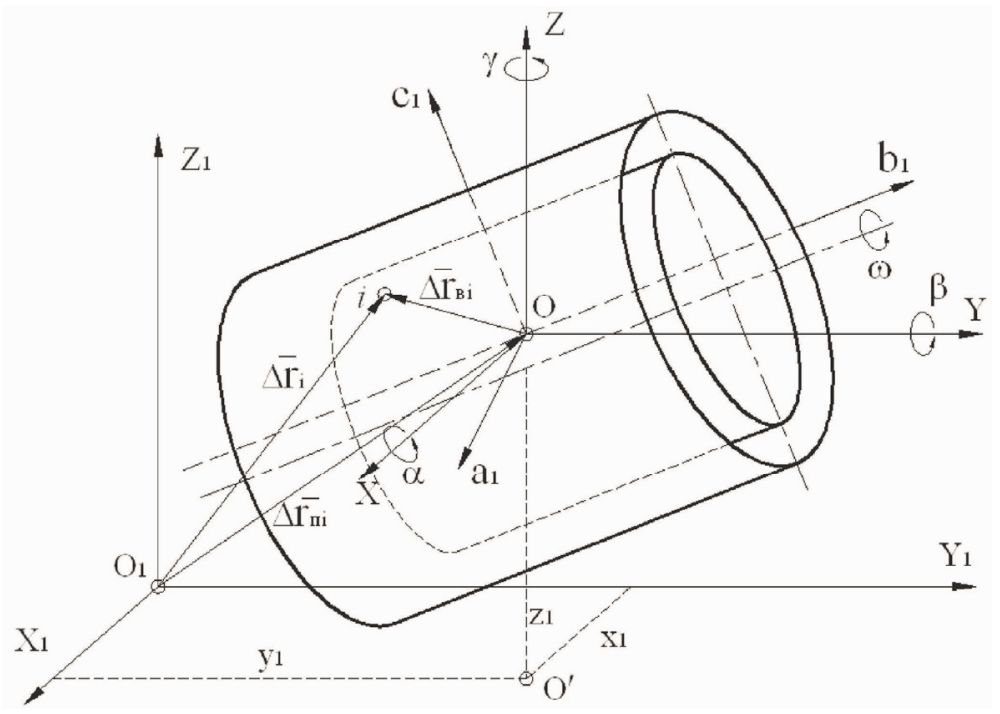

Fig. 2. Design diagram

As generalized coordinates, presetting system's location in space, three Cartesian rectangular coordinates of the machine drum inertia centre (axes $a_{1}, b_{1}, c_{1}$, serving as principal central axes) and three angles $\alpha, \beta, \gamma$, specifying rotations of these axes of coordinates regarding to the immovable, connected with the frame, coordinate axes $X_{1}, Y_{1}, Z_{1}$, or parallel to them axes $X, Y$, $Z$, coinciding in the drum's centre of mass $\mathrm{O}$, generally not laying on the drum's rotation axis, are assumed.

In such coordinate system oscillations can be presented as a superposition of six helicoidal motions with the propellers' immovable axes $X_{1}, Y_{1}, Z_{1}$, and generally the system of tank-drum carries out six-unit oscillations.

In order to constitute differential equations of the system's motion we will use second type Lagrange.

$m_{1}$ - mass of the tank; $J_{a_{1}}^{(1)}, J_{b_{1}}^{(1)}, J_{c_{1}}^{(1)}$ - tank inertia moments with respect to the main central axes $a_{1}, b_{1}, c_{1}$ accordingly; $m_{2}-$ mass of the drum.

Tank-drum system's kinetic energy can be calculated as a sum:

$T=T_{1}+T_{2}$

where, $T_{1}+T_{2}$ - kinetic energies of the tank and drum, correspondingly.

According to Konig's theorem kinetic energy of the tank [36] can be written as:

$T_{1}=\frac{1}{2}\left[m_{1}\left(\dot{x}_{1}^{2}+\dot{y}_{1}^{2}+\dot{z}_{1}^{2}\right)+J_{a 1}^{(1)} \dot{\psi}_{a 1}^{2}+J_{b 1}^{(1)} \dot{\psi}_{b 1}^{2}+J_{c 1}^{(1)} \dot{\psi}_{c 1}^{2}\right]$

where $\dot{\psi}_{a 1}^{2}, \dot{\psi}_{b 1}^{2}, \dot{\psi}_{c 1}^{2}-$ vector projections of angular velocity $\bar{\psi}=\bar{\alpha}+\bar{\beta}+\bar{\gamma}$ to the axes $a_{1}$, $b_{1}, c_{1}$.

In order to constitute expression of the drum kinetic energy we need to study its movement in general case (Fig. 3), when the centre of the drum mass in point $\mathrm{S}$ does not agree with the centre of the tank masses at the point O. Let's designate drum eccentricity by a letter $e$.

Let us introduce extra coordinates of the system into examination: performing the 
reciprocating movement $X_{2}, Y_{2}, Z_{2}$ with start at the point $\mathrm{S}$ and $X_{3}, Y_{3}, Z_{3}$ with start at the point $\mathrm{D}$, firmly connected with the drum.

The coordinate axes $a_{2}, b_{2}, c_{2}$ are considered as main central axes of drum inertia.

The start point for data recording D we receive as a result of drum's rotation axis intersection with a plane, passing at the point $S$ through the centre of masses perpendicularly to the drum rotation axis. In the reference state and in the case of eccentricity absence $(e=0)$ the point $S$ is congruent with the point $\mathrm{D}$.

It is presumed that in the initial state the axes $Y, Y_{1}, Y_{2}, Y_{3}$ and $b_{1}, b_{2}, b_{3}$ is parallel to the axis of drum rotation.

In the examined coordinate system the movement of the drum generally can be represented as a complex motion: reciprocating movement at the point $\mathrm{S}$ with the centre of masses and rotation around this centre with the angular velocity of $\bar{\Omega}=\bar{\psi}+\bar{\omega}$.

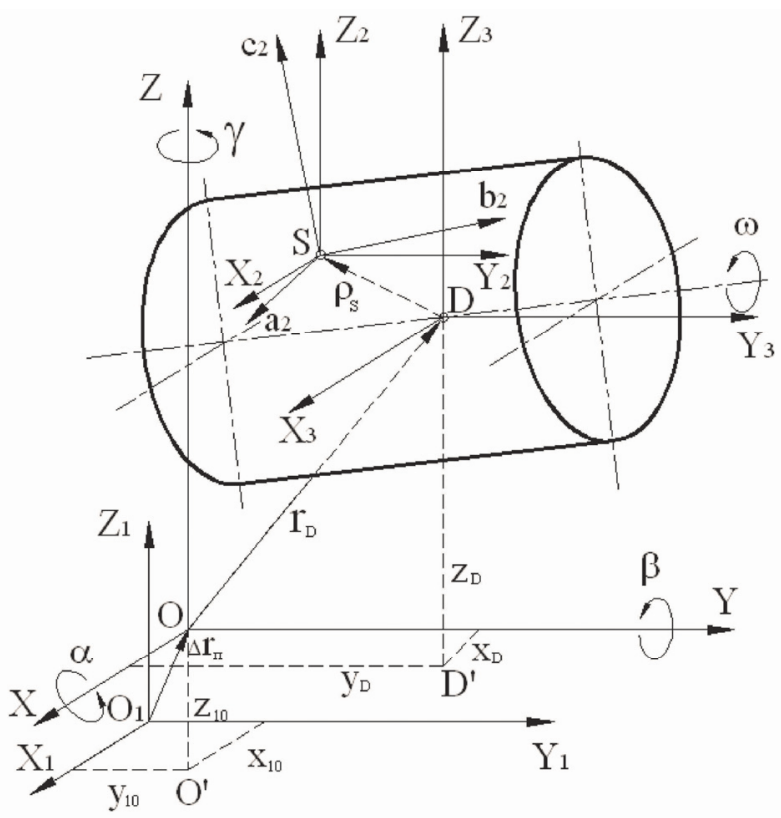

Fig. 3. Movement of the drum in general case

Using the Konig's theorem we can write the expression for the kinetic energy of the drum:

$T_{2}=\frac{1}{2}\left[m_{2}\left(V_{S x 1}^{2}+V_{S y 1}^{2}+V_{S z 1}^{2}\right)+I_{a 2}^{(2)}\left(\Omega_{a 2}^{2}+\Omega_{c 2}^{2}\right)+I_{b 2}^{(2)} \Omega_{b 2}^{2}\right]$.

Expression for the kinetic energy of the tank-drum system will be following:

$$
\begin{aligned}
T & =\frac{1}{2}\left[m_{1}\left(\dot{x}_{1}^{2}+\dot{y}_{1}^{2}+\dot{z}_{1}^{2}\right)+I_{a 1} \dot{\alpha}^{2}+I_{b 1} \dot{\beta}^{2}+I_{c 1} \dot{\gamma}_{c 1}^{2}\right] \\
& +m_{2} \dot{x_{1}}\left(\dot{\beta} z_{D}-\dot{\gamma} y_{D}+\omega e \cos \omega t\right)-m_{2} \dot{\gamma} y_{D}\left(\dot{\beta} z_{D}+\omega e \cos \omega t\right) \\
& +m_{2} \dot{\beta} \omega e\left(z_{D} \cos \omega t+x_{D} \sin \omega t\right)+m_{2} \omega^{2} e^{2} \cos \omega t \\
& +m_{2} \dot{y}_{1}\left(\dot{\gamma} x_{D}-\dot{\alpha} z_{D}\right)-m_{2} \dot{\gamma} \dot{\alpha} x_{D} z_{D}+m_{2} \dot{z}_{1}\left(\dot{\alpha} y_{D}-\dot{\beta} x_{D}-\omega e \sin \omega t\right) \\
& -m_{2} \dot{\alpha} y_{D}\left(\dot{\beta} x_{D}+\omega e \sin \omega t\right)+\frac{1}{2} I_{b 2}^{(2)} \omega(\omega+2(\dot{\beta}+\alpha \dot{\gamma}-\dot{\alpha} \gamma)) .
\end{aligned}
$$

Let us find the expressions for the potential energy and the energy dissipation within dampers 
of the tank-drum system.

Oscillating tank-drum system potential energy is determined by supports elastic deformations.

It is presumed that the tank-drum system is joined with the body of washing-machine through $n$ elastic elements and $m$ dampers.

In order to simplify dependencies, we will presume that the principal rigidity axes and constants of viscous friction of all elastic elements or dampers are parallel to the main central inertia axes of the tank-drum system.

Then projections of the rigidity vector of $i$-th elastic element to the coordinate axes $X_{1}, Y_{1}, Z_{1}$, which represent their main rigidities, will be $C_{x i}, C_{y i}, C_{z i}$, and for every i-th damper as projections of vector of constants of viscous friction are $h_{x i}, h_{y i}$ and $h_{z i}$, moreover the latter also represent main constants of viscous friction. Such simplification is practically compatible with the structural composition of the elastic elements in existing washing-machines, and their other compositions do not promise any additional advantages.

Under these conditions following expression will represent the equation of potential energy of the tank-drum system:

$\Pi=\frac{1}{2} \sum_{1}^{n}\left(C_{x_{i}} \Delta r_{x_{1 i}}^{2}+C_{y_{i}} \Delta r_{y_{1 i}}^{2}+C_{z_{i}} \Delta r_{z_{1 i}}^{2}\right)$,

where $\Delta r_{x_{1 i}}, \Delta r_{y_{1 i}}, \Delta r_{z_{1 i}}$ - fastening points movements along the axes $X_{1}, Y_{1}, Z_{1}$ relatively to the movable system of elastic elements; $n$ - number of elastic elements of the tank-drum system.

Dissipation energy in the dampers owing to action of viscous friction, which depends on the velocity of motion of the points, will be following:

$D=\frac{1}{2} \sum_{1}^{m}\left(h_{x_{i}} \Delta \dot{r}_{x_{1 i}}^{2}+h_{y_{i}} \Delta \dot{r}_{y_{1 i}}^{2}+h_{z_{i}} \Delta \dot{r}_{z_{1 i}}^{2}\right)$,

where $\Delta \dot{r}_{x_{1 i}}, \Delta \dot{r}_{y_{1 i}}, \Delta \dot{r}_{z_{1 i}}-$ velocities along the axes $X_{1}, Y_{1}, Z_{1}$ of connection points of the dampers to the tank-drum system; $m$ - number of dampers in the tank-drum system.

Differential equations of the tank-drum system oscillation will be received by applying II type Langrage equations and taking into account the energy dissipation by assuming Rayleigh type damping [37]:

$\frac{d}{d t}\left(\frac{\partial T}{\partial \dot{q}_{j}}\right)-\frac{\partial T}{\partial q_{j}}+\frac{\partial \Pi}{\partial q_{j}}+\frac{\partial D}{\partial \dot{q}_{j}}=0$,

where $j$-number of generalized coordinates, which in this case is equal to 6 .

By carrying out prescribed by the equations of Lagrange Eq. (7) according to six generalized coordinates, i.e. $X_{1}, Y_{1}, Z_{1}, \alpha, \beta, \gamma$, mathematical operations, which assume that $\omega=$ const and by omitting indexes at $X_{1}, Y_{1}, Z_{1}$, we receive a system of six differential equations:

$$
\begin{aligned}
& m \ddot{x}+m_{2} \ddot{\beta} z_{D}-m_{2} \ddot{\gamma} y_{D}+x \sum_{1}^{n} c_{x_{i}}+\beta \sum_{1}^{n} c_{x_{i}} z_{i}-\gamma \sum_{1}^{n} c_{x_{i}} y_{i} \\
& +\dot{x} \sum_{1}^{m} h_{x_{i}}+\dot{\beta} \sum_{1}^{m} h_{x_{i}} z_{i}-\dot{\gamma} \sum_{1}^{m} h_{x_{i}} y_{i}=m_{2} \omega^{2} e \sin \omega t
\end{aligned}
$$




$$
\begin{aligned}
& m \ddot{y}-m_{2} \ddot{\alpha} z_{D}+m_{2} \ddot{\gamma} x_{D}+y \sum_{1}^{n} c_{y_{i}}-\alpha \sum_{1}^{n} c_{y_{i}} z_{i}+\gamma \sum_{1}^{n} c_{y_{i}} x_{i} \\
& \quad+\dot{y} \sum_{1}^{m} h_{y_{i}}-\dot{\alpha} \sum_{1}^{m} h_{y_{i}} z_{i}+\dot{\gamma} \sum_{1}^{m} h_{y_{i}} x_{i}=0 \\
& m \ddot{z}+m_{2} \ddot{\alpha} y_{D}-m_{2} \ddot{\beta} x_{D}+z \sum_{1}^{n} c_{z_{i}}+\alpha \sum_{1}^{n} c_{z_{i}} y_{i}-\beta \sum_{1}^{n} c_{z_{i}} x_{i} \\
& \quad+\dot{z} \sum_{1}^{m} h_{z_{i}}+\dot{\alpha} \sum_{1}^{m} h_{z_{i}} y_{i}-\dot{\beta} \sum_{1}^{m} h_{z_{i}} x_{i}=m_{2} \omega^{2} e \cos \omega t \\
& J_{a_{1}} \ddot{\alpha}-m_{2} \ddot{y} z_{D}+m_{2} \ddot{z} y_{D}-m_{2} \ddot{\beta} y_{D} x_{D}-m_{2} \ddot{\gamma} x_{D} z_{D}-2 J_{b_{2}}^{(2)} \omega \dot{\gamma} \\
& \quad-y \sum_{1}^{n} c_{y_{i}} z_{i}+z \sum_{1}^{n} c_{z_{i}} y_{i}+\alpha\left(\sum_{1}^{n} c_{z_{i}} y_{i}^{2}+\sum_{1}^{m} c_{y_{i}} z_{i}^{2}\right) \\
& \quad-\beta \sum_{1}^{m} c_{z_{i}} x_{i} y_{i}-\gamma \sum_{1}^{m} c_{y_{i}} x_{i} y_{i}-\dot{y} \sum_{1}^{m} h_{y_{i}} z_{i}+\dot{z} \sum_{1}^{m} h_{z_{i}} y_{i} \\
& \quad+\dot{\alpha}\left(\sum_{1}^{m} h_{z_{i}} y_{i}^{2}+\sum_{1}^{m} h_{y_{i}} z_{i}^{2}\right)-\dot{\beta} \sum_{1}^{m} h_{z_{i}} x_{i} y_{i}-\dot{\gamma} \sum_{1}^{m} h_{y_{i}} x_{i} y_{i}=m_{2} y_{D} \omega^{2} e \cos \omega t,
\end{aligned}
$$

$J_{b_{1}} \ddot{\beta}+m_{2} \ddot{x} z_{D}-m_{2} \ddot{z} x_{D}-m_{2} \ddot{\alpha} y_{D} x_{D}-m_{2} \ddot{\gamma} y_{D} z_{D}$

$$
\begin{aligned}
& +x \sum_{1}^{n} c_{x_{i}} z_{i}-z \sum_{1}^{n} c_{z_{i}} x_{i}-\alpha \sum_{1}^{n} c_{z_{i}} y_{i} x_{i}+\beta\left(\sum_{1}^{n} c_{x_{i}} z_{i}^{2}+\sum_{1}^{n} c_{z_{i}} x_{i}^{2}\right) \\
& -\gamma \sum_{1}^{n} c_{x_{i}} y_{i} z_{i}+\dot{x} \sum_{1}^{m} h_{x_{i}} z_{i}-\dot{z} \sum_{1}^{m} h_{z_{i}} y_{i}-\dot{\alpha} \sum_{1}^{m} h_{z_{i}} y_{i} x_{i} \\
& +\dot{\beta}\left(\sum_{1}^{m} h_{x_{i}} z_{i}^{2}+\sum_{1}^{m} h_{z_{i}} x_{i}^{2}\right)-\dot{\gamma} \sum_{1}^{m} h_{x_{i}} y_{i} z_{i}=-m_{2} \omega^{2} e\left(x_{D} \cos \omega t-z_{D} \sin \omega t\right), \\
& J_{c_{1}} \ddot{\gamma}-m_{2} \ddot{x} y_{D}+m_{2} \ddot{y} x_{D}-m_{2} \ddot{\alpha} x_{D} z_{D}-m_{2} \ddot{\beta} y_{D} z_{D}+2 J_{b_{2}}^{(2)} \omega \dot{\alpha}
\end{aligned}
$$

$$
\begin{aligned}
& -x \sum_{1}^{n} c_{x_{i}} y_{i}+y \sum_{1}^{n} c_{y_{i}} x_{i}-\alpha \sum_{1}^{n} c_{y_{i}} z_{i} x_{i}-\beta \sum_{1}^{n} c_{x_{i}} z_{i} y_{i} \\
& +\dot{\gamma}\left(\sum_{1}^{n} c_{y_{i}} x_{i}{ }^{2}+\sum_{1}^{m} c_{x_{i}} y_{i}{ }^{2}\right)-\dot{x} \sum_{1}^{m} h_{x_{i}} y_{i}+\dot{\gamma} \sum_{1}^{m} h_{y_{i}} x_{i} \\
& -\dot{\alpha} \sum_{1}^{m} h_{y_{i}} z_{i} x_{i}-\dot{\beta} \sum_{1}^{m} h_{x_{i}} z_{i} x_{i}+\dot{\gamma}\left(\sum_{1}^{m} h_{y_{i}} x_{i}{ }^{2}+\sum_{1}^{m} h_{x_{i}} y_{i}{ }^{2}\right)=-m_{2} \omega^{2} e y_{D} \sin \omega t .
\end{aligned}
$$

Differential equations in matrix form are:

$[M] \ddot{q}+([G]+[D]) \dot{q}+[A] q=[Q]$

where $[M]=\left\|P_{i j}\right\|_{1}^{6}-$ matrix of inertia coefficients; $[G]=\left\|q_{i j}\right\|_{1}^{6}-$ matrix of gyroscopic coefficients; $[D]=\left\|\alpha_{i j}\right\|_{1}^{6}-$ matrix of coefficients of damping; $[A]=\left\|\alpha_{i j}\right\|_{1}^{6}-$ matrix of coefficients of rigidity; $q=\{x, y, z, \alpha, \beta, \gamma\}^{T}$ - matrix-column of generalized coordinates; $Q=\left\{Q_{x}, Q_{y}, Q_{z}, Q_{\alpha}, Q_{\beta}, Q_{\gamma}\right\}^{T}-$ matrix-column of generalized factors of forces.

Also, the coefficients, for example, of the matrix $A$ have the following expressions: 


$$
\begin{aligned}
& a_{11}=\sum_{i=1}^{n} c_{x_{i}}, \quad a_{15}=a_{51}=\sum_{i=1}^{n} c_{x_{i}} z_{i}, \quad a_{16}=a_{61}=-\sum_{i=1}^{n} c_{x_{i}} y_{i}, \\
& a_{22}=\sum_{i=1}^{n} c_{y_{i}}, \quad a_{25}=a_{52}=-\sum_{i=1}^{n} c_{y_{i}} z_{i}, \quad a_{26}=a_{62}=\sum_{i=1}^{n} c_{y_{i}} x_{i}, \\
& a_{33}=\sum_{i=1}^{n} c_{z_{i}}, \quad a_{34}=a_{43}=\sum_{i=1}^{n} c_{z_{i}} y_{i}, \quad a_{35}=a_{53}=-\sum_{i=1}^{n} c_{z_{i}} x_{i}, \\
& a_{44}=\sum_{i=1}^{n} c_{z_{i}} y_{i}^{2}+\sum_{i=1}^{n} c_{y_{i}} z_{i}^{2}, \quad a_{45}=a_{54}=-\sum_{i=1}^{n} c_{z_{i}} x_{i} y_{i}, \quad a_{46}=a_{64}=-\sum_{i=1}^{n} c_{y_{i}} x_{i} z_{i}, \\
& a_{55}=\sum_{i=1}^{n} c_{x_{i}} z_{i}^{2}+\sum_{i=1}^{n} c_{z_{i}} x_{i}^{2}, \quad a_{56}=a_{65}=-\sum_{i=1}^{n} c_{x_{i}} y_{i} z_{i}, \\
& a_{66}=\sum_{i=1}^{n} c_{y_{i}} x_{i}^{2}+\sum_{i=1}^{n} c_{x_{i}} y_{i}^{2}, \quad a_{12}=a_{13}=a_{23}=a_{24}=a_{36}=0,
\end{aligned}
$$

where $c_{x_{i}}, c_{y_{i}}, c_{z_{i}}$ - projections of the stiffness vector of the $i$-th elastic element to the coordinate axes $X_{1}, Y_{1}, Z_{1}$.

According to their structure elements $a_{i j}$ of the matrix $A$ can be divided into four groups and named analogously to the components of the inertia tensor. The first group comprises from elements $a_{i j}$, those which have $i, j \leq 3$ and $I=j$. They represent summarized rigidity, which values are essentially positive.

The second group includes elements $a_{i j}$, having $i, j>3$ and $i=j$. They represent torsional rigidity of the tank-drum system suspension. According to signs they are similar to the inertia moments with respect to the coordinate axes, in other words they are always positive.

The third group includes elements $a_{i j}$, having $i, j \leq 3$ and $i \neq j$. They represent static moments of rigidity with respect to system's coordinate planes. According to signs they are similar to the static moments of masses with respect to coordinate planes, in other words they can be positive, negative and zero.

And, finally, the forth group includes elements $i, j>3$, having $i \neq j$. They represent centrifugal moments of rigidity with respect to pairs of coordinate planes. According to signs they are similar to the centrifugal moments of inertia, in other words they can be positive, negative and zero.

Such analogy provides a possibility for developing simple rules, at which the non-diagonal elements of the rigidity matrix - static and centrifugal moments of rigidity will become zero, and it is necessary for separation of the system's oscillations. Particularly, static and centrifugal moments of rigidity will become zero, if coordinate planes with respect to which they have been determined, will be planes of symmetry of the tank-drum system suspension. Analogous assumptions remain valid about the elements structure of the damping matrix $D$.

For complete separation of free oscillations (when the drum is not rotating), in addition to the rigidity matrix $A$, it is necessary that the matrix of inertia coefficients $M$ would have a diagonal form, and it is possible when center of mass of the tank coincidence with the centre of mass of the balanced drum.

However in practice because of linen random distribution inside the drum it is impossible to receive complete coincidence between center of mass of the balanced drum and tank. However, for reducing vibroactivity of the washing-machine it is essential to seek that the centre of mass of the tank would be on the rotation axis of the drum and that they would be as much as possible closer to its centre of mass at uniform positioning of linen.

Realized analysis of differential equations of motion allowed to formularize requirements 
concerning the washing-machine structure, which should be followed during the design stage: the centre of the tank mass must be on the rotation axis of the drum; rotation axis of the drum must be the main central axis of drum inertia; there must be a coincidence between centre of mass of the tank and drum; there must be a coincidence between the rigidity centre of the system of elastic supports with the gravity centre of the tank and between main rigidity axes and main central axes of tank's inertia. The main axes of viscous friction constants must coincide with the main central axes of tank's inertia.

The system of Eq. (8) considered above has both solutions qualitative and quantitative. Carried out calculation referring machines "Viatka" and "Volga" is about determining resonances and providing their adjustment, on the first stage by separating oscillations by means of repositioning of masses and rigidity of the whole machine, and then - shifting every single resonance be variation of masses and rigidities, influencing only on the value of this resonance, have corroborated validity of formularizes requirements to the design of machines with low vibroactivity.

Nevertheless, calculations are not always successful. And the matter here is not related with the incomplete design diagrams and equations of oscillations, but it is connected with the fact that looking only on the drafts of the machine it is difficult to precisely determine exact values of rigidity coefficients, damping, inertia and masses, that are included into the equations, which are adduced for chosen design diagram. A lot of elements of the machine should be considered at the same time as mass, and rigidity, and also as vibration exciter (resonator) and vibration suppressor (damper). Under conditions of high amplitudes of oscillations some of components, that are considered as being rigid under conditions of lower amplitudes by right, deform, involve adjacent elements into oscillation, fastening to themselves a certain part of their mass and rigidity, in other words they change the initial conditions of the problem and, therefore, values of designed shapes and oscillation frequencies.

All of this requires the development of equipment and experimental methods of machine dynamics research in order to accelerate their adjustment, examination and specifying calculations, identifying parameters of masses, rigidity and damping, that are included in equations of effective search for the reasons of occurring defects and efficiency checking of measures taken for their elimination.

\section{Relationship between the rotor balance and operability of rolling bearings}

Problems of balancing rotors and dynamics of machines are closely connected with the work of bearings and ensuring their efficiency.

On the one hand, the design of the bearing unit, the stiffness of the coupling with the parts clamps, the mounting gaps, the organization of lubrication, its viscosity and temperature affect the overall dynamic state of the machine and the movement of the rotor, and on the other hand, the imbalance determines the value of loads on the bearing and specifies, depending on listed above factors the nature of the movement of the pin in the bearing, the mode of its operation, causes one or another type of its destruction.

The practice of washing machines of various designs has shown that the rolling bearings are often out of order in the drum mounting unit to the body.

In most cases, the breakdowns are the malfunctioning of treadmills, balls and (or) breakage of the separator of bearings. The purpose of experimental research of bearings is to search for possible reasons for their unsatisfactory work.

Most bearing defects are due to their overload, unsatisfactory organization and the composition of lubrication.

In a much lesser extent, the robotability of bearings at low loads is studied and in this section attention is drawn to this fact, since it is related and was discovered during the study of balancing rotors.

In the study of the dynamics of washing machines, there was a discrepancy between the 
working capacity of roller bearings, the balance of the drum and the load on the bearing.

It is known that the normal mode of the ball bearing operation corresponds to a mode in which the balls roll without slipping $[1,2]$. In the presence of slipping the balls in the bearing, the rolling friction is replaced by sliding friction, which leads to an increase in the wear of both running tracks and the balls themselves, and in the final result - to jamming of the balls, breakage of the separator, etc. It is precisely this case that occurs in carefully balanced machines in the mode of washing and other modes with small vibrations.

In this connection, the research of the working capacity of roller bearings was reduced to determining the value of the slipping of balls at different values of the drum imbalance.

\section{Presentation of research materials}

The evaluation of the ball bearings was performed on the basis of measurements of the speed of the separator and shaft and the comparison of the measured values with the theoretical ones. In a perfectly selected bearing, the number of revolutions of the separator and the shaft of the rotor on which the inner races of bearing are located, expressed by the ratio:

$n_{c}=\frac{n_{s}}{2} \cdot \frac{d_{0}-d_{b} \cos \beta}{d_{0}}$

where $n_{s}$ - number of rotations of the shaft of the engine rotor; $d_{0}$ - diameter of placement of centers of rolling bodies; $d_{b}$ - diameter of the rolling body (balls or roller); $\beta$ - the contact angle of the body of rolling with the track of the inner races.

For a ball bearing in the absence of an axial force, the contact angle $\beta=0$, then Eq. (9) will take the form:

$n_{c}=\frac{n_{s}}{2}$

Eq. (10) is valid in the case if the balls in the bearing roll without slip [3].

Measurement of the number of revolutions of the separator of bearings was performed at an imbalance of the rotor $22000 \mathrm{~g} \cdot \mathrm{cm}$, equal to the maximum possible imbalance in the nominal drum load (4 kg of dry linen), without an imbalance and when acting on the drum only the axial force. To get dependence $n_{c}=f\left(n_{s}\right)$ of separator rotation frequency $\left(n_{c}\right)$ on the rotational speed of the shaft $\left(n_{s}\right)$ the record was performed on the passage from 0 to maximum engine speed. Depending on the received values, dependency charts were constructed $n_{c}=f\left(n_{s}\right)$. The same graphs were applied theoretical dependence Eq. (10).

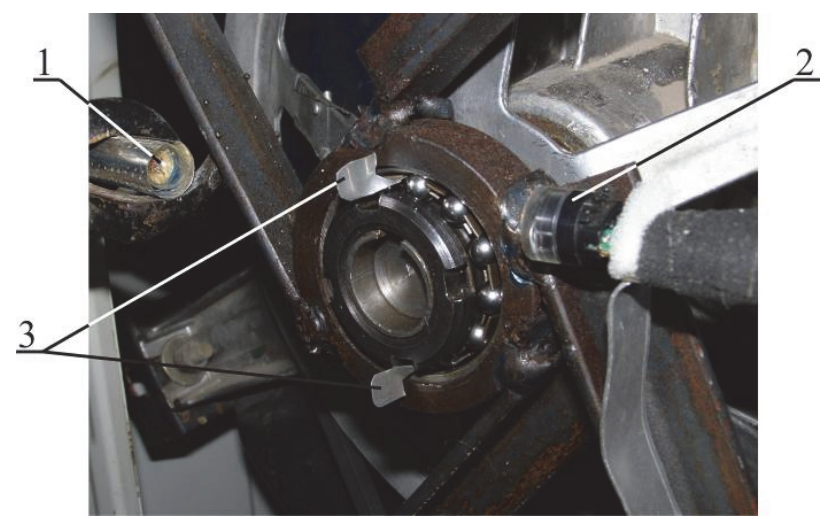

Fig. 4. Device for measuring the number of revolutions of the bearing separator: 1 - incandescent lamp; 2 - photodiode FD-10K; 3 - blinds 
Taking into account that at the point of attachment of the drum, ball bearings are located in hard-to-reach places with limited space, a special device for measuring the rotation of the separator has been developed.

The device is an optoelectronic pair consisting of a miniature incandescent lamp 1 (Fig. 4) and a photodiode FD-10K 2 with a small size of photosensitive area. To modulate the light coming from the lamp on the photodiode on the separator of the investigated bearing, special curtains are installed 3. The lamp and the photodiode were installed in such a way that the beam of light during the rotation of the separator was covered with blinds.

The photodiode was connected through a matching device to the "Amphiton" amplifier, signals from which were fixed to the photocell of the oscilloscope H071.4M (Fig. 5).

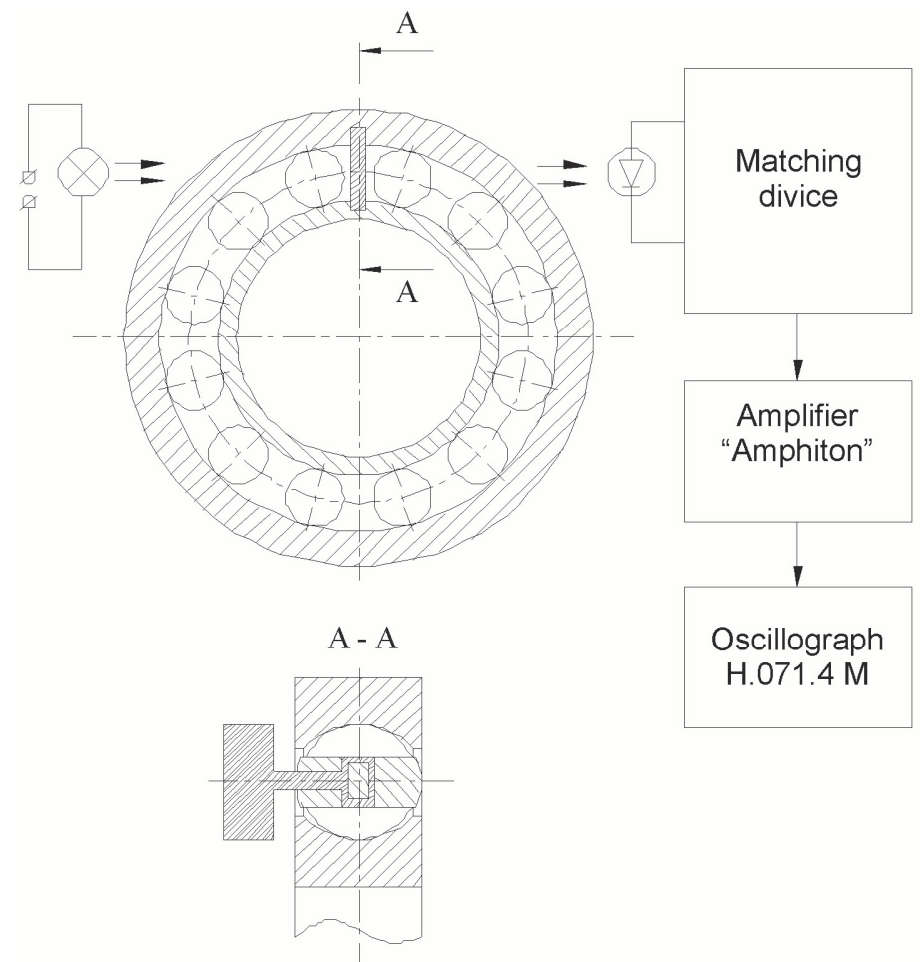

Fig. 5. Block diagram of measuring the rotational speed of the ball bearing separator

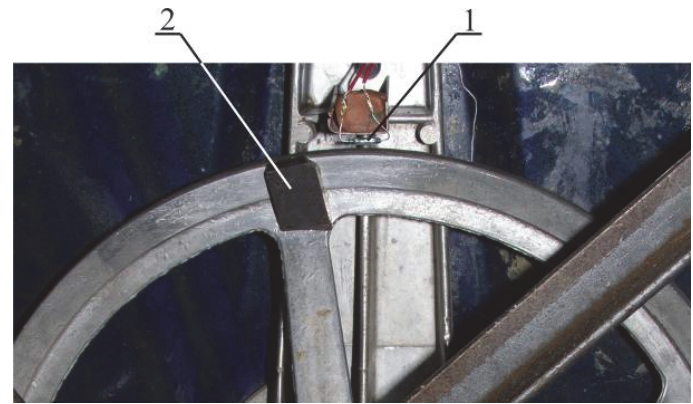

Fig. 6. Rotary shaft sensor: 1 - reed switch; 2 - magnet

Simultaneously, on the oscilloscope's film, the speed of the drum shaft was recorded with the help of a speed sensor. The sensor was made on the basis of the reed switch, which has the ability to lock the electric circuit when passing through a magnetic field. Practically this was done as 
follows. On the plate, which was rigidly connected with the installation case, a reed switch 1 (Fig. 6) was stuck with soldered to the ends of its conductors, and the magnet 2 was stuck on the pulp of the unit.

Since the pulley is rigidly connected to the rotor shaft, the speed of their rotation was the same. Thus, with every revolution of the shaft, the magnet, fixed on the pulley, and the magnetic field formed by it, approached the reed switch and locked its contacts. In a closed reed switch on the electric circle, the current, which was the signal of the speed indicator, began to flow.

Experiments were carried out on the washing machine "Aisha" with new and worn bearings.

In order to reduce the random error of measurements, each experiment was conducted several times.

After decoding the received records of the rotational numbers of the bearing separator and the drum shaft, dependences were constructed $n_{c}=f\left(n_{s}\right)$ shown in Figs. 7 and 8.
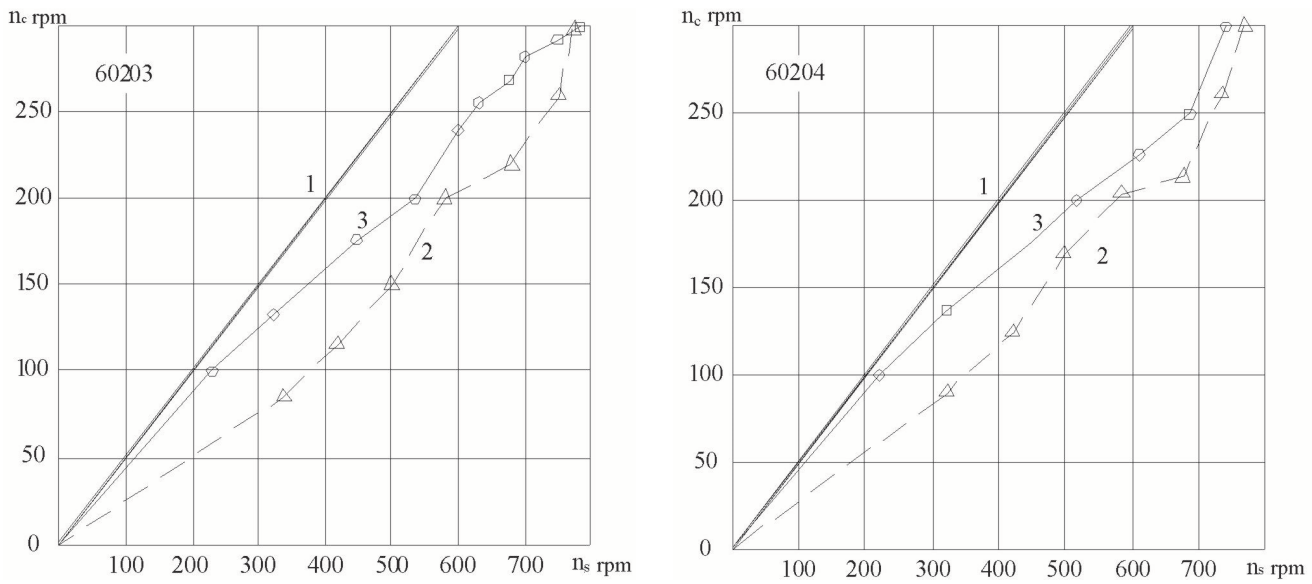

Fig. 7. Dependence of the rotor speed of the bearing separator on the rotational speed of the shaft (non-worn bearings): 1 - theoretical dependence; 2 - without drum loading; 3 - with imbalance $22000 \mathrm{~g} \cdot \mathrm{cm}$
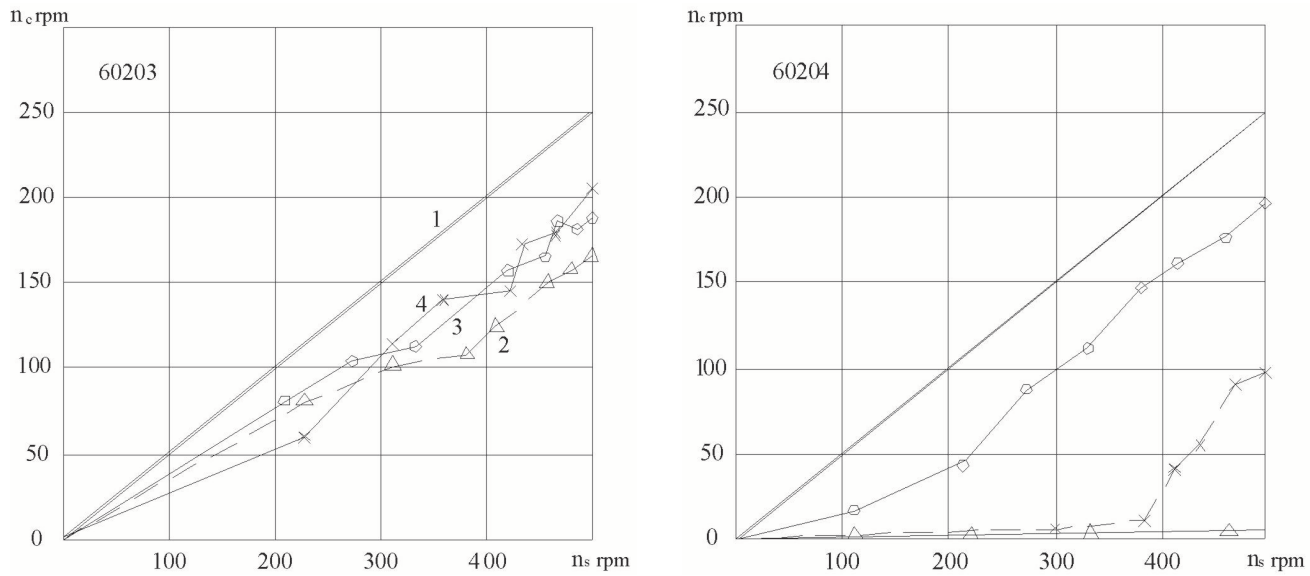

Fig. 8. Dependence of the rotor speed of the bearing separator on the rotational speed of the shaft (worn bearings): 1 - theoretical dependence; 2 - without drum loading; 3 - with an imbalance of $22000 \mathrm{~g} \cdot \mathrm{cm} ; 4$ - with an axial load of $4 \mathrm{~kg}$

As can be seen from the received graphs, for new and worn bearings, there is a considerable lag behind the number of revolutions of the separator from its theoretical value. When slipping the balls in the bearing, the friction of the roller is replaced by sliding friction, which can lead to 
an increase in wear, both in treadmills and in the balls itself. Significant interest is also the fact that when the load on the bearings slipping decreases. On the basis of the obtained results it can be concluded that in order to reduce the size of the slider of balls in bearings, bearings with some initial load, for example, creating some preliminary axial load, should be used.

In the course of this study it was found that roller bearings create noise and high-frequency perturbations, in which certain elements of the machine resonate.

These disadvantages are forcing to find ways to reduce the number of roller bearings in the design of the machine or complete relief from them.

On the basis of the obtained results it can be concluded that for reducing the size of the balls slip, it is necessary to use bearings with some initial load, for example, creating some preliminary axial load or switching to the ball bearings of a lighter series.

In addition, studies have shown that decreasing the drum imbalance, for example, with the help of the ABD, leads to an increase in the degree of slipping of the balls. Therefore, it seems advisable, together with the installation in the drum of the machine ABD or the implementation of other measures that reduce the imbalance of the drum to move on the bearings of a lighter series or bearings of slip.

In this study, in lieu of four ball bearings No. 602031 Fig. 9, the knot of the tank of the washing machine to the housing, were made and tested bearings of sliding 2, 3 in the form of 2 cylindrical bushes, the working drawing is depicted in Fig. 7 from self-lubricating material "Hraphiton-K". The technical characteristics of which are given in Table 1.

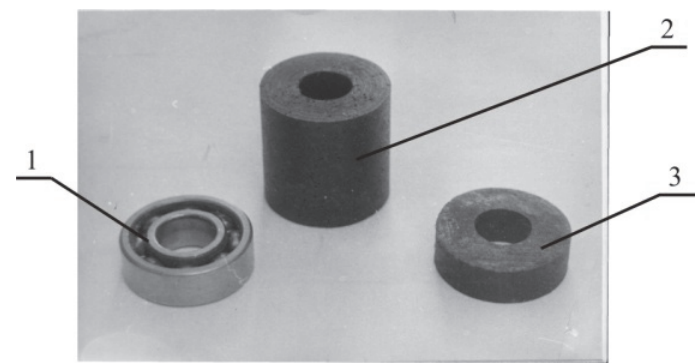

Fig. 9. Bearings of the tank mounting unit for the washing machine to the housing:

1 - ball bearing No. 60203; 2 - cylindrical bush is made of "Hraphiton" (performance 2);

3 - cylindrical bush is made of "Hraphiton" (performance 1)

Table 1. Basic statistical characteristics of the antifriction properties of the material "Hraphiton-K"

\begin{tabular}{|l|c|}
\hline \multicolumn{1}{|c|}{ The name of the characteristic } & Value \\
\hline Density, $\mathrm{kg} / \mathrm{m}^{3}$ & 1080 \\
\hline Strength, MPa when stretching & 46 \\
\hline Strength, MPa with compression & 81 \\
\hline Thermal conductivity at $50-125^{\circ} \mathrm{C} \mathrm{W} / \mathrm{m} \cdot \mathrm{K}$ & $0,88-1,02$ \\
\hline Heat capacity at $50-125^{\circ} \mathrm{C} \mathrm{KJ} \cdot \mathrm{kg} \cdot \mathrm{K}$ & $1,88-2,35$ \\
\hline Friction coefficient without lubrication & $0,22-0,35$ \\
\hline Friction coefficient with limited lubrication $10 \%$ & 0,08 \\
\hline Friction coefficient with limited lubrication $50 \%$ & 0,07 \\
\hline Intensity of wear $\left(\times 10^{-7}\right) \mathrm{mm}^{3} / \mathrm{N} \cdot \mathrm{m}$ without lubrication & 100 \\
\hline Intensity of wear $\left(\times 10^{-7}\right) \mathrm{mm}^{3} / \mathrm{N} \cdot \mathrm{m}$ with limited lubrication $10 \%$ & 5,1 \\
\hline Intensity of wear $\left(\times 10^{-7}\right) \mathrm{mm} 3 / \mathrm{N} \cdot \mathrm{m}$ with limited lubrication $50 \%$ & 4,0 \\
\hline
\end{tabular}

Detailed researches of the first variant (replacement of all rolling bearings with sliding bearings) showed the presence of wear in the form of an enlarged radius of 1-1.5 mm rounding the transition from the end part to the creature's inner surface from the side of the end closest to the pulley. The reason for such wear was a one-sided, almost static load transmitted by the pulley from the tension of the pass. 
When working with similar bearings in a machine, when there is a flexible coupling between the pulley and the shaft of the drum and the effort on the side of the pulley is absent, the observed defect was not observed. However, the aforesaid wear was not reflected in the performance of the machine and may be permissible.

In this case, if the customer acknowledges the specified wear as inadmissible, we have conducted a third-version study, when one ball bearing closer to the pulp, stayed in place, and instead of the other three, the bushes of the material Hraphiton were delivered.

Long-lasting, at least 300-hour machine research in the course of experimental studies has shown the possibility of using this version as well, since none of the roller bearing or slip bearings were defective.

At the same time, in the zone of high sound frequencies (above $200 \mathrm{~Hz}$ ), the sound power level decreased by a total of $20 \%$, which also confirms the expediency of the transition to these bearings.

All of the foregoing suggests the replacement of at least three rolling bearings 1 Fig. 9 of the Aisha washing machine reel bearings in the form of bushes 2 or 3 of Fig. 9 made of Hraphiton material in accordance with the drawing (Fig. 10), such material or ready-made bushings may be made by Khmelnytskyi National University.

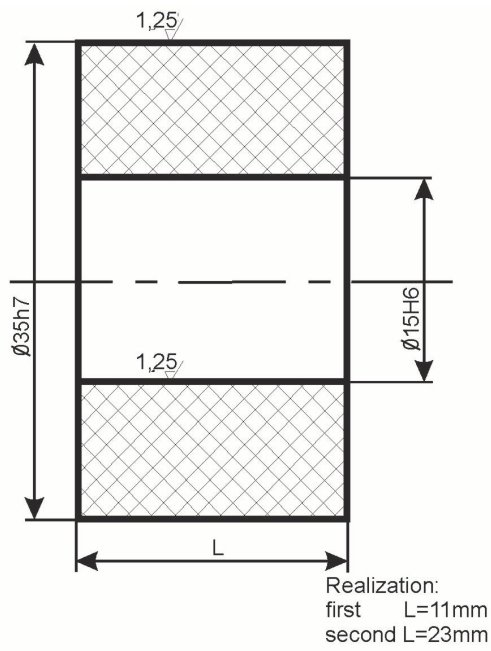

Fig. 10. Working drawing of the bush

Conclusions that we can draw include the following:

1. A device and method for contactless measurement of the value of slipping balls in roller bearings were developed by measuring the frequency of rotation of the separator by a photoelectric sensor.

2. The connection between the magnitude of the imbalance in the drum and the working capacity of rolling bearings is investigated.

3. It is shown that the decrease of the unbalance value leads to an increase in the value of the slipping of balls in the bearing.

4. It was found that when increasing the radial load on the bearing or in the presence of an axial load, the slip of the balls decreases.

5. On the basis of the conducted research it was established that simultaneously with the decrease in the value of disturbing forces, for example, due to the establishment of the ABD, it is advisable to switch to bearings of slip or ball bearings of a lighter series.

Let's consider in more detail one of modern methods of elimination of negative influence of vibration of rotary machines (in particular washing machines of a drum type) - passive automatic balancing by liquid working bodies. 


\section{Theoretical part}

Developing mathematical model describing behavior of liquid in ABU uses the properties of liquid listed below:

- whatever volume of liquid can have any change of its shape being forced by whatever small load;

- viscosity forces are considered only for fast motion when shears in liquid change fast; those forces are not considered for solving problems of liquid balance.

Equation describing condition of stationary motion of solid body with liquid in its hollow [38] in accordance to the principle of least action in the form of Hamilton-Ostrogradski is:

$\delta W=-\frac{1}{2} \frac{k_{0}^{2}}{I_{0}^{2}} \delta I+\delta P=0$,

where $W=\frac{1}{2} \frac{k_{0}^{2}}{I}+P$ - varied potential energy of the system; $P$ - potential energy of forces acting on the system: gravity and centripetal forces, $k_{0}$ - value of the constant $k$ in case of uniform rotation of the whole system as one solid body around some immobile line with angular speed $\omega$, $I_{0}-$ value of $I$ (moment of inertia of system) for stabilized motion.

Thus, in case of stationary motion of system equation $W$ has extreme value.

Let's assume a solid body being in uniform field of gravity and centripetal forces and having one fixed point $\mathrm{O}$, cylindrical hollow with radius $R$ and height $h$, and being partially filled with liquid. Axis $x_{3}^{\prime \prime}$ of the immobile system with coordinate origin in fixed point of the body is directed vertically. Mobile axes fixed with body will be drawn so that the axis $x_{3}$ would go through gravity center of the system. Gravity center of the system is shifted at the value e relative to geometric axis of the body.

To consider mechanical system without surface tension of liquid potential energy of system and inertia moment of system relative to axis $x_{3}^{\prime \prime}$ are defined by the formulas:

$P=M g\left(x_{c 1} \gamma_{1}+x_{c 2} \gamma_{2}+x_{c 3} \gamma_{3}\right)$,

$I=A \gamma_{12}+B \gamma_{22}+C \gamma_{32}-2 D \gamma_{2} \gamma_{3}-2 E \gamma_{1} \gamma_{3}-2 F \gamma_{1} \gamma_{2}$

where $A, B, C$ - axial inertia moments, $D, E, F$ - centripetal inertia moments of system; $M$ system mass; $x_{c 1}, x_{c 2}, x_{c 3}$ - coordinates of gravity center of system; $\gamma_{i}$ - projections of ort $i_{3}^{\prime \prime}$ of immobile axis $x_{3}^{\prime \prime}$ to mobile axis are connected by equation:

$\gamma_{12}+\gamma_{22}+\gamma_{32}=1$

Condition Eq. (11) considering $\omega I=k$ gives equations in generalized coordinates of solid body in stationary motion:

$\frac{\partial W}{\partial \gamma_{1}}=-\omega^{2}\left[(A-C) \gamma_{1}+D \gamma_{1} \gamma_{2} \gamma_{3}^{-1}-E\left(\gamma_{3}-\gamma_{1}^{2} \gamma_{3}^{-1}\right)-F \gamma_{2}\right]+M g\left(x_{c 1}-x_{c 3} \gamma_{1} \gamma_{3}^{-1}\right)=0$
$\frac{\partial W}{\partial \gamma_{2}}=-\omega^{2}\left[(B-C) \gamma_{2}-E\left(\gamma_{3}-\gamma_{2}^{2} \gamma_{3}^{-1}\right)+E \gamma_{1} \gamma_{2} \gamma_{3}^{-1}-F \gamma_{1}\right]+M g\left(x_{c 2}-x_{c 3} \gamma_{2} \gamma_{3}^{-1}\right)=0$

Eq. (13) lets solution:

$\gamma_{1}=\gamma_{2}=0, \quad \gamma_{3}=1$,

which will be true at any value of $\omega$, if gravity center of system is located on the axis $x_{3}$, which is in turn the general symmetry axis, in condition that: 
$x_{c 1}=x_{c 2}=0, \quad D=E=0$.

Solving Eq. (14) corresponds to uniform rotation of the whole system around axis $x_{3}^{\prime}$ aligned with axis $x_{3}^{\prime \prime}$, with angular speed $\omega$, and axis $x_{3}^{\prime}$ being general inertial axis of system, what is condition of auto-balancing. In this condition free surface of liquid will have the shape of revolving paraboloid:

$\frac{1}{2} \frac{\gamma \omega^{2}}{g}\left(x_{1}^{2}+\left(x_{2}+e\right)^{2}\right)-\gamma x_{3}-p_{0}=C^{*}$,

where $\gamma=g \rho-$ volume weight of liquid.

Let the point $\mathrm{O}^{\prime}$ be the coordinate origin, axis $x_{3}^{\prime}$ be directed as revolution axis, and axis $x_{2}^{\prime}$ go through points $\mathrm{O}$ and $\mathrm{O}^{\prime}$. Although another coordinate system $O x_{1} x_{2} x_{3}$ will be considered, in which axis $x_{3}$ is geometric axis, axis $x_{2}$ coincides with axis $x_{2}^{\prime}$.

Free surface has the shape of revolution paraboloid with parameter $1 / \beta=2 g / \gamma \omega^{2}$, what depends on angular rotation speed; growing angular rotation speed makes parameter decrease and paraboloid be similar to surface of circular cylinder: $r^{2}=x_{1}{ }^{2}+\left(x_{2}+e\right)^{2}=b^{2}$ and in limit when $2 g / \gamma \omega^{2} \rightarrow 0$ it becomes a circular cylinder. Correspondently, in horizontal section with axis $x_{3}=0$ that coincides with lower base of the hollow, free surface of liquid has circle shape with center $\mathrm{O}$ and radius $R_{1}$. Let's find relation between radius $R_{1}$ and quantity of liquid in the cylindrical hollow $V$. Therefore, we'll find constant $C^{*}$ in Eq. (16) in condition that volume of liquid retains in rest (when $\omega=0$ ) and in revolution.

There are three cases of liquid position in the hollow which depend on relation between liquid and imbalance:

1) quantity of liquid is greater than it's needed to balance imbalance (Fig. 11);

2) quantity of liquid is that liquid can balance imbalance in considered frequencies (Fig. 12);

3) quantity of liquid is not enough to balance imbalance (Fig. 13).

Thus, the free surface of heavy liquid revolving around vertical eccentrical axis will be described by equation:

$x_{3}=\frac{\omega^{2}}{2 g}\left(x_{1}{ }^{2}+\left(x_{2}+e\right)^{2}\right)-\left(\frac{\omega^{2}}{2 g} R^{2}-\frac{\omega^{2} V}{2 \pi g h}-\frac{h}{2}\right)$.

Considering horizontal section (Fig. 11), it's possible to make conclusion that liquid displaces towards to rotor axis, opposite to imbalance. Its motion stops when total imbalance of liquid and rotor becomes equal zero, that's the balance where principal inertia axis of rotor system coincides with revolution axis. It's automatic balancing when liquid has such shape.

If equation of free surface of liquid Eq. (16) is known one can define equation for liquid optimal volume what is necessary for accurate balancing imbalance in considered frequencies of rotation of system with given physical and geometrical parameters:

$$
\begin{aligned}
& \left(R^{2} h-\frac{h+\beta(e-R)^{2}}{4}\left(\sqrt{\frac{h}{\beta}+(e-R)^{2}}-e+R\right)^{2}+\frac{3 \beta}{32}\left(\sqrt{\frac{h}{\beta}+(e-R)^{2}}-e+R\right)^{4}\right. \\
& \left.+\frac{(R-e)^{4} \beta}{2}\right) \pi=V .
\end{aligned}
$$

In this case radius $\alpha$ of free liquid surface in horizontal section made by the plain $x_{3}=0$ equals: 
$R_{1}=R-e$.

It explains that thickness of the layer in horizontal section made by plain $x_{3}=0$ opposite to imbalance shouldn't exceed $2 e$. Thus, only thin layer of liquid takes part in changing imbalanced state of rotating system.

In the third case (Fig. 13) constant $C^{*}$ will be found from Eq. (20).

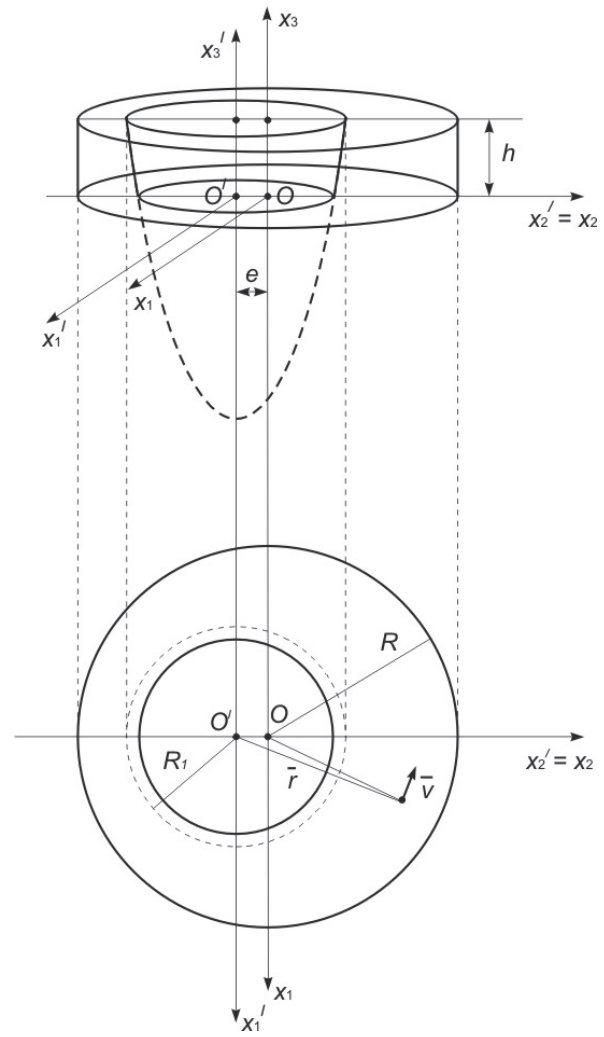

Fig. 11. Position of liquid in the hollow (case 1)

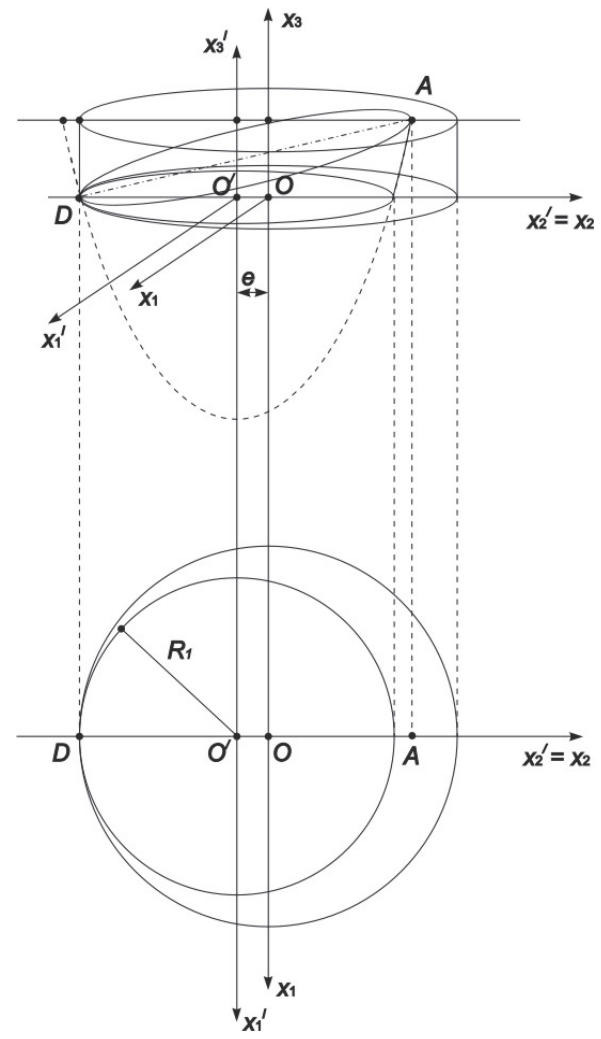

Fig. 12. Position of liquid in the hollow (case 2)

In Fig. 13 thickness of the layer in horizontal section having area $x_{3}=0$ opposite to imbalance is much less than $2 e$. Thus, volume of the liquid is not enough to reduce deviation of longitudinal axis of the rotor from revolution axis. Research of liquid's free surface helps make conclusion that not the whole volume of liquid is involved into balancing of imbalance. Optimal volume of liquid to balance imbalance in the given revolution frequencies is estimated by Eq. (18):

$$
\begin{gathered}
\pi R^{2} h-\left[\frac{R^{2} h}{2} \cdot \operatorname{arcos}\left(\frac{C-\beta\left(R^{2}+e^{2}\right)}{2 e R \beta}\right)-\frac{h\left(-C^{2}+2 C \beta\left(R^{2}+e^{2}\right)-\beta^{2}\left(R^{2}-e^{2}\right)^{2}\right)}{4 e \beta^{2}}\right. \\
\left.+\frac{h(h+2 C)}{2 \beta} \cdot\left(\pi-\operatorname{tg}\left(\frac{-C^{2}+2 C \beta\left(R^{2}+e^{2}\right)-\beta^{2}\left(R^{2}-e^{2}\right)^{2}}{2 e \beta\left(C-\beta\left(R^{2}+e^{2}\right)\right)}\right)\right)\right]=V .
\end{gathered}
$$

This value may be treated as maximal volume of liquid necessary for accurate balancing at the given geometric dimensions of cylindrical surface. Eq. (19) delivers that thickness of the layer in horizontal section having area $x_{3}=0$ opposite to imbalance shouldn't exceed $2 e$. Thus, only thin layer of liquid takes part in changing imbalance of revolving system. Finding this fact confirms experimental conclusion about highest effectiveness of using multichamber ABU. 
Hence if to set liquid $\mathrm{ABU}$ on the rotor, then at any angular speed $\omega$ of stationary motion of system liquid, which moves around absolute rotation axis under action of centripetal forces, flows towards to the side opposite to imbalance and tends to bring the common gravity center to rotation axis.

Value of the constant $C^{*}$ in the right part of the Eq. (16) is estimated by volume of liquid in the hollow of solid body (ABU).

Motion stability of heavy body with hollow partially filled with liquid demands minimum $W[38]$.

Condition of minimum $W$ in our case is narrowed to inequality:

$$
\left(C_{0}-A_{0}\right) \omega^{2}-M g x_{c 3}^{0}-a>0,
$$

where $A_{0}=B_{0}$ (because hollow is cylindrical), $A_{0}, B_{0}, C_{0}$ - general inertia moments of body having unperturbed motion:

$a=\rho g \int_{0}^{2 \pi} \int_{R_{1}-e \cos \varphi}^{R}\left[\frac{\omega^{2}}{g^{2}}\left(\frac{\omega^{2}}{2} r^{2}-C\right)+1\right]^{2} r^{3} d r$.

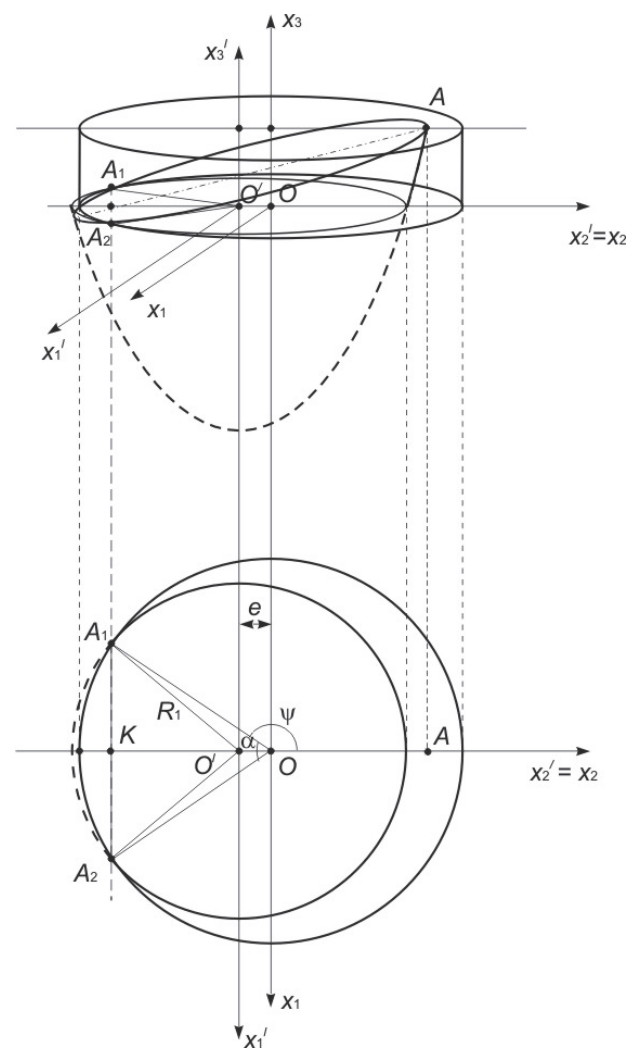

Fig. 13. Position of liquid in the hollow (case 3 )

Matching condition Eq. (11) with condition $\left(C_{0}-A_{0}\right) \omega^{2}-M g x_{c 3}^{9}>0$ of stability of uniform vertical rotation of solid body with liquid in case when its hollow is completely filled up with liquid shows that having free surface of the liquid destabilizes stationary rotation of the system.

Stability condition Eq. (21) can still be tolerated at high-speed rotation if hollow with liquid is oblate enough $\left(A_{0}<C_{0}\right)$ when the height of cylindrical hollow $h$ is much greater its radius $R$. 
Condition Eq. (21) is valid if liquid that fills the hollow has ultimate viscosity. In case considered here when liquid rotates together with body as one, liquid's viscosity has no effect on its motion.

In case of nonstationnary motion of hollow, which is partially filled with liquid, results look different: one should consider oscillation of free surface of liquid. Some simplifying assumptions [38] show that there's always risk of losing stability when natural frequency of any form of natural oscillation of liquid is close to nutation frequency of the body carrying liquid. Infinite range of forms of natural oscillation of liquid corresponds to infinite range of instability regions, however practice testifies that only a few first regions can be practicable. One may assume that internal friction damping natural oscillations of liquid neutralizes regions of instability of higher orders. Natural frequencies and hence regions of instability depend on how much the hollow is filled up.

Research of this space model describing steady motion of rotor with cylindrical hollow which is partly filled with liquid indicates that liquid auto-balancing is possible on steady motion of the system with any angular velocity.

However, offered model doesn't explain how exactly and under action of what forces liquid moves inside the ABU chamber.

Therefore, next step of the research was creating mathematical model for liquid motion in ABU chamber being under force of change of total system imbalance and bend.

\section{Physical content of fluid balancing}

In the absence of an $\mathrm{ABD}$, the value of the system imbalance remains unchanged in magnitude and direction, and since there is a relationship between the deflection of the shaft, the magnitude of the imbalance and the angular velocity of rotation:

$f=e_{0} \frac{\left(\omega / \omega_{k r}\right)^{2}}{1-\left(\omega / \omega_{k r}\right)^{2}}$

then, obviously, with the increase of the angular velocity $\omega$, the deflection $f$ is continuously increases by subcritical rotation of the shaft.

In the case of an $\mathrm{ABD}$, the imbalance of the system $\overline{e_{C}}$ consists of a stationary imbalance created by the unbalance $\overline{e_{0}}$ of the shaft, and an imbalance created by the liquid $\bar{e}$. At $\omega=0$, the liquid imbalance is zero (Fig. 14(a)).

At the initial moment of rotation, the unbalance of the shaft results in a deflection $f$, which coincides in the direction with the rotor imbalance (Fig. 14(b)). The centrifugal forces reject fluid to the walls of the ABD and its bulk is concentrated in the deflection, as shown by the established law of pressure distribution in the liquid. With an increase in the speed of rotation of the rotor, the direction of the deflection begins to lag behind the direction of the imbalance at the angle $\delta$ (in the presence of fluid in the ABD chamber, the angle $\delta$ should be deducted from the total imbalance of the rotor) (Fig. 14(c)). The liquid tends to occupy the position at the furthest point from the axis of rotation, namely in the trough, and moves along with it from the imbalance. This in turn leads to a change in the total imbalance of the system in terms of direction and magnitude (increases as the value of the total imbalance is defined as the geometric sum of the vectors $\overline{e_{0}}$ and $\bar{e}$ and depends on the angle between these vectors $\alpha$ ). This change leads to an increase in deflection, and an increase in the speed of rotation results in an increase in the angle $\delta$. The fluid following the deflection changes the total imbalance by increasing the angle $\alpha$ and decreasing the total imbalance (Fig. 14(d)). That in turn leads to a decrease in the value of the deflection. Consequently, with an increase in the speed of rotation due to the decrease $e_{C}$, we have a decrease in the deflection by magnitude even in the subcritical rotor rotation mode.

Because: 
$\operatorname{tg} \delta=\frac{2 n \omega}{\omega_{\text {res }}^{2}-\omega^{2}}$

then when the speed of rotation approaches the critical $\delta$, it increases and goes to $90^{\circ}$.

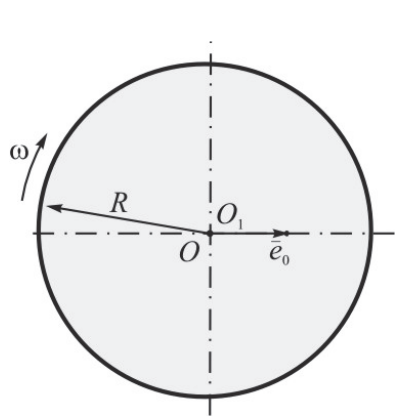

a)

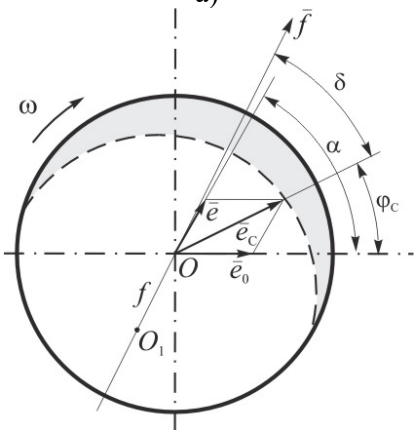

d)

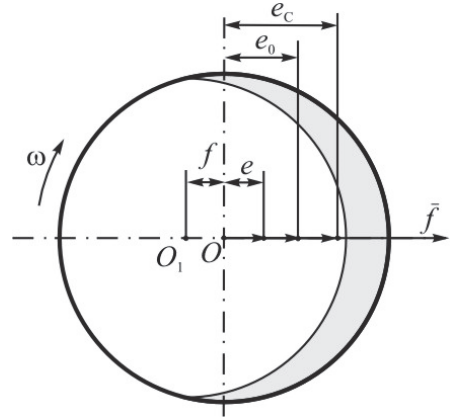

b)

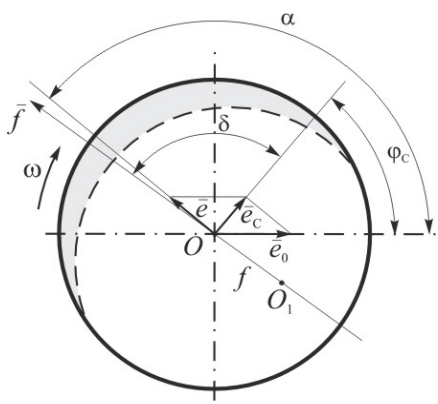

e)

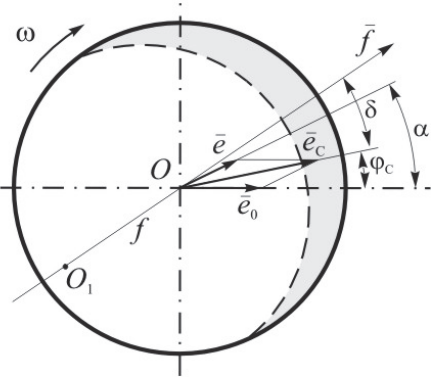

c)

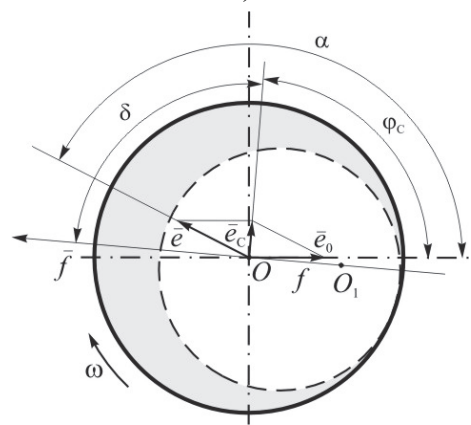

f)

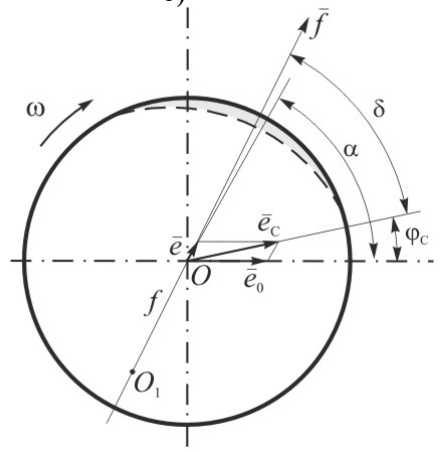

g)

Fig. 14. Vector model of process of auto-balance liquid

Because $\delta$ is the angle between the deflection and the total imbalance, and $\varphi C$ is the angle between the stationary and total imbalances, the angle between the deflection and the stationary imbalance when rotating the rotor with the angular velocity is equal to $90^{\circ}+\varphi C$ (Fig. 5).

In the case where the stationary imbalance and the imbalance created by the liquid are equal to the magnitude of $e_{0}=e$, then $\varphi C$ will be approximately equal to $\delta$ (Fig. 6). And so, the angle between the deflection and the stationary imbalance will be directed to $180^{\circ}$. Consequently, when passing the resonance speed of rotor rotation, the fluid along with the deflection of the shaft will be shifted to an angle of $180^{\circ}$ relative to the stationary imbalance. After this, there will be an automatic balancing of the shaft imbalance with the fluid, which will lead to a significant reduction in the rotor's vibrations. 


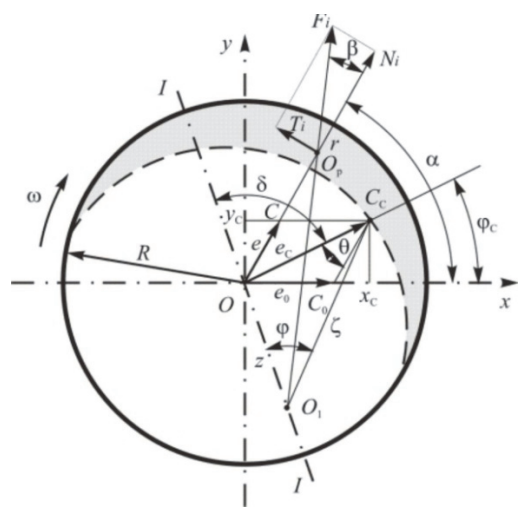

Fig. 15. Forces acting in the SBD in the presence of resistance

With a further increase in angular velocity, a balanced system is rotated without changing the relative position of the liquid and stationary imbalance.

With a small imbalance created by the liquid $e \ll e_{0}$, the total imbalance of the system will mainly be determined by the magnitude and direction of the stationary imbalance and will not change significantly with the increase of the angle $\delta$ (Fig. 7). Consequently, the size of the deflection of the shaft, due to the total imbalance of the rotor, will not be significantly reduced.

Therefore, the amount of liquid that creates a small imbalance in comparison with the stationary imbalance will not be sufficient to balance the system. To increase the imbalance generated by the liquid, it is possible by increasing its mass or the radius of the chamber ABD.

With constant parameters of ABD increasing the mass of fluid can achieve the equilibrium of liquid and shaft, in which we will have the maximum balance of the system.

A further increase in the mass of the liquid will not affect the imbalance created by it, and, consequently, on the balance of the system, because the liquid will be located concentrically around the axis of rotation, which in this case is already the main axis of inertia of the system.

The mass of fluid in the ABD can significantly affect the stability of its placement in relation to stationary imbalances. Since, due to the hydraulic properties of the liquid medium, which can arbitrarily change its shape under the influence of any small forces, the emergence of random minor external perturbations can significantly affect the location of its center of mass, and, consequently, change the state of equilibrium of the system. Moreover, the larger the mass of the liquid, the larger the direction and direction will be the change of the vector of its imbalance.

The basic idea is that at rotation velocity of the system $\omega<\omega_{\text {rot }}$ external resistance (friction forces in bearings, friction forces of cylinder against the air etc.) conditions bend plane (I-I) delay against total imbalance plane (OCc) for the phase angle $\delta$ (Fig. 15) and appearing in this condition tangential force $T$ of centrifugal inertial force $F$, and specific properties of liquid (fluidity, ability of any liquid volume to freely change its shape being forced by irrespectively low force, viscosity forces are considered only for quite fast motion when liquid displacements change quite fast) enable drawing liquid in ABU chamber to position that reduces the total imbalance of the system even at pre-critical rotation velocity. Analysis of geometric model (Fig. 15) shows that when external resistance is sufficient liquid that is in stable balance can balance rotor at pre-critical angular velocity.

It will be shown analytically.

Total rotor eccentricity with liquid is defined as:

$e_{c}=\sqrt{x_{c}^{2}+y_{c}^{2}}=e \sqrt{1+2 k \cos \alpha+k^{2}}$,

where $k=D_{0} / D-$ relation of rotor and liquid imbalance.

Analysis of geometric model (Fig. 15) shows that balance condition that doesn't take into 
account tension forces is missing tangential force: either $\operatorname{tg} \beta=0$, or:

$f\left[\operatorname{tg} \alpha \cos \left(\delta+\varphi_{c}\right)-\sin \left(\delta+\varphi_{c}\right)\right]=0$.

The last equation is decomposed into two conditions (zero index corresponds to angles for liquid balance $(\beta=0))$ :

$f=0$,

$\operatorname{tg} \alpha_{0} \cos \left(\delta+\varphi_{0_{c}}\right)-\sin \left(\delta+\varphi_{0_{c}}\right)=0$.

Condition Eq. (23a) corresponds to no deflection condition that contradicts condition of the problem of elastically strained rotor. If liquid is in balance:

$\operatorname{tg} \alpha_{0}=\operatorname{tg}\left(\delta+\varphi_{0_{c}}\right)$,

what comes from condition Eq. (23b).

Taking into account values of $e, e / e_{0}, e c$ and $\theta=\alpha-\varphi c[4]$ :

$\sin \alpha_{0}=\frac{1}{k} \sin \delta \sqrt{1+2 k \cos \alpha_{0}+k^{2}}$.

It appears from Eq. (24) that if external resistance is missing in the system $(\delta=0, k \neq 0)$ liquid balance coincides by the angle with rotor imbalance $(\alpha=0)$, what is approved by the conclusion that liquid increases imbalance in system without external damping.

The expression for angle $\alpha_{0}$ corresponding liquid balance $(\beta=0)$ is obtained through algebraic transformations of Eq. (24) when $\delta \neq 0, k \neq 0$ :

$\alpha_{0}=\pi-\arccos \left(\sin ^{2} \delta+\sqrt{\left(\sin ^{2} \delta-1\right) \cdot\left(\sin ^{2} \delta-k^{2}\right)}\right)$.

Analyzing Eq. (25) gives positive arccosine argument and that's why even in pre-critical frequency of the rotor when $\delta \in(0, \pi / 2)$ angle $\alpha_{0} \in(\pi / 2, \pi)$.

Effectiveness of balancing is characterized by relation of misalignment with rotational axis of mass center of the system with liquid and without it $\lambda_{0}$ :

$\lambda_{0}=\frac{\sqrt{1+2 k \cos \alpha_{0}+k^{2}}}{k}$

if $1+2 k \cos \alpha_{0}+k_{2}=0$ balance will be complete, therefrom the only solution will be $k=1$ if $\cos \alpha_{0}=-1$, and if $\alpha_{0}=\pi$. Hence, the greatest balancing effect will be reached when imbalance of liquid that is involved into balancing is close to initial imbalance of the rotor $(k \approx 1)$. Liquid which takes part in balancing makes concentric circles what has no influence on total imbalance of the system.

Eqs. (24) and (26) contain relations of angular velocity to critical one, external resistance factor, relation of initial imbalance to liquid imbalance and relative dimensions of ABU. Obtained analytical relationships between these parameters allow solving applied problems to select optimal parameters of liquid $\mathrm{ABU}$ for particular rotor system, find effect of varying parameters of the system rotor $-\mathrm{ABU}-$ liquid on effectiveness of balancing.

Internal friction between layers of real liquid hinders its motion and creates delay effect of viscous fluid engaging auto-balancing. Tangential component of centripetal force $T$ is decreasing by decreasing angle $\beta$ and in some moment can't overcome friction force. That's why viscosity makes balancing incomplete. 
Results of theoretical researches for vertical rotor arrangement are also applied for the case of horizontally arranged rotor under the mode of liquid capture (when sum of centrifugal force and friction force are bigger then gravity).

Theoretical results were proved by experimental researches.

\section{Experimental part}

\subsection{Case of vertically arranged rotor}

Equipment, apparatus and methods of research of working bodies conduct in SBD.

Research installation is the system rotor-SBD with the vertical axis of rotation.

1. Breadboard model of automatic balancing device. For the investigation of working bodies conduct in the automatic balancing device in under-resonance and over-resonance zones of the rotor rotation a breadboard model of automatic balancing device was elaborated and made of optically-transparent material, which enables to keep up with the work of the SBD in all modes of operation visually or by the help of modern means of video recording.

The breadboard model of SBD is the ring with external diameter of $\varnothing 400 \mathrm{~mm}$, two concentric partitions with diameters of $\varnothing 300$ and $\varnothing 200 \mathrm{~mm}$ which form three concentric chambers for arrangement of working bodies. General appearance of the elaborated breadboard model of SBD is shown in Fig. 16.

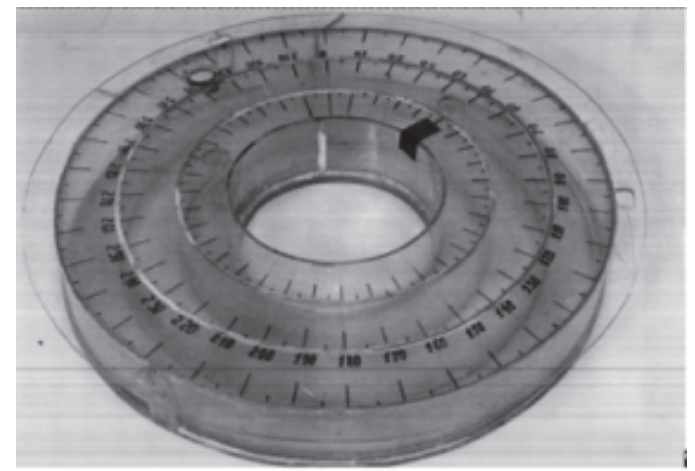

Fig. 16. General appearance of the SBD breadboard model

All three chambers are isolated from each other which allow using simultaneously both fluid and rigid working bodies in the SBD. Colored water in different colors as fluid working body was used for each chamber of the SBD. Metal and plastic balls with diameters of 6, 8, 12 and $16 \mathrm{~mm}$ were used as rigid working bodies. Metal and plastic balls with diameters of $1 ; 1,5 ; 2 ; 4 \mathrm{~mm}$ were used as dry substances. The scale with scale factor of 5 grades was marked on the surface of the ring edge to determine the places of arrangement of working bodies in the SBD during the experiment. The position of misbalanced mass (misbalance) is fixed on this scale with the help of a thin color strip, of size $10 \times 20 \mathrm{~mm}$ with adhesive layer on one of its surfaces. The bottom of the SBD is mat to increase the contrast of visual observation of the working bodies' position. Fixing rods are made on the external side surface of the SBD at angle $120^{\circ}$ to fix the breadboard model on the rotor.

2. The construction of the research installation. The research installation is a rigid cantilever vertical rotor, resiliently hung to the frame.

In order to provide sufficient rigidity of both the rotor and the SBD, fixed on it, the first one is made in the form of a hollow cylinder of stainless steel plate with the bottom on one side which is rigidly fixed on the axis of rotation.

Rotor axis through swinging bearings is connected with a rigid platform which is joined to the frame of the installation by means of four resiliently damper brackets (typical meaning of the 
proper vibration frequency of all the system on the brackets is equal to 2-2,5 Hz). The SBD breadboard model is set on the free end of the hollow cylinder (further - a drum). It is put into the drum and is fixed to it on its external diameter. Due to this, necessary rigidity of fixing of the SBD on the rotor and conformity of axes are ensured. The SBD breadboard model has an inner aperture, enough for free access to the inner cavity of the drum which enables to change the rotor misbalance without demounting the SBD both on the place of arrangement and on the size by means of fixing to the inner side the given load which is additionally held by forces during experiments.

Such construction of rotor provides necessary level of security of personnel against occasional tear of the load-misbalance or weakening of the SBD fastening.

Rotor drive is operated by a synchronous electric motor with power on the shaft equal to $180 \mathrm{~W}$ through the lowering V-belt transmission. The motor is fed with the alternative current which power can be changed in a wide range with the help of automatic transformer with the operation system. Due to it the installation enables to rotate the rotor at various frequencies in scope of 0 , 5-30 Hz (30-1800 rpm), to set different angular accelerations at rotor dispersal, to stop at under-resonance, resonance and over-resonance zones of the rotor rotation for a given period of time with the aim of detailed investigation of the SBD operation.

For a high-velocity video recording the inner surface of the drum was lightened by incandescent lamp of $200 \mathrm{~W}$, arranged with the help of bracket in the inner cavity of the rotor. To obtain steady light the inner surface of the drum was covered by mat color.

3. Measuring, registering equipment and preparation of installation Demonstrating auto balancing process as a whole, high-speed video shooting of working bodies' behavior is suggested to be used. For this aim, we have elaborated a testing stand to analyze auto balancing process with applied video and computer techniques. Block scheme of the stand is found at Fig. 17.

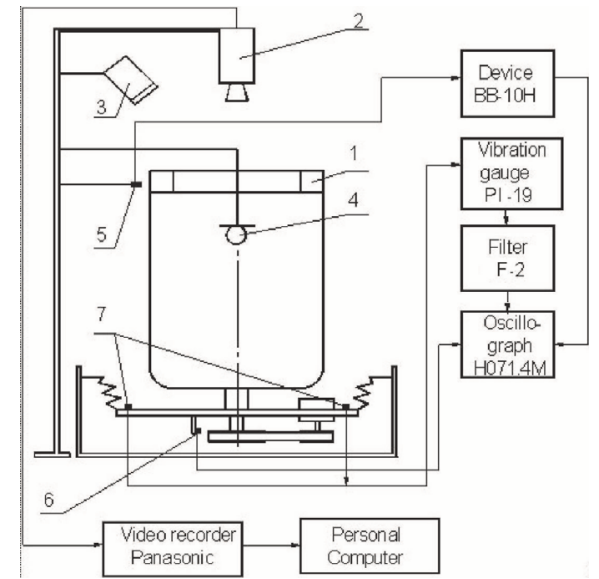

Fig. 17. Bloc-scheme of the equipment connecting: 1 - the SBD breadboard model, 2 - video camera, 3 - stroboscope, 4 - incandescent lamp, 5 - induction data unit, 6 - revolutions data unit, 7 - accelerometers

The velocity of the rotor rotation was measured by means of the noncontact marker of revolutions elaborated on the basis of Hall's data unit.

Accelerometers were installed on the rotor platform. Signal from the accelerometers was carried to vibration gauges PI-19 where it was integrated twice after intensification in order to obtain amplitudes of the shifting of the system platform-rotor.

Amplitudes of rotor vibrations proper were measured by means of the elaborated optical electronic device.

Video camera was placed vertically over the SBD on the axis of rotation of the rotor. Video recording of balancing was carried out synchronically with the recording of vibrations level of the rotor. 
The most interesting and representative slides were recorded in the computer in BMP format and were processed further with the help of graphic packages ACAD and Photopaint.

4. Methods of high-velocity video recording of the SBD operation.

The methods of high-velocity video recording of the SBD operation on the vertical rotor consist of three stages: preparatory, investigation of transition modes, investigation of stationary and quasi-stationary modes.

4.1. Preparatory stage includes:

- Balancing of rotor with the SBD breadboard model in the absence of working bodies in it;

- Selection of dimension and place of arrangement of the rotor artificial disbalance;

- Determination of optimum volume or quantity of working bodies in the SBD;

- Determination of optimum light of the SBD;

- Determination of necessary movement velocity of magnetic videotape;

- Determination of optimum position of video cameras (choice of sight angle and distance to the SBD;

- Test starting of all system for coordination of interaction of its separate sections.

Balancing of the rotor with the SBD breadboard model in the absence of working bodies in it was carried out using the method of rounds of test load at the working frequency of the rotor rotation.

Meaning of the rotor artificial misbalance was determined at its working frequencies of rotation in the following way. The mass of artificial misbalance was increased gradually with step of $5 \mathrm{~g}$ from starting to start and the rotor vibration level was measured.

Critical maximum meaning of misbalance mass was considered to be that one at which vibrations amplitude reached $35 \mathrm{~mm}$ (higher meanings in the given construction of the installation cause impacts of moveable elements with the frame) or when the electric motor is not able to increase revolutions during transition through system resonance and rotor "is suspended" at the critical rotation frequency.

The meaning of misbalance mass obtained according to these criteria, reduced for $5 \mathrm{~g}$ (the size of one step) and which is equal to $30 \mathrm{~g}$ was considered as the initial maximum meaning for further investigation.

Criterion of optimum volume of working fluid or of quantity of working bodies in the SBD was considered to be visibility of the process of automatic balancing on all revolutions of the rotor, i.e., when the position of working bodies or fluid in relation to the place of arrangement of artificial misbalance was clearly visual in the light of stroboscope and on the videotape. Determination of such meanings of volume or quantity was carried out by means of experiments.

As a result of such investigation volumes fluid (colored water) were obtained which constituted $0.45,0.3$ and 0.251 for external, medium and internal chambers of the SBD correspondently.

Maximum quantity of working bodies separately for each of diameters of balls was obtained in the same way.

4.2. Video recording of movement of working bodies in the SBD in stationary modes of the rotor rotation.

Resonance angular velocity of the rotor rotation is equal to $\omega_{\text {res }}=12^{-16} \mathrm{~s}^{-1}$. For the investigation of working bodies conduct in the SBD at under-resonance, resonance and over-resonance frequencies of the rotor rotation, the latter was given angular velocity of $0.5 \omega_{\text {res }}$ and $0.75 \omega_{\text {res }}$ (under-resonance zone), $\omega_{\text {res }}$ (resonance zone), $0.5 \omega_{r}$ and $\omega_{r}$ (over-resonance zone), where $\omega_{r}$ is equal $90 \mathrm{~s}^{-1}-$ nominal frequency of the rotor rotation.

Video camera and equipment were switched on in the period of time equal $\Delta t=10^{-15} \mathrm{~s}$ of the rotor delay at the determined frequency of rotation when position of working bodies relatively to marks on the SBD frame stabilized.

Video recording at each of the above mentioned frequencies had been carried out for 20-30 s - time sufficient for recording not less than 10-20 full revolutions of the rotor. 
Experimental research of the SBD operation and its results.

Fig. 4 shows the most typical and representative computer slides of the video recording of the fluid position in the SBD at under-resonance (Fig. 18(a), $\omega=8 \mathrm{~s}^{-1}$ ), resonance (Fig. 18(b), $\omega=12.5 \mathrm{~s}^{-1}$ ) and over-resonance (Fig. 18(c), $\omega=90 \mathrm{~s}^{-1}$ ) frequencies of the rotation of the rotor with the SBD.

Analysis of these and other slides shows that fluid compensates rotor misbalance at under-resonance, resonance and over-resonance frequencies of its rotation. Theoretical explanation of the fluid's position in relation to misbalance, witnessed by video-recording, can be given if one takes into consideration of hydromechanical properties of the fluid and damping of the rotor system.

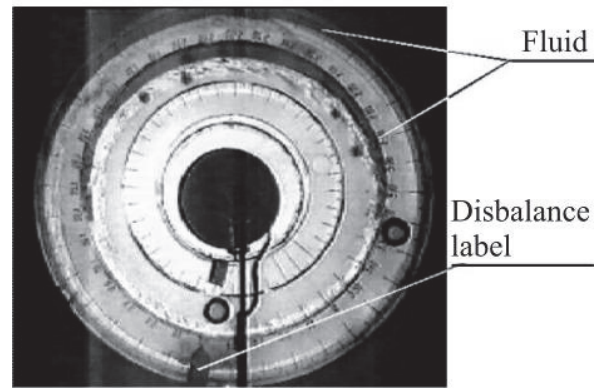

a)

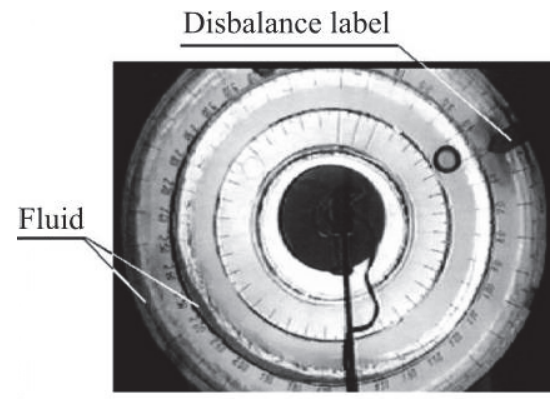

b)

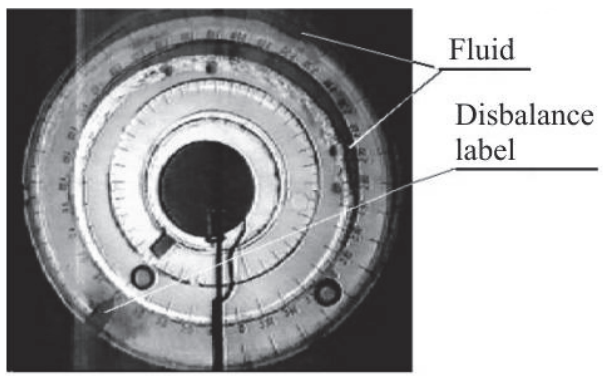

c)

Fig. 18. Video shots: a) before resonance, b) at resonance, c) beyond resonance

\subsection{Case of horizontal rotor}

For simplification of understanding of results presented in the article we will remind on which physical phenomena existing theories of self-balancing by passive fluid SBD are based. For this we will examine weightless horizontal bank with self-balancing disk with mass $M$ set up on it (Fig. 19). Rotation axis (bearing axis) is marked as $O_{1} O_{1}$ (Fig. 19), self-balancing disk center of mass $-\mathrm{C}$, geometric center of disk - $\mathrm{O}$. In composure state geometrical center $\mathrm{O}$ lies on bearing axis $O_{1}$ and center of mass $\mathrm{C}$, if there is unbalanced state, is shifted on $e$ distance. In this case rotor's imbalance equals $\bar{D}=\bar{e} M$. Rotor passing through resonance has next theoretical explanation. After the beginning of the movement as a consequence of centrifugal force action $F_{i}=M e \omega_{2}$, occurs bending deflection of rotor $\mathrm{y}$, which depends on hardness of rotor $\mathrm{c}$ and it is increased when angle speed is increasing according to the formula $y=M e \omega^{2} /\left(c-M \omega^{2}\right)$. When angle speed is approaching to resonance the value of bending deflection considerably increases and tends to $+\infty$ (Fig. 20(a)) [36].

After passing critical speed (resonance) self-centering of rotor, decreasing of bending deflection from $-\infty$ to $-e$ happens. It is worth noting that if the speed is critical changing of bending deflection from $+\infty$ to $-\infty$ happens, and also relative position of rotor's points mentioned above is changed. (Fig. 19(c)). So far as fluid in self-balancing device will be trying to locate in 
most distant from the rotation axis place, that is it will coincide with direction with bending deflection, summary center of mass of Cc disk-fluid system will be shifted to side of eccentricity increasing (imbalance). That is self-balancing by passive fluid SBD is effective on above resonance rotation frequencies [36].

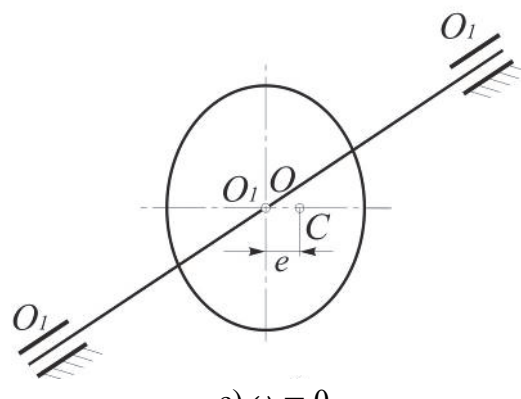

c) $\omega=0$

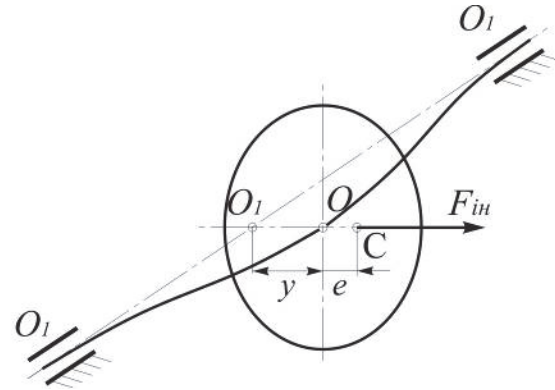

b) $\omega<\omega_{\text {res }}$

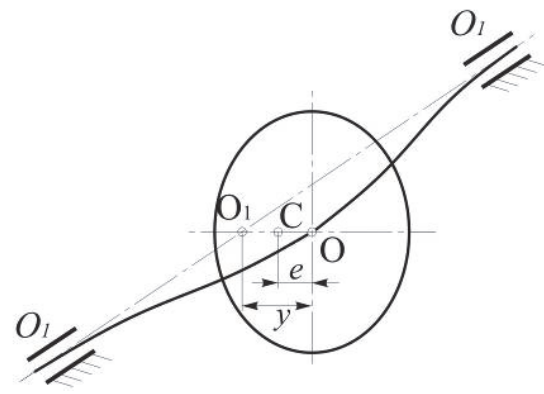

c) $\omega>\omega_{\text {res }}$

Fig. 19. Bank with self-balanced disk, which is set up on footholds

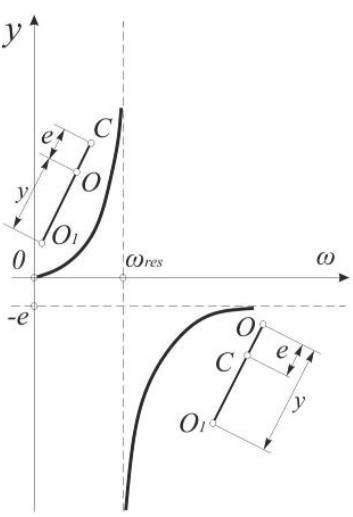

a)

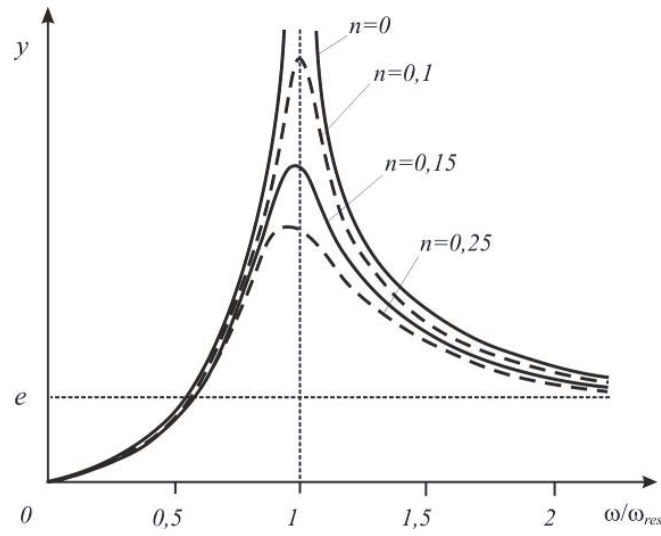

b)

Fig. 20. a) Graph of rotor's bending deflection dependence on angle rotation speed;

b) AFC of rotor when damping coefficients are different

When studying already existing theoretical points of rotor self-balancing authors had wish to examine what is happening with rotor in resonance zone, as bending deflection cannot be endless and also instant change of rotor's bending deflection from $+\infty$ to $-\infty$ is impossible. While studying works about researches of rotor vibration when passing through resonance it was determined that real AFC of rotor systems on which forces of viscous (Fig. 20(a)).

Vibration amplitudes are equal not to infinity but friction influence (Fig. 20(b)), are different from theoretical specific values which are indicated by damping coefficient. With damping 
increasing not only vibration decreasing will happen but also shifting of maximum in the zone of lower frequencies of rotor rotation.

In addition, authors pay attention to the fact that change of bending defection direction relatively to rotation axis occurs on resonance. Relative position of eccentricity and bending deflection are also changed (Fig. 19(c)). That is if the distance from rotation axis $O_{1}$ to center of mass $C$ was indicated as sum $y+e$ in below resonance zone, then this distance $\left(O_{1} C\right)$ is indicated as disparity $y-e$ in above resonance zone.

Authors made an assumption that change of relative position of bending deflection and eccentricity happens not instantly at the resonant speed, but progressively and begins at below resonance frequencies of rotation and it happens like that. When approaching the resonance in consequence of damping action (external and internal friction forces) the lag of bending deflection plane from centrifugal force is happening, because rotor is not able to overcome large distance in limited time. Eccentricity direction begins to pass ahead plane of bending deflection on phase angle $\delta$ (Fig. 21), which increases with speed increasing. After resonance angle between eccentricity and bending deflection direction becomes $180^{\circ}$. When rotor is passing resonance, it is rotated both around bearing axis $O_{1}(\omega)$ and geometric center $O\left(\omega_{1}\right)$, that is bank twisting is happening. It means that redistribution of tensions, mutual change of squeezing and extension tensions happen in fibers of rotor.

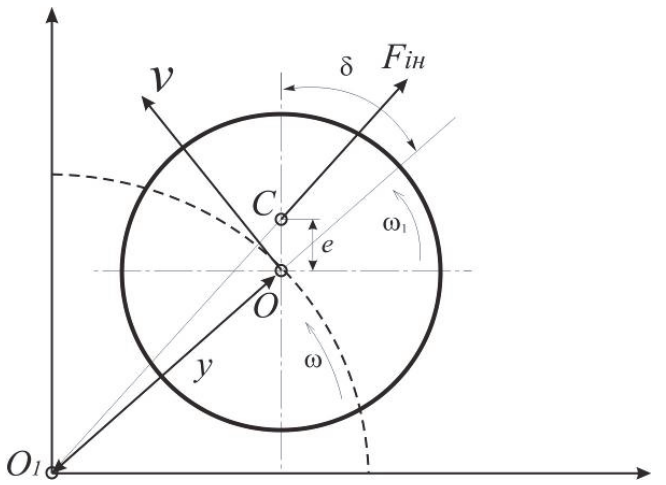

Fig. 21. Rotor movement when passing resonant speed

If fluid exists in self-balancing disk under influence of centrifugal force it tries to take up a position in most distant place from rotation axis, which is saved in direction of bending deflection. It leads to changing of summary system imbalance, which consist of rotor imbalance and fluid imbalance in size and in direction. So far as for certain constant angle speed lag of bending deflection from summary imbalance $\delta$ is constant, with changing summary imbalance direction the position of bending deflection relatively to initial eccentricity (that is angle of lag relatively to initial imbalance is increased). Fluid follows the bending deflection, and changes again summary imbalance, which leads to further increasing of lag angle $\delta$. Process can continue until angle will not acquire $180^{\circ}$, and it will lead to decreasing of bending deflection value and changing of its position relatively to $e$.

For verification of these assumptions authors made a decision to conduct experimental studies on improved laboratory plant for demonstration of critical speeds of bank. When working in educational laboratories of the university self-balanced device (SBD) was made and set up on bearing. Bank of the equipment if located horizontally and bounded with bank of electric motor through flexible connector. Bank has two supports on frictionless bearings.

Created SBD presents a disk with external diameter $150 \mathrm{~mm}$ made of steel, with two circular isolated one from another camera with diameters 100 and $130 \mathrm{~mm}$, for placing in them working mediums, and set up imbalance. For possibility of observation of working mediums behavior front wall (cover) of self-balancing device is made of optically transparent material - Plexiglas. This 
SBD was fastened by collet clamp, provided in construction of SBD. General look of SBD is given on Fig. 22.

Rotor's drive is made by electric motor of alternating current with power 180 watt though muff coupling. Due to autotransformer and control system the plant has possibility to define rotor rotation with different frequencies, which are in range from 0 to $5000 \mathrm{rpm}$. Also, it gives possibilities to make acceleration with different age accelerations of the rotor and allows it to rotate with constant angle speed in below critical, critical and above critical zones.

Unbalancing the rotor was made with the help of load (imbalance) fastened with the help of threaded connection on back butt of SBD. SBD has two geometrically isolated cameras, which helps to use fluid as working medium in each of them simultaneously and separately. Colored water was used as a working medium.
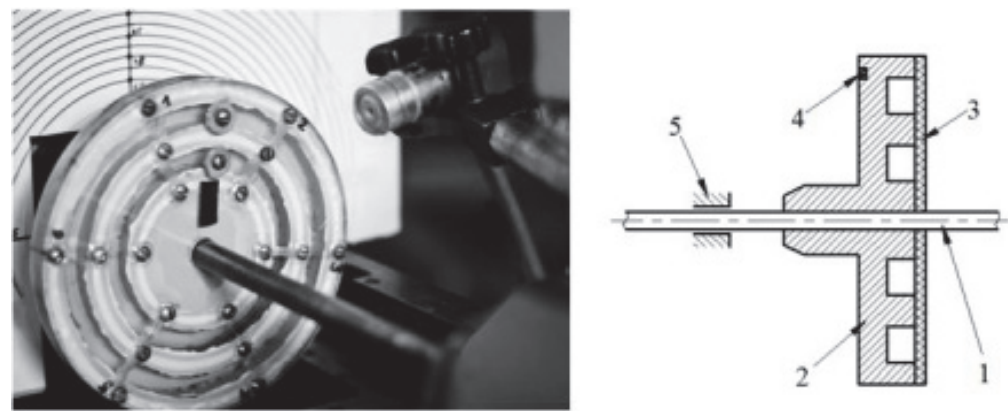

Fig. 22. Scheme of mounting SBD: 1 - bank, 2 -SBD, 3 - cover, 4 - imbalance, 5 - bearings

Position of unbalanced mass (eccentricity) was marked on front butt of SBD with the help of thin black stripe with dimensions $(10 \times 20) \mathrm{mm}$ and sticky layer on one of its surfaces.

Created plant allowed to make experimental researches of self-balancing of the rotor with horizontal rotation axis and to observe fluid behavior in SBD.

Research of self-balancing process and fluid behavior in SBD were made on described plant with using next special devices:

- Video camera Panasonic AG-DVX100BE;

- Lamps with halogen lamps (power - 1000 watt each);

- Inductive sensor, marker of turns;

- ADC (analog-to-digital converter);

- Notebook computer.

When video filming is fast it is necessary to have high illumination. That is why two lamps with halogen lamps of power 1000 watt each were used for illumination of the object. Lamps were directly set up close to object of the filming. Video camera was placed horizontally, frontally and in the direction of rotor rotation axis.

Methodology of conducting researches of the SBD work on horizontal rotor with the help of speed filming consisted of:

- Rotor balancing with SBD model without fluid in it;

- Matching value and place of artificial imbalance of the rotor;

- Determination of optimal fluid volume in SBD;

- Synchronous recording of the fluid position in SBD and signal of vibration detector.

Rotor balancing with SBD model without fluid in it was made by using cargo workaround method [36] on working frequency of rotor rotation. Then plant was turned on and vibration recording on computer were made in conditions of smooth rotor acceleration from 0 to $2000 \mathrm{rpm}$. After rotor of the plant was unbalanced by mounting cargo with mass $10 \mathrm{~g}$. on the butt of SBD with the help of threaded connection. Cargo on SBD was marked with the help of color stripe, then the plant was turned on and vibration recording on computer were made in conditions of smooth rotor acceleration from 0 to $2000 \mathrm{rpm}$. 
2.1. SYSTEM ANALYSIS OF AUTOMATIC BALANCING (SELF BALANCING) MACHINE ROTORS WITH LIQUID WORKING BODIES ON THE EXAMPLE OF DRUM TYPE WASHING MACHINES. ILONA DRACH, VILEN ROYZMAN, VITALII TKACHUK, ANDRII GOROSHKO

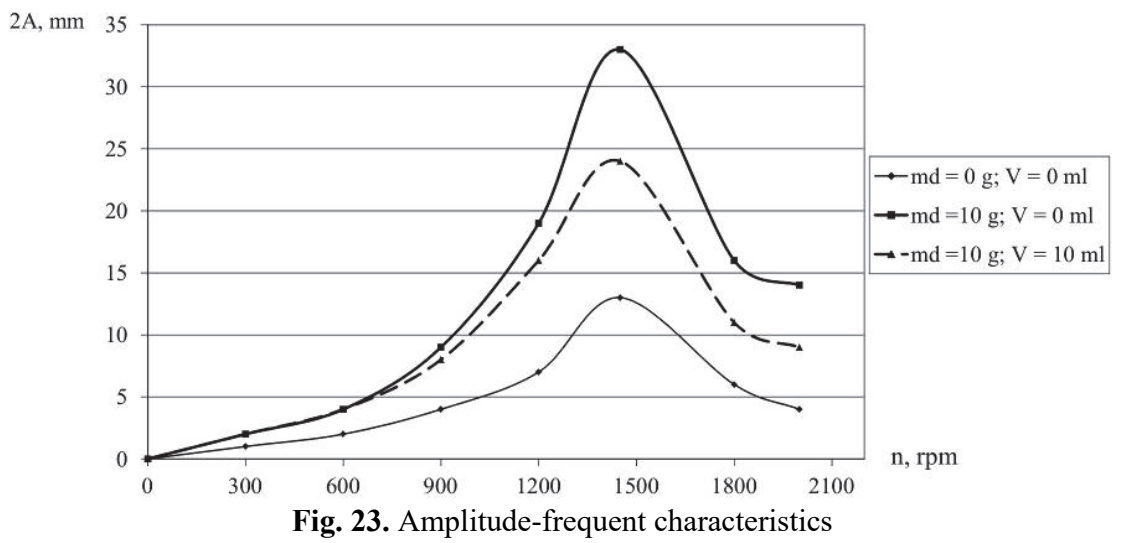

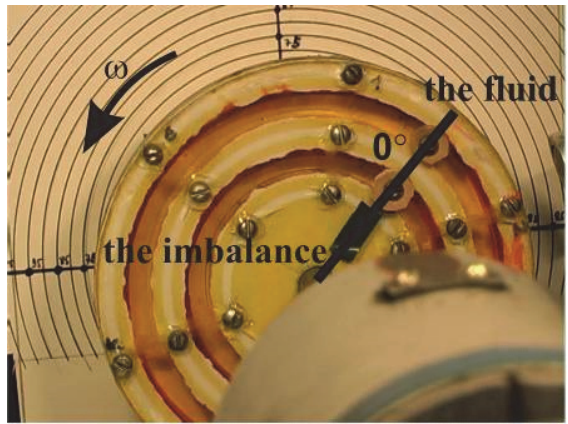

a)

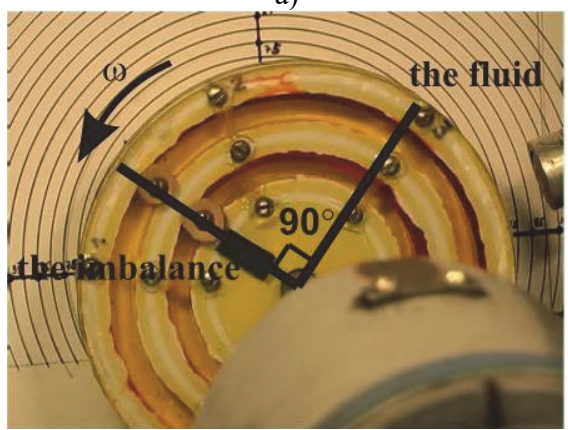

c)

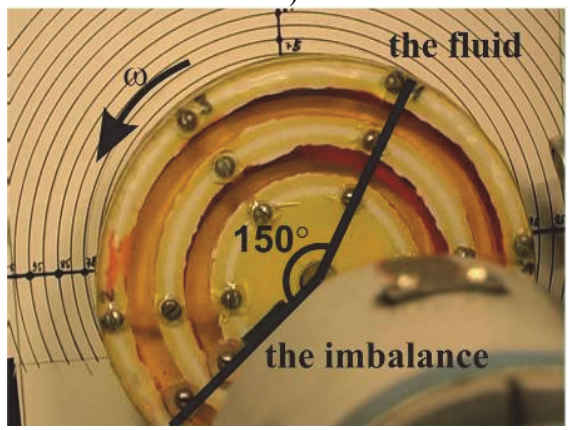

e)

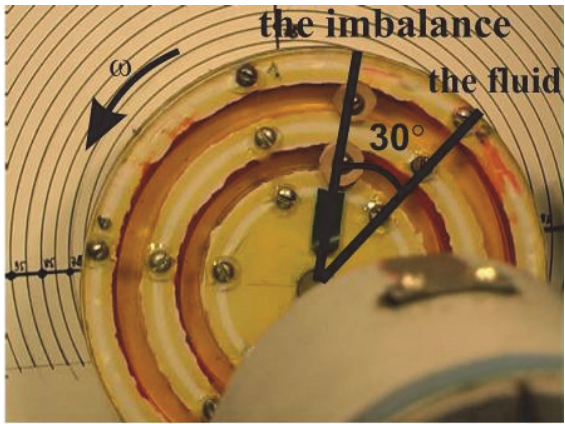

b)

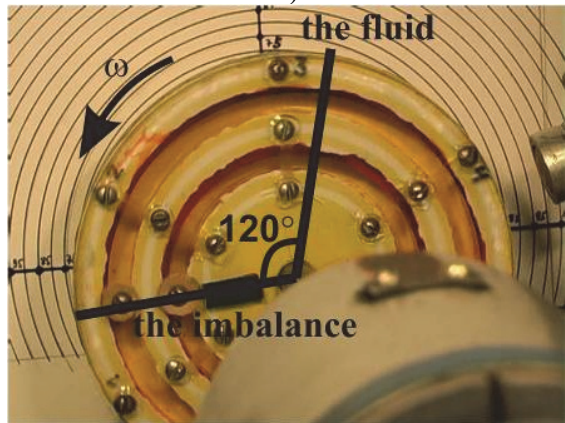

d)

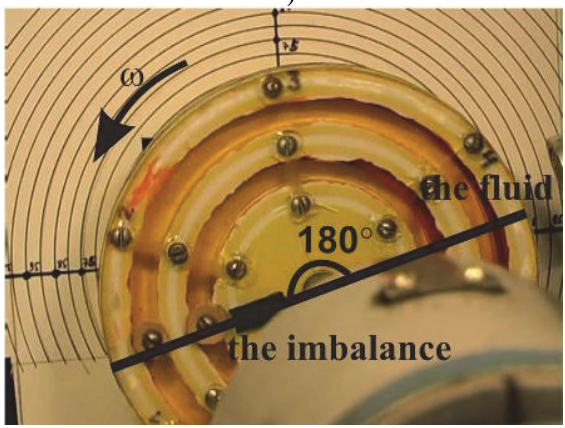

f)

Fig. 24. Shots of video recording of fluid position in SBD when passing through resonance: a) $\delta=0^{\circ}(n=600 \mathrm{rpm})$; b) $\delta=30^{\circ}(n=900 \mathrm{rpm})$; c) $\delta=90^{\circ}(n=1000 \mathrm{rpm})$; d) $\delta=120^{\circ}(n=1100 \mathrm{rpm})$; e) $\delta=150^{\circ}(n=1200 \mathrm{rpm})$; f) $\delta=180^{\circ}(n=1300 \mathrm{rpm})$ 
By filling in each camera of SBD with $10 \mathrm{ml}$ of water, without changing position or mass of imbalance, the plant was turned on again. Rotor vibrations were recorded on computer in text file in conditions of smooth rotor acceleration from 0 to $2000 \mathrm{rpm}$. Simultaneously were made video record of fluid behavior in SBD.

Amplitude-frequent characteristics of rotor when passing through resonance are illustrated on Fig. 23. Shots of created video film with angles of fluid progressive shift aside opposite to imbalance are shown on the Fig. 24.

\section{Conclusions}

In Article theoretically obtained and experimentally verified main requirements for the structure of washing-machines. On the basis of investigations of mathematical model vibration of washing machines a number of recommendations for the design of washing machines were provided:

- Center of mass of the tank needs to be the drum's rotation axis;

- Rotation axis of the drum needs to be the main axis of tank's inertia;

- There needs to be a coincidence between drum's and tank's center of mass;

- Rigidity center of elastic supports system needs to coincide with tank's center of gravity, and main rigidity axes - with main central axes of tank's inertia;

- Main axes of constants of viscous friction need to coincide with main central axes of tank's inertia.

It was also investigated that bearings are the most widespread and most vulnerable elements of any rotary mechanism. Bearings shall spatial fixing rotors and accept most of the static and dynamic forces arising in the mechanism. Therefore, the technical condition of bearings is the major component determining working capacity of the whole mechanism. In article researches, a stock of working capacity of the of rolling and plain bearings by means of the experimental stand are described.

The current work is devoted to system research of the fundamental natural phenomenon - rotor self-balancing with the help of passive auto-balancing units (ABD) which look like cavity chambers, partly filled with working media (liquid) being passive regulators of direct action which don't need supply of energy and control system for correcting masses movement.

The results of experimental and theoretical research of fluid behavior in passive self-balancing devices, which are installed in rotors with vertical and horizontal rotation axes when passing the resonance, are given in this article. Received relations showed that automatic balancing by fluid is effective for elastically deformed rotors or (and) rotor on elastic supports, where exists the difference in phases between the direction of centrifugal force and flexure (or movement) of the rotor. As experimental results showed, this difference in phases occurs when rotor reaches resonance speed and increases up to $180^{\circ}$ when passing the resonance. In this case the tangential force occurs, under the influence of which the fluid moves to the side of flexure, opposite to imbalance, and further equilibration of the rotor already at resonance rotation frequency. The influence of gravity under conditions of horizontal arrangement of SBD rotation axis, is in decreasing of tangential component, which moves fluid in position opposite to imbalance and leads to decreasing of balancing accuracy in comparison with vertical rotation axis.

\section{References}

[1] Racic Z., Racic M. Practical approach for solving vibrations of large turbine and generator rotors reconciling the discord between theory and practice. Proceedings of the 10th International Conference on Vibrations in Rotating Machinery, Graz, Austria, 2017.

[2] L'vov M., Uryev E. Use of modal weight sets for residual modal unbalance assessment of high speed balance quality of flexible rotors. Proceedings of the 10th International Conference on Vibrations in Rotating Machinery, London, 2012. 
[3] Chen Hai-Wei, Ji Wei-Xi, Fan Sheng-Yao A method for vibration isolation of a vertical axis automatic washing machine with a hydraulic balancer. Journal of Mechanical Science and Technology, Vol. 26, Issue 2, 2012, p. 335-343.

[4] Chen Hai-Wei, Zhang Qiu-Ju Stability analyses of a vertical axis automatic washing machine with a hydraulic balancer. Mechanism and Machine Theory, Vol. 46, Issue 7, 2011, p. 910-926.

[5] Chen Hai-Wei, Zhang Qiu-Ju Dynamic analysis and design of a balancer for a three-column centrifuge. Shock and Vibration, Vol. 2016, 2016, p. 7957821.

[6] Jung C. H., Kim C. S., Choi Y. H. A dynamic model and numerical study on the liquid balancer used in an automatic washing machine. Journal of Mechanical Science and Technology, Vol. 22, Issue 9, 2008, p. 1843-1852.

[7] Alyokhin S., Petrosov S., Zheltushkin L., Alyokhin A. Self-balancing equipment of drum-type washing machines. Young scientist USA, Vol. 1, Issue 5, 2014, p. 9-11.

[8] Royzman V., Bubulis A., Drach I. System analysis of automatic balancing (self-balancing) machine rotors with liquid working bodies. Solid State Phenomena, Vol. 147, Issue 149, 2009, p. 374-379.

[9] Rankine W. J. On the centrifugal whirling of shaft. The Engineer, Vol. 27, 1869, p. 249.

[10] Stodola A. Dampf und Gasturbinen. Auflage Edition, Springer, Berlin, 1924, p. 1157.

[11] Royzman V., Drach I., Bubulis A. Movement of working fluid in the field of centrifugal forces and forces of weight. Proceedings of the 21st International Scientific Conference: Mechanika, Kaunas, 2016.

[12] Kushul Ya M. Self-Oscillations of Rotor. AS USSR, 1963, p. 168.

[13] Anikeev G. I. Transitional Almost Periodical Oscillations of Rotors. Nauka, Moscow, 1979, p. 136.

[14] Ragulskis K. M., Ionushas R. D., Bakshis A. K. Vibrations of Rotor Systems. Mokslas, Vilnius, 1976, p. 413.

[15] Siminovskiy V. I. Steadiness and Nonlinear Oscillations of Rotors of Centrifugal Machines. Kharkiv University, Kharkiv, 1986, p. 120.

[16] Kalmens Ya V. Provision of Vibrostability of Rotor Machines on the Basis of Similitude and Modeling Methods. RAN StP., 1992, p. 372.

[17] Kelzon A. S., Malinin L. M. Controlling Oscillations of Rotor. Polytechnics, 1992, p. 118.

[18] Kim Keunjoo, Park Seungchul, Kim Jongryong Balancing Unit and Laundry Treatment Apparatus. Patent No. EP3085827A1, LG Electronics, 2016.

[19] Ostdiek Stephen D., Vishal Verma Laundry Treating Appliance with Balancing System. Patent No. US20120144598A1, Whirlpool Corporation, 2012.

[20] SKF-Automatic balancing units, http://www.skf.com/us/industry-solutions/portable-powertools/applications/grinders-planners-and-sanders/automatic-balancing-unit/index.html.

[21] Kim Yong Kwon, Lee Kyu Chai, Ko Hong Seok, Lee Sang Up Washing Machine, Inner Tub of Washing Machine and Balancer Connection and Assembly Method Thereof. Patent No. US8984918B2 Samsung Electronics Co., 2015.

[22] Leblanc M. Automatic balancer for rotating bodies. Patent No. US1209730, Expl Des Procedes Westinghouse Leblanc Sa, 1916.

[23] Thearle E. L. A new type of dynamic-balancing machine. Transactions ASME, Vol. 54, 1932, p. 131-141.

[24] Den Hartor J.-P. Mechanical Oscillations. Phismathgiz, Moscow, 1960, p. 574, (in Russian).

[25] Dyer J. B. Domestic Appliance. Patent No. US2375635A, Motors Liquidation Co, 1945.

[26] Kasahara M., Kaneko S., Oshita K., Ishii H. Experiments of liquid motion in a whirling ring. Proceedings of the Dynamics and Design Conference, Tokyo, Japan, 2000.

[27] Nakamura T. Study on the improvement of the fluid balancer of washing machines. Proceedings of the 13th Asia-Pacific Vibrations Conference, New Zealand, 2009.

[28] Bae S., Lee J. M., Kang Y. J., Kang J. S., Yun J. R. Dynamic analysis of an automatic washing machine with a hydraulic balancer. Journal of Sound and Vibration, Vol. 257, 2002, p. 3-18.

[29] Majewski T. Fluid balancer for a washing machine. Proceedings of the 16th International Congress, 2010.

[30] Urbiola Soto L., Lopez Parra M. Dynamic performance of the Leblanc balancer for automatic washing machines. Journal of Vibration and Acoustics, Vol. 133, Issue 4, 2011, p. 41014.

[31] Gusarov A. A. Balancing Machine Rotors. Book 2, Nauka, Moscow, 2004, p. 266.

[32] Nesterenko V. P. Theory and practice of devices for automatic balancing of rotors. Thesis, Novosibirsk Electrical Engineering Institute, Novosibirsk, 1990, p. 24-30. 
[33] Pashkov E., Martyushev N., Ponomarev A. Efficiency of balancing by liquid-type automatic balancing devices. Advanced Materials Research, Vol. 1040, 2014, p. 858-863.

[34] Langthjem M. A., Nakamura T. Dynamics of the fluid balancer: perturbation solution of a forced Korteweg-de Vries-Burgers equation. RIMS, Kyoto University, Vol. 1847, 2013, p. 73-85.

[35] Marlon Wesley Machado Cunico Characterization and modelling of Leblanc hydrodynamic stabilizer: a novel approach for steady and transient state models. Modelling and Simulation in Engineering, Vol. 2015, 2015, p. 729582.

[36] Dimentberg F. M., Shatalov K. T., Husarov A. A. Oscillations of Machines. Mashinostroenie, Moscow, 1964, p. 256-291.

[37] Pars L. A. Analytical Dynamics. Nauka, Moscow, 1971, p. 636.

[38] Moiseev N., Rumyantsev V. Dynamics of the Body with Cavities Containing Liquid. Nauka, Moscow, 1965, p. 440, (in Russian).

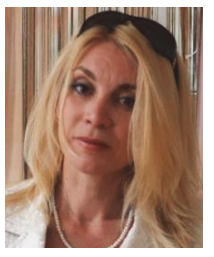

Ilona Drach Ph.D., Associate Professor of the Software Engineering Department of the Khmelnytsky National University. After graduation from Ivan Franko Lviv State University she was qualified as a mathematician. In 2008 she defended his thesis. In 2010 awarded the title of Associate Professor. Scientific interests: engineering (balancing, reducing vibration), mathematics, mathematical modelling.

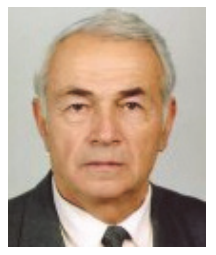

Vilen Royzman received Doctoral degree in Machine Engineering Institute of Science Academy of USSR, Moscow, Russia, in 1979. In 1980 he received title of Professor. Now he works at Khmelnytsky National University as Head of Department of Telecommunication and Radio Engineering. His current research interests include vibrations, rotor balancing, inverse problems and strength in electronics.

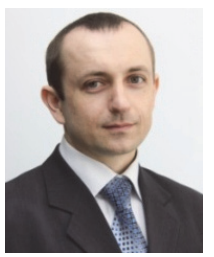

Vitalii Tkachuk Ph.D. of the Engineering. Born in 1981 in the Khmelnytsky region. In the period of 1998-2003 he studied at the Khmelnytsky National University. In 2011 he defended his thesis. In 2014 awarded the title of Associate Professor. Works as the Associate Professor of Department of Engineering Techniques, Khmelnitskiy National University. Scientific interests: balancing, reducing vibration, engineering analysis, $\mathrm{CNC}$ machines

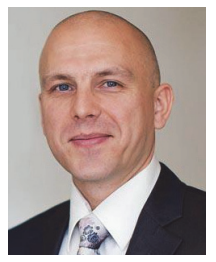

Andrii Goroshko received Doctoral degree of dynamics and strength of machines in Lviv Polytechnic National University in 2017. Now he works at Khmelnytsky National University as Professor of Department of Physics and Electrical Engineering. His current research interests include vibrations, rotor balancing, strength, inverse problems, statistics. 


\title{
2.2. Spindle units on rolling bearings diagnostics by indirect measurement of spindle vibration
}

\author{
Yuriy Danylchenko ${ }^{1}$, Andriy Petryshyn ${ }^{2}$ \\ National Technical University of Ukraine "Igor Sikorsky Kyiv Polytechnic Institute”, Kyiv, Ukraine

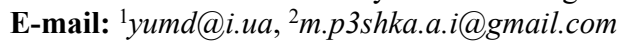

\begin{abstract}
A method of indirect measurement of spindle vibrations based on the results of measuring the vibrations of the spindle unit housing and theoretical compliance function has been developed. Method is based on performing experimental and theoretical studies of the spindle unit dynamic behavior, mounted on a special test rig with a low natural frequency. Test rig consists of the frame, elastically fixed on a massive foundation and spindle unit that is attached to the frame. Dynamic model of the test rig is presented in a form of a complex vibratory mechanical system which consists of four subsystems. For system decomposition, method of dynamic compliance is used, and for subsystems dynamic characteristics calculation-transfer matrix method. Theoretical compliance function is considered as the ratio of dynamic compliances of the subsystems at the points of calculation and measurement of vibrations. This method was used for assembling and manufacturing faults diagnostic of spindle units on rolling bearings that cause low-frequency vibrations and its effectiveness has been confirmed.
\end{abstract}

Keywords: spindle unit, rolling bearings, assembling and manufacturing faults, vibration diagnostic, mechanical vibratory system, dynamical compliance.

\section{Introduction}

The most common methods for rotary machines technical condition evaluation, including spindle units, are methods of vibration diagnostic.

The advantages of these methods are explained by the sufficiently high degree of mechanical vibrations determinacy in frequency composition, which allows us to perform identification their causes and sources with sufficient confidence.

In the vibrations spectrum of the rotor mounted on rolling bearings, it is possible to distinguish 4 main ranges that characterize [1]: 1) the presence and magnitude of the imbalance (vibration with a frequency equal to the rotor running speed); 2) bearings misalignment due to assembling faults or external loads on the rotor (vibration with a rotor running speed and twice rotor running speed frequency); 3) quality of the rotary drive (vibration at the characteristic frequencies of rotary drive); 4) manufacturing defects of races and rolling elements (so-called "bearing vibrations" associated with frequencies, determined by the bearing kinematics). Vibration diagnostics methodology of rotor systems is based on the evaluation of the vibration magnitude at these frequencies.

Vibration diagnostics systems of spindle units (SU) of modern metal-cutting machines are aimed at spindle error motion control [2-4] and fault detection of its bearings [5, 6].

Modern methods of spindle error motion control are built on the patterns of non-contact measurement of spindle linear displacements on the reference surface for measurements of a spindle or artifact $[3,4]$. In this case, it is necessary to extract from the measurement results a component, which is caused by the error on reference surface for measurements. The accuracy of the spindle error motion determining depends on the method of solving this problem [4]. This is especially true for precision SU, the error motion of which is commensurable with the error on reference surface for measurements [7]. Such a procedure for spindle error motion determination requires ensuring special conditions for the measurement, which makes it problematic to use for error motion determination of fixed in spindle workpiece or tool, in the cutting zone during machining. An alternative to direct measurement of spindle vibrations can be the measurement of vibrations on the spindle unit housing unit with subsequent identification of these vibrations according to the theoretically established compliance function. 
The most commonly used type in SU are rolling bearings. The vibration signal of a faulty rolling bearing includes deterministic signals, bearing element defect signals and noise [8]. Deterministic signals are, as a rule, low-frequency signals caused manufacturing faults of recess and rolling elements of bearings. The fault signals of the bearing elements are periodic resonance vibrations of the carrier elements resulting from short pulses generated each time the defect hits its matched element [5]. It is this difference in signals that makes it possible to extract signals of bearing elements faults from the general signal. This difference also determines the effectiveness of methods for condition monitoring and fault diagnostics of rolling bearings [9].

The most effective methods for bearing faults diagnostics are methods of high-frequency excitation, known as high frequency resonance technique (HFRT), which is also known as envelope analysis, and shock pulse method (SPM) $[5,8,9]$. The use of low-frequency excitation to detect faults in of low-speed bearings elements is considered almost impossible. The main reason for this considered to be limitation on the energy level, generated by faults in the bearing elements [10].

The carried-out analysis indicates significant advantages of vibration diagnostics of the SU from measurements of vibrations in resonant zones and the prospects of creating tools and methods for conducting such diagnostics.

This study is devoted to the development of a method for indirect measurement of spindle vibrations and its application for estimating assembling and manufacturing faults of the SU on rolling supports.

\section{The main idea of the method of indirect measurement of spindle vibrations}

The method of indirect measurement of the spindle vibrations is based on measuring the vibrations on the spindle unit housing with the subsequent calculation of the spindle axis vibration (fixed in the spindle mandrel) according to the theoretical compliance function $W(\omega)$. For the measurement schemes shown in Fig. 1, compliance function $W(\omega)$ associates the spindle housing vibrations, at the place of measurement (position 1) and the axis of the tool mandrel (position 2).

In common case compliance function $W(\omega)$ is defined as:

$W(\omega)=\frac{q_{2}(\omega)}{q_{1}(\omega)^{\prime}}$

where $q_{2}(\omega)$ and $q_{1}(\omega)$ - calculated functions of tool holder displacement at point 2 and the spindle housing at point 1 under unit harmonic force applied at point 3 (Fig. 1); $\omega$ - excitation frequency.

The position of point 3 is determined by the objective. In case of evaluating assembling and manufacturing faults of the SU on rolling-contact bearings, point 3 is located on the axis of the spindle under the leading bearing (Fig. 1(a)). In case of evaluating spindle error motion during machining, point 3 is placed on the axis of the tool holder under cutting zone, that is, the positions of points 2 and 3 coincide (Fig. 1(b)).

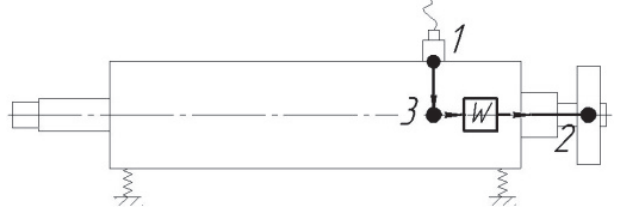

a)

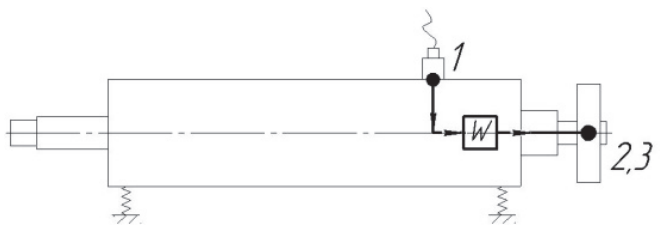

b)

Fig. 1. Measurement scheme for the assembling and manufacturing faults estimation of SU on rolling bearings a) and spindle vibrations b): 1 - point of vibration measurement of the spindle housing; 2 - point of vibration calculation of the spindle axis (tool-holder); 3 - point of the unit harmonic force application 
Vibration spectrum calculation of the tool-holder from measured vibrations of the spindle housing is performed according to:

$A_{2}\left(\omega_{i}\right)=A_{1}\left(\omega_{i}\right) \cdot W\left(\omega_{i}\right)$

where $A_{1}\left(\omega_{i}\right)$ and $A_{2}\left(\omega_{i}\right)$ - amplitudes of the $i$-th spectral components of the experimental vibrations spectrum of the spindle housing and theoretical vibration spectrum tool-older axis, respectively.

These are the spectrums of absolute vibrations. Axis vibration spectrum of the tool-holder relative to the spindle housing is obtained as a result of vector subtraction of the corresponding spectrums of absolute vibrations taking into account phase angles of the spectral components.

Proposed theoretical-experimental method allows simplifying the procedure for spindle unit condition monitoring and evaluation. In addition, it does not require the use of high-precision measuring surfaces for spindle vibration determination.

\section{Test rig}

\subsection{Test rig design}

When evaluating level of vibrations at characteristic frequencies, it should not be forgotten that there is a complex interaction of disturbing forces in the bearing with natural frequencies of the entire unit [11].

However, this SU property can be used to detect the incipient faults of the rolling bearings [12]. For this, it is necessary that the frequencies of the shock pulses that occur when the faults of the bearing elements periodically enter the contact zone coincide with the same resonant frequency of the mechanical system.

In turn, it should be noted that the resonant frequencies of the spindle units are quite high. For example, for a vertical milling machine [13], the first natural frequency of the spindle head is $315 \mathrm{~Hz}$, and the spindle frequency is $1410 \mathrm{~Hz}$. Such values of natural frequencies do not allow using the results of measuring resonance vibrations of the machine units for diagnostic of faults that cause low-frequency vibrations. However, this can be realized on special test rigs, with sufficiently lower natural frequencies of the carrier elements [14].

Test rig [14] is a frame elastically mounted on a massive foundation, with the possibility of oscillating motion in a vertical plane. The angular stiffness of the frame is regulated by changing the working length of the spring or by installing a rigid beam instead. By adjusting the angular rigidity of the frame, it is possible to change the value of its first natural frequency in the range 5-37 Hz. Spindle unit is elastically mounted on the frame. Test rig design is shown in Fig. 2.

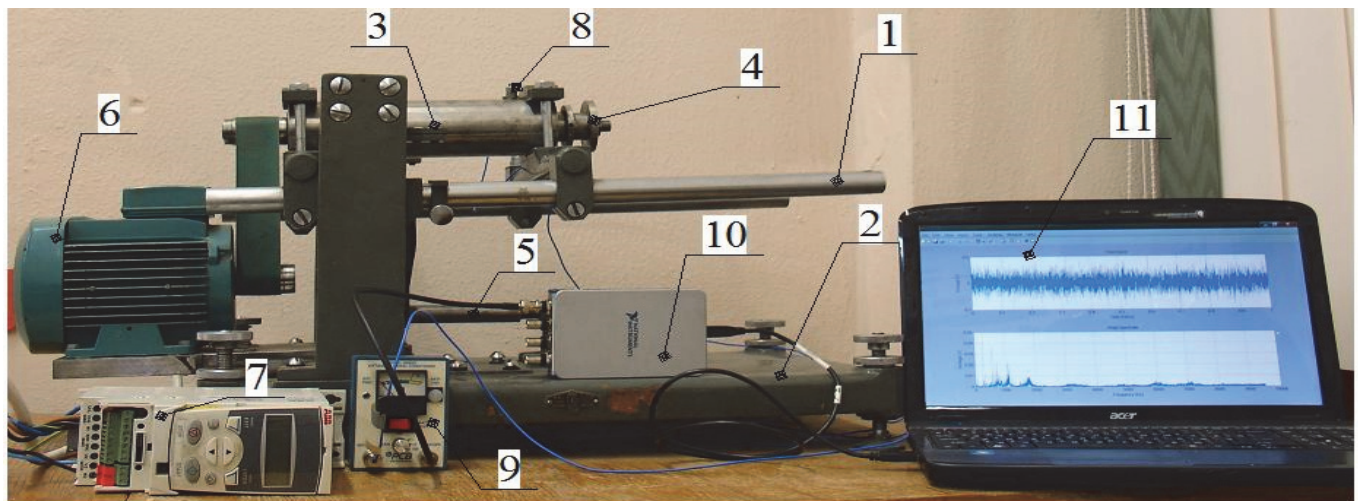

Fig. 2. Test rig design: 1 - frame; 2 - foundation; 3 - SU; 4 - tool-holder; 5 - spring/beam; 6 - motor; 7 - frequency converter; 8 - sensor; 9 - amplifier; 10 - AD converter; 11 - PC 


\section{Test rig dynamic model}

The implementation of the method of indirect measuring of spindle vibrations on a test rig requires determination of its dynamic characteristics. In order to perform that, it is necessary to create its dynamic model and experimentally confirm its adequacy.

When studying the dynamic behavior of complex mechanical systems, it is considered reasonable to separate them (decomposition) into simpler subsystems [15, 16]. Separation of subsystems from each other is ensured either by the introduction of additional connections that prohibit movement of common points (nodes) of subsystems (dynamic stiffness method) or, on the contrary, by eliminating constrains between them (method of dynamic compliances).

For rotary machines dynamic characteristics calculation, where SU belongs to, it is more convenient to use the method of dynamic compliances [16]. When system is decomposed using the method of dynamic compliances, harmonic reactions are applied to the discarded constrains, which are then determined from the deformation compatibility condition of subsystems, that is, the conditions for the amplitude balance of generalized displacements (linear and angular) at the separation points of the subsystems.

The sequence of dynamic model development of the mechanical vibratory system that includes several subsystems is described in detail in [17].

Using the recommendations of [17], we will present a test rig with a mounted SU in the form of a mechanical vibratory system which consists of 4 subsystems (Fig. 3).

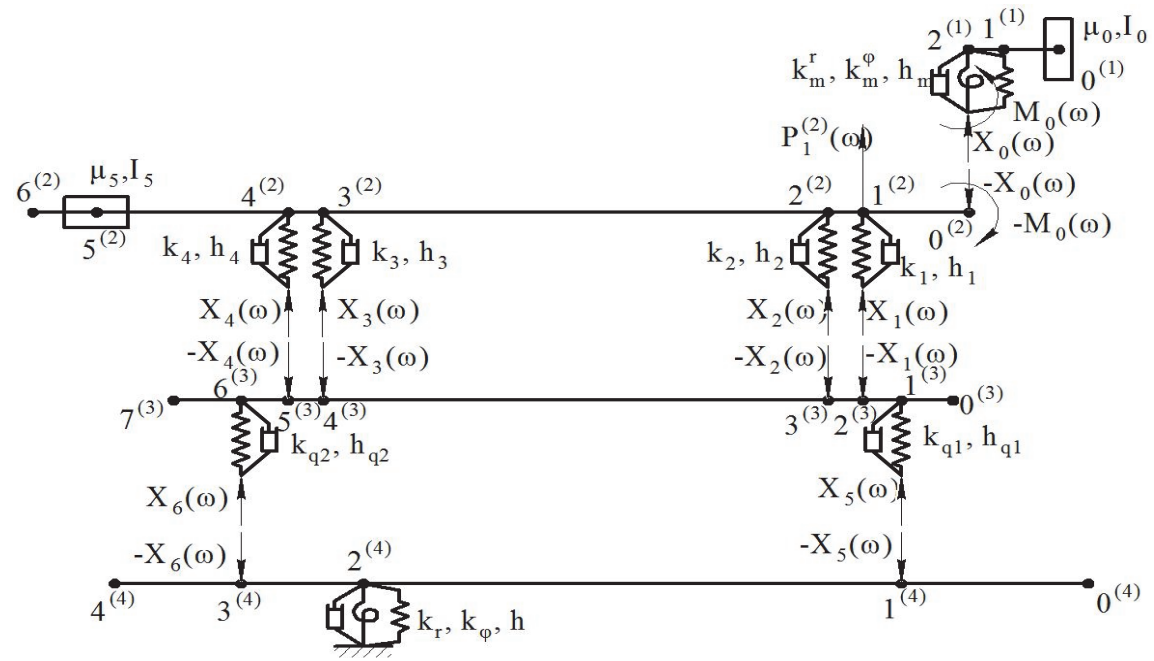

Fig. 3. Design models of test rig subsystems

First subsystem (tool-holder, index $s=1$ ) is considered as a beam, that consists of two sections with distributed mass. On the front end of tool-holder (cross-section $0^{(1)}$ ) concentrated weight is located (disc). In section $2^{(1)}$ the tool-holder is connected with spindle (spindle cross-section $0^{(2)}$ ), which is taken into account by an elastic connection with radial $k_{m}^{r}$ and angular $k_{m}^{\phi}$ stiffness.

Second subsystem (spindle, index $s=2$ ) is considered as a beam, which consists of six sections with distributed mass. In cross-sections $1^{(2)}, 2^{(2)}, 3^{(2)}$ and $4^{(2)}$ bearings are located, which is taken into account by elastic connections of spindle and spindle housing with stiffness $k_{1}, k_{2}, k_{3}$ and $k_{4}$ accordingly. In cross-section $5^{(2)}$ concentrated weight is located (pulley wheel).

Third subsystem (spindle housing, index $s=3$ ) is considered as a hollow beam, which consists of seven sections with distributed mass. In cross-sections $2^{(3)}, 3^{(3)}, 4^{(3)}$ and $5^{(3)}$ spindle housing is connected with spindle. In cross-sections $1^{(3)}$ and $6^{(3)}$ its connected to the frame, that 
is taken into account by supports with stiffness $k_{q 1}$ and $k_{q 2}$.

Fourth subsystem (frame, index $s=4$ ) is considered as an equivalent beam, which consists of four sections with distributed mass. In cross-sections $1^{(4)}, 3^{(4)}$ of the frame spindle unit is mounted, in cross-section $2^{(4)}$ radial and angular joins is located, which connect frame with test rig foundation, and taken into account by radial $k_{r}$ and angular $k_{\phi}$ stiffness.

The connections of the subsystems are: connection of tool-holder with spindle for subsystems 1 and 2; spindle bearings for subsystems 2 and 3; fixtures for subsystems 3 and 4 . All connections have elastic and dissipative properties.

To determine the compliance functions $W(\omega)$ for different system elements vibrations, design model (Fig. 3) should take into account the necessity of a unit harmonic load $P_{i}^{(s)}(\omega)=1 \cdot \sin \omega t$ application at certain points of the subsystems. Thus, the scheme shown in Fig. 3 is designed to solve the problem of assembling and manufacturing faults estimation of SU (Fig. 1(a)). Therefore, in the design model (Fig. 3) a unit harmonic load $P_{1}^{(2)}(\omega)=1 \cdot \sin \omega t$ is applied to the spindle section.

According to decomposition scheme (Fig. 3), deformation compatibility conditions in subsystem separation points are:

- For subsystems 1 and 2:

$$
\left\{\begin{array}{l}
\alpha_{00}^{12} X_{0}+\gamma_{00}^{12} M_{0}-\alpha_{01}^{(2)} X_{1}-\alpha_{02}^{(2)} X_{2}-\alpha_{03}^{(2)} X_{3}-\alpha_{04}^{(2)} X_{4}=\alpha_{0 P}^{(2)} \\
\beta_{00}^{12} X_{0}+\phi_{00}^{12} M_{0}-\beta_{01}^{(2)} X_{1}-\beta_{02}^{(2)} X_{2}-\beta_{03}^{(2)} X_{3}-\beta_{04}^{(2)} X_{4}=\beta_{0 P}^{(2)}
\end{array}\right.
$$

- For subsystems 2 and 3:

$\left\{\begin{array}{l}-\alpha_{10}^{(2)} X_{0}-\gamma_{10}^{(2)} M_{0}+\alpha_{11}^{23} X_{1}+\alpha_{12}^{23} X_{2}+\alpha_{13}^{23} X_{3}+\alpha_{14}^{23} X_{4}-\alpha_{21}^{(3)} X_{5}-\alpha_{26}^{(3)} X_{6}=-\alpha_{1 P}^{(2)}, \\ -\alpha_{20}^{(2)} X_{0}-\gamma_{20}^{(2)} M_{0}+\alpha_{21}^{23} X_{1}+\alpha_{22}^{23} X_{2}+\alpha_{23}^{23} X_{3}+\alpha_{24}^{23} X_{4}-\alpha_{31}^{(3)} X_{5}-\alpha_{36}^{(3)} X_{6}=-\alpha_{2 P}^{(2)}, \\ -\alpha_{30}^{(2)} X_{0}-\gamma_{30}^{(2)} M_{0}+\alpha_{31}^{23} X_{1}+\alpha_{32}^{23} X_{2}+\alpha_{33}^{23} X_{3}+\alpha_{34}^{23} X_{4}-\alpha_{41}^{(3)} X_{5}-\alpha_{46}^{(3)} X_{6}=-\alpha_{3 P}^{(2)}, \\ -\alpha_{40}^{(2)} X_{0}-\gamma_{40}^{(2)} M_{0}+\alpha_{41}^{23} X_{1}+\alpha_{42}^{23} X_{2}+\alpha_{43}^{23} X_{3}+\alpha_{44}^{23} X_{4}-\alpha_{51}^{(3)} X_{5}-\alpha_{56}^{(3)} X_{6}=-\alpha_{4 P}^{(2)}\end{array}\right.$,

- For subsystems 3 and 4:

$\left\{\begin{array}{l}-\alpha_{12}^{(3)} X_{1}-\alpha_{13}^{(3)} X_{2}-\alpha_{14}^{(3)} X_{3}-\alpha_{15}^{(3)} X_{4}+\alpha_{11}^{34} X_{5}+\alpha_{16}^{34} X_{6}=0, \\ -\alpha_{62}^{(3)} X_{1}-\alpha_{63}^{(3)} X_{2}-\alpha_{64}^{(3)} X_{3}-\alpha_{65}^{(3)} X_{4}+\alpha_{61}^{34} X_{5}+\alpha_{66}^{34} X_{6}=0,\end{array}\right.$

or in a matrix form:

$[\mathbf{D}(\omega)] \cdot \mathbf{F}=\Delta_{\mathbf{P}}$

where $X_{i}, M_{i}$ - harmonic reactions in discarded connections; $\alpha_{i j}^{(s)}, \beta_{i j}^{(s)}, \gamma_{i j}^{(s)}, \phi_{i j}^{(s)}-$ dynamic compliances of subsystems $s: \alpha_{i j}^{(s)}$ and $\beta_{i j}^{(s)}$ - accordingly displacement and angle of rotation in point $i$ from unit harmonic force applied in point $j ; \gamma_{i j}^{(s)}$ and $\phi_{i j}^{(s)}$ - accordingly displacement and angle of rotation in point $i$ from unit harmonic moment applied in point $j ; \alpha_{i P}^{(2)}$ and $\beta_{i P}^{(2)}-$ generalized displacements (linear and angular) from unit harmonic load $P_{1}^{(2)}, \alpha_{i P}^{(2)}=\alpha_{i 1}^{(2)} P_{1}^{(2)}$, $\beta_{0 P}^{(2)}=\beta_{01}^{(2)} P_{1}^{(2)}, i=0,1,2,3,4 ; \mathbf{R}$ - vector of harmonic reactions in discarded connections, $\mathbf{R}=\left(X_{0}, M_{0}, X_{1}, X_{2}, X_{3}, X_{4}, X_{5}, X_{6}\right)^{T} ; \Delta_{\mathbf{P}}-$ generalized displacements vector:

$\Delta_{\mathbf{P}}=\left(\alpha_{0 P}^{(2)}, \beta_{0 P}^{(2)},-\alpha_{1 P}^{(2)},-\alpha_{2 P}^{(2)},-\alpha_{3 P}^{(2)},-\alpha_{4 P}^{(2)}, 0,0\right)^{T}$, 
and $[\mathbf{D}(\omega)]$ - dynamic compliance martix:

$$
\mathbf{D}(\omega)=\left(\begin{array}{cccccccc}
\alpha_{00}^{12} & \gamma_{00}^{12} & -\alpha_{01}^{(2)} & -\alpha_{02}^{(2)} & -\alpha_{03}^{(2)} & -\alpha_{04}^{(2)} & 0 & 0 \\
\beta_{00}^{12} & \phi_{00}^{12} & -\beta_{01}^{(2)} & -\beta_{02}^{(2)} & -\beta_{03}^{(2)} & -\beta_{04}^{(2)} & 0 & 0 \\
-\alpha_{10}^{(2)} & -\gamma_{10}^{(2)} & \alpha_{11}^{23} & \alpha_{12}^{23} & \alpha_{13}^{23} & \alpha_{14}^{23} & -\alpha_{21}^{(3)} & -\alpha_{26}^{(3)} \\
-\alpha_{20}^{(2)} & -\gamma_{20}^{(2)} & \alpha_{21}^{23} & \alpha_{22}^{23} & \alpha_{23}^{23} & \alpha_{24}^{23} & -\alpha_{31}^{(3)} & -\alpha_{36}^{(3)} \\
-\alpha_{30}^{(2)} & -\gamma_{30}^{(2)} & \alpha_{31}^{23} & \alpha_{32}^{23} & \alpha_{33}^{23} & \alpha_{34}^{23} & -\alpha_{41}^{(3)} & -\alpha_{46}^{(3)} \\
-\alpha_{40}^{(2)} & -\gamma_{40}^{(2)} & \alpha_{41}^{23} & \alpha_{42}^{23} & \alpha_{43}^{23} & \alpha_{44}^{23} & -\alpha_{51}^{(3)} & -\alpha_{56}^{(3)} \\
0 & 0 & -\alpha_{12}^{(3)} & -\alpha_{13}^{(3)} & -\alpha_{14}^{(3)} & -\alpha_{15}^{(3)} & \alpha_{11}^{34} & \alpha_{16}^{34} \\
0 & 0 & -\alpha_{62}^{(3)} & -\alpha_{63}^{(3)} & -\alpha_{64}^{(3)} & -\alpha_{65}^{(3)} & \alpha_{61}^{34} & \alpha_{66}^{34}
\end{array}\right),
$$

where:

$$
\begin{aligned}
& \alpha_{00}^{12}=\alpha_{22}^{(1)}+\alpha_{00}^{(2)}+\frac{1}{k_{m}^{r}}, \quad \beta_{00}^{12}=\beta_{22}^{(1)}+\beta_{00}^{(2)}, \quad \gamma_{00}^{12}=\gamma_{22}^{(1)}+\gamma_{00}^{(2)}, \\
& \phi_{00}^{12}=\phi_{22}^{(1)}+\phi_{00}^{(2)}+\frac{1}{k_{m}^{\phi},} \quad \alpha_{11}^{23}=\alpha_{11}^{(2)}+\alpha_{22}^{(3)}+\frac{1}{k_{1}}, \quad \alpha_{12}^{23}=\alpha_{12}^{(2)}+\alpha_{23}^{(3)}, \\
& \alpha_{13}^{23}=\alpha_{13}^{(2)}+\alpha_{24}^{(3)}, \quad \alpha_{14}^{23}=\alpha_{14}^{(2)}+\alpha_{25}^{(3)}, \quad \alpha_{21}^{23}=\alpha_{21}^{(2)}+\alpha_{32}^{(3)}, \\
& \alpha_{22}^{23}=\alpha_{22}^{(2)}+\alpha_{33}^{(3)}+\frac{1}{k_{2}}, \quad \alpha_{23}^{23}=\alpha_{23}^{(2)}+\alpha_{34}^{(3)}, \quad \alpha_{24}^{23}=\alpha_{24}^{(2)}+\alpha_{35}^{(3)}, \\
& \alpha_{31}^{23}=\alpha_{31}^{(2)}+\alpha_{42}^{(3)}, \quad \alpha_{32}^{23}=\alpha_{32}^{(2)}+\alpha_{43}^{(3)}, \quad \alpha_{33}^{23}=\alpha_{33}^{(2)}+\alpha_{44}^{(3)}+\frac{1}{k_{3}}, \\
& \alpha_{34}^{23}=\alpha_{34}^{(2)}+\alpha_{45}^{(3)}, \quad \alpha_{41}^{23}=\alpha_{41}^{(2)}+\alpha_{52}^{(3)}, \quad \alpha_{42}^{23}=\alpha_{42}^{(2)}+\alpha_{53}^{(3)}, \\
& \alpha_{43}^{23}=\alpha_{43}^{(2)}+\alpha_{54}^{(3)}, \quad \alpha_{44}^{23}=\alpha_{44}^{(2)}+\alpha_{55}^{(3)}+\frac{1}{k_{4}}, \quad \alpha_{11}^{34}=\alpha_{11}^{(3)}+\alpha_{11}^{(4)}+\frac{1}{k_{q 1}}, \\
& \alpha_{16}^{34}=\alpha_{16}^{(3)}+\alpha_{13}^{(4)}, \quad \alpha_{61}^{34}=\alpha_{61}^{(3)}+\alpha_{31}^{(4)}, \quad \alpha_{66}^{34}=\alpha_{66}^{(3)}+\alpha_{33}^{(4)}+\frac{1}{k_{q 2}} .
\end{aligned}
$$

Dynamic compliances $\alpha_{i j}^{(s)}, \beta_{i j}^{(s)}, \gamma_{i j}^{(s)}, \phi_{i j}^{(s)}$ are determined according to methodology, presented in [17] using the transfer matrix method [18, 19].

Subsystems transfer matrices $\Pi^{(s)}$ are written in accordance with decomposition scheme (Fig. 3 ) by the methodology [17]:

For tool-holder $(s=1, u=2)$ :

$$
\Pi^{(1)}=\prod_{i=2}^{0} \Pi_{i}^{(1)}=\mathbf{U}_{2}^{(1)} \cdot \mathbf{U}_{1}^{(1)} \cdot \mathbf{G}_{0}^{(1)} \text {. }
$$

For spindle $(s=2, u=6)$ :

$$
\Pi^{(2)}=\prod_{i=6}^{0} \Pi_{i}^{(2)}=\mathbf{U}_{6}^{(2)} \cdot \mathbf{G}_{5}^{(2)} \cdot \mathbf{U}_{5}^{(2)} \cdot \mathbf{U}_{4}^{(2)} \cdot \mathbf{U}_{3}^{(2)} \cdot \mathbf{U}_{2}^{(2)} \cdot \mathbf{U}_{1}^{(2)} .
$$

For spindle housing $(s=3, u=7)$ : 
$\Pi^{(3)}=\prod_{i=7}^{0} \Pi_{i}^{(3)}=\mathbf{U}_{7}^{(3)} \cdot \mathbf{U}_{6}^{(3)} \cdot \mathbf{U}_{5}^{(3)} \cdot \mathbf{U}_{4}^{(3)} \cdot \mathbf{U}_{3}^{(3)} \cdot \mathbf{U}_{2}^{(3)} \cdot \mathbf{U}_{1}^{(3)}$

For frame $(s=4, u=4)$ :

$\Pi^{(4)}=\prod_{i=4}^{0} \Pi_{i}^{(4)}=\mathbf{U}_{4}^{(4)} \cdot \mathbf{U}_{3}^{(4)} \cdot \mathbf{R}_{2}^{(4)} \cdot \mathbf{U}_{2}^{(4)} \cdot \mathbf{U}_{1}^{(4)}$,

where $\Pi^{(s)}$ - transfer matrix of the beam system, equal to the product of transfer matrices of all sections between the $u$-th and the 0 -th sections of the system's beam, $\Pi^{(s)}=\prod_{i=u}^{0} \Pi_{i}^{(s)} ; \Pi_{i}^{(s)}$ transfer matrix of the $\mathrm{i}$-th section, equal to the product of all transfer matrices on this section, $\Pi_{i}^{(s)}=\mathbf{G}_{i}^{(s)} \cdot \mathbf{R}_{i}^{(s)} \cdot \mathbf{U}_{i}^{(s)} ; \mathbf{G}_{i}$ - mass-inertia matrix of the concentrated weight; $\mathbf{R}_{i}$ - matrix of elastic-dissipative linear and angular support; $\mathbf{U}_{i}$ - matrix of the beam section with distributed mass, $i$ - number of the subsystem section; $u$ - general number of subsystem sections. Transfer matrices $\mathbf{G}_{i}, \mathbf{R}_{i}$ and $\mathbf{U}_{i}$ are determined by dependencies [18, 19].

From the system of equations (6), the reactions of the discarded connections are determined, and then the amplitudes $q_{i}^{(s)}(i=0, \ldots, u)$ of the transverse (radial) displacements of the characteristic points of the subsystems:

Tool-holder $(s=1, i=0, \ldots, 2)$ :

$q_{i}^{(1)}=\alpha_{i 2}^{(1)} X_{0}+\gamma_{i 2}^{(1)} M_{0}$.

Spindle $(s=2, i=0, \ldots, 6)$ :

$q_{\mathrm{i}}^{(2)}=-\alpha_{\mathrm{i} 0}^{(2)} X_{0}-\gamma_{\mathrm{i} 0}^{(2)} M_{0}+\alpha_{\mathrm{i} 1}^{(2)} X_{1}+\alpha_{\mathrm{i} 2}^{(2)} X_{2}+\alpha_{\mathrm{i} 3}^{(2)} X_{3}+\alpha_{\mathrm{i} 4}^{(2)} X_{4}+\alpha_{\mathrm{i} 1}^{(2)} P_{1}^{(2)}$.

Spindle housing $(s=3, i=0, \ldots, 7)$ :

$q_{i}^{(3)}=-\alpha_{i 2}^{(3)} X_{1}-\alpha_{i 3}^{(3)} X_{2}-\alpha_{i 4}^{(3)} X_{3}-\alpha_{i 5}^{(3)} X_{4}+\alpha_{i 5}^{(3)} X_{5}+\alpha_{i 6}^{(3)} X_{6}$.

Frame $(s=4, i=0, \ldots, 4)$ :

$q_{i}^{(4)}=-\alpha_{i 1}^{(4)} X_{5}-\alpha_{i 3}^{(4)} X_{6}$.

Compliance function $W(\omega)_{13}$, connecting the vibration of the point $0^{(1)}$ of the tool-holder subsystem $(s=1)$ with the vibrations of the spindle housing $(s=3)$ at the point $2^{(3)}$ under unit harmonic load applied at this point (Fig. 3 ) is determined as:

$W(\omega)_{13}=\frac{q_{0}^{(1)}(\omega)}{q_{2}^{(3)}(\omega)}=\frac{\alpha_{02}^{(1)} X_{0}+\gamma_{02}^{(1)} M_{0}}{-\alpha_{22}^{(3)} X_{1}-\alpha_{23}^{(3)} X_{2}-\alpha_{24}^{(3)} X_{3}-\alpha_{25}^{(3)} X_{4}+\alpha_{25}^{(3)} X_{5}+\alpha_{26}^{(3)} X_{6}}$.

\subsection{Experimental verification of the test rig dynamic model adequacy}

As the tested SU grinding head were taken. The SU housing is made in the form of a quill with an external diameter of $65 \mathrm{~mm}$ and a length of $250 \mathrm{~mm}$. The spindle shaft is mounted on duplex angular-contact ball bearings type 7004, assembled according to the "tandem-X" scheme.

Measurement of vibration at the test rig was carried out using a piezoelectric accelerometer. PCB 353B15 and amplifier PCB 480E09. The signal was registered using the NI USB-9215 AD 
converter and processed in Matlab.

The experimental verification consists in obtaining and analyzing of system harmonic response to the impact load. Studies were carried out for four different versions of the system: 1) test rig with mounted SU and attached tool-holder, the angular rigidity of the system is set by an extension spring; 2) test rig without SU, angular rigidity of system is set by an extension spring; 3) stand with mounted SU and attached tool-holder, the angular rigidity of the system is set by a rigid beam, installed instead of a spring; 4) stand without SU, angular rigidity of system is set by a rigid beam, installed instead of a spring.

For the first and third versions, measurement were taken at the end of the tool-holder (Fig. 4(a)), the impulse load was applied to the frame.

For the second and fourth variants, measurements were taken at the place where the spindle unit was attached to the frame (Fig. 4(b)).

The natural frequencies values of the test rig with mounted SU (Fig. 4(a)) are determined using Eq. (7) from the condition $\operatorname{det}[\mathbf{D}(\omega)]=0$. Natural frequencies of the frame (Fig. 4(b)) are determined from the calculation of frame transfer matrix Eq. (11) using the dependency given in [19].

The results of comparison are shown in Fig. $5 . P_{i}^{(s)} i$ th natural frequency of the subsystem $s$. The left values of the natural frequency correspond to the experimental values, the right ones calculated.

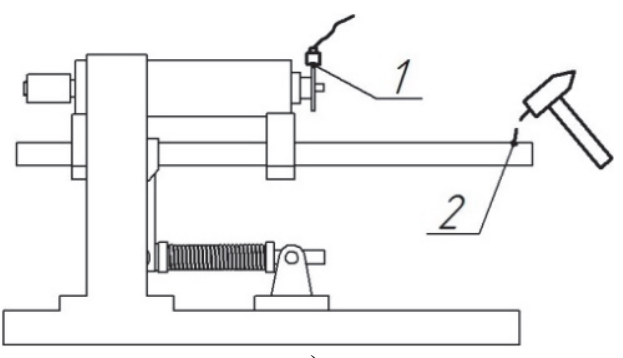

a)

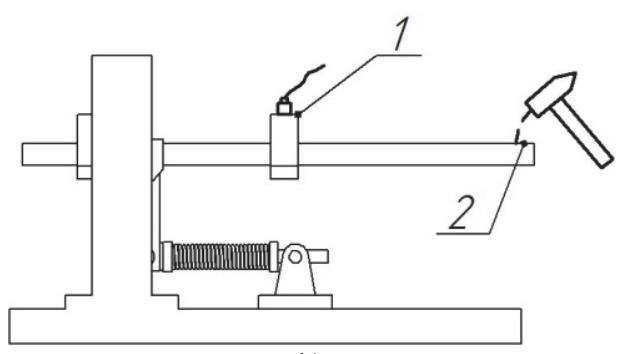

b)

Fig. 4. Schemes for the sensor installation and applied impact load for measuring natural frequencies spectrum of the system: 1 - sensor, 2 - place of load application

\section{An example of using the method of indirect measurement of spindle vibrations}

\subsection{The research procedure at estimation of assembling and manufacturing faults of SU on rolling bearings}

The use of the method involves experimental and theoretical studies of dynamic behavior of the SU, mounted on the test rig.

The experimental part of the study (Fig. 1(a)) provides:

1. Determination of the first natural frequency $P_{1}^{(4)}$ of the test rig frame $\left(P_{1}^{(4)}=33 \mathrm{~Hz}\right.$, Fig. 5(c)).

2. Determination of the spindle rotation frequencies corresponding to the conditions for the resonance vibrations of the frame due to occurrence of assembling and manufacturing faults of the SU.

3. Measurement of spindle housing vibrations at these speeds.

The spindle rotation frequencies at which the resonance vibrations of the frame occurs are determined by the condition of the frame first natural frequency matching with the frequencies of the forced vibrations caused by assembling and manufacturing faults of the SU on rolling bearings. The characteristic frequencies of forced vibrations and rotational speeds of the spindle, corresponding to the conditions of frame resonance vibrations, are given in Table 1. 


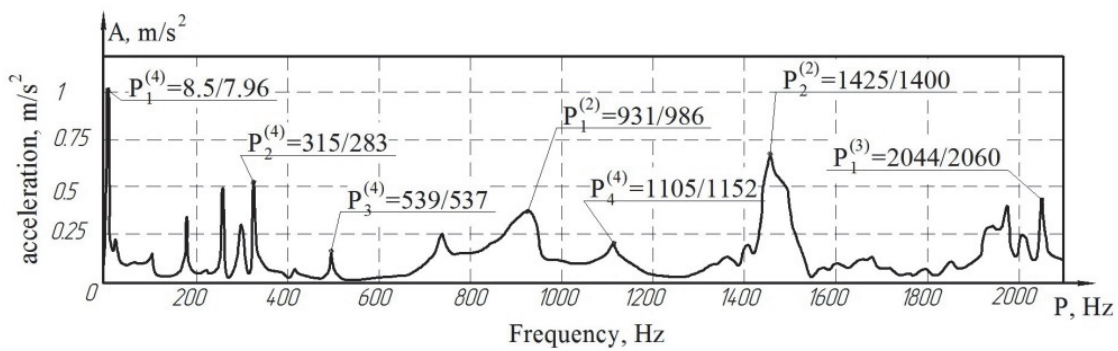

a)

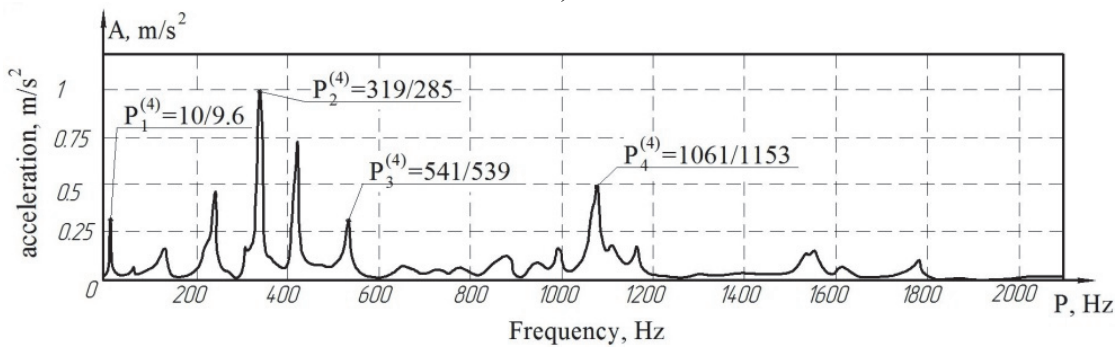

b)

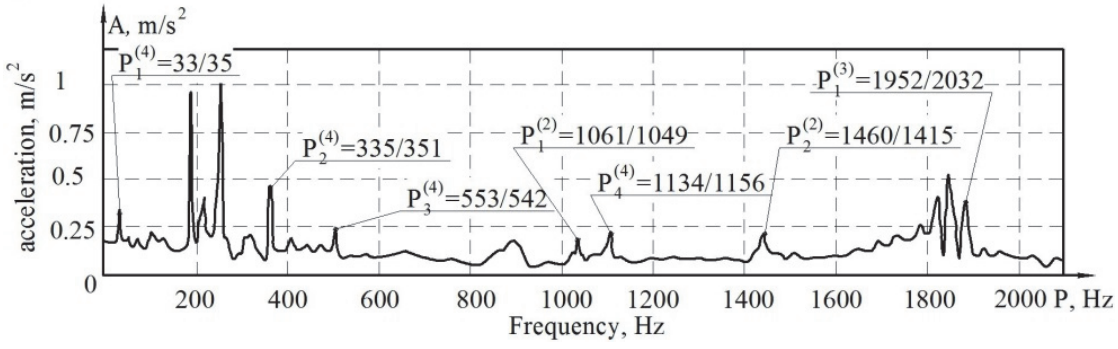

c)

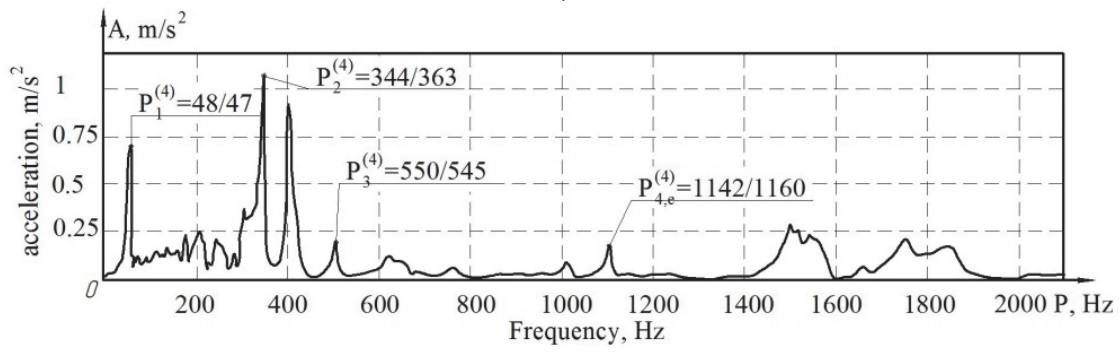

d)

Fig. 5. Natural frequencies spectrums of the test rig for different variants of test rig: a) with extension spring and $\mathrm{SU}$; b) with extension spring without $\mathrm{SU}$; c) with rigid beam and $\mathrm{SU}$; d) with rigid beam without $\mathrm{SU}$

Theoretical part of the study:

1. Calculation of the displacement functions of $q_{0}^{(1)}(\omega)$ and $q_{2}^{(3)}(\omega)$ of point $0^{(1)}$ (tool-holder) and point $2^{(3)}$ (spindle housing) under unit harmonic load applied to the spindle at the location of the front support leading bearing (point $1^{(2)}$ ). These functions, in fact, are dynamic compliances of the tool-holder and spindle housing subsystems and can be calculated from the Eq. (12) and Eq. (14).

2. Calculation of the compliance function $W(\omega)_{13}$ that connects vibration of points $0^{(1)}$ of the tool-holder and point $2^{(3)}$ of the spindle housing Eq. (16).

3. Calculation of tool-holder axis vibration spectrum at characteristic frequencies of spindle 
(Table 1), according to experimental vibration spectrum of spindle housing Eq. (2).

4. Calculation of the spectrum of tool-holder and spindle housing relative vibration and determination of relative vibration amplitudes of tool-holder point $0^{(1)}$ at characteristic frequencies of forced vibrations.

Table 1. Main frequencies of forced vibrations caused by assembling and manufacturing faults of SU on rolling bearings

\begin{tabular}{|c|c|c|}
\hline No. & Forced vibration frequency & $\begin{array}{c}\text { Spindle rotation frequency } f_{r} \text { corresponding to } \\
\text { the frame resonance vibrations } P_{1}^{(4)}, \mathrm{Hz}\end{array}$ \\
\hline 1 & Spindle rotation frequency, $f_{r}$ & $f_{r 1}=33$ \\
\hline 2 & Spindle double rotation frequency, $2 f_{r}$ & $f_{r 2}=16.5$ \\
\hline 3 & Spindle triple rotation frequency, $3 f_{r}$ & $f_{r 3}=11$ \\
\hline 4 & FTF, $f_{F T F}=\frac{1}{2} \cdot f_{r} \cdot\left(1-\frac{d_{B d}}{d_{P d}} \cdot \cos \theta\right)$ & $f_{r 4}=85.77$ \\
\hline 5 & BPFO, $f_{B P F O}=f_{F T F} \cdot N b$ & $f_{r 5}=9.55$ \\
\hline 6 & BPFI, $f_{B P F I}=\left(f_{r}-f_{F T F}\right) \cdot N b$ & $f_{r 6}=5.96$ \\
\hline 7 & BSF, $f_{B S F}=\frac{1}{2} \cdot f_{r} \cdot \frac{d_{P d}}{d_{B d}} \cdot\left(1-\frac{d_{B d}^{2}}{d_{P d}^{2}} \cdot \cos ^{2} \theta\right)$ & $f_{r 7}=16.1$ \\
\hline $\begin{array}{l}\text { Note: } d_{B d}-\text { ball or roller diameter, mm; } N b-\text { number of rolling elements in one row; } \\
d_{P d}-\text { bearing pitch diameter, mm; } \theta-\text { bearing contact angle. }\end{array}$ \\
\hline
\end{tabular}

\subsection{Research results}

Results of theoretical determination of tool-holder and spindle housing dynamic characteristics are shown in Figs. 6-9.

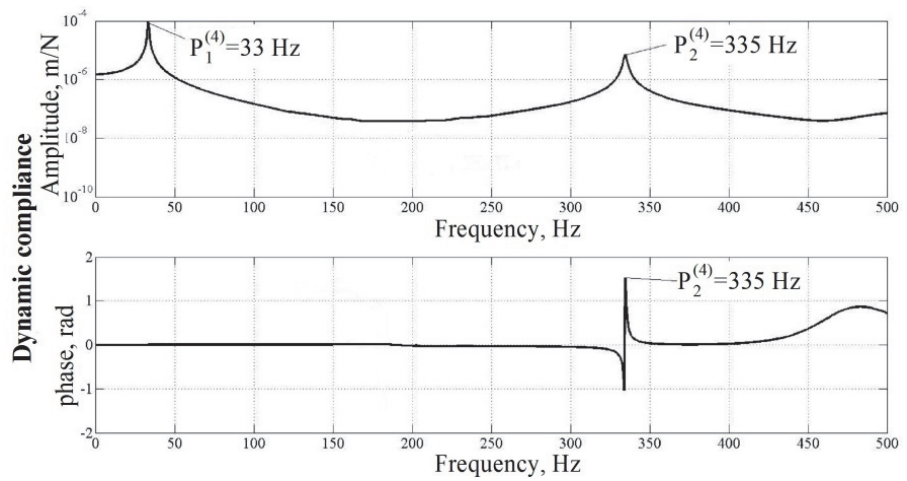

a)

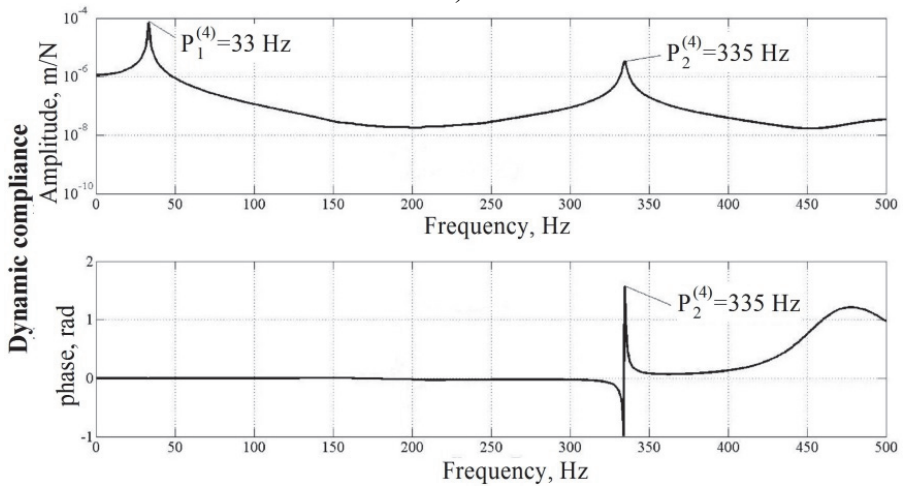

b)

Fig. 6. a) Dynamic compliances of tool-holder and b) spindle housing 
Fig. 6 presents the amplitude-frequency and phase-frequency characteristics of the tool-holder $q_{0}^{(1)}(\omega)$ and spindle housing $q_{2}^{(3)}(\omega)$ dynamic compliances. Fig. 7 illustrates the amplitude-frequency and phase-frequency characteristics of the compliance function. Fig. 8 illustrates experimentally obtained spectrum of the spindle housing absolute vibrations at point $2^{(3)}$ and the corresponding calculated spectrum of the tool-holder absolute vibrations at point $1^{(2)}$ and obtained at spindle speed $n=660 \mathrm{RPM}\left(f_{r}=11 \mathrm{~Hz}\right)$. Fig. 9 shows the calculated spectrum of the relative vibrations of the tool-holder end at spindle speed corresponding to the frame resonances at characteristic frequencies of assembling and manufacturing faults of the SU.

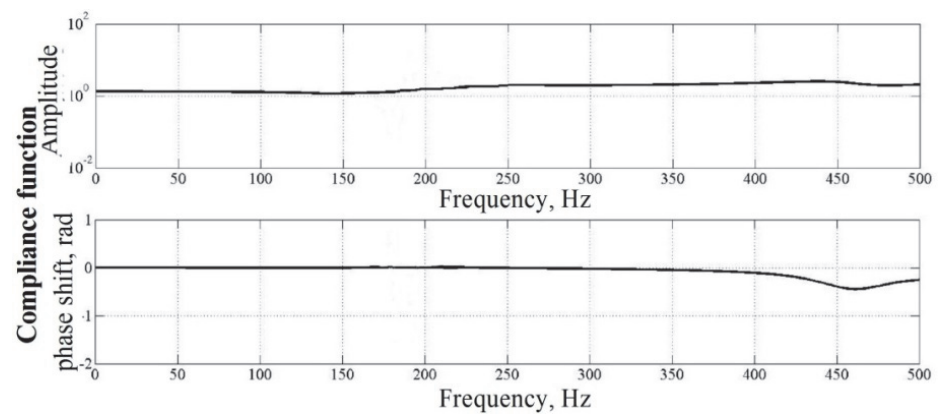

Fig. 7. Compliance function $W(\omega)_{13}$

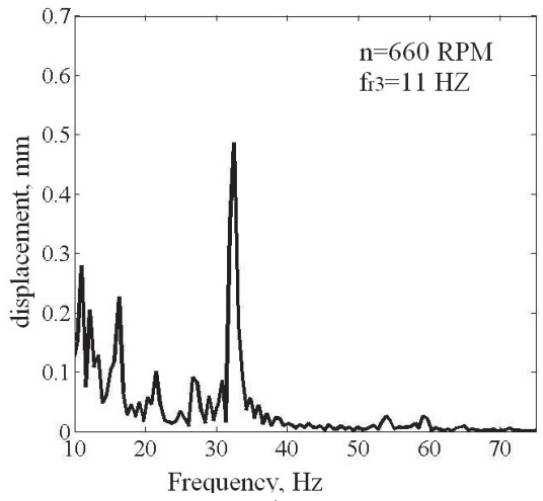

a)

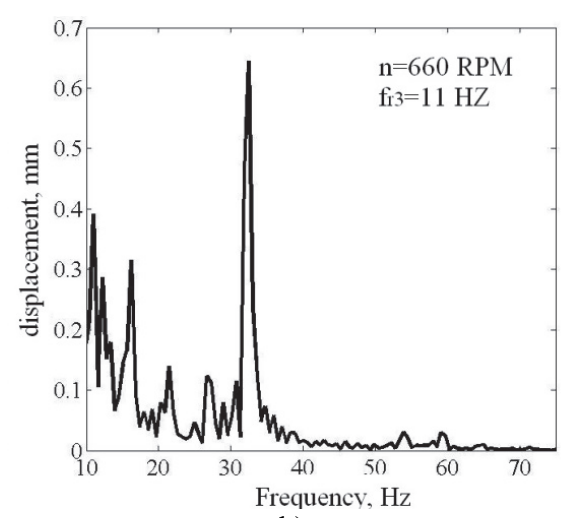

b)

Fig. 8. Absolute vibration spectrums: a) spindle housing - experimental; b) tool-holder - calculated

\subsection{Discussion}

As can be seen from Fig. 7, in the low-frequency range (up to $70 \mathrm{~Hz}$ ) there are two resonance zones corresponding to the first $P_{1}^{(4)}=33 \mathrm{~Hz}$ and second $P_{2}^{(4)}=335 \mathrm{~Hz}$ natural frequencies of the test rig frame. In this case, the compliance function $W(\omega)_{13}$ (Fig. 7) is practically linear. This means that the nature of the absolute vibrations of spindle housing and tool-holder is determined by the vibrations of the frame. This is confirmed by the similarity of the absolute vibration experimental spectrum of the spindle housing and the corresponding calculated tool-holder absolute vibration spectrum (Fig. 8).

From the calculated spectrum of the tool-holder relative vibration (Fig. 10), we can make the following conclusions:

- in the non-resonance mode at low spindle speeds, the effect of assembling and manufacturing faults of spindle units on the formation of tool-holder vibration spectrum is not observed (Fig. 9(a));

- high amplitudes of resonance vibrations at first $f_{r}$ (Fig. 10(f)), second $2 f_{r}$ (Fig. 10(e)) and 
third $3 f_{r}$ (Fig. 10, d) harmonics of spindle rotation frequency indicate the presence of a significant spindle imbalance and misalignment of bearing seats [20];

- a high amplitude of resonance vibrations at $f_{B P F O}$ frequency indicate the presence of a significant defect on the bearing outer ring (Fig. 10(c));

- there are no significant defects on the bearing inner ring (Fig. 10(b)).

This confirms the high information value of the compliance function $W(\omega)_{13}$ and prospective of developed method of indirect measurement of spindle vibrations for the assembling and manufacturing faults diagnostics of SU on rolling bearings that cause low-frequency vibrations.

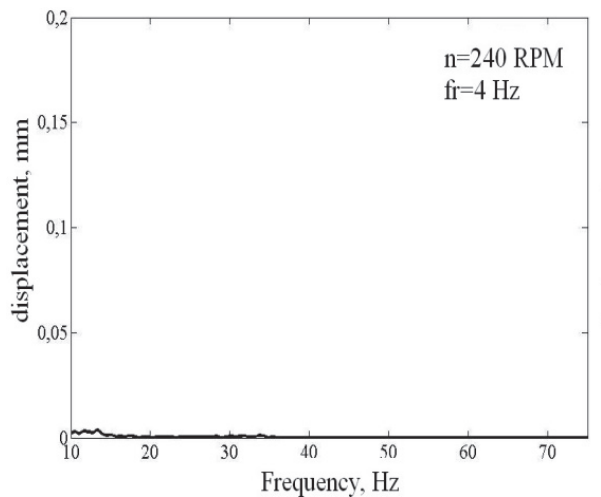

a)

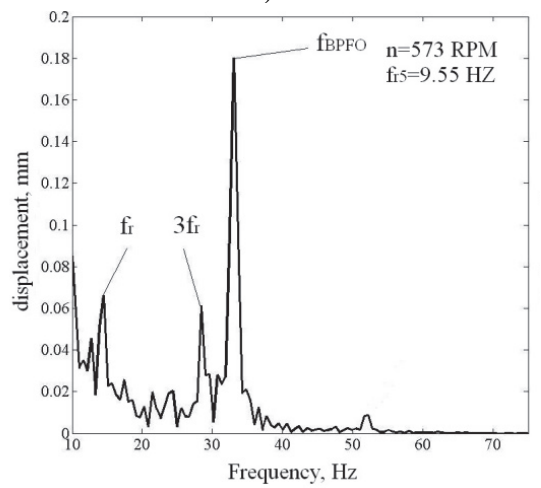

c)

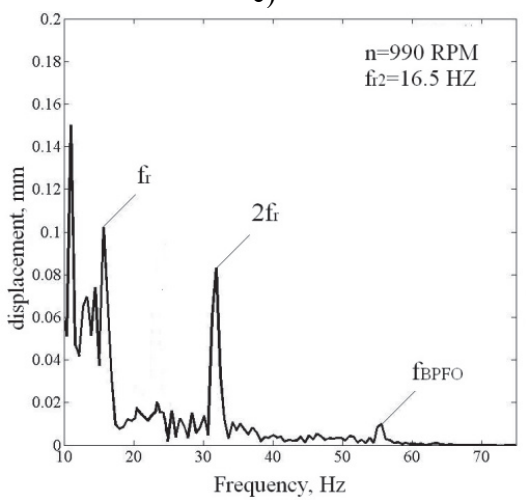

e)

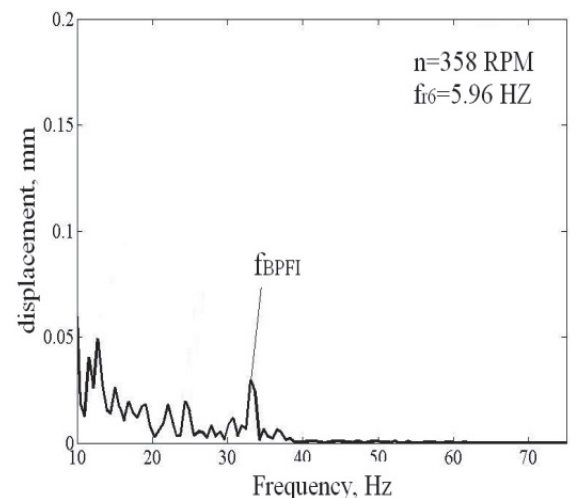

b)

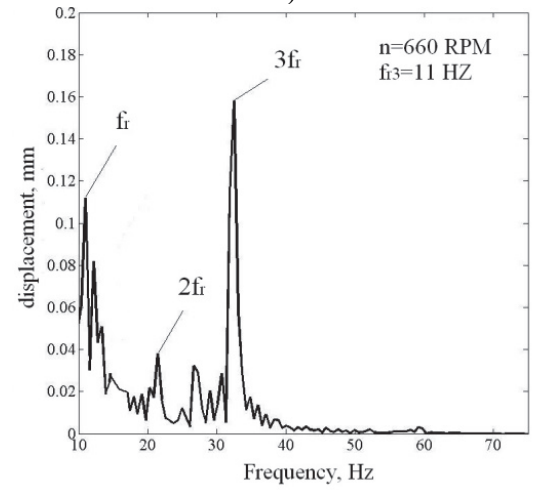

d)

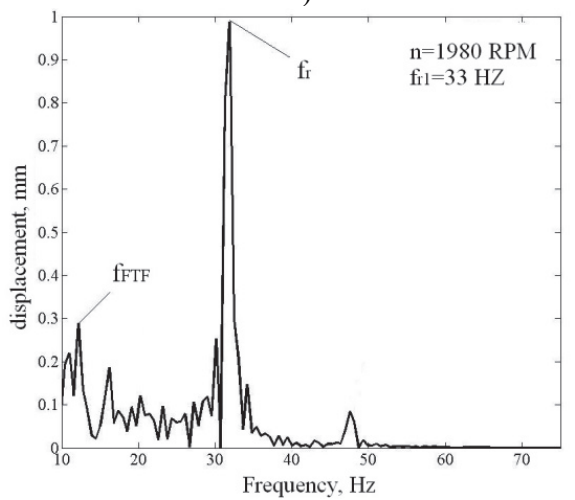

f)

Fig. 9. Calculated spectrums of tool-holder relative vibrations at spindle speeds, corresponding to frame resonance at characteristic frequencies of forced vibrations:

a) non-resonance mode, b) $f_{B P F I}$, c) $f_{B P F O}$, d) $3 f_{r}$; e) $2 f_{r}$; f) $f_{r}$ 


\section{Conclusions}

1) An alternative to direct measurement of spindle vibrations (or tool-holder) may be their identification based on the results of measuring the vibrations of the spindle housing using the calculated compliance function characterizing the ratio of vibrations at the points of measurement and identification. It was this idea that formed the basis for creating a method for indirect measurement of spindle vibrations.

2) When developing a design scheme of a spindle unit mounted on the test rig as a complex mechanical vibratory system, the theoretical compliance function will be the ratio of the dynamic compliances of the corresponding subsystems at the points of calculation and vibration measurement.

3) The application of the dynamic compliance method for the decomposition of a complex mechanical vibratory system, which contains a spindle unit, in combination with the transfer matrix method, used for calculation of subsystems dynamic characteristics, makes it possible to obtain acceptable modeling results with the relative simplicity of design schemes and algorithms computation.

4) The results of the study indicate a high degree of information value of compliance function and prospects of developed method application for the assembling and manufacturing faults diagnostics of SU on rolling bearings in the low-frequency region. It is established that the compliance function gives a clear idea of the relationship between the displacements at measurement and identification points, as well as reasons of these relations variation.

\section{References}

[1] Vibration Diagnostic Guide. SKF Condition Monitoring, CM5003, 2000, p. 28.

[2] Axes of Rotation, Methods for Specifying and Testing. An American National Standard, ASME B89.3.4, 2010.

[3] Graham T. Smith Machine Tool Metrology. An Industrial Handbook. Springer International Publishing Switzerland, 2016, p. 700.

[4] Kok You Cheng Review of Machine Tool Metrology (Spindle). National University of Singapore, Department of Mechanical Engineering, 2011/2012, p. 40.

[5] Trivedi P., Bharti P. K. Study of bearing rolling element defect using empirical mode decomposition technique. International Journal of Engineering Development and Research (IJEDR), Vol. 5, Issue 2, 2017, p. 553-565.

[6] Shakya P., Darpe A. K., Kulkarni M. S. Vibration based fault diagnosis in rolling element bearings: ranking of various time, frequency and time-frequency domain data-based damage identification parameters. International Journal of Condition Monitoring, Vol. 3, Issue 2, 2013, p. 53-62.

[7] Nahata S., Anandan K. P., Ozdoganlar O. B. LDV-based spindle metrology for ultra-high-speed micromachining spindles. North American Manufacturing Research Conference, 2013.

[8] Barszcz T., Sawalhi N. Fault detection enhancement in rolling element bearings using the minimum entropy deconvolution. Archives of Acoustics, Vol. 37, Issue 2, 2012, p. 131-141.

[9] Barzdaitis V., Zemaitis V., Zebelys K., Pocius Z., Mazeika P. Condition monitoring of roller bearings using different diagnostic methods. Diagnostyka, Vol. 30, Issue 1, 2004, p. 53-60.

[10] Jamaludin N., Mba D., Bannister R. H. Condition monitoring of slow-speed rolling element bearings using stress waves. Proceedings of the Institution of Mechanical Engineers, Part E: Journal of Process Mechanical Engineering, Vol. 215, Issue 4, 2001, p. 245-271.

[11] Kozochkin M. P., Sabirov F. S. Detection of spindle units defects by vibration acoustic methods. Vestnik UGATU - Ufa: UGATU, Vol. 13, Issue 1, 2009, p. 133-137.

[12] Denisenko A. F., Gasparov E. S. Vibration characteristics analysis of spindle unit bearings. Vestnik Samarskoko GTU, Technical Science, Vol. 2, Issue 34, 2012, p. 103-108.

[13] Jui Hung P., Yuan Lai L., Tzuo Luo L., Hsi Hsiao H. Prediction of the dynamic characteristics of a milling machine using the integrated model of machine frame and spindle unit. World Academy of Science, Engineering and Technology, Vol. 6, 2012, p. 626-632.

[14] Danylchenko Yu M., Petryshyn A. I., Danylchenko M. A. Test Bench for Assembling and Manufacturing Faults Diagnostics of Spindle Sleeve. Patent No. UA112210U, 2016. 
[15] Banakh L. Y., Kempner M. L. Vibrations of Mechanical Systems with Regular Structure. Springer, 2010, p. 250.

[16] Biderman V. L. Applied Theory of Mechanical Vibrations. Mashinostroenie, Moscow, 1972, p. 416.

[17] Danylchenko Yu M., Dorozhko A. O., Petryshyn A. I. Research of the dynamical characteristics of "spindle unit" mechanical system. Vestnik MSTU STANKIN, Vol. 28, Issue 1, 2014, p. 81-91.

[18] Hatter D. J. Matrix Computer Methods of Vibration Analysis. Butterworth, London, 1973, p. 206.

[19] Ivovych V. A. Transfer Matrices in Elastic Systems Dynamic. Handbook, Mashinostroenie, Moscow, 1981, p. 183.

[20] Balitckiy A. V., Barkov A. V., Barkov N. A., et al. Vibration-Based Diagnostics. Non-destructive testing: Handbook, Vol. 7, Book 2, Mashinostroenie, Moscow, 2005, p. 829.

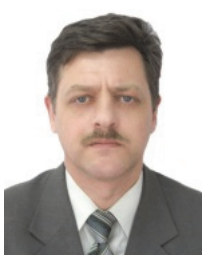

Yuriy Danylchenko received Doctor of Science in engineering in National Technical University of Ukraine "Kyiv Politechnic Institute", Kyiv, Ukraine, in 2004, where he works to the present day. His current research interests include dynamic and spindle units accuracy, rolling bearings rotating accuracy, vibration monitoring of spindle units on rolling bearings.

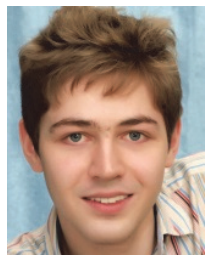

Andriy Petryshyn received Ph.D. degree in National Technical University of Ukraine "Kyiv Politechnic Institute", Kyiv, Ukraine, in 2016, where he works to the present day. His current research interests include control, spindle units dynamics and fault diagnosis. 


\title{
2.3. Methods of ensuring the required dynamics of an AMB supported axial LVAD rotor
}

\author{
Yulia Bogdanova ${ }^{1}$, Alexander Gouskov ${ }^{2}$ \\ ${ }_{1,2}$ Bauman Moscow State Technical University, Moscow, Russia \\ ${ }^{1}$ National Research Center "Kurchatov Institute", Moscow, Russia \\ ${ }^{2}$ Vibrations Laboratory, RAS Institute of Machine Science, Moscow, Russia \\ E-mail: 'bogdanova.bmstu@gmail.com, ${ }^{2}$ gouskov_am@mail.ru
}

\begin{abstract}
Left ventricular assist device is a main alternative to donor heart transplantation today. The third-generation of devices includes continuous-flow pumps with an impeller or rotor suspended in the blood flow path using a magnetic bearing design. Magnetic bearings offer the advantages of non-contact suspension, thus, there is no wear over time and no friction, and large clearances. Active magnetic bearings are a mechatronic product in which the rotor dynamics can be controlled actively through the bearings. The main problem of non-contact suspension is to ensure a stability of rotor position. Besides researches of the last years have shown that with prolonged use of pumps with the constant blood flow, i.e. without pulsations, patients have complications associated with a cardiac atrophy, a coronary artery disorder, an embrittlement of vessels and, as a result, an internal bleeding. In this regard, the creation of blood flow pulsations and the provision of the stable controlled behavior of the rotor in pulsating modes of functioning of the axial LVAD with magnetic bearings are relevant and proposed in this article.
\end{abstract}

Keywords: nonlinear dynamics, rotor stabilization, attractor, invariant, synergetic control theory, left ventricular assist device.

\section{Introduction}

Cardiovascular diseases are a major cause of death worldwide. If medical treatments fail to restore an adequate blood flow in a patient, a mechanical support is needed.

Left ventricular assist device (LVAD) re-establish sufficient perfusion in patients with advanced heart failure when medication and other surgical therapies, such as valve replacement or ventricular reconstruction, have reached their limits. An implanted device draws blood from the left ventricle into the aorta. Energy is supplied from an external battery and transferred to the LVAD through a percutaneous cable [1].

Unfortunately, the base of donor hearts is small even in the most developed countries. Annually in the countries of Europe and the USA about 3500 transplantations are performed, whereas in Russia the number of transplantations per year is about 165 [2]. Today LVAD is the basic alternative to heart transplantation [3].

Patients are forced to be with implanted blood pumps for a long time. Increasingly, patients abandon the donor heart in favor of mechanical support [4]. Therefore, the main task of the development of LVAD is to improve the quality of the device.

To date, different types of blood pumps have been developed, but only a few are clinically available. The development of LVAD is a highly complex and interdisciplinary process with many requirements to be fulfilled [1]. Generally, LVADs can broadly be divided into first-, second and third-generation devices.

The first-generation of devices includes pulsatile membrane displacement pumps. These devices are engineered with a pumping chamber and two valves that fill and empty cyclically. Disadvantages of such blood pumps are large size, unreliable and an external position [5].

The second-generation devices include implantable, continuous flow, rotary pumps that offer several advantages over pulsatile flow pumps. Some of the advantages are the smaller size that reduces the risk of infections and simpler implantation. These pumps have an internal rotor within the blood flow path that is suspended by contact bearings $[5,6]$.

But in this design, there are some problems. Ball bearings are lubricated directly with blood 
that leading to extensive damage to the red blood cells, called hemolysis [7, 8]. Seal failures resulted in many early pump failures. Additionally, seals accumulate thrombosis. Bloodlubricated bearings tend to be subject to high shear due to the high rotational speeds and small clearances [9]. So the most important problems associated with the development of the LVAD are the blood trauma (hemolysis), thrombosis, unreliability of the devices, lack of pulsations.

The third-generation of devices includes continuous-flow pumps with an impeller or rotor suspended in the blood flow path using a magnetic bearing design. Magnetic bearings offer the advantages of non-contact suspension, thus, there is no wear over time and no friction, and large clearances $[10,11]$.

The large clearance allows the rotor to rotate at high speeds with the appearance of low shear stresses that reduces the rate of hemolysis and thrombosis. The magnetic bearings provide the durability and the operational reliability of the blood pump. Moreover, there is no need for maintenance. In addition, active magnetic bearings (AMB) are controlled by electronic control scheme with feedback. This provides a higher positioning accuracy of the rotor [12]. The development of LVAD of axial type with magnetic bearings suspension becomes perspective.

Currently the most popular blood pump of axial type with magnetic bearings introduced into medical practice is Incor (BerlinHeart Company). Incor as well as other pumps of the second and third generation has a continuous blood flow. But researches of the last years have shown that with prolonged use of pumps with the constant blood flow, i.e. without pulsations, patients have complications associated with a cardiac atrophy, a coronary artery disorder, an embrittlement of vessels and, as a result, an internal bleeding [13, 14]. In this regard, a task of the creation of pulsations at the outflow from the pump chamber becomes one of the priority when developing the pump of a new generation.

There is not the axial blood pump of pulsating type in the world market yet. In contrast to the huge number of researches devoted to the study of the blood flow in the pump chamber, the influence of shear stresses on erythrocytes, the design of the geometry of the flow part, there are practically no studies related to the dynamics of the rotor with active magnetic supports in conditions of blood flow and pump functioning regimes. The problem of absence of mathematical models of the nonlinear rotor dynamics of the LVAD with magnetic bearings remains open. AMB are widely used in machine-tool constructions, in vacuum systems, in high-speed engineering [15-17]. As a rule, objects have a small gap, and in most works the magnetic suspension model linearized at the equilibrium position is used. However, the nonlinear properties of magnetic bearings can lead to the dynamics of the rotor, whose motion is completely different from that predicted by the linear model [18-22].

The development of reliable, designed for long-term mechanical support, domestic LVAD of the new generation seems perspective. The analysis of researches allows to state that to date there are still no scientifically based methodologies for designing such complex system as blood pump. Especially it concerns the design techniques of the rotor-magnetic part of the pump. Therefore, the creation of blood flow pulsations and the provision of the stable controlled behavior of the rotor in pulsating modes of functioning of the axial LVAD with AMB are relevant.

\section{Equations of motion}

The rigid rotor with mass $m$ of the axial LVAD rotates with the constant angular velocity $\Omega$ in two radial active magnetic bearings AMB A and AMB B (Fig. 1(a)). Magnetic bearings consist of four identical contours (Fig. 1(b)), which are located symmetrically opposite each other.

The small motions of the rotor are described by the displacements $x^{o}$ and $y^{o}$ of its geometric center $O$ with respect to the inertial reference $I X Y Z$ and by inclinations of axes $O x^{o} y^{o} z^{o}$ (Fig. 2). These inclinations are described by two angles $\alpha$ and $\beta$. The axis $O z^{o}$ coincides with the geometrical longitudinal axis of the rotor and the axis $O x^{o}$ passes through the center of mass of the rotor $C . C x^{\prime} y^{\prime} z^{\prime}$ is the rotor-fixed system of principal axes with $I_{x^{\prime}}=I_{y^{\prime}}=I_{x}$ and $I_{z^{\prime}}=I_{z}$ 
moments of inertia. The rotor motion in the longitudinal direction is fixed by permanent magnets and therefore is not considered. Rotor has a static and dynamic unbalances. The static unbalance is characterized by the eccentricity $O C=e$. The dynamic unbalance is characterized by the angle $\gamma_{x}=\gamma[23,24]$. In the nominal reference position of the rotor, axes of $O x^{o} y^{o} z^{o}$ system coincide with the origin of the inertially fixed coordinate system $I X Y Z$.

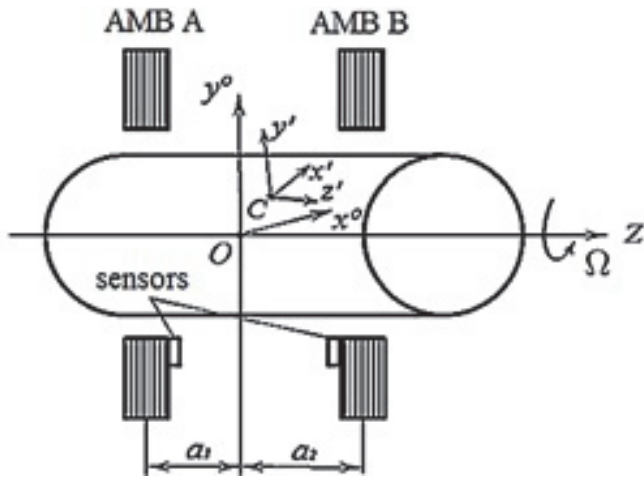

a)

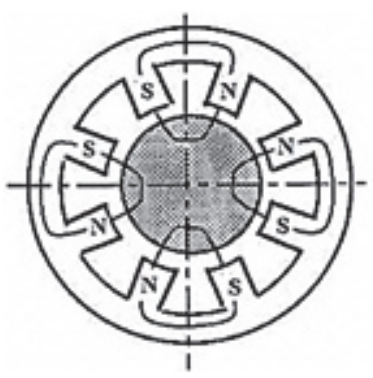

b)

Fig. 1. a) Symmetrical rigid rotor in two radial active magnetic bearings,

b) geometry of the radial bearing magnet

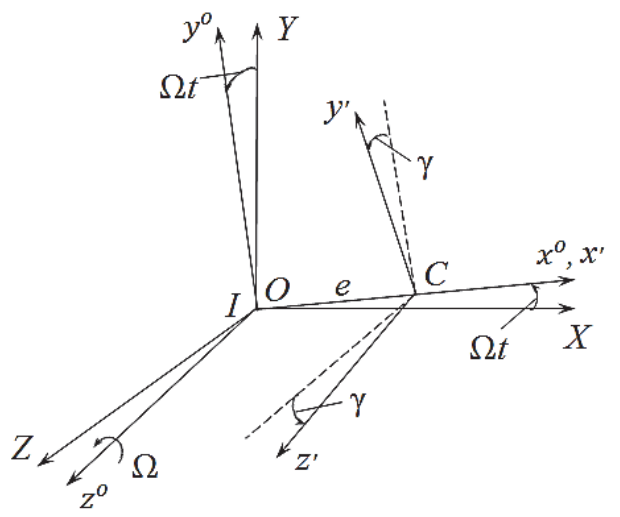

Fig. 2. Relationship of coordinate systems

The angular velocity $\Omega$ of the rotor about its longitudinal axis is assumed to be constant. The equations of motion for the variables:

$\mathbf{q}=\left\{x_{o}, y_{o}, \alpha, \beta\right\}^{T}$.

Follow from Lagrange's equations:

$\frac{d}{d t}\left(\frac{\partial T}{\partial \dot{q}_{i}}\right)-\frac{\partial T}{\partial q_{i}}=Q_{i}, \quad i=1, \ldots, 4$

With the kinetic energy $T$ and the generalized forces $Q_{i}$.

The kinetic energy $T$ is:

$T=\frac{1}{2} m\left(x_{0}^{2}+y_{0}^{2}\right)+m W e\left(-x_{o} \sin W t+y_{0} \cos W t\right)+\frac{1}{2} \mathbf{\Omega}^{T} \mathbf{I}_{o} \mathbf{\Omega}$, 
where $\mathbf{I}_{o}$ is the matrix of the inertia tensor and $\boldsymbol{\Omega}$ is expressed [17]:

$\boldsymbol{\Omega}=\left\{\begin{array}{l}\Omega_{x^{o}} \\ \Omega_{y^{o}} \\ \Omega_{z^{o}}\end{array}\right\}=\left[\begin{array}{c}\dot{\alpha} \cos (\Omega t)+\dot{\beta} \sin (\Omega t) \\ -\dot{\alpha} \sin (\Omega t)+\dot{\beta} \cos (\Omega t) \\ \Omega-\alpha \dot{\beta}\end{array}\right]$.

Hence, the equations of motion follow in the form [17]:

$$
\begin{aligned}
& m \ddot{x}_{o}=F_{1}+F_{\text {ext } 1}+m \Omega^{2} e \cos (\Omega t), \\
& m \ddot{y}_{o}=F_{2}+F_{\text {ext } 2}+m \Omega^{2} e \sin (\Omega t), \\
& I_{x} \ddot{\alpha}+I_{z} \Omega \dot{\beta}=F_{3}+F_{\text {ext } 3}+\Omega^{2} \gamma\left(I_{x}-I_{z}\right) \cos (\Omega t), \\
& I_{x} \ddot{\beta}-I_{z} \Omega \dot{\alpha}=F_{4}+F_{\text {ext } 4}+\Omega^{2} \gamma\left(I_{x}-I_{z}\right) \sin (\Omega t) .
\end{aligned}
$$

The first two equations in Eq. (5) describe the translational motion of the rotor. The last two represent angular motions, with the second terms in the left-hand sides characterizing the gyroscopic effects. As can be seen from equations Eq. (5), the parameters of unbalances play the role of an external periodic load.

In matrix form the equations of motion are:

$$
\mathbf{M} \ddot{\mathbf{q}}+\Omega \mathbf{G} \dot{\mathbf{q}}=\mathbf{F}+\mathbf{F}_{\text {ext }}+\Omega^{2} \mathbf{Q}_{v}(t),
$$

where $\mathbf{M}=\operatorname{diag}\left(m, m, I_{x}, I_{x}\right)$ is the inertia matrix, $\mathbf{G}$ is the skew-symmetric gyroscopic matrix with two non-zero elements $(\mathbf{G})_{34}=-(\mathbf{G})_{43}=I_{z}, \mathbf{F}=\left\{F_{1}, \ldots, F_{4}\right\}^{T}, \mathbf{F}_{\text {ext }}=\left\{F_{\text {ext } 1}, \ldots, F_{\text {ext } 4}\right\}^{T}$ are the electromagnetic reactions of the suspension and the generalized external forces respectively, $\mathbf{Q}_{v}(t)$ is the generalized excitation loads which expressed:

$$
\mathbf{Q}_{v}(t)=\left\{\begin{array}{c}
\operatorname{mecos}(\Omega t) \\
\operatorname{mesin}(\Omega t) \\
\gamma\left(I_{x}-I_{z}\right) \cos (\Omega t) \\
\gamma\left(I_{x}-I_{z}\right) \sin (\Omega t)
\end{array}\right\} .
$$

The generalized electromagnetic reactions of the suspension are created by controlling magnetic bearings forces:

$\mathbf{F}_{A M B}=\left\{F_{A x}, F_{A y}, F_{B x}, F_{B y}\right\}^{T}$,

which are applied to the rotor in the control points of AMB A and AMB B at the distance $a_{1}$ and $a_{2}$ from the geometric center $O$. The sensors have the same longitudinal coordinates as the centers of the support points of the rotor, that is, the control points coincide with the measurement points.

Vectors $\mathbf{F}$ and $\mathbf{F}_{A M B}$ are related by:

$\mathbf{F}=\mathbf{T}_{b}{ }^{T} \mathbf{F}_{A M B}$

where $\mathbf{T}_{b}$ is the transformation matrix:

$$
\mathbf{T}_{b}=\left[\begin{array}{cccc}
1 & 0 & 0 & -a_{1} \\
0 & 1 & a_{1} & 0 \\
1 & 0 & 0 & a_{2} \\
0 & 1 & -a_{2} & 0
\end{array}\right] .
$$

This leads to the following form for the equations of motion: 
$\mathbf{M} \ddot{\mathbf{q}}+\Omega \mathbf{G} \dot{\mathbf{q}}=\mathbf{T}_{b}^{T} \mathbf{F}_{A M B}+\mathbf{F}_{\text {ext }}+\Omega^{2} \mathbf{Q}_{v}(t)$.

As a rule, the magnetic suspension equations are linearized at the equilibrium position [25]. Then the linear model is adequate only for small deviations of the variables from their nominal values or displacement values. In fact, these deviations can be considerable. In some works, it is shown, if the deflections of the rotor exceed half of the gap, the net magnetic force which is produced by a pair of opposite electromagnets changes more than $44 \%$ from the linear approximation of itself [20]. Therefore, the magnetic bearings operation changes from the equilibrium point. For small air gaps and large control currents the nonlinear characteristics become very significant. Dynamic behavior of the rotor can be changed from the linear model result because of the nonlinear characteristics of magnetic bearings. The nonlinear model of bearings forces has a form:

$$
\begin{aligned}
& F_{A x}=F_{A x 1}-F_{A x 2}=k_{A}\left[\left(\frac{i_{0}+i_{A x}}{\delta-x_{b A}}\right)^{2}-\left(\frac{i_{0}-i_{A x}}{\delta+x_{b A}}\right)^{2}\right], \\
& F_{A y}=F_{A y 1}-F_{A y 2}=k_{A}\left[\left(\frac{i_{0}+i_{A y}}{\delta-y_{b A}}\right)^{2}-\left(\frac{i_{0}-i_{A y}}{\delta+y_{b A}}\right)^{2}\right], \\
& F_{B x}=F_{B x 1}-F_{B x 2}=k_{B}\left[\left(\frac{i_{0}+i_{B x}}{\delta-x_{b B}}\right)^{2}-\left(\frac{i_{0}-i_{B x}}{\delta+x_{b B}}\right)^{2}\right], \\
& F_{B y}=F_{B y 1}-F_{B y 2}=k_{B}\left[\left(\frac{i_{0}+i_{B y}}{\delta-y_{b B}}\right)^{2}-\left(\frac{i_{0}-i_{B y}}{\delta+y_{b B}}\right)^{2}\right],
\end{aligned}
$$

with the bias current $i_{0}$, the constructive parameter $k_{A}=k_{B}=\frac{\mu_{0} n^{2} A}{2} \cos (\chi)\left(\mu_{0}-\right.$ magnetic constant, $n$-number of winding turns, $A$ - winding area, $\chi$ - angle of deviation of magnetic force from vertical/horizontal equal to $22.5^{\circ}$ ) and the radial gap $\delta$. The currents $i_{A x}$ and $i_{B x}$ create the magnetic forces $F_{A x}$ and $F_{B x}$ in the $X$ direction and the currents $i_{A y}, i_{B y}$ create the magnetic forces $F_{A y}$ and $F_{B y}$ in the direction of the axis $Y$ (Fig. 3).
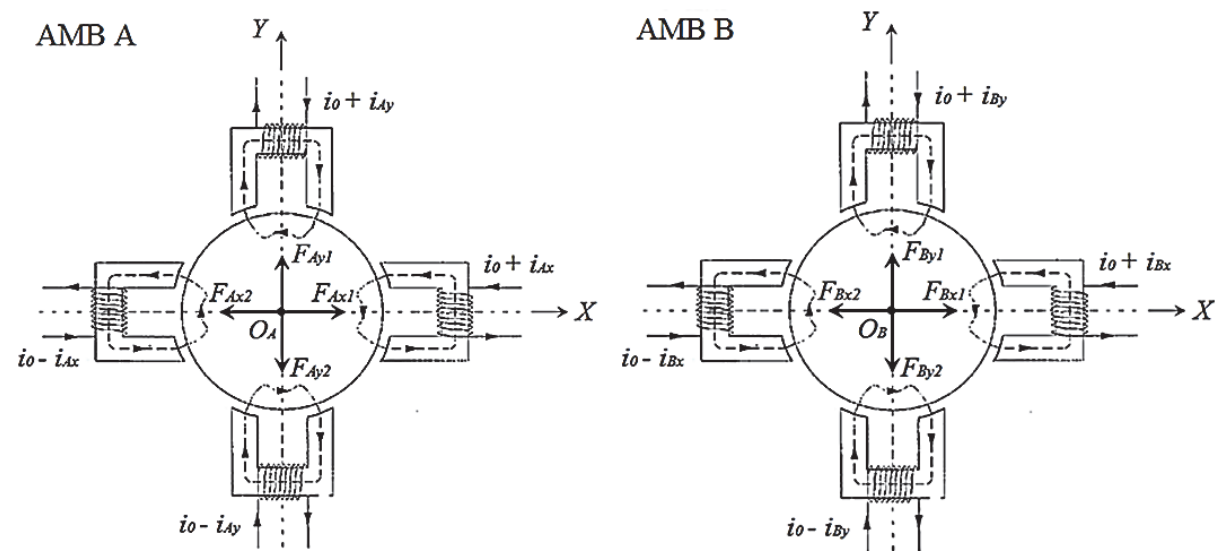

Fig. 3. Principle of radial force generation

The vector:

$\mathbf{q}_{b}=\left\{\begin{array}{l}x_{b A} \\ y_{b A} \\ x_{b B} \\ y_{b B}\end{array}\right\}=\left\{\begin{array}{l}x_{o}-a_{1} \beta \\ y_{o}+a_{1} \alpha \\ x_{o}+a_{2} \beta \\ y_{o}-a_{2} \alpha\end{array}\right\}$. 
Comprises the rotor displacements within the magnetic bearings, whereas the vector $\mathbf{i}=\left\{i_{A x}, i_{B x}, i_{A y}, i_{B y}\right\}^{T}$ contains the individual coil control currents of all four bearing magnets.

At a switched-on electric motor the right-hand side of the equations of motion Eq. (11) will include a matrix of positional forces $\mathbf{C}_{m}[17]$ :

$\mathbf{M} \ddot{\mathbf{q}}+\Omega \mathbf{G} \dot{\mathbf{q}}=\mathbf{T}_{b}^{T} \mathbf{F}_{A M B}+\mathbf{C}_{m} \mathbf{q}+\mathbf{F}_{e x t}+\Omega^{2} \mathbf{Q}_{v}(t)$.

For the motor with a length $l_{m}$ arranged in a symmetrical suspension, the matrix, taking into account its effect on the suspension, has the form:

$\mathbf{C}=\frac{c_{m}}{4}\left[\begin{array}{cccc}1+k_{m} & 0 & 1-k_{m} & 0 \\ 0 & 1+k_{m} & 0 & 1-k_{m} \\ 1-k_{m} & 0 & 1+k_{m} & 0 \\ 0 & 1-k_{m} & 0 & 1+k_{m}\end{array}\right]$

where $c_{m}$ is the motor stiffness, $k_{m}=\frac{l_{m}^{2}}{3\left(a_{1}+a_{2}\right)^{2}}$ is the motor design parameter (constant value).

Matrices $\mathbf{C}$ and $\mathbf{C}_{m}$ are related by:

$\mathbf{C}_{m}=\mathbf{T}_{b}^{T} \mathbf{C} \mathbf{T}_{b}$

Then the equations of motion follow in the form:

$\mathbf{M} \ddot{\mathbf{q}}+\Omega \mathbf{G} \dot{\mathbf{q}}+\left(-\mathbf{T}_{b}^{T} \mathbf{C} \mathbf{T}_{b}\right) \mathbf{q}=\mathbf{T}_{b}^{T} \mathbf{F}_{A M B}+\mathbf{F}_{\text {ext }}+\Omega^{2} \mathbf{Q}_{v}(t)$.

Rotation in the blood flow is associated with the hydrodynamic resistance. Since the rotor is screw and has a non-constant shape, the standard theory of hydrodynamic lubrication cannot be applied. From the theory of similarity and dimension vector of hydrodynamic resistance can take the form:

$\left\{\begin{array}{l}M_{H x} \\ M_{H y} \\ M_{H z}\end{array}\right\}=c_{w} \eta \pi r^{2} l\left\{\begin{array}{c}-\dot{\alpha}-\Omega \beta \\ -\dot{\beta}+\Omega \alpha \\ -\Omega\end{array}\right\}$

with the coefficient of medium resistance $c_{w}$ and the dynamic viscosity of blood $\eta$. The radius and the length of the rotor are indicated $r$ and $l$ respectively. The relation (18) can be written in the form:

$\begin{aligned} \mathbf{M}_{H} & =\mathbf{H}_{D} \dot{\mathbf{q}}+\Omega \mathbf{H}_{S} \mathbf{q}, \\ \mathbf{H}_{D} & =\left[\begin{array}{cccc}0 & 0 & 0 & 0 \\ 0 & 0 & 0 & 0 \\ 0 & 0 & -c_{w} \eta \pi r^{2} l & 0 \\ 0 & 0 & 0 & -c_{w} \eta \pi r^{2} l\end{array}\right], \quad \mathbf{H}_{S}=\left[\begin{array}{cccc}0 & 0 & 0 & 0 \\ 0 & 0 & 0 & 0 \\ 0 & 0 & 0 & -c_{w} \eta \pi r^{2} l \\ 0 & 0 & c_{w} \eta \pi r^{2} l & 0\end{array}\right] .\end{aligned}$

With considering Eq. (19) the equations of motion are expressed:

$\mathbf{M} \ddot{\mathbf{q}}+\left(\Omega \mathbf{G}-\mathbf{H}_{D}\right) \dot{\mathbf{q}}+\left(-\mathbf{T}_{b}^{T} \mathbf{C} \mathbf{T}_{b}-\Omega \mathbf{H}_{S}\right) \mathbf{q}=\mathbf{T}_{b}^{T} \mathbf{F}_{A M B}+\mathbf{F}_{e x t}+\Omega^{2} \mathbf{Q}_{v}(t)$.

Influence of the various accelerations that a person experiences in everyday life and in professional activity is taken into account in the vector of the generalized external forces. External inertial loads can be either piecewise-constant or harmonic: 
$\mathbf{F}_{e x t}=\left\{\begin{array}{l}F_{x}+A_{x} \sin (p t) \\ F_{y}+A_{y} \sin (p t) \\ M_{x}+B_{x} \sin (p t) \\ M_{y}+B_{y} \sin (p t)\end{array}\right\}$,

where $F_{x}, F_{y}, M_{x}, M_{y}$ - external piecewise-constant loads, $A_{x}, A_{y}, B_{x}, B_{y}, p$ - amplitudes and frequency of external harmonic influence.

There is interest to present the equations of motion in a dimensionless form [26, 27], highlighting the dimensionless complexes (similarity criteria), that characterize a whole class of an artificial circulatory supports. Applying the change of variables:

$x_{1}=x_{o}, \quad y_{1}=y_{o}, \quad \alpha_{1}=\alpha, \quad \beta_{1}=\beta$,

$x_{2}=\dot{x}_{1}, \quad y_{2}=\dot{y}_{1}, \quad \alpha_{2}=\dot{\alpha}_{1}, \quad \beta_{2}=\dot{\beta}_{1}$,

and formulas for the transition to dimensionless quantities $t=\bar{t} S_{t}, x_{1}=\bar{x}_{1} S_{x}, y_{1}=\bar{y}_{1} S_{x}$, $\alpha_{1}=\bar{\alpha}_{1} S_{\beta}, \beta_{1}=\bar{\beta}_{1} S_{\beta}, a_{1}=\bar{a}_{1} S_{L}, a_{2}=\bar{a}_{2} S_{L}, i_{0}=\bar{\imath}_{0} S_{i}, i_{A x}=\bar{\imath}_{A x} S_{i}, i_{A y}=\bar{\imath}_{A y} S_{i}, i_{B x}=\bar{\imath}_{B x} S_{i}$, $\delta=\bar{\delta} S_{x}, k_{m}=\bar{k}_{m}, F_{x}=\bar{F}_{x} S_{F}, F_{y}=\bar{F}_{y} S_{F}, M_{x}=\bar{M}_{x} S_{M}, M_{y}=\bar{M}_{y} S_{M}, r=\bar{r} S_{L}, l=\bar{l} S_{L}$, $e=\bar{e} S_{x}, A_{x}=\bar{A}_{x} S_{F}, A_{y}=\bar{A}_{y} S_{F}, B_{x}=\bar{B}_{x} S_{M}, B_{y}=\bar{B}_{y} S_{M}, \Omega=\bar{\Omega} S_{\Omega}, p=\bar{p} S_{\Omega}, \gamma=\bar{\gamma} S_{\beta}$ lead to the equations of motion in the dimensionless form (dimensionless quantities are with a dash, ()$\left.^{\prime}=d / d \bar{t}\right)$ :

$\bar{x}_{1}^{\prime}=\bar{x}_{2}$,

$\bar{x}_{2}^{\prime}=\bar{x}_{1}+\frac{\left(-\bar{a}_{1}+\bar{a}_{2}\right)}{2} \bar{\beta}_{1}+\bar{F}_{A x}+\bar{F}_{B x}+\bar{F}_{x}+\bar{A}_{x} \sin (\bar{p} \bar{t})+\bar{\Omega}^{2} \bar{e} \cos (\bar{\Omega} \bar{t})$,

$\bar{y}_{1}^{\prime}=\bar{y}_{2}$,

$\bar{y}_{2}^{\prime}=\bar{y}_{1}+\frac{\left(\bar{a}_{1}-\bar{a}_{2}\right)}{2} \bar{\alpha}_{1}+\bar{F}_{A y}+\bar{F}_{B y}+\bar{F}_{y}+\bar{A}_{y} \sin (\bar{p} \bar{t})+\bar{\Omega}^{2} \bar{e} \sin (\bar{\Omega} \bar{t})$,

$\bar{\alpha}_{1}^{\prime}=\bar{\alpha}_{2}$,

$\bar{\alpha}_{2}^{\prime \prime}=-\Pi_{H} \bar{\alpha}_{2}-\Pi_{1} \bar{\Omega} \bar{\beta}_{2}+\frac{\Pi_{2}}{2}\left(\bar{a}_{1}-\bar{a}_{2}\right) \bar{y}_{1}-\Pi_{H} \bar{\Omega} \bar{\beta}_{1}+\bar{a}_{1} \Pi_{2} \bar{F}_{A y}-\bar{a}_{2} \Pi_{2} \bar{F}_{B y}+\ldots$

$+\frac{\Pi_{2}}{4}\left(\left(\bar{a}_{1}^{2}+\bar{a}_{2}^{2}\right)\left(1+\bar{k}_{m}\right)-2 \bar{a}_{1} \bar{a}_{2}\left(1-\bar{k}_{m}\right)\right) \bar{\alpha}_{1}+\bar{M}_{x}+\bar{B}_{x} \sin (\bar{p} \bar{t})$

$+\bar{\Omega}^{2} \bar{\gamma}\left(1-\Pi_{1}\right) \cos (\bar{\Omega} \bar{t})$,

$\bar{\beta}_{1}^{\prime}=\bar{\beta}_{2}$,

$\bar{\beta}_{2}^{\prime}=-\Pi_{H} \bar{\beta}_{2}+\Pi_{1} \bar{\Omega} \bar{\alpha}_{2}+\frac{\Pi_{2}}{2}\left(-\bar{a}_{1}+\bar{a}_{2}\right) \bar{x}_{1}+\Pi_{H} \bar{\Omega} \bar{\alpha}_{1}-\bar{a}_{1} \Pi_{2} \bar{F}_{A x}+\bar{a}_{2} \Pi_{2} \bar{F}_{B x}+\ldots$

$+\frac{\Pi_{2}}{4}\left(\left(\bar{a}_{1}^{2}+\bar{a}_{2}^{2}\right)\left(1+\bar{k}_{m}\right)-2 \bar{a}_{1} \bar{a}_{2}\left(1-\bar{k}_{m}\right)\right) \bar{\beta}_{1}+\bar{M}_{y}+\bar{B}_{y} \sin (\bar{p} \bar{t})$

$+\bar{\Omega}^{2} \bar{\gamma}\left(1-\Pi_{1}\right) \sin (\bar{\Omega} \bar{t})$,

where:

$\bar{F}_{A x}=\Pi_{K}\left[\left(\frac{\bar{\imath}_{0}+\bar{\imath}_{A x}}{\bar{\delta}-\left(\bar{x}_{1}-\bar{a}_{1} \bar{\beta}_{1}\right)}\right)^{2}-\left(\frac{\bar{l}_{0}-\bar{l}_{A x}}{\bar{\delta}+\left(\bar{x}_{1}-\bar{a}_{1} \bar{\beta}_{1}\right)}\right)^{2}\right]$,
$\bar{F}_{B x}=\Pi_{K}\left[\left(\frac{\bar{l}_{0}+\bar{l}_{B x}}{\bar{\delta}-\left(\bar{x}_{1}+\bar{a}_{2} \bar{\beta}_{1}\right)}\right)^{2}-\left(\frac{\bar{\imath}_{0}-\bar{l}_{B x}}{\bar{\delta}+\left(\bar{x}_{1}+\bar{a}_{2} \bar{\beta}_{1}\right)}\right)^{2}\right]$, 


$$
\begin{aligned}
& \bar{F}_{A y}=\Pi_{K}\left[\left(\frac{\bar{\imath}_{0}+\bar{\imath}_{A y}}{\bar{\delta}-\left(\bar{y}_{1}+\bar{a}_{1} \bar{\alpha}_{1}\right)}\right)^{2}-\left(\frac{\bar{\imath}_{0}-\bar{\imath}_{A y}}{\bar{\delta}+\left(\bar{y}_{1}+\bar{a}_{1} \bar{\alpha}_{1}\right)}\right)^{2}\right], \\
& \bar{F}_{B y}=\Pi_{K}\left[\left(\frac{\bar{\imath}_{0}+\bar{l}_{B y}}{\bar{\delta}-\left(\bar{y}_{1}-\bar{a}_{2} \bar{\alpha}_{1}\right)}\right)^{2}-\left(\frac{\bar{\imath}_{0}-\bar{l}_{B y}}{\bar{\delta}+\left(\bar{y}_{1}-\bar{a}_{2} \bar{\alpha}_{1}\right)}\right)^{2}\right] .
\end{aligned}
$$

As independent units of dimension, the following scales were chosen:

$$
S_{x}=\delta, \quad S_{L}=l, \quad S_{t}=\sqrt{\frac{m}{c_{m}}}, \quad S_{i}=\sqrt{\frac{P}{R}}
$$

where $P$ is the electric power and $R$ is the resistance. Two scales - meter-introduced because of the presence of anisotropy in the longitudinal and transverse dimensions of the research object and are related to each other by the ratio $\frac{S_{x}}{S_{L}}=\frac{\delta}{l}=\varepsilon$. The remaining scales are dependent on the dimension and are expressed through the selected ones and listed above. In the process of reducing the rotor model to a dimensionless form, dimensionless complexes were identified - the similarity criteria:

$\Pi_{1}=\frac{I_{z}}{I_{x}}, \quad \Pi_{2}=\frac{m l^{2}}{I_{x}}, \quad \Pi_{H}=\frac{c_{w} \eta \pi l^{3} \bar{r}^{2} \bar{l} \sqrt{m / c_{m}}}{I_{x}}, \quad \Pi_{K}=\frac{k_{A} P}{c_{m} R \delta^{3}}$.

It is necessary to create the blood flow pulsations as it was said earlier. From Pressure-Flow characteristic it can be seen that different rotational speeds can provide the same flow rate, depending on what pressure rise is required [28]. As is known a pressure of 120 to $80 \mathrm{mmHg}$ is normal. The idea of the approach to create the blood flow pulsations is to change pressure rise in accordance with the change in the rotational speed. To approbate this method let change the rotational speed vary according to the harmonic law $\bar{\Omega}=\bar{A} \sin (\bar{\omega} \bar{t})+\bar{\Omega}_{0}$ with a given amplitude and frequency. It should be noted, that the non-constant rotational speed can lead to additional accelerations and as a result other equations of motion. However, it was shown that the influence of these additional accelerations on the results of calculations is infinitely small and may not be taken into account.

\section{Synergetic synthesis of control}

The LVAD system is characterized by the following features: structural complexity (nonlinearity, large dimension, multiply connection and parametric uncertainty); multi-mode operation and lack of time for reconfiguration; nonlinear properties in modes of large deviations; the presence of external disturbances, which can lead to emergency situations; the requirement of energy efficiency.

The most appropriate method has been chosen, which makes it possible to solve immediately a set of stated problems. This is a method of analytical design of aggregated regulators (ADAR) of synergetic control theory (SCT) [29-36].

SCT is a new direction in control science. In general, SCT provides methods for designing optimal controllers for dynamical systems, where the controllers are coordinated with internal expectations of the systems. In SCT control goals are written in the form of invariants (technological, energy, chemical etc.). For the control goals were fulfilled, it is necessary that these invariants become attractors of the dynamical system. An attractor is a region in the state space of a dynamical system that pulls the trajectories from nearby areas of the state space. Whatever the initial conditions are, the system moves towards one of the attractors and remains 
there infinitely. So, the control goals are fulfilled on this attractor. Procedure of synergetic synthesis is the search for control laws under which these invariants become attractors of the dynamical system. Introducing such areas of attraction, or attractors, to the dynamical system, is one of the main concepts of the SCT. The resulting dynamical systems with their controllers have areas of attraction that correspond to the control goals.

The model of the rotor dynamics in active magnetic bearings Eq. (23) includes external disturbances, which can lead to malfunctioning or failure of the system. Thus, effective control of the magnetic suspension should have the ability to compensate for their undesirable effect. For this, the principle of integral adaptation of ADAR method can be applied. The integral adaptation implies that to parry external disturbances it is necessary to represent each of them in the form of a dynamic model at the output of which an approximate perturbation of the corresponding class is formed. The original system Eq. (23) can be extended by introducing the perturbation estimates:

$\bar{z}_{1}=\bar{F}_{x}+\bar{A}_{x} \sin (\bar{p} \bar{t})+\bar{\Omega}^{2} \bar{e} \cos (\bar{\Omega} \bar{t})$,

$\bar{z}_{2}=\bar{F}_{y}+\bar{A}_{y} \sin (\bar{p} \bar{t})+\bar{\Omega}^{2} \bar{e} \sin (\bar{\Omega} \bar{t})$,

$\bar{z}_{3}=\bar{M}_{x}+\bar{B}_{x} \sin (\bar{p} \bar{t})+\bar{\Omega}^{2} \bar{\gamma}\left(1-\Pi_{1}\right) \cos (\bar{\Omega} \bar{t})$,

$\bar{z}_{4}=\bar{M}_{y}+\bar{B}_{y} \sin (\bar{p} \bar{t})+\bar{\Omega}^{2} \bar{\gamma}\left(1-\Pi_{1}\right) \sin (\bar{\Omega} \bar{t})$,

and by introducing the perturbation model for each new variable in the form of integrators:

$\frac{d \bar{z}_{1}}{d \bar{t}}=\mu_{1} \Phi_{t e c h 1}, \frac{d \bar{z}_{2}}{d \bar{t}}=\mu_{2} \Phi_{t e c h 2}, \frac{d \bar{z}_{3}}{d \bar{t}}=\mu_{3} \Phi_{t e c h 3}, \frac{d \bar{z}_{4}}{d \bar{t}}=\mu_{4} \Phi_{t e c h 4}$,

with $\mu_{1}, \ldots, \mu_{4}$ - constant coefficients. For the rotor stabilization the control goals can be written in the form of invariants (technological invariants):

$\Phi_{\text {tech } 1}=\bar{x}_{1}-\bar{x}_{1}^{0}=0, \quad \Phi_{\text {tech } 2}=\bar{y}_{1}-\bar{y}_{1}^{0}=0$,

$\Phi_{\text {tech } 3}=\bar{\alpha}_{1}-\bar{\alpha}_{1}^{0}=0, \quad \Phi_{\text {tech } 4}=\bar{\beta}_{1}-\bar{\beta}_{1}^{0}=0$,

where $\bar{x}_{1}^{0}, \bar{y}_{1}^{0}, \bar{\alpha}_{1}^{0}, \bar{\beta}_{1}^{0}$ are desired values of the state coordinates. With Eq. (26) and Eq. (27) the system of equations Eq. (23) is expressed:

$$
\begin{aligned}
\bar{x}_{1}^{\prime} & =\bar{x}_{2}, \\
\bar{x}_{2}^{\prime} & =\bar{x}_{1}+\frac{\left(-\bar{a}_{1}+\bar{a}_{2}\right)}{2} \bar{\beta}_{1}+\bar{S}_{A x}+\bar{S}_{B x}+\bar{z}_{1}, \\
\bar{y}_{1}^{\prime} & =\bar{y}_{2}, \\
\bar{y}_{2}^{\prime} & =\bar{y}_{1}+\frac{\left(\bar{a}_{1}-\bar{a}_{2}\right)}{2} \bar{\alpha}_{1}+\bar{S}_{A y}+\bar{S}_{B y}+\bar{z}_{2}, \\
\bar{\alpha}_{1}^{\prime} & =\bar{\alpha}_{2} \\
\bar{\alpha}_{2}^{\prime} & =-\Pi_{H} \bar{\alpha}_{2}-\Pi_{1} \bar{\theta}_{2} \bar{\beta}_{2}+\frac{\Pi_{2}}{2}\left(\bar{a}_{1}-\bar{a}_{2}\right) \bar{y}_{1}-\Pi_{H} \bar{\theta}_{2} \bar{\beta}_{1}+\bar{a}_{1} \Pi_{2} \bar{S}_{A y}-\bar{a}_{2} \Pi_{2} \bar{S}_{B y}+\ldots \\
& +\frac{\Pi_{2}}{4}\left(\left(\bar{a}_{1}^{2}+\bar{a}_{2}^{2}\right)\left(1+\bar{k}_{m}\right)-2 \bar{a}_{1} \bar{a}_{2}\left(1-\bar{k}_{m}\right)\right) \bar{\alpha}_{1}+\bar{z}_{3}, \\
\bar{\beta}_{1}^{\prime} & =\bar{\beta}_{2}, \\
\bar{\beta}_{2}^{\prime} & =-\Pi_{H} \bar{\beta}_{2}+\Pi_{1} \bar{\theta}_{2} \bar{\alpha}_{2}+\frac{\Pi_{2}}{2}\left(-\bar{a}_{1}+\bar{a}_{2}\right) \bar{x}_{1}+\Pi_{H} \bar{\theta}_{2} \bar{\alpha}_{1}-\bar{a}_{1} \Pi_{2} \bar{S}_{A x}+\bar{a}_{2} \Pi_{2} \bar{S}_{B x}+\ldots \\
& +\frac{\Pi_{2}}{4}\left(\left(\bar{a}_{1}^{2}+\bar{a}_{2}^{2}\right)\left(1+\bar{k}_{m}\right)-2 \bar{a}_{1} \bar{a}_{2}\left(1-\bar{k}_{m}\right)\right) \bar{\beta}_{1}+\bar{z}_{4}, \\
\dot{\bar{z}}_{1} & =\mu_{1} \Phi_{\text {tech } 1}, \quad \dot{\bar{z}}_{2}=\mu_{2} \Phi_{\text {tech } 2}, \quad \dot{\bar{z}}_{3}=\mu_{3} \Phi_{\text {tech } 3}, \quad \dot{\bar{z}}_{4}=\mu_{4} \Phi_{\text {tech } 4},
\end{aligned}
$$


where:

$$
\begin{aligned}
& \bar{S}_{A x}=\Pi_{K}\left[\left(\frac{\bar{\imath}_{0}+\bar{u}_{1}}{\bar{\delta}-\left(\bar{x}_{1}-\bar{a}_{1} \bar{\beta}_{1}\right)}\right)^{2}-\left(\frac{\bar{l}_{0}-\bar{u}_{1}}{\bar{\delta}+\left(\bar{x}_{1}-\bar{a}_{1} \bar{\beta}_{1}\right)}\right)^{2}\right], \\
& \bar{S}_{B x}=\Pi_{K}\left[\left(\frac{\bar{\imath}_{0}+\bar{u}_{2}}{\bar{\delta}-\left(\bar{x}_{1}+\bar{a}_{2} \bar{\beta}_{1}\right)}\right)^{2}-\left(\frac{\bar{l}_{0}-\bar{u}_{2}}{\bar{\delta}+\left(\bar{x}_{1}+\bar{a}_{2} \bar{\beta}_{1}\right)}\right)^{2}\right], \\
& \bar{S}_{A y}=\Pi_{K}\left[\left(\frac{\bar{\imath}_{0}+\bar{u}_{3}}{\bar{\delta}-\left(\bar{y}_{1}+\bar{a}_{1} \bar{\alpha}_{1}\right)}\right)^{2}-\left(\frac{\bar{l}_{0}-\bar{u}_{3}}{\bar{\delta}+\left(\bar{y}_{1}+\bar{a}_{1} \bar{\alpha}_{1}\right)}\right)^{2}\right], \\
& \bar{S}_{B y}=\Pi_{K}\left[\left(\frac{\bar{l}_{0}+\bar{u}_{4}}{\bar{\delta}-\left(\bar{y}_{1}-\bar{a}_{2} \bar{\alpha}_{1}\right)}\right)^{2}-\left(\frac{\bar{l}_{0}-\bar{u}_{4}}{\bar{\delta}+\left(\bar{y}_{1}-\bar{a}_{2} \bar{\alpha}_{1}\right)}\right)^{2}\right] .
\end{aligned}
$$

The control currents $\bar{\imath}_{A x}, \bar{l}_{B x}, \bar{l}_{A y}, \bar{l}_{B y}$ are control channels $\bar{u}_{1}=\bar{l}_{A x}, \bar{u}_{2}=\bar{l}_{B x}, \bar{u}_{3}=\bar{l}_{A y}$, $\bar{u}_{4}=\bar{l}_{B y}$. To further synthesize the control laws a parallel totality of macrovariables can be selected in the following form [32]:

$\psi_{1}=\bar{x}_{2}-\varphi_{1}, \quad \psi_{2}=\bar{y}_{2}-\varphi_{2}, \quad \psi_{3}=\bar{\alpha}_{2}-\varphi_{3}, \quad \psi_{4}=\bar{\beta}_{2}-\varphi_{4}$.

$\varphi_{1}, \varphi_{2}, \varphi_{3}, \varphi_{4}$ represent the internal controls and are determined in the course of further synthesis. For the macrovariables Eq. (30) to be invariant manifolds it is necessary that $\psi_{1, \ldots, 4}=0$. For this the introduced set Eq. (30) must satisfy the solution of the system of basic functional equations of the ADAR method [32-34]:

$T_{1} \dot{\psi}_{1}+\psi_{1}=0, \quad T_{2} \dot{\psi}_{2}+\psi_{2}=0, \quad T_{3} \dot{\psi}_{3}+\psi_{3}=0, \quad T_{4} \dot{\psi}_{4}+\psi_{4}=0$,

where $T_{1}, T_{2}, T_{3}, T_{4}>0$ are time constants that affect the quality of the processes dynamics in a closed-loop system. Substituting expressions Eq. (30) and the complete derivatives of expressions Eq. (30) into equations Eq. (31) we have:

$$
\begin{array}{ll}
T_{1}\left[\dot{\bar{x}}_{2}-\dot{\varphi}_{1}\right]+\bar{x}_{2}-\varphi_{1}=0, & T_{2}\left[\dot{\bar{y}}_{2}-\dot{\varphi}_{2}\right]+\bar{y}_{2}-\varphi_{2}=0, \\
T_{3}\left[\dot{\bar{\alpha}}_{2}-\dot{\varphi}_{3}\right]+\bar{\alpha}_{2}-\varphi_{3}=0, & T_{4}\left[\dot{\bar{\beta}}_{2}-\dot{\varphi}_{4}\right]+\bar{\beta}_{2}-\varphi_{4}=0 .
\end{array}
$$

The controls $\bar{u}_{1}, \bar{u}_{2}, \bar{u}_{3}, \bar{u}_{4}$ can be obtained from the system Eq. (32) when substituting the right-hand sides of the equations for variables $\dot{\bar{x}}_{2}, \dot{\bar{y}}_{2}, \dot{\bar{\alpha}}_{2}, \dot{\bar{\beta}}_{2}$ in Eq. (29). The obtained control laws depend on $\varphi_{1, \ldots, 4}$ and $\dot{\varphi}_{1, \ldots, 4}$. Under the control laws $\bar{u}_{1}, \bar{u}_{2}, \bar{u}_{3}, \bar{u}_{4}$ the represented point (RP) of the dynamical system hits the intersection of manifolds $\psi_{1, \ldots, 4}=0$ and then moving along it to a given position of the phase space. When RP hits the intersection of manifolds Eq. (30), the system Eq. (29) is decomposed dynamically. The motion of the RP along the intersection of manifolds $\psi_{1, \ldots ., 4}=0$ is described by the equations of "internal" dynamics - the decomposed system:

$$
\begin{array}{ll}
\dot{\bar{x}}_{1}=\varphi_{1}, & \dot{\bar{z}}_{1}=\mu_{1}\left(\bar{x}_{1}-\bar{x}_{1}^{0}\right), \\
\dot{\bar{y}}_{1}=\varphi_{2}, & \dot{\bar{z}}_{2}=\mu_{2}\left(\bar{y}_{1}-\bar{y}_{1}^{0}\right), \\
\dot{\bar{\alpha}}_{1}=\varphi_{3}, & \dot{\bar{z}}_{3}=\mu_{3}\left(\bar{\alpha}_{1}-\bar{\alpha}_{1}^{0}\right), \\
\dot{\bar{\beta}}_{1}=\varphi_{4}, & \dot{\bar{z}}_{4}=\mu_{4}\left(\bar{\beta}_{1}-\bar{\beta}_{1}^{0}\right) .
\end{array}
$$

For system Eq. (33), introduced earlier and called "internal" controls $\varphi_{1}, \varphi_{2}, \varphi_{3}, \varphi_{4}$ are external. Then, to find them explicitly, we introduce another parallel set of macrovariables: 
$\psi_{5}=\left(\bar{x}_{1}-\bar{x}_{1}^{0}\right)+h_{1} \bar{z}_{1}, \quad \psi_{6}=\left(\bar{y}_{1}-\bar{y}_{1}^{0}\right)+h_{2} \bar{z}_{2}$,

$\psi_{7}=\left(\bar{\alpha}_{1}-\bar{\alpha}_{1}^{0}\right)+h_{3} \bar{z}_{3}, \quad \psi_{8}=\left(\bar{\beta}_{1}-\bar{\beta}_{1}^{0}\right)+h_{4} \bar{z}_{4}$,

where $h_{j}, j=1, \ldots, 4$ - constant coefficients. The structure of macrovariables Eq. (34) includes the desired technological invariants Eq. (28), which determine the control goals. The introduced set of macrovariables must satisfy the solution of the system of basic functional equations of the ADAR method:

$T_{5} \dot{\psi}_{5}+\psi_{5}=0, \quad T_{6} \dot{\psi}_{6}+\psi_{6}=0, \quad T_{7} \dot{\psi}_{7}+\psi_{7}=0, \quad T_{8} \dot{\psi}_{8}+\psi_{8}=0$,

where $T_{5}, T_{6}, T_{7}, T_{8}>0$ are time constants. To explicitly define the functions $\varphi_{1}, \varphi_{2}, \varphi_{3}, \varphi_{4}$ it is necessary to differentiate the macrovariables Eq. (34) and substitute the expressions for $\psi_{5, \ldots, 8}$ and their derivatives with the right-hand sides of the system Eq. (33) into the functional equations Eq. (32). Under the control laws $\varphi_{1}, \varphi_{2}, \varphi_{3}, \varphi_{4}$ the RP of the dynamical system will fall on the intersection of manifolds $\psi_{5, \ldots, 8}=0$ on which the control goals (technological invariants) will occur. A prerequisite for this is the tendency to zero $\bar{z}_{i}, i=1, \ldots, 4$. This is clearly seen from the decomposed system:

$\dot{\bar{z}}_{1}=-\mu_{1} h_{1} \bar{z}_{1}, \quad \dot{\bar{z}}_{2}=-\mu_{2} h_{2} \bar{z}_{2}, \quad \dot{\bar{z}}_{3}=-\mu_{3} h_{3} \bar{z}_{3}, \quad \dot{\bar{z}}_{4}=-\mu_{4} h_{4} \bar{z}_{4}$.

The stability of the degenerate system Eq. (36) is given by simple conditions: $\mu_{1} h_{1}>0, \mu_{2} h_{2}>0, \mu_{3} h_{3}>0$ and $\mu_{4} h_{4}>0$. The expressions for $\varphi_{1, \ldots, 4}$ obtained in explicit form are substituted into the $\bar{u}_{1}, \bar{u}_{2}, \bar{u}_{3}, \bar{u}_{4}$.

Table 1. Model data for dynamic analysis

\begin{tabular}{|c|c|}
\hline Parameter & Symbol/ Value/ Unit \\
\hline Diameter of the flow part & $D=16 \cdot 10^{-3} \mathrm{~m}$ \\
\hline Rotor diameter & $d=15.6 \cdot 10^{-3} \mathrm{~m}$ \\
\hline Radial gap & $\delta=0.2 \cdot 10^{-3} \mathrm{~m}$ \\
\hline Rotor length & $m=22 \cdot 10^{-3} \mathrm{~m}$ \\
\hline Rotor mass & $m=12.42 \cdot 10^{-3} \mathrm{~kg}$ \\
\hline Moments of inertia & $I_{x}=I_{y}=6.9 \cdot 10^{-7} \mathrm{~kg} \mathrm{~m}^{2}, I_{z}=3.78 \cdot 10^{-7} \mathrm{~kg} \mathrm{~m}^{2}$ \\
\hline Bearings coordinates & $a_{1}=a_{2}=9 \cdot 10^{-3} \mathrm{~m}$ \\
\hline Motor stiffness & $c_{m}=6000 \mathrm{~N} / \mathrm{m}$ \\
\hline Motor design parameter & $k_{m}=0.202$ \\
\hline Unbalances parameters & $e=4.27 \cdot 10^{-7} \mathrm{~m}, \gamma=2.15 \cdot 10^{-5} \mathrm{rad}$ \\
\hline Dimensionless complex & $\Pi_{1}=I_{z} / I_{x}=0.548$ \\
\hline Dimensionless complex & $\Pi_{2}=m l^{2} / I_{x}=8.714$ \\
\hline Dimensionless complex & $\Pi_{K}=k_{A} P / c_{m} R \delta^{3}=10^{5}$ \\
\hline Dimensionless complex & $\Pi_{H}=\frac{c_{w} \eta \pi l^{3} \bar{r}^{2} \bar{l} \sqrt{m / c_{m}}}{I_{x}}=1.48 \cdot 10^{-7}$ \\
\hline $\begin{array}{c}\text { Blood viscosity at } 37{ }^{\circ} \mathrm{C} \\
\text { (non-Newtonian model of blood) }\end{array}$ & $\eta=0.056 \mathrm{~Pa} \mathrm{~s}$ \\
\hline Coefficient of medium resistance & $c_{w}=0.91$ \\
\hline
\end{tabular}

\section{Research results}

Fig. 4 shows transients of the closed-loop system under external piecewise constant and harmonic loads. The transient time is $0.4 \mathrm{~s}$ and the output to the steady state occurs without additional oscillations and overregulation. The requirement of the accuracy of the rotor positioning is $4 \mu \mathrm{m}$ and due to compensation of external disturbances is fulfilled. System parameters are given 
by Table 1 .

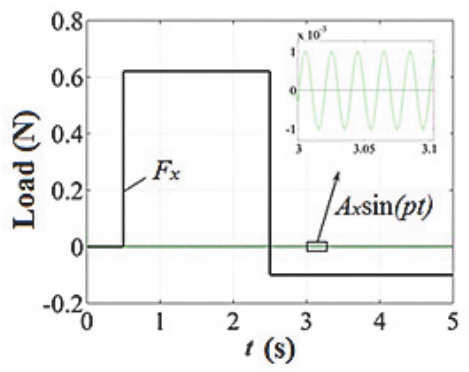

a)

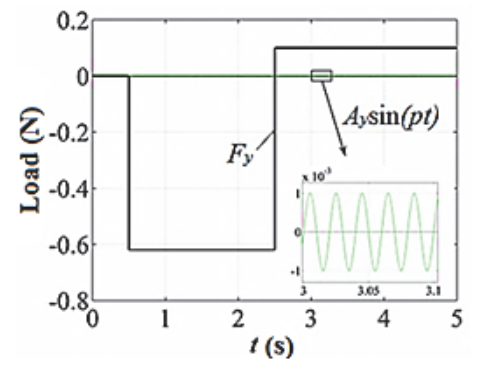

c)

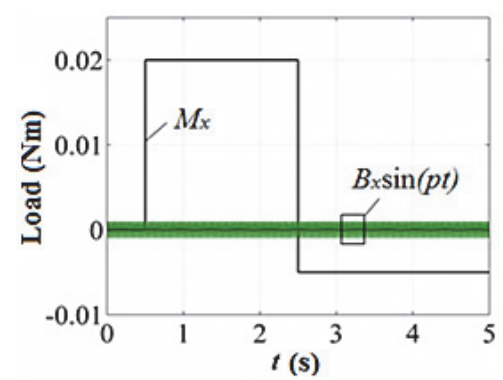

e)

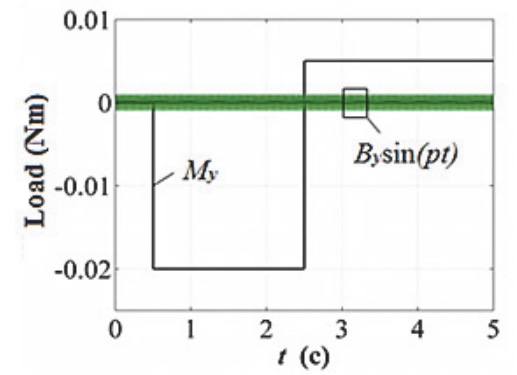

g)

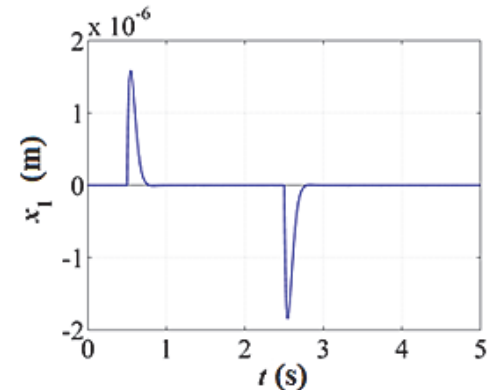

b)

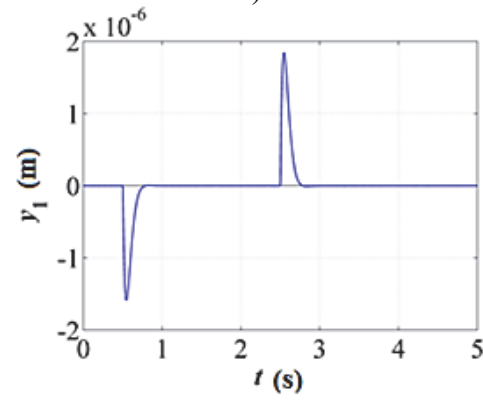

d)

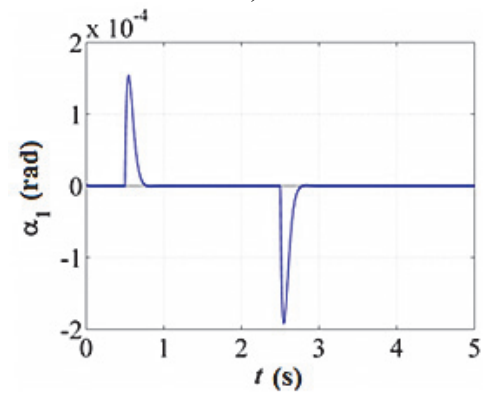

f)

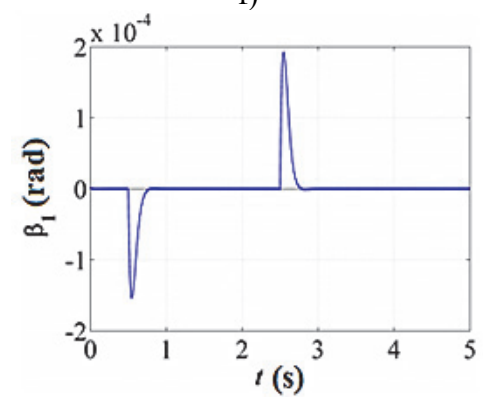

h)

Fig. 4. State variables transients

Dynamic analysis was performed for the following coefficients of control: $T_{1,2, \ldots, 8}=35, h_{1,2,3,4}=4000, \mu_{1}=\mu_{2}=0.2, \mu_{3}=\mu_{4}=1.2$ and zero initial values for the state coordinates. From the Pressure-Flow characteristic, constructed for the flow part optimal geometry of the blood pump [28], the following values of the rotational speed were received: 
$A=\frac{700 \cdot \pi}{30} \mathrm{rad} / \mathrm{s}, \omega=2 \pi \mathrm{rad} / \mathrm{s}, \Omega_{0}=\frac{9000 \cdot \pi}{30} \mathrm{rad} / \mathrm{s}$. Values of the piecewise constant load are reference data and taken from the biomechanical analysis of human organism behavior at various accelerations [37]. Harmonic inertial load, to which the patient is exposed, for example, in transport, has low frequency (about $50 \mathrm{~Hz}$ ).

Phase portraits (Fig. 5) demonstrate the contraction of the phase trajectories of the dynamical system to the target attractor under various initial conditions - something that is difficult to provide with other control methods. This fact also confirms the modeling of the "levitation startup" mode of the rotor from the safety bearings when the suspension is switched on, i.e. with the maximum possible deviation of the rotor from the zero-equilibrium position (Fig. 6(a)). The range of possible rotor positions is the region of attraction to the equilibrium position. The synthesized control laws guarantee the asymptotic stability of the closed-loop system and the parametric robustness of the dynamical system. The study of the parametric robustness was carried out by double increasing and decreasing each of the following parameters: $\Pi_{1}, \Pi_{2}, \Pi_{H}, \bar{a}_{1}, \bar{a}_{2}, \bar{e}, \bar{\gamma}$. The maximum value of the control currents is $5.4 \mathrm{~mA}$ (Fig. 6(b)). This is two times less than with linear PID control.

To test the effectiveness of the approach to creating the blood flow pulsations due to change in rotational speed computational fluid dynamics (CFD) analysis was carried out $[38,39]$. Pressure rise changes in accordance with the change in the rotational speed (Fig. 7).

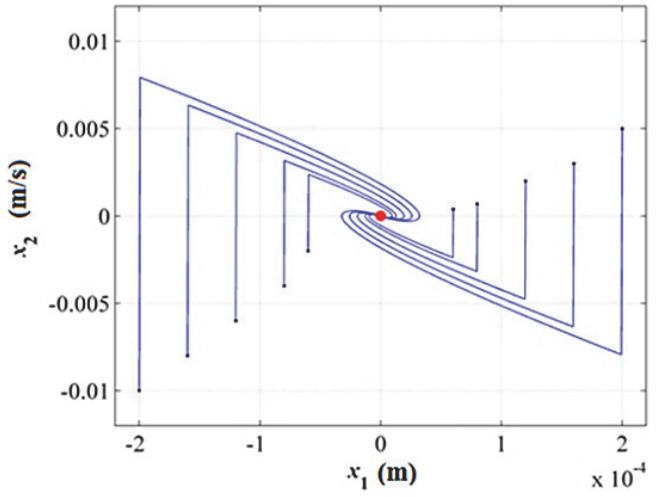

a)

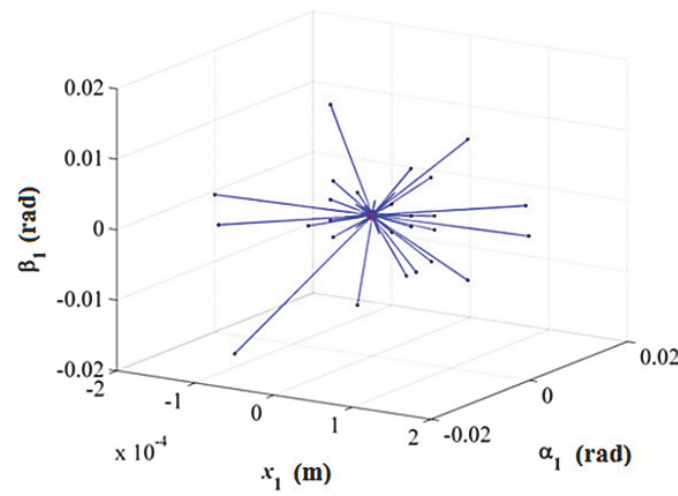

b)

Fig. 5. Phase portraits

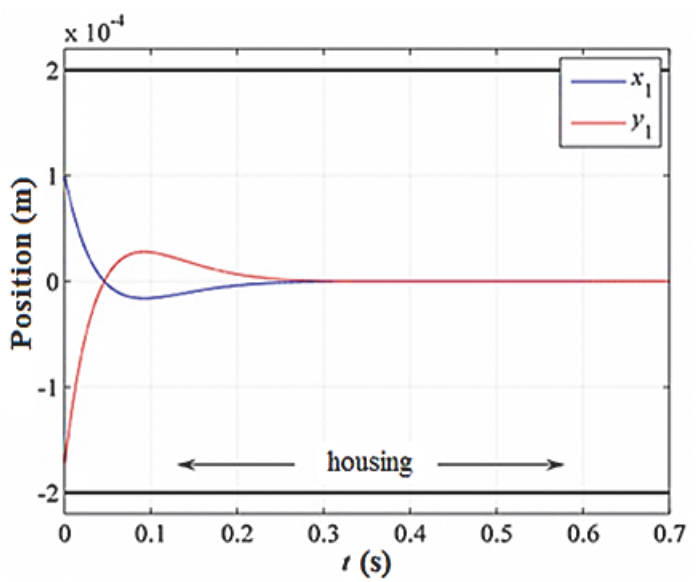

a) 


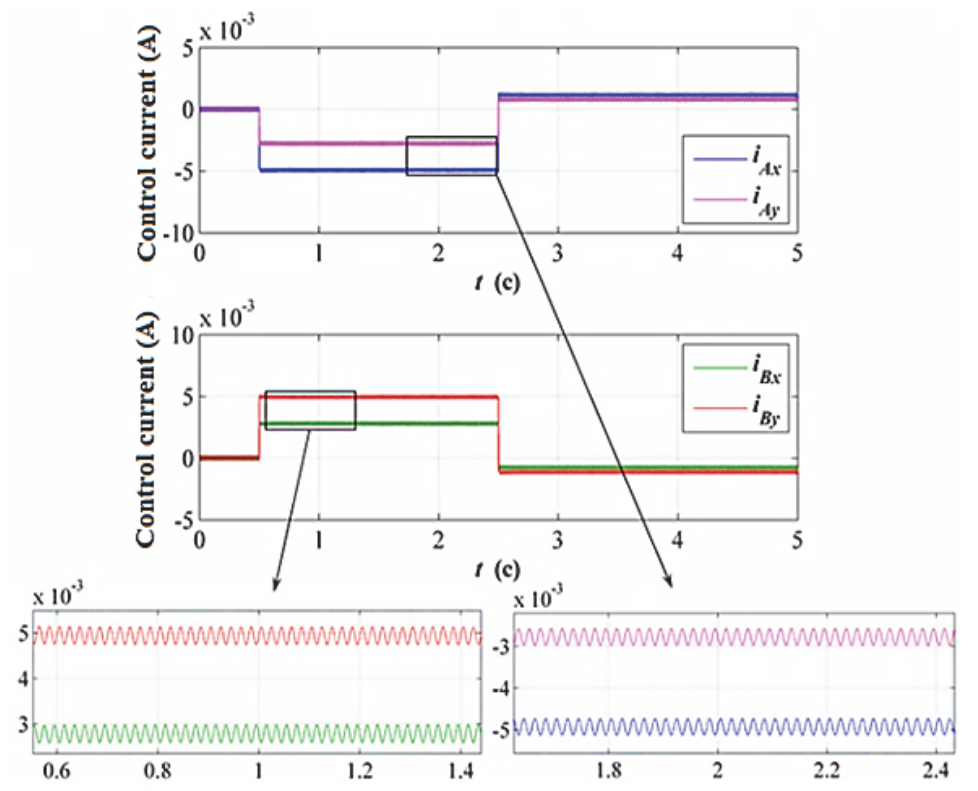

b)

Fig. 6. a) Levitation startup time history, b) value of control currents

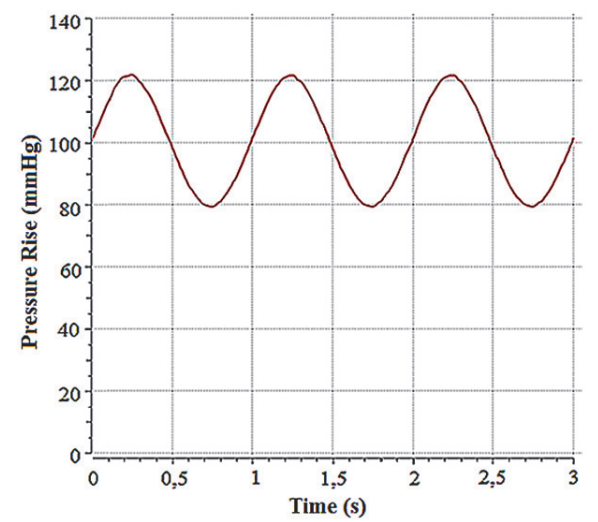

a)

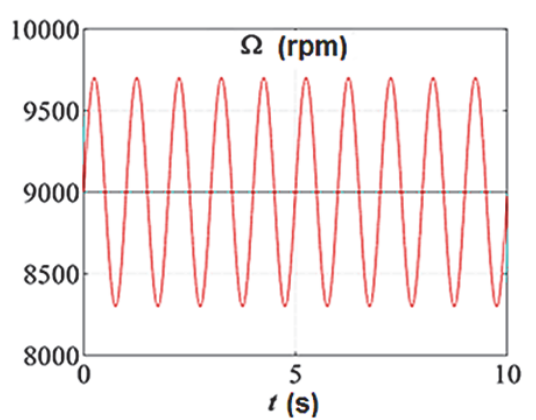

b)

Fig. 7. The change in pressure rise (CFD analysis result) a) according to change in rotational speed b)

\section{Conclusions}

The effective nonlinear control laws for stabilizing with high accuracy the rotor suspended by active magnetic bearings in axial LVAD that guarantee the provision of the required rotor dynamics under the conditions of various perturbations and changes in the internal parameters of the model were synthesized in this research. For this the basic method and principle of the synergetic control theory were applied. The developed methods for ensuring the required rotor dynamics are accurate, fast, economical in the sense of the values of control currents, and most importantly reliability, which in general ensures an increase in the safety of the life support system - left ventricular assist device. The designed synergetic control laws ensure the blood flow pulsations regime and compensate unwanted external disturbances as well as internal parametric perturbations. For the proposed LVAD with magnetic bearings of the new generation, which is the complex biomechanical system, with a wide spread of physical parameters and performance characteristics, the synergetic approach, unlike classical control methods, allows performing 
highly efficient "non-power" control, consistent with a substantially nonlinear and multiply connected structure of control object.

\section{Acknowledgements}

This work was supported by the Russian Foundation for Basic Research (Grant No. 15-29-01085 ofi_m).

\section{References}

[1] Finocchiaro T., et al. Methods of design, simulation, and control for the development of new VAD/TAH concepts. Biomedical Engineering, Vol. 54, Issue 5, 2009, p. 269-281.

[2] Gautier S. V., et al. Donation and organ transplantation in the Russian federation in 2013. Bulletin of Transplantology and Artificial Organs, Vol. 2, 2014, p. 5-23.

[3] Sajgalik P., et al. Current status of left ventricular assist device therap. Mayo Clinic Proceedings, Vol. 91, Issue 7, 2016, p. 927-940.

[4] Birks E. J. The comparative use of ventricular assist device. 18th Annual THI Summit, 2010, p. 565-567.

[5] Alba A. C., Delgado D. H. The future is here: ventricular assist devices for the failing heart. Expert Review of Cardiovascular Therapy, Vol. 7, Issue 9, 2009, p. 1067-1077.

[6] Rau G. Research Report 2001/2002. Institute for Biomedical Technologies, Aachen University, 2002, p. 101.

[7] Wurzinger L. J., Opitz R., Eckstein H. Mechanical blood trauma: an overview. Angiology, Vol. 38, 1986, p. 81-97.

[8] Allaire P. E., et al. Design of a magnetic bearing-supported prototype centrifugal artificial heart pump. Tribology Transactions, Vol. 39, Issue 3, 1996, p. 663-669.

[9] Yu J., Zhang X. Hydrodynamic and hemolysis analysis on distance and clearance between impeller and diffuser of axial blood pump. Journal of Mechanics in Medicine and Biology, Vol. 16, Issue 2, 2016, p. 1650014.

[10] Hoshi H., Shinshi T., Takatani S. Third-generation blood pumps with mechanical noncontact magnetic bearings. Artificial Organs, Vol. 30, Issue 5, 2006, p. 324-338.

[11] Pagani F. D. Continuous-flow rotary left ventricular assist devices with "3rd generation" design. Seminars in Thoracic and Cardiovascular Surgery. WB Saunders, Vol. 20, Issue 3, 2008, p. 255-263.

[12] Bogdanova Yu V., Gouskov A. M. Left ventricular assist device (LVAD) design features: literature review. Science and Education: Scientific Publication of the Bauman Moscow State Technical University, Vol. 3, 2014, p. 162-187.

[13] Crow S., et al. Gastrointestinal bleeding rates in recipients of nonpulsatile and pulsatile left ventricular assist devices. The Journal of Thoracic and Cardiovascular Surgery, Vol. 137, Issue 1, 2009, p. 208-215.

[14] John R., et al. A decade of experience with continuous-flow left ventricular assist devices. Seminars in Thoracic and Cardiovascular Surgery. WB Saunders, Vol. 28, Issue 2, 2016, p. 363-375.

[15] Chiba A., et al. Magnetic Bearings and Bearingless Drives. Elsevier, 2005, p. 400.

[16] Schweitzer G., Maslen E. H. Magnetic Bearings: Theory, Design, and Application to Rotating Machinery. Springer-Verlag Berlin Heidelberg, 2009, p. 541.

[17] Zhuravlev Yu N. Active Magnetic Bearings. Polytechnic, 2002, p. 206.

[18] Esfahani A. Chaotic Behaviour of Active Magnetic Bearing System by Time Series Analysis. Thesis, Lappeenranta University of Technology, 2012, p. 104.

[19] Gerami A., Allaire P., Fittro R. Nonlinear modeling and control of a magnetic bearing with material saturation. Proceedings of the 14th International Conference on Magnetic Bearings, Linz, Austria, 2014.

[20] Ji J. C., Hansen C. H., Zander A. C. Nonlinear dynamics of magnetic bearing systems. Journal of Intelligent Material Systems and Structures, Vol. 19, Issue 12, 2008, p. 1471-1491.

[21] Kang K. Nonlinear Dynamics of a Rotor Supported by Homopolar Magnetic Bearings with Saturation. Thesis, Texas A\&M University, 2010, p. 109.

[22] Steinschaden N., Springer H. Some nonlinear effects of magnetic bearings. Proceedings of the DETC ASME Conference, 1999.

[23] Vibration. Requirements for Balancing Rigid Rotors: Part 1. GOST ISO 1940-1-2007, 2007. 
[24] Vibration. Vibration of Rotary Action Machines with Active Magnetic Bearings: Part 2. GOST R ISO 14839-2-2011, 2011.

[25] Gomez A. D. Control of a magnetically levitated ventricular assist device. Thesis, Rochester Institute of Technology, 2009, p. 140.

[26] Barenblatt G. I. Scaling, Self-Similarity, and Intermediate Asymptotics: Dimensional Analysis and Intermediate Asymptotics. Cambridge University Press, 1996, p. 388.

[27] Sedov L. I. Similarity and Dimension Methods in Mechanics. Elsevier, 1977, p. 376.

[28] Gouskov A. M., et al. Mathematical modeling of the blood flow in the flowing part of the axial pump of the artificial ventricle of the heart. Science and Education: Scientific Publication of the Bauman Moscow State Technical University, Vol. 10, 2015, p. 473-488.

[29] Bogdanova Yu, Gouskov A., Guskov M. Synergetic approach to control of axial left ventricular assist device rotor supported by magnetic bearings. Vibroengineering PROCEDIA, Vol. 8, 2016, p. 340-345.

[30] Bounasla N., Hemsas K., Mellah H. Synergetic and sliding mode controls of a PMSM: a comparative study. Journal of Electrical and Electronics Engineering, Vol. 3, 2015, p. 22-26.

[31] Genta G. Vibration Dynamics and Control. Springer Science, 2009, p. 812.

[32] Kolesnikov A. A., et al. Modern Applied Control Theory. Part II: The Synergetic Approach in Control Theory. TSET Publishing House, Moscow-Taganrog, 2000, p. 559.

[33] Kolesnikov A. A. Synergetic Methods of Managing Complex Systems: Mechanical and Electromechanical Systems. Librokom, Moscow, 2012, p. 300.

[34] Kolesnikov A. A. Synergetic Methods for Controlling Complex Systems: The Theory of System Synthesis. Energoatomizdat, Moscow, 2012, p. 340.

[35] Lidozzi A., Solero L., Taddia P. Synergetic control for axial-flux PM motor drives. 40th IAS Annual Meeting. Conference Record of the 2005 Industry Applications Conference, 2005.

[36] Wang Z., Zhao Y. The speed mode synergetic control approach for magnetic suspended reaction flywheel. Applied Mathematics and Information Sciences, Vol. 7, 2013, p. 107-111.

[37] Rabinovich B. A. Safety of a Person under Acceleration. Biomechanical Analysis, Moscow, 2006, p. 208.

[38] Apel J., Neudel F., Reul H. Computational fluid dynamics and experimental validation of a microaxial blood pump. ASAIO Journal, Vol. 47, Issue 5, 2001, p. 552-558.

[39] Behbahani M., et al. A review of computational fluid dynamics analysis of blood pumps. European Journal of Applied Mathematics, Vol. 20, Issue 4, 2009, p. 363-397.

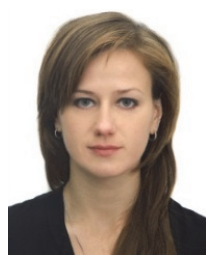

Yulia Bogdanova received the Specialist degree in applied mechanics (2013) and the Candidate of Science degree in mechanical engineering (2017) from Bauman Moscow State Technical University, Russia. During her postgraduate studies at Bauman Technical University, Yulia has focused on nonlinear dynamics, control theory and rotordynamics. In parallel she works as an engineer of the Robotics Laboratory in the NRC "Kurchatov Institute".

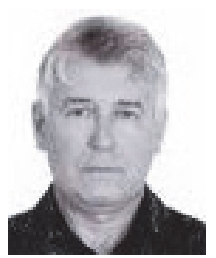

Alexander Guskov is the Doctor of Science degree in mechanical engineering and Professor at the Department of Applied Mechanics in Bauman Moscow State Technical University and in the Vibrations Laboratory in the RAS Institute of Machine Science, Russia. His current research interests include nonlinear dynamics, stability theory, rotordynamics, control theory. 


\title{
2.4. Increasing of efficiency of parts turning at spindle speed from 5,000 to $10,000 \mathrm{rpm}$ by controlling the dynamics of machining
}

\author{
Wiliam Zaloga ${ }^{1}$, Yurii Shapoval ${ }^{2}$, Vitalii Kolesnyk ${ }^{3}$ \\ Sumy State University, Sumy, Ukraine \\ E-mail: ${ }^{1}$ zalogav@gmail.com, ${ }^{2}$ diyyura@gmail.com, ${ }^{3}$ v.kolesnik@tmvi.sumdu.edu.ua
}

\begin{abstract}
The article deals with the issues of increasing the productivity of turning parts at rotational speed up to $10,000 \mathrm{rpm}$. The reasons for reducing roughness of machined surface when approaching the resonant rotational speeds are analysed. The hypothesis about the dependence of the vibrational component of roughness of the machined surface on the external generating forces and natural oscillation modes and frequencies of technological system is set-up. The methods of studying the dynamic behaviour of the lathe are reviewed, as well as the way of changing system properties to improve stability in the increased cutting conditions and productivity. The authors prove the possibility of a local reduction in the oscillation amplitude of a tool by changing the machine natural oscillation modes in order to increase rotational speed during the continuous machining.
\end{abstract}

Keywords: turning at 5-10 thousand rpm, turning dynamics, stability.

\section{Introduction}

In the machine building, instrumentation, aircraft industry and other industries due to the reduction in machines and mechanisms, the proportion of parts representing the rotation bodies up to $20 \mathrm{~mm}$ is constantly increasing (bodies of measuring detectors, short axes of devices, plungers of hydraulic dispensers and power units, tips for current collectors of welding equipment, pins, etc.), manufacturing technology of which requires turning. Besides, the quality requirements for the machined surfaces of these parts are increasing, which is stipulated by the necessity to provide the relevant performance characteristics of the item, particularly strength in case of its overall dimensions reduction, durability, fatigue resistance, increase of contact stiffness, vibration stability, etc. The need to ensure a constantly changing demand for a small-diameter parts creates preconditions for increasing the process productivity of machining. Increasing the productivity of cutting off the dimensional allowance during parts turning with surface diameters of up to $20 \mathrm{~mm}$, due to their relatively low rigidity, is usually possible by increasing the cutting speed.

Turning technology of machining parts made of different structural materials with relatively high cutting speeds during turning can provide the necessary quality according to requirements and in many cases eliminate the need for additional finishing. This became possible with the development of tool materials for edge cutting machining, for example, hard alloys, particularly with wear-resistant coatings which allowed to machine ferrous metal parts (steels, alloys, cast iron, etc.) at cutting speeds over $500 \mathrm{~m} / \mathrm{min}$, and for non-ferrous (aluminium, copper, etc.) metals and alloys at speeds above $1000 \mathrm{~m} / \mathrm{min}$. Thus, one can say that the potential reserves for increasing productivity during turning are the modern tool materials' ability to cut at high speed, and for parts with small dimensions (diameters up to $20 \mathrm{~mm}$ ) with rotational speeds of $6000 \mathrm{rpm}$ and more.

The analysis shows that in order to increase the cutting speed of small diameter parts by increasing rotational speed to obtain the required (given in a drawing) accuracy and roughness of the machined surfaces, it is necessary to provide a stable dynamic behaviour of machines. In this case, the influence machines' part oscillations on the cutting process should be minimal, and dynamic characteristics of movable operating elements should ensure the exact and synchronous operation of the whole technological system.

Today a sufficient number of modern machines and machining centres provide maximum spindle speed up to 5000-6000 rpm. At the same time, one of the important issues during their 
application is to ensure the stable dynamic behaviour of the spindle unite, sliding carriage, auxiliary devices, etc.

Increasing of rotational speeds when machining parts with a diameter of up to $20 \mathrm{~mm}$ can reduce the cutting speed to the values recommended by tool manufacturers (for example, machining of steel by carbide tools require cutting speed $250-300 \mathrm{~m} / \mathrm{min}$ ), which can lead to the loss of dynamic stability of the spindle unite and sliding carriage decline in the corresponding quality rating. It happens due to the fact that the high rotational speed significantly increases the imbalance of "spindle - device - workpiece" system and as a result, the power of the imbalance can often significantly exceed the power of cutting and therefore significantly affect the dynamic behaviour of the entire technological system.

Thus, the development of the principles of controlling dynamics machining during turning parts with diameter up to $20 \mathrm{~mm}$ at high rotational speeds is an important scientific and technical task, the solution of which will improve performance of machining these parts while ensuring the drawing requirements for roughness and tolerance.

\section{State of the art}

In the nearest future the technical capabilities of turning lathes and machining centres will allow to machine on higher speeds compared to rotational speeds which are traditionally used in modern manufacturing. This is due to the fact that machines of other groups (for example, milling machines, grinding machines, etc.) are capable of carrying out the main motion at rotational speeds of up to $100.000 \mathrm{rpm}$. At the same time, even this is not enough to use all the capabilities of a modern cutting tool, for example, diamond or cubic boron nitride, especially for machining parts of non-ferrous metals with small diameters.

Traditionally, the scheme for small-diameter parts turning is a machining with automatic lathes and $\mathrm{CNC}$ machines. The worldwide manufacturing leaders of such machines are Star CNC Machine Tool Corp, Index Corporation, Haas Automation, Okuma, DMG, Mori Seiki. Serially produced machines which can provide the following maximum speeds: Haas OL-1, Haas ST-10 - 6000 rpm, Okuma MULTUS B200II - 5000 rpm, DMG Nef400 - 4500 rpm, Mori Seiki NLX 2500/750 - 3500 rpm, Yamazaki Mazak Quick turn smart 100s - 6000 rpm, Index c100 $6000 \mathrm{rpm}$. Today only the following machine station models allow the maximum possible rotational speed for the workpiece: Star micronics AG SR20RIII - 10,000 rpm and Star micronics AG SR-10j - 15,000 rpm, Tornos SwissNano 16,000 rpm, NomuraDS NN - 10EX 15,000 rpm, HanwhaXD20V 10,000 rpm [1].

The analysis shows that during parts machining with a diameter of up to $20 \mathrm{~mm}$ reaching the optimum cutting speed recommended by the tool manufacturer on the mass-produced machines even at sufficiently high spindle rotational speed is very difficult due to the common use of a long bar as a workpiece in batch manufacturing. Usage of long bars generate unbalance due to its out-of-roundness and other reasons, often causing an unacceptable level of vibration (Fig. 1). For example, when turning a part with a diameter of $20 \mathrm{~mm}$ in order to reach the speed of $300 \mathrm{~m} / \mathrm{min}$, it is necessary to provide its rotation speed of almost $5000 \mathrm{rpm}$, which can be done on almost all modern turning lathes. However, only certain specialized models of lath machines for example, STAR machines, can provide the cutting speed of $500 \mathrm{~m} / \mathrm{min}$. But even they do not allow to reach high cutting speeds when turning parts with surfaces less than $10 \mathrm{~mm}$ in diameter.

Analysis of the catalogues of machine tool manufacturers showed that solutions to these problems have already been outlined. This includes, for example, length reduction of the rotating part of the bar, the use of automatic spindle balancing devices, active control of natural oscillations frequencies and modes of the machine. Thus, there are methods for automatic balancing of rotary bodies, which are described in US2331756A [2], US3953074A [3], US4043221A [4], US4075909A [5], US4755006A [6], US5460017A [7], US5605078A [8].

They have argued their efficiency in systems where a rapidly rotating body does not change the value of the speed for a long time. However, their use in machines where the rotational speed 
during machining is constantly change is not directly possible.

Turning at high cutting speeds will help to increase efficiency of machining and quality of the machined surface, but only if the vibration level when during machining is not significant. The proximity of the rotational speed to the natural oscillation modes of the technological system causes concerns of increasing vibrations and deterioration of quality of the machined surface. That is why there is a necessity of the development of method of controlling dynamic behavior of technological system in order to establish it stability under the oscillation when machining.

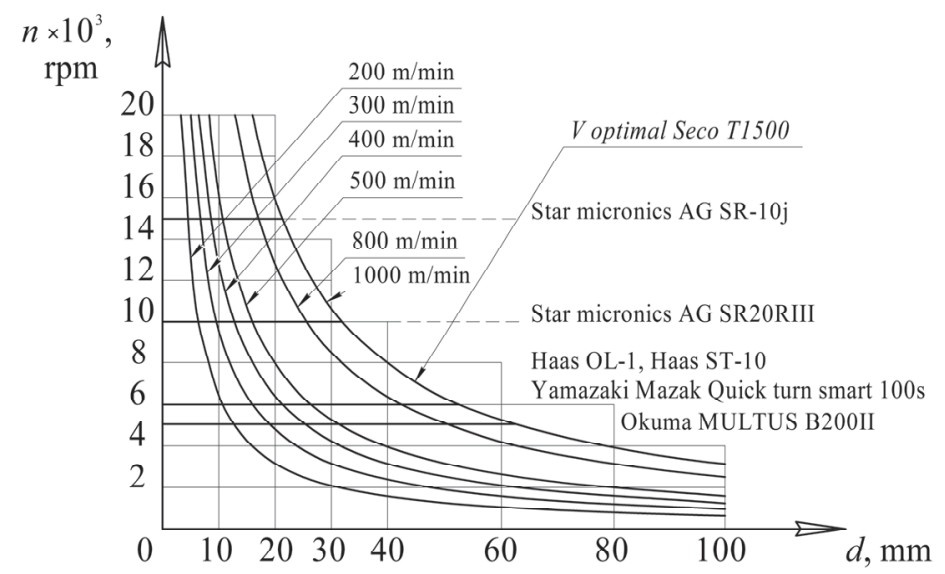

Fig. 1. Effect of rough workpiece diameter on the spindle rotation speed, providing different cutting speeds [1]

The methods of dynamics control of machine processing were studied in the works by Yu. M. Vnukov [9, 10], I. H. Zharkov [11], M. F. Kashirin [12], S. S. Kedrov [13], V. O. Kudinov [14], Yu. M. Kuznietsov [15], I. V. Lutsiv [16], P. P. Melnychuk [17], V. M. Poduraiev [18], V. M. Svinin [19], V. B. Strutynskyi [17], N. I. Tashlitskiy [20], Otto O. [21], J. S. Park [22] and others. The researches of these scientists have proved that increasing rotational speed tends to result in insufficient vibrational stability of cutting, which results in poor roughness of the machined surface, and, therefore, a decrease in the productivity of the machining. It was experimentally found that the roughness of the machined surface is most sensitive to vibrations in technological system than other quality parameters, since the relative oscillation of the tool and spindle along with the workpiece is precisely reflected on microgeometry of machined surface. For example, it is known that with a size tolerance for the 6th quality grade, the roundness oscillation within $2-3 \mu \mathrm{m}$ is not critical, while an increase in roughness of $2-3 \mu \mathrm{m}$ is inadmissible.

Along with the analytical and experimental methods of modelling the dynamical behaviour of technological systems, numerical methods for modelling dynamic processes in technical systems, in particular during machining at lathe modelling, have now become widely used. The most significant achievements in this direction were made by such Ukrainian researchers as N. E. Veselovska, Yu. M. Kuznietsov, I. V. Lutsiv, P. P. Melnychuk, V. B. Strutynskyi, V. M. Chupryna and others. Most works are dedicated to the development of mathematical models of the lathe elastic system that consider spatial rigidity of the individual subsystems of the lathe. In mathematical modelling of separate subsystems at a cyclic load it is suggested to perform an influence analysis of the correlation of rigidity of the technological system elements on the deformation of the subsystem "tool-workpiece", which allowed to determine the trajectories of mutual movement of the tool and the part and determine the degree of influence of the system dynamic behaviour on the accuracy and roughness of the machined surface. Prof. V. M. Svinin proved that reducing vibrations in the technical system is possible by varying the frequency and depth of modulation (change) of cutting speed.

In experimental and numerical studies, the existence of a displacements plane attached to the 
tool edge point was revealed. This plane proves to be inclined compared to the machines tool axes, which contains an ellipse that is the place of the points of the tool tip displacements. It was established that the tool tip point describes an ellipse. This ellipse is very small and can be considered as a small straight-line segment for the stable cutting process (without vibrations). The ellipse of displacement became more visible in unstable mode (with vibrations). A difference in phase occurred between the tool tip displacements on the radial direction and in the cutting direction which was almost in phase to feed motion direction. The ratio of small and long ellipse axes increases proportionally to feed rate. A weak growth $(6 \%)$ of the long and small axes ratio is obtained when the feed rate value decreases. The axis that goes through the stiffness center and the tool tip represents the maximum stiffness direction. The maximum (resp. minimum) stiffness axis of the tool is perpendicular to the large (resp. small) ellipse displacements axis. The selfexcited vibrations appearance is strongly influenced by the system stiffness values, their ration and their direction. FFT analysis of the accelerometers signals allows to reach several important parameters and establish coherent correlations between tool tip displacements and the static elastic characteristics of the machine tool components was tested [23].

M. Siddhpura and R. Paurobally summarized that chatter vibrations are present in almost all cutting operations and they are major obstacles in achieving desired productivity. Vibration between the tool and the workpiece forced regenerative chatter, resulting in a poor surface finish, high-pitch noise and accelerated tool wear which in turn reduces machine tool life, reliability and safety of the machining operation. [24].

G. Urbikain, F. J. Campa, et al. proposed two numerical methods which are combined with multimode analysis to predict chatter in big horizontal lathes. First, a traditional single frequency model (SFM) is used. Secondly, the modern collocation method based on the Chebyshev polynomials (CCM) is alternatively studied. This model can be used to identify the machine design features limiting lathe productivity, as well as the threshold values for choosing good cutting parameters [25].

H. Liu, S. Tang, et al. developed indirect measurement method: the vibration of the tool tip is obtained from the vibration of the measurement point on the turret. The relationship between the tool tip vibration and the turret measurement point vibration is obtained through impact tests. The vibration of the measurement point on the turret is measured via an accelerometer. The measurement point is so far away from the cutting area and chip evacuation zone that the accelerometer and its measurement results will not be affected by the chips and cutting fluid. The accuracy of the measurement method in study was checked by comparing the calculated tool tip vibration calculated from the vibration of the turret measurement point to the directly measured results, which was measured via an accelerometer installed below the tool tip. The comparison results show that the calculated tool tip vibration at main frequencies as well as low frequencies has a high accuracy. Moreover, the effectiveness of the measurement method is verified through machined surface profile [26].

However, not all the important issues are reflected in modern literature. Therefore, the implemented analysis allowed to formulate the purpose and main objectives of the study.

\section{Aim and objectives of study}

The aim of reported study was to increase efficiency of parts machining on turning lathes by controlling the dynamic behaviour of the technological system to ensure roughness of the surface finish in according to drawing requirements.

To reach this purpose the following objectives were set and realized:

- Analyse the possibility to increase machining efficiency of parts with a diameter of 5-20 mm and to establish the source of influence on roughness of the surface finish during turning at high rotational speed.

- Methodology of oscillations study of turning lathe during cutting process with high rotational speed was developed and implemented at experimental set-up. 
- Finite element model of the bench and experimentally verify the effect of natural oscillation frequencies and modes of machine elements on roughness of the surface finish was developed.

- Ways of oscillation amplitudes reduction of the turning lathe working parts during machining at high rotation speed was identified.

- Develop practical recommendations for research results implementation in order to increase productivity of processing by turning parts with relatively small diameters, while complying with drawing requirements used for processed surfaces roughness.

Object of study - dynamics of turning process.

Subject of study - dynamic behaviour of a turning lathe during turning surfaces of the parts with a small diameter at high rotational speed and with set roughness of the surface finish.

\section{Methodology of experimental study}

Experimental studies were carried out on an experimental set-up (Fig. 2) consisting of: an upgraded high-accuracy turning lathe with computer numerical control (CNC) 1700BФ30 with increased power of main motion actuator up to $1500 \mathrm{~W}$ which allowed to increase rotational speed in the range from 300 to $10.000 \mathrm{rpm}$; vibration diagnostic equipment which made it possible to measure and digitize signals of oscillation amplitudes of the parts and the cutting tool, and also to perform a modal analysis of the experimental set up, determine its frequency response, and signal modulator of the task (Fig. 2). The modulator allowed to control rotational speed according to the sinusoidal law during the workpiece turning by a left bent straight turning tool with main angle in the plane of $93^{\circ}$ equipped with a rhombic throwaway indexable carbide cutting insert CT35M with coating.

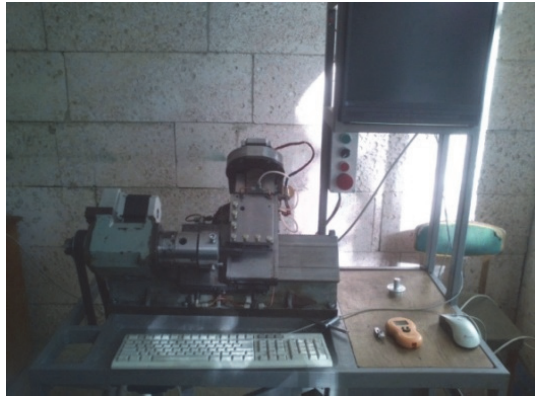

a)

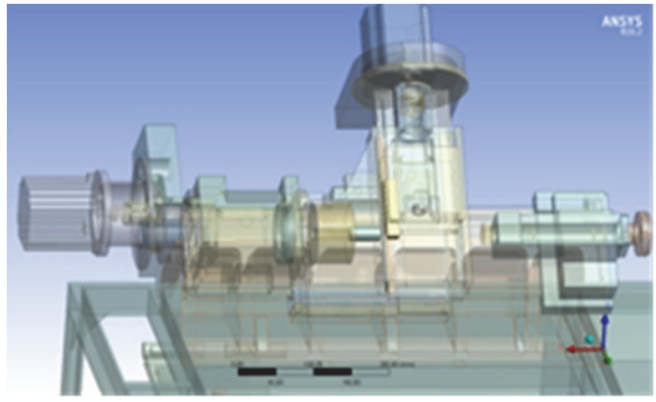

b)

Fig. 2. General view of the bench

The signal modulator is designed to change rotational speed according to the harmonic law. The device changes signal of the task that comes from CNC system to power converter of the main motion actuator (Fig. 3). For rotation of a spindle with a given frequency, CNC system produces a signal of $U_{3} \mathrm{DC}$ task which is proportional to the required spindle rotational speed. The modulator consists of two main components - generator and adder.

The generator produces sinusoidal signal the amplitude of which is proportional to the depth of spindle rotation modulation, while signal frequency is proportional to modulation frequency. With the help of the adder, these two signals are combined and sent to power converter of the main motion actuator. Thus, using a modulator, it is possible to change spindle rotational frequency with set values of modulation depth and frequency, which provides the ability to control the disruption of regeneration of self-oscillations due to the reconstruction of the "trace" cutting.

The vibrational acceleration of the frame was measured by standard Analog Device accelerometer ADXL 321, mounted on a spindle assembly next to spindle bearing and serves as a basis for measuring vibrational acceleration of the tool using the ADXL326 sensor. The vibratory displacement values are determined by further double integration with trapezoidal methods. 
Spindle displacement relative to the head stock is measured with the help of the contactless inductive displacement sensor XS1 M18AB120.

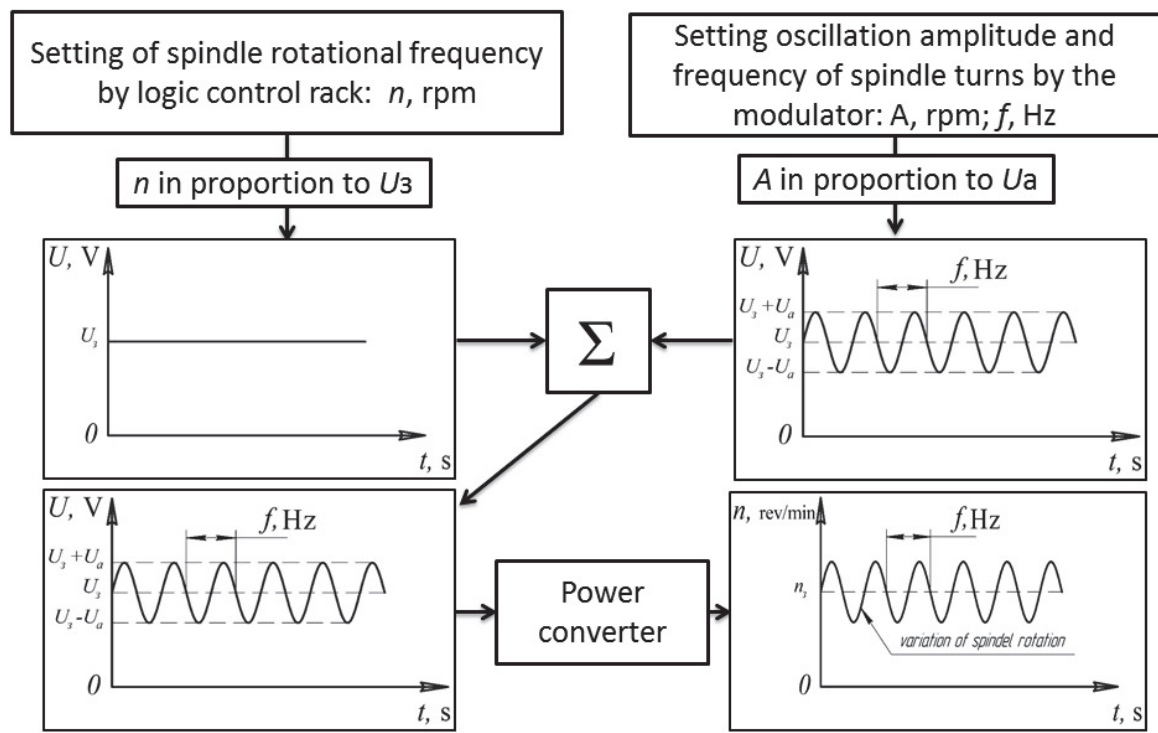

Fig. 3. The basic diagram of the modulator work

The coordination of the operation of all sensors in time occurs on the front edge of a zero-tag of spindle rotation sensor. The signals of all sensors are digitized and transferred to a personal computer (PC) with the help of a four-channel analog-to-digital converter (ADC) with independent inputs for LT-22 model L-CARD vibroacoustic researches (Fig. 4) [27].

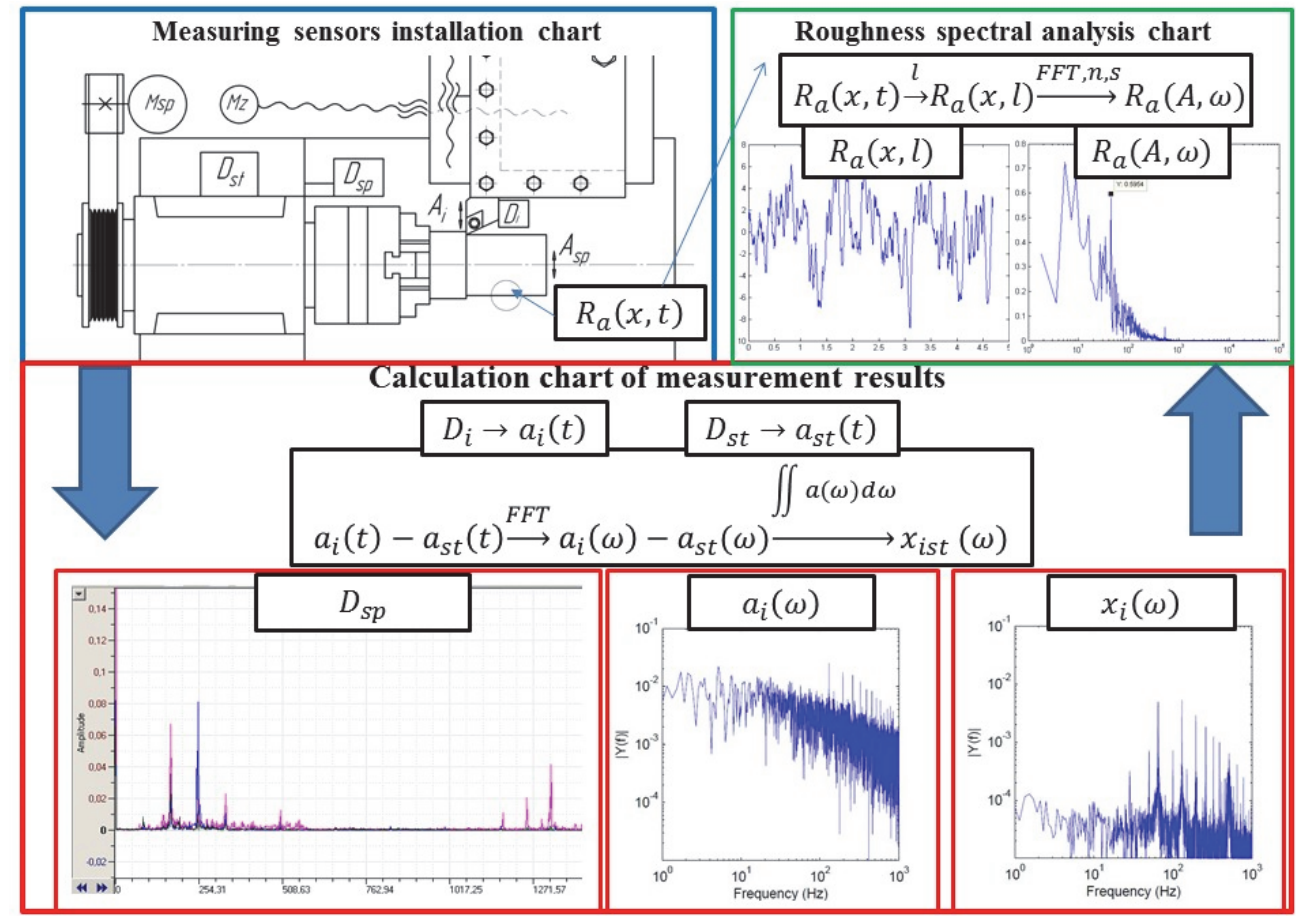

Fig. 4. Methodology of dynamic behaviour study 
The quality assessment of the surface finish was carried out according to the roughness parameter $R a$ using a modernized profilograph-profilometer $\Pi-283$ to a precision of $12 \%$ in accordance with GOST 27964-88 and ISO/R-468 methods. The modernization of the П-283 profile recorder (Fig. 5) was meant to equip it with a sensor to measure the real length of the surface of the part 4, which allowed tracing the cutter tip displacement in relation to the part at set spindle rotational frequency and feed when turning the test samples of different machined materials (steel, aluminum alloy, etc.). The modernization with a description of hardware being subjected to modernization procedure was implemented by connecting RPM sensor of the drive engine 5, which moves the probe head 2 via reducer 3 . The digital signal received was transmitted through the ADC LTR-22 7 to PC 9, which allowed performing a spectral roughness analysis of processed surface 1. The actual roughness value is recorded from the instrument scale 6 (Fig. 4) [28].

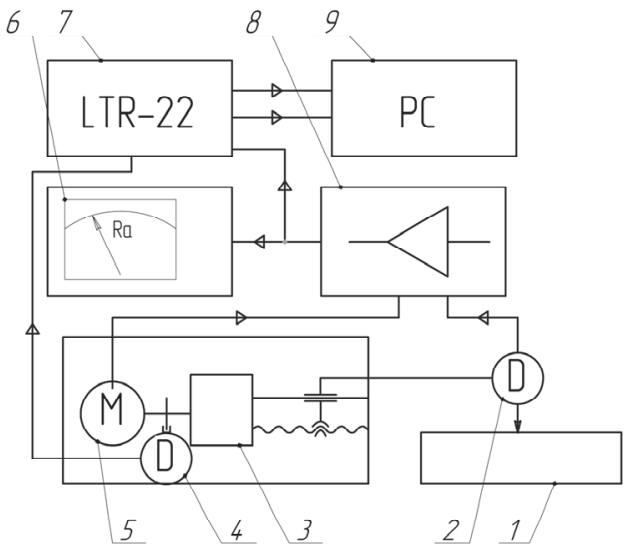

a)

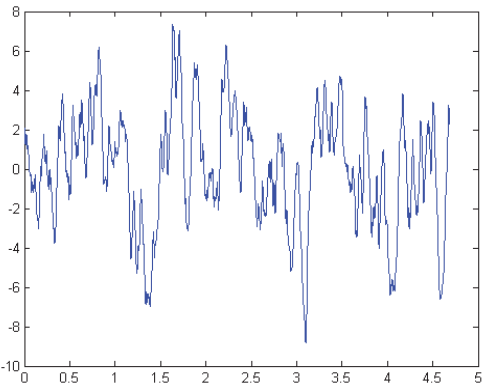

b)

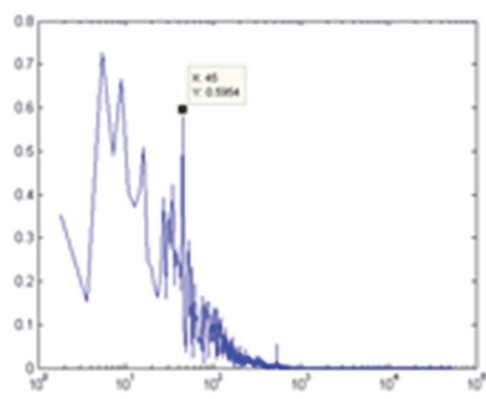

c)

Fig. 5. a) Profilograph-profilometer $\Pi-283$, b) profile record, c) spectral analysis

\section{Results}

During the turning with dynamic variation of rotational speed, it had been experimentally proven that the maximum achievable depth of modulation depending on the mass of rotating parts of the main actuator of the lathe and engine power essentially depends on the average rotational speed and the required modulation frequency. Thus, the lathe $16 \mathrm{~K} 20 \mathrm{~T} 1$ at the rotational speed of $800 \mathrm{rpm}$ and the modulation frequency of $4 \mathrm{~Hz}$ can maximally change its rotational speed by $180 \mathrm{rpm}$, and the lathe $1700 \mathrm{~B} Ф 30$ at rotational speed of $1900-7000 \mathrm{rpm}$ at a frequency of modulation of $5 \mathrm{~Hz}$ maximally changes the frequency by $50 \mathrm{rpm}$, and for the modulation frequency of $3 \mathrm{~Hz}$ by $150 \mathrm{rpm}$.

While researching the failure of regeneration of secondary self-oscillations due to the reconstruction of the "trace" cutting on the lathe 16K20T1 during the processing of the $60 \mathrm{~mm}$ 
diameter rough workpiece from $40 \mathrm{X}$ steel, with a hardness of $58 \mathrm{HRC}$ at cutting speed of $40 \mathrm{~m} / \mathrm{min}$, depth of $0.5 \mathrm{~mm}$ and a feed of $0.2 \mathrm{~mm}$ for normal turning, the roughness value was Ra $1.7 \mu \mathrm{m}$ with visible traces of self-oscillations. When machining with modulation frequency of $1 \mathrm{~Hz}$ at a modulation depth of $60 \mathrm{rpm}$ with same cutting parameters surface finish decreased to $\mathrm{Ra}$ $0.8 \mu \mathrm{m}$ without the traces of self-oscillations on the surface.

Conducting the same research on the 1700BФ30 lathe when machining D16T aluminum alloy workpiece at rotational speed of 4500-6000 rpm did not give a clear result. During parts machining with a modulation frequency of $1-5 \mathrm{~Hz}$ and a maximum modulation depth of $50-150 \mathrm{rpm}$ at frequencies of 4500-6800 rpm, the roughness values of the surface finish were within the same class and there was a tendency toward its small oscillation (within the measurement error of profilometer П-283) (Fig. 6) [29].

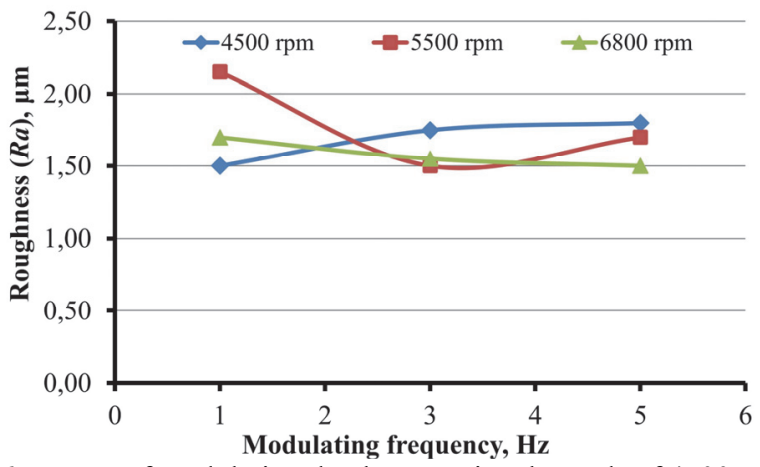

Fig. 6. Impact of modulation depth at rotational speeds of 4500, 5500, $6800 \mathrm{rpm}$ on roughness of the processed surface

During the machining the qualitative dependence of surface finish on the amplitude of the spindle mutual oscillations with the workpiece and the point of cutting insert depending on rotational speed was experimentally determined, and the oscillation amplitude of spindle and the tool was determined depending on rotational speed at idle (Fig. 7) [30].

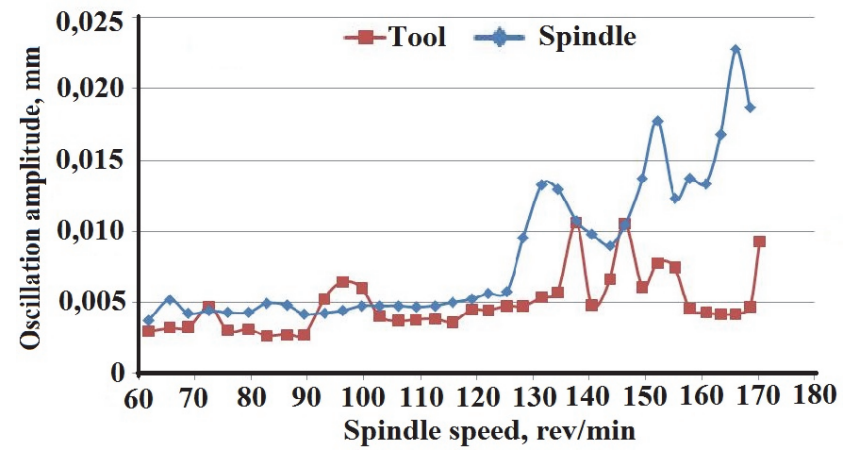

Fig. 7. Oscillation amplitude of a spindle and a tool tip depending on spindle rotational speed at idle

It has been established that the oscillations amplitude significantly increases at the resonance frequencies of the technological system: 135,152 and $166 \mathrm{~Hz}$, amplitude is $0.013,0.017$, and $0.022 \mathrm{~mm}$ respectively. At intermediate values between the resonance rotational speed (143 and $160 \mathrm{~Hz}$ ), the oscillations amplitude decreases to 0.009 and $0.012 \mathrm{~mm}$ respectively. Similarly, the oscillation amplitude of the tool under 138 and $146 \mathrm{~Hz}$ is about $0.01 \mathrm{~mm}$, and for other modulation frequencies, including large ones, is about $0.005 \mathrm{~mm}$.

By mean of finite element modelling, the amplitude of the mutual oscillation of spindle with the workpiece and blade tip of the tool was determined by analysing the Frequency Response of 
the Ansys Software (Fig. 8) and compared with the experimental studies of the effect of rotational speed during steady cutting parameters $(V=500 \mathrm{~m} / \mathrm{min}, S=0.05 \mathrm{~mm} / \mathrm{rev}, t=0.5 \mathrm{~mm})$ on the roughness of the surface finish.

Comparing the charts, one can see that the mutual oscillations amplitudes of the tool and spindle, as well as roughness depending on rotational speed used during the machining are similar. Thus, it can be argued that the amplitude of the relative oscillations of spindle with the workpiece and the blade tip of the tool is displayed on the machines surface of the part as a vibration component of roughness [29].

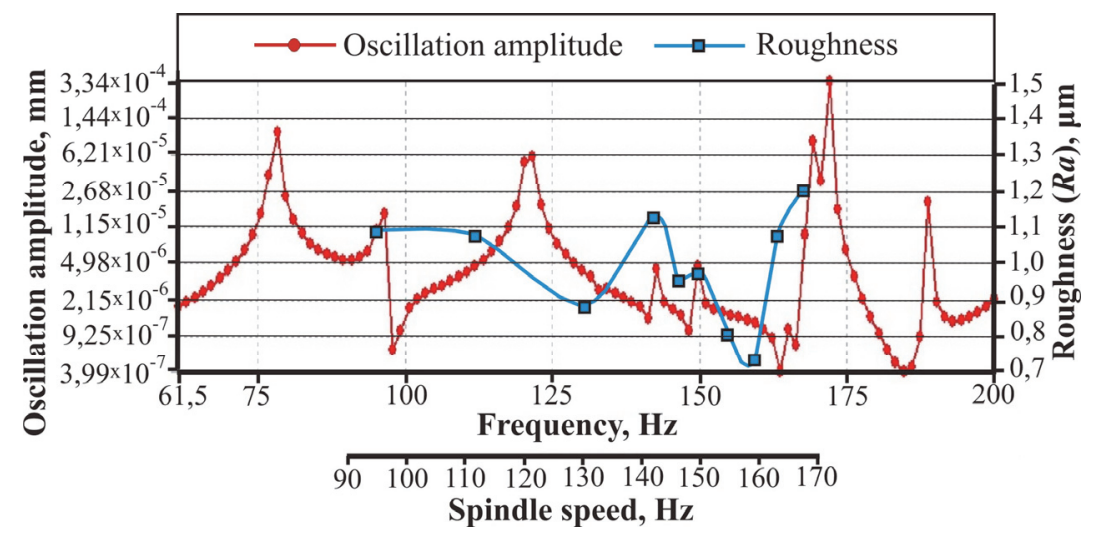

Fig. 8. The effect of mutual oscillations amplitude of spindle with the workpiece and blade tip of the tool on the roughness of the processed surface under various spindle rotational speeds

Experiment has been carried out to confirm the effect of natural oscillations modes of technological system on the mutual oscillations of a tool point and a workpiece. The oscillations amplitudes of the tool at the sliding carriage positions at a distance of $20 \mathrm{~mm}$ and $200 \mathrm{~mm}$ from the spindle front face, as well as at a distance of $20 \mathrm{~mm}$ from the spindle front face, are determined with a foot block mounted on the base, weighing $8 \mathrm{~kg}$ or without it. The oscillation amplitudes of the tool under different rotational speeds and tool positions are shown in Fig. 9. It has been proved that the additional mass leads to a change in the amplitude-frequency characteristic of cutter oscillation in relation to spindle. In this case, the maxima of the oscillations amplitudes are shifted. During turning without mass (without dead head), the minimum oscillations amplitude in the fixed position of the cutter occurs at frequencies of 6800 and $8800 \mathrm{rpm}$, and with mass - at frequencies of 4800 and $7800 \mathrm{rpm}$. Thus, by changing the position of the tool relative to the frame, and also by setting the additional controlled mass to the stand, it is possible to change the tool oscillation amplitude (for this lathe in the range from 0.2 to $0.45 \mu \mathrm{m}$ ), which will be reflected on processed surface roughness [29].

One of the effective methods for improving dynamic control of the lathe support oscillations is the method of implementation of additional actions that allows tracking oscillations amplitude of the sliding carriage by increasing the inertial properties of lathe and reducing logarithmic oscillation decrement of the cutter, which improves accuracy of machining and roughness of the surface finish at higher spindle rotation speeds. Oscillation control sensor (cutter displacement) at coordinate X located on a cutter connects to the control unit. During machining at first the minimum oscillation amplitude of the cutter is determined, then the signal from oscillation control sensor of the cutter is sent to the additional mass actuator, with which it moves along the frame at a distance proportional to the minimum amplitude of cutter oscillation. This method of dynamic control of oscillations in the cutting could be realised and experimental set-up which consists of: 1 - cutter; 2 - vibratory acceleration sensor; 3 - sliding carriage; 4 - communication channel between sensor and control unit; 5 - control unit; 6 - additional mass actuator communication channel; 7, 8 - tool oscillation vectors; 9 - additional mass (Fig. 10) [29]. 


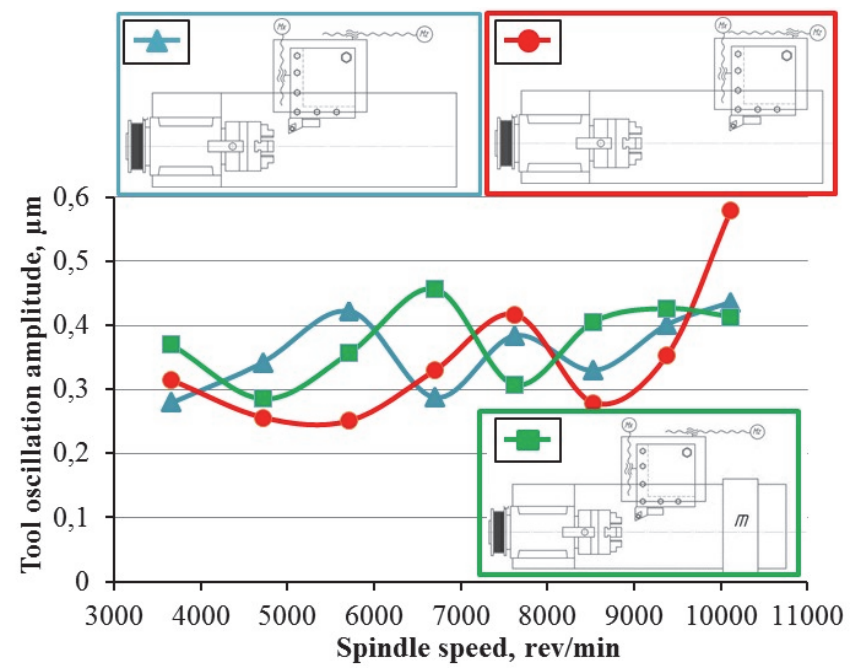

Fig. 9. Tool oscillation amplitudes at different spindle rotation speeds and tool positions

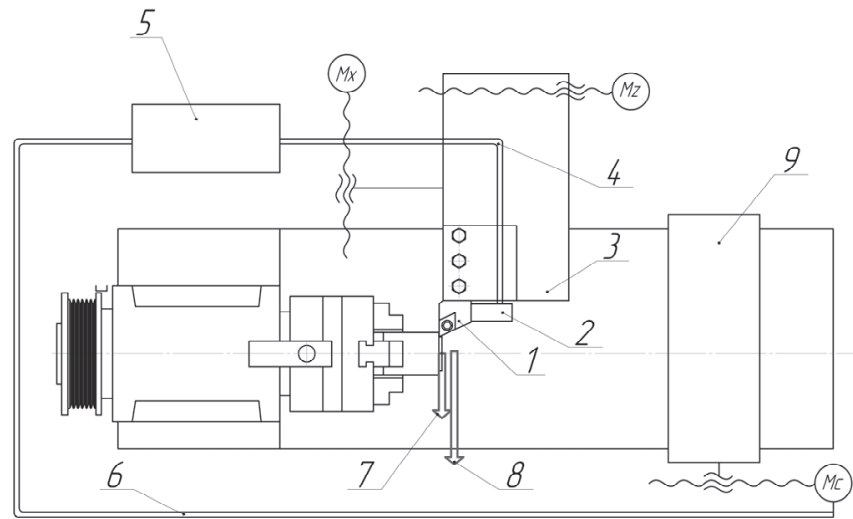

Fig. 10. Method of dynamic control of lathe support oscillations

An equally effective method of controlling the dynamic state of the technological system is the method of loading the metal cutting machine by a bar, when a long bar stock is cut into shorter bars using a special device located on the side of spindle opposite the operating area. In this case, the bar part (1) of the required length is clamped in spindle (2) and the rest of the bar is cut off next to the back side (3) of the spindle assembly (4). In addition, the rest of the bar is connected to the loading device (6) till the rod (7) and used as an elongated size rod. After the last cutting, the end of the bar remainder is disconnected with the loading device rod and sent to spindle as rod for machining fixed by clamping device (8). Feature machining is realised be combination of main rotational motion of the spindle and axillary motion of cutoff carriage (9) tool support bar (10) and cutter (11) (Fig. 11).

The suggested method of loading a metal-cutting lathe with a bar is aimed at solve a technical task of reducing dynamic loads at higher rotation speeds when lengthy bars are present in the loading mechanism by using the bar itself as an elongated rod for loading bars of reduced length and address the need for equipment for preparing (slicing) bars of the required length [32].

\section{Scientific novelty of the results obtained}

- For the first time, for the turning process at high rotation speeds, functional dependence of vibration component of roughness of the surface finish on the external driving forces and natural 
oscillation modes and frequencies of the TL (turning lathe) was established, which made it possible to effectively manage the machining.

- For the first time it has been established that the combination of the oscillation node of the system by moving the additional mass with the processing area during turning at high spindle rotational speed leads to a decrease in roughness of working surface by means of reducing the oscillation amplitude of the tool, due to changes in natural oscillation modes of the TL.

- Further development of the definition of application limits of inhibition effect of regenerative oscillations using the method of dynamic variation of rotational speed in connection with the change in ratio between the oscillation wave length of cutting edge and the distance covered by it during this time along the surface of part.

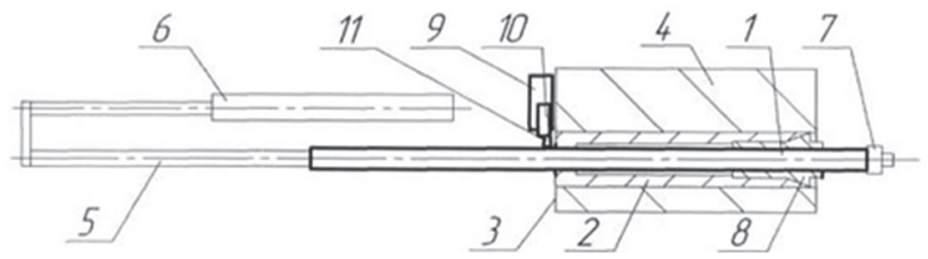

Fig. 11. Metal-cutting lathe loading method with a bar

\section{Conclusions}

In this work the scientific and practical task of increasing efficiency of machining parts on turning lathes is solved by controlling the process dynamics at higher rotational speeds to ensure the cutting speed recommended by tool material manufacturer and drawing requirements for accuracy and roughness of surfaces finish.

1. The reasoned assumption is made stating that increasing the efficiency of machining parts on turning lathes is possible by controlling the process dynamics through improving productivity at increased rotation speeds to meet the drawing requirements for roughness of the machined processed surface of parts with a diameter of 5-20 mm using cutting speeds recommended by tools manufacturers.

2. The method and testing bench developed on the basis of the turning lathe model 1700ВФ30 allow: a) to provide a rotational speed of up to $10,000 \mathrm{rpm}$; b) measure the immediate value of vibratory displacement and vibration acceleration of tool spindle and tool point in real time; c) perform spectral analysis of the profile record of the processed surface; d) establish the relationship between the profile of the machined surface and oscillations of spindle and tool.

3. In experimental study it has been established that the main sources of tool oscillations are self-oscillation and oscillations from excitatory force in the system "spindle-device-workpiece", which limit the maximum rotation speed with the workpiece by increasing roughness of the surface finish. It is proved that the minimum roughness of the surfaces finish is achieved by reducing the system response to the force in the cutting area by:

- Modulation of rotational speed with frequency from 1 to $5 \mathrm{~Hz}$, which allowed to control failure of self-oscillations regeneration due to the reconstruction of the "trace" cutting.

- The combination of node of natural oscillation modes and the cutting area, due to dynamic control of oscillation of turning lathe sliding carriage by increasing inertial properties of lathe and reducing the oscillation amplitude of the cutter, which allows to improve the processing accuracy and reduce roughness of the surface finish at higher rotation speeds.

It has been proven that for small rotational speeds, in particular the coercive force frequency, the possibility of reducing vibrations in technological system at the expense of mass redistribution is difficult to implement, at the same time, at relatively high spindle rotational speeds (more than $6,000 \mathrm{rpm}$ ) it is possible to substantially affect the dynamic behaviour of the technological system, because even small masses lead to changes in modes and frequencies of its natural oscillations, which are close to oscillation frequencies of coercive force. 


\section{References}

[1] Zaloga W. A., Zinchenko R. N., Shapoval Y. V. Machining of part with small diameter when turning with high rotational speed. Problems and development perspective. Modern Technologies in Manufacturing Engineering, Vol. 9, 2014, p. 50-63.

[2] Zobel R. Automatic Balancing Device. US2331756A, United States Patent Office Claims, 1942.

[3] Wilson Cox W. Automatic and Substantially Permanent Wheel Balancing Device. US3953074A, United States Patent Office Claims, 1975.

[4] Peschel D., Funke D. Mass-Imbalance Oscillation Generator. US4043221A, United States Patent and Trademark Office, 1974.

[5] Deakin J. E. Automatic Shaft Balancer. US4075909A, United States Patent and Trademark Office, 1976.

[6] Clay S. C., Clay R. A. Dynamic Wheel Balancing Device. US4755006A, United States Patent and Trademark Office, 1986.

[7] Taylor G. R. Weight Compensating Apparatus. US5460017A, United States Patent and Trademark Office, 1992.

[8] Taylor G. R., Wierzba P., Hannah R. C. Weight Compensating Method and Apparatus. US5605078A, United States Patent and Trademark Office, 1992.

[9] Kuchuhurov M. V., Vnukov Yu N., Diadia S. Y. Method and device for studying regenerative self-oscillations during turning. Cutting and Tools in Technological Systems, ONPU, Odesa, Vol. 83, 2013, p. 42-54, (in Russian).

[10] Vnukov Yu. N. Germashev A. I. Komorkin P. A. Kozlova E. B. Estimation of efficiency and quality of processing of thin-walled parts at end milling. Information Technologies in Education, Science and Production, Vol. 7, Issue 2, 2014, p. 97-108, (in Russian).

[11] Zharkov I. G. Vibrations During Processing with a Blade Tool. Mashinostroyeniye, 1986, p. 184, (in Russian).

[12] Kashirin M. F. Investigation of Vibrations during Cutting. Nauka Publishing, USSR, 1944, p. 262, (in Russian).

[13] Kedrov S. S. Oscillations of Metal-Cutting Machines. Mashinostroyeniye, Moscow, 1978, p. 198 (in Russian).

[14] Kudinov V. A. Dynamics of Machines. Mashinostroyeniye, Moscow, 1967, p. 367, (in Russian)

[15] Podolskyi M. I., Kuznietsov Yu M., Dmytriiev D. O., Sidorov V. A. Experimental studies of dynamic parameters of a rod support system of a multipurpose lathe during cutting technological complexes. Mizhnarodnyi naukovyi zhurnal, Vol. 10, Issue 2, 2014, p. 175-181, (in Ukrainian).

[16] Anelchyk Ie D., Shvets S. V., Lutsiv I. V., Dubetskyi I. D. Cutting System: Physical Basics of Optimization. TDTU, Odesa-Ternopyl, 2000, p. 145, (in Ukrainian).

[17] Strutynskyi V. B. Mathematical Modeling of Metal-Cutting Machines. ZhITI, Zhytomyr, 2002, p. 570, (in Ukrainian).

[18] Podurayev V. N. Processing by Cutting with Vibration. Mashinostroyeniye, Moscow, 1970, p. 350, (in Russian).

[19] Svinin V. M. Milling with a Modulated Cutting Speed. IrSTU Publishing, Irkutsk, 2007, p. 304, (in Russian).

[20] Tashlitskiy N. I. The primary source of excitation energy of self-oscillations during metal cutting. Vestnik Mashinostroyeniya, Vol. 2, 1960, p. 10-20.

[21] Otto A., Radons G. Application of spindle speed variation for chatter suppression in turning. CIRP Journal of Manufacturing Science and Technology, Vol. 6, Issue 2, 2013, p. 102-109.

[22] Park J. S. The prediction of chatter stability in hard turning. Ph.D. Thesis in Mechanical Engineering, Atlanta, 2004.

[23] Bisu C. F., Darnis P., G'erard A., et al. Displacements analysis of self-excited vibrations in turning. The International Journal of Advanced Manufacturing Technology, Vol. 44, Issues 1-2, 2009, p. 1-16.

[24] Siddhpura M., Paurobally R. A review of chatter vibration research in turning. International Journal of Machine Tools and Manufacture, Vol. 61, 2012, p. 27-47.

[25] Urbikaina G., Campaa F.-J., Zulaikab J.-J. Preventing chatter vibrations in heavy-duty turning operations in large horizontal lathes. Journal of Sound and Vibration. Vol. 340, 2015, p. 317-330.

[26] Liu H., Tang S., He S. A method of measuring tool tip vibration in turning operations. The International Journal of Advanced Manufacturing Technology, Vol. 85, Issues 5-8, 2016, p. $1325-1337$. 
[27] Zaloga W. A., Zinchenko R. N., Shapoval Y. V. Increasing stability of cutting process by varying cutting speed when machining. 23rd International Technical Science Seminar, 2015, (in Ukrainian).

[28] Zaloga W. A., Shapoval Y. V. Methodology of evaluation of dynamic behavior of cutting system by determining geometric parameters of surface finish quality. Cutting and Tool in Technological System, Vol. 87, 2017, p. 63-81, (in Ukrainian).

[29] Zaloga W. A., Shapoval Y. V. Increasing efficiency of machining parts by controlling dynamics of turning with high rotational speed. Herald of Zhitomyr State Technical University, Vol. 80, Issue 2, 2017, p. 175-184, (in Ukrainian).

[30] Zaloga W. A., Kryvoruchko D. V., Shapoval Y. V., Drofa K. Dynamic control of oscillation when turning. Mechanics and Advanced Technologies, Vol. 79, 2017, p. 100-107, (in Ukrainian).

[31] Korotun N. N., Kryvoruchko D. V., Shapoval Y. V. Dynamic Control of Sliding Carrier Oscillation. Patent No. UA107301U, 2016, (in Ukrainian).

[32] Kryvoruchko D. V., Shapoval Y. V., Korotun N. N. Method of Loading of Machine Tools Rod. Patent No. UA103688U, 2015, (in Ukrainian).

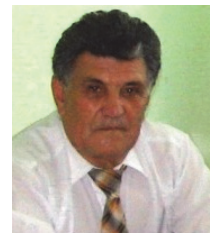

Zaloha William Oleksandrovych born 29 August, 1936, in Kyiv, Ukrainian. Started working in 1954 at Kuianivska sugar plant in Sumy oblast. In 1954 graduated from Kharkiv Polytechnic Institute (KhPI) where he studied machine building. In 1965-1969 worked by the assignment at a plant (military base 44528, Bataysk, Rostov Oblast) as a master workman, manufacturing technician, and deputy shop foreman. Has been working in Sumy State University since 1969 as an Assistant, senior teacher, Docent, Professor, and head of a department. In 1970-1973 pursued postgraduate studies in KhPI under the supervision of professor M.F. Semko, Doctor of Engineering Science, and docent A.I. Hrabchenko, Candidate of Engineering Science. He became Candidate of Engineering Science himself in 1974. In 1977 was awarded with the title of Assistant Professor. From 1978 to 1990 was a head of the Metal-cutting machines and tools (MRV) department and starting from 1993 has been working as a professor. In 1995 was given a title of Professor in MRV department. In 2000 he passed his Doctor of Engineering Science. Defence in the speciality 05.03.01 - Machining Processes, Lathes and Tools. Since 2002 worked as a head of MRV department again in Sumy State University, and since 2007, after the unification of the departments Manufacturing Engineering and Metal-cutting machines and systems, works as the head of the department Manufacturing Engineering, Machines and Tools.

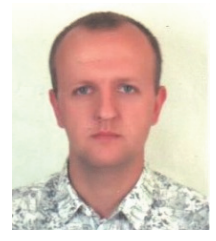

Shapoval Yurii Volodymyrovych born 28 of April, 1982, in Sumy. In 1999, he graduated from the Physics and Mathematics class of Sumy Educational Complex No. 7 with a silver medal. In 2004 graduated with honour from Sumy State University, receiving a Master's degree in metal-cutting machines and systems. Started to work in Sumy Scientific and Production Association named after Frunze as designing engineer at the Industrial Electronics Administration where he was engaged in repairing and modernization of $\mathrm{CNC}$ machines. In 2008, he worked as the Chief Mechanic of the Kiev Drilling Equipment Plant. In 2012-2015 pursued postgraduate studies at Sumy State University, where he has been working since 2015 as an Assistant of the department Technology of Machine-Building, Machines and Tools.

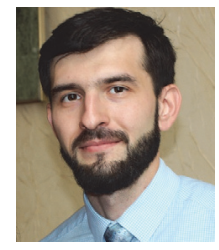

Kolesnyk Vitalii Oleksandrovych born 31 of May, 1988, in Sumy. In 2010 graduated from Sumy State University, receiving a Master's degree in manufacturing engineering. Started to work in Sumy Plant "Nasosenegomash" as Manufacturing Engineer at the Chief Manufacturing Engineer office where he was engaged in manufacturing preparation of production. In 2011-2014 pursued postgraduate studies at Sumy State University, where he has been working since 2015 as an senior teacher of the Department Manufacturing Engineering, Machines and Tools. In 2016 defended a Ph.D. thesis in manufacturing engineering. His research interests include machining of CFRP/Ti alloy stacks, insurance of accuracy and quality of machining. 


\section{Chapter 3}

Increasing the strength of machines under static, cyclic and dynamic loads 


\title{
3.1. Increase of reliability and wear resistance of cylindrical blocks with surface plastic deformation
}

\author{
Aleksandr Dykha ${ }^{1}$, Dmitry Marchenko² \\ ${ }^{1}$ Khmelnitsky National University, Khmelnitsky, Ukraine \\ ${ }^{2}$ Mykolaiv National Agrarian University, Mykolaiv, Ukraine \\ E-mail: ${ }^{1}$ tribosenator@gmail.com, ${ }^{2}$ marchenkodd1984@gmail.com
}

\begin{abstract}
The study of the efficiency of hardening the parts working in spalling conditions through reeling with rollers were performed with the help of physical simulation and showed the high effect of hardening cast steels. Electrography examination has shown that an increase in the degree of work hardening when reeling with a needle roller manifests itself in a higher dislocation density and cell size decrease in the substructure of ferrite grains. Diffusion of chemical elements in the surface layer in the process of surface deformation was studied with an analysis of the change of the surface microhardness. The process of the contact friction surface wear during reeling with consideration of slippage was investigated. It was proved that roughness of the friction surfaces affects the coefficient of friction and the rate of tribo-contact wear during reeling with slippage. A procedure for determining conditions of reeling with a wedge roller was developed. Optimal reeling conditions were found due to experiment planning using the steep convergence method. The obtained results of calculations will serve as initial data in designing working elements of the reeling devices and developing technological processes for strengthening parts. The conducted studies will find their future use in evaluation of the wear processes taking.
\end{abstract}

Keywords: contact strength, wear, surface plastic deformation, wedge roller, rope block.

\section{Introduction}

The problem of increasing wear resistance, contact strength and resistance to contact spalling is becoming increasingly relevant as intensity of handling equipment operation is constantly increasing. The most economical extension of the rope block service life is possible by improving properties of the surface layer. Surface properties can be controlled by changing the surface layer structure and physical and mechanical properties.

When operating equipment for general technical purposes, large-sized steel parts that perceive contact loads, such as cable blocks, tackle blocks, pulleys, etc. often fail. Significant work forces in the presence of misalignment of interconnected parts often bring about spalling and wear of the working friction surfaces, shape distortion, alteration of clearances between the parts. As a result, service life of handling equipment and machinery units in general is reduced. Application of thermal or thermochemical strengthening methods in the manufacture of large size rope blocks is limited by their dimension and weight. The most simple, available, and often solely possible, method for strengthening such blocks is surface processing by cold plastic working, namely reeling or hammering. To improve appearance and increase wear resistance of the surface layer, finishing surface plastic deformation (SPW) is applied and in order to increase fatigue strength and contact strength of parts, work hardening is used. Therefore, the study of the effect of the reeled surface layer properties on tribotechnical characteristics is an urgent scientific and technical problem.

\section{Literature review and problem statement}

During operation of rope blocks, rope interacts with the surface of the block groove. Because of elastic deformation and torsion of the rope under load, it slips and rotates round its axis. This brings about various types of damage: the groove wear, appearance of cracks, collar spalling, general deformations, and other flaws. A fundamental contribution to the theory, calculation, and design of a "rope block - rope" friction couple is made in [2]. The proposed theoretical 
calculations in the design of the "rope block - rope" friction couple have low accuracy because of numerous simplifications and assumptions in the calculation method.

The issues of wear, study of surfaces subjected to contact loading as well as improvement of physical properties of surface layers are considered in [3-5]. These studies do not take into account the phenomenon of slippage of contact friction surfaces which in turn affects the mechanism of wear of the tribological couple.

The use of cast iron for casting the blocks increases wear resistance of the block by $10-12 \%$ compared to the steel blocks. Worn cast-iron (SCh 15-32) rope blocks are replaced by blocks of steel (25L) [6]. However, the proposed methods of hardening and restoring rope blocks, namely, restoration of the blocks with the help of automatic surfacing, welding, electromechanical processing, electroplating, etc. are very costly and of a low material-output ratio. Therefore, it is more advisable to take measures to harden the rope blocks and increase their longevity, especially with the help of SFW.

When deciding on the expediency of hardening and restoration of parts, one should proceed from the technical feasibility of the enterprise in question. It is necessary to ensure performance of the part after its hardening and restoration for the entire inter-recouple service life of the unit encompassing this part and economic feasibility of hardening and restoration.

Solution of the tribo-contact problem for a friction couple was made on the basis of the wear model in [7]. The proposed solution is quite complex for practical implementation since it requires division of the wear area into discrete sections. It does not take into consideration technological features and the form of the contact friction couple.

Solution of an inverse wear contact problem for identifying parameters of the dependence of wear intensity on pressure and speed of sliding taking into account their distribution over the contact spot has been made in [8]. Based on the wear experiment by the "finger-disk" scheme, expressions for determining these parameters were obtained. However, assumption of stability of the wear spot accepted in the work in accordance with the test scheme does not make it possible to use the obtained solution for the test schemes with a variable contact (wear) spot.

A study of wear of a working "rope block - rope" friction couple caused by difference in diameters is presented in [9]. It was established on the basis of this study that the difference between the radii of the rope and the rope block profile leads to a change in distribution of contact pressures, and a phenomenon of axial slippage of the rope occurs which in turn leads to wear of the contact friction surfaces. However, the article does not present the dependence on quality of processing the friction surfaces of the "rope block - rope" couple.

The mechanism of wear of the "rope block - rope" friction couple and distribution of residual stresses in the substrate surface were studied in [10]. The results of this study indicate that the rate of wear of the alloyed steel depends on diameter of the rope wires. The mechanism of wear of annealed low-alloy steel is characterized by fatigue and abrasive wear but the wire demonstrates partial adhesion because of microabrasive wear when a fatigue crack appears. Distribution of the residual stress contacts does not depend on the motion speed. Therefore, there are constant values in the contact surface.

Analysis of the nature of failure of the parts that wear from the contact load of the friction surfaces showed that failure of rope blocks begins mostly in the surface layer and the resistance to this failure is determined by quality of the surface layer. Therefore, it is possible to influence quality of the surface layer by changing methods of surface treatment to provide predefined tribological characteristics to the friction surfaces.

Thus, further theoretical and experimental studies of physical and mechanical properties of the surface layer and microstructural studies of steel specimens after reeling them with rollers are needed. It is necessary to investigate diffusion of chemical elements in the surface layer during surface deformation by means of microchemical analysis and carry out tribological studies of wear of contact friction surfaces during reeling with allowance for slippage. All these measures will make it possible to create a method, a process, and a device for strengthening the rope blocks by 
reeling and solve production problems during its implementation.

\section{Materials and methods used in the tribological study of the effect of reeling parts on the contact strength}

Electron microscopy studies were carried out using a UEMV-100K microscope on thin foils prepared from the specimens taken at various distances from the surface and then thinned to a thickness transparent for electrons.

Depth and degree of work hardening during plastic deformation of the surface layer were estimated using the regression analysis method. To this end, rupture tests were conducted on $0.2, \ldots, 0.4 \mathrm{~mm}$ thick flat specimens cut from a part at various distances from the part surface with the help of Shovenar machine with film recording of specimen deformation versus load diagrams.

The study of the degree of hardening with analysis of the change of microhardness and diffusion of chemical elements in the surface layer in the process of SFW was carried out as follows.

Microhardness after reeling the specimens in various conditions was determined using the PMT-3 microhardness meter. Determination of microstructure was carried out with the help of the KMT-1 device. Investigation of chemical distribution of strengthening elements by means of microchemical analysis was carried out on a raster electron microscope with the Superprobe-733 micro-X-ray micro-chemical analyzer from Jeol Company (Japan).

The study of pressure on the surface of plastic contact of the roller with the part during work hardening was carried out on $6.4 \mathrm{~mm}$ thick models prepared of an optically sensitive material ED6-M with the PPU-4 polarization installation (Russia).

The TRB-S-DE tribometer (Switzerland) was used in the study of wear of the contact friction surfaces during reeling taking into consideration slippage to determine tribotechnical characteristics of the "disk-sphere" friction couple and the MI wear machine was used for modeling slippage with its upper shaft rotatable to ensure transverse slip. The VLR-200 (Russia) laboratory scale was used to measure weight loss in specimens.

Experimental studies of the technological process of hardening the rope block surface by reeling were carried out on a universal $1 \mathrm{~K} 65$ screw-cutting machine. The technological process was recorded on Panasonic SDR-S26 video camera with a subsequent frame-by-frame examination. Force on the roller varied within $\pm 5 \%$ since the friction force of the rolling bearings was not more than 0.008 . This provided a uniform deformation of the surface layer in the rope block groove.

The degree of work hardening was measured and determined after reeling with the help of TIME Hardness Tester TH130 (India) universal integral dynamic hardness meter.

Roughness of the rope block working surface before reeling was measured using reference roughness comparison specimens OSh (GOST 9378-93 made in accordance with requirements of GOST 2789-73). After reeling, roughness was measured with the help of replicas prepared from self-curing Protacril-M plastic using the profilographer-profilometer of A1 type (GOST 19299-73 and GOST 19300-73), model 252 manufactured by Caliber Enterprise (Russia).

The reeled rope blocks were tested for spalling with ropes in production conditions on KRUPP ship reloaders (Germany) with a hoisting capacity of 40 tons, MGZ OJSC, and KS-3575 autocranes with a hoisting capacity of 10 tons, Mykolayivbudmekhanizatsiya JSC (Ukraine).

\section{Results of tribological tests and process parameters of reeling steel parts with rollers}

Vickers hardness under force of $0.10 \mathrm{kN}$ and mechanical characteristics $\sigma_{B}, \sigma_{0,2}$ and $\delta$ were determined on specimens taken from a shaft made of 40 grade steel and reeled under a reeling force of $50.0 \mathrm{kN}$ (Fig. 1).

Statistical estimation of accuracy in determining the depth of work hardening was made proceeding from the changes in hardness HV10 and the yield strength of the work hardened metal 
layer. A hypothesis of normality of distribution of measurements of initial hardness and yield strength was verified by the Kolmogorov criterion. After that, the results of hardness tests were statistically analyzed by the small specimen technique.

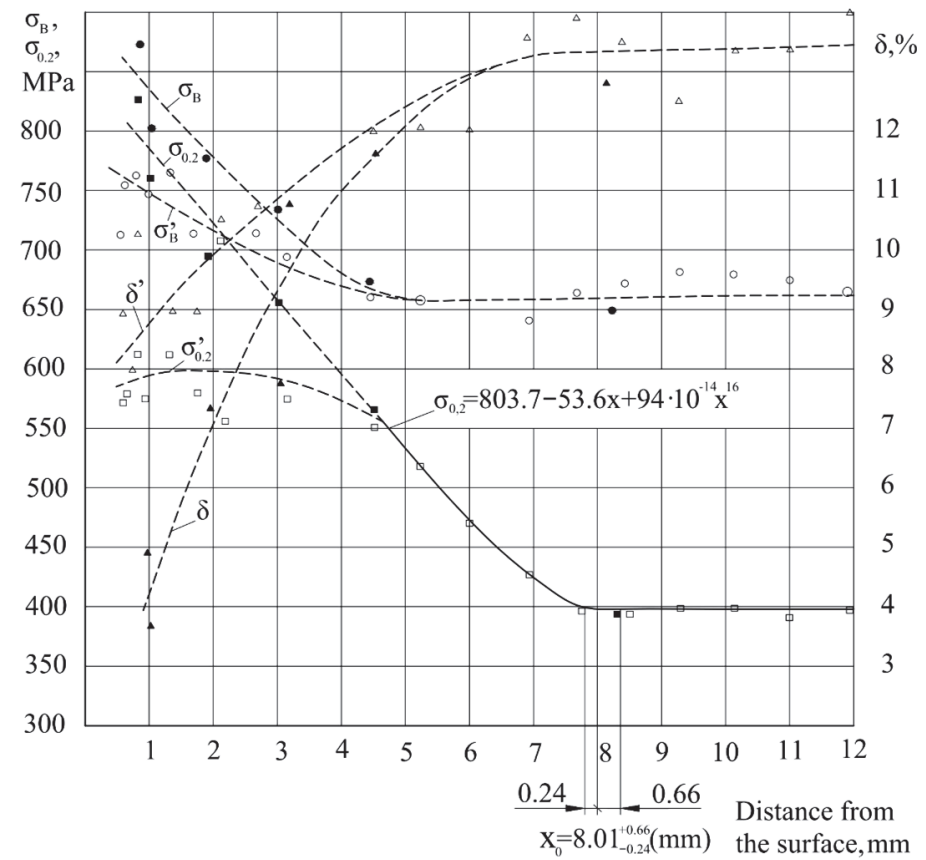

Fig. 1. Mechanical properties of the surface layer of the shafts reeled with toroidal rollers with diameter $D_{p}=105 \mathrm{~mm}$, profile radius $r_{p}=10 \mathrm{~mm}: \sigma_{B}, \sigma_{0,2}$ and $\delta$ are properties in a circular direction; $\sigma_{B}, \sigma_{0,2}, \delta$ are properties in the axial direction

Nonlinear regression analysis was used to determine the depth of work hardening (estimated by the start of change in mechanical properties HV10 and $\sigma_{0,2}$ of the deformed layer) and estimate accuracy of determining the depth of work hardening.

To describe the regression lines, the functions were taken [1]:

$y=a_{1}+b_{1} x+b_{2} x^{m}$,

$y=a_{1}+b_{1} x+b_{2} \lg x$

where $y$ is hardness or yield strength; $x$ is the distance of the measuring point from the reeled surface.

Statistical processing of the results obtained in determining the coefficients $a_{1}, b_{1}, b_{2}$ in the regression equations was performed according to the least squares method. Dispersion coefficients, work hardening depth and its confidence intervals and the standard deviation were estimated as well.

In the process of the regression analysis, the values of coefficients of the regression Eqs. (1), (2) and the abscissa of the extremum point were initially calculated taking into account the values of mechanical properties which differed significantly from the initial ones. After the points of measuring initial mechanical properties were shifted in the direction of the $x$-axis, up to coincidence of their abscissas with the abscissa of the extremum point of the regression line, solution was performed again, but with taking into consideration measurements of the initial mechanical properties altered in this way.

The solution was repeated until the difference between abscissas of the points of extremum of 
two last regression lines did not exceed a specified number equal to $0.01 \mathrm{~mm}$. Calculations were carried out on a PC. Coefficient $\mathrm{m}$ of the parabolic regression line acquired values from 2 to 25 .

The line having smallest residual dispersion S2 was selected from various regression lines. The best regression line has appeared to be a parabolic dependence. For all tested specimens, the hypothesis of equality of mean values of work hardening depth determined by the results of measuring hardness and yield strength checked by the Student's criterion was not confirmed.

The study results have shown that yield strength of the work hardened layer increased to a greater extent than hardness (100-130\% versus $20-60 \%)$. Due to this, the boundary of the work hardened layer was more clearly defined by the change in the yield strength. Use of cylindrical needle rollers of a small diameter leads to a sharp increase in the deformation ratio in a thin surface layer which was indicated by grain elongation in the direction of reeling seen in the optical microphotographs.

Accuracy of determining boundary of the work hardened layer by regression analysis according to the results of measurement of yield strength is twice as high in comparison with those obtained in Vickers hardness tests. The $95 \%$ confidence intervals for the work hardening depth calculated from the results of measurement of yield strength make up 11-36 \% of the work hardening depth and 32-75\% for the hardness tests. The depth of work hardening which is determined by variations in the yield strength was $25-50 \%$ more than the depth determined by Vickers hardness tests. This difference increases with a decrease in the degree of work hardening. The depth of work hardening determined according to the values of yield strength for circular and close to them imprints $(b / a \leq 2)$ corresponded to that calculated by Heifets method even with a rather small reduced curvature of the contact between the roller and the part $\left(k=0.0835 \mathrm{~mm}^{-1}\right)$. It has been established that the yield strength was a more sensitive mechanical characteristic for determining the depth of plastic deformation than hardness. The depth of penetration of compressive stresses was close to the depth of work hardening determined by the yield strength.

Influence of conditions of reeling with rollers on the change of microstructure of the worked metal was studied in reeling with cylindrical and toroidal rollers of small diameter. Microstructure of the specimens prior to surface work hardening consists of pearlite grains surrounded by hypo-eutectoid ferrite (Fig. 2(a)). After reeling, changes in the microstructure observed on optical-digital microphotographs could only be found in the surface layers of the shaft reeled with a roller (Fig. 2(b)). The changes were seen as a considerable elongation of both ferrite and perlite grains in a circular direction of reeling. It was established that at the distance from the surface $(16.42 \mathrm{~mm})$, the ferrite plates of perlite din not contain dislocations. In some locations, there were isolated dislocations in the ferrite-cementite interface. Ferrite grains in the specimens were constrained by flat straight boundaries. Inside the grains, there was a three-dimensional net of low density dislocations.

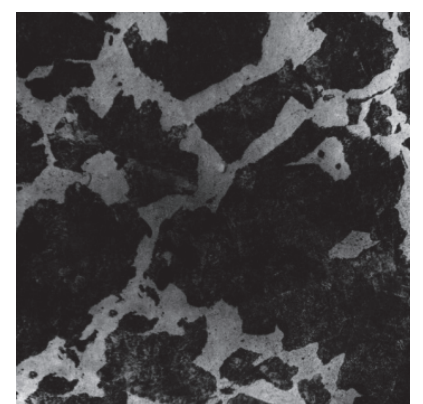

a)

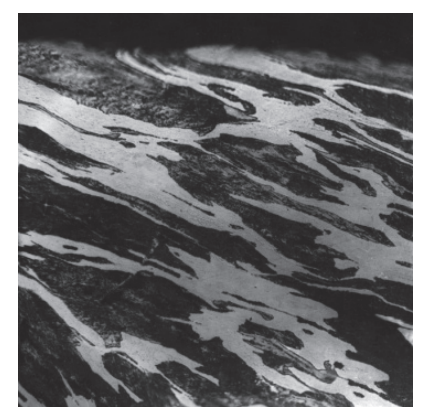

b)

Fig. 2. Microstructure of specimens of the surface layer of normalized 40 grade steel: a) before reeling, $b)$ after reeling $(\times 300)$

As the electron-diffraction examination has shown, strengthening of the surface layers during 
reeling shafts with a roller was mainly due to emergence of dislocation cells in the structure of the excessive ferrite grains. Higher degree of work hardening when reeling with a needle roller manifested itself by a higher density of dislocations and a reduced size of cells in the substructure of ferrite grains and a thicker grid of dislocations in the ferrite plates of perlite. In some locations, bend and fracture of cementite plates was observed which indicated the limit degree of plastic deformation of the surface layer. This was confirmed by the beginning of peeling of the surface reeled with a five-millimeter roller.

Distribution of microhardness $H_{\mu}$ in the depth of three specimens is shown in Fig. 3.

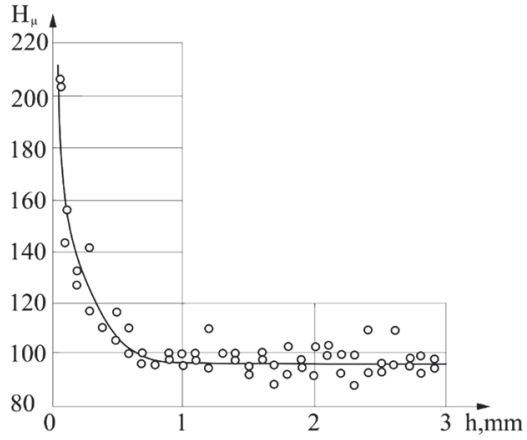

a)

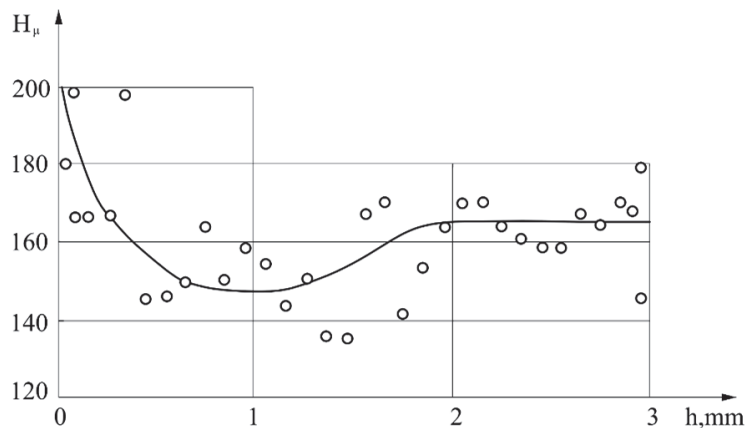

b)

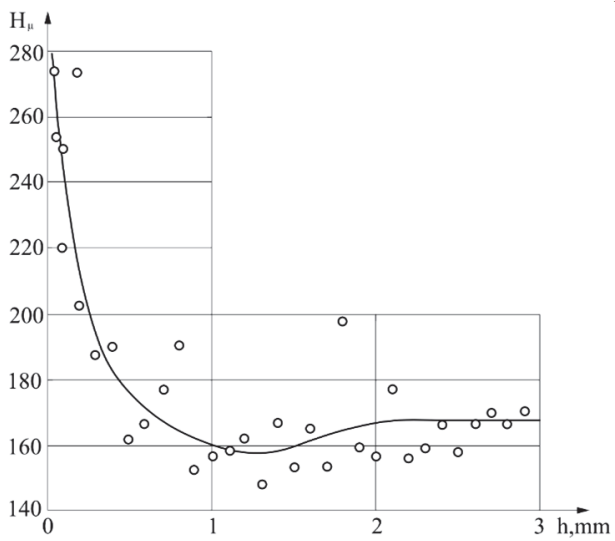

c)

Fig. 3. Distribution of microhardness in the depth of the hardened layer: a) Armco iron, $r_{p}=2.5 \mathrm{~mm}$, $P=1.5 \mathrm{kN}$, b) 45 grade steel, $\left.r_{p}=5 \mathrm{~mm}, P=5 \mathrm{kN}, \mathrm{c}\right) 40 \mathrm{X}$ grade steel, $r_{p}=2.5 \mathrm{~mm}, P=5.5 \mathrm{kN}$

In the graphs of Fig. 3(b), (c) for the specimens of 45 grade and 40X grade steel, a decrease in microhardness $H_{\mu(t . z .)}$ in the transition zone between the hardened layer and the initial metal with microhardness $H_{\mu(i .)}$ was found. The average values of $H_{\mu(t . z .)}$ and $H_{\mu(i .)}$ of normally distributed values were compared with the help of $t$, the Student criterion.

For this purpose, the reduced variance was determined:

$$
\begin{aligned}
& S^{2}=\frac{\left(n_{1}-1\right) S_{1}^{2}+\left(n_{2}-1\right) S_{2}^{2}}{t^{2}+n_{2}-2}, \\
& S \sqrt{\frac{1}{n_{1}}+\frac{1}{x_{2}}}
\end{aligned}
$$


where $n_{1}, n_{2}$ is the number of measurements of $H_{\mu}$ in the transition zone and in the initial metal, respectively; $S_{1}^{2}, S_{2}^{2}$ is dispersion of $H_{\mu(t . z .)}$ and $H_{\mu(i .)}$ values, respectively; $\overline{x_{1}}, \overline{x_{2}}$ is the mean value of $H_{\mu(t . z .)}$ and $H_{\mu(i .)}$, respectively.

If $|t| \geq t_{\alpha}, K$, then difference between mean values is significant. The value of the confidence probability was taken equal to $0.95,(\alpha=0,05)$, the number of degrees of freedom was determined from the expression $K=n_{1}+n_{2}-2$.

It was established that the content of $C_{r}$ and $C$ in the transition zone decreased by $20-30 \%$ and increased to $10-15 \%$ in the hardened layer. In reeling $40 \mathrm{X}$ grade steel and 45 grade steel, a significant reduction of microhardness was observed in the transition zone between the hardened layer and the initial metal. When Armco iron was reeled, such decrease was not detected (Fig. 3(a)). Based on these studies, a hypothesis of diffusion of strengthening chemical elements $(\mathrm{Cr}, \mathrm{C})$ from the intermediate layer to the part surface was advanced. The main mechanism of diffusion during SFW was the gradient of dislocation density.

The method of photoelasticity has shown that during elastoplastic deformations, stresses in the surface of contact of the roller with the part were distributed along a curve close to elliptic, the difference was not more than $7 \%$.

It was established that when slippage was up to $2 \%$, a sharp change in the coefficient of friction could be observed after which it remained practically constant due to the spread of slippage over the entire contact area: an obvious dependence of the value of the maximum friction coefficient on the state of the friction surface. For example, in the process of abrupt change of the coefficient of friction for hardened and not hardened specimens, with lubrication and without it, the zone (crosshatched area) was detected when running-in was achieved faster for the specimens reeled with rollers (Fig. 4(a)). It can be stated that the surface roughness only affects at small slips (up to $3 \%$ ) while reeling with rollers creates specified tribotechnical properties with reduced wear indicators in the surface layer.

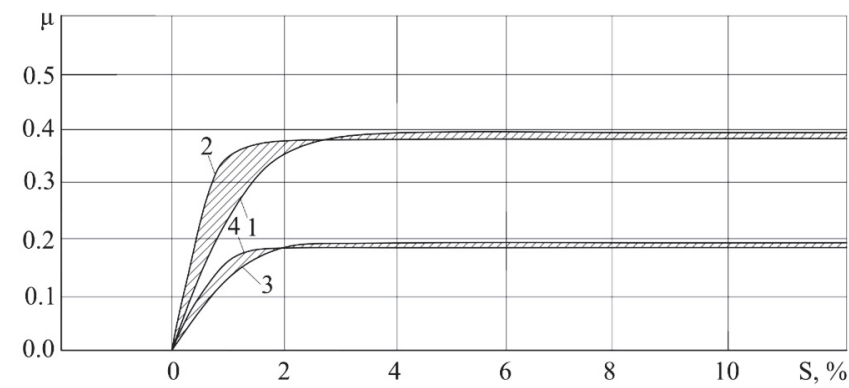

a)

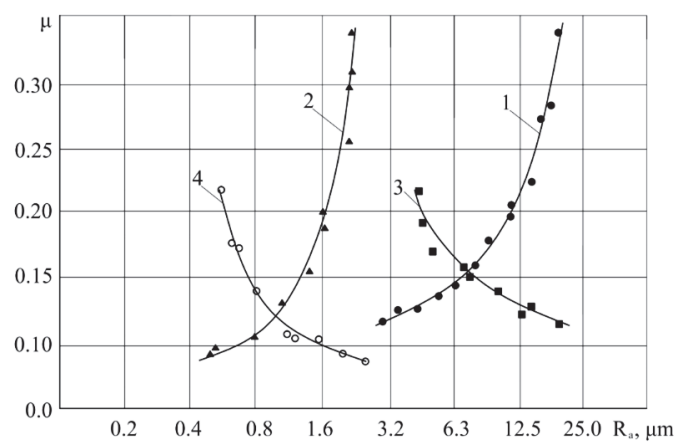

b)

Fig. 4. a) Dependence of the coefficient of friction: on the magnitude of slip, b) on the surface roughness: non-reeled nonlubricated specimen (1); the nonlubricated specimen reeled with a roller (2); the non-reeled lubricated specimen (3); the lubricated specimen reeled with a roller (4) 
Fig. 4(b) shows dependence of the coefficient of friction on the surface roughness for the specimens before reeling and after reeling with a roller and Torsiol-55 lubricant applied. It was established that with a decrease in surface roughness after reeling with rollers, lubricated specimens had a decreased coefficient of friction.

When reeling with slippage, the main mechanisms of wear were oxidizing and fatigue (spalling) wear $[11,12]$. Spalling deformation increased with an increase in slippage if tangential stresses were sufficiently large.

A procedure for determining the force of reeling with a wedge roller was developed. In order to prevent excessive work hardening and peeling of the reeled metal, a limitation for the reeling force at an average angle of indentation not more than $5^{\circ}$ was introduced. With the help of nomogram, rolling force was determined as dependent on the size of the part and the round roller and the reduced profile radius of the roller were chosen to determine the design parameters. The method of reeling steel parts [13] was developed and a theoretical analysis of geometrical parameters was made for reeling the rope blocks with a wedge roller. For this case, beating, eccentricity and diameter of the roller, its curvature, curvature of the reeled part and normal strengthening between the roller and the part in the deformation zone at all points of the profile were calculated. Calculations have shown that in order to prevent formation of waviness on the reeled surface, the profile radius $r_{p}$ is made of a variable value which ensures constancy of the angle $\varphi=5^{\circ}$ of the roller indentation in all sections of the part profile.

The design of the device for reeling the rope blocks is shown in Fig. 5. It was patented, and invention patents were granted $[13,14]$.

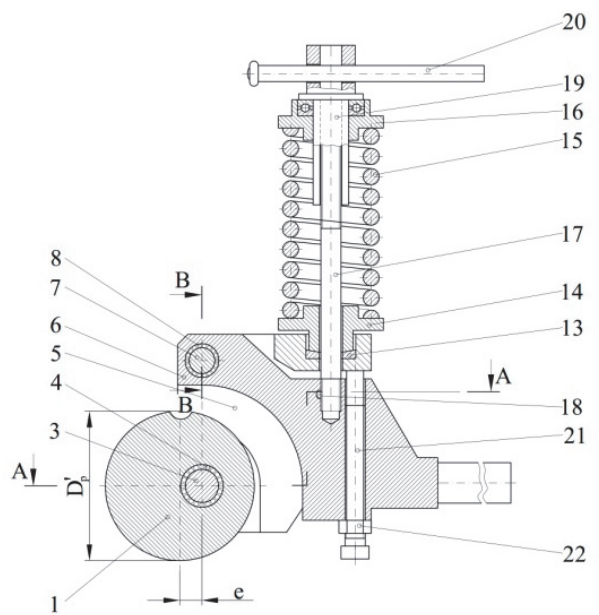

a)

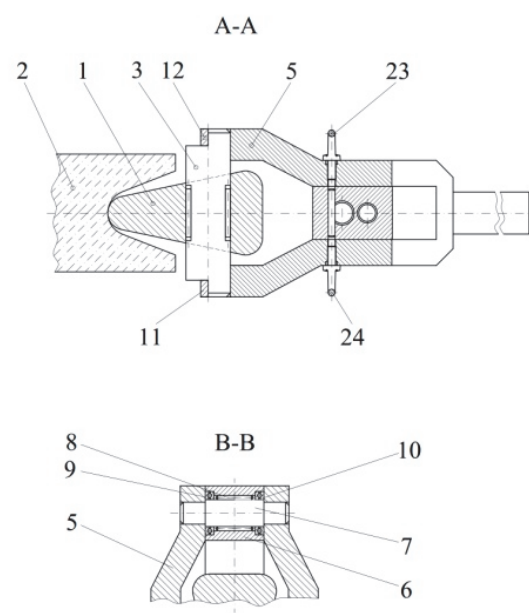

b)

Fig. 5. The device for reeling rope blocks with a wedge roller: roller (1); reeled part $(2)$; shaft $(3,7)$; bearings $(4,8,9,10)$; lever $(5)$; bracket $(6)$; plates $(11,12)$; spherical washer $(13)$; sleeve $(14)$; spring $(15)$; sleeve (16); pulling rod (17); pin (18); nuts $(19,22)$; handle $(20)$; screw $(21)$; eye bolts $(23,24)$

The developed device and technology of reeling [14] the rope blocks by a wedge roller provide low roughness and a high degree of surface work hardening. This effect is achieved by maintaining constant the average angle $\varphi$ of the roller indentation into the surface to be worked and mounting of the roller unit on the rolling bearings. This contributes to a uniform deformation of the surface layer in absence of waviness and leads to an increase in durability of the workpiece. In the course of the experimental study, estimation of dependence of performance of the reeling process on the angle of indentation of the roller, profile radius of the roller, reeling speed and the number of revolutions of the rope block was made.

When plotting the diagram, factors were applied on the abscissa axis in an order of decreasing their rank and the sum of the ranks for the corresponding factor was applied on the ordinate axis. 
With the help of the obtained diagram, an assessment of significance of the factors was made. To determine the factors that do not influence the technological process, the Student criterion $(t$ criterion) was used. After analysis and rejection of the mentioned minor factors, a classical diagram of the ranks with a decrease in their magnitude depending on the influence of the factor on the quality of realization of the technological process was constructed. The check of significance of the expert opinions consisted in election of 10 experts, with the number of factors in the matrix being 12 and the number of degrees of freedom $f=11$.

Analysis of expert opinions ("psychological experiment") and statistical processing have allowed us to conclude on the impact on the course and quality of implementation of the technological process. The four factors had the most affect: roller profile radius $X_{1}, \mathrm{~mm}$; speed of reeling $X_{2}, \mathrm{~m} / \mathrm{min}$; angle of the roller indentation $X_{3}$, degrees; number of the rope block revolutions $X_{4}$, rev. The three-level, four-factor Box plan of the second order was used.

After statistical processing of the experimental data on a PC with the help of Statistica and Excel programs, mathematical models were obtained. These models were obtained for surface roughness (SR) and hardening degree (HD) which describe the technological process of reeling the rope blocks using a wedge roller device. The regression equations take the form:

$$
\begin{aligned}
& S R=1,9224-0,2789 \cdot X_{1}+0,2520 \cdot X_{2}-0,5837 \cdot X_{3}-0,4970 \cdot X_{4} \\
& \quad-0,014 \cdot X_{1} \cdot X_{2}+0,280 \cdot X_{1} \cdot X_{3}-0,002 \cdot X_{1} \cdot X_{4}-0,154 \cdot X_{2} \cdot X_{3} \\
& \quad-0,522 \cdot X_{2}^{2}+1,405 \cdot X_{3}^{2}-1,280 \cdot X_{4}^{2} \\
& H D=47,5008-0,2578 \cdot X_{1}-0,7167 \cdot X_{2}+0,135 \cdot X_{3}+0,1157 \cdot X_{4}-0,127 \cdot X_{1} \cdot X_{2} \\
& \quad+0,236 \cdot X_{1} \cdot X_{3}-0,535 \cdot X_{1} \cdot X_{4}-0,124 \cdot X_{2} \cdot X_{3}+0,115 \cdot X_{2} \cdot X_{4}-1,062 \cdot X_{3} \cdot X_{4} \\
& \quad-0,483 \cdot X_{1}^{2}-0,106 \cdot X_{2}^{2}-0,925 \cdot X_{3}^{2}+0,931 \cdot X_{4}^{2} .
\end{aligned}
$$

After statistical processing, analysis of the obtained regression equations was made with encoded values of factors (Table 1). Study of optimization criteria depending on the change of independent factors was carried out using the method of two-dimensional sections.

Table 1. Data of encoding the test results

\begin{tabular}{|c|c|c|c|}
\hline Indicator & -1 & 0 & 1 \\
\hline$X_{1}-$ The roller profile radius, mm & 10 & 15 & 20 \\
\hline$X_{2}-$ Speed of reeling, m/min. & 20 & 50 & 80 \\
\hline$X_{3}-$ Angle of roller indentation, deg. & 2.5 & 5 & 7.5 \\
\hline$X_{4}-$ Number of the rope block revolutions, rev. & 100 & 200 & 300 \\
\hline
\end{tabular}

The mathematical models were checked for the rope blocks made of steel. When analyzing the values of coefficients at factors in the regression Eq. (5), a conclusion can be drawn that the process quality is most influenced by the angle of roller indentation $\left(X_{3}\right)$ and the number of revolutions of the rope block $\left(X_{4}\right)$. Similarly, it can be seen from Eq. (6) that the most important are the roller profile radius $\left(X_{1}\right)$ and the speed of reeling $\left(X_{2}\right)$. For a more thorough study of the process, an analysis of possible combinations of pairwise combination of factors was made.

Two factors were equalized in turn to zero leaving the other two unequal to the target value. Regression equation for surface roughness and the degree of hardening at possible combinations of factors was obtained.

Equating to zero the value of the angle of roller indentation $\left(X_{3}\right)$ and the number of revolutions of the rope block $\left(X_{4}\right)$, regression equations were obtained in the form:

$$
\begin{aligned}
& S R=1,9224-0,2789 \cdot X_{1}+0,2520 \cdot X_{2}-0,014 \cdot X_{1} \cdot X_{2}-0,094 \cdot X_{1}^{2}-0,522 \cdot X_{2}^{2} \\
& H D=47,5008-0,2578 \cdot X_{1}-0,7167 \cdot X_{2}-0,127 \cdot X_{1} \cdot X_{2}-0,483 \cdot X_{1}^{2}-0,106 \cdot X_{2}^{2} .
\end{aligned}
$$

Let us take partial $X_{1}$ and $X_{2}$ derivatives and obtain a system of equations for each of the optimization criteria: 


$$
\begin{aligned}
& \left\{\begin{array}{l}
\frac{\partial S R}{\partial X_{1}}=-0,0144 \cdot X_{2}-0,1886 \cdot X_{1}-0,2789=0, \\
\frac{\partial S R}{\partial X_{2}}=-1,0452 \cdot X_{2}-0,01440 \cdot X_{1}+0,25204=0,
\end{array}\right. \\
& \left\{\begin{array}{l}
\frac{\partial H D}{\partial X_{1}}=-0,12771 \cdot X_{2}-0,96658 \cdot X_{1}-0,25778=0, \\
\frac{\partial H D}{\partial X_{2}}=-0,21326 \cdot X_{2}-0,12771 \cdot X_{1}-0,71667=0 .
\end{array}\right.
\end{aligned}
$$

After solving the system of equations by each of the mathematical models, coordinates of the centers of surface response were determined for each of the optimization criteria and the value of the target function in the found YS center.

The angle of rotation of the axes in the origin of coordinates of the mathematical model was determined in a canonical form by formula:

$\operatorname{arctg} 2 \alpha=\frac{B_{12}}{B_{11}-B_{22}}$

Coordinates of response of the surface centers were calculated: for the surface roughness: $X_{1}=-1.49 ; X_{2}=0.26 ; \alpha=-0.96^{\circ} ; Y S=2.16$; for the degree of hardening: $X_{1}=0.19$; $\mathrm{X}_{2}=-3.47 ; \alpha=9.36^{\circ} ; Y S=48.72$.

Coefficients of the regression equations of the characteristic equations for each of the optimization criteria were determined in a canonical form:

$f(\lambda)=\left|\begin{array}{cc}B_{11}-\lambda & B_{12} / 2 \\ B_{21} / 2 & B_{22}-\lambda\end{array}\right|=0$,

to that end, the equations were reduced to the form:

$\lambda^{2}-I \cdot \lambda+D=0$

Roots of this equation are the coefficients of a mathematical model in a canonical form. After the calculations, the regression equations were obtained in a canonical form:

for the surface roughness:

$S R-2,164=-0,094 \cdot X_{1}^{2}-0,522 \cdot X_{2}^{2}$,

For the degree of hardening:

$H D-48,7=-0,096 \cdot X_{1}^{2}-0,493 \cdot X_{2}^{2}$.

The results obtained by combining the $X_{1}$ and $X_{2}$ factors are shown in Fig. 6 .

Consideration of the constructed diagrams makes it possible to draw a conclusion that the zone of optimal combination of factors is constrained by SR and HD curves at A, B, C, F, G points. In this case, the surface roughness will lie within $1.2 \mu \mathrm{m}<S R<1.4 \mu \mathrm{m}$, and the degree of hardening $44 \%<H D<45 \%$.

With such indicators of the optimization criteria, the value of the roller profile radius should be $16, \ldots, 20 \mathrm{~mm}$ and the speed of reeling $27, \ldots, 36 \mathrm{~m} \mathrm{~min}$.

Consecutively changing the combination of factors, two-dimensional sections of the response surfaces were obtained for all possible combinations of factors. 


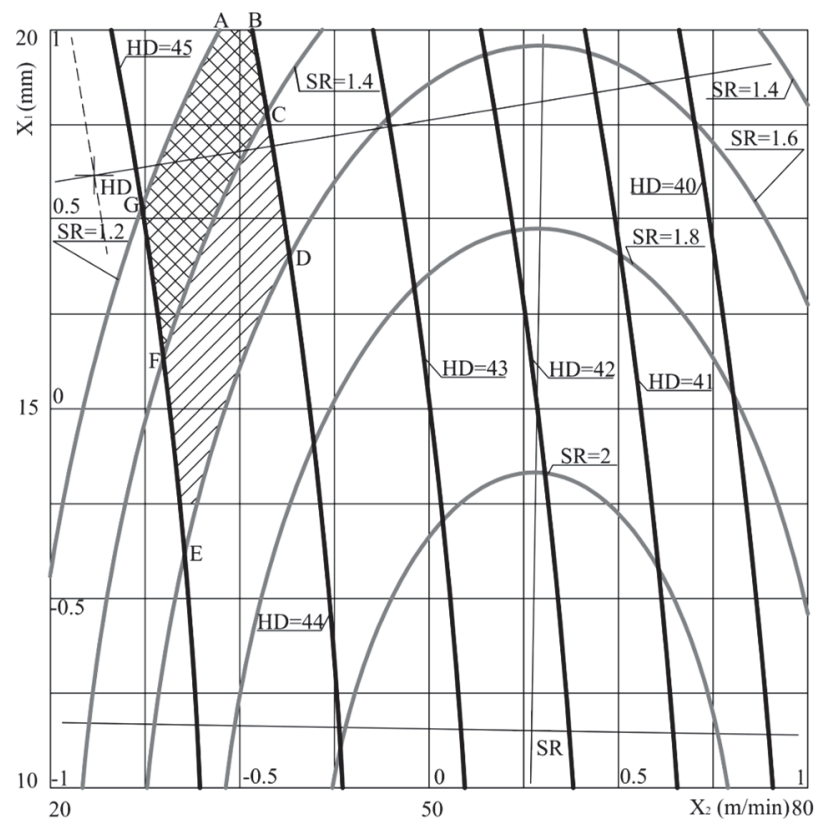

Fig. 6. Two-dimensional cross sections of the response surface after combination of the $X_{1}$ and $X_{2}$ factors at $X_{3}=0$ and $X_{4}=0$

For example, by combining the values of the angle of indentation $\left(X_{3}\right)$ and the number of revolutions of the rope block $\left(X_{4}\right)$ at $X_{1}=0$ (the roller profile radius) and $X_{2}=0$ (speed of reeling), the following regression equations were obtained:

$S R=1,9224-0,5837 \cdot X_{3}-0,4970 \cdot X_{4}+0,359 \cdot X_{3} \cdot X_{4}+1,405 \cdot X_{3}^{2}-1,280 \cdot X_{4}^{2}$
$H D=47,5008+0,135 \cdot X_{3}+0,1157 \cdot X_{4}-1,062 \cdot X_{3} \cdot X_{4}-0,925 \cdot X_{3}^{2}+0,931 \cdot X_{4}^{2}$.

Solution to the system of equations has produced coordinates of the centers of the response surfaces:

- for the surface roughness: $X_{3}=0.22 ; X_{4}=-0.16 ; \alpha=3.81^{\circ} ; Y S=1.89$;

- for the degree of hardening: $X_{3}=0.08 ; X_{4}=-0.01 ; \alpha=14.8^{\circ} ; Y S=47.50$.

Fig. 7 shows results obtained for Eqs. (16) and (17) from which it is evident that the zones of the optimal combination of factors are constrained by the SR and HD curves in A, B, C and C, D, E points. In this case, surface roughness in both zones is about $1 \mu \mathrm{m}$, the degree of hardening is $47 \%$ at the roll indentation angle of $4^{\circ}, \ldots, 7^{\circ}$ and the number of the rope block revolutions of $265, \ldots, 300 \mathrm{rev}$.

Significance of the obtained estimates of coefficients and the adequacy of the model was checked using the Cochran's G-criterion and the Fisher's F-criterion.

When optimizing the technological process of reeling the rope block with a wedge roller, optimum working conditions were obtained by planning the experiment. They have appeared to be the following: the roll profile radius $\left(X_{1}\right) 15 \mathrm{~mm}$, the reeling speed $\left(X_{2}\right) 40-50 \mathrm{~m} / \mathrm{min}$, the roll indentation angle $\left(X_{3}\right) 5$ degrees, the number of the rope block revolutions $\left(X_{4}\right)$ 160-180 rev. (Figs. 6, 7).

Their optimal combination forms quality of the technological process of steel part surface hardening by reeling with rollers at indicators of surface roughness (SR) of $1, \ldots, 1.9 \mu \mathrm{m}$ and the hardening degree (HD) of $46.5, \ldots, 56 \%$.

Data on wear are given in Table 2. 


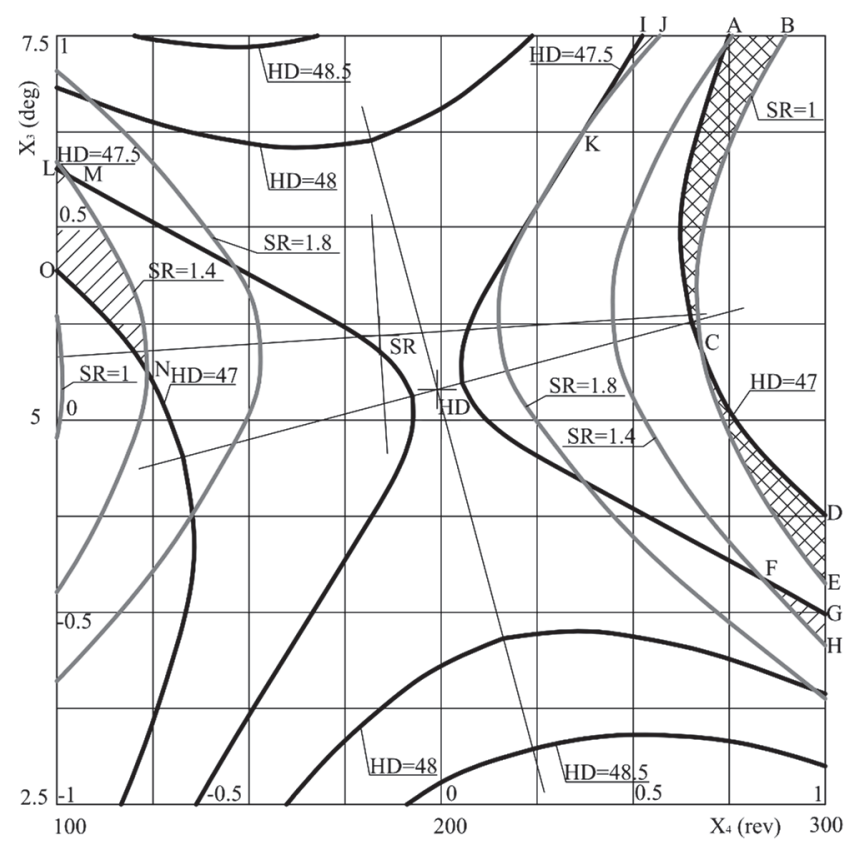

Fig. 7. Two-dimensional cross sections of the response surfaces for combining $X_{3}$ and $X_{4}$ factors at $X_{1}=0, X_{2}=0$

Table 2. Spalling the rope block working surface (800/120) of the KRUPP ship reloader

\begin{tabular}{|c|c|c|c|}
\hline Wearing part & Wear, $\mathrm{mm}$ & Operation period, mos. & Average wear, $\mathrm{mm} / \mathrm{mo}$ \\
\hline \multirow{2}{*}{ Non-reeled block } & 4.3 & 4 & 1.0 \\
\cline { 2 - 4 } & 3.8 & 3 & 1.26 \\
\hline \multirow{2}{*}{ Reeled block } & 3.9 & 16 & 0.24 \\
\cline { 2 - 4 } & 3.2 & 12 & 0.26 \\
\hline
\end{tabular}

It has been shown that reeling of the rope block working profile with rollers increases the contact strength and therefore imparts a 3 to 4 times higher durability. Accordingly, durability of ropes also improves as bending of the rope wires on irregularities of the worn rope block is eliminated.

The procedure of the rope block reeling to increase wear resistance and contact strength has been implemented on Krupp ship reloaders (Germany) in the cargo port of Mykolayiv Alumina Plant OJSC (Ukraine) and the auto cranes KS-3575 built by Mykolayivbudmechanizatsiya JSC (Ukraine).

\section{Discussion of results of tribological studies and process conditions of reeling steel parts with rolls}

Yield strength of the work hardened layer increases to a greater extent than hardness (100-130\% versus 20-60\%). Due to this, boundary of the deformed layer is more clearly found by the change of the yield strength. Use of cylindrical needle rollers of small diameter in the reeling process results in a sharp increase in the degree of deformation in a thin surface layer which is recorded on optical microphotographs as grain elongation in the direction of reeling.

Accuracy of determining boundary of the work hardened layer by the method of regression analysis using the results of measuring the yield strength is two times higher than in the case of using the results of Vickers hardness tests. The $95 \%$ confidence intervals for the depth of work hardening with toroidal and cylindrical rollers calculated on the basis of the results of measuring 
the yield strength make up 11-36\% of the depth of work hardening and the corresponding figures for hardness tests are $32-75 \%$.

Strengthening of the surface layers by reeling the shafts with a roller is mainly due to the emergence of dislocation cells in the structure of grains of excess ferrite. Ferrite plates of perlite show smaller deformation. Deformations of cementite plates during reeling with toroidal rollers were not detected.

An increase in the degree of work hardening by reeling with a needle roller manifests itself in a higher density of dislocations and the reduced cell size in the substructure of ferrite grains, as well as in a thicker grid of dislocations in ferrite plates of perlite. In some locations, bending and fracture of cementite plates occur indicating the extreme degree of plastic deformation of the surface layer. This is confirmed by the onset of peeling on the surface reeled with a $5 \mathrm{~mm}$ roller.

Diffusion of chemical elements of the surface layer in the process of surface deformation was studied. It was established that the content of $\mathrm{Cr}$ and $\mathrm{C}$ in the transition zone was 20-30\% lower and $10-15 \%$ higher in the hardened layer. Based on these studies, a hypothesis of diffusion of strengthening chemical elements $(\mathrm{Cr}, \mathrm{C})$ from the intermediate layer to the surface of the part was put forward. The main mechanism of diffusion during SFW is the dislocation density gradient.

When reeling proceeds with slippage, the main mechanisms of wear are oxidizing and fatigue (spalling) wear. Spalling deformation increases with the growth of slippage if tangential stresses are sufficiently high.

A procedure for determining conditions of reeling the rope blocks with a wedge roller was developed. In order to prevent excessive hardening and scaling of the reeled metal, a limitation to the reeling force with an average angle of indentation not more than $5^{\circ}$ was introduced.

The method of reeling the rope blocks and other steel parts of rotation having a complex profile was developed to improve wear resistance and contact strength [11]. Advantage of the wedge roller consists in equilibrium of the axial component of the reeling force and reeling of the working profile of the rope block is possible on horizontal and vertical lathes in a single positioning.

The device and technology of the rope block reeling with a wedge roller have been developed to provide low roughness and high degree of work hardening of the surface [12]. A set of laboratory devices was developed to determine roughness of the reeled surface by means of replicas and the degree of work hardening of the working surface. This will assist in studying physical and mechanical properties of steel parts of a complex shape working under a contact loading, including the rope blocks. This ensures obtaining of correct indicators of the technological process of work hardening using the designed device with a wedge roller.

Tribotechnical characteristics of the reeled rope blocks were defined with the help of a device with a wedge roller: the angle of indentation and the profile radius of the wedge roller, the number of revolutions of the steel rope block and speed of reeling. This has made it possible to ascertain possible limits of variation of main design and technological conditions of strengthening with the proposed device and elucidate shape of the wedge roller.

As a result of experimental study with the use of the method of steep convergence, optimal design and kinematic parameters of the reeling process were determined. Optimum working conditions were obtained by planning the experiment with optimization of the technological process of reeling the rope block with a wedge roller. They are the roller indentation angle: $5^{\circ}$, the roller profile radius: $15 \mathrm{~mm}$, the number of the rope block revolutions: $160-180 \mathrm{rev}$., the speed of reeling: $40-50 \mathrm{~m} / \mathrm{min}$.

The optimum combination forms quality of the technological process of surface hardening of the rope blocks by reeling with rollers with the following indicators: surface roughness of $1, \ldots, 1.9 \mu \mathrm{m}$ and hardening degree of $46.5, \ldots, 56 \%$.

The conducted experimental studies have proved adequacy of the results obtained in physical and mathematical modeling of the tribotechnical processes occurring during reeling the working friction surface of the rope block with a wedge roller. This allows us to recommend the developed mathematical models for their application in hardening steel parts working in conditions of wear 
and action of contact forces in the mechanical engineering and other industry sectors.

It has been shown that reeling of the working profile of the rope blocks ensure a 3-4 times higher durability and contact strength and therefore service life. Accordingly, service life of ropes improves as well due to elimination of the rope wire bending over the surface irregularities of the worn rope block.

The proposed hardening technology can be used for other large-sized steel parts working in wear conditions in various industries. Tribological studies of the "rope block - rope" friction couple during reeling taking into account slippage do not provide absolute correspondence with the actual course of the wear process but is a necessary step in the development of computational engineering methods for predicting wear resistance of friction units which will facilitate development of techniques to improve their service life. Further studies in this direction should be conducted by dissemination of the proposed technology to other machinery friction units and their corresponding laboratory wear test patterns.

\section{Conclusions}

1. With application of the regression analysis methods, the actual accuracy of determining the depth of hardening has been estimated using the data on changes in mechanical properties of the surface layer metal. It was established that accuracy of determining the boundary of the work hardened layer according to the yield strength change is twice as high as that according to the hardness change. It was shown that the depth of work hardening which is determined according to the changes in the yield strength is $25-50 \%$ greater than the depth determined according to the Vickers hardness change. This difference grows with the decrease in the work hardening degree.

2. Diffusion of strengthening chemical elements taking place in the surface layer during the process of surface deformation was studied. It was established that the content of $\mathrm{Cr}$ and $\mathrm{C}$ decreased by $20-30 \%$ in the transition zone and increased to $10-15 \%$ in the hardened layer. When reeling steel of $40 \mathrm{X}$ and 45 grades with rollers, a significant reduction of microhardness in the transition zone between the hardened layer and the initial metal was observed. When reeling Armco iron, this reduction cession was not detected. Based on these studies, a hypothesis of diffusion of the strengthening chemical elements $C_{r}$ and $C$ from the intermediate layer to the surface of the part due to the dislocation density gradient has been advanced.

3. A procedure for calculating the depth of work hardening for the case of reeling the part friction surface with a wedge roller was proposed and the limits of its rational application were set. An original technique of reeling steel parts was developed in order to increase their contact strength and wear resistance. This technique makes it possible to intensify the process and optimize conditions of plastic deformation, increase productivity of the reeling process, and achieve specified tribotechnical properties of the friction surfaces in large-sized steel parts.

\section{References}

[1] Babei Y. I., Butakov B. I., Sysoev V. G. Analysis of piezomotor driver for laser beam deflection. Journal of Vibroengineering, Vol. 11, Issue 1, 2009, p. 17-26.

[2] Kindrachuk M. V., Kornienko A. O., Fedorchuk S. V., Tisov O. V. Stress-deformed state of composite material loaded with friction and temperature. Problems of Tribology, Vol. 1, 2006, p. $153-157$.

[3] Aulin V., Arifa W., Lysenko S., Kuzyk A. Improving of the wear resistance of working parts agricultural machinery by the implementation of the effect of self-sharpening. International Journal of Engineering and Technology (UAE), Vol. 5, Issue 4, 2016, p. 126-130.

[4] Kuzmenko A. G., Dykha A. V. Contact, Friction and Wear of Oiled Surfaces. KhNU, Khmelnitsky, 2007, p. 344.

[5] Braude B. I., Gohberg M. M., Zvyagin I. E. Handbook for Cranes. Mechanical Engineering. Characteristics of Materials and Loads. Basics of Calculation of Cranes, Their Drives and Metal Constructions, 1988, p. 536. 
[6] Chernets M. V. Prediction of the life of a sliding bearing based on a cumulative wear model taking into account the lobing of the shaft contour. Journal of Friction and Wear, Vol. 36, Issue 2, 2015, p. $163-169$.

[7] Soldatenkov I. A. Evolution of contact pressure during wear of the coating in a thrust sliding bearing. Journal of Friction and Wear, Vol. 31, Issue 2, 2010, p. 102-106.

[8] Togawa K., Arai S., Uwatoko M. Influence of traction sheave P.C.D. difference on sheave and rope. Journal of the Proceedings of the Elevator, Escalator and Amusement Rides Conference, 2012, p. 31-34.

[9] Ryu J. B., Chae Y. H., Kim S. S. A fundamental study of the tribological characteristics of sheave steel against a wire rope. Journal of the Key Engineering Materials, Vol. 297-300, 2005, p. 1382-1387.

[10] Dykha A. V., Kuzmenko A. G. Solution to the problem of contact wear for four-ball wear-testing scheme. Journal of Friction and Wear, Vol. 36, Issue 2, 2015, p. 138-143.

[11] Dykha A., Aulin V., Makovkin O., Posonskiy S. Determining the characteristics of viscous friction in the sliding supports using the method of pendulum. Eastern-European Journal of Enterprise Technologies, Vol. 7, Issue 87, 2017, p. 4-10.

[12] Butakov B. I., Shebanin V. S., Butakova G. S., Marchenko D. D. A Method of Finishing and Strengthening the Processing of Surfaces of the Bodies of Rotation of a Complex Profile and a Device for Its Implementation. Patent No. 93252, Ukraine, Bulletin No. 13, 2010.

[13] Butakov B. I., Shebanin V. S., Butakova G. S., Marchenko D. D. A Device for Finishing and Strengthening the Surfaces of Bodies of Rotation of a Complex Profile. Patent No. 2493954 Russian Federation, Bulletin No. 27, 2013.

[14] Marchenko D. D., Butakov B. I. Promoting contact strength of steel by rolling. Journal of Friction and Wear, Vol. 34, Issue 4, 2013, p. 308-316.

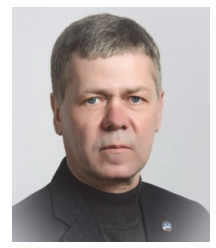

Aleksandr Dykha received Doctor of Science degree in Khmelnytsky National University, Ukraine, in 2009. Graduated from the Kyiv Polytechnic Institute in 1989 with a degree in mechanical engineering. Now he works at Khmelnytsky National University, head of the department of wear and reliability. He is editor-in-chief of international scientific journal "Problems of tribology". His current research interests include modeling of friction and wear of machines, calculation models of reliability.

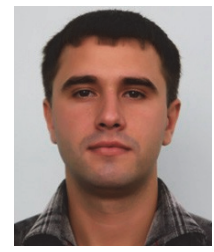

Marchenko Dmitry received Ph.D. degree in Khmelnytsky National University, Ukraine, in 2014. Graduated from the Mykolaiv National Agrarian University in 2007 with a degree in mechanization of agricultural production and hydro-melioration works. Now he works at Mykolaiv National Agrarian University, department of tractors and agricultural machinery, operation and maintenance, associate professor. His current research interests include technologies of surface hardening of parts of agricultural machines 


\title{
3.2. Problems of high-rate deformation of elements of modern technology under the influence of impact and impulse loads
}

\author{
Yurii Vorobiov ${ }^{1}$, Nataliia Ovcharova ${ }^{2}$ \\ A. Podgorny Institute for Mechanical Engineering Problems National Academy of Sciences of Ukraine, \\ Kharkov, Ukraine \\ E-mail: ${ }^{1}$ vorobiev@ipmach.kharkov.ua, ${ }^{2}$ Natka.Ovch@gmail.com
}

\begin{abstract}
The high-rate elastic-plastic deformation of the elements of modern technology is considered, it is taking into account the change of the properties of materials in the process of deformation under the action of impact and impulse loads. The use of experimental studies to determine the parameters of the dynamic properties of the material is shown. On the basis of three-dimensional models and numerical methods, an analysis of the stress-strain state of protective elements for vehicles and gas turbine engines corps under impact loads was carried out. It is shown that multilayered protective elements more effectively withstand impact loads. The analysis of parameters of technological processes of connection and separation of elements due to explosion energy is carried out. Calculation-experimental methods for analysis of deformation of cylindrical elements under impact loads are considered. The results of the research are used to ensure the dynamic strength of elements of real structures.
\end{abstract}

Keywords: shock, protective elements, high-rate deformation, dynamic material properties, experiment, three-dimensional models, numerical methods, technological processes, calculation and experimental methods.

\section{Introduction}

Many objects of modern technology are exposed to shock and impulse loads. Therefore, protective elements play an important role for energy, transport, aerospace and military systems. Like a protective element can be used the cladding elements of vehicles, gas turbine engine corps, protective boxes, process chambers, protective elements of military facilities, personal protective equipment, etc. Under the influence of local shock loads, a three-dimensional dynamic stress-strain state arises in them. The processes of dynamic deformation proceed in the elastic-plastic stage. In this case, it is necessary to take into account the dynamic properties of the material, which depend on deformations and strain rates [1-4]. At intense impact loads, finite deformations and displacements is occurring. Therefore, the analysis of dynamic stress-strain state (SSS) is an actual and complex problem. It requires the construction of three-dimensional mathematical models that take into account all the features of the process of high-rate deformation.

Solving the problem of the protection of civil and military installations include experimental studies of dynamic material properties, numerical studies of impact loads on the critical objects of modern technology and the development of measures to reduce the danger of dynamic loads.

\section{Determination of the dynamic properties of materials}

Dependencies for the dynamic properties of the material are built on the basis of experimental data. Experimental studies are carried out on flat and cylindrical samples on special stands in the range of strain rates up $5 \cdot 10$ to $5 \cdot 10^{3} \mathrm{~s}^{-1}$ (Fig. 1). In this case, it is necessary to take into account the variability of the deformation rate and the real dynamic loads that are registered by the inertia-free sensors.

Researches in the field of high-rate deformation of structural elements are impossible without experimental data of dynamic characteristics of materials, which reflect relationships of stresses $\sigma$ and strain $\varepsilon$, and strain rate $\dot{\varepsilon}=\frac{d \varepsilon}{d t}: \sigma=\sigma(\varepsilon, \dot{\varepsilon})$. Beside that complexity of processes of 
high-rate deformation occurring in the elastoplastic range requires periodical experimental check of new theoretical results in this field $[3,5]$.

The experimental base includes several unique installations and measuring devices (Fig. 2).

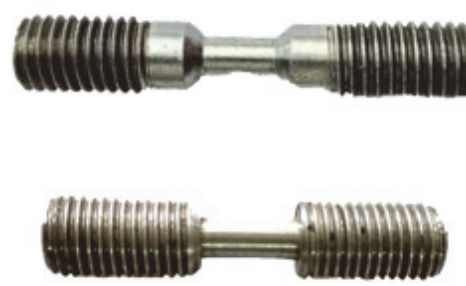

a)

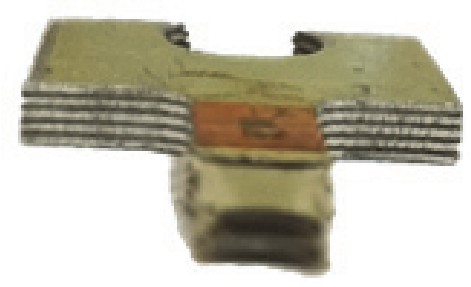

b)

Fig. 1. Samples for testing: a) cylindrical specimens; b) a flat multilayered specimen after failure

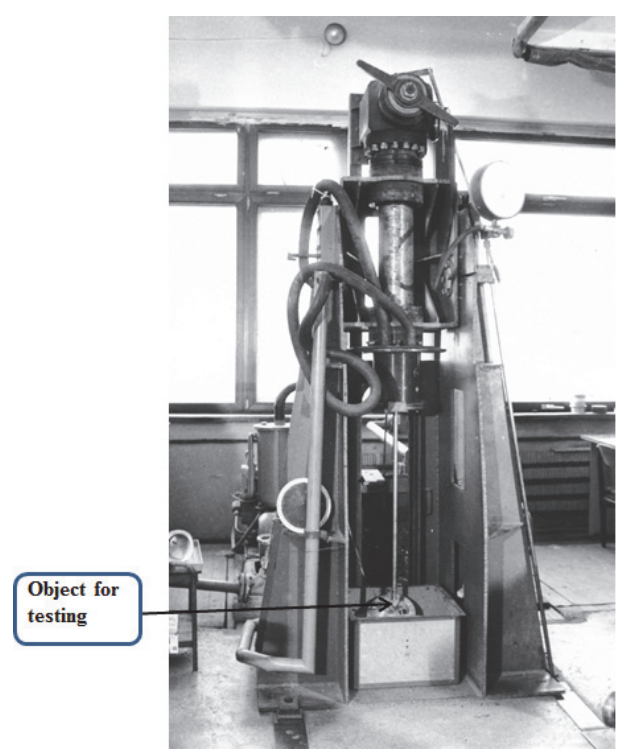

a)

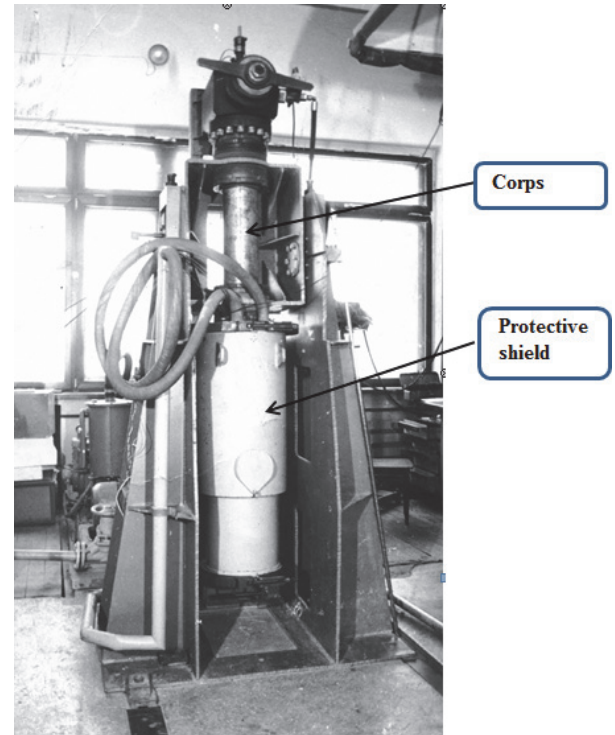

b)

Fig. 2. General view of the overspeed for testing samples for high-rate deformation

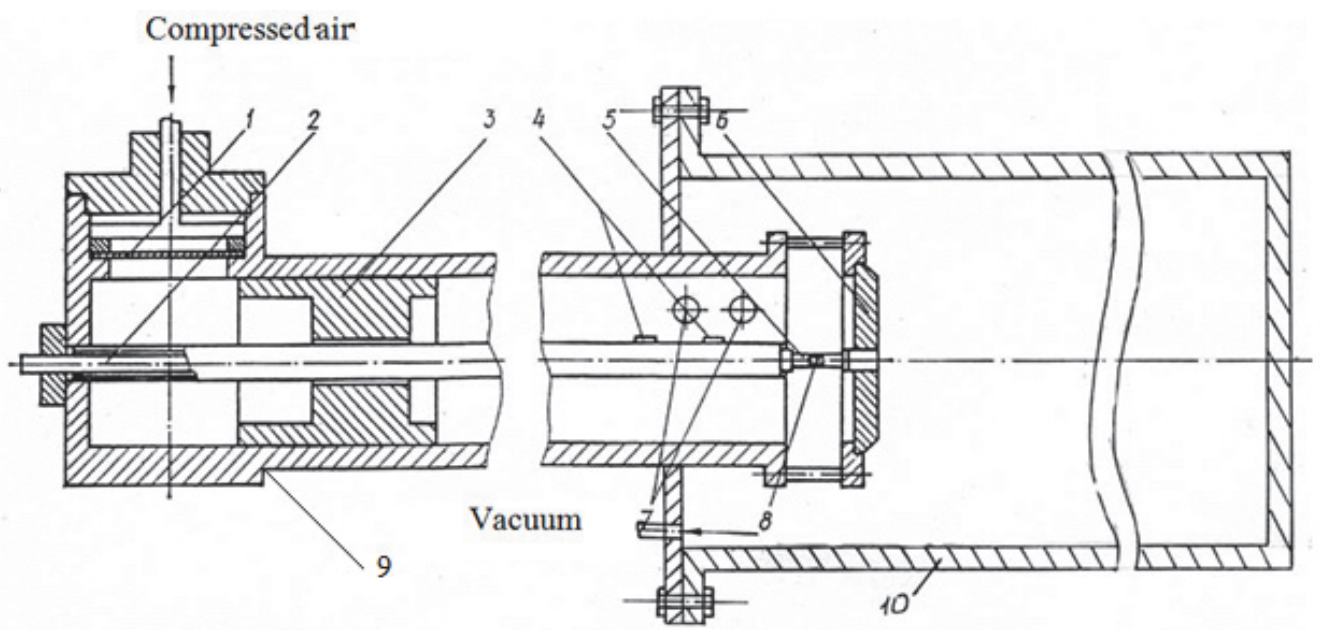

Fig. 3. Installation scheme for testing samples for high-rate deformation 
Strains and strain rates are registered by small-base foil, wire or semiconductor (silicic) strain sensors. They are practically inertialess. Foil sensors operates in the rage of deformations of $\varepsilon=0.02-0.1$ (up to $10 \%$ ) in the rage of temperatures from -200 to $+500{ }^{\circ} \mathrm{C}$. Acceleration is measured by piezoceramic sensors. Contactless vortex sensors are applied for measurement of motions. Sensors are of modulating type and operate in composition of bridges with active and reactive constituents [5].

The linear flat and cylindrical short-base specimens are tested under the conditions of high-rate uniaxial tension by the overspeed installation for determination of dynamic characteristics of material

The installation scheme is shown in Fig. 3.

Compressed air presses on the round membrane (1) with stress concentrator. The plate breaks at the predetermined pressure because of the concentrator and it provides velocities of impactor in the wide range $(10-300 \mathrm{~m} / \mathrm{s})$. Under the action of compressed air hammer (3) moves in the casing (9) of 125-mm diameter till the shock on anvil (6). Specimen (5) clamped in the anvil and rod-dynamometer (2) is loaded when hammer shocks anvil. Simultaneously elastic deformations of dynamometer are registered by strain sensors (4), elastic-plastic deformations of specimen - by foil sensors (8), and hammer's speed - by pair of vortex sensors (7) and contact sensors operating from fracture. Working zone of installation is coved with protective shield (10), in which the vacuum of $10 \mathrm{~Pa}$ is created. Diameter of rod-dynamometer is designed of such a value that it deforms only in the elastic region and gives a force of specimen deformation $N(t)$, from which stress in specimen $\sigma(t)$ is determined. And sensor located on the specimen gives a strain of its working length $\varepsilon(t)$ [5]. Thus, relationship $\sigma=\sigma(\varepsilon, \dot{\varepsilon})$ can be plotted from the results of direct measurements without previous assumptions about the nature of specimen deformation (Fig. 4).

It is often assumed that the rate of deformation of a specimen is constant, on the basis that the kinetic energy of the impactor is an order of magnitude greater than the strain energy of the specimen. However, the process records show that this assumption is valid not at all stages of the deformation of the specimen $[3,5]$. So, in Fig. 4 nonlinear sections are visible, when the sample is deformed. At hammer's speeds provided by compressed air the strain rates of $6 \cdot 10^{2}-6 \cdot 10^{4} \mathrm{~s}^{-1}$ are realized.

$\varepsilon \cdot 10^{6}$ Strain gauge reading at the specimen

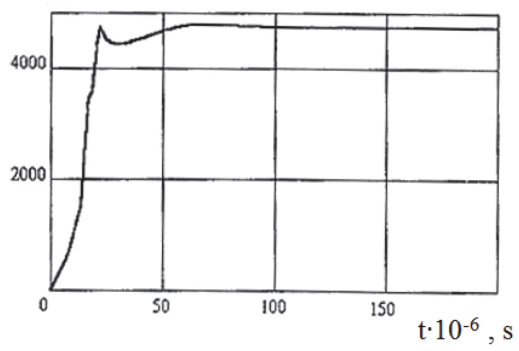

a)

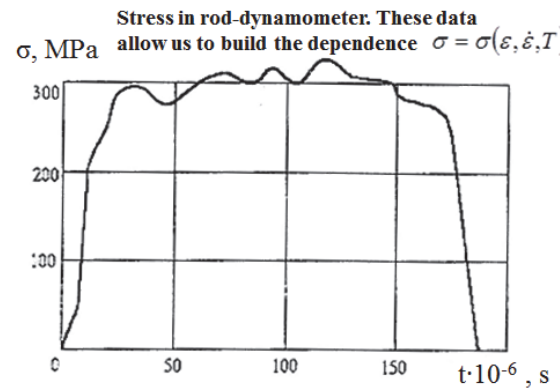

b)

Fig. 4. Changes of the: a) deformation of the specimen, b) stresses in the rod-dynamometer in time

To refine the dynamic characteristics of the material, the change in its cross section during deformation is takes into account (Fig. 4). Large strains (more than $10 \%$ ) when the neck is formed and the fracture of specimen are determined as the difference in the displacements of the two sections, referred to the distance between these sections $\varepsilon \approx \Delta u / \Delta x$.

Based on this measurement results, a spatial dynamic diagram is plotted in coordinates $\sigma, \varepsilon, \dot{\varepsilon}$. (Fig. 5).

It visually demonstrates the way of deformation of the specimen and explains the possibility of large residual deformations $\varepsilon_{f}$ at a high-rate loading of the structure.

It can be seen, that the elastic limit increase with increasing deformation rate (2). 


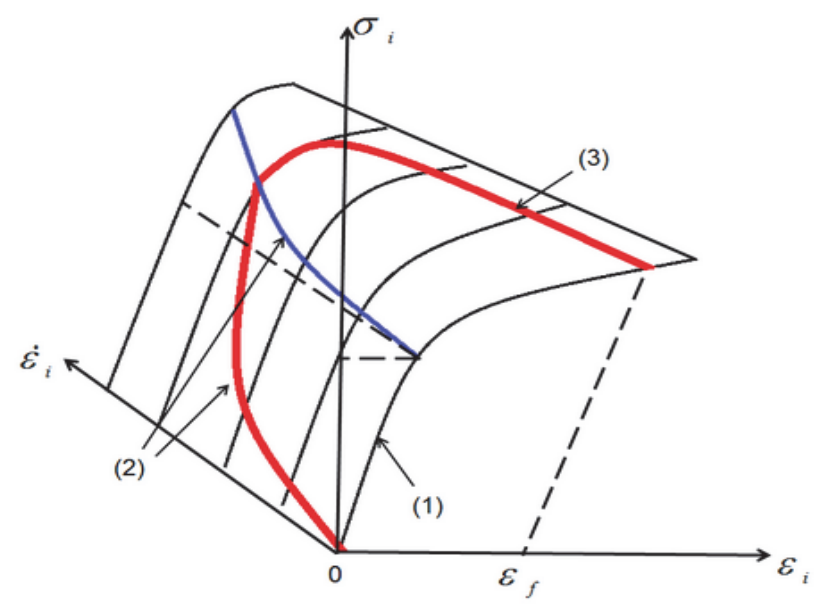

Fig. 5. The relationship between stress intensity, strain and strain rate: static deformation of the sample (1), deformation with variable speed (2), sample unloading (3),

$$
\text { (1) } \dot{\varepsilon}_{i}=0 \text {; (2) } 0 \leq \dot{\varepsilon}_{i} \leq \varepsilon_{S}\left[1+\left(\frac{\dot{\varepsilon}_{i}}{\gamma}\right)^{\frac{1}{n}}\right] \text {; (3) } \Delta \varepsilon_{i} \leq 0
$$

For ease of use in the calculation of the measurement results are processed and presented in the form of a power-law dependence [5]:

$$
\begin{aligned}
& \sigma_{i}=\sigma_{S}\left[1+\left(\frac{\dot{\varepsilon}_{i}}{D}\right)^{\frac{1}{n}}\right]+\sigma_{S}\left\{\frac{\varepsilon_{i}}{\varepsilon_{S}}\left[1+\left(\frac{\dot{\varepsilon}_{i}}{D}\right)^{\frac{1}{n}}\right]\right\}^{m}, \\
& \sigma_{i}=\sigma_{S} \cdot K\left(\dot{\varepsilon}_{i}\right)+\sigma_{S}\left[\frac{\varepsilon_{i}}{\varepsilon_{S}}-K\left(\dot{\varepsilon}_{i}\right)\right]^{m},
\end{aligned}
$$

where $\sigma_{S}, \varepsilon_{S}-$ static yield stresses for stresses and strains, $D, n, m$ - parameters of high-rate material hardening.

Coefficient $K\left(\dot{\varepsilon}_{i}\right)=\left[1+\left(\frac{\dot{\varepsilon}_{i}}{D}\right)^{\frac{1}{n}}\right]$ can be used to determine the dynamic yield stresses, strength, etc. according to their static values $\sigma_{d}=\sigma_{S} \cdot K\left(\dot{\varepsilon}_{i}\right)$.

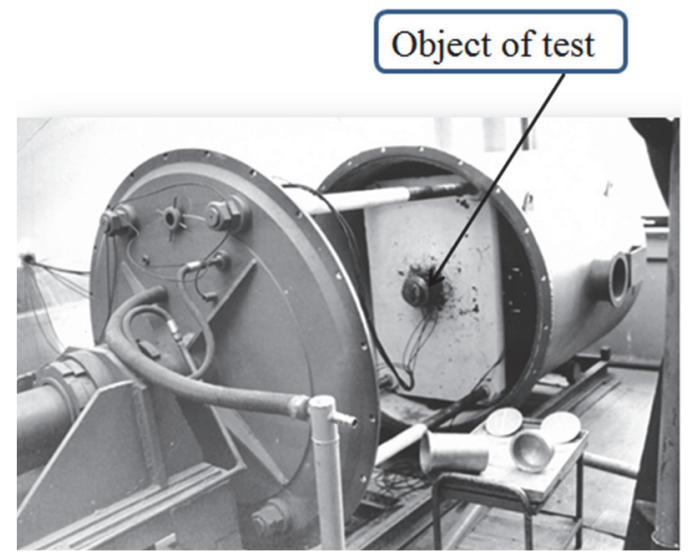

a)

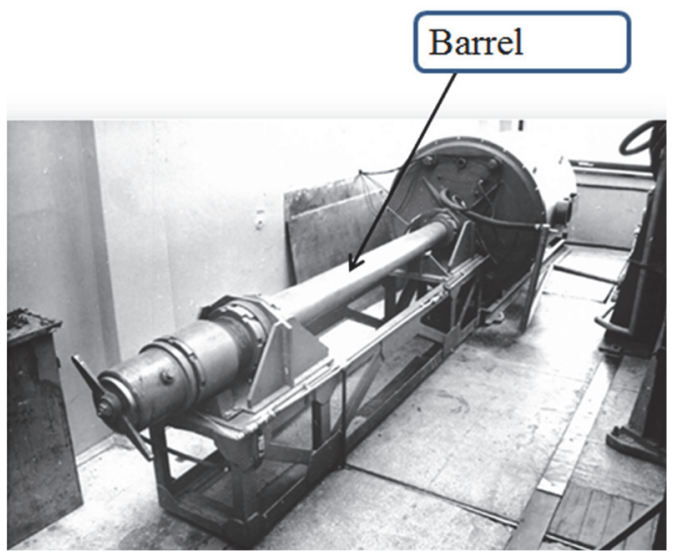

b)

Fig. 6. General view of overspeed installations 
The installation also allows for the connection of the impactor with the deformable structure to perform a shock test, for example, shells with the ground or liquid.

For the experimental analysis of structural elements under the influence of shock loads and verification of the results of numerical analysis of the processes of high-rate deformation, a horizontal shock loading installation is used.

The general view of the overspeed installation of high-rate shock and aerodynamic loading of structural elements is shown in Fig. 6. In the shock loading mode, the impactor moves in the barrel, and in the aerodynamic loading mode, the high-rate flow of air moves in the barrel.

The diagram of the installation for aerodynamic loading is shown in Fig. 7.

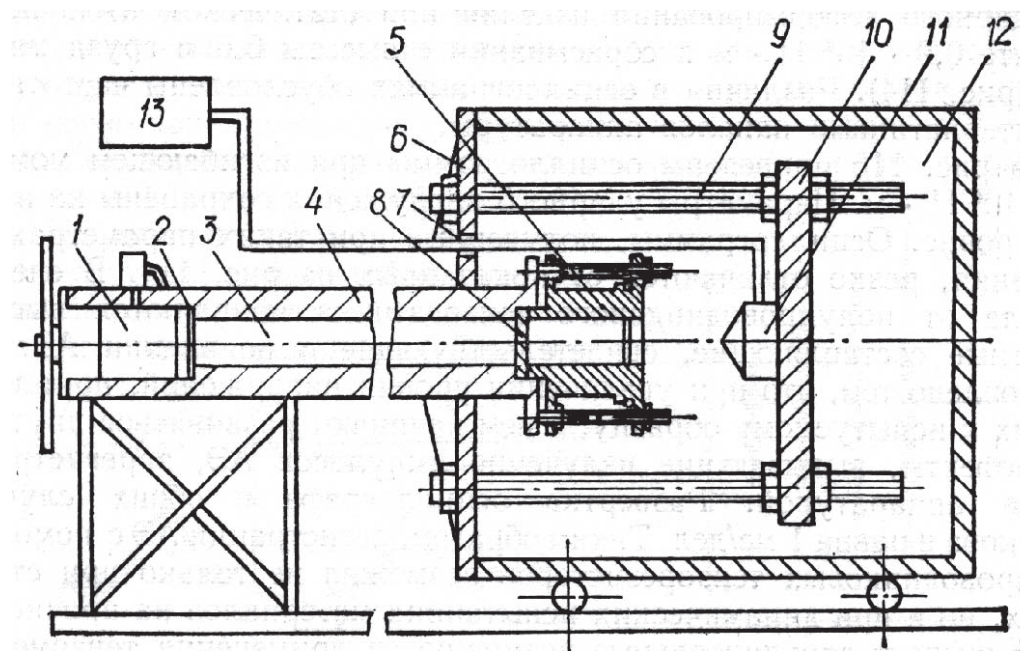

Fig. 7. Installation scheme for the aerodynamic loading regime

In the mode of shock loading the compressed air is fed into high-pressure chamber (1). At the predetermined pressure the diaphragm (2) fractures and hammer moves along the barrel of $125 \mathrm{~mm}$ diameter. Subject of test (11) and mounting plate (10) are located in the expansion chamber (12), connected to the barrel by flange (6). The chamber is of $1.6 \mathrm{~m}$ diameter and $2.2 \mathrm{~m}$ length with wall thickness of $\delta=0.014 \mathrm{~mm}$ allows accommodating testing subjects of sufficient dimensions. Chamber is supplied with vacuum of $10 \mathrm{~Pa}$ and protection of room. Compressed air provides hammer's speed up to $300 \mathrm{~m} / \mathrm{s}$ [5]. The results are recorded in the measuring device (13).

In the mode aerodynamic loading the installation provides two-stage acceleration of flow by means of two diaphragms (2.8). Turbulator may be mounted on the way of aerodynamic flow.

\section{Analysis of high-rate deformation of structural elements under impact.}

Analytical solutions for the analysis of high-rate deformation of protective elements are achieved only in simple special cases $[3,5]$. The main methods of research in this area are numerical. Ones of the most effective methods are the finite element method and the finite difference method, which take into account the specific nature of this problem. In this case, the conditions at the nodes of the finite elements and the forms functions must ensure a smooth variation of the dynamic stresses. Under the action of local loads, the zone of intense stress is very limited. Outside this zone, the stresses decrease rapidly in space and in time. Calculations in this zone can be performed with a denser grid. High-rate deformation elements of structures under the action of short-term loads of various physical nature (mechanical, impulse, shock, moving, magneto-impulse, thermal, etc.) takes place in the elastoplastic stage. It is necessary to take into account the dynamic characteristics of materials of the type $\sigma=\sigma(\varepsilon, \dot{\varepsilon}, T)$, that are obtained experimentally. 
In the beginning, the behavior of elements from various materials under impact loads is studied. So, in Fig. 8 the changes of equivalent stresses in elements from various materials under the action of impactor weighing $100 \mathrm{~g}$ at a speed of $200 \mathrm{~m} / \mathrm{s}$ are presented $[6,7]$.

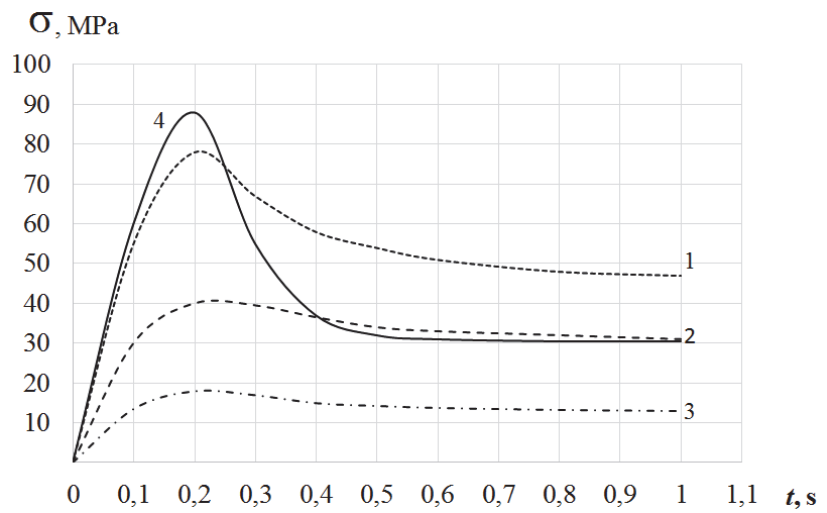

Fig. 8. Comparison of the variation of the maximum equivalent stresses for different materials:

1 - steel element; 2 - aluminum alloy, 3 - composite, 4 - titanium alloy

In the elements of steel and titanium alloy, the maximum equivalent stresses and the greatest localization of stresses are observed. The lowest stresses are observed in composite elements due to the least stiffness and large displacements.

It is allowing to select the material for protective elements or use the properties of materials of existing structures. It is shown that the elements of titanium alloys have the greatest resistance to impact loads and the growth of dynamic hardening with the impact speed, even greater than the steel samples. At the same time, the composite materials with a sufficient resistance to impact loads have the least weight.

For examples, the protective properties of vehicle lining are considered. So, for the lining of locomotives (electric locomotives), the effects of a standard impactor with weight of $100 \mathrm{~g}$ and with a locomotive speed of $80 \mathrm{~km} / \mathrm{h}$ are considered. Also, a double impact speed, when moving oncoming trains, are interest. In Fig. 9 shows the distribution of equivalent stresses when the impactor hits an $8 \mathrm{~mm}$ thick fiberglass element [7].
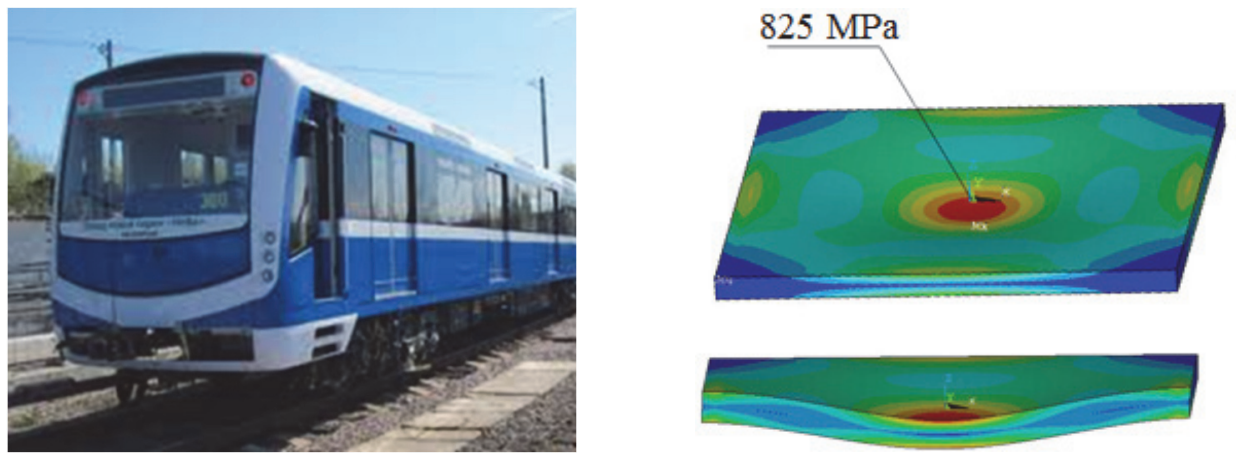

Fig. 9. The view of a locomotive and the distribution of equivalent stresses in the element of its lining under the action of impactor at a speed of $180 \mathrm{~km} / \mathrm{h}$

In this case, the largest equivalent stresses do not exceed $900 \mathrm{MPa}$, and the displacements are $1.2 \mathrm{~mm}$. It can be seen that this element provides dynamic strength at speeds of up to $180 \mathrm{~km} / \mathrm{h}$.

For the facing of vehicles, the action of impactors at a speed of $60 \mathrm{~km} / \mathrm{h}$, but at different angles of encounter is considered. So, in Fig. 10 shows the displacements and stresses fields in the element of corps at an angle of $45^{\circ}[7,8]$. 


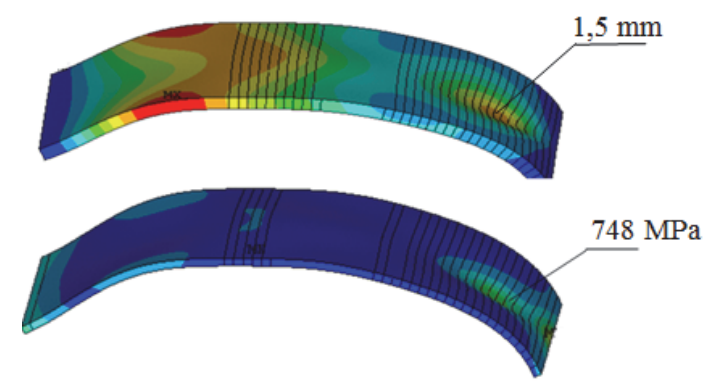

Fig. 10. Displacements and stresses in the element of the motor transport after encounter with impactor with a speed of $60 \mathrm{~km} / \mathrm{h}$ at an angle of $45^{\circ}$

A series of similar studies show that, under impact action, the local stresses in motor vehicle corps reach significant values (up to $900 \mathrm{MPa}$ ) and significant deformations of the lining, but the construction retains the main operational properties at speeds of up to $60 \mathrm{~km} / \mathrm{h}$.

Of great interest is the analysis of the impact on the elements of the gas turbine engine corps of foreign objects and fragments of the compressor blades and fan blades. The effect of these impactors is interrelated. During take-off and landing of the aircraft, parts of the runway and other objects are drawn into the gas-air path of the engine, and particles of ice, moisture, sand, etc., can be drawn into when flying. These particles can cause damage of the blades. Fragments of the blades and foreign objects under the action of centrifugal forces cause impact on the engine corps. Therefore, protective elements in the form of reinforcing linings made of steel or composite materials are used in dangerous locations. Below are the results of the impact of some fragment with weight $100 \mathrm{~g}$, caused by rotation of the rotor, on the protective elements of the housing. Single-layer and two-layered elements of corps are considered.

In Fig. 11 shows the distribution of equivalent stresses arising from the action of fragment of the fan blades on a protective element from composite at different times. Characteristic dimensions of the protective element: thickness $30 \mathrm{~mm}$, diameter $800 \mathrm{~mm}$. The rotor speed is $8500 \mathrm{rpm}[7,8]$.

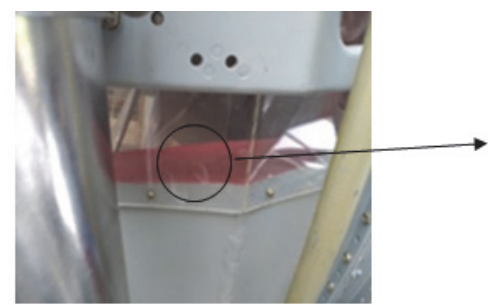

a)

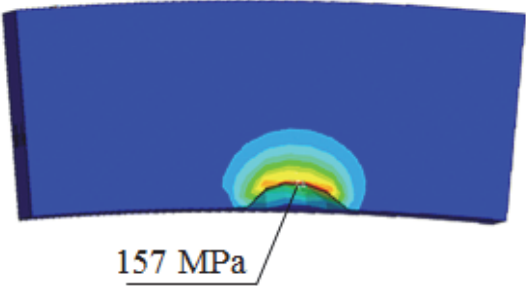

c)

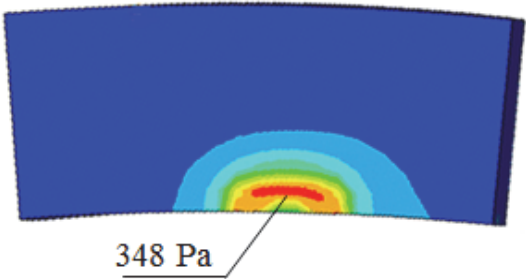

b)

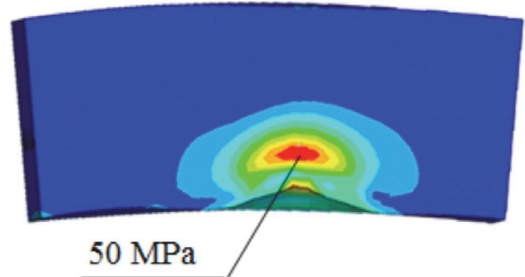

d)

Fig. 11. Distribution of stresses in the protective element from the composite at different times at impact with a speed of $400 \mathrm{~m} / \mathrm{s}$ : a) protective element from composite, b) $t=0,001 \mathrm{~s}$; c) $t=0,01 \mathrm{~s}$; d) $t=0,1 \mathrm{~s}$

It can be seen an increase of the equivalent stresses in local zone and the formation of residual stresses and damage. The protective element does not destroy and can perform its functions even 
further until repair.

At the location of the compressor blades, the elements of corps are made from steel with a thickness of $8 \mathrm{~mm}$ and a diameter of $415 \mathrm{~mm}$. It can withstand the action of the identical impactor at a speed of $350 \mathrm{~m} / \mathrm{s}$. In this case the maximum equivalent stresses reach values of $140 \mathrm{MPa}$, and displacement of $2 \mathrm{~mm}$. It is interesting, that the use of linings and double-layered protective elements significantly increases their effectiveness.

In Fig. 12 shows the distribution of equivalent stresses and displacements in a two-layer element with thicknesses of $1.4 \mathrm{~mm}$ and $2 \mathrm{~mm}$ with the same impact.

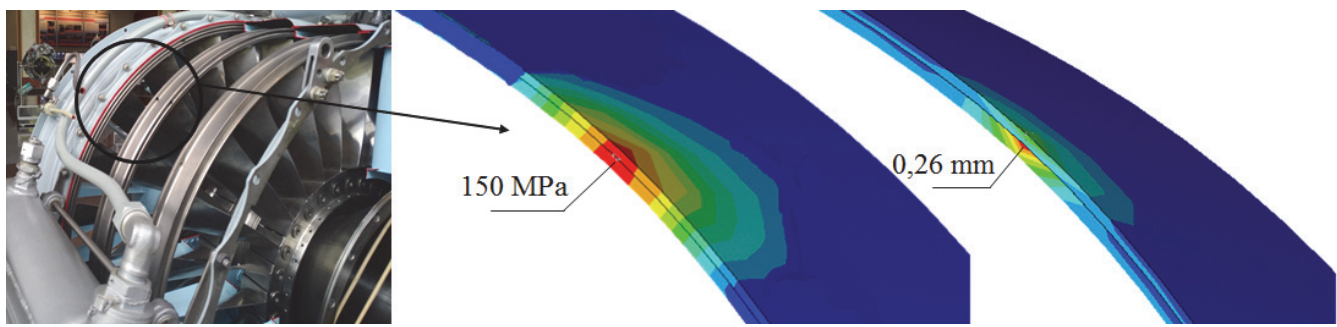

Fig. 12. Double-layered protective element and equivalent stresses and displacements in it

The maximum stresses in this case reach $150 \mathrm{MPa}$, but the total thickness of the protective element is much less than the thickness of a single-layer element $[7,8]$. As the speed of the impactor increases, elastic-plastic deformations of the protective element and its damage arise (Fig. 13). For a better visualization of stresses and deformations in the element, the impactor is not shown in the figure.

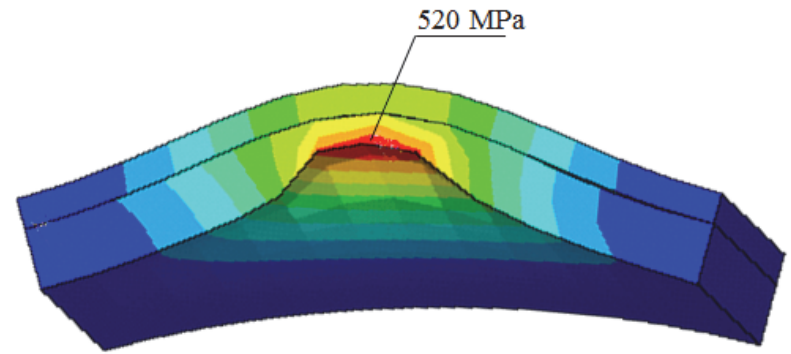

Fig. 13. Equivalent stresses in a two-layer protective element, when the impactor speed up to $1000 \mathrm{~m} / \mathrm{s}$

The presented studies show the expediency of using multilayer elements. As an example, some results of this study is given in Fig. 14. The weight of impactor is $100 \mathrm{~g}$, a speed of $400 \mathrm{~m} / \mathrm{s}$; the characteristic dimensions of a three-layer target-element is $100 \times 100 \mathrm{~mm}$ and the thickness of two outer layers from titanium alloy $2 \mathrm{~mm}$ and inner ceramic layer $3.2 \mathrm{~mm}$
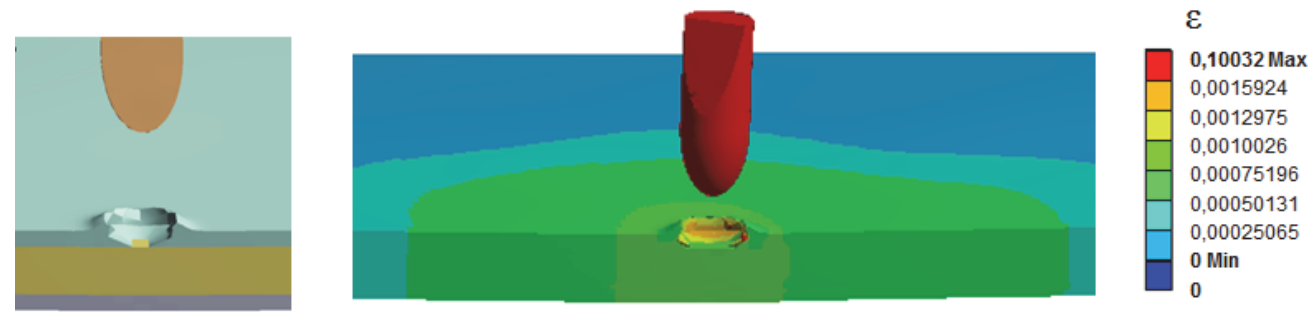

Fig. 14. Displacements and deformations in a three-layer element when the impactor speed of $400 \mathrm{~m} / \mathrm{s}$

In the top titanium layer, an area of local damage occurs, and crater formations are observed. Deformations pass into the plastic stage, and the maximum equivalent stresses reach $1330 \mathrm{MPa}$. 
Local deformations in the top layer lead to the distribution of deformation in a wide region of the ceramic layer, and the stresses decrease accordingly. As a result, the impact energy is absorbed in the ceramic layer. Despite the damage of the top layer, the three-layer element retains its protective properties. A new impact occurs, as a rule, in a new location, and the element can again withstand impact. Such elements are advisable to use for light-armored vehicle and in other cases [9].

Studies have also been conducted to ensure safety during technological processes of compound and separating structural elements. For example, the processes of compounds elements by explosive energy are considered [5].

The compound of two cylindrical layers is considered. These studies should be conducted before the implementation of the relevant processes. Connections of elements occur by means of explosion welding, when the layer being thrown is connected to the target layer at a speed $3 \cdot 10^{3}-9 \cdot 10^{3} \mathrm{~m} / \mathrm{s}$ due to the energy of explosive charge in sheet form, which provide a short-term $\left(10^{-6} \mathrm{~s}\right)$ pressure $1.3 \cdot 10^{10} \mathrm{~Pa}$. The radial displacement of the layers has the order of $10^{-4} \mathrm{~m}$. The distribution of axial displacements is shown in Fig. 15.

In Fig. 16 shows the diagram of elastic-plastic deformation in the axial direction from the loading zone and the diagram of residual deformations.
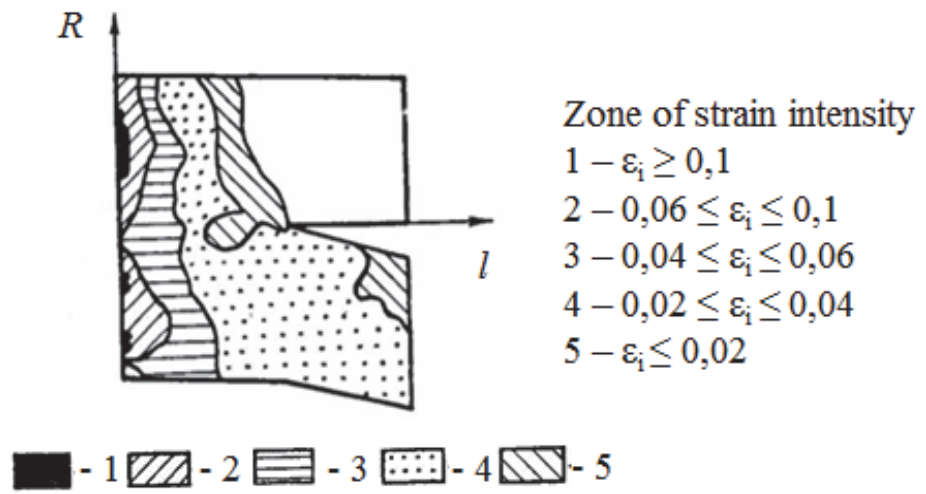

Fig. 15. The distribution of the strain intensities for the high-rate joining of two layers

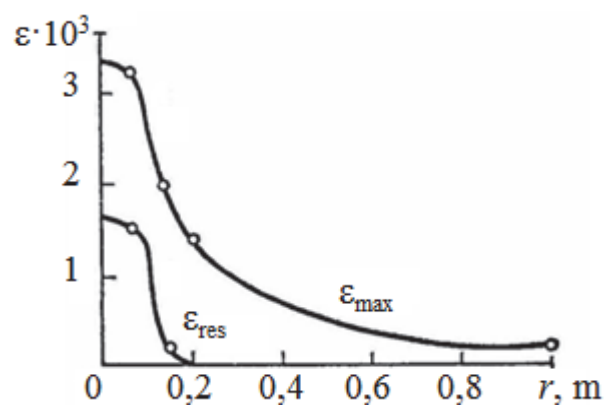

Fig. 16. The distribution of maximal $\left(\varepsilon_{\max }\right)$ and residual $\left(\varepsilon_{\text {res }}\right)$ deformations depending on the loading zone

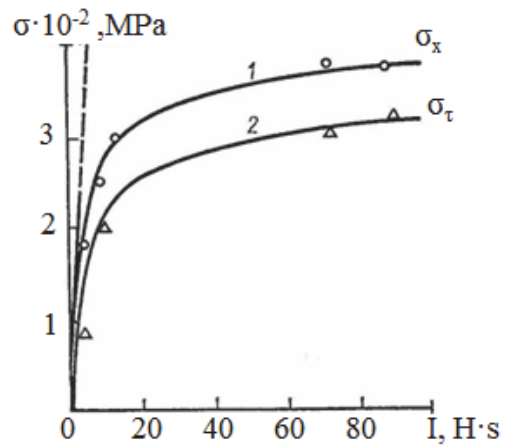

Fig. 17. Dependences of the axial (1) and circumferential (2) stresses on the loading pulse

These graphs are the boundaries in which deformations can occur during this process [5]. The process is non-linear. The dependences of the axial (1) and circumferential (2) stresses on the loading pulse $I$ are shown in Fig. 17.

For comparison, the dotted line represents a linear relationship. The processes of explosion welding were used to combine parts of heat exchangers. A fragment of the heat exchanger at the place of compound of the tube with the elements of construction is shown in Fig. 18. 


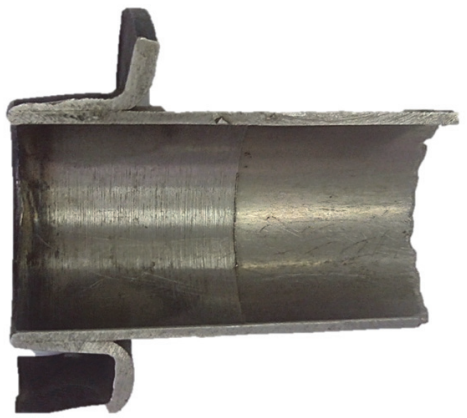

Fig. 18. A fragment of the heat exchanger at the junction of its elements by explosion welding

Also, processes of separation of elements using explosion energy are often used. In such processes, after the application of concentrators, cord explosive charges are used. In Fig. 19 shows isolines of stresses near the concentrator after $5 \mu$ s after the action of the impulse [10].

Separation processes by explosion energy were used for pulsed controlled separation of elements of space technology.

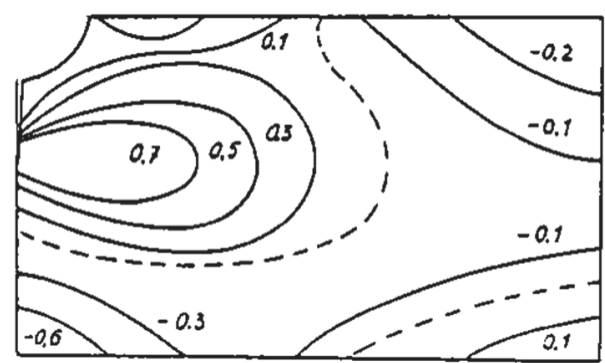

Fig. 19. The Isolines of stress $(\sigma, \mathrm{GPa})$ near the concentrator

\section{Use of the calculation-experimental method}

If the properties of the materials are not sufficiently known, then the problem can be solved with the help of calculation-experimental methods. In this case, for characteristic elements, multivariate experimental studies are carried out under the actions of local loads. As a result of the processing of the experimental data, spatial dependences of the maximum values of the strain intensities on the magnitude of the impact pulse, the location of the load application, and the characteristics of the element are constructed. The methodology is based on the principles of regression analysis $[11,12]$. The results of the studies are illustrated with concrete examples. As a characteristic element, cylindrical shells are chosen. Experimental studies were carried out with shells of different relative thicknesses $h / R$, where $h$ - thickness, and $R$ - shell radius. The loading was carried out with a series of loads with mass $m$, which were dropped on the shell from different heights, which provide different speeds of impact $V$, and the impulses of load $m V$. The deformations of the shells were measured using a system of sockets of strain sensors, which were located in the axial and circumferential directions from the loading point. Based on a series of experimental studies, a system of spatial graphs of the dependence of deformations on the parameters $h / R, I=m V$ and coordinates in the axial $x$ and the circumferential $y$ directions. It was assumed that the impulse load is distributed over the area of the contact region of radius $c$ unevenly in accordance with the law:

$P(x, y, t)=P(t) \sqrt{1-\left(\frac{x}{c}\right)^{2}-\left(\frac{y}{c}\right)^{2}}$. 
The problem of the stress-strain state is solved on the basis of Timoshenko's functional equation, the Fourier cosine-transform method with respect to the $x$ and $y$ coordinates, and the Laplace transform with respect to time $t$ [11]. The structure of the solution shows that it does not have a clearly multiplicative character. Therefore, based on the processing of experimental studies, the dependence of the strain intensity $\varepsilon_{i}(t)$ on the parameters $t, m, V, h / R, x$ was build. In Fig. 20 shows the spatial graphs of the dependence for $\varepsilon_{i}(t)$, on the surface of which lie the solution of problems for specific cases of loading.

To assess the reliability of the results, repeated experiments were carried out. Verification of the adequacy of the constructed dependence to the actual process was performed on the basis of the coefficient of multiple correlations and the Fisher test [12].

The case of impact loads on the elements of vehicles when the road milling cutters the layer of road surface is considered. When the working speed of the milling cutter is $350 \mathrm{rpm}$, the speed of the fragments is $62 \mathrm{~m} / \mathrm{s}$. Approximate weight of a typical fragment is $0.22 \mathrm{~kg}$. The analysis of the deformation of gas cylinders of different sizes, which use on vehicles, under the action of shock loads was carried out (Fig. 21).
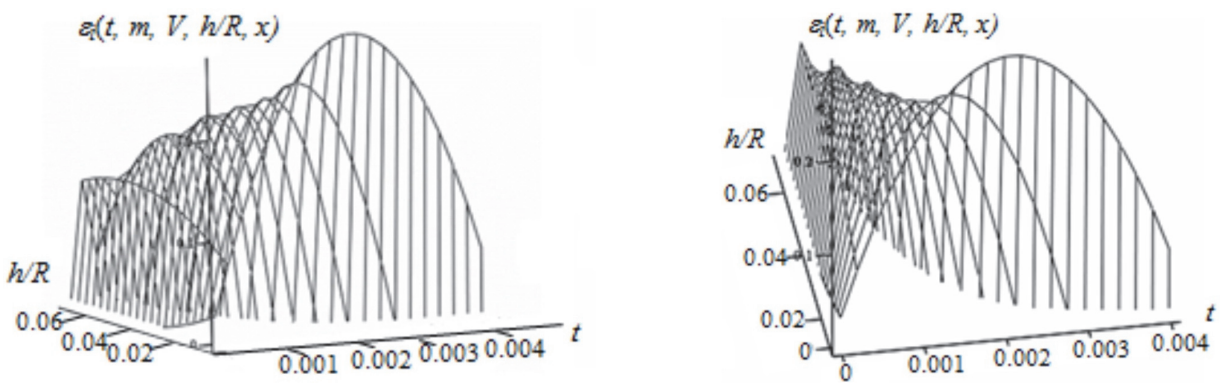

Fig. 20. Examples of surfaces on which solutions lie for different load cases
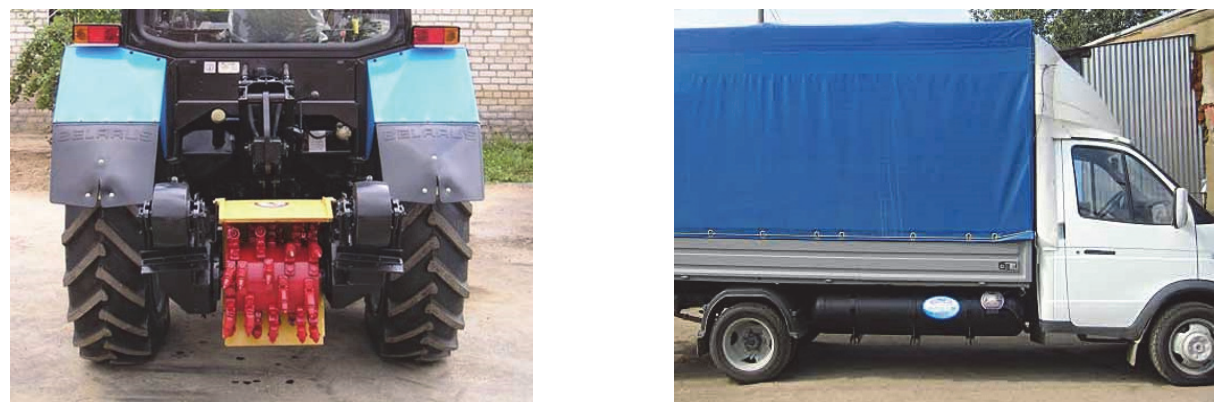

Fig. 21. General view of a road mill and vehicle with cylindrical balloon for gas
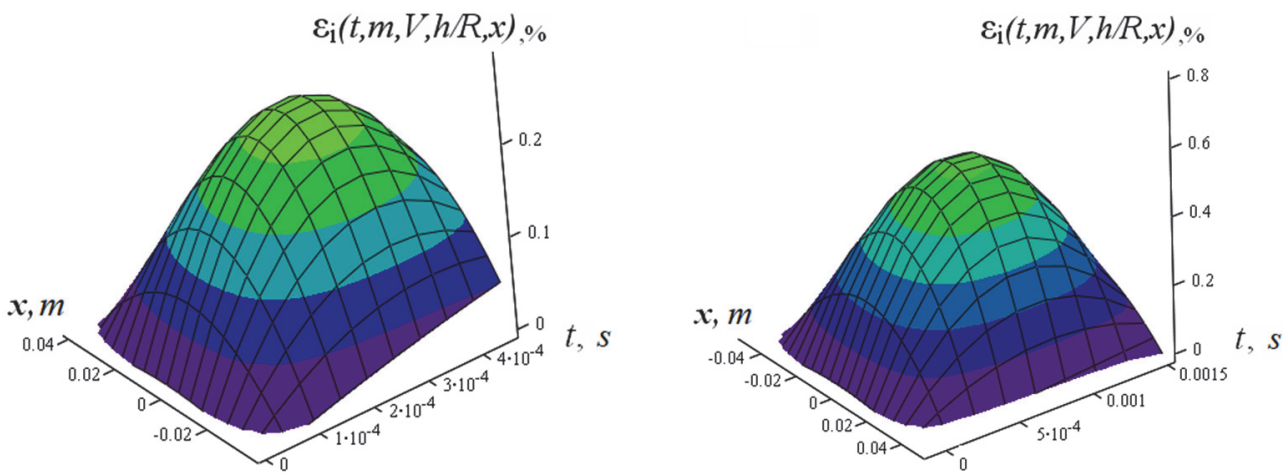

Fig. 22. Distribution of deformations around the impact zone on balloons 
In Fig. 22 shows the distribution of deformations around the impact area. As an example, cylinders of different diameters and thicknesses are considered.

The maximum deformations reach $0.72 \%$, which is below the dynamic limit of the elasticity of the material of the cylinders. There are examples of calculations for shock loads of other cylindrical structures. Thus, by means of the calculation-experimental method, effective studies of the strength of the elements of shell structures are provided.

\section{Conclusions}

It is shown, that the high-rate deformation of the elements of modern structures under shock loads occurs in the elastic-plastic stage with finite displacements and changes in the properties of materials during deformation. Experiential installations for the high-rate deformation of specimens allow to determine the characteristics of the dynamic properties of materials. These characteristics are presented in the form of dependencies of stress intensities on the strain intensities and strain rates. All characteristics can change during deformation.

Investigations of the variation of equivalent stresses under shock loads in time have been carried out, which facilitates the choice of material for protective structures. The action of impact loads on protective elements is carried out using FEM based on three-dimensional models. A series of numerical studies of the dynamic stress-strain state of protective elements for vehicles and gas turbine engine corps have shown their ability to withstand shock loads in operating conditions. The advantage of multi-layered protective elements, that can withstand repeated loads, is revealed. The parameters of technological processes of compound and separation of elements with the help of explosion energy are estimated. The efficiency of the calculation-experimental method is shown, when estimating the high-rate deformation of cylindrical protective elements.

The results of the investigations make it possible to give recommendations on increasing the efficiency of protective elements of critical structures under impact and impulse loads.

\section{References}

[1] Stepanov G. V., Babutsky A. I. Influence of a High-Density Pulsed Electric Current on the Stress-Strain State of Metal Structural Materials. Naukova Dumka, Kiev, 2011.

[2] Stepanov G. V., Kharchenko V. V. Dynamic Plasticity. Mechanics of Inhomogeneous Environments. Novosibirsk, 1989, p. 285-299.

[3] Pisarenko G. S. Strength of Materials and Structural Elements in Extreme Conditions. Naukova Dumka, Kiev, 1980.

[4] Meyers M. A. Dynamics Behavior of Materials. Wiley, New York, 1994.

[5] Vorobiov Y. S., Kolodyazhny A. V., Sevryukov V. I., Yanyutin E. G. High-Rate Deformation of Structure Elements. Naukova Dumka, Kiev, 1989.

[6] Vorobiov Iu. S., Kruszka L., Ovcharova N. Y. FEM analysis of cylindrical structural elements under local shock loading. Applied Mechanics and Material, Vol. 566, 2014, p. 499-504.

[7] Vorobiov Iu. S., Kruszka L., Ovcharova N. Y. Sensitivity of high strain rate of structural elements in relation to dynamics properties of material. European Physical Journal Web of Conferences, Vol. 94, 2015, p. 1-3.

[8] Vorobiov Iu. S., Kruszka L., Ovcharova N. Y. The resistance of structural elements to impact and shock-wave load. Key Engineering Materials, Vol. 715, 2016, p. 216-221.

[9] Vorobiov Iu. S., Ovcharova N. Y. High-rate deformation of multilayer elements under contact impact Journal Technical Mechanics, Dnipro, Vol. 3, 2016, p. 17-24.

[10] Vorobiov Y. S., Sevryukov V. I. Separation of construction elements at impulse loading. Computational Plasticity, Barcelona, Spain, 1992, p. 1883-1890.

[11] Vorobiov Y. S., Kolodyazhny A. V., Yaryzhko A. V. High-rate elastic-plastic deformation of a cylindrical shell at a local impact. Journal Dynamics and Strength of Machines. Vol. 36, 2006, p. $40-48$.

[12] Yaryzhko A. V. Calculation-experimental method for determine dynamics strain state of shell structures under impact load. Journal of P. Vasilenka KhNTUA. Vol. 100, 2010, p. 233-239. 


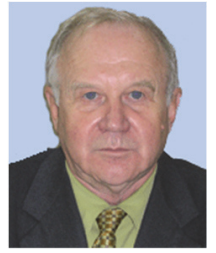

Yuri Vorobiov received Dr. Sc. in dynamic and strength of machine in 1979 and Prof. in 1984 National Technical University "Kharkovskiy Polytechnic Institute", Kharkov, Ukraine. His current research interests include dynamics and strength of machines, turbomachines blading, high-rate deformation of structural elements under shocks and pulse loading taking into account elastic-plastic deformation and dynamic properties of material. He is a Secretariat member of the Ukrainian National Committee on Theoretical and Applied Mechanics; Academic of Ukraine Universities Academy; Academic of Ukraine Engineering Academy; Member of Technical Committee Rotor Dynamics of IFFToMM

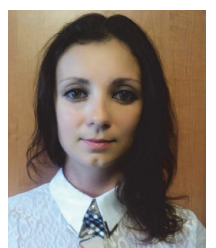

Nataliia Ovcharova received Ph.D. in dynamic and strength of machine National Technical University "Kharkovskiy Polytechnic Institute", Kharkov, Ukraine in 2018. His current research interests include high-rate deformation of structural elements under shocks and pulse loading taking into account elastic-plastic deformation and dynamic properties of material. She also took part in research vibrations stress localization complex engineering constructions and turbomachines blading vibrations. 



\section{Chapter 4}

Creation of new materials 


\title{
4.1. Study of energy ions, their varieties and charge on temperature, rate of temperature rise, thermal stresses, and also on the size, amount of volume, number of particles in nanostructures and effective current density for nanostructures
}

\author{
Gennadiy Kostyuk \\ Department of Theoretical Mechanical Engineering and Robotic-Mechanics Systems, \\ National Aerospace University named by N. Ye. Zhukovsky "KhAI”, Kharkov, Ukraine \\ E-mail: gennadiykostyuk206@gmail.com
}

\begin{abstract}
On the basis of joint decisions of the problem of heat conduction and thermoelasticity obtained temperature field and thermal stress in which the zones where the nanostructures are formed. For a large number of different ions and charge rules 1 to 3 were obtained according to the volume of the nanostructure, and the depth range, which are implemented by the nanostructure ion energy and charge, determined the effective current density.
\end{abstract}

Keywords: temperature, thermal stresses, nanostructures, effective current density.

\section{Introduction}

For nanocrystalline structures on the surface, as is well known, we need a certain temperature (or its rate of increase) in the required amount of pressure and having a metal atom around which the nanostructure.

As shown in [1-11], the action of charged particles on construction materials leads to the appearance of the material to a depth of rather high temperatures under the influence of individual ions of different varieties and heat affected zone has the probability of occurrence of thermal stresses of considerable magnitude, which confirms the possibility of local zones, where it reaches the conditions for the emergence of nanostructures.

To these conditions were realized in a large volume (high temperatures and voltages) is also necessary to ensure maximum filling of the temperature field with high temperatures and at the same time maintain the zone with the highest temperature gradients when implemented high values of thermal stresses. Clearly, a simple increase of ion current density to achieve this would be impossible, as the current density increases the temperature gradients in the adjacent area of the particles is reduced, and hence, reduces the amount of stress and temperature conditions for the formation of nanostructures not performed.

\section{Status of the question}

Due to the action of ions of various sorts, energy, battery, current densities it is possible to maintain a sufficient temperature (due to the corresponding temperature distribution of the temperature field in depth ensures a high average temperature), at the same time, choosing the location of the field depth (varying power, battery, and type of particle), provides high temperature gradients. Consequently, a fairly large amount of material will act on significant voltage value, i.e. will run conditions for obtaining the crystal structures.

Therefore, several upgraded models of the individual particles of structural materials [1-11], get a model that takes into account relevant factors (in particular, the model does not take into account the charge of the ion and the complex nature of their interference in the rather large amount of consideration). 


\section{Statement of the research task}

Taking as criteria for obtaining nanostructures desired temperature range, the rate of rise of temperature, pressure (thermal stress) and the presence of the catalyst [2], we can consider the possibility of obtaining nanostructures depending on the physical and technological parameters of flow during processing, which will analyze some of the theoretical and technical task (Fig. 1):

I. The model of interaction of streams of ions of different sorts, energy, charge and current densities, which allows:

1) to obtain the depth range, which is likely to receive for each of the nanostructures streams separate and evaluate the effect of flow depth;

2) calculate the distribution of temperature fields and thermal stresses in the amount of detail;

3) to determine the grain size;

4) organize the flow of particles so that the maximum amount of items has been filled the fields of temperature and thermal stress (theoretically and technologically);

$5)$ determine the amount theoretically nanocluster particles in a quantity of nanoclusters formed in unit time.

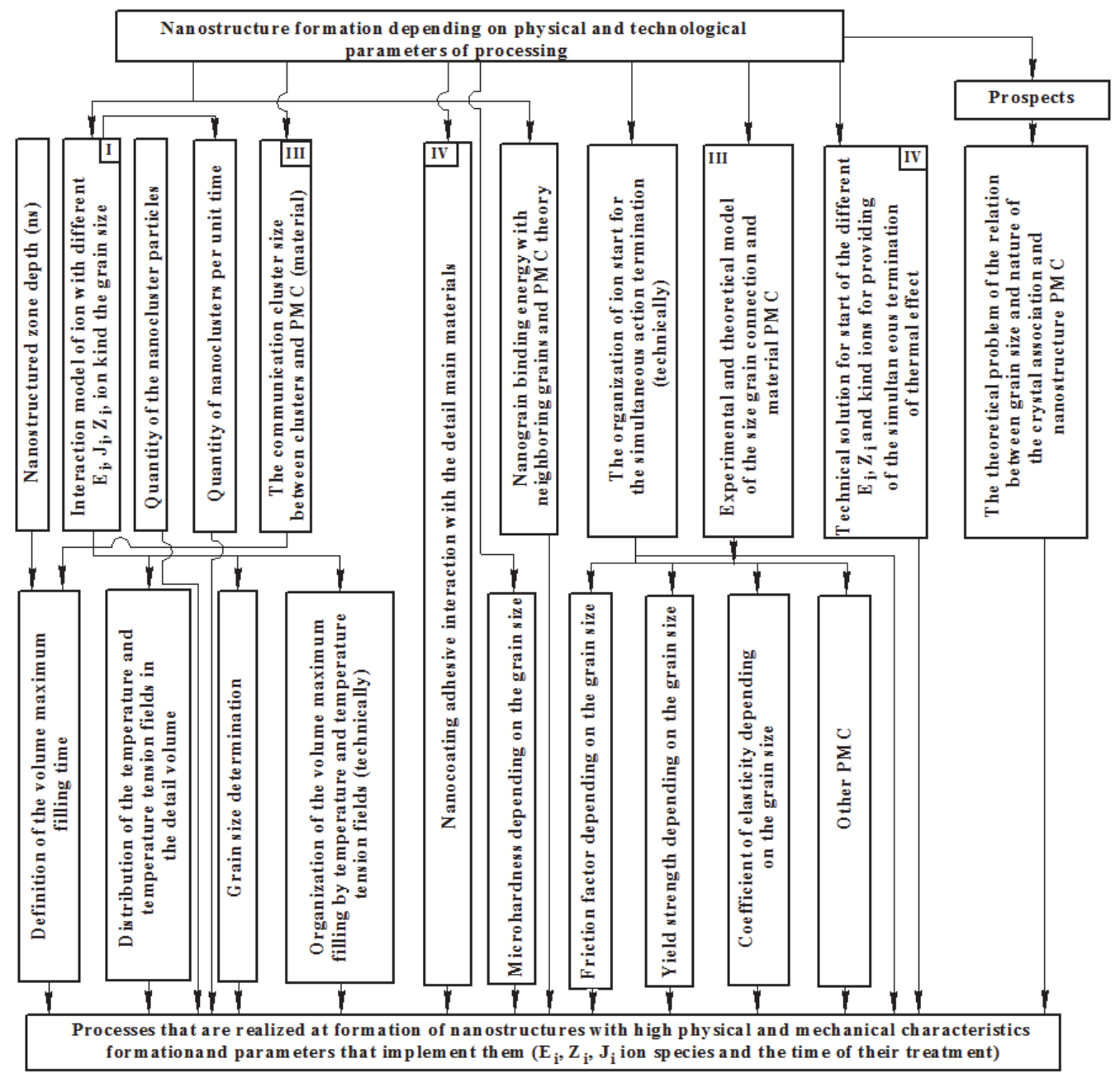

Fig. 1. Processes that are realized at formation of nanostructures with high physical and mechanical characteristics formation 
II. The theory of the interaction of nanoclusters (nanocrystals) their size, power relations between them and the physical and mechanical properties of nanostructures, which allows you to:

1) calculate the size of nanoclusters energy ties with neighboring nanoclusters (grains) and physic-mechanical properties of the nanomaterial;

2) to obtain the theoretical dependence of physical and mechanical characteristics of the National Assembly on the grain size, location and nature of their interactions, completeness volume nanostructures.

III. Experimental and theoretical model of grain size due to the FMH:

1) microhardness;

2) the coefficient of friction;

3) the yield strength;

4) tensile strength;

5) The modulus of elasticity;

6) wear;

7) resistance of cutting tools (RI).

1. Adhesion bond nano-coatings to the base material part (theory).

2. Technical solution: software start flow of ions of different sorts, energy, charge and current densities.

Solving these problems will help you find the physical and technological parameters of ion fluxes, which will provide the desired physical and mechanical properties of nanostructures and nanomaterials.

Now consider the real possibility of implementing these tasks.

\section{Model of the action of an individual ion on a structural material}

The interaction of charged particles and plasma flows with structural materials related to the implementation of a wide range of processes: collisional, thermal, thermomechanical thermal fatigue, diffusion, thermochemical and plasma, but there are currently no job, taking into account these factors and the more their relationship. These processes influence both on the nature of heat transfer in the target volume and on the surface, so keeping these processes needed in the balance of energy in a volume element of a metal target and a target heat exchange surface, i.e. in boundary conditions.

\subsection{Heat balance in an elementary volume of a part}

Change the amount of heat per unit volume (the first term in the left-hand side of Eq. (1)) can be realized by moving the particle flow along the surface to be treated or moving target with respect to the plasma flow at a speed $V_{p}$ (second term), thermal processes influence on heat transfer finite speed of propagation of heat (third term), thermal conductivity (the first term on the right), the displacement of the evaporation front (second term), point (third term); collisional processes: volumetric heat source due to the action of the particle (the fourth term), energy consumption in the displacement of atoms (fifth term); thermo-elastic, thermoplastic and thermal fatigue processes that determine the energy of deformation of the material elementary volume (six terms); diffusion processes that determine the heat transfer diffusing material (seventh term); thermochemical processes related to the implementation of chemical reactions between the material of the target and bombarding ions or between the alloy components in composite materials and Lentz-Joule heating due to the spreading of the ion current: 


$$
\begin{aligned}
C[T] \cdot \gamma[T] \frac{d T(x, y, z, t)}{d t}+C[T] \cdot \gamma[T] \frac{\partial T(x, y, z, t)}{\partial y} \cdot V_{n} \\
\quad+C[T] \cdot \gamma[T] \cdot \tau_{r t} \frac{d^{2} T(x, y, z, t)}{d t^{2}}=\nabla \lambda[T \cdot \nabla T](x, y, z, t)+C[T] \cdot \gamma[T] \cdot V \frac{\partial T(x, y, z, t)}{\partial x} \\
\quad-A \cdot L_{m . m} \cdot \gamma[T] \frac{d V_{m . m}}{d t}+B \cdot j_{i, e} \cdot \mu_{i, e} \frac{\partial E_{i, e}}{\partial x}-E_{d i s} \cdot S_{d i s} \frac{j_{i, e}}{\lambda_{c p i, e}} \pm D \frac{d W(x, y, z, t)}{d t} \\
\quad \pm m_{a} \cdot C_{a}\left[T_{a}\right] \frac{d n_{a}}{d t}\left(T_{a}-T(x, y, z, t)\right) \pm P_{T . C .}\left(n_{A}, n_{B}, T, t_{e}\right) \frac{d n_{A(B)}}{d t} L_{T . C .} \\
\quad+\rho[T] \cdot j^{2}(x, y, z, t),
\end{aligned}
$$

where $C[T]$ and $\gamma[T]$ - specific heat and density of the target material corresponding to a temperature $T ; \tau_{r}$ - the relaxation time of one Kelvin temperature; $V_{n}$ - ion flux moving speed or the target relative thereto; $L_{m . m}$ and $L_{\text {T.C. }}-$ specific heat of fusion and thermochemical reaction; $V_{f}[T]$ - the rate of displacement of the evaporation front; $V_{m . m}$ - the volume of molten metal; $j_{i, e}$ - the current density of ions, electrons or plasma flow; $\mu_{i, e}-$ accommodation coefficient ion and an electron; $-\partial E_{i, e} / \partial x$ loss of ion energy and electron at a depth of target; $E_{\text {dis }}-$ the energy of the atomic displacements; $S_{d i s}$ - the number of displaced atoms as a result of ion or electron; $\lambda_{c p i, e}$ - linear average free path of ions and electrons in a target material; $W(x, y, z, t)$ - the strain energy per unit volume of the target; $m_{a}$ - mass diffusing atom; $C_{a}\left[T_{a}\right]$ - diffusing the heat capacity of the material at a temperature $T_{a} ; P_{T . C .}\left(n_{A}, n_{B}, T, t_{B 3}\right)$ - chance of thermochemical reactions, depending on the concentration of the reactants $n_{A}$ and $n_{B}$, temperature $T$ and reaction time $t_{B 3} ; n_{A}$ and $n_{B}$ - reagent concentration, determines the possible reactions; $\rho[T]-$ electrical resistivity at a temperature $T ; j(x, y, z, t)$ - the current density based on current spreading.

Strain energy per unit volume is defined as:

$$
\begin{aligned}
W & =G\left[\varepsilon_{x x}^{2}+\varepsilon_{y y}^{2}+\varepsilon_{z z}^{2}+2\left(\varepsilon_{x y}^{2}+\varepsilon_{y z}^{2}+\varepsilon_{z x}^{2}\right)+\frac{\mu}{1-\mu} l^{2}\right. \\
& \left.-\frac{2(1+\mu) \alpha_{1}}{1-2 \mu} l(T(x, y, z, t)-T)\right],
\end{aligned}
$$

where $2 \varepsilon_{i k}=2 \varepsilon_{k i}=\frac{\partial u_{k}}{\partial i}+\frac{\partial u_{i}}{\partial k}(k, i=x, y, z) ; l=\varepsilon_{x x}+\varepsilon_{y y}+\varepsilon_{z z}, \varepsilon_{x x}, \varepsilon_{y y}, \varepsilon_{z z}-$ extension; $\varepsilon_{x y}, \varepsilon_{y z}, \varepsilon_{z x}$ - shifts relative to the respective axes; $u_{x}, u_{y}, u_{z}$ - movement about respective axes; $\alpha_{1}$ - the coefficient of linear expansion of the target material; $\mu$ - Poisson's ratio (the ratio of transverse strain to longitudinal $\mu$ values are between 0 and 0.5 ); $G$ - the shear modulus (modulus of the second kind); $T_{n}$ - initial temperature. for iron $G=3,5, \ldots, 5,3 \cdot 1010 \mathrm{~N} / \mathrm{m}^{2}$, $\mu=0,23, \ldots, 0,31$.

Shear strain $\varepsilon_{i k}$ can not be set arbitrarily, they are connected by differential relations conditions for compatibility:

$$
\begin{array}{ll}
\frac{\partial^{2} \varepsilon_{x x}}{\partial y^{2}}+\frac{\partial^{2} \varepsilon_{y y}}{\partial x^{2}}=2 \frac{\partial^{2} \varepsilon_{x y}}{\partial x \partial y}, & \frac{\partial^{2} \varepsilon_{x x}}{\partial y \partial z}=\frac{\partial}{\partial x}\left[-\frac{\partial \varepsilon_{y z}}{\partial x}+\frac{\partial \varepsilon_{z x}}{\partial y}+\frac{\partial \varepsilon_{x y}}{\partial z}\right], \\
\frac{\partial^{2} \varepsilon_{y y}}{\partial z^{2}}+\frac{\partial^{2} \varepsilon_{z z}}{\partial y^{2}}=2 \frac{\partial^{2} \varepsilon_{x y}}{\partial z \partial y}, & \frac{\partial^{2} \varepsilon_{y y}}{x y \partial z}=\frac{\partial}{\partial y}\left[-\frac{\partial \varepsilon_{z x}}{\partial y}+\frac{\partial \varepsilon_{x y}}{\partial z}+\frac{\partial \varepsilon_{y z}}{\partial x}\right], \\
\frac{\partial^{2} \varepsilon_{x x}}{\partial z^{2}}+\frac{\partial^{2} \varepsilon_{z z}}{\partial x^{2}}=2 \frac{\partial^{2} \varepsilon_{z x}}{x z}, & \frac{\partial^{2} \varepsilon_{z z}}{\partial y \partial x}=\frac{\partial}{\partial z}\left[-\frac{\partial \varepsilon_{y x}}{\partial z}+\frac{\partial \varepsilon_{y z}}{\partial x}+\frac{\partial \varepsilon_{x z}}{\partial y}\right] .
\end{array}
$$

These conditions verified the correctness of the extensions and changes, and their adjustment the introduction of additional stresses. 
To determine elongations $\varepsilon_{x x}, \varepsilon_{y y}, \varepsilon_{z z}$ and shifts $\varepsilon_{x y}, \varepsilon_{y z}, \varepsilon_{z x}$. We use the expression thermoelastic potential of displacement $\Phi$ :

$\Delta \Phi-\frac{1-2 \mu}{2(1-\mu)} \cdot \frac{\gamma}{G} \cdot \frac{\partial^{2} \Phi}{\partial t^{2}}=\frac{1+\mu}{1-\mu} \cdot \alpha_{1} \cdot[T(x, y, z, t)-T]$,

where $\gamma$ - density target material.

Considering that the solution of the problem is carried out in a moving coordinate system and output to a landline or close to it mode, the second term in the left side of the Eq. (4) becomes negligible, we obtain:

$\Delta \Phi=\frac{1+\mu}{1-\mu} \cdot \alpha_{1} \cdot[T(x, y, z, t)-T]$

The magnitude of thermoelastic potential of displacement and the known ratio is the elongation and shifts:

$\varepsilon_{i k}=\frac{\partial^{2} \Phi}{\partial i \partial k}, \quad(i, k=x, y, z)$

The values of thermal stress is defined by the expression:

$\sigma_{i k}=2 G\left(\frac{\partial^{2} \Phi}{\partial i \partial k}-\Delta \Phi \cdot \delta_{i k}\right)$

where $\delta_{i k}$ subject to the conditions $\delta_{i k}=0$ at $i \neq k(i, k=x, y, z), \delta_{i k}=1$ at $i=k$.

The term of the Eq. (1) takes into account the heat transfer diffusing material, the mass of the diffusing atom is defined as:

$m_{a}=M \cdot m_{p}$

where $M$ - the atomic weight of the coating material; $m_{p}$ - proton mass.

The change in concentration of the diffusing atoms per unit time in $t<t_{k}$ defined as:

$\frac{d n_{a}}{d t}=\frac{j}{e \cdot z \cdot \sqrt{\alpha \tau}}$

At $t \geq t_{k}$ :

$\frac{d n_{a}}{d t}=\frac{j}{e \cdot z \cdot L_{D}}$

where $j$ - current density; $e$ - electron charge; $z$ - atomic number material applied; $L_{D}-$ part thickness; $t_{k}-$ the time in which the item is to warm up the entire thickness, $t_{k}=L_{D 2} / \alpha$; $\alpha$ - thermal diffusivity.

The formula to calculate the diffusion coefficient is given by:

$K_{\mathrm{dif}}=a_{\mathrm{d}} \cdot d_{\mathrm{e}}^{2} \cdot V_{0} \cdot \exp \left(-\frac{U}{(k T)}\right)$

where $a_{d}$ - factor on the order of 0.1 , determined by the type of crystal lattice; $d_{e}-$ the distance 
between the closest equivalent to the provisions of vacancies in the crystal; $V_{0}$ - the order of the frequency of atomic vibrations in a crystal $\left(10^{12}, \ldots, 10^{14} \mathrm{~s}^{-1}\right) ; U-$ the potential barrier that must be overcome job when skewed to the adjacent position; $k$ - the Boltzmann constant; $T-$ the absolute temperature $T$.

\subsection{Heat transfer on the surface of a part}

The heat flux on the target surface created by:

1. Collisional processes heat is generated on the surface due to the action of an electron or ion (the first term on the right), is given the heat flux from the sputtered particles (second term), with thermionic (third term) for the ions is given - heat flux from ion-ion emission or secondary ion emission (fifth term), the potential of the ion-electron emission (six terms), the kinetic ion-electron emission (seventh term), the characteristic X-ray radiation (eighth term), bremsstrahlung (ninth term), transition radiation (tenth term ).

2. Thermophysical processes assigned to the heat flow from the vaporized material (eleventh term), departed from the material in the liquid phase, if the conditions for its release (twelfth term), the thermal radiation of the heated surface (thirteenth term) and condensed atoms, previously steamed (fourteenth term).

3. The plasma-chemical processes, realized due to the reactions of the plasma flow and the flow of ions and spray vaporized target material or adsorbed gases (fifteenth term); This energy is transferred by radiation.

The transfer of energy is also carried out by the radiation flux of ions, electrons or plasma (the last term in Eq. (11)):

$$
\begin{aligned}
-\lambda & {[T] \frac{\partial T(x, y, z, t)}{\partial x}=F_{i, e}-F_{e v}-F_{m . e} } \\
& -\left[F_{i i . e}+F_{i . p . e}+F_{i . e . e}+F_{k . e}+F_{c . r e}+F_{t h r}+F_{t r}\right]_{i} \\
& -F_{e v}-F_{m . m}-\sigma \varepsilon T^{*}(0, y, z, t) \pm F_{c o n d}+F_{n x}+\sigma \varepsilon_{c} T_{c}^{*},
\end{aligned}
$$

where $\sigma-$ the Stefan Boltzmann.

To address the issue of effective filling volume nanostructures is necessary to determine the energy charges and ion species in which this condition is satisfied. It should be performed for each of the streams of ions, and it is desirable that the depth ranges where the nanostructures are realized, complement each other. Therefore, such a study is conducted.

\section{The study of temperature and rate of rise of temperature depending on the ion energy and charge the different varieties}

The study area size, wherein the nanostructure can be formed, allowing the temperature to identify the boundary of the formation of the nanostructure, and the maximum instantaneous temperature in the area.

Analysis Figs. 2-7 shows that an increase in energy and charge the maximum temperature in the area of origin of nanoclusters grow, and reach temperatures of up to $10^{6} \mathrm{~K}$, but the duration of these temperatures $-10^{-14}, \ldots, 10^{-9}{ }^{\circ} \mathrm{C}$ during heating and cooling, which does not lead to melting of the material is even. The temperatures at the border of the zone within the formation range of the definition, i.e. near the error of their determination. 

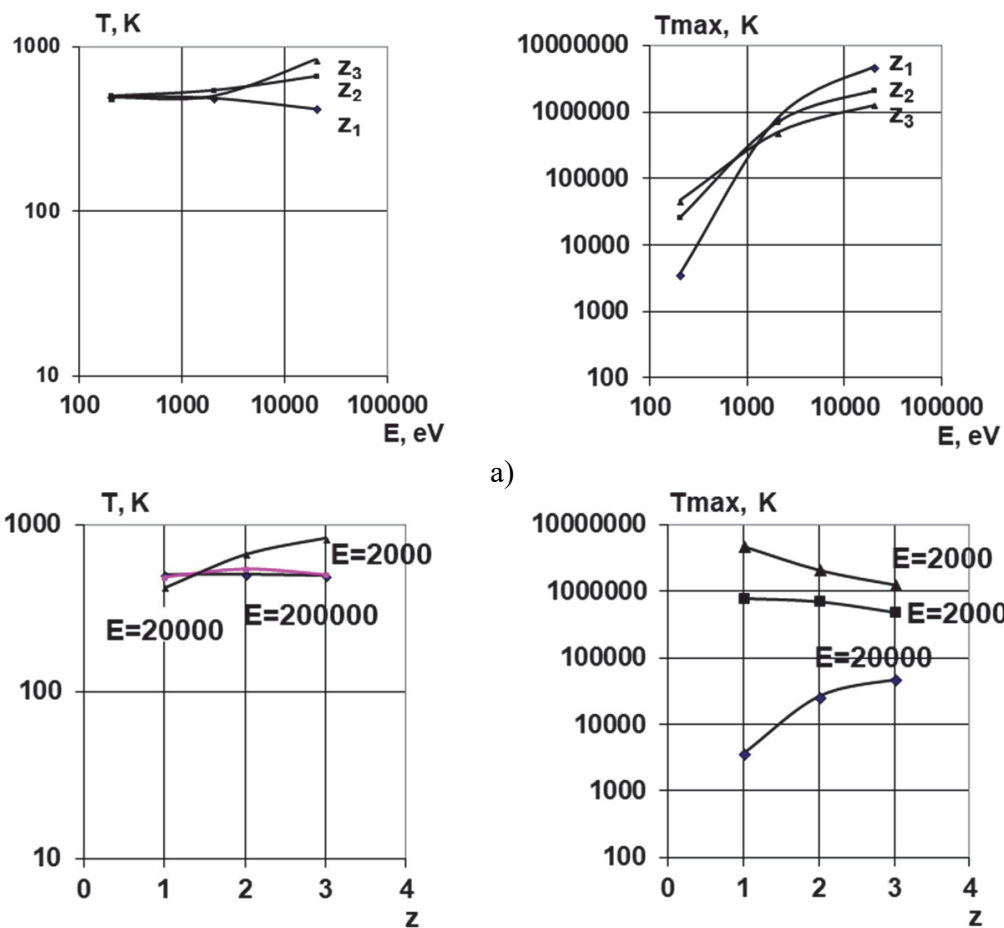

a)

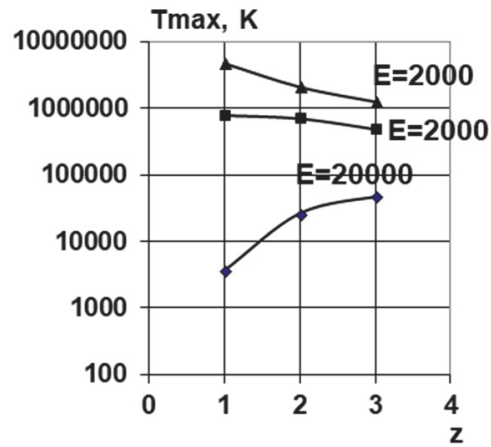

b)

Fig. 2. Plots of $T$ and $T$ the maximum energy from the a) ion charge and b) for the ions $\mathrm{C}, \mathrm{B}, \mathrm{N}$
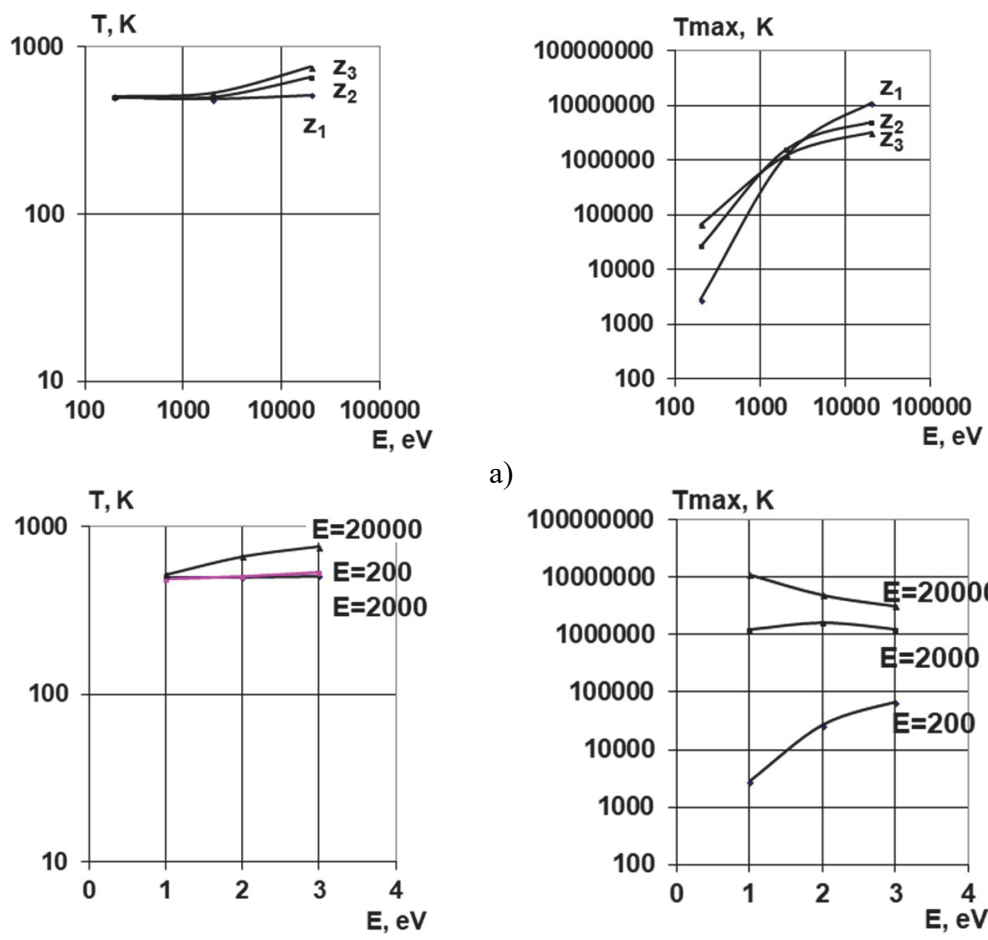

a)

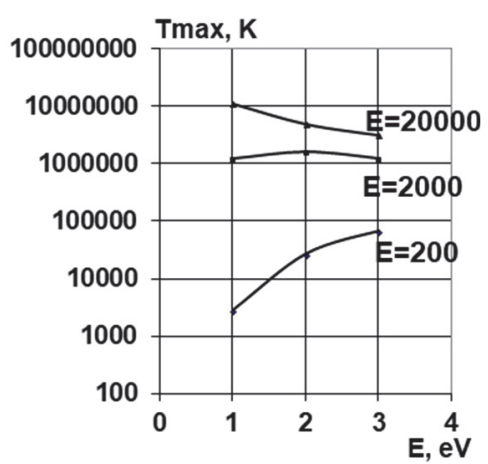

b)

Fig. 3. Plots of $T$ and $T$ the maximum energy from the a) ion charge and b) the ion $\mathrm{Al}$ 

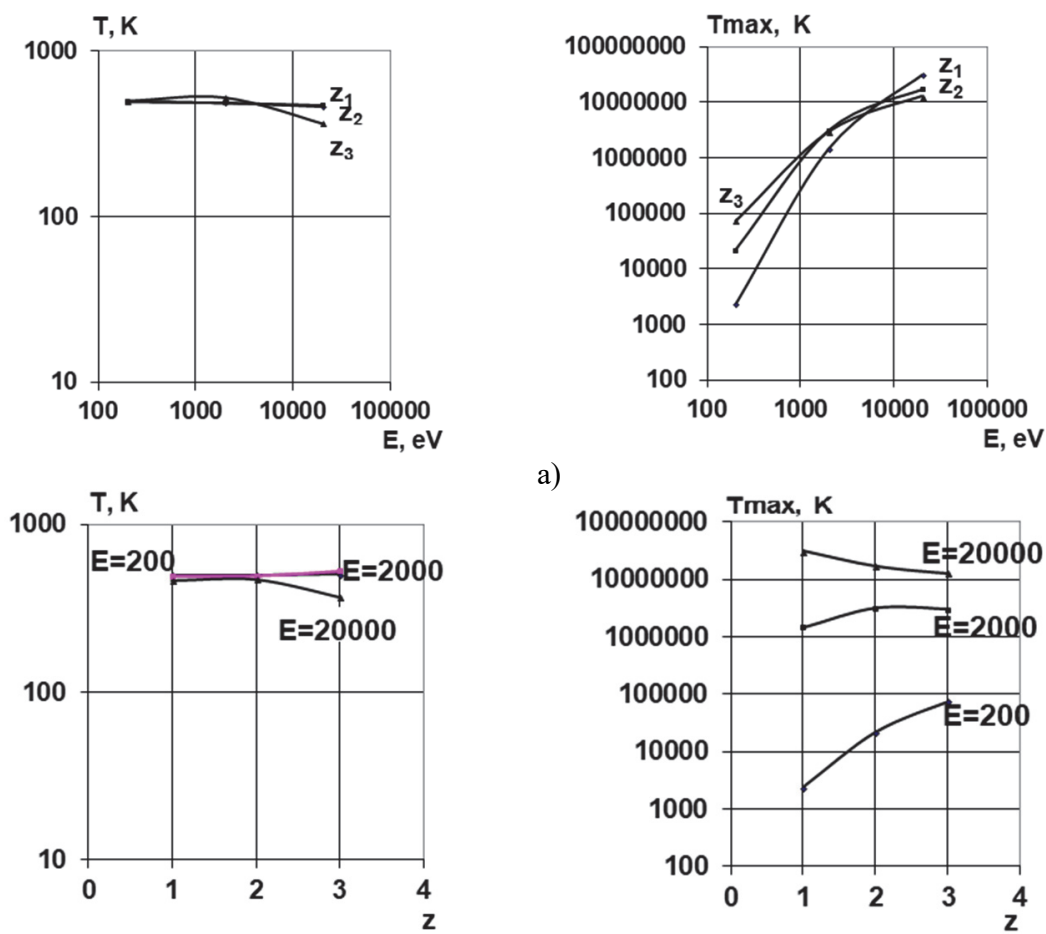

a)

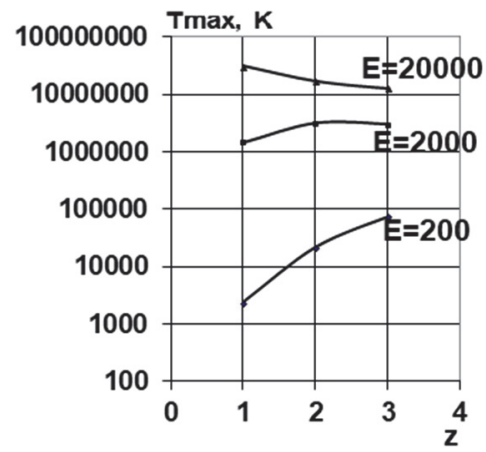

b)

Fig. 4. Plots of $T$ and $T$ the maximum energy from the a) ion charge and b) for the ions $\mathrm{Ti}, \mathrm{V}, \mathrm{Cr}$
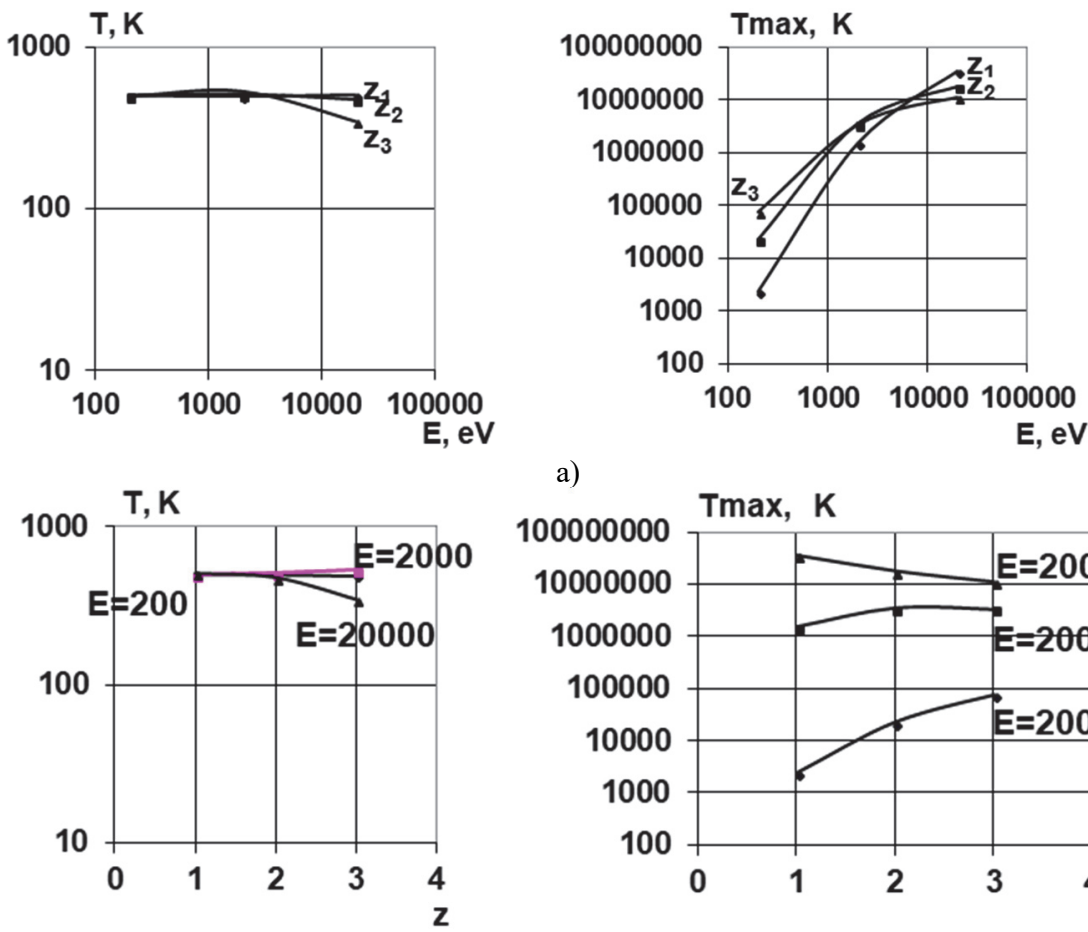

a)

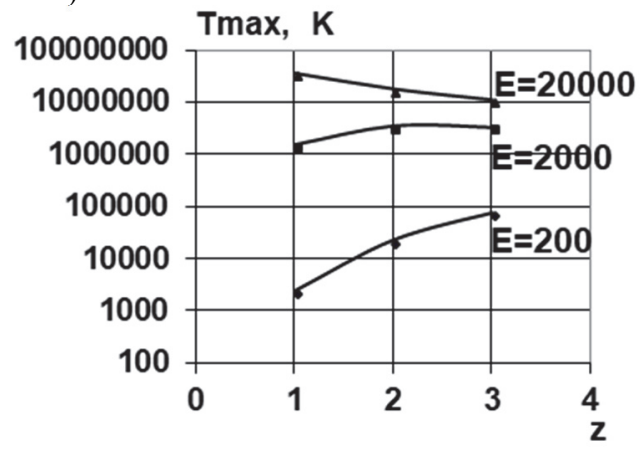

b)

Fig. 5. Plots of $T$ and $T$ the maximum energy from the a) ion charge and b) for the ions $\mathrm{Fe}, \mathrm{Ni}, \mathrm{Co}$ 

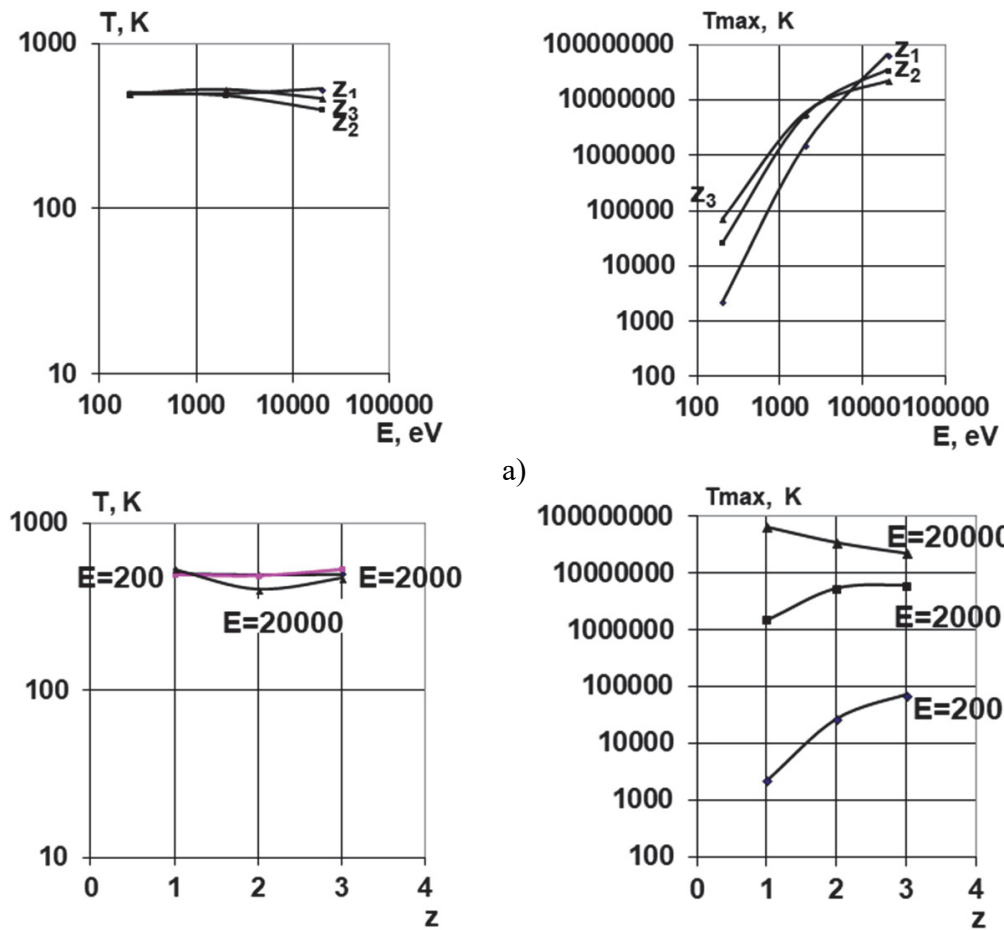

a)

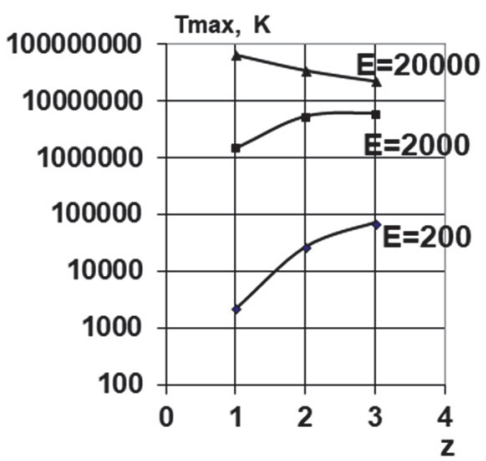

b)

Fig. 6. Plots of $T$ and $T$ the maximum energy from the a) ion charge and b) for the ions $\mathrm{Y}, \mathrm{Zr}, \mathrm{Nb}, \mathrm{Mo}$
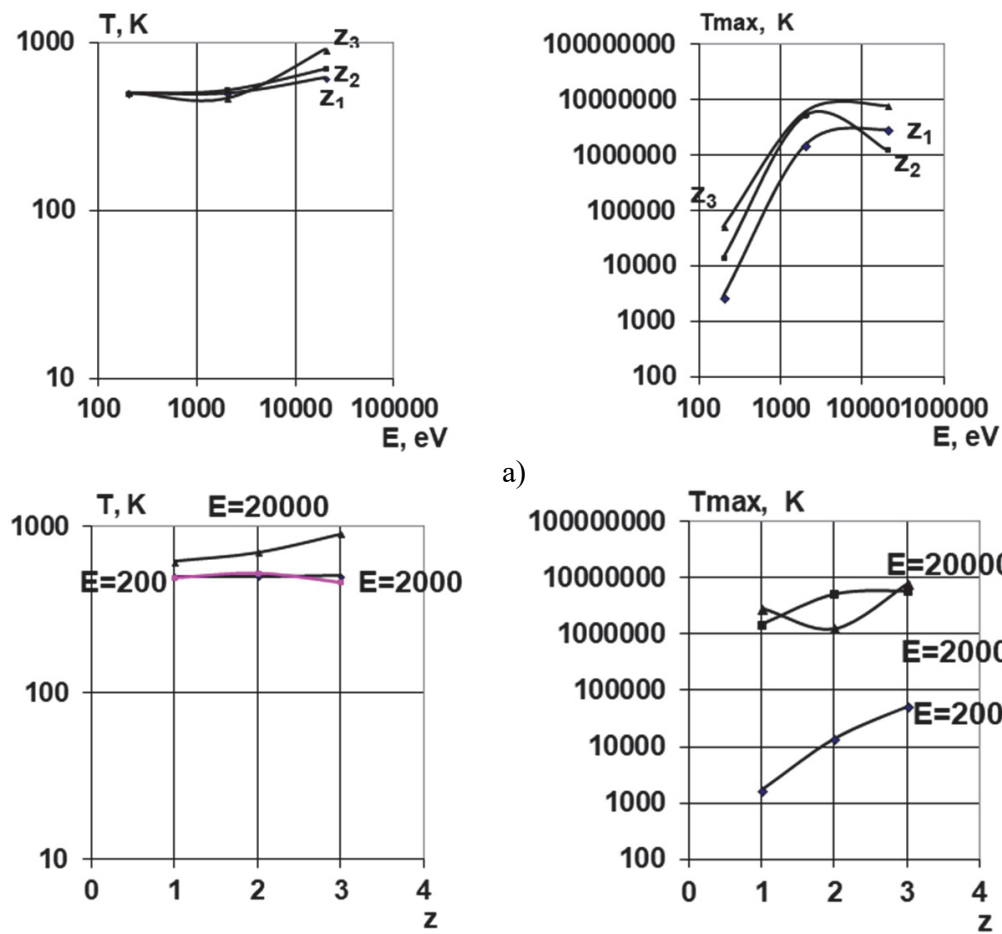

a)

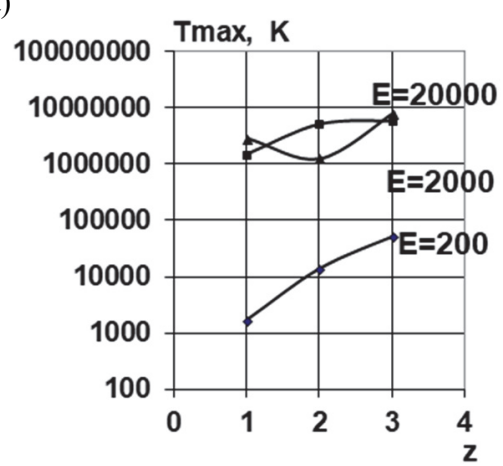

b)

Fig. 7. Plots of $T$ and $T$ the maximum energy from the a) ion charge and b) ion $\mathrm{Hf}, \mathrm{Ta}, \mathrm{W}, \mathrm{Pt}$ 


\subsection{Research results}

The rate of rise in temperature is an important factor in the possibility of forming a nanostructure which is formed at the rate of increase in temperature greater than $10^{7} \mathrm{~K} / \mathrm{s}$. The calculation results are obtained depending on the rate of rise of temperature on the energy and charge are shown in Figs. 8, 9. It is seen that with increasing energy and charge increases the rate of rise in temperature, ranging from $10^{16}$ to $10^{18} \mathrm{~K} / \mathrm{s}$.
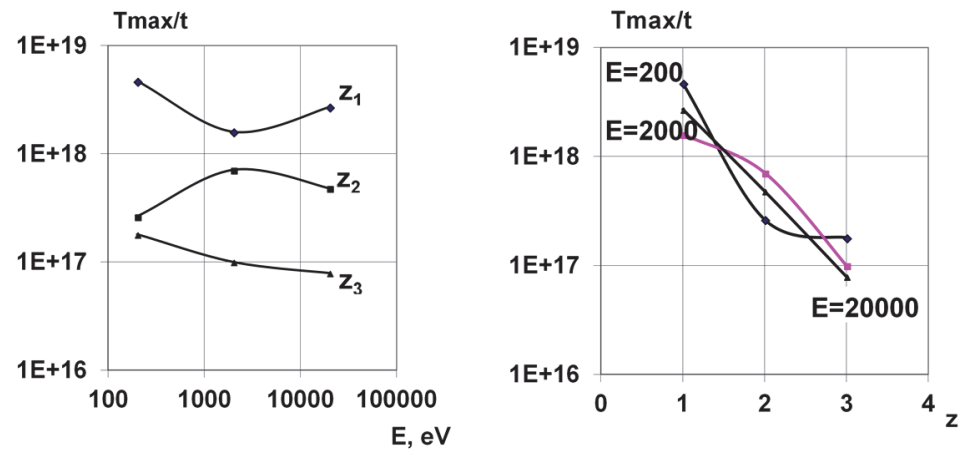

a)
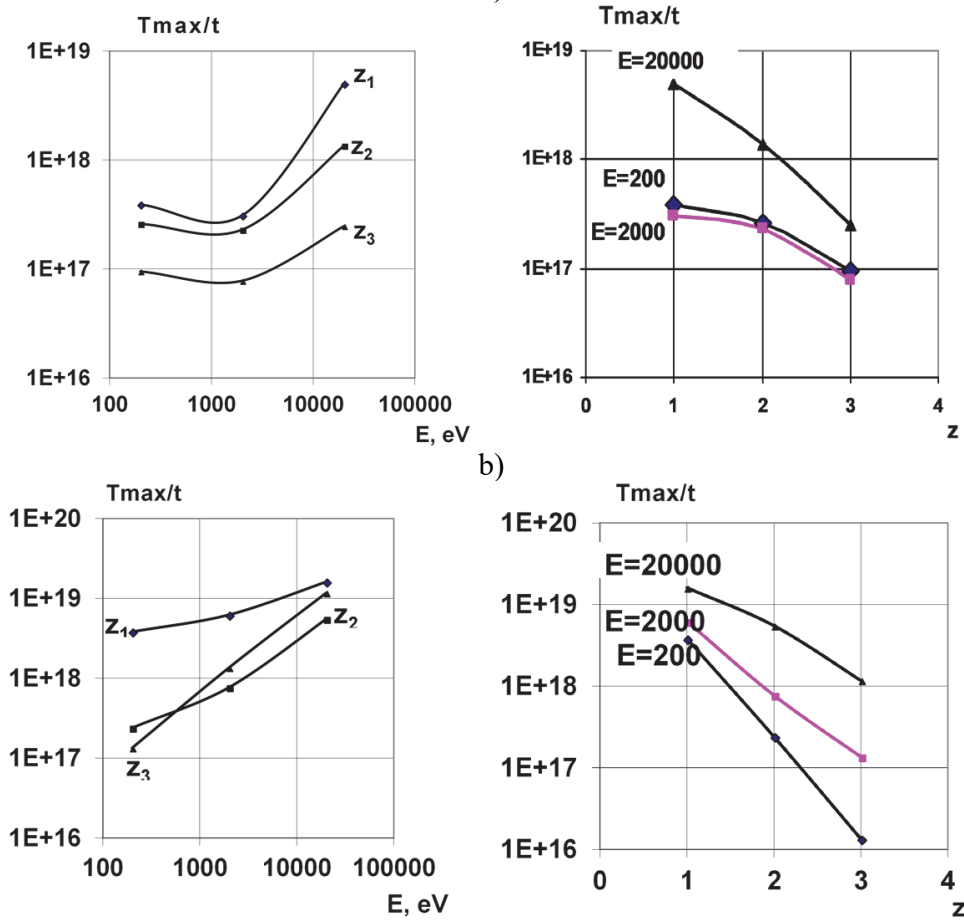

b)

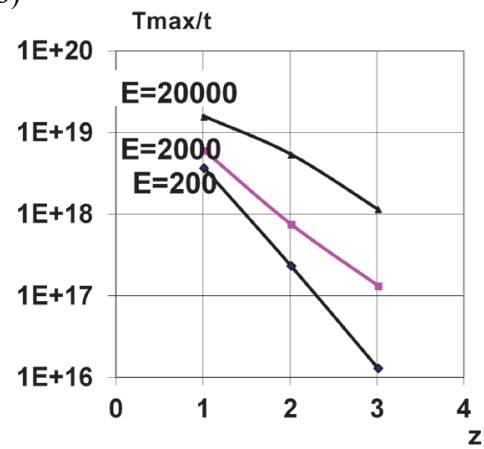

c)

Fig. 8. The graphs of the rate of rise in temperature the energy and charge of ions:

a) $\mathrm{C}, \mathrm{B}, \mathrm{N}$; b) $\mathrm{Al}$; c) $\mathrm{Ti}, \mathrm{V}, \mathrm{Cr}$

\subsection{Conclusions}

The dependence of the maximum temperature of energy and ion charge in steel treatment showed that their growth is increasing the maximum temperature for all investigated types of ions.

Investigation of the temperature dependence of the rate of increase of energy and charge of the 
ions showed that with increasing energy and charge the rate of rise in temperature increases and reaches the maximum values for ions.

The values obtained for the temperature and rate of rise of temperature confirm the reality of the formation of nanoclusters in filling a number of layers of nanostructures by the simultaneous action of different kinds of ions, energy and charge and receive nanolayer sufficient surface detail.
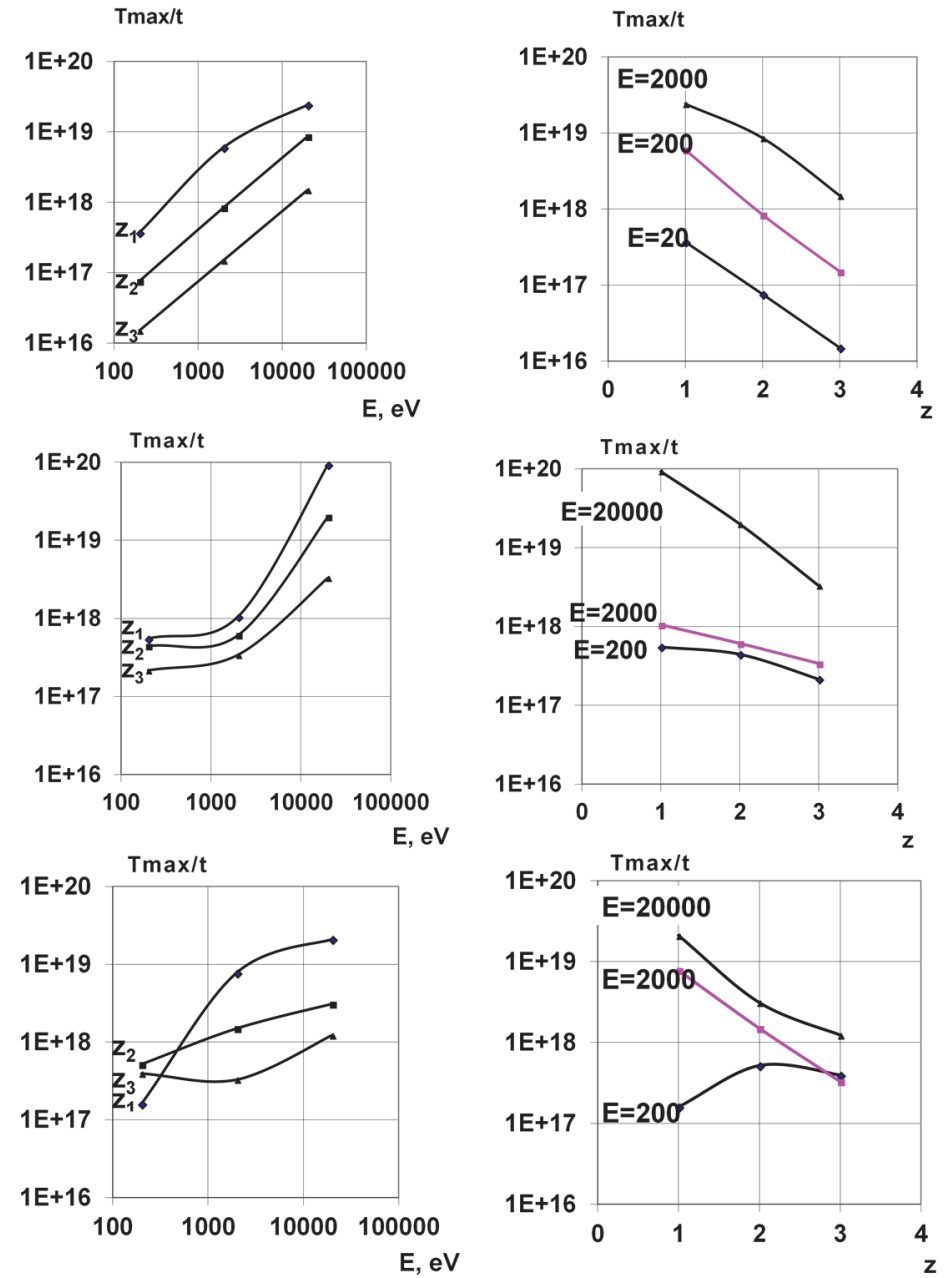

Fig. 9. The graphs of the rate of rise in temperature of energy and charge of the ions:

a) Fe, Ni, Co; b) Y, Zr, Mo; c) Hf, Ta, W, Pt

\section{Research quantities of thermal stresses in the zone of ions of various sorts, energy and charge for steel}

In addition to the required temperature and rate of rise of temperature important factor is the presence of pressure (thermal stress) in the area of formation of nanostructures. In the first two criteria defined area nanocluster. Conduct a review of the results of calculations of thermal stresses on the boundary of the formation of nanostructures on the first two criteria $-\sigma_{h 1}$ and the maximum value of thermal stress $-\sigma_{h 2}$ under various charges of 1 to 3, Energy $-200,2000$ and $20000 \mathrm{eV}$ (the actual technologies for hardening) ions $\mathrm{C}, \mathrm{B}, \mathrm{N}, \mathrm{Al}, \mathrm{Ti}, \mathrm{V}, \mathrm{Cr}, \mathrm{Fe}, \mathrm{Ni}, \mathrm{Co}, \mathrm{Y}, \mathrm{Zr}, \mathrm{Nb}, \mathrm{Mo}, \mathrm{Hf}$, Ta, W, Pt. 


\subsection{Research results}

The results of investigations of temperature stresses on the boundary of the formation of nanostructures are shown in Fig. 10 for the ions: a) C, B, N; b) $\mathrm{Al}$; in c) Ti, V, Cr, and Fig. 11 ions: a) $\mathrm{Fe}, \mathrm{Ni}, \mathrm{Co}$; b) $\mathrm{Y}, \mathrm{Zr}, \mathrm{Nb}, \mathrm{Mo}, \mathrm{c}) \mathrm{Hf}, \mathrm{Ta}, \mathrm{W}$, Pt. It is seen that with increasing ion charge and ion energy value of their increased slightly, with the exception of aluminum ions, for which the voltage increases to energy $2000 \mathrm{eV}$, then decline. The voltages range from $10^{6}$ to $10^{8} \mathrm{H} / \mathrm{m}^{2}$, maximum values are realized for triply charged ions of $\mathrm{Hf}, \mathrm{Ta}, \mathrm{W}, \mathrm{Pt}$ and constitute $5,85 \cdot 10^{8} \mathrm{H} / \mathrm{m}^{2}$. The values of thermal stresses on the boundary of the formation of nanostructures even by themselves are not sufficient to produce nanostructures, but together with the action of the other two factors - the temperature and the rate of increase in temperature will significantly accelerate the emergence of nanostructures on the border zone.
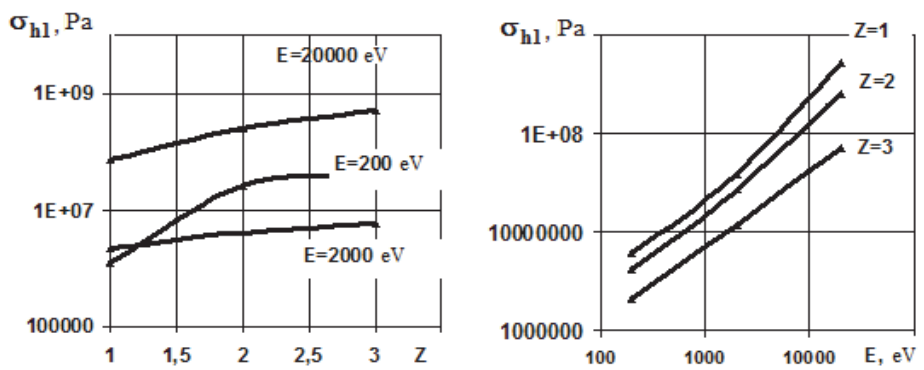

a)
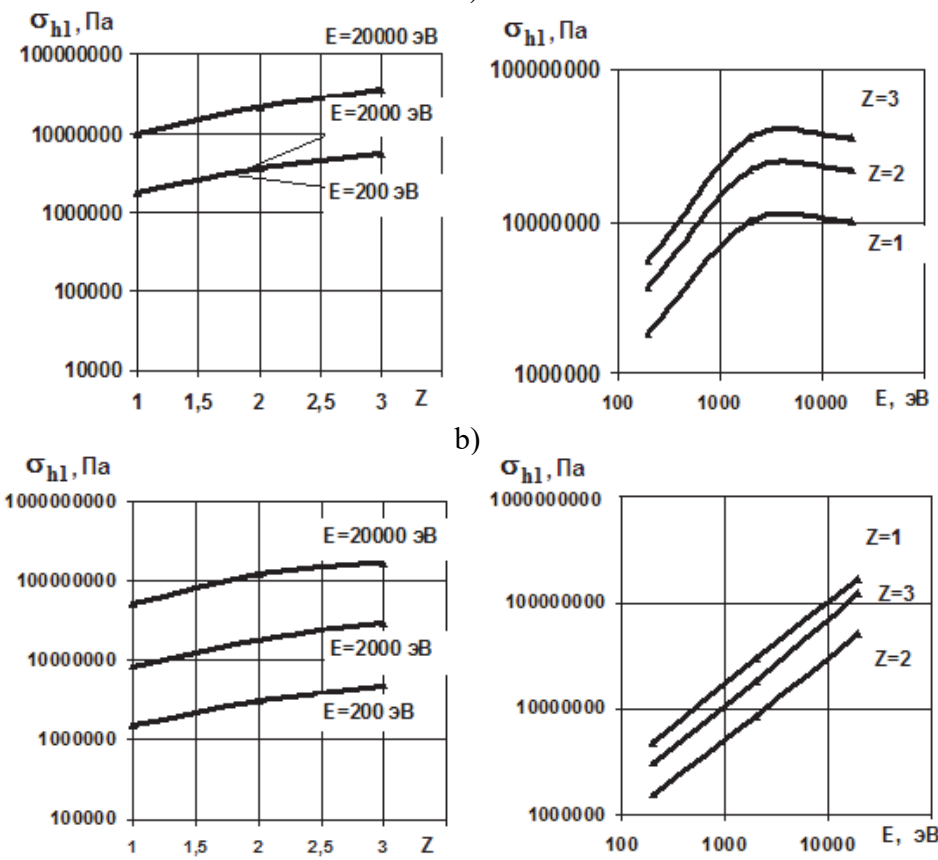

b)

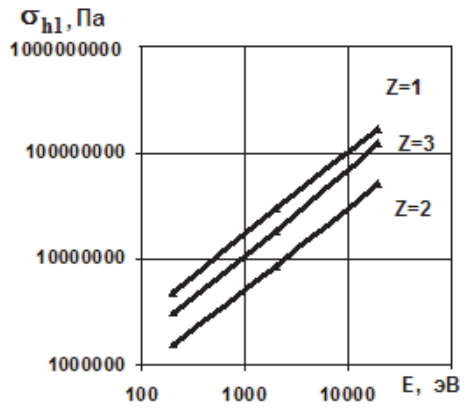

c)

Fig. 10. Plots of the values of thermal stress on the border of nanostructure formation on the charge and energy of the ions: a) $\mathrm{C}, \mathrm{B}, \mathrm{N}$; b) Al; c) Ti, $\mathrm{V}, \mathrm{Cr}$

The findings of maximum thermal stress $\sigma_{h 2}$ in nanoclusters are shown in Fig. 12 ions: a) C, B, N; b) Al; c) Ti, V, Cr and in Fig. 13 for ions: a) Fe, Ni, Co; b) Y, Zr, Nb, Mo, c) Hf, Ta, W, Pt. It can be seen that the magnitude of the stresses increased significantly, about more than an order of magnitude, which, depending on the charge (to an energy of $200 \mathrm{eV}$ ) increases, i.e., with 
increasing charge voltage temperature rise, whereas with increasing energy from $2 \cdot 10^{3}$ to $2 \cdot 10^{4} \mathrm{eV}$ and increasing the value of the charge of thermal stresses are reduced. Exceptions are ions $\mathrm{Hf}$, Ta, $\mathrm{W}, \mathrm{Pt}$, where the energies of $200 \mathrm{eV}$ and 2000, an increase in thermal stress.
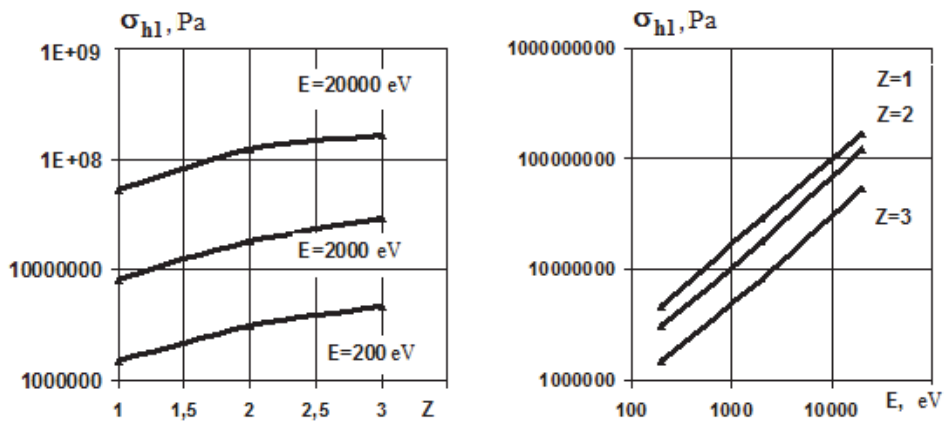

a)
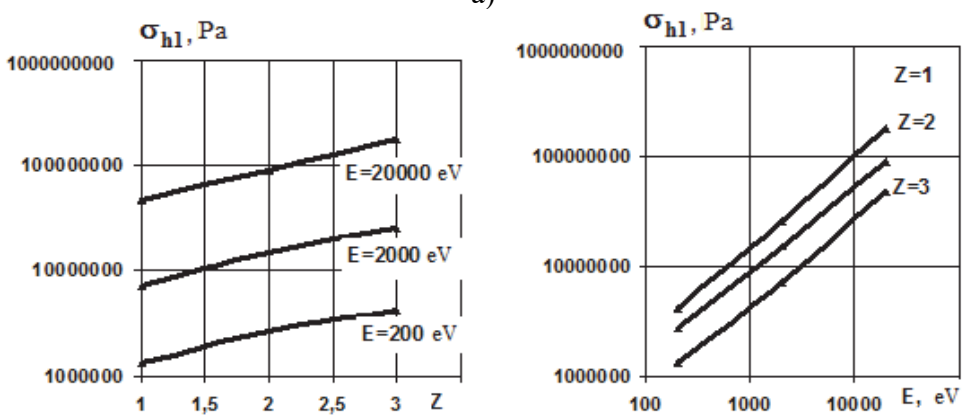

b)
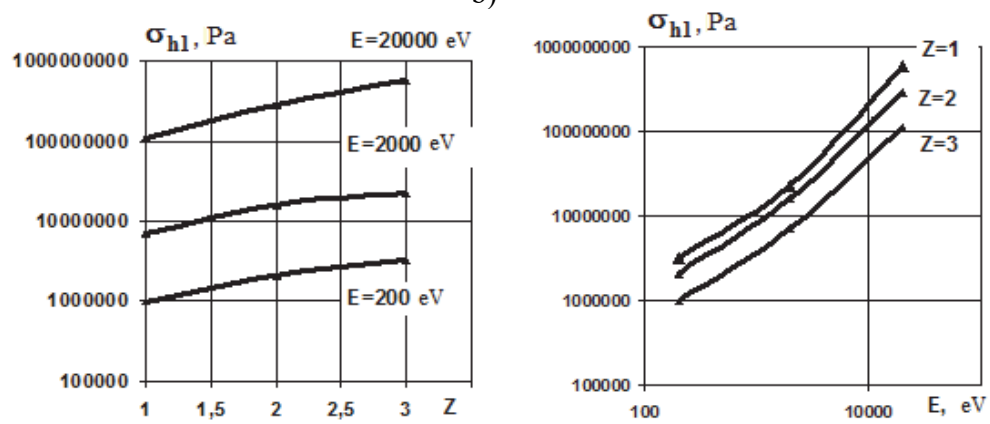

c)

Fig. 11. Plots of the values of thermal stress on the border of nanostructure formation on the charge and energy of the ions: a) Fe, Ni, Co; b) Y, Zr, Mo; c) Hf, Ta, W, Pt

The dependence of the maximum thermal stresses $\sigma_{h 2}$ the energy for almost all of the charges at a maximum ion energy of ions $2 \cdot 10^{3} \mathrm{eV}$, with the exception of dependence $\sigma_{h 2}=f\left(E_{i}\right)$ for singly charged ions of $\mathrm{Ti}, \mathrm{V}, \mathrm{Cr}, \mathrm{Fe}, \mathrm{Ni}, \mathrm{Co}, \mathrm{Y}, \mathrm{Zr}, \mathrm{Mo}$, where the energy increases the value of thermal stress are increasing, due to the small area of the nanostructure formation (nanocluster).

The values of the maximum thermal stress range from $10^{7}$ to $10^{10} \mathrm{~N} / \mathrm{m}^{2}$, the maximum value of thermal stress is $2.68 \cdot 10^{10} \mathrm{~N} / \mathrm{m}^{2}$ for singly charged ions $\mathrm{Y}, \mathrm{Zr}$, Mo.

It is seen that the maximum values of temperature and stress mostly do not reach the required pressure value for the formation of nanostructures, but together with the action required temperatures and temperature growth will accelerate or enhance the probability of formation of nanostructures.

Perhaps the emergence of an independent nanostructures by action of singly charged ions $Y$, 
$Z_{r}, M_{o} 2 \cdot 10^{4} \mathrm{eV}$ of energy when the temperature voltage reaches the necessary pressure for the formation of nanostructures.
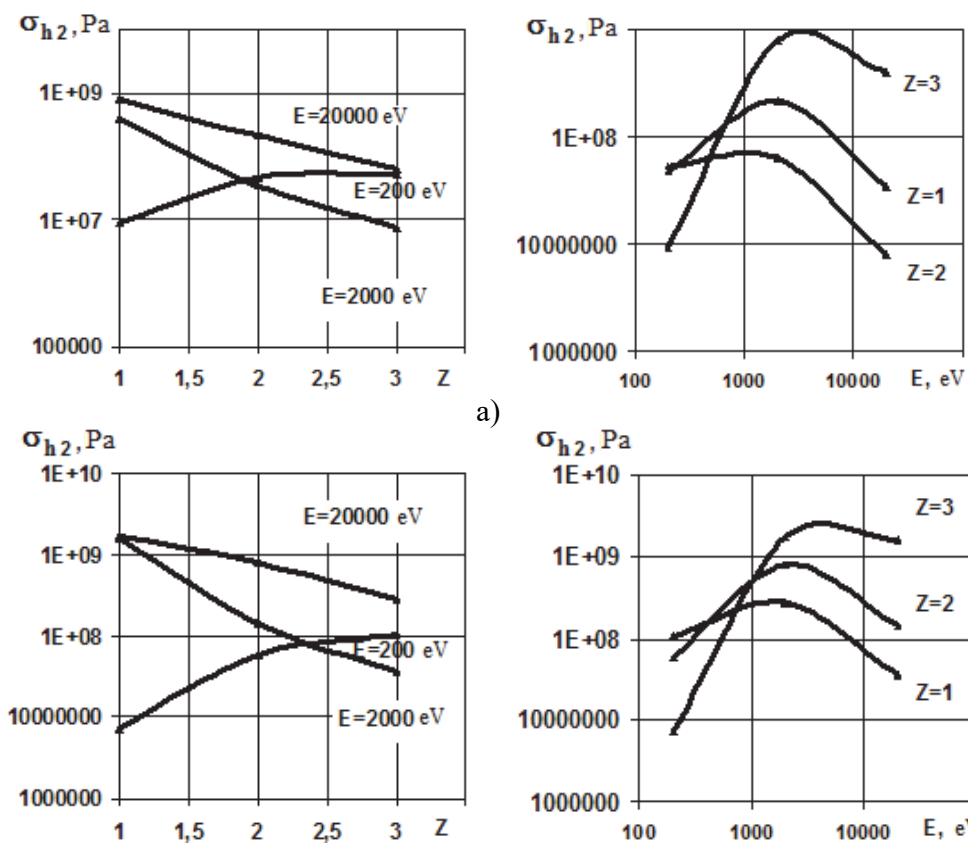

a)
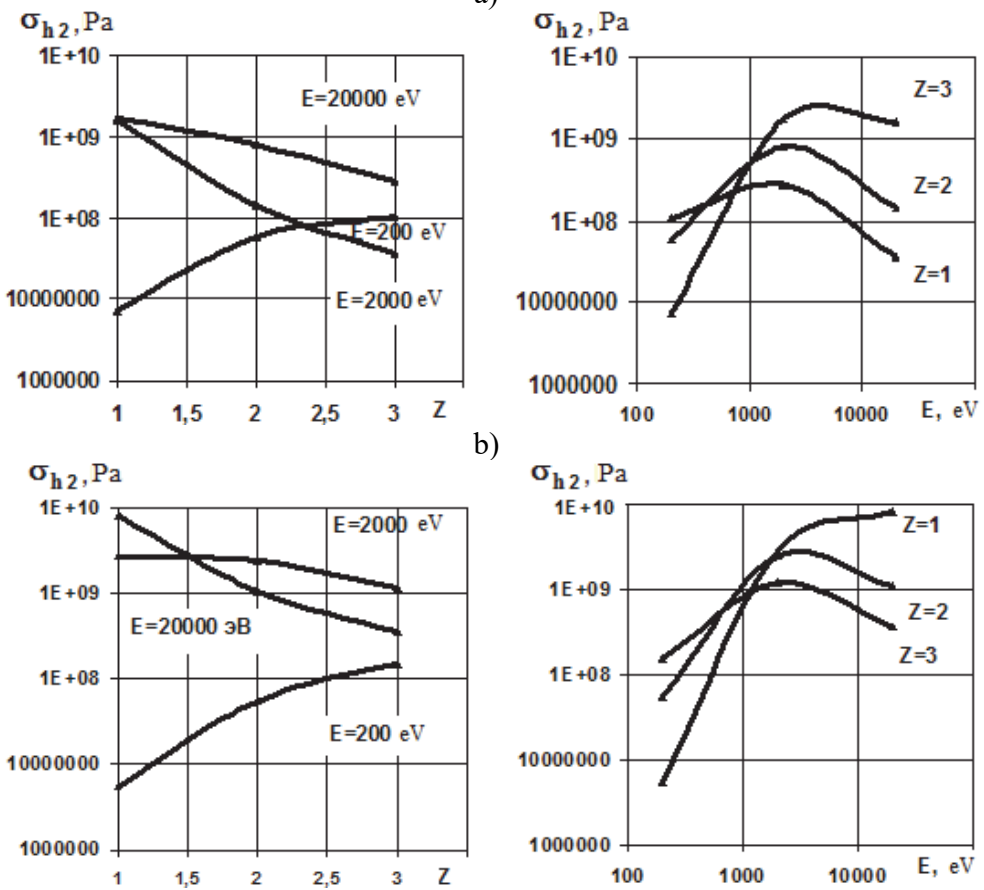

b)

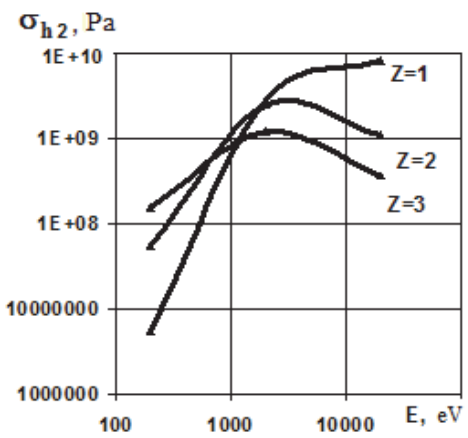

c)

Fig. 12. The graphs of the maximum values of temperature stresses on the charge and energy of the ions: a) $\mathrm{C}, \mathrm{B}, \mathrm{N}$; b) $\mathrm{Al}$; c) $\mathrm{Ti}, \mathrm{V}, \mathrm{Cr}$

\subsection{Conclusions}

1. In the example of processing steel the possibility substantial acceleration of nanostructure formation by the action of thermal stresses on the boundary of the nanocluster and in its entirety.

2. The possibility of formation of nanostructures only by the action of thermal stresses of singly charged ions $\mathrm{Y}, \mathrm{Zr}$, Mo at energies eV $2 \cdot 10^{4}$ when thermal stresses exceed $10^{10} \mathrm{~N} / \mathrm{m}^{2}$.

3 . The resulting thermal stresses depending on the zone boundary nanostructure formation and maximum thermal stress on the charge and energy allows for a wide range of ions $\mathrm{C}, \mathrm{B}, \mathrm{N}, \mathrm{Al}, \mathrm{Ti}$, $\mathrm{V}, \mathrm{Cr}, \mathrm{Fe}, \mathrm{Ni}, \mathrm{Co}, \mathrm{Y}, \mathrm{Zr}, \mathrm{Nb}, \mathrm{Mo}, \mathrm{Hf}, \mathrm{Ta}, \mathrm{W}, \mathrm{Pt}$ in the processing of steel to choose the process conditions and the type of ions to obtain the desired thermal stress, accelerating the formation of nanostructures. 


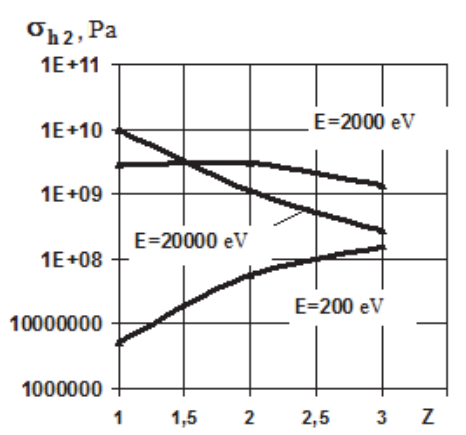

a)

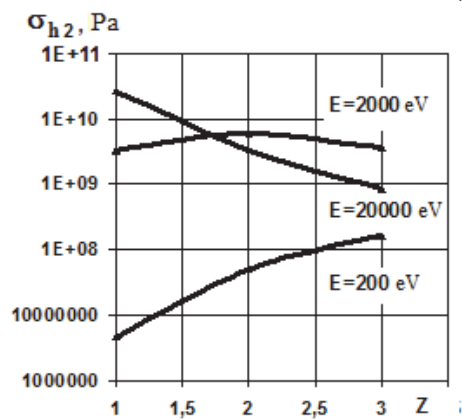

b)

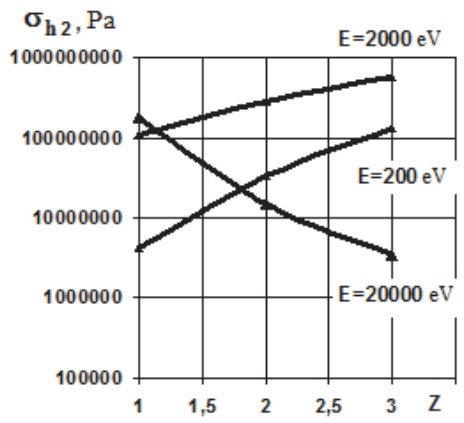

c)
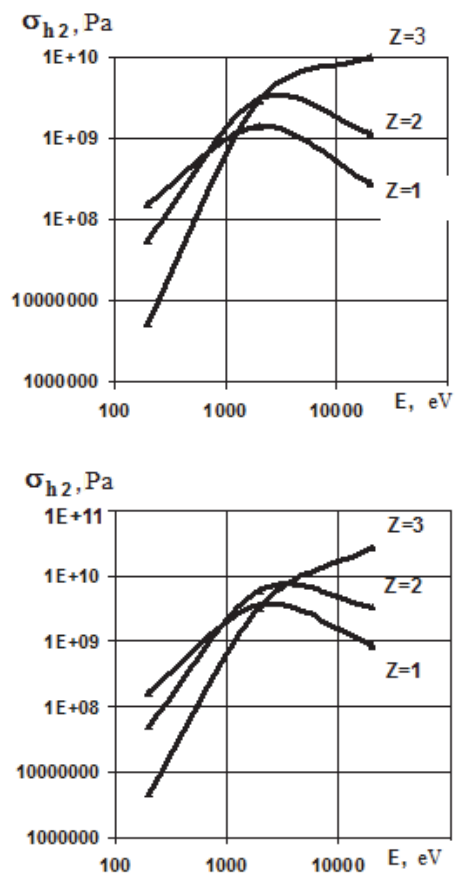

$\sigma_{\mathrm{h} 2}, \mathrm{~Pa}$

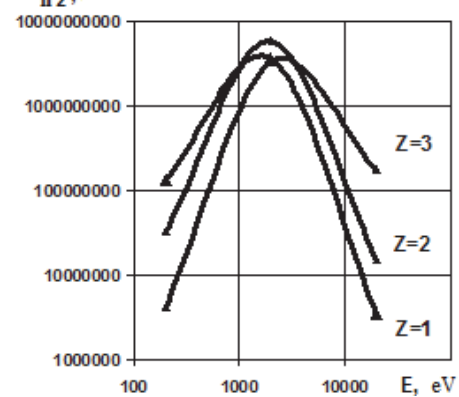

Fig. 13. The graphs of the maximum values of temperature stresses on the charge and energy of the ions: a) Fe, Ni, Co; b) Y, Zr, Mo; c) Hf, Ta, W, Pt

\section{Influence of the type of ion, its energy and charge on the grain size and number of particles in nanocluster}

The size of the grains of the material defined area where temperatures exceed 500-1500 K, the rate of rise in temperature over $10^{7} \mathrm{~K} / \mathrm{s}$ and pressure values (thermal stress) about $10^{7}, \ldots, 10^{10} \mathrm{~Pa}$. In the fields of temperature, operating by the end of the thermal action of the ion, it highlights a zone where the temperature exceeded the necessary temperature rise rate for almost all studied types of ions, and the values of thermal stresses are close to or above required for current densities less than or equal to the first critical (for the duration of the heat source particles is observed imposing temperature fields of action of neighboring particles).

Given these criteria formation of nanostructures were obtained depending on the grain size of the ion energy (energy of $2 \cdot 10^{2}, 2 \cdot 10^{3}$ and $\left.2 \cdot 10^{4} \mathrm{eV}\right)$ at different charge number of ions $(z=1,2$ and 3$)$ and the grain size of the ion charge $(z=1,2$ and 3$)$ for different ion energies $\left(2 \cdot 10^{2}, 2 \cdot 10^{3}\right.$ and $2 \cdot 10^{4} \mathrm{eV}$ ) ions for the groups that differ little by weight of $1-\mathrm{P}, \mathrm{B}, \mathrm{N} ; 2-\mathrm{AI} ; 3-\mathrm{Ti}, \mathrm{V}, \mathrm{Cr}$; $4-\mathrm{Fe}, \mathrm{Ni}, \mathrm{Co} ; 5-\mathrm{Y}, \mathrm{Zr}$, Mo; $6-\mathrm{Hf}$, Ta, W, Pt, which are shown in Figs. 14-25. It is seen that 
with increasing ion charge the grain size increases for all examined types of ions, but the absolute value decreases during the transition from the lighter ions $(N)$ to heavier $\left(P_{t}\right)$ (Fig. 25).

The increase in grain size with increasing ion charge due to the fact that with increasing ion charge increases mileage and hence the growing zone in which the subject field of temperature and stress, and hence the area where they exceed the criterial value for nanostructures. By increasing the ion energy increases the grain size (cluster) that is also associated with increasing mileage, and hence the size of the area in which it is possible obtaining nanostructures.

Knowing the size of the grains, one can predict the microhardness, tensile strength, friction coefficient, wear resistance and other physical and mechanical performance. For example, in Fig. 26 and 27 are generalized dependence of the microhardness and yield stress of the grain size derived from surveys [12] or from [3,4]. These values can give a forecast of the microhardness and yield stress. Using the data book [1].

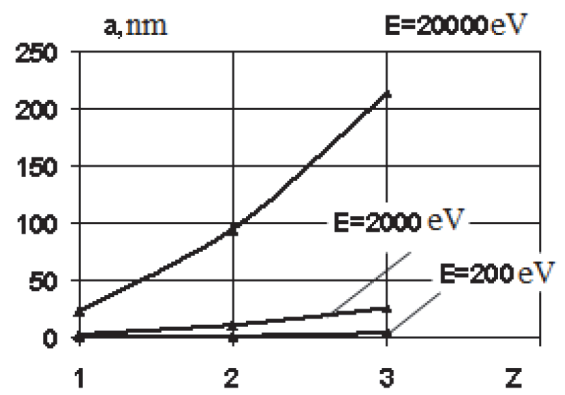

Fig. 14. The plots of the grain size of the ion charge $\mathrm{C}, \mathrm{B}, \mathrm{N}$ at energies $2 \cdot 10^{2}, 2 \cdot 10^{3}$ and $2 \cdot 10^{4} \mathrm{eV}$

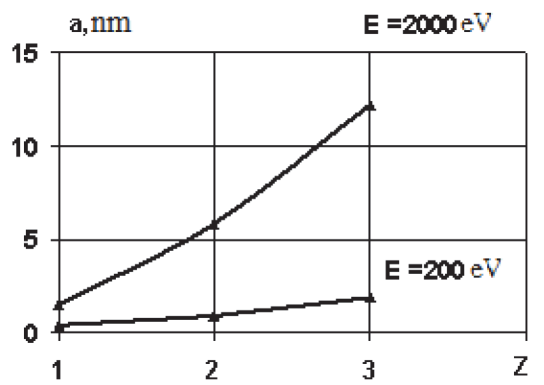

a)

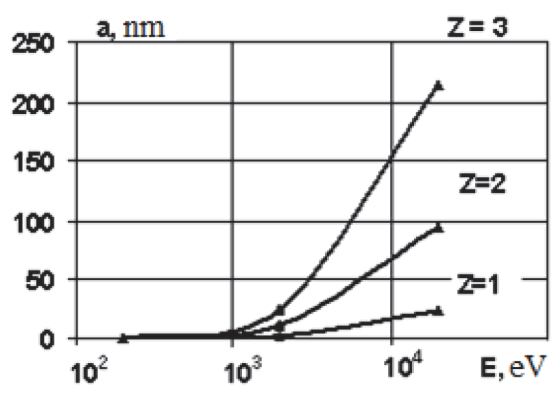

Fig. 15. The plots of the grain size of the ion energy $\mathrm{C}, \mathrm{B}, \mathrm{N}$ at the charges 1,2 and 3

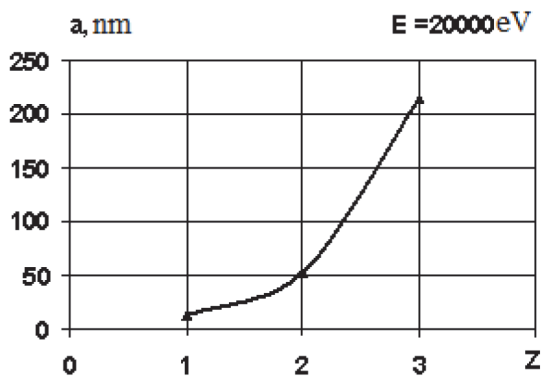

b)

Fig. 16. The plots of the grain size of the charge of $\mathrm{Al}$ ions at energies a) $2 \cdot 10^{2}, 2 \cdot 10^{3}$ and b) $2 \cdot 10^{4} \mathrm{eV}$

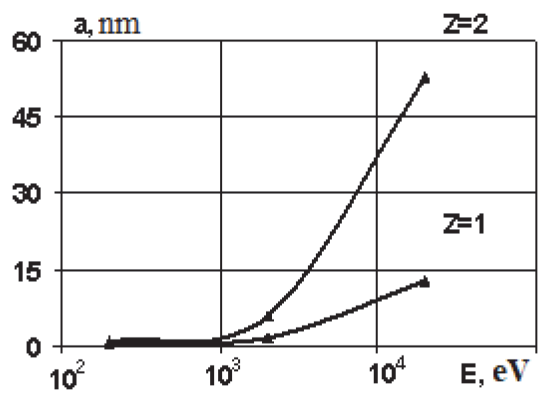

a)

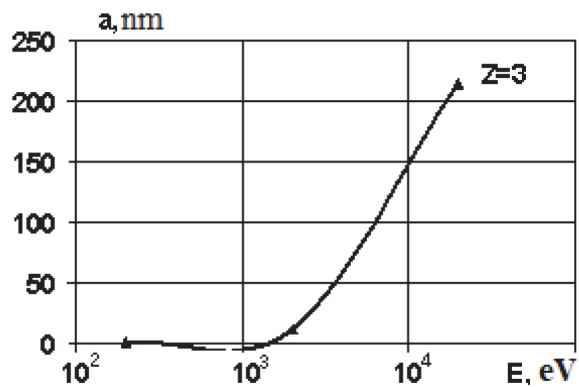

b)

Fig. 17. The plots of the grain size of the energy of $\mathrm{Al}$ ions when a) charges 1,2 and b) 3 


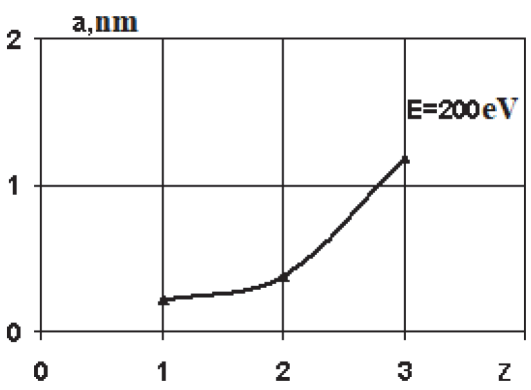

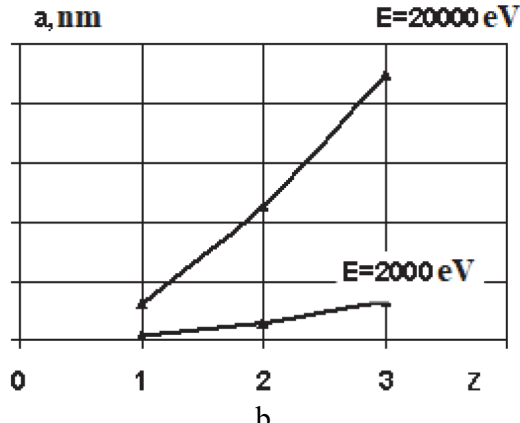

b

Fig. 18. The plots of the grain size of the charge of the ions Ti, V, Cr at energies a) $2 \cdot 10^{2} \mathrm{eV}$ and b) $2 \cdot 10^{3}, 2 \cdot 10^{4} \mathrm{eV}$

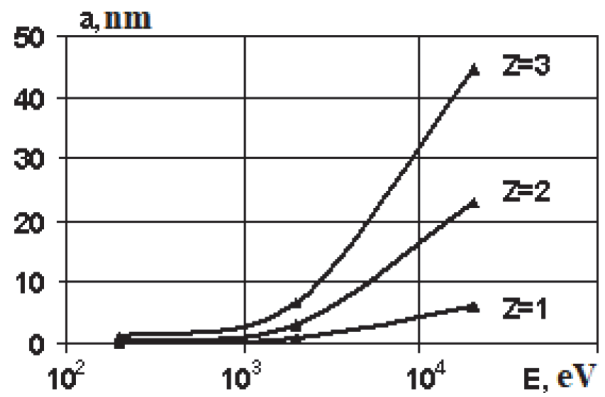

Fig. 19. The plots of the grain size of the ion energy Ti, V, Cr charges at 1,2 and 3
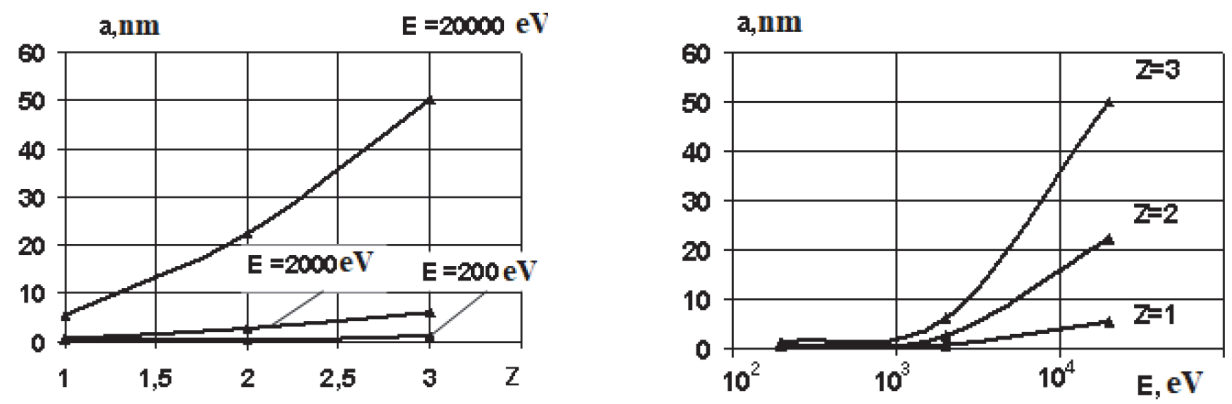

Fig. 20. The plots of the grain size of the charge of the Fig. 21. The plots of the grain size of the energy of ions $\mathrm{Fe}, \mathrm{Ni}$, Co at energies $2 \cdot 10^{2}, 2 \cdot 10^{3}, 2 \cdot 10^{4} \mathrm{eV}$ the ions $\mathrm{Fe}, \mathrm{Ni}$, Co when charging 1,2 and 3
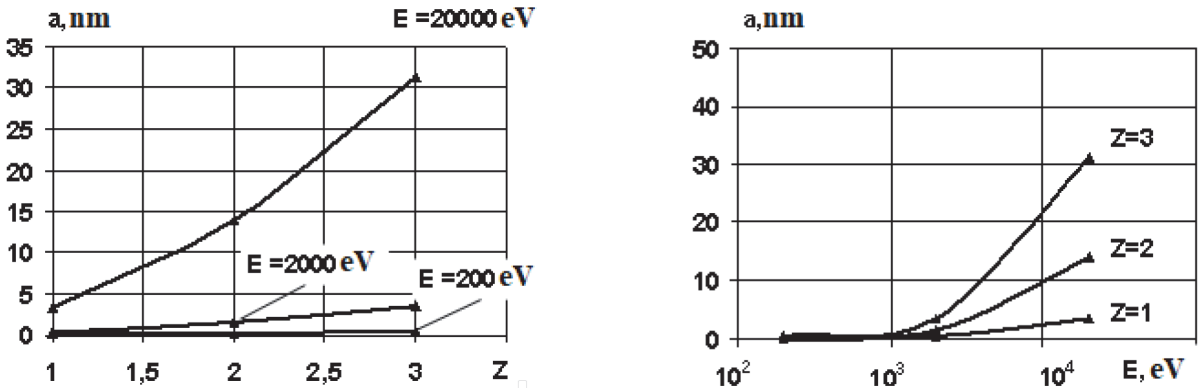

Fig. 22. The plots of the grain size of the charge of the Fig. 23. The plots of the grain size of the energy of ions $\mathrm{Y}, \mathrm{Zr}$, Mo at energies $2 \cdot 10^{2}, 2 \cdot 10^{3}, 2 \cdot 10^{4} \mathrm{eV}$ the ions $\mathrm{Y}, \mathrm{Zr}$, Mo when charging 1.2 and 3 


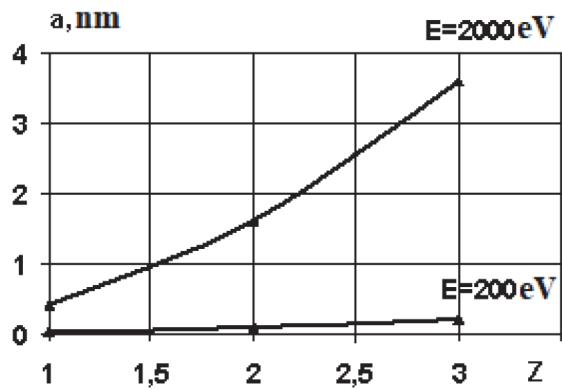

a)

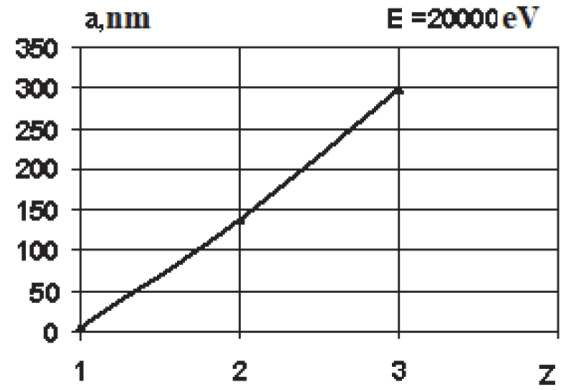

b)

Fig. 24. The plots of the grain size of the charge of the ions $\mathrm{Hf}, \mathrm{Ta}, \mathrm{W}, \mathrm{Pt}$ at energies a) $2 \cdot 10^{2}, 2 \cdot 10^{3}$ and b) $2 \cdot 10^{4} \mathrm{eV}$

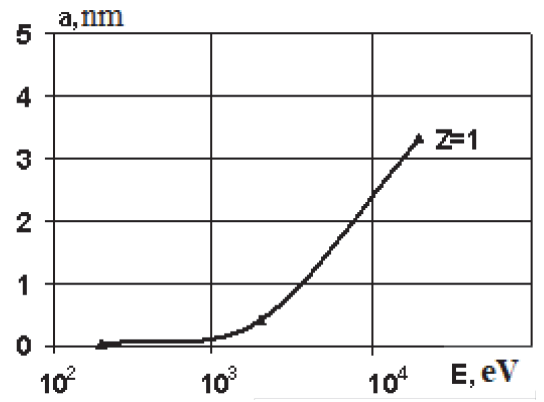

a)

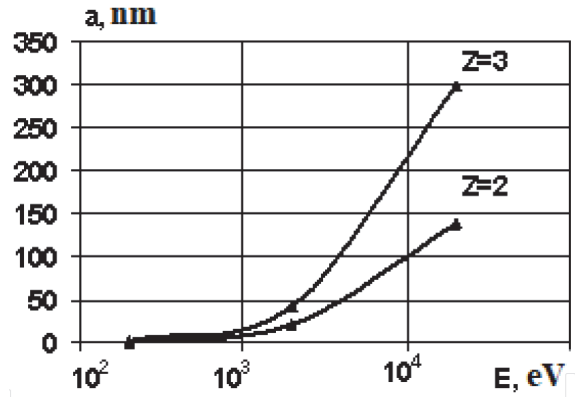

b)

Fig. 25. Plots of the grain size of the ion energy Hf, Ta, W, Pt when charges a) 1 and b) 2,3

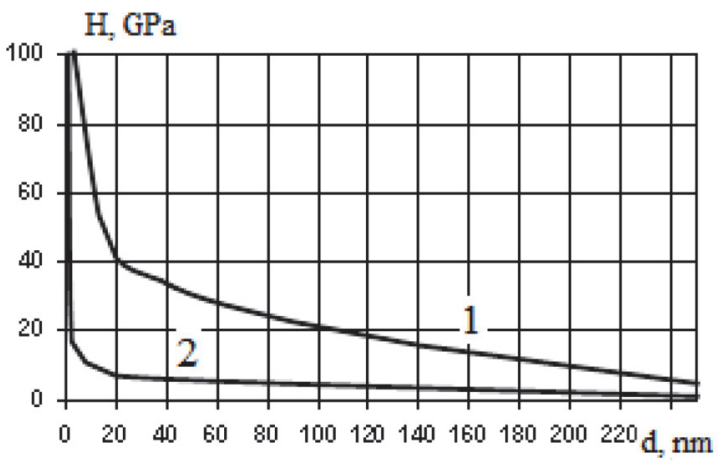

Fig. 26. The results generalize the microhardness grain size: 1 - maximum values; 2 - minimum

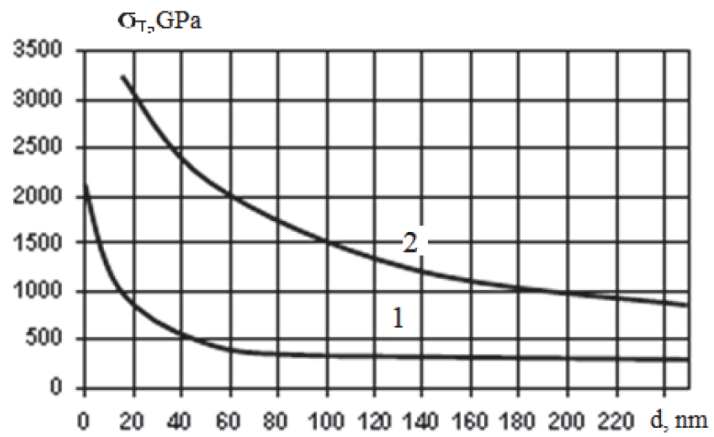

Fig. 27. The results are generalizations based yield strength the grain size of the 1.2 


\section{Investigation of the effect of the charge and grade ion energy the number of particles in nanocluster}

The number of particles in the nanocluster nanocluster determines the possibility of obtaining with the usual bars, when the number of particles greater than 4 , or deformed bars nanoscale $n<4$ (special nanostructures), so this study is necessary.
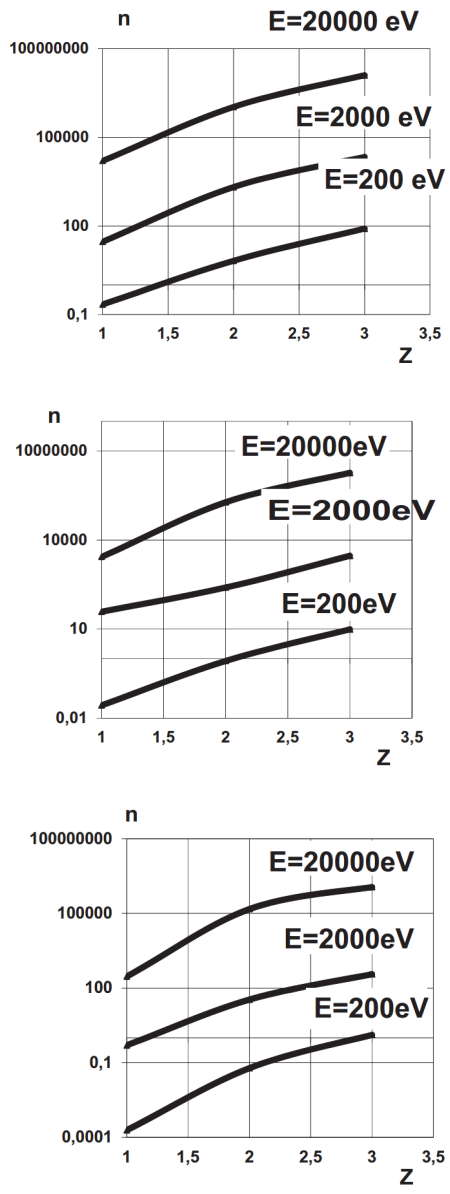

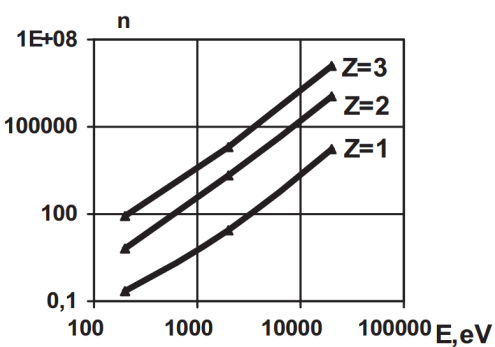

a)

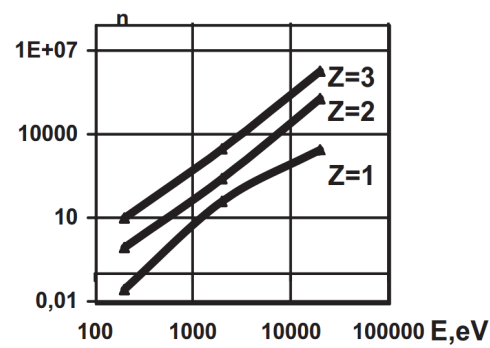

b)

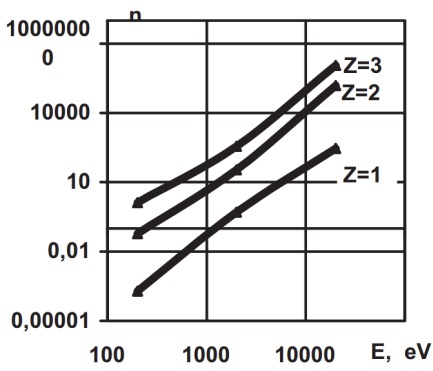

c)

Fig. 28. The graphs of the number of particles in nanoclusters on the charge $\mathrm{z}$, and ion energy Ei ions: a) C, B, N; b) Al; c) Ti, V, Cr

The calculation results of the particles in the cluster, depending on the charge and energy of the ions are shown in Fig. 28, for ions a) C, B, N, b) Al, c) Ti, V, Cr and in Fig. 29 for ions a) Fe, $\mathrm{Ni}, \mathrm{Co}$; b) Y, Zr, Mo and c) Hf, Ta, W, Pt. It is seen that with increasing ion charge increases the number of particles in the cluster, which is associated with an increase in area covered by a temperature sufficient to produce the nanostructures. The rate of temperature increase to virtually all ions than necessary $-10^{7} \mathrm{~K} / \mathrm{s}$, and the values of thermal stress (the required pressure) of the order of from $10^{7}$ to $10^{10} \mathrm{~Pa}$.

It is seen that with increasing mass ions occurs more problematic modes where nanostructures are formed only on the surface with a deformed grain, i.e. major contribution to nanograin give the incident ions at the same time for light ions at energy used in processing plants, it is possible to obtain the nanostructure in the base material parts, at the same time it is possible, for ion 
energies $2 \cdot 10^{4} \mathrm{eV}$ and lower energies but to charge number 3 (sometimes 2).

All of this suggests the need to estimate the number of particles in a nanocluster.
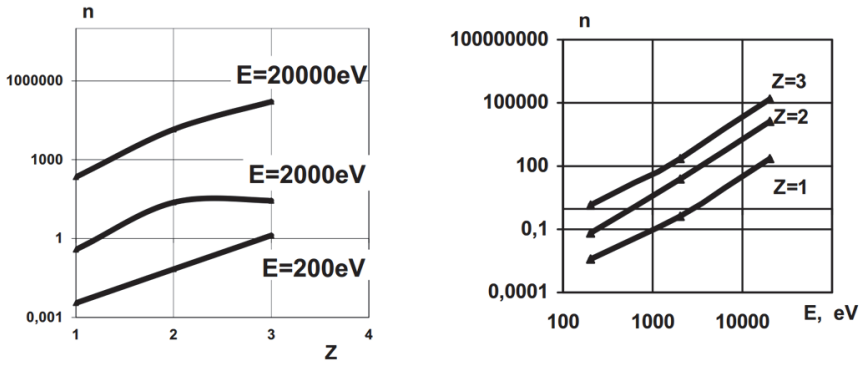

a)
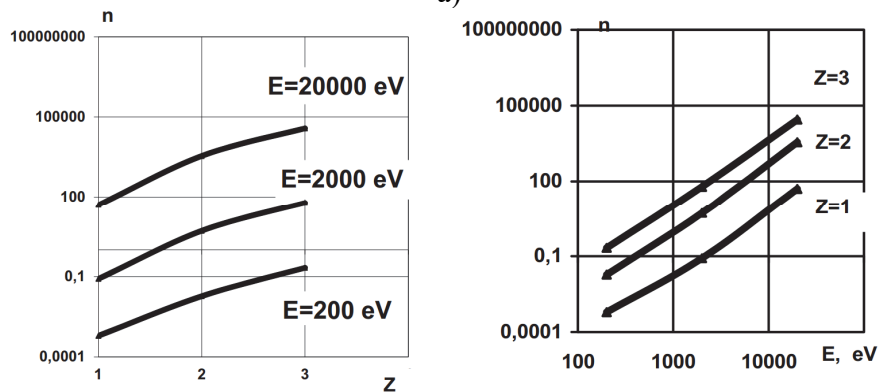

b)
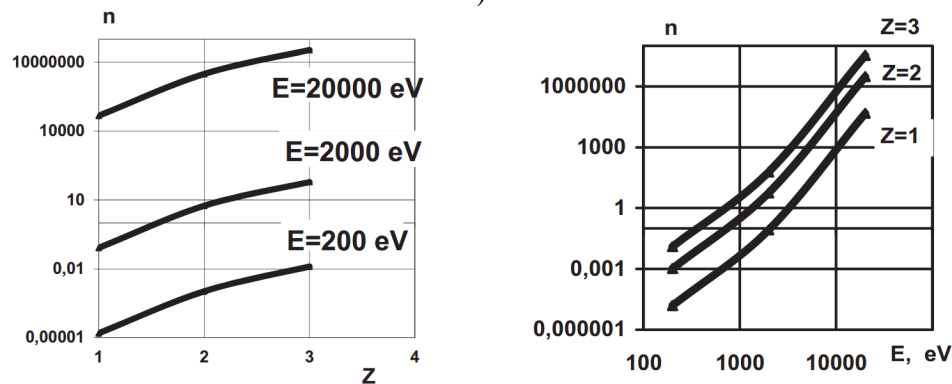

c)

Fig. 29. The graphs of the number of particles in the charge of the nanocluster charge $z$ ion energy and ion Ei: a) Fe, Ni, Co; b) Y, Zr, Mo; c) Hf, Ta, W, Pt

\subsection{Conclusions}

Revealed power class and the charges of the ions, in which the nanoclusters are implemented and are within the scope of the nanostructure as surface structures, i.e. when the base material is not involved in the details of processing.

Solution of the problem allowed us to find the extent to which conditions can be implemented to produce nanostructures. Therefore, it is possible to determine the actual number of particles that can participate in the creation of volume or flat nanostructures.

\section{Effect of the atomic mass of the ion in the grain size and number of particles in nanocluster}

Results of the study of influence of the mass of the bombarding ions on the grain size are shown in Fig. 30, and 31, which shows that for low energies 200 and $2000 \mathrm{eV}$, the charge number 1 and 2 are unlikely to provide the bulk of nanostructures in detail, whereas the energy they produced $2 \times 10^{4} \mathrm{eV}$ for all ion masses (Fig. 30(a)) when the charge number 2 has, for the energy 
of 2000 and $20000 \mathrm{eV}$ actually get the bulk of the nanostructure on the details, whereas at $E_{i}=200 \mathrm{eV}$ may receive only surface nanostructures (Fig. 30(b)) an increase in the number of charge up to 3 leads to an improvement of the situation, but to an energy of $200 \mathrm{eV}$ at ion mass more than $26 \mathrm{amu}$ implemented only the surface of the nanostructure.

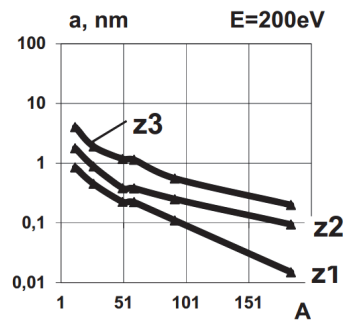

a)

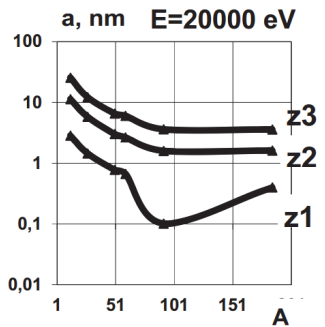

b)

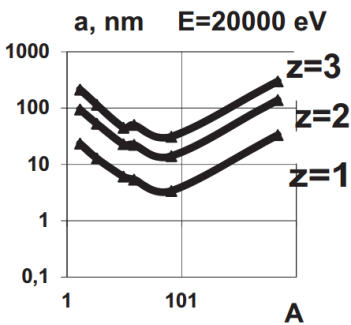

c)

Fig. 30. The plots of the grain size and the atomic mass of the incoming particles for various charges of ions and energy: a) $200 \mathrm{eV}$; b) $2000 \mathrm{eV}$ and c) $20000 \mathrm{eV}$

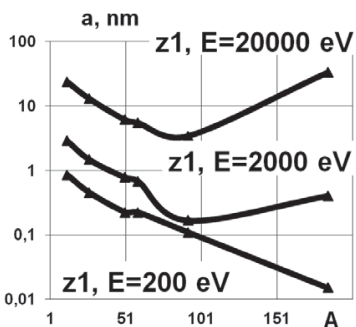

a)

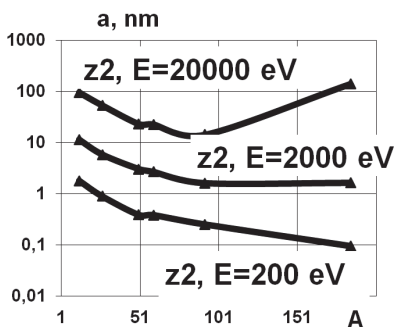

b)

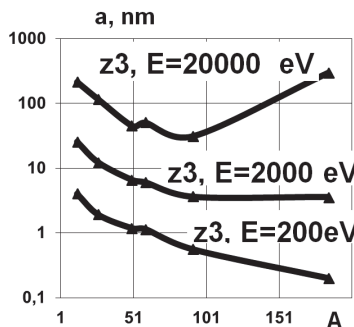

c)

Fig. 31. The plots of the grain size and the atomic mass of the ion at different energies $(200,2000$ and $20000 \mathrm{eV})$, and the charge of all particles: a) 1 ; b) 2 ; c) 3

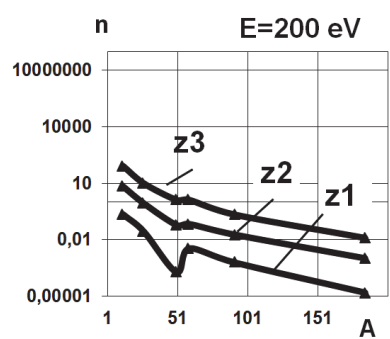

a)

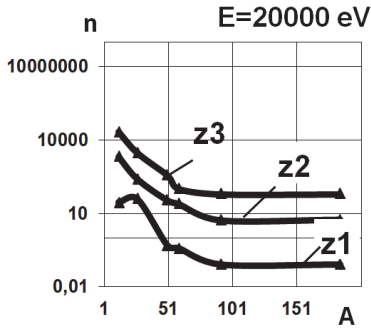

b)

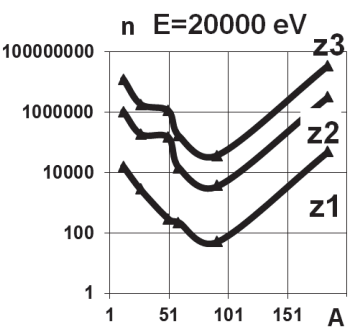

c)

Fig. 32. The graphs of the number of particles in the cluster $\mathrm{n}$ from the atomic mass of the ion $\mathrm{A}$ with an ion energy of a) $200 \mathrm{eV}$, b) $2000 \mathrm{eV}$, and c) $2 \cdot 10^{4} \mathrm{eV}$ charges for various ions $z$

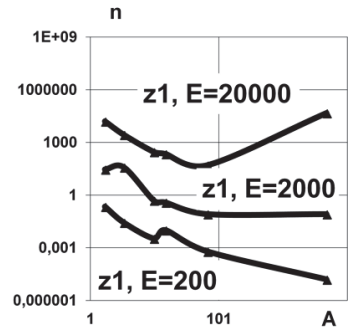

a)

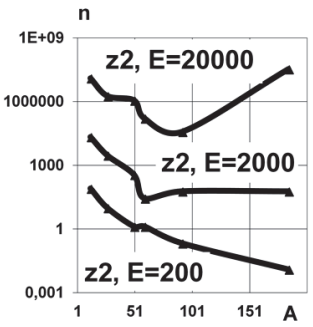

b)

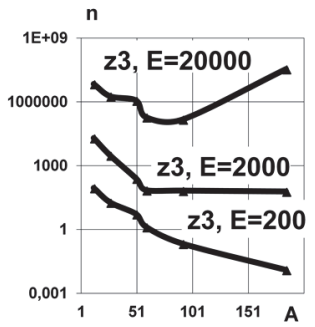

c)

Fig. 33. The graphs of the number of particles in the cluster from the atomic mass $n$ ion A ions at different energies of the different charges: a) $z=1$; b) $z=2$; c) $z=3$ 
Analysis of the dependency of the grain size on the ion mass suggests that the nanostructure is obtained or not. So out Fig. 31 and seen that at an energy of $200 \mathrm{eV}$ only if the charge number 3, and then only for a very low-mass ions can obtain a nanostructure in the amount of detail, whereas the energy of $2000 \mathrm{eV}$ can be guaranteed to get nanostructures at atomic number 2 and 3, and the charge number 1 is problematic.

At ion energies $2 \cdot 10^{4} \mathrm{eV}$ for all chargers numbers can be guaranteed to get a real three-dimensional nanostructures.

\subsection{Conclusions}

The studies allow us to determine the critical values of the atomic mass of the ion, after which it is unlikely to obtain bulk nanostructures.

\section{Effect of ion energy, their variety and magnitude of the charge on the volume of nanostructure and determining the effective current density for obtaining nanostructures}

The value for a unit volume of na'nostructures ion action allows to predict the required density of the ion current to flow to completely fill the layer where formation of this ion to the corresponding sort, energy and charge.
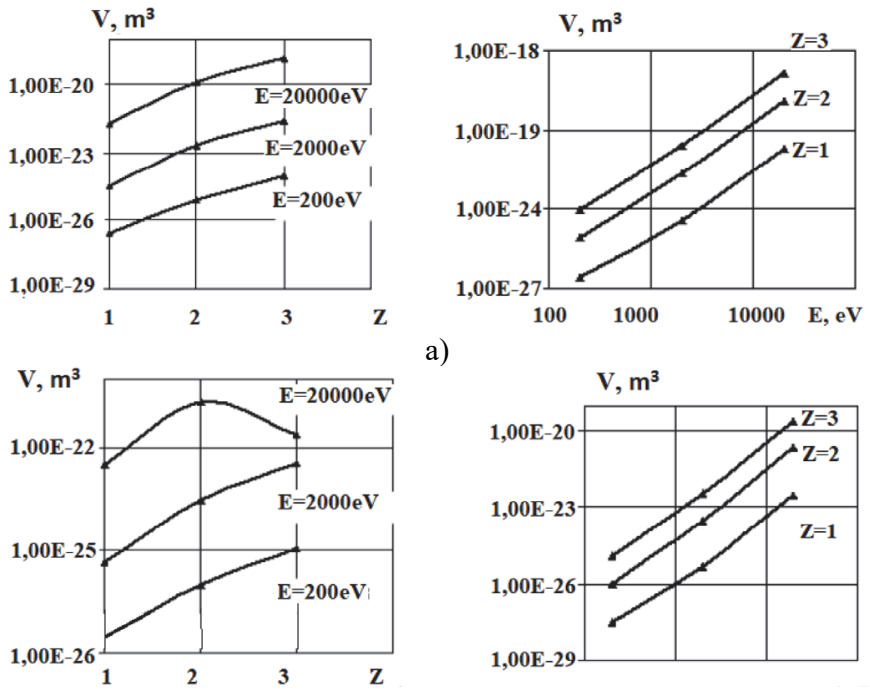

a)

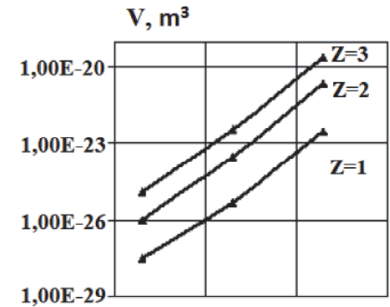

b)
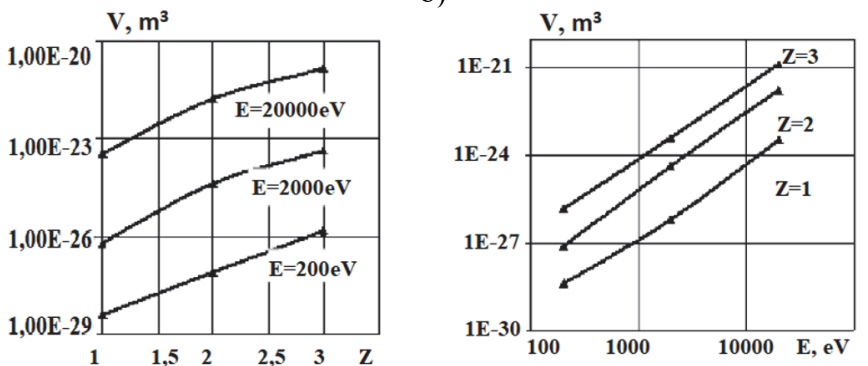

c)

Fig. 34. Plots of the extent to which implemented nanostructures on the charge number and energy of the ions: a) C, B, N, b) Al, c) Ti, V, Cr

Thus, in Fig. 34 shows a graph of the volume of nanoclusters on the charge number $z$ and the 
ion energy E: C, B, N - well; b) Al; a) Ti, V, Cr similar function for the ions Fe, Ni, Co - Fig. 35, and; Y, Zr, Nb, Mo - Fig. 35(b); Hf, Ta, W, Pt - Fig. 35 in. It is seen that with increasing energy of the ions and their charges there is a significant increase in the charge of ions affects less.
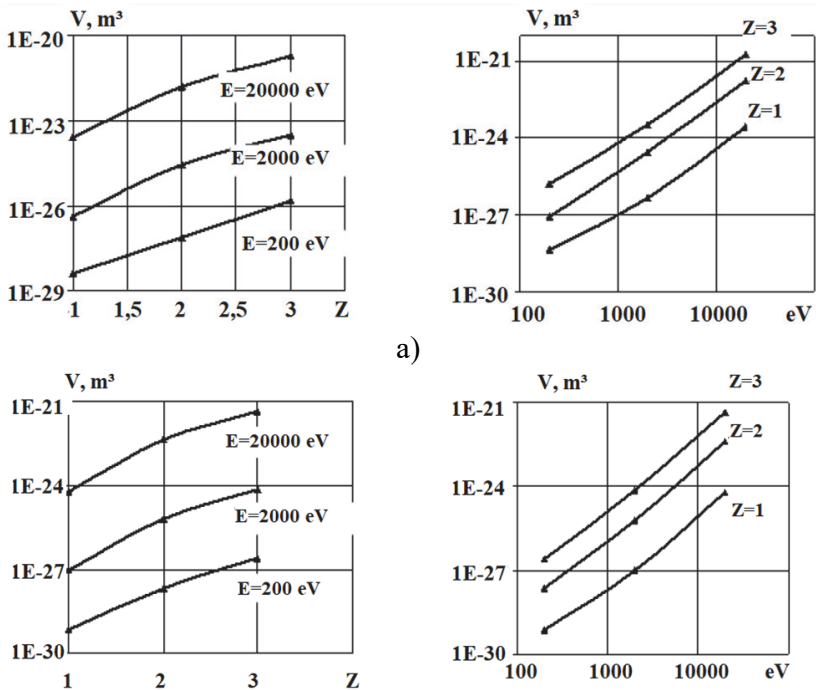

a)

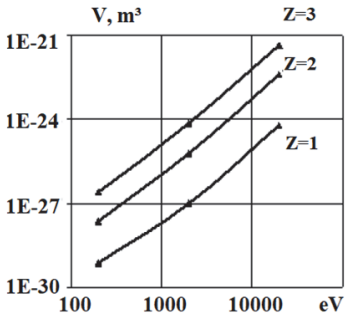

b)
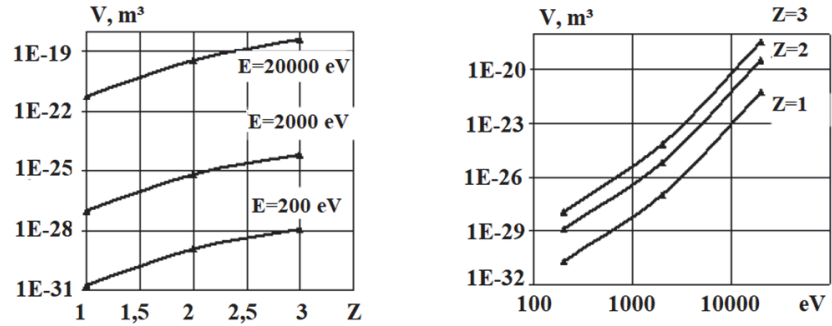

c)

Fig. 35. Plots of the volume of nanostructures on the charge and energy of the ions a) $\mathrm{Fe}, \mathrm{Ni}, \mathrm{Co}, \mathrm{b}) \mathrm{Y}, \mathrm{Zr}, \mathrm{Mo}, \mathrm{c}) \mathrm{Hf}, \mathrm{Ta}, \mathrm{W}, \mathrm{Pt}$

These dependencies are required to identify the needs of the current density of the variety, charge and energy to completely fill the layer nanostructures, and selecting appropriate energy charges and the type of ions to produce nanostructures generally required thickness, similarly assesses the current densities required in each layer.

Knowing the volume occupied by the nanostructure in the corresponding energies, grades and charge of the ions and a range of depths at which this volume is obviously possible to estimate the ion current density.

Then the known range of depths of bedding areas, where the image of the nanostructure, select power, variety and ion charge to completely fill the volume of depth, $i_{e}$ so that they complement each other. Next, determine the current density of each of the varieties of ion charge and to maximize the amount of fill required parts of the surface layer nanostructures (preferably to the end of the first layer was the beginning of the second end of the second the start of the third layer, and so on). Then for each $i$ th layer of the current density is defined as:

$j_{i}=\frac{h_{i 2}-h_{i 1}}{V_{H C i}} z_{i} e$,

where $h_{i 1}$ and $h_{i 2}$ - starting and ending coordinates of the zone where the nanostructures are 
realized for the $i$ th ion (Figs. 36-41); $V_{H C i}$ - File size area, where the image of the nanostructure; $z_{i}$ - charge number of $i$ th ion: $e$ - electron charge.
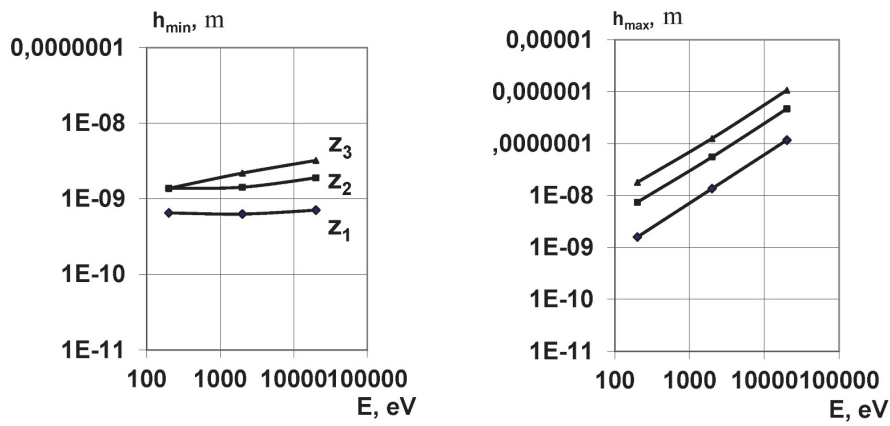

a)
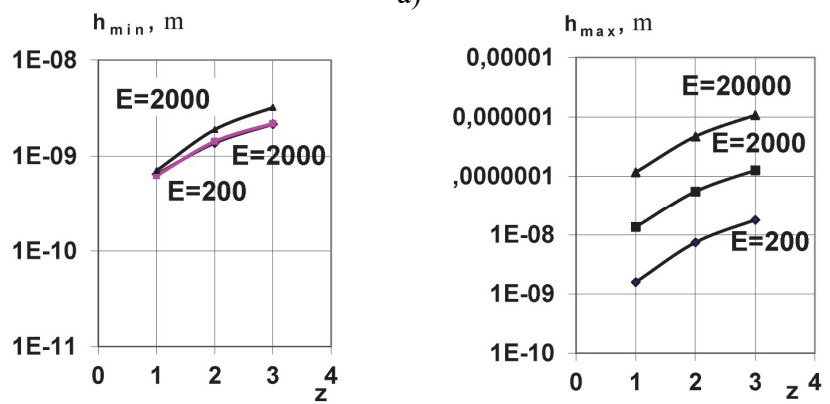

b)

Fig. 36. The graphs of the maximum and minimum $h 1 h 2$ Depth nanolayer obtain the a) energy and b) ion charge (ions $\mathrm{C}, \mathrm{B}, \mathrm{N}$ )
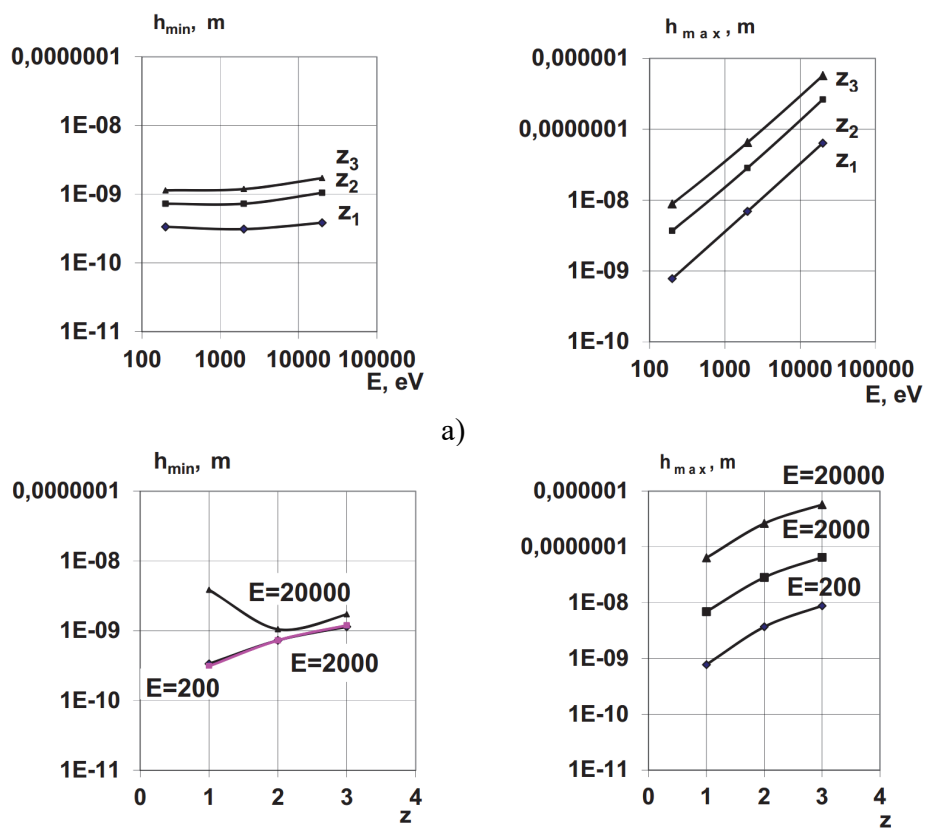

b)

Fig. 37. Graphs of the dependence of the minimum $h 1$ and maximum $h 2$ of the depth of production of the nanolayer on a) energy and $\mathrm{b}$ ) ion charge ( $\mathrm{Al}$ ion) 

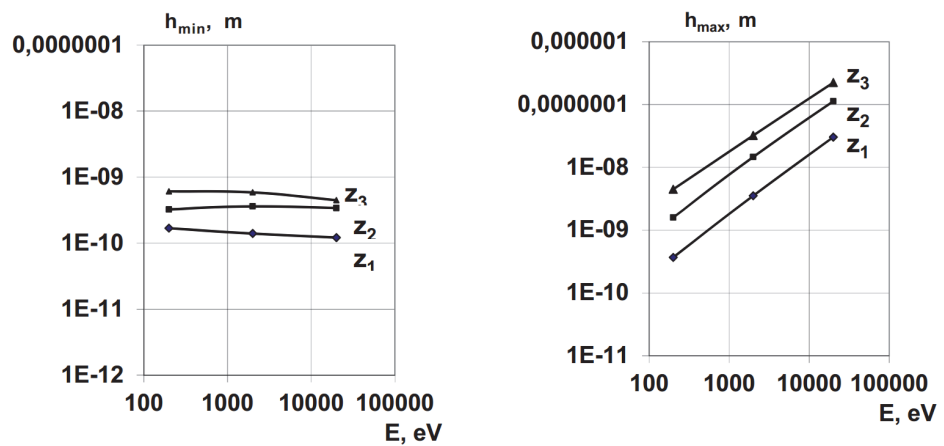

a)
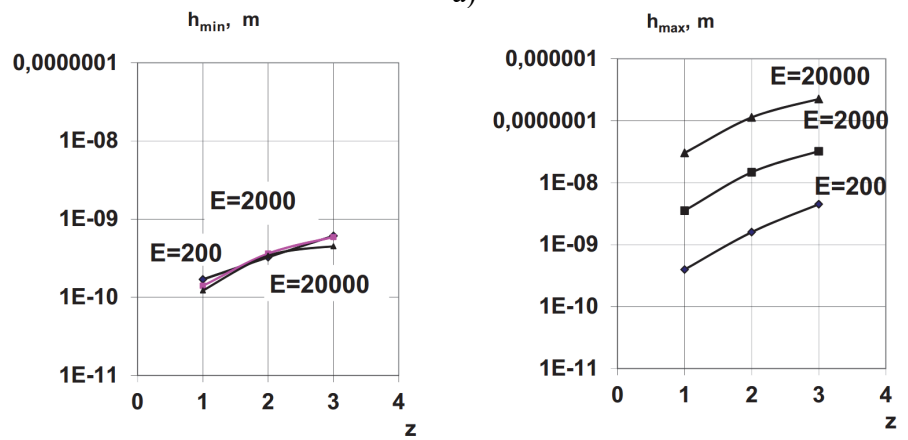

b)

Fig. 38. The graphs of the maximum and minimum $h 1 h 2$ Depth nanolayer obtain the a) energy and b) ion charge (ions $\mathrm{Ti}, \mathrm{V}, \mathrm{Cr}$ )
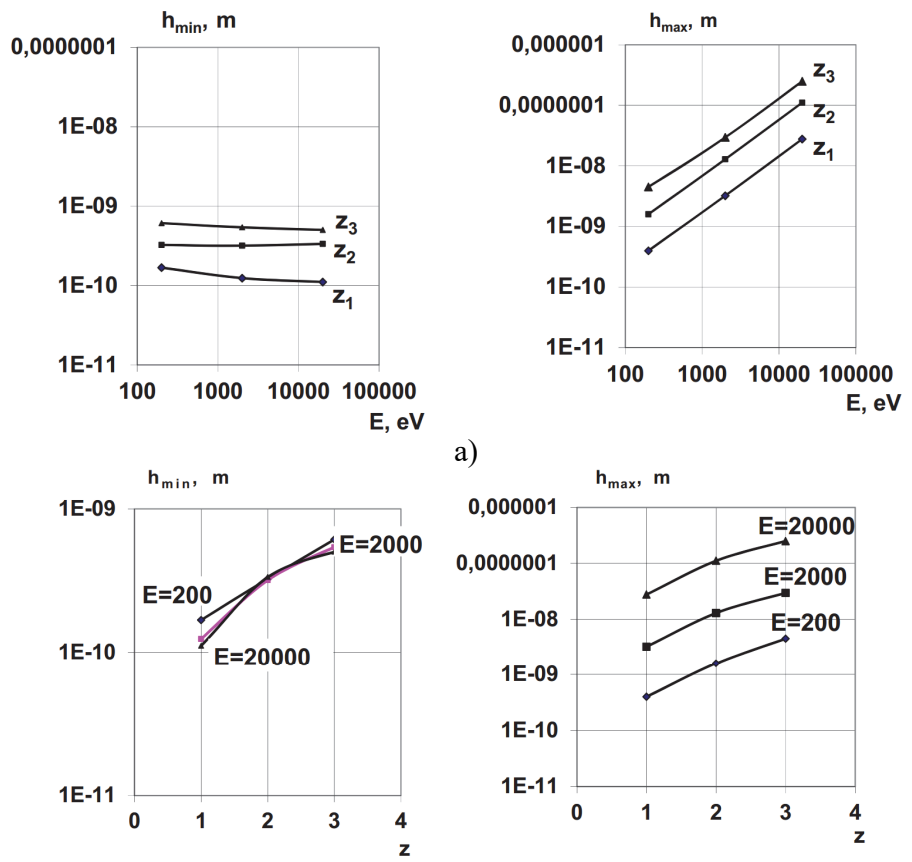

a)

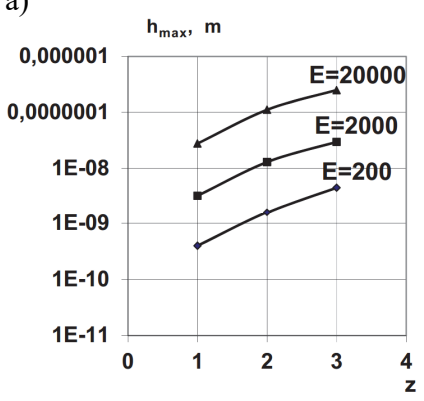

b)

Fig. 39. The graphs of the maximum and minimum $h 1 h 2$ Depth nanolayer obtain the a) energy and b) ion charge (ions $\mathrm{Fe}, \mathrm{Ni}, \mathrm{Co}$ ) 

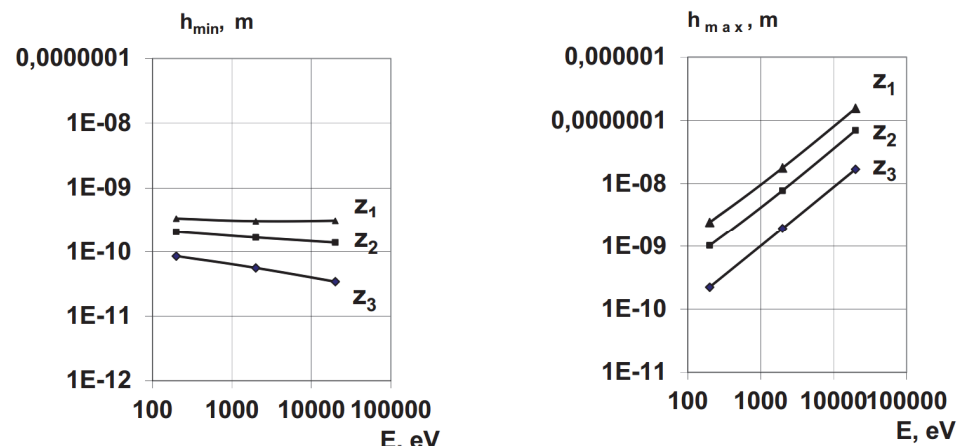

a)
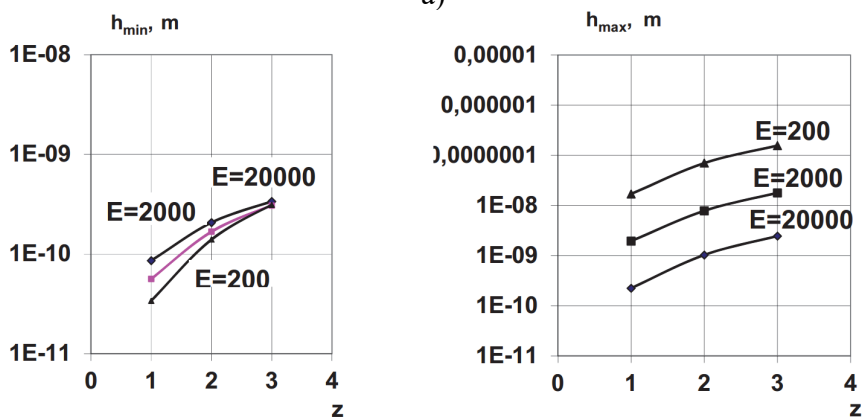

b)

Fig. 40. The graphs of the maximum and minimum $h 1 h 2$ Depth nanolayer obtain the a) energy and b) ion charge (ions $\mathrm{Y}, \mathrm{Zr}, \mathrm{Nb}, \mathrm{Mo}$ )
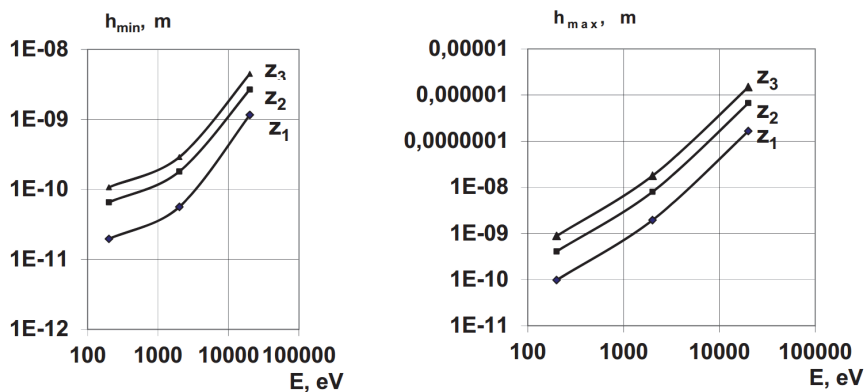

a)
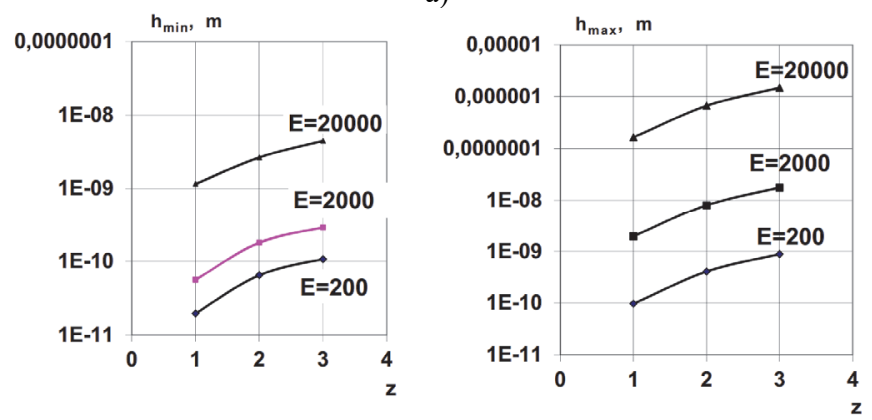

b)

Fig. 41. The graphs of the maximum and minimum $h 1 h 2$ Depth nanolayer obtain the a) energy and b) ion charge (ions $\mathrm{Hf}, \mathrm{Ta}, \mathrm{W}, \mathrm{Pt}$ )

Knowing $j_{i}, V_{H C i}, h_{i 1}, h_{i 2}, E_{i}$, we can create the necessary number of streams of ions that 
provide a nanostructured layer of the desired thickness, and with the technical task of forming nemonoenergetichnyh raznozaryadovyh ion fluxes can solve the problem of technological parameters of ion treatment.

\subsection{Conclusion}

On the basis of studies of the effect of energy, variety and ion charge the possibility of obtaining the ion current density at which you can ensure the best possible filling of nanostructures on the layer depth range (minimum and maximum $\left(h_{1}\right.$ and $\left.h_{2}\right)$ occurrence of nanostructures.

The possibility of receiving the necessary layers of nanostructures and thickness through the use of ion beams with different energy charge and variety.

Construction of nanostructures using different streams of ions, is provided as follows: the maximum depth of the first layer should be close to the minimum depth of the second layer and so on.

\section{Selection of technological parameters of ion fluxes different varieties, energy, charge and current densities for nanostructures}

Ensuring nanostructures by controlling the flow of ions simultaneously hit and chargers to the workpiece is carried out under the conditions.

Terms and conditions: both ions hit:

$$
\begin{aligned}
& \tau_{\max _{1}}\left[E_{\min _{T}}, Z_{\min _{T}}\right]=\tau_{2}+\tau_{c 3}+\tau_{H 2}=\tau_{3}+2 \tau_{c 3}+\tau_{H 3}=\tau_{4}+3 \tau_{c 3}+\tau_{H 4} \\
& \quad=\tau_{5}+3 \tau_{c 3}+\tau_{H 5}=\cdots
\end{aligned}
$$

where $\mathrm{E}_{\min _{\mathrm{T}}}, Z_{\min _{T}}$ - energy and the charge of the heavy ion; $*(L)$ - distance from the control grid to detail; $\tau_{\max _{1}}\left[E_{\min _{T}}, Z_{\min _{T}}\right]$ - the time of flight of the heavy-ion charger with minimum ( $z=1$ ) minimum energy; $\tau_{2}, \tau_{3}, \tau_{4}, \tau_{5}$ - time span the distance $L$ ions of different energies, battery grade and increasing energy and charge; $\left(\tau_{2}>\tau_{3}>\tau_{4}>\tau_{5}\right)-$ composition $E \cdot z ; \tau_{c 3}-$ while the interaction of ions with the workpiece; $\left(\tau_{H 2}, \tau_{H 3}, \tau_{H 4}, \tau_{H 5}\right)$ - the launch of the second, third, fourth, fifth ions $\left(\tau_{H 1}=0\right)$ with respect to the first and determined:

$$
\begin{aligned}
& \tau_{H 2}=\tau_{\max }\left[E_{\min }, E_{\min _{T}}\right] \tau_{2}-\tau_{c 3}=\frac{L}{\sqrt{\frac{2 E_{\min }}{m_{i_{T}}} z_{\min }}}-\frac{L}{\sqrt{\frac{2 E_{2}}{m_{i_{2}}} Z_{2}}}-\tau_{c 3}, \\
& \tau_{H 3}=\frac{\mathrm{L}}{\sqrt{\frac{2 E_{\min }}{m_{i_{T}}} z_{\min }}}-\frac{L}{\sqrt{\frac{2 E_{3}}{m_{i_{3}}} z_{3}}}-2 \tau_{c 3}, \\
& \tau_{H 4}=\frac{\mathrm{L}}{\sqrt{\frac{2 E_{\min }}{m_{i_{T}}} Z_{\min }}}-\frac{L}{\sqrt{\frac{2 E_{4}}{m_{i_{4}}} Z_{4}}}-3 \tau_{c 3}, \\
& \tau_{H_{\mathrm{J}}}=\frac{\mathrm{L}}{\sqrt{\frac{2 E_{\min }}{m_{i_{T}}} Z_{\min }}}-\frac{L}{\sqrt{\frac{2 E_{j}}{m_{i_{j}}}} z_{j}}-(j-1) \tau_{c j} .
\end{aligned}
$$

Contact with ions of various sorts, and recharging energy can be arranged simultaneously using one or two of the ion source, pulling ions through the grid by using nanosecond pulse voltage, 
wherein the first pulse is supplied to the lower amplitude more later, i.e. increasing amplitudes as they are ascending:

$$
C_{n s}^{\prime}=V_{n s} \frac{j}{e z}, \quad S_{l . t} l_{n s}=V_{t r}
$$

$t=\frac{S_{d e t} \cdot l_{n s} \cdot e Z}{V_{n s} \cdot j_{i} \cdot S_{l . t}}-$ the time of receipt of the nanostructure in a layer $l_{n s}$ on the surface area $S_{d e t} ;$ $j_{i_{c . d}}=\frac{S_{d e t} \cdot l_{n s} \cdot e z}{t_{l . t} \cdot V_{n s} \cdot S_{p r}}-$ current density required to obtain a limited time for the desired layer $l_{n s}$ the surface area of the details $S_{d e t} ; j_{i_{c . d}} \geq j_{c r}^{I}$, therefore, the calculations can be taken a current density equal to the first critical.

The action is not mono-energetic ion flux of different varieties and recharging the critical current density for such flows can be obtained as described in $[2,3]$ or estimated from particle (ion) with the highest mileage (less heavy ion), and large work. Define the first critical current density, assuming that the remaining ions fill the volume between the zones of action of these ions, which is close to the real picture as shown by studies of the distribution of temperature fields and thermal stresses [1].

Then, for the action of the ions of different sorts, energy, charge and current densities while receiving nanostructure layer of the desired thickness can be obtained as:

$t=\frac{S_{d e t} \cdot l_{n s}}{\left(\sum V_{n s} \frac{j_{c r}^{I}}{e z_{i}}\right) \cdot S_{p r}}$,

or in the case can be used to simplify the expression:

$t=\frac{S_{d e t} \cdot l_{n s} \cdot e z_{m . m}}{V_{n s} \cdot k_{a s} \cdot j_{c r_{m . m}}^{I}}$

where $z_{m . m}$ and $j_{c r_{m . m}}^{I}$ - the first charge and the critical current density of the ions with the maximum mileage, $k_{a s}=1.2-2$ to increase the filling ratio by volume of particles with smaller runs and, more important factor corresponds to a greater number of additional streams of ions. Examples of calculation of the duty ratio of volume and temperature fields, thermal stresses are presented in [8] for the different laws of distribution of the ions in space parts of various laws (spiral of Archimedes, cellular location and uniform rectangular arrangement of the particles).

Organization of simultaneous ingress of ions of different sorts of energy and recharging the surface of the parts is carried out under the conditions.

Contact with ions of various sorts, energy and recharging at the same time can be organized using one or two of the ion source, pulling them through the grid by using nanostructured voltage pulses. And the first pulse is applied to the lower amplitude, and later with large amplitudes with increasing.

\subsection{Conclusions}

The analysis allows to find treatment regimens in which is possible to obtain nanostructures.

The possibility of the simultaneous implementation of the end of the thermal springs of action-particles, which improve the quality of treatment.

\section{References}

[1] Kostyuk G. I. Nanotechnology: Theory, Experiment, Technology, Perspectives. Academy of Sciences and Innovation, 2012, p. 648. 
[2] Kostyuk G. I. Physical and Technical Principles of Coating, Ion Implantation and Ion Doping, Laser Processing and Hardening, Combined Technologies. Book 1: Physical Processes of Plasma-Ion, Ion-Beam, Plasma, Light-Beam and Combined Technologies. Publishing House of AIU, 2002, p. 596.

[3] Kostyuk G. I. Physical and Technical Principles of Coating, Ion Implantation and Ion Doping, Laser Processing and Hardening, Combined Technologies. Book 2: Handbook for Calculating the Basic Physical and Technological Parameters, Assessing the Possibilities, Choosing the Type of Technology and Equipment. Publishing House of the AAS, 2002, p. 482.

[4] Kostyuk G. I. Nanostructures and Nanocoatings: Perspectives and Reality. University of Kharkov, 2009, p. 406.

[5] Kostyuk G. I. Scientific Foundations of the Creation of Modern Technologies. University of Kharkov, 2008, p. 552.

[6] Kostyuk G. I. Effective Cutting Tool with a Coating and a Reinforced Layer. University of Kharkov, 2007, p. 633.

[7] Kostyuk G. I. Effective Cutting Tool with a Coating and a Reinforced Layer. View of AINU, 2003, p. 412.

[8] Kostyuk G. I. Physicotechnical Foundations of Robot Production. University of Kharkov, 2006, p. 614 .

[9] Grechikhin L. I. Physics of Nanoparticles and Nanotechnologies. Technoprint, 2004, p. 397.

[10] Schneider P. Engineering Problems of Heat Conduction. Izd-vo Inostr. Lit., Moscow, 1960, p. 488.

[11] Aksenov I. I. Vacuum Arc in Erosion Sources of Plasma. Publishing House of the Scientific Research Institute "KIPT", 2005, p. 211.

[12] Hayakawa S. Nuclear-physical Aspect. Mir, Moscow, 1973, p. 701, (in 2 Books).

[13] Gott Yu. V. Interaction of Particles with Matter in Plasma Studie. Atomizdat, Moscow, 1978, p. 271.

[14] Gusev A. I. Nanocrystalline Materials: Methods of Obtaining and Properties. Publishing House of the Russian Academy of Sciences, Ural Division, Ekaterinburg, 1998, p. 302.

[15] Gusev A. I. Nanomaterials of Nanostructure, Nanotechnology. Fizmatlit, Moscow, 2005, p. 416.

[16] Reshetnyak E. N. Synthesis of hardening nanostructured coatings. Questions of Atomic Science and Technology, Vol. 2, 2008, p. 119-130.

[17] Andrievsky R. A. Nanomaterials: the concept and modern problems. Physics of Metals and Metallography, Vol. 91, Issue 1, 2003, p. 50-56.

[18] Goncharov A. A., Ignatenko P. I., Petukhov V. V., et al. Composition, structure and properties of nanostructured tantalum boride films. Zhurnal Tekhnicheskoǐ Fiziki, Vol. 76, Issue 10, 2006, p. 87-90.

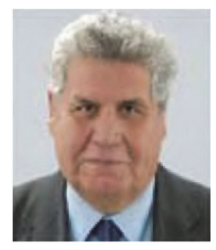

Kostyuk Gennady Igorevich Professor of the Department of Theoretical Mechanics, Machine Science and Robot Mechanics Systems of the National Aerospace University. N. E. Zhukovsky "KhAI", vice-president of the International Academy of Sciences and Innovative Technologies. Doctor of Technical Sciences, Professor, Academician of the Academy of Engineering Sciences and the Academy of Sciences of Technological Cybernetics of Ukraine. The scientific heritage of G. I. Kostyuk counts 1070 scientific works (including 210 works in the far abroad), 8 of them are monographs, 32 textbooks and textbooks, 12 patents for inventions and 13 copyright certificates. Under his leadership is protected 4 doctoral and 25 master's theses. 


\title{
4.2. Prediction of the physical and mechanical characteristics, the removable material volume for the durability period, cutting tools durability and processing productivity depending on the grain size of the coating or cutting tool base material
}

\author{
Gennadiy Kostyuk \\ National Aerospace University named by N. Ye. Zhukovsky, KhAI, Kharkov, Ukraine \\ E-mail: gennadiykostyuk206@gmail.com
}

\begin{abstract}
The microhardness and yield strength decrease with grain size increasing for wide range of the $0.2 \mathrm{HfN}+0.8 \mathrm{ZrN}$ coated materials (K40, Sandvik Koromant, MS221) was disclosed. Two-layer $\mathrm{Al}_{2} \mathrm{O}_{3}+(0.2 \mathrm{HfN}+0.8 \mathrm{ZrN})$ coated Sandvik Koromant plate microhardness changes slightly (due to the fact that almost always nanostructures implemented) with the grain growth 33.7-63.8 nm, $H_{\mu}=16.08-17.14 \mathrm{GPa}$. The coating effective application criterion will condition: the coating grain size should be less than the cutting tool base material grain size. Maximum microhardness is realized at the TiN concentrations in diborides from 50 to $80 \%$ and grain sizes range from 5 to $13 \mathrm{~nm}$. It is proved that modulus of elasticity increases with the grain size growth. It is shown that at the effectiveness and efficiency assessing of the coated hard alloys at the K19195 hardened materials and G10450 steel processing it is necessary to take into account the coating grain size at that to the smaller grain size is generally (but not always) corresponds to more effective processing (the maximum removable material volume for the durability period) and its working capacity. For G10450 steel effective processing can be used $0.18 \mathrm{HfN}+0.82 \mathrm{ZrN}$ coated Sandvik Koromant company solid alloy, MS221 and K40, which will be both effective and operable. It found that at the cast iron processing the coating adhesive interaction with the manufacturable material is an important characteristic which provide unique value of removable volume for durability period of the (1.3-1.8) $10^{7} \mathrm{~mm}^{3}$ order. The maximum durability $(19,000-14,000 \mathrm{sec})$ also realized in this case, maximum productivity is realized at the minimum $\mathrm{G}$ and $\mathrm{T}$ values. Performed investigations allow to predict the removable material volume over the durability period, cutting tool durability and processing productivity depending on the coating grain size. It is important to select the cutting tool coating and processing mode.
\end{abstract}

Keywords: cutting tools, microhardness, nanostructures, nanotechnologies.

\section{Introduction}

The experimental results presented in the previous parts showed that the grain size of the coating or the main cutting tool material affects on the physical-mechanical characteristics of the material cutting tools, its efficiency and productivity. Experiment results summary from the more than 500 references showed the following.

The grain size effect on the physical-mechanical and electrical properties of the materials were studied in [1-3]. The microhardness maximum presence, depending on the grain size (Figs. 1-5) was found in [1-3]. Weak grain size influence on the microhardness in magnetron nitride nanocomposites nc-TiN $/ \alpha \mathrm{Si}_{3} \mathrm{~N}_{4}$ at the different annealing temperatures is observed in [2], whereas for vacuum-arc nanocomposites maximum microhardness realized for certain grain sizes (Fig. 5).

In [4-8] were obtained high microhardness values up to $45 \mathrm{GPa}$ for $0.8 \mathrm{ZrN}+0.2 \mathrm{HfN}$ coating at the combine processing, increased cutting tools wear resistance and durability up to 20 times, and at the only $0.8 \mathrm{ZrN}+0.2 \mathrm{HfN}$ coating deposition the microhardness was about $35 \mathrm{GPa}$, which is more than twice higher than the individual components microhardness. All this allows to suppose that nanostructures appearance is realized even at the only coating deposition while additional electron-beam modification will provide more effective nanostructures with improved 
properties and in the considerable depth $(0.1 \mathrm{~mm})$.
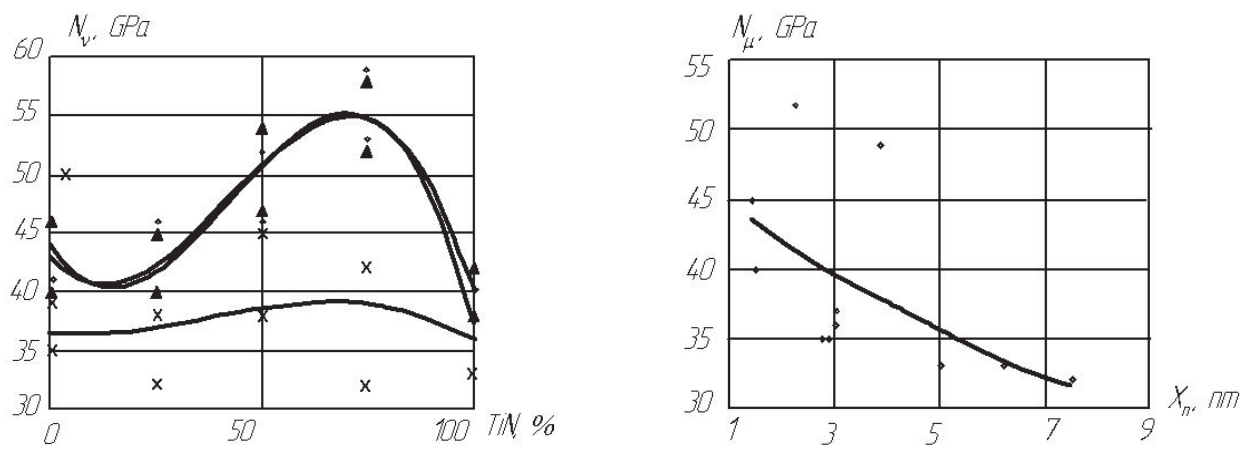

Fig. 1. The coating microhardness dependence on the Fig. 2. The coating microhardness depending on the TiN percentage composition in the TiB2 + TiN coating (crystal size 3-10.2 nm) [2]
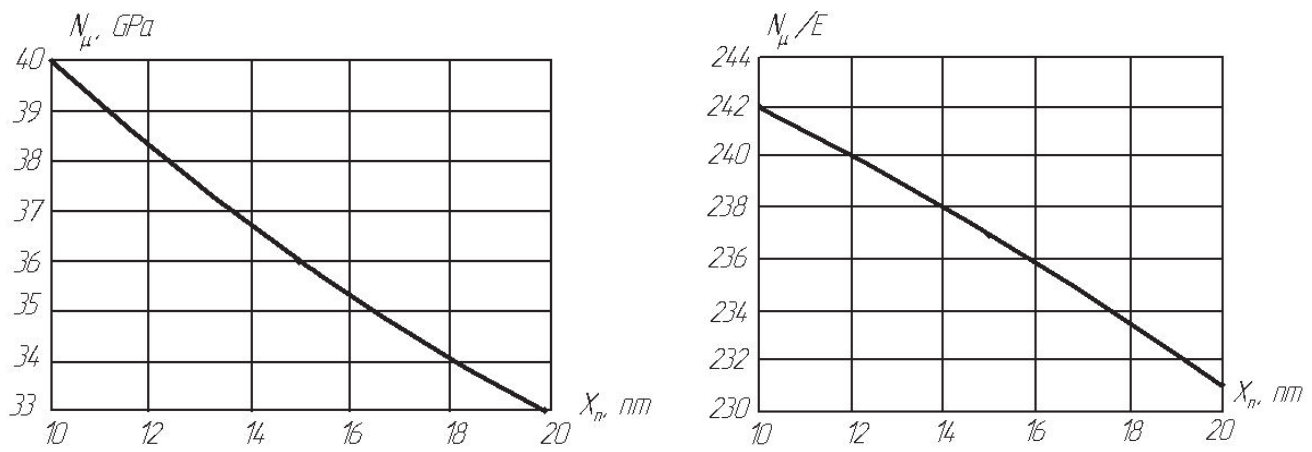

Fig. 3. The coating microhardness dependence on the grain size according to [3]

Fig. 4. The coating microhardness dependence on the grain size according to [3]

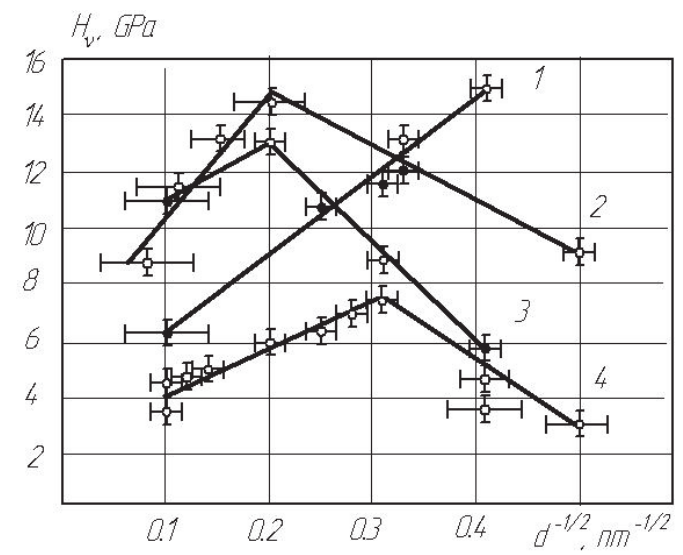

Fig. 5. The coating microhardness $H_{v}$ dependence on the grain size of the dispersed phase, released in nanocrystalline alloys obtained by amorphous alloys crystallization:

$1-\mathrm{Fe}_{73.5} \mathrm{Cu}_{1} \mathrm{Nb}_{3} \mathrm{Si}_{13.5} \mathrm{~B}_{9} ; 2-\mathrm{Fe}_{81} \mathrm{Si}_{7} \mathrm{~B}_{12} ; 3-\mathrm{Fe}_{5} \mathrm{Co}_{70} \mathrm{Si}_{15} \mathrm{~B}_{10} ; 4-\mathrm{Pd}_{81} \mathrm{Cu}_{7} \mathrm{Si}_{12}$ [4]

The grains size knowledge enables to predict the microhardness, yield strength, friction coefficient, wear resistance and other physical-mechanical and operational characteristics. For example, generalized microhardness and yield stress dependences on the size from the Fig. 7 and 6 are obtained from reviews [2] or papers [4-8]. The microhardness and yield strength data forecast 
can be performed on these values or at the monograph [8] using.

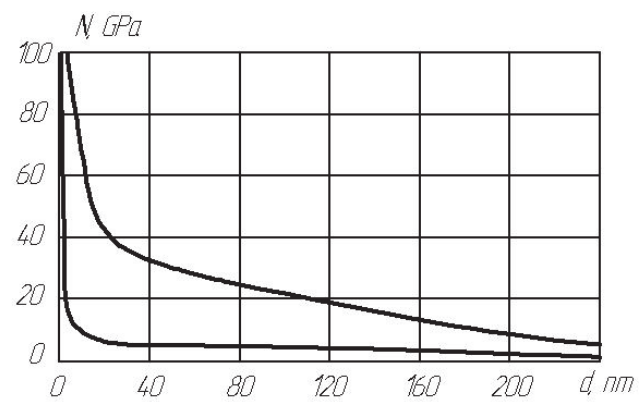

Fig. 6. The generalization results of the microhardness dependence on the grain size:

top - maximum values; bottom - minimum values

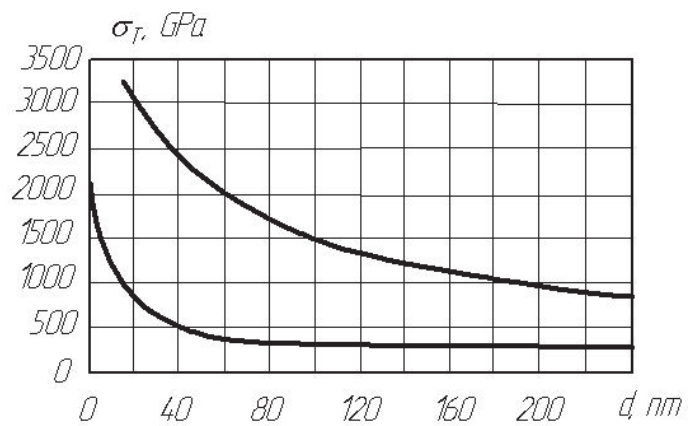

Fig. 7. The generalization results of the yield strength dependence on the grain size according to [9]

The most important nanotechnology mission is the required physical and mechanical characteristics obtaining on the cutting tools and details surface layers coating and directly in the cutting tools and details surface layer. Theoretical prediction of the physical and mechanical characteristics is not yet fully possible due to the complexity and diversity of the processes involved in the certain physical and mechanical characteristics formation. In this case it is possible with a reliability high degree to determine theoretically the grain size, and in future, using the experimental dependence of certain physical and mechanical characteristics on the grain size, to estimate its values for the given technological and physical parameters. Using such dependencies or extrapolate these dependencies relationships for other materials or modes, we can predict with reasonable accuracy the process parameters that provide the necessary characteristics.

\section{Microhardness prediction in dependence on the grain size in the $0.2 \mathrm{HfN}+0.8 \mathrm{ZrN}$ single-layer coating hard alloy cutting tool}

Microhardness of the $0.2 \mathrm{HfN}+0.8 \mathrm{ZrN}$ coated hard alloys cutting tools is measured on the PMT-3 microdurometer: the average value was selected from five measurements. The grain size was measured by the REM-106 electron microscope for $0.2 \mathrm{HfN}+0$. coating $8 \mathrm{ZrN}$ on plates made of VK-8 hard alloy (production of the USSR), Sandvik Koromant (H13 plate) and a bilayer coated Sandvik Koromant: $\mathrm{Al}_{2} \mathrm{O}_{3}$ (front surface layer) and $0.2 \mathrm{HfN}+0.8 \mathrm{ZrN}$ (layer directly on the plate front surface, plate side surfaces and cutting tool back surface) (Fig. 8).

The $0.2 \mathrm{HfN}+0.8 \mathrm{ZrN}$ coated MS 221 hard alloy plates (production of the Russia) and coated and uncoated Sandvik Koromant (H13) plates were investigated also. 


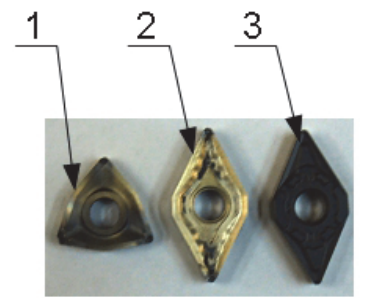

Fig. 8. Appearance plates: $1-\mathrm{VK} 8 ; 2-0.2 \mathrm{HfN}+0.8 \mathrm{ZrN}$ coated Sandvik Koromant (H13); 3 - two-layer coated $\mathrm{Al}_{2} \mathrm{O}_{3}+0.2 \mathrm{HfN}+0.8 \mathrm{ZrN}$ Sandvik Koromant

The most characteristic parts of the cutting tool coating surface are shown on the Figs. 9-13; the sizes of the typical grains for the coating each type are also given. It can be seen that the minimum grain sizes were realized on the two-layer coating $\mathrm{Al}_{2} \mathrm{O}_{3}+0.2 \mathrm{HfN}+0.8 \mathrm{ZrN}$ $(33, \ldots, 63 \mathrm{~nm})$ (Fig. 9).

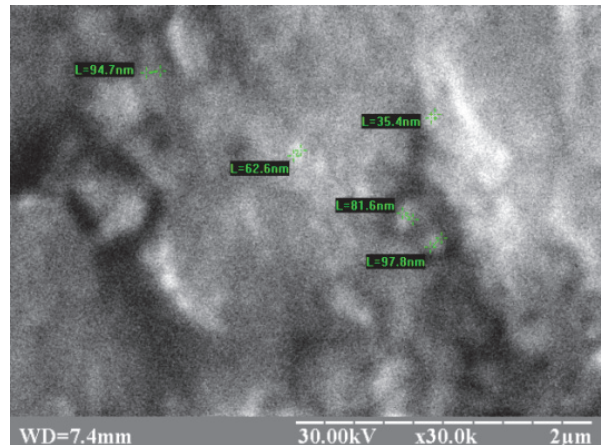

a)

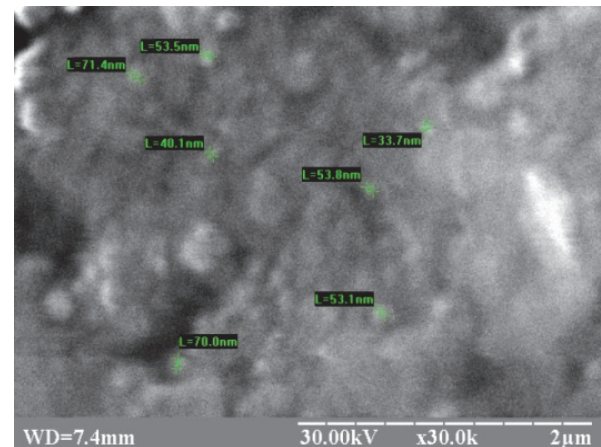

b)

Fig. 9. The micrograph of the surface $\mathrm{Al}_{2} \mathrm{O}_{3}+0.2 \mathrm{HfN}+0.8 \mathrm{ZrN}$ coating on the Sandvik Koromant hard alloy obtained by the electron microscope ( $\mathrm{Cu}$ coating was used to reduce the charge effect on the $\mathrm{Al}_{2} \mathrm{O}_{3}$ )

for the front surface different zones: a) near the top; b) in the middle part of the plate front surface

Relatively low grain size in the $0.2 \mathrm{HfN}+0.8 \mathrm{ZrN}$ coating is realized on the $\mathrm{VK} 8$ hard alloy plates; its value is in $84, \ldots, 119 \mathrm{~nm}$ range, i.e. were realized both nanostructures $\alpha \leq 100 \mathrm{~nm}$ ) and submicrostructure with grain sizes range from 100 to $119 \mathrm{~nm}$ (Fig. 10(a, b)).

Photo of the $0.2 \mathrm{HfN}+0.8 \mathrm{ZrN}$ coating fracture enables to estimate its thickness which in the wearing zone of the coating is $\approx 1, \ldots, 2$ microns, whereas in the non-intensive wear zone it is close to the original value of 10 microns order (Fig. 10(c)).

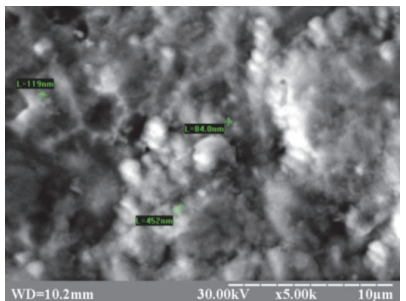

a)

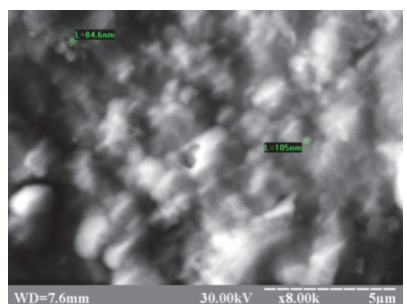

b)

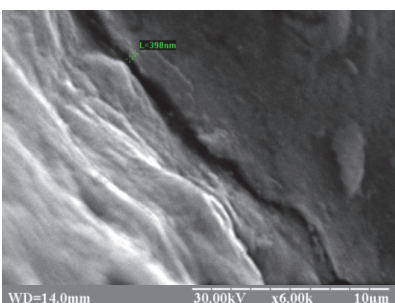

c)

Fig. 10. Photomicrographs of the $0.2 \mathrm{HfN}+0.8 \mathrm{ZrN}$ coated VK 8 plates:

a) near the top; b) in the plate 4 middle; c) near the coating fracture zone

For the hard alloy Sandvik Koromant $(\mathrm{H} 13)$ plates with $0.2 \mathrm{HfN}+0.8 \mathrm{ZrN}$ coating grain sizes is from 84 to $200 \mathrm{~nm}$, where the submicrostructure grain size 100 to $200 \mathrm{~nm}$ prevails, but there are a significant number of the nanoclusters grain size from 84 to $100 \mathrm{~nm}$ (Fig. 11). 


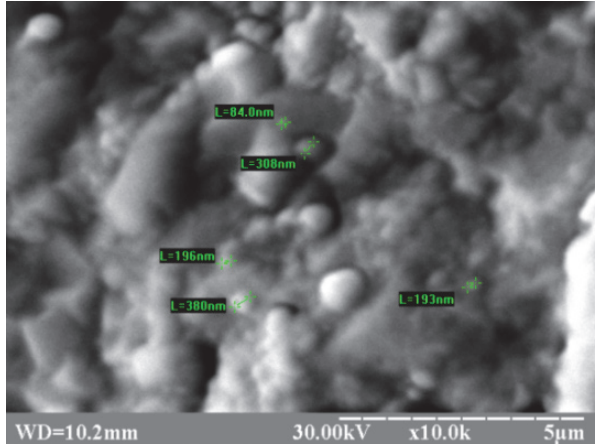

a)

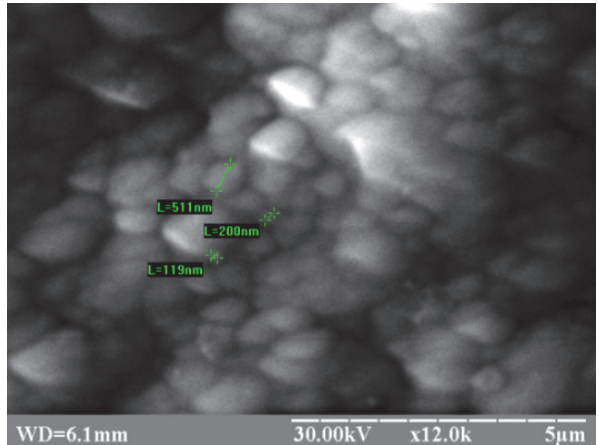

b)

Fig. 11. Photomicrographs of the $0.2 \mathrm{HfN}+0.8 \mathrm{ZrN}$ coated Sandvik Koromant plates: a) near the top; b) in the middle part of the plate front surface

Photos of the MS221 hard alloy coating are shown in Fig. 12. Obviously that grain size is in the range from 36.5 to $105 \mathrm{~nm}$, the grains part with size of $100, \ldots, 105 \mathrm{~nm}$ is very small, the grains vast majority has size from 36 to $58 \mathrm{~nm}$, i.e. practically all coating is nanostructured.

Photos of the uncoated Sandvik Koromant hard alloy are shown in Fig. 13. Obviously that grain size is in the range from 35.6 to $115 \mathrm{~nm}$, the grains significant amount has size from 40 to $88 \mathrm{~nm}$, i.e. nanostructures are realized in the sufficiently large portion of the plate volume.

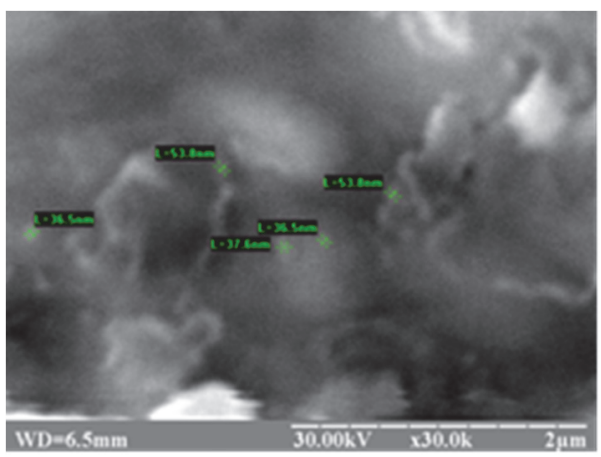

a)

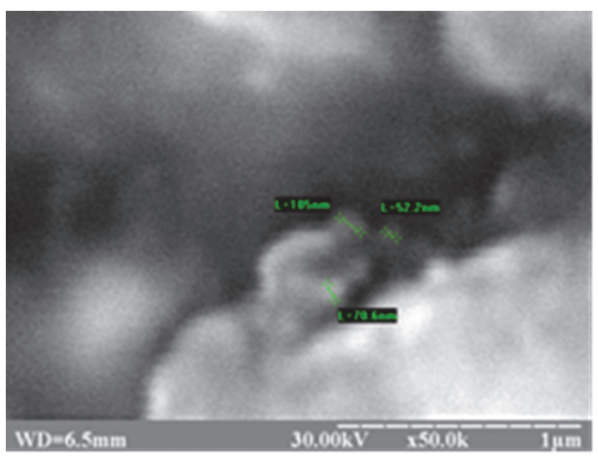

b)

Fig. 12. Photomicrograph of the $0.2 \mathrm{HfN}+0.8 \mathrm{ZrN}$ coatingon the MS221 hard alloy

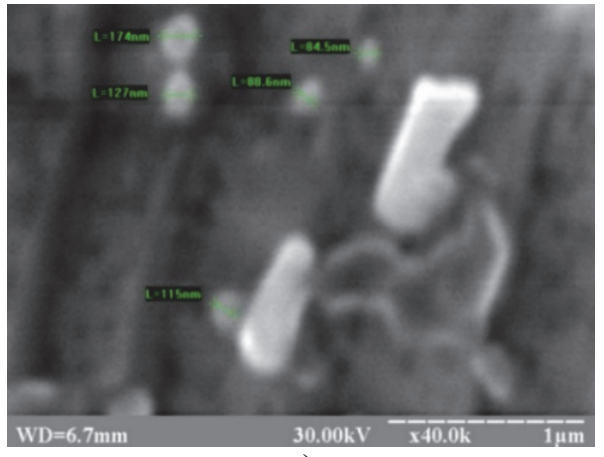

a)

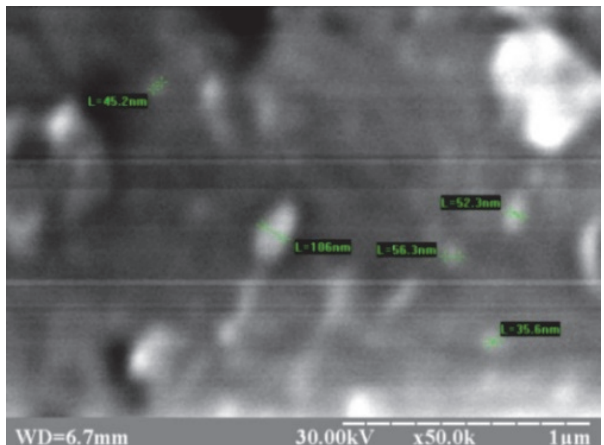

b)

Fig. 13. Photomicrograph of the uncoated Sandvik Koromant (H13) hard alloy Photomicrograph of the uncoated Sandvik Koromant (H13) hard alloy

All the above suggests that it is really to receive nanostructures not only in the coating but also 
in the base material of the cutting tool.

These coated plates microhardness dependence on the grain size are shown in Fig. 14, where microhardness decrease at the grain size increasing. VK8 plates (production of the USSR) are characterized by the highest microhardness, but at the same time they are characterized by the fastest microhardness value decline to $14.67 \mathrm{GPa}(\alpha=119 \mathrm{~nm})$. For $0.2 \mathrm{HfN}+0.8 \mathrm{ZrN}$ coated Sandvik Koromant plate microhardness decrease to the almost the same value (14 GPa) at the $200 \mathrm{~nm}$ grain size. For two-layer coating $\mathrm{Al}_{2} \mathrm{O}_{3}+0.2 \mathrm{HfN}+0.8 \mathrm{ZrN}$ Sandvik Koromant plates microhardness varies within small range 16.08-17.14 $\mathrm{GPa}$ for grain size variation between $33.7, \ldots, 63.8 \mathrm{~nm}$, i.e. almost always nanostructures are realized.

For coated MS221 cutting tools microhardness is $22.28 \mathrm{GPa}$ at the grain size of $36 \mathrm{~nm}$; microhardness reduces with grain size increasing and with for grain size of $105 \mathrm{~nm}$ microhardness becomes equal 16.08 GPa (Fig. 14, curve 5).

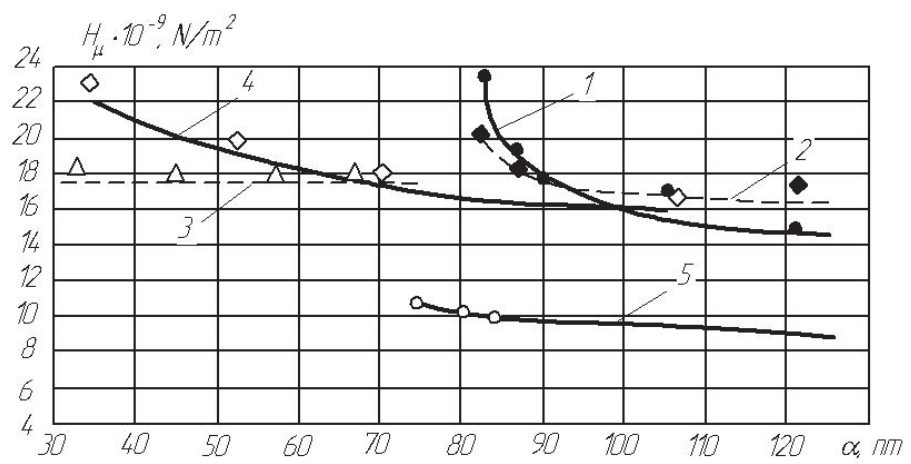

Fig. 14. The microhardness dependence on the grain size for coating: $1-0.2 \mathrm{HfN}+0.8 \mathrm{ZrN}$ on the $\mathrm{K} 40$; 2 - on the Sandvik Koromant (H13 plates); 3 - for the $\mathrm{Al}_{2} \mathrm{O}_{3}+0.2 \mathrm{HfN}+0.8 \mathrm{ZrN}$ two-layer coating on the Sandvik Koromant; $4-0.2 \mathrm{HfN}+0.8 \mathrm{ZrN}$ coating on the MS221; 5 - for the uncoated Sandvik Koromant (H13)

Microhardness changes relatively slightly on the grain size of the uncoated Sandvik Koromant plates (see Fig. 14). It can be seen that the grain size variation from 36 to $176 \mathrm{~nm}$ reduces microhardness from 8.9 to $10.42 \mathrm{GPa}$.

Also, the Sandvik Koromant hard alloy microhardness small change shows the sufficiently high isotropic structure, and hence the of the plate manufacturing quality technology (see Fig. 14, curve 5).

The similar microhardness dependences on the grain size for the $0.18 \mathrm{HfN}+0.82 \mathrm{ZrN}$ coating on the T15K6 plate are shown in Fig. 15 (grain size was considered as average statistical, measured by the REM-106, microhardness was measured by the PMT-3 instrument).

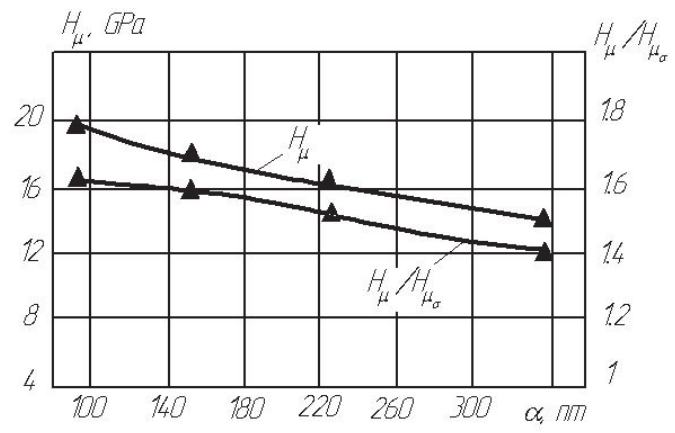

Fig. 15. $0.18 \mathrm{HfN}+0.82 \mathrm{ZrN}$ coated $\mathrm{P} 40$ plate microhardness $H_{\mu_{n}}$ and relative microhardness dependence on the grain size $a, \mathrm{~nm}$ 
It can be seen that at the grain size increasing the coating microhardness is reduced, but still remain larger than microhardness of the hafnium or zirconium nitrides. The microhardness relative change (coated cutting tool with respect to the uncoated cutting tool) on grain size is shown in Fig. 15. It is seen that the microhardness maximum increase is realized for the nanostructured coatings case.

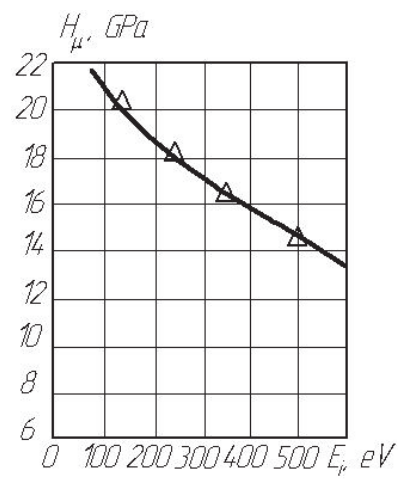

Fig. 16. The microhardness dependence on the ion energy

As result of the nanostructures realization only for one mode at the $110 \mathrm{eV}$ ion energy (Fig. 16), it is not possible to expect effective work of the T15K6 cutting tool.

All this confirms the thesis that the nanostructures significantly increase the microhardness.

From the preceding it follows that for the effective coatings use such condition is necessary: the coating grain size should be less than in the cutting tool base material, and preferably nanostructure realization.

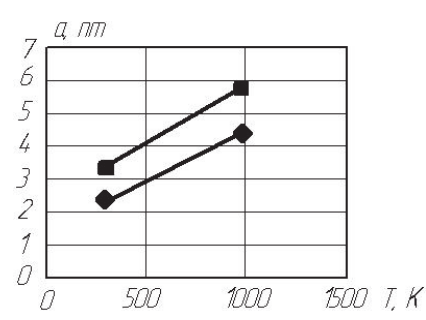

a)

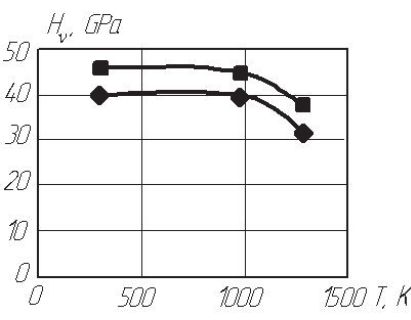

b)

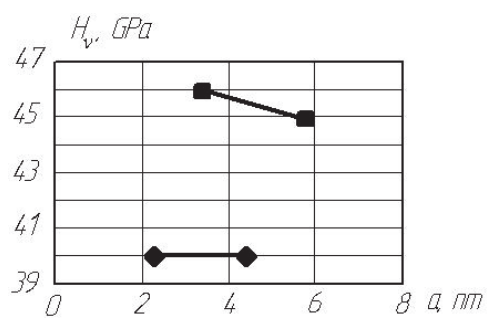

c)

Fig. 17. Dependence of the a) grain size and b) microhardness on the temperature, c) microhardness dependence on the grain size $(\boldsymbol{\square}$ - maximum value, $\bullet-$ minimum value $)$ for $\mathrm{TiB}_{2}+0.25 \mathrm{TiN}$ nanostructure

On the study basis [2] annealing temperature influence on the grain size and microhardness and the microhardness dependence on the crystallite size were analyzed (Fig. 17) for diboride. Investigations of the samples with the obtained in the experiment maximum and minimum microhardness were conducted. These curves analysis shows that the grain size is in the range $2.3, \ldots, 19.6 \mathrm{~nm}$, at that for many diborides grain size maximum values depend on the temperature.

The maximum microhardness values are realized at the temperatures close to normal $T=273 \mathrm{~K}$, at the temperature increase to $1000 \mathrm{~K}$, microhardness values reduced insignificantly, while the further temperature increase significantly reduces the microhardness from 46 to $32 \mathrm{GPa}$ $\left(\mathrm{TiB}_{2}+0.25 \mathrm{TiN}\right)$, Fig. 17.

For $\mathrm{TiB}_{2}+0.5 \mathrm{TiN}$ coating the maximum grain size value increases at the temperature growth and minimum size value has maximum; grain size is in the range from 2.7 to $5.5 \mathrm{~nm}$. The maximum microhardness value are realized at the temperature close to $1000 \mathrm{~K}$, microhardness decreases at the further temperature increase. The microhardness is also reduced with the grain 
size growth (Fig. 18).

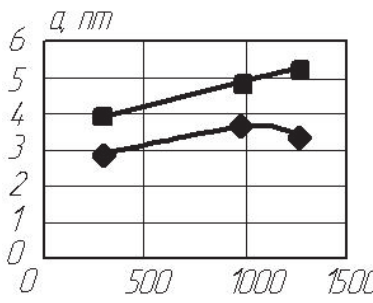

a)

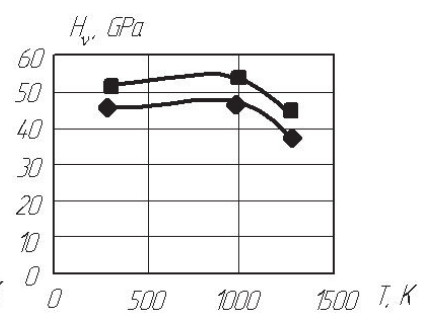

b)

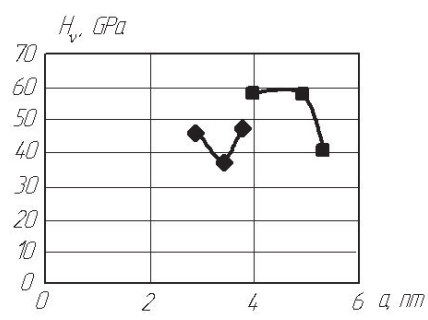

c)

Fig. 18. Dependence of a) the grain size and b) microhardness on the temperature, c) microhardness dependence on the grain size ( - maximum value, - minimum value) for $\mathrm{TiB}_{2}+0.5 \mathrm{TiN}$ nanostructure

Diboride $\mathrm{TiB}_{2}+0.75 \mathrm{TiN}$ research showed that at the temperature growth to $1000 \mathrm{~K}$, the grain size increases significantly, then the grain growth is slowing. Microhardness decreases slightly in the range temperature up to $1000 \mathrm{~K}$ and later it decreases considerably. The microhardness dependence on the grain size allows to predict it in dependence on the diboride grain size (Fig. 19).

Similar studies of the titanium nitride (TiN) showed that it behaves much the same way, but the grain size is slightly higher and reaches above $20 \mathrm{~nm}$, which is realized at the temperature of $1000 \mathrm{~K}$ (Fig. 20(a)). The maximum microhardness is also realized at the temperature of $1000 \mathrm{~K}$. At the further temperature growth microhardness also reduced, though not as fast as for diborides (Fig. 20(b)). The microhardness dependence on the grain size allows to predict the microhardness value on the grain size.

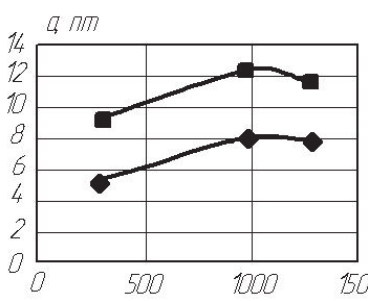

a)

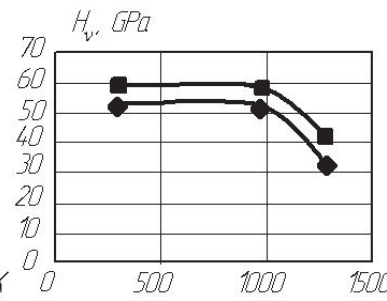

b)

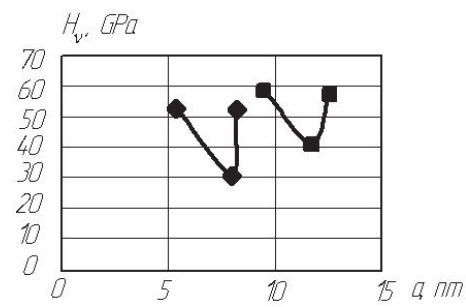

c)

Fig. 19. Dependence of a) the grain size and b) microhardness on the temperature, c) microhardness dependence on the grain size ( - maximum value, - minimum value) for $\mathrm{TiB}_{2}+0.75 \mathrm{TiN}$ nanostructure

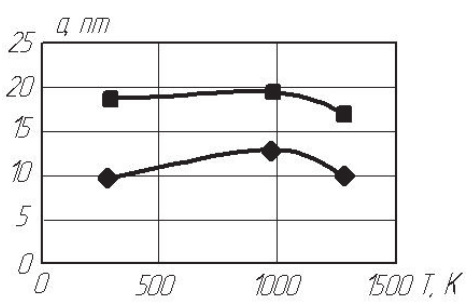

a)

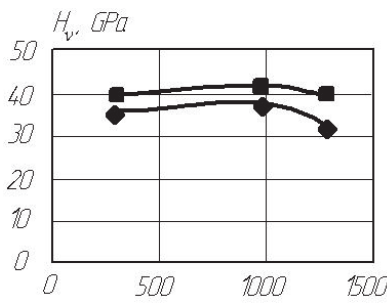

b)

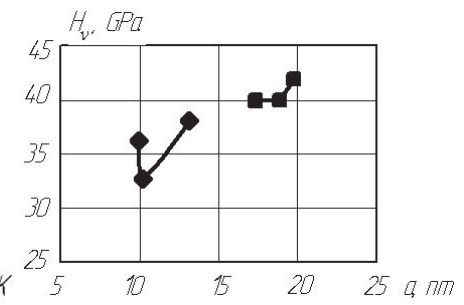

c)

Fig. 20. Dependence of a) the grain size and b) microhardness on the temperature, c) microhardness dependence on the grain size ( $\square$ - maximum value, - minimum value) for TiN nanostructure

The microhardness dependences on the temperature were obtained for $\mathrm{TiB}_{2}$, maximum microhardness value is realized at the temperature close to $1000 \mathrm{~K}$. Later the temperature decreases (Fig. 21).

The grain size and microhardness as a function of the TiN percentage (Fig. 22(a), (b)) and the 
microhardness dependence on the coating grain size (Fig. 22(c)) were also studied. It is seen that at the TiN concentration growth the grain size increases up to $18 \mathrm{~nm}$ (maximum size) and $10 \mathrm{~nm}$ (minimum size). The maximum microhardness is realized at the near $77 \% \mathrm{TiN}$ concentration. The microhardness dependence on the grain size (Fig. 22(c)) shows that the maximum its value is realized at the about $10 \mathrm{~nm}$ grain size at $T=273 \mathrm{~K}$.

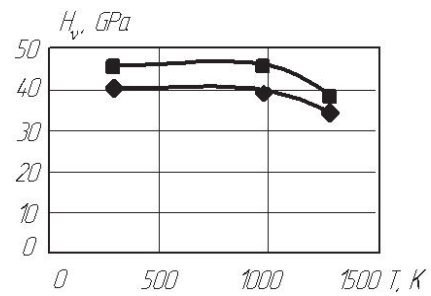

Fig. 21. Dependence of the microhardness on the temperature ( - maximum value, - minimum value) for $\mathrm{TiB}_{2}$ nanostructure

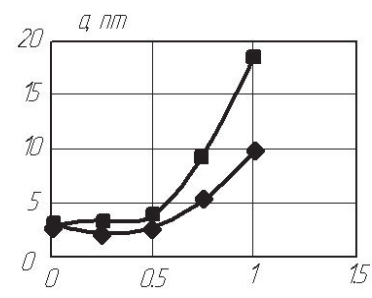

a)

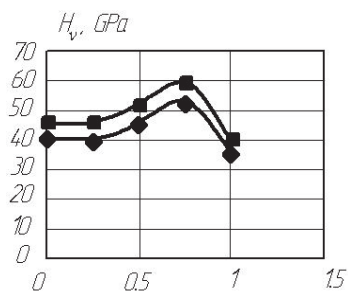

b)

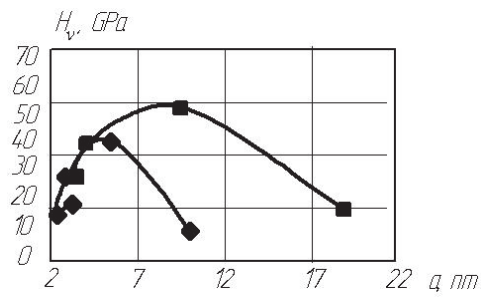

c)

Fig. 22. Dependence of a) the grain size and b) microhardness on the TiN percentage, c) microhardness dependence on the grain size ( - maximum value, - minimum value) at the temperature $T=273 \mathrm{~K}$ for diborides

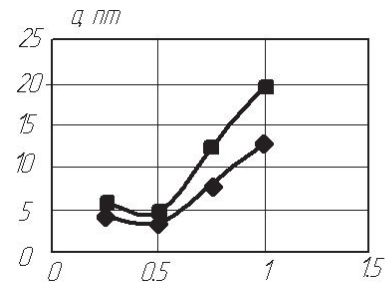

a)

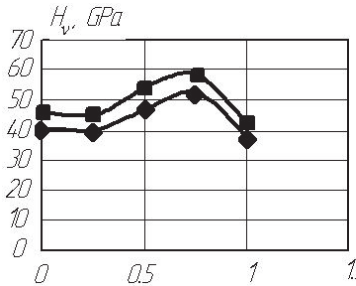

b)

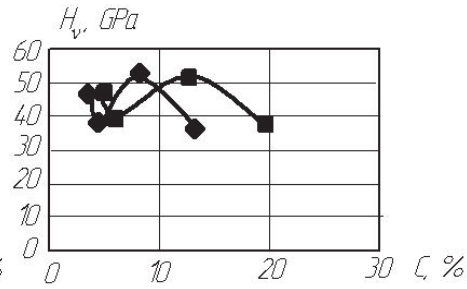

c)

Fig. 23. Dependence of a) the grain size and b) microhardness on the TiN percentage, c) microhardness dependence on the grain size ( - maximum value, - minimum value) at the temperature $T=973 \mathrm{~K}$ for diborides

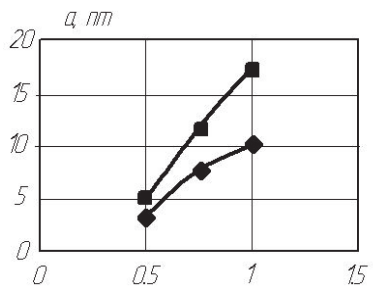

a)

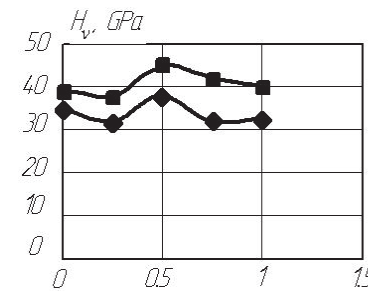

b)

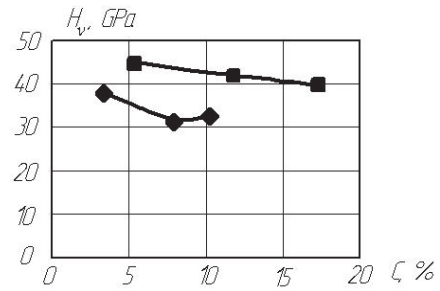

c)

Fig. 24. Dependence of a) the grain size and b) microhardness on the TiN percentage, c) microhardness dependence on the grain size ( - maximum value, - minimum value) at the temperature $T=1273 \mathrm{~K}$ for diborides

The grain size and microhardness dependence on the TiN percentage (Fig. 23(a), (b)) and this 
coating microhardness dependence on the grain size (Fig. 23(c)) were examined. It is seen that at the TiN concentration growth the grain size increases up to $20 \mathrm{~nm}$ (maximum size) and $13 \mathrm{~nm}$ (minimum size). The maximum microhardness is achieved at the near $80 \%$ TiN concentration. The microhardness dependence on the grain size (Fig. 2(c)) shows that the maximum value is realized at the about $10 \mathrm{~nm}$ grain size at $T=973 \mathrm{~K}$.

Similar dependences for $T=1273 \mathrm{~K}$ are shown on Fig. 24. It is seen that at the TiN percentage growth the grain size increases and achieves maximum value $17.10 \mathrm{~nm}$ for $100 \%$ TiN concentration (Fig. 24(a)). The microhardness dependence on the TiN coating percentage is shown in Fig. 24(b). It is seen that microhardness maximum value is realized at the $50 \% \mathrm{TiN}$ concentration and near $5 \mathrm{~nm}$ grain size (Fig. 24(c)). The grain size influence on the elastic modulus (Young's modulus) was studied in [8] (Fig. 25). The figure shows that the Young's modulus increases with grain size growth for $\mathrm{Ni}+2 \%$ (mass.) $\mathrm{P}$ (Fig. 25(a)) [37] and for $\mathrm{ZrO}_{2}+3 \%$ (mass.) $\mathrm{Y}_{2} \mathrm{O}_{3}$ (Fig. 25(b)).

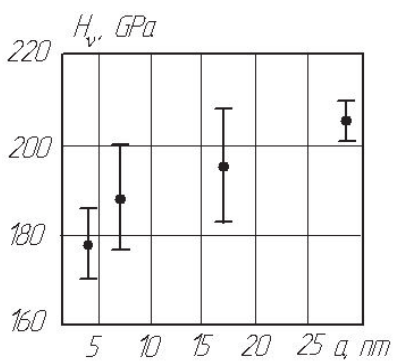

a)

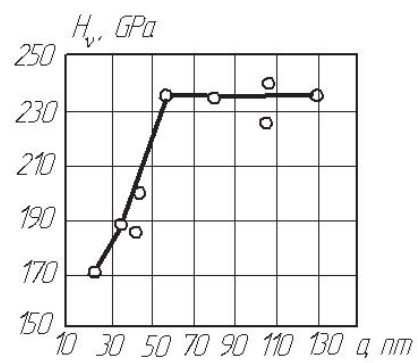

b)

Fig. 25. Grain size influence on the Young's modulus for a) $\mathrm{Ni}+2 \%$ (mass.) $\mathrm{P}$ [37] and b) $\mathrm{ZrO}_{2}+3 \%$ (mass.) $\mathrm{Y}_{2} \mathrm{O}_{3}[6]$

\subsection{Conclusions}

The microhardness decrease with grain size increasing for $0.2 \mathrm{HfN}+0.8 \mathrm{ZrN}$ coated $\mathrm{K} 40$, Sandvik Koromant, MS221 materials was disclosed.

Two-layer $\mathrm{Al}_{2} \mathrm{O}_{3}+(0.2 \mathrm{HfN}+0.8 \mathrm{ZrN})$ coated Sandvik Koromant plate microhardness changes slightly (due to the fact that almost always nanostructures realized) with the grain growth (33.7-63.8 nm, $H_{\mu}=16.08-17.14 \mathrm{GPa}$.

The $0.2 \mathrm{HfN}+0.8 \mathrm{ZrN}$ coated $\mathrm{P} 40$ plate microhardness is lower than for other hard alloys with the same coating, that is associated with significantly large grain size, which from nanostructured at the $E_{i}=110 \mathrm{eV}$ transforms to submicrostructured at the $E_{i}=250-500 \mathrm{eV}$.

The coating effective application criterion will such condition: the coating grain size should be less than the cutting tool base material grain size.

The most effective functioning of the nanostructured coatings is shown.

The grain size increases and the microhardness decreases at the temperature growth.

The maximum microhardness values implemented are realized at the grain sizes in the range of 5 to $10 \mathrm{~nm}$.

The TiN concentration growth in the diboride increases the grain size.

The maximum microhardness is realized at the TiN concentration from $50 \%$ to $80 \%$.

The maximum microhardness for the diborides are realized in the grains sizes range from 5 to $13 \mathrm{~nm}$.

It has been proved that modulus of elasticity increases at the grain size growth. 


\section{Coating grain size influence on the material removable volume for the resistance period, the cutting tool durability and the hardened K19195 steels and G10450 steel machining productivity}

In order to evaluate the grain size influence on the efficiency of the K19195 hardened steel machining were conducted the investigations of the grain size in the $0.18 \mathrm{HfN}+0.82 \mathrm{ZrN}$ coating on the P40, K40 (USSR), K40 (Russia), MS221 hard alloys and VOK 60 ultrahard material.

Surface layer composition (a) of the coating and cutting tool, this layer percentage, plate photograph (b) and the surface layer photomicrograph with grain sizes are shown on the Figs. 11-15. It is seen that the grain size for P40 is in the range of 75.1-106 nm for plates processed in the plasma flow center, and in the range of 113-266 nm for the plate on the flow periphery at the $250 \mathrm{eV}$ ion energy. In this case, we observed $32.04 \% \mathrm{Zr}$ and $24.95 \% \mathrm{Hf}$ in the surface layer. It can be seen that $\mathrm{Hf}$ to $\mathrm{Zr}$ ratio greatly exceeds the cathode elements ratio what, obviously, due to the zirconium evaporation nature from the plate surface layer, which is larger than hafnium evaporation. As a result, a surface layer is zirconium-depleted, as evidenced by the surface layer percentage composition.

Similar studies for K40 (USSR) plate with the same coating are shown on the Fig. 26. It is seen that the grain size is in the range of $106, \ldots, 219 \mathrm{~nm}$. If the plates are located on the ions flow axis, the zirconium percentage composition is $56.5 \%$ and hafnium percentage composition is $38.48 \%$. Whereas if they are located on the periphery, then $\mathrm{Zr}$ and Hf percentage is $55.29 \%$ and $37.67 \%$ accordingly. It is seen that in this case the surface layer zirconium-hafnium ratio is increased in relation to hafnium (relative to its cathode portion). It is shown that the coating grain size on the K40 plate is slightly higher than for P40 plate. In this case is realized submicrostructure grain and the surface layer percentage determines the zirconium and hafnium evaporation nature ( $\mathrm{Zr}$ evaporates faster than $\mathrm{Hf}$ )

Investigations of the grain size influence on the removable material volume during the durability period, the durability of the $0.18 \mathrm{HfN}+0.82 \mathrm{ZrN}$ coated K40 (USSR) cutting tool and K19195 hardened steel processing performance shown (Fig. 26), that material removable volume for durability period $G$ has maximum value $1.8 \cdot 10^{4} \mathrm{~mm}^{3}$, the cutting tool durability has relatively low value $T=600 \mathrm{~s}$ and the processing capacity $P=40 \mathrm{~mm}^{3} / \mathrm{s}$.

It is obvious that, although this cutting tool effectiveness at the unhardened steel and cast iron processing is high enough, it is small for K19195 hardened steel.

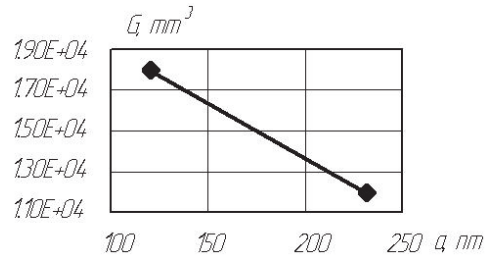

a)

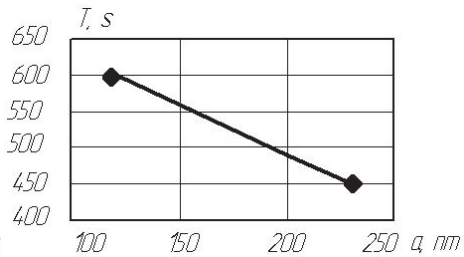

b)

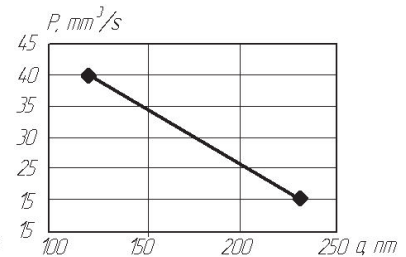

c)

Fig. 26. Dependencies of a) the K19195 hardened steel removable volume for durability period, b) the $0.18 \mathrm{HfN}+0.82 \mathrm{ZrN}$ coated $\mathrm{K} 40$ (USSR) cutting tool durability and

c) processing performance from the grain size

Investigation of the K40 (Russia) plate cutting tool (Fig. 27) revealed that the grain size becomes somewhat larger than K40 (USSR) plate grain size and lies in the range 108-303 nm for the plates on the flow axis and in the range $188-370 \mathrm{~nm}$ for plates on the flow periphery. In the first case, the zirconium/hafnium composition ratio is 67.48/28.42. In this case, the percentage compositions ratio is closer to the cathode original ratio. For the periphery plates ratio is close to the P40 ratio and equal 59.16/36.31, i.e. in this case zirconium selective evaporation has large influence on the coating properties (Fig. 27).

Similar dependences for the case of the ShKh15 hardened steel processing by the K40 (Russia) 
cutting tool with the same coating (Fig. 27) allow to provide the same volume of the removable material over the durability period $3.5 \cdot 10^{7} \mathrm{~mm}^{3}$ and at the almost nanostructured grain size 106 $\mathrm{nm}$, the grain size growth decreases rapidly to $2.5 \cdot 10^{4} \mathrm{~mm}^{3}$. The maximum cutting tool durability is realized with another grain size (in the $140-210 \mathrm{~nm}$ range) and at the further grain growth cutting tool durability is reduced. Processing performance dependence from the grain size repeats dependence for $G$ and the maximum value is $320 \mathrm{~mm}^{3} / \mathrm{s}$ (Fig. 27).

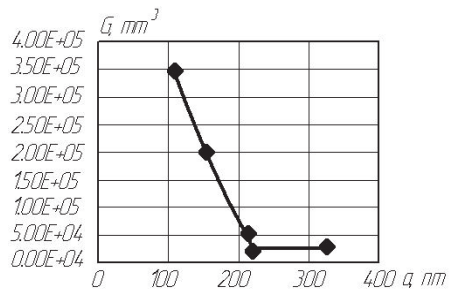

a)

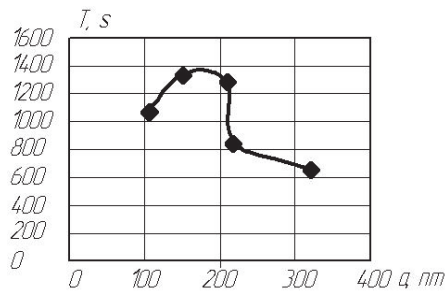

b)

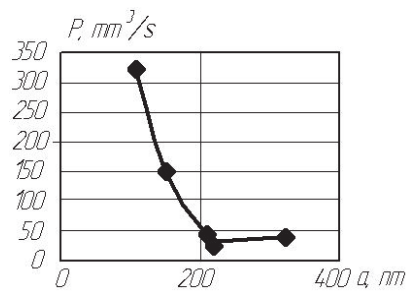

c)

Fig. 27. Dependencies of a) the K19195 hardened steel removable volume for durability period, b) the $0.18 \mathrm{HfN}+0.82 \mathrm{ZrN}$ coated $\mathrm{K} 40$ (Russia) cutting tool durability and c) processing performance from the grain size

The spectral composition and percentage analysis of the coating on the MS221 plate didn't performed unfortunately. The grain size investigations in the MS221 plate coating is shown on the Fig. 12. Grain size is in the range of $110-229 \mathrm{~nm}$ for plates on the flow axis and grain size is $128-428 \mathrm{~nm}$ on the flow periphery. In this case microhardness were measured, which, depending on the grain size, in the first instance was in the range 1270-1413 $\mathrm{Hv}$ and a larger value corresponds to a smaller grain size, while for the plates, located on the periphery, microhardness ranges is in the range from 915 to $1184 \mathrm{Hv}$. Measurements were performed by the PMT-3 instrument. In the last case lower microhardness values Hv from 732 to 891 were observed, which are associated with the submicrostructure grain size presence, which turn into microstructure grain. In this case, the microhardness minimum values were implemented.

Analysis of the removable volume dependencies for the durability period $G$, durability $T$ and processing performance $P$ for K19195 hardened steel turning by the cutting tools from $0.18 \mathrm{HfN}+0.82 \mathrm{ZrN}$ coated MS221 plate shows that the maximum value of the removable volume $G=5.5 \cdot 10^{5} \mathrm{~mm}^{3}$ is obtained with $120 \mathrm{~nm}$ grain size, cutting tool durability $T=870 \mathrm{~s}$ and processing capacity $P=64 \mathrm{~mm}^{3} / \mathrm{s}$ at the same grain size. It can be seen that $0.18 \mathrm{HfN}+0.82 \mathrm{ZrN}$ coated MS221 cutting tool allows to obtain acceptable results at the ShKh15 hardened steel processing (Fig. 28).

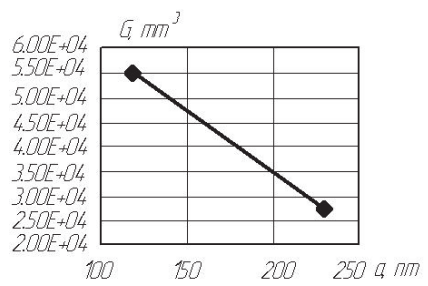

a)

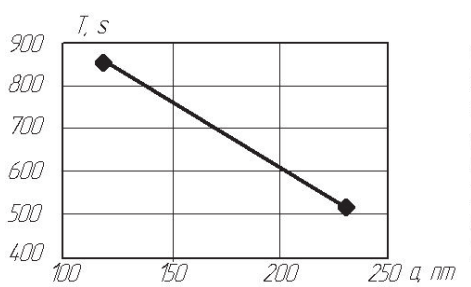

b)

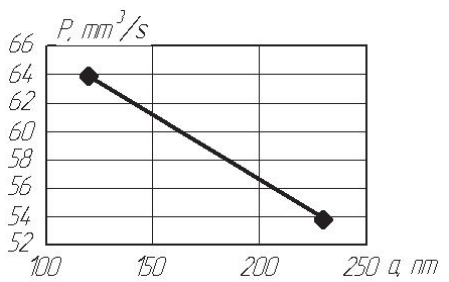

c)

Fig. 28. Dependencies of a) the K19195 hardened steel removable volume for durability period,

b) the $0.18 \mathrm{HfN}+0.82 \mathrm{ZrN}$ coated MS221 cutting tool durability and

c) processing performance from the grain size

If we analyze Table 1 [1] in respect to coating efficiency rating, we find that the coating on the K40 plate with minimal grain size has maximum removable material volume during the durability period, although in this case its durability is somewhat different from the maximum. For the 
MS221 plate the mode with fairly high rating on the removable material volume for durability period is realized too and has third rating (Table. 1). Obvious that this material may be used for the ShKh15 hardened steel processing.

VOK60 superhard material use for ShKh15 hardened steel processing showed that the maximum removable material for the durability period $G=2.6 \cdot 10^{5} \mathrm{~mm}^{3}$, the maximum durability $-T=200 \mathrm{~s}$ (implemented at $a \approx 70 \mathrm{~nm}$ ), and the maximum processing performance is provided at the grain size $103 \mathrm{~nm}$. It can be seen that the VOK60 superhard material is not effective at the ShKh15 hardened steel processing (Fig. 29).

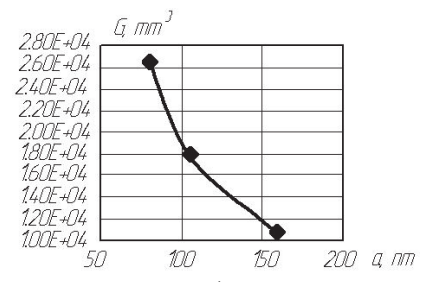

a)

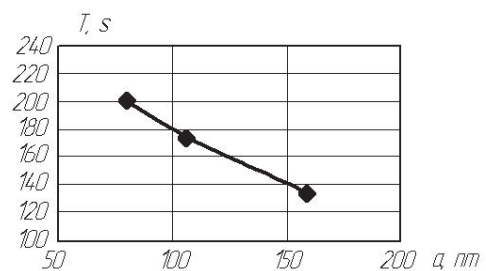

b)

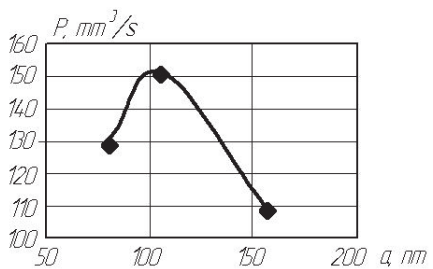

c)

Fig. 29. Dependencies of a) the K19195 hardened steel removable volume for durability period, b) the $0.18 \mathrm{HfN}+0.82 \mathrm{ZrN}$ coated VOK 60 cutting tool durability and c) processing performance from the grain size

Despite the fact that for coated P40 plate we have the grain size in the surface layer close to nanostructured size, the hardened steel processing tool performance is not high and has only tenth rating. Obviously, it's connected with P40 relatively low microhardness in comparison with K40 that reduces $\mathrm{P} 40$ efficiency. Performed investigations of the hard alloy cutting tools performance were also continued for the hardened steel turning; the results are shown on the Figs. 30-36. In this case, as the cutting tool were used $0.18 \mathrm{HfN}+0.82 \mathrm{ZrN}$ coated carbide Sandvik Koromant, two-layer $\mathrm{Al}_{2} \mathrm{O}_{3}+(0.18 \mathrm{HfN}+0.82 \mathrm{ZrN})$ coated Sandvik Koromant and hard alloys P40, MS221. Compared with the previous variants have been added $\mathrm{Al}_{2} \mathrm{O}_{3}+(0.18 \mathrm{HfN}+0.82 \mathrm{ZrN})$ coating, for which we have grain sizes from 75.1 to $159 \mathrm{~nm}$, i.e. in this case nanostructured grain was realized, which should provide a high rating. Unfortunately, this coating has fourteenth rating and, in principle, it is don't make sense to use it for the hardened steels processing due to the small $\mathrm{Al}_{2} \mathrm{O}_{3}$ coating surface layer microhardness. At the same time the $0.18 \mathrm{HfN}+0.82 \mathrm{ZrN}$ coating on the Sandvik Koromant plate provides maximum efficiency rating and the maximum cutting tool durability. This suggests that cutting tool operability and efficiency provides not only the minimum grain size, but the cutting tool surface layer microhardness, which is equal $35 \mathrm{GPa}$ for this coating.

For the same coating on the MS221 plate is realized the second mode on the material removable volume for the durability period, although the overall rating based on durability and productivity, takes on the maximum value, and this coated hard alloy can be used in all cases for the G10450 hardened steel processing.

Unfortunately, $0.18 \mathrm{HfN}+0.82 \mathrm{Zrn}$ coated $\mathrm{P} 40$ hard alloy can't be used effectively both for G10450 hardened steel machining, and for K19195 hardened steel machining.

Analysis of the $0.18 \mathrm{HfN}+0.82 \mathrm{ZrN}$ coated MS222 cutting tool research results showed that the maximum removable material volume (45 hardened steel) for the durability period $G=1.1 \cdot 10^{5} \mathrm{~mm}^{3}$ at the $a=70 \mathrm{~nm}$, the maximum durability $T=800 \mathrm{~s}$ at the $a=95 \mathrm{~nm}$ and the productivity $P=30 \mathrm{~mm}^{3} / \mathrm{s}$ at the $a=150 \mathrm{~nm}$. This suggests that each of the productivity and efficiency criteria has own grain size (Fig. 30).

Similar investigations were carried out for the MS221 cutting tool with the same coating (Fig. 34). The maximum value is realized for $G=1.05 \cdot 10^{5} \mathrm{~mm}^{3}$ at the $a=123 \mathrm{~nm}$, for $T=850 \mathrm{~s}$ at the $a=123 \mathrm{~nm}$ and for $P=40 \mathrm{~mm}^{3} / \mathrm{s}$ at the $a=210 \mathrm{~nm}$. For the same MS221 coated cutting tool are realized more efficient modes $G_{1}=7.2 \cdot 10^{5} \mathrm{~mm}^{3}$ at the $a=105 \mathrm{~nm}$ (Fig. 32) and 
$G_{2}=5.7 \cdot 10^{5} \mathrm{~mm}^{3}$ for the $a=73 \mathrm{~nm}$ (Fig. 33) and $G_{3}=8 \cdot 10^{5} \mathrm{~mm}^{3}$ (Fig. 34), $T_{1}=2500 \mathrm{~s}$, $T_{2}=1900 \mathrm{~s}$ and $T_{3}=800 \mathrm{~s}$, and $P_{1}=400 \mathrm{~mm}^{3} / \mathrm{s}, P_{2}=30 \mathrm{~mm}^{3} / \mathrm{s}$ and $P_{3}=15 \mathrm{~mm}^{3} / \mathrm{s}$, respectively. It can be seen that the first mode is more preferred, then the second mode can be used for employment.

For $0.18 \mathrm{HfN}+0.82 \mathrm{ZrN}$ coated Sandvik Koromant cutting tool results of the 45 hardened steel turning are shown on the Fig. 35 and 36. It was found that $G_{1}=1.27 \cdot 10^{5} \mathrm{~mm}^{3}$ is realized at the $a=110 \mathrm{~nm}$, the $G_{2}=5 \cdot 10^{5} \mathrm{~mm}^{3}$ for $a=300 \mathrm{~nm} ; T_{1}=4500 \mathrm{~s}, T_{2}=4000 \mathrm{~s}$, and $P_{1}=50 \mathrm{~mm}^{3} / \mathrm{s}$ and $P_{2}=70 \mathrm{~mm}^{3} / \mathrm{s}$. It is evident that with the grain size growth all parameters, except $P$, are reduced, and $P$ increases and reaches a maximum at the $a=560 \mathrm{~nm}$. All this suggests that, despite the fact that the first mode for Sandvik Koromant cutting tool is successful on the removable volume for the durability period and cutting tool durability, but the mode with $560 \mathrm{~nm}$ large grain size is more rational on the productivity.

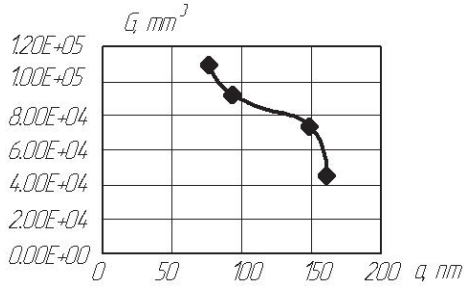

a)

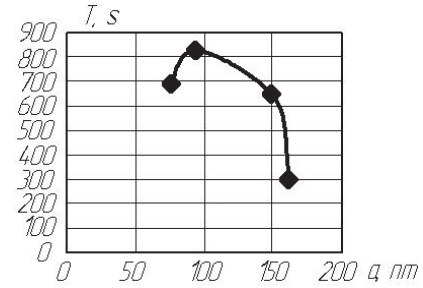

b)

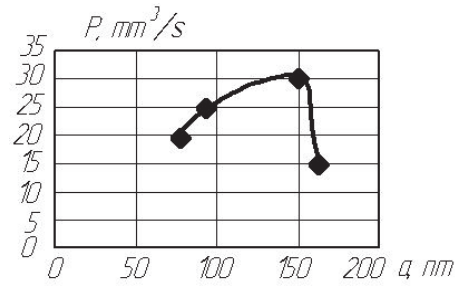

c)

Fig. 30. Dependencies of a) the manufacturable G10450 hardened steel removable volume for durability period, b) the $0.18 \mathrm{HfN}+0.82 \mathrm{ZrN}$ coated MS222 cutting tool durability and

c) processing performance from the grain size

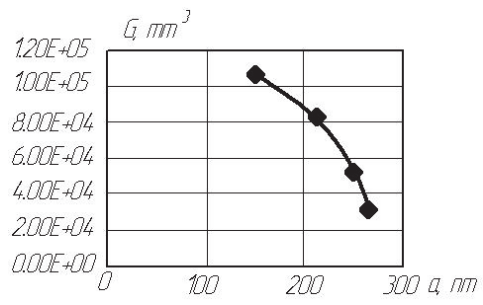

a)

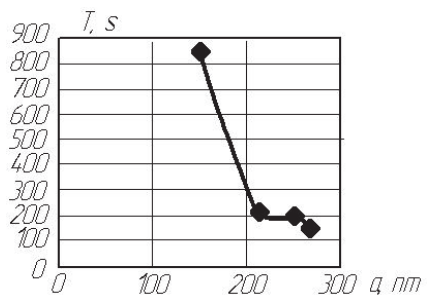

b)

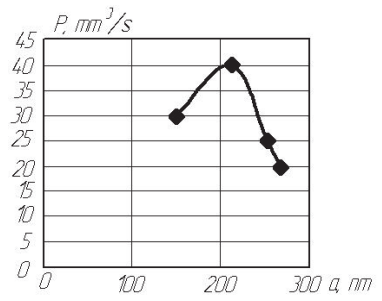

c)

Fig. 31. Dependencies of a) the manufacturable G10450 hardened steel removable volume for durability period, $b$ ) the $0.18 \mathrm{HfN}+0.82 \mathrm{ZrN}$ coated MS221 cutting tool durability and

c) processing performance from the grain size

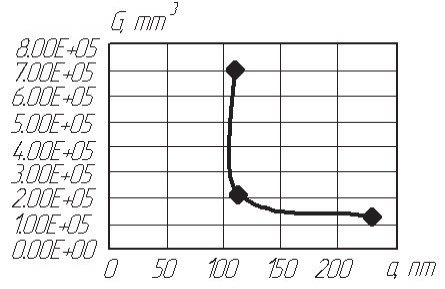

a)

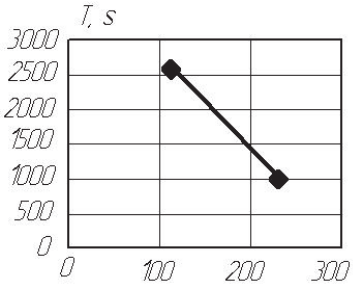

b)

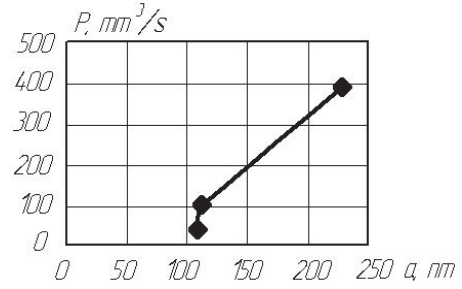

c)

Fig. 32. Dependencies of a) the manufacturable G10450 hardened steel removable volume for durability period, b) the $0.2 \mathrm{HfN}+0.8 \mathrm{ZrN}$ coated MS221 cutting tool durability and

c) processing performance from the grain size 


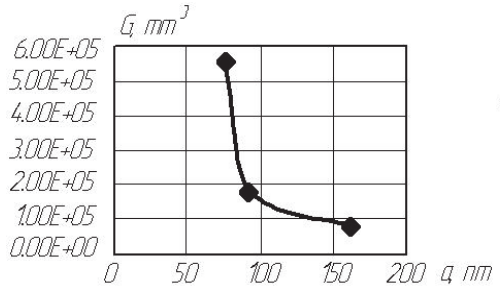

a)

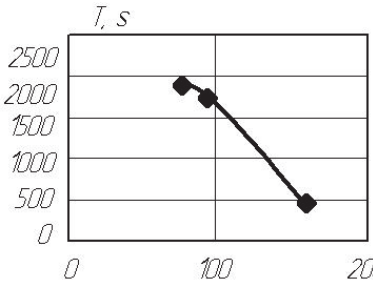

b)

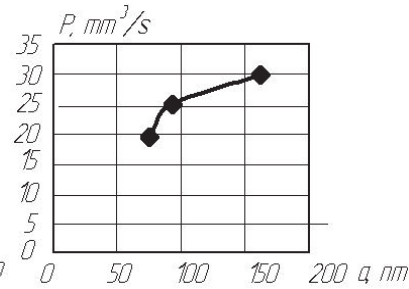

c)

Fig. 33. Dependencies of a) the manufacturable G10450 hardened steel removable volume for durability period, b) the $0.2 \mathrm{HfN}+0.8 \mathrm{ZrN}$ coated MS221 cutting tool durability and

c) processing performance from the grain size

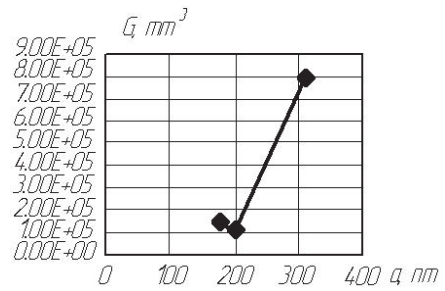

a)

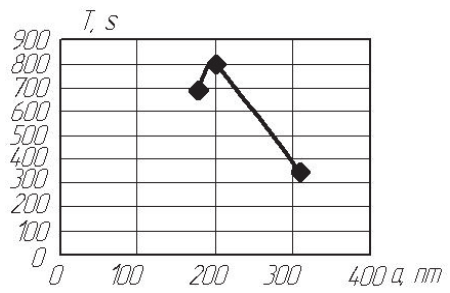

b)

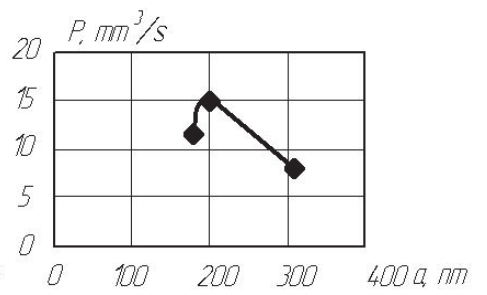

c)

Fig. 34. Dependencies of a) the manufacturable G10450 hardened steel removable volume for durability period, b) the $0.2 \mathrm{HfN}+0.8 \mathrm{ZrN}$ coated MS221 cutting tool durability and

c) processing performance from the grain size

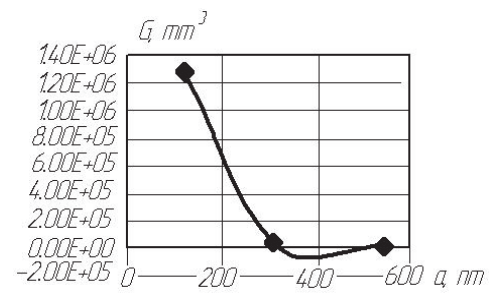

a)

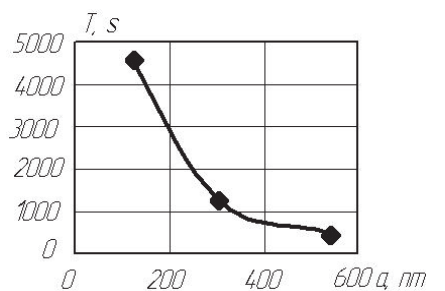

b)

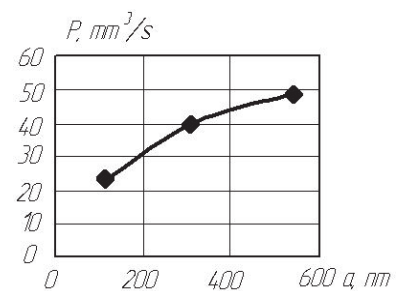

c)

Fig. 35. Dependencies of a) the manufacturable G10450 hardened steel removable volume for durability period, b) the $0.2 \mathrm{HfN}+0.8 \mathrm{ZrN}$ coated Sandvik Koromant cutting tool durability and

c) processing performance from the grain size

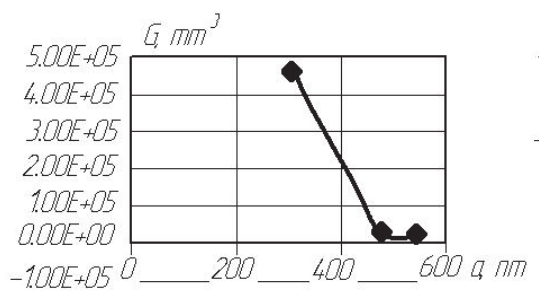

a)

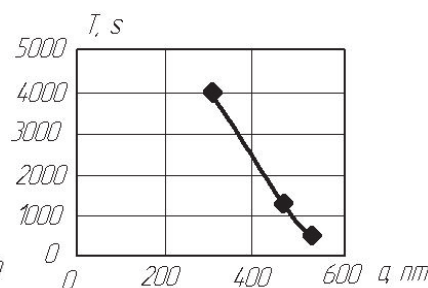

b)

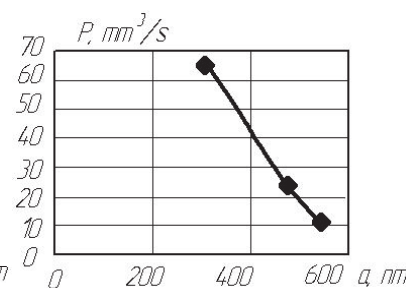

c)

Fig. 36. Dependencies of a) the manufacturable G10450 hardened steel removable volume for durability period, b) the $0.2 \mathrm{HfN}+0.8 \mathrm{ZrN}$ coated Sandvik Koromant cutting tool durability and

c) processing performance from the grain size

\subsection{Conclusions}

1) It is shown that at the effectiveness and efficiency assessing of the coated hard alloys at the K19195 hardened materials and G10450 steel processing it is necessary to take into account the coating grain size at that to the smaller grain size is generally (but not always) corresponds to more 
effective processing (the maximum removable material volume for the durability period) and its working capacity.

2) For 45 steel effective processing can be used hard alloy of the Sandvik Koromant company with $0.18 \mathrm{HfN}+0.82 \mathrm{ZrN}$ coating and MS221 hard alloy, which will be both effective and serviceable.

3) It was found that $0.18 \mathrm{HfN}+0.82 \mathrm{ZrN}$ coated $\mathrm{K} 40$ hard alloy and MS221 plate with the same coating have greater efficiency for the K19195 hardened steel processing.

\section{The cast iron machining effectiveness by the nano- and submicrostructure coated cutting tool}

Investigation results of the material removable volume for the durability period $G$, cutting tool durability and iron cast machining performance $P$ are shown on the Figs. 37-46. Thus, in the special cast iron machining case by the $0.2 \mathrm{HfN}+0.8 \mathrm{ZnN}$ coated Sandvik Koromant company cutting tool at the coating deposition displacement potentials are $U=250 \mathrm{~V}$ and $U=500 \mathrm{~V}$ (Figs. 37 and 38 respectively). Obviously, that at the smaller values of $U=250 \mathrm{~V}$ removable volumes value significantly greater than for $U=500 \mathrm{~V}$; also the cutting tool durability values are higher, which with the grain size growth is reduced substantially: from $G=1.2 \cdot 10^{6}$ to $2 \cdot 10^{5} \mathrm{~mm}^{3}$ $(U=250 \mathrm{~V})$ and $T=1700$ to $200 \mathrm{~s}(U=250 \mathrm{~V})$ and from $G=7.2 \cdot 10^{5}$ to $1.8 \cdot 10^{5} \mathrm{~mm}^{3}$ and $T=770$ to $T=270 \mathrm{~s}(U=500 \mathrm{~V})$. All this confirms the theoretical predictions that these values are associated with cutting tool material physical and mathematical properties, which with the grain size growth is reduced, that leads to these quantities dependence on the grain size. The maximum processing performance for $U=250 \mathrm{~V}$ is realized at the grain size near $110 \mathrm{~nm}$, while for $U=500 \mathrm{~V}$ it realized at the grain size about $150 \mathrm{~nm}$. Material removable volume over the durability period, cutting tool durability and machining performance which can be used at the processing by $0.2 \mathrm{HfN}+0.8 \mathrm{ZnN}$ coated Sandvik Koromant tool may be estimated on these dependencies.

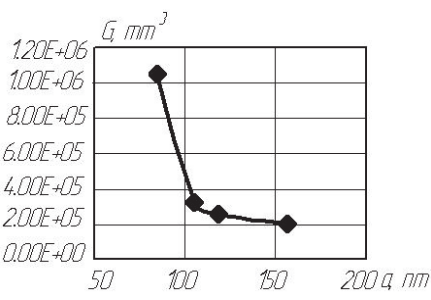

a)

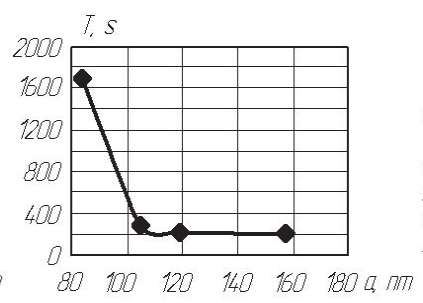

b)

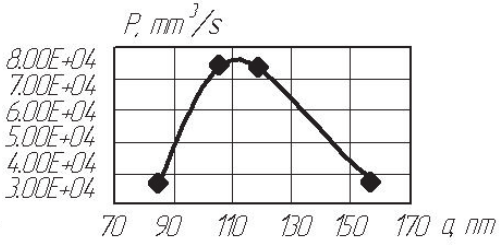

c)

Fig. 37. Dependencies of a) the manufacturable special cast iron (NiW) removable volume for durability period, b) the $0.2 \mathrm{HfN}+0.8 \mathrm{ZrN}$ coated Sandvik Koromant cutting tool durability at the displacement potential $(U=250 \mathrm{~V})$ and c) processing performance from the grain size

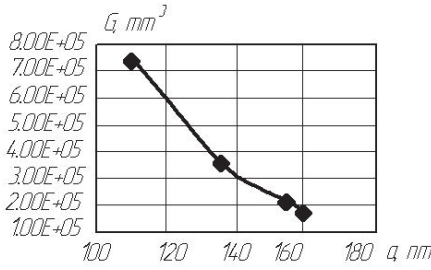

a)

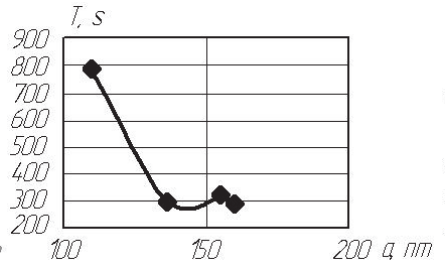

b)

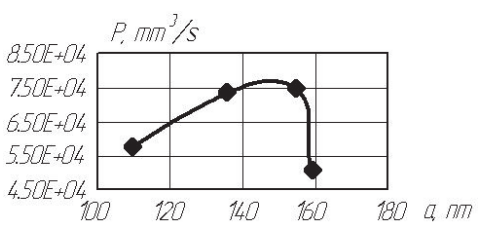

c)

Fig. 38. Dependencies of a) the manufacturable special cast iron (NiW) removable volume for durability period, b) the $0.2 \mathrm{HfN}+0.8 \mathrm{ZrN}$ coated Sandvik Koromant cutting tool durability at the displacement potential $(U=500 \mathrm{~V})$ and $\mathrm{c})$ processing performance from the grain size 


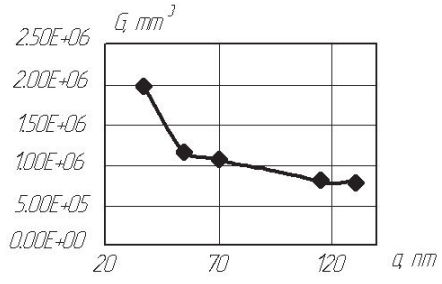

a)

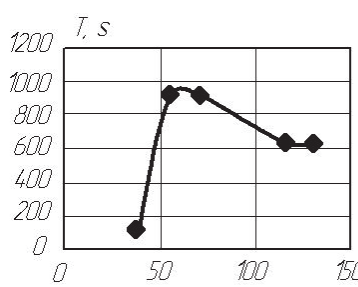

b)

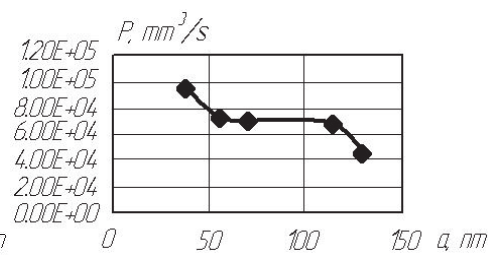

c)

Fig. 39. Dependencies of a) the manufacturable special cast iron (NiW) removable volume for durability period, b) the $0.2 \mathrm{HfN}+0.8 \mathrm{ZrN}$ coated MS221 cutting tool durability at the displacement potential $(U=250 \mathrm{~V})$ and c) processing performance from the grain size

Similar dependences for the case of the cast iron machining by $0.2 \mathrm{HfN}+0.8 \mathrm{ZnN}$ coated MS221 cutting tool at the displacement potential $U=250 \mathrm{~V}$ are shown in Fig. 39. Evidently that the removable volume and productivity are reduced with the grain size growth, while the cutting tool durability has maximum value, for nanostructured grain $(a=35 \mathrm{~nm})$ productivity $G$ reaches $2 \cdot 10^{6} \mathrm{~mm}^{3}$ and then decreases to $7.5 \cdot 10^{5} \mathrm{~mm}^{3}$ at the $a=130 \mathrm{~nm}$. Durability maximum is realized at the $60 \mathrm{~nm}$ grain size, and at the smaller and larger grain size durability is somewhat lower. Productivity for nanostructured grains is $10^{5} \mathrm{~mm}^{3} / \mathrm{s}$, i.e. presented dependences allow to evaluate the performance of the coated MC221 tricarbidic hard alloy at the cast iron processing.

A similar dependences for processing of the of special cast iron by $0.2 \mathrm{HfN}+0.8 \mathrm{ZnN}$ coated K40 (Russia) cutting tool at the displacement potential $U=250 \mathrm{~V}$ are shown on the Fig. 40 . Evidently, that the removable volume is significantly reduced with increasing grain size and at the $130 \mathrm{~nm}$ grain size the cutting modes are almost unacceptable for processing. At the same time durability dependence has nonmonotonic character: at first it reduced, then it rises, and the minimum durability is realized at the $120 \mathrm{~nm}$ grain size. Productivity behaves similarly - it is maximal both at the small grain size $(80 \mathrm{~nm})$ and large grain size $(120 \mathrm{~nm})$.

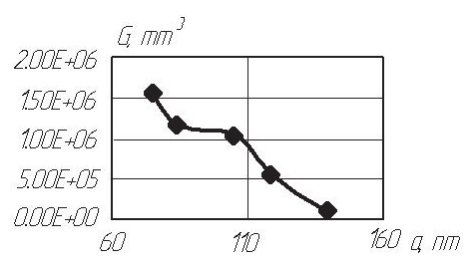

a)

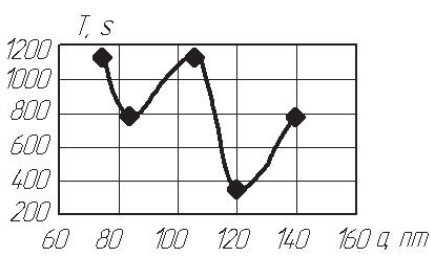

b)

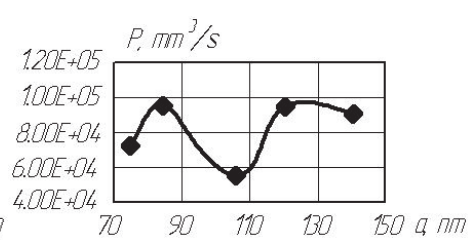

c)

Fig. 40. Dependencies of a) the manufacturable special cast iron (NiW) removable volume for durability period, b) the $0.2 \mathrm{HfN}+0.8 \mathrm{ZrN}$ coated $\mathrm{K} 40$ (Russia) cutting tool durability at the displacement potential $(U=250 \mathrm{~V})$ and $\mathrm{c})$ processing performance from the grain size

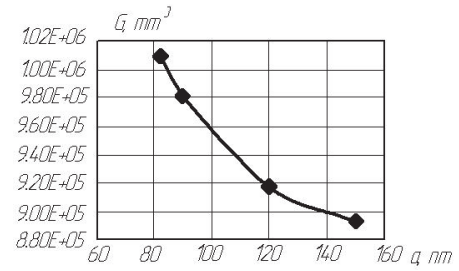

a)

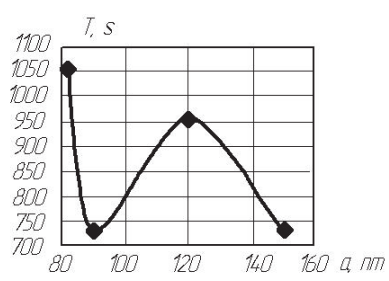

b)

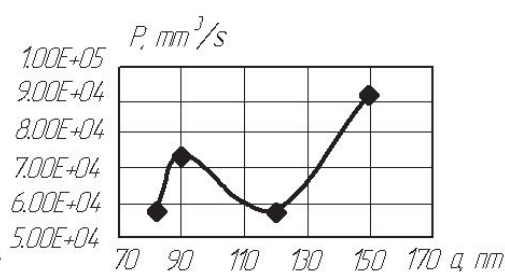

c)

Fig. 41. Dependencies of a) the manufacturable special cast iron (NiW) removable volume for durability period, b) the $0.2 \mathrm{HfN}+0.8 \mathrm{ZrN}$ coated K40 (Russia) cutting tool durability at the displacement potential $(U=500 \mathrm{~V})$ and c) processing performance from the grain size

For the same cutting tool with the same coating but at the $500 \mathrm{~V}$ potential (Fig. 41) maximum removable volume significantly decreased, although durability remained practically permanent. 
$\mathrm{Al}_{2} \mathrm{O}_{3}$, durability and productivity changes have undulating character. The maximum grain size is equal $120 \mathrm{~nm}$.

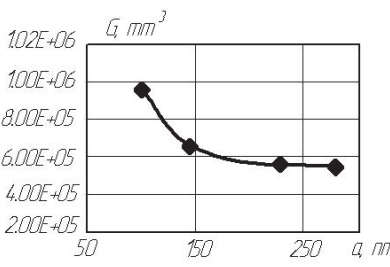

a)

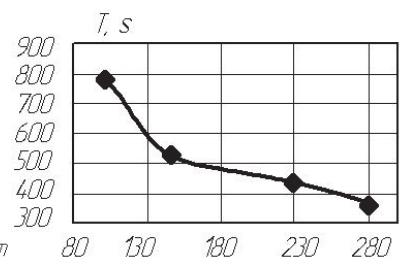

b)

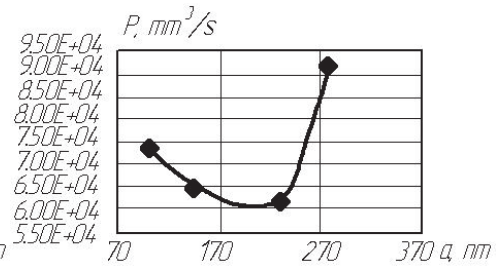

c)

Fig. 42. Dependencies of a) the manufacturable special cast iron (NiW) removable volume for durability period, b) the $0.2 \mathrm{HfN}+0.8 \mathrm{ZrN}$ coated $\mathrm{K} 40$ (Russia) cutting tool durability at the displacement potential $(U=110 \mathrm{~V})$ and $\mathrm{c})$ processing performance from the grain size

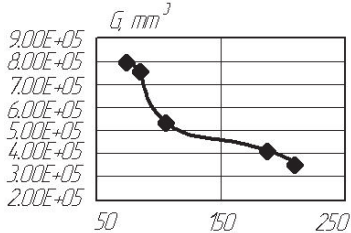

a)

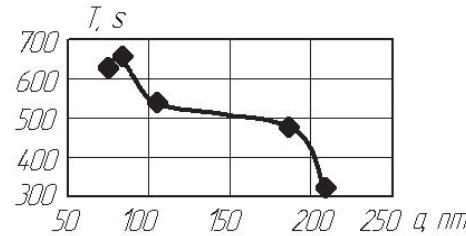

b)

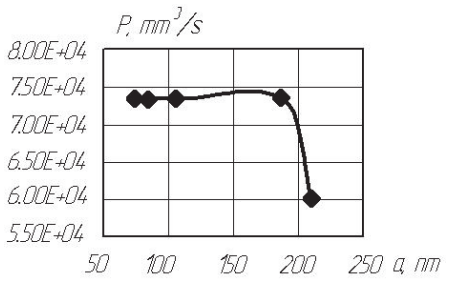

c)

Fig. 43. Dependencies of a) the manufacturable special cast iron (NiW) removable volume for durability period, b) the $0.2 \mathrm{HfN}+0.8 \mathrm{ZrN}$ coated $\mathrm{P} 40$ cutting tool durability at the displacement potential $(U=250 \mathrm{~V})$ and c) processing performance from the grain size

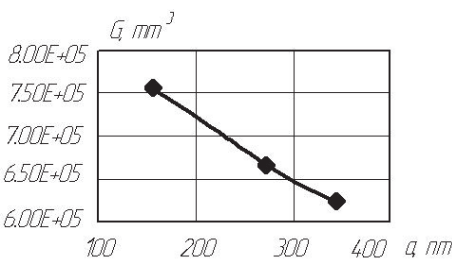

a)

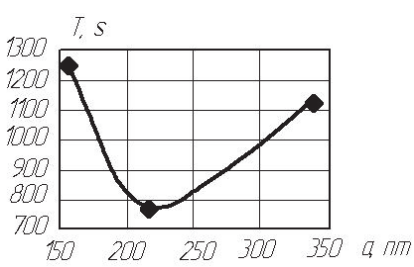

b)

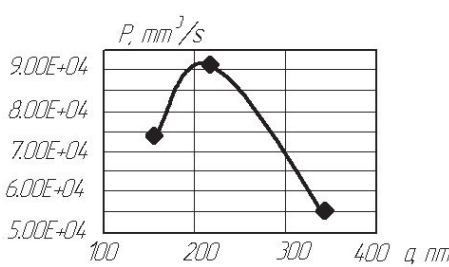

c)

Fig. 44. Dependencies of a) the manufacturable special cast iron (NiW) removable volume for durability period, $b$ ) the $0.2 \mathrm{HfN}+0.8 \mathrm{ZrN}$ coated $\mathrm{K} 40$ (USSR) cutting tool durability at the displacement potential $(U=250 \mathrm{~V})$ and $\mathrm{c})$ processing performance from the grain size

For similar conditions, but with displacement potential equal $110 \mathrm{~V}$, removable volume decreased, durability also decreases relatively smoothly and the productivity is reduced firstly and then reaches to the maximum value at the $275 \mathrm{~nm}$ size - Fig. 42. The cast iron processing effectiveness by P40 cutting tool with the same coating when the displacement potential is $250 \mathrm{~V}$ is low, since removable volume is reduced to $8 \cdot 10^{5} \mathrm{~mm}^{3}$, the maximum durability is $660 \mathrm{~s}$, but the productivity is maintained at the high level in the grain sizes range from 70 to $180 \mathrm{~nm}$ and equal $7.4 \cdot 10^{4} \mathrm{~mm}^{3} / \mathrm{s}$ (ris.43). For the case of the special cast iron processing by K40 (USSR) cutting tool with the same coating at the $250 \mathrm{~V}$ displacement potential results are shown on Figs. 42-44. It can be seen that the removable volume is approximately the same as for P40 plate and decreases with grain size increasing due to rather high grain size values. At the grain growth the durability at first decreases and then slightly increases (minimum value at the $215 \mathrm{~nm}$ grain size), whereas productivity in this case has maximum value (Fig. 44).

At the cast iron processing by the TH20 cutting tool with the same coating at $250 \mathrm{~V}$ displacement potential the removable volume is relatively little, durability is low too and the maximum productivity is realized at the $240-320 \mathrm{~nm}$ grain size and equal $9.3 \cdot 10^{4} \mathrm{~mm}^{3} / \mathrm{s}$, i.e. this 
mode is favorable for the maximum processing productivity case (Fig. 45).

Similar dependences for the special cast iron processing case by $\mathrm{Al}_{2} \mathrm{O}_{3}+0.2 \mathrm{HfN}+0.8 \mathrm{ZrN}$ coated Sandvik Koromant cutting tools show that the maximum removable volume increased by more than an order of magnitude. This is due to the fact that the $\mathrm{Al}_{2} \mathrm{O}_{3}$ coating (cutting tool outer coating) has minimal adhesive interaction with cast iron, what provides minimum adhesive wear and friction coefficient significant reduction, but even with the grain size growth in the nanostructured range (from 37 to $95 \mathrm{~nm}$ ), removed metal volume value still decreases. Durability also decreases with grain size increasing, but productivity increases and reaches maximum value at the $95 \mathrm{~nm}$ grain size. Obviously that the two-layer coating at the cast iron processing has large advantages, since adhesive wear is the main wear form and $\mathrm{Al}_{2} \mathrm{O}_{3}$ outer coating significantly reduces its (Fig. 46).

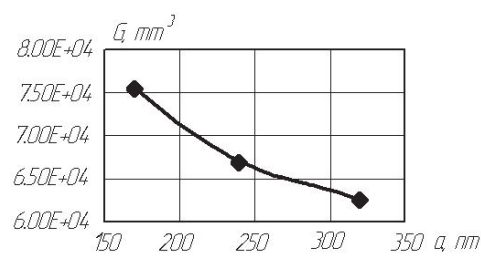

a)

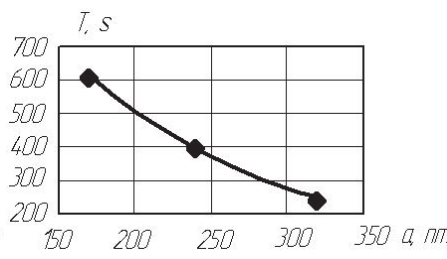

b)

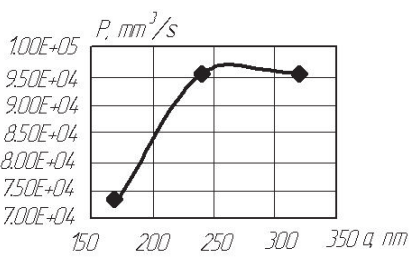

c)

Fig. 45. Dependencies of a) the manufacturable special cast iron (NiW) removable volume for durability period, b) the $0.2 \mathrm{HfN}+0.8 \mathrm{ZrN}$ coated TH20 cutting tool durability at the displacement potential $(U=250 \mathrm{~V})$ and c) processing performance from the grain size

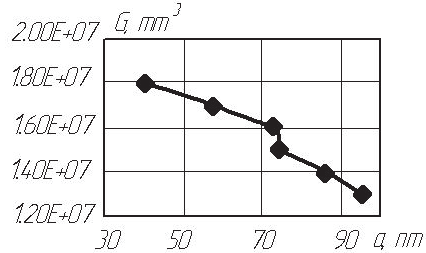

a)

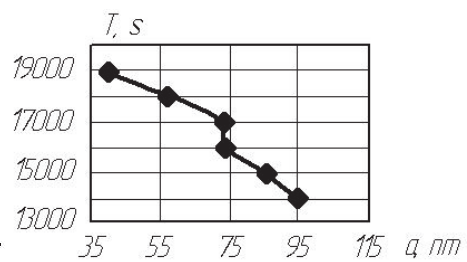

b)

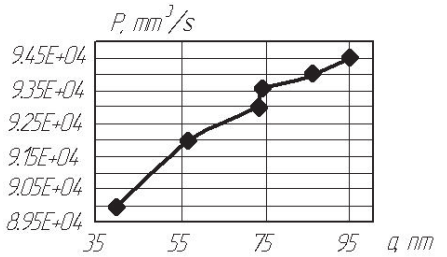

c)

Fig. 46. Dependencies of a) the manufacturable special cast iron (NiW) removable volume for durability period, b) the $\mathrm{Al}_{2} \mathrm{O}_{3}+0.2 \mathrm{HfN}+0.8 \mathrm{ZrN}$ coated Sandvik Koromant cutting tool durability and

c) processing performance from the grain size

It has been shown that at the cast iron processing the coating adhesive interaction with the manufacturable material is an important characteristic which provide unique value of removable volume for durability period of the $1.3 \cdot 10^{7}-1.810^{7} \mathrm{~mm}^{3}$ order. The maximum durability $(19,000-14,000 \mathrm{~s})$ also realized in this case, maximum productivity is realized at the minimum $G$ and $T$ values.

MS221 $(U=250 \mathrm{~V})$, Sandvik Koromant $(U=250 \mathrm{~V})$ and VK8 (Russia) $(U=250 \mathrm{~V})$ cutting tools have acceptable processing modes. All other cutting tools and coating deposition modes are less effective.

Performed investigations allow to predict the removable material volume over the durability period, cutting tool durability and processing productivity depending on the coating grain size. It is important to select the cutting tool coating and processing mode.

\section{Conclusions}

The microhardness and yield strength decrease with grain size increasing for wide range of the $0.2 \mathrm{HfN}+0.8 \mathrm{ZrN}$ coated materials (K40, Sandvik Koromant, MS221) was disclosed.

Two-layer $\mathrm{Al}_{2} \mathrm{O}_{3}+(0.2 \mathrm{HfN}+0.8 \mathrm{ZrN})$ coated Sandvik Koromant plate microhardness changes slightly (due to the fact that almost always nanostructures implemented) with the grain growth 


\section{(33.7-63.8 nm, $H_{\mu}=16.08-17.14 \mathrm{GPa}$.}

The coating effective application criterion will condition: the coating grain size should be less than the cutting tool base material grain size.

Maximum microhardness is realized at the TiN concentrations in diborides from 50 to $80 \%$ and grain sizes range from 5 to $13 \mathrm{~nm}$.

It is proved that modulus of elasticity increases with the grain size growth.

It is shown that at the effectiveness and efficiency assessing of the coated hard alloys at the K19195 hardened materials and G10450 steel processing it is necessary to take into account the coating grain size at that to the smaller grain size is generally (but not always) corresponds to more effective processing (the maximum removable material volume for the durability period) and its working capacity.

For G10450 steel effective processing can be used $0.18 \mathrm{HfN}+0.82 \mathrm{ZrN}$ coated Sandvik Koromant company solid alloy, MS221 and K40, which will be both effective and operable.

It found that at the cast iron processing the coating adhesive interaction with the manufacturable material is an important characteristic which provide unique value of removable volume for durability period of the $1.310^{7}-1.810^{7} \mathrm{~mm}^{3}$ order. The maximum durability $(19,000-14,000 \mathrm{~s})$ also realized in this case, maximum productivity is realized at the minimum $G$ and $T$ values.

Performed investigations allow to predict the removable material volume over the durability period, cutting tool durability and processing productivity depending on the coating grain size. It is important to select the cutting tool coating and processing mode.

\section{References}

[1] Gusev A. I. Nanocrystalline Materials: Methods of Obtaining and Properties. Publishing House of RAS, Ekaterinburg, 1998, p. 302.

[2] Andrievsky R. A. Nanomaterials: the concept and modern problems. Physics of Metals and Metallography, Vol. 91, Issue 1, 2003, p. 50-56.

[3] Gusev A. I. Nanomaterials, Nanostructures, Nanotechnologies. Fizmatlit, Moscow, 2005, p. 416.

[4] Kostyuk G. I. Nanotechnology: Selection of Technological Parameters and Installations, Processing Capacity, Physical and Mechanical Characteristics of Nanostructures. Publishing House of the International Academy of Science and Innovate Technologies, 2014, p. 479.

[5] Kostyuk G. I. Nanotechnology: Theory, Experiment, Technology, Perspectives. Technologies, 2012, p. 648 .

[6] Kostyuk G. I. Nanostructures and Nanocoatings: Perspectives and Reality. Zhukovsky "KhAI”, 2009, p. 406 .

[7] Kostyuk G. I. Physical and technical principles of coating, ion implantation and ion doping, laser processing and hardening, combined technologies. Book 1. Physical Processes of Plasma-Ion, Ion-Beam, Plasma, Light-Beam and Combined Technologies. Publishing House of the AINU, 2002, p. 596.

[8] Kostyuk G. I. Physical and Technical Principles of Coating, Ion Implantation and Ion Doping, Laser Processing and Hardening, Combined Technologies. Book 2. Handbook for Calculating the Basic Physical and Technological Parameters, Assessing the Possibilities, Choosing the Type of Technology and Equipment. Publishing House of the AAS, 2002, p. 482.

[9] Kostyuk G. I. Effective Tool with Nanocoatings and Nanostructured Modification Layers. Book. 1. Plasma-Ion and Ion-Beam Technologies, Publishing House "Planet-print", 2016, p. 735.

[10] Kostyuk G. I. Effective Tool with Nanocoatings and Nanostructured Modification Layers. Book. 2. Laser Technologies, Publishing House "Planet-print", 2017, p. 507.

[11] Kostyuk G. I. Effective Coatings and Modified Hardened Layers on Cutting Tools. Publishing House of the International Academy of Science and Innovate Technologies, 2012, p. 728. 
Kostyuk Gennady Igorevich Professor of the Department of Theoretical Mechanics, Machine Science and Robot Mechanics Systems of the National Aerospace University. N. E. Zhukovsky "KhAI", vice-president of the International Academy of Sciences and Innovative Technologies. Doctor of Technical Sciences, Professor, Academician of the Academy of Engineering Sciences and the Academy of Sciences of Technological Cybernetics of Ukraine. The scientific heritage of G. I. Kostyuk counts 1070 scientific works (including 210 works in the far abroad), 8 of them are monographs, 32 textbooks and textbooks, 12 patents for inventions and 13 copyright certificates. Under his leadership is protected 4 doctoral and 25 master's theses. 


\title{
4.3. Effect of technological factors on electromagnetic wave absorption properties of the polymer composites
}

\author{
Tamaz Natriashvili ${ }^{1}$, Jimsher Aneli ${ }^{2}$, Lana Shamanauri ${ }^{3}$ \\ R. Dvali Institute of Machine Mechanics, 10 Mindeli Str., Tbilisi 0186, Georgia \\ E-mail: ${ }^{1} T$ natriashvili@yahoo.com, ${ }^{2}$ jimaneli@yahoo.com, ${ }^{3}$ lana-Shamanauri@mail.ru
}

\begin{abstract}
Effect of different technological factors on the electromagnetic wave (EMW) with frequencies (3-10 GHz) absorbing properties of polymer composites based on epoxy and silicon resin with electric conducting (carbon black, graphite) and magnetic (nickel, cobalt, ferrite) nanofillers have been investigated. There are obtained the composites with one and binary fillers, concentration of which changes in the wide range. Relatively high absorption is manifested for composites containing 40-50 $\mathrm{wt} \%$ filler. It is established that the high frequency absorption capability of investigated materials essentially increases with the degree of homogeneity of distribution of filler particles in the polymer matrix. For the composites with ferrite and graphite so called synergistic effect (non-additive increasing of some parameters at definite proportion of two or more fillers) was observed. By variation of the absorber profile the composite with maximal absorption of EMW has been selected. Besides of the effect of electric and magnetic fields at hardening of the composites on the wave absorbing capability has been investigated. It is established that the level of the EMW absorption may be regulated by collection of the sandwich type absorber contained several absorbing films with different distribution and content of these films.
\end{abstract}

Keywords: polymer composite, electric and magnetic fillers, absorption of electromagnetic waves, synergistic effect, sandwich type absorber.

\section{Introduction}

Currently a great interest causes the problem of the absorption of high and ultrahigh frequency electromagnetic waves (from the range hundred millions to several ten milliards Herz) by different materials, because this problem is significant at solution of many practical tasks $[1,2]$. These materials in the form of thin plates often are used in design of the cameras without echo with radio-engineering destination. These materials are used also for the protection of communication equipment's and staff, etc. [2-4]. In general, the absorbers of EMW present the ceramic and polymer composites filled with different effectively absorbing fine dispersed fillers or constructions, at interaction with which the intensity of these waves decreases essentially [5]. The absorbers of EMW waves are used widely for design of the complexes including antenna system [6]. The materials absorbing EMW are used also in the sphere of sertification of compatibility with electromagnetic waves [7].

The EMW absorbers on a physical basis divided on three groups:

1. Materials and installations, in which the decreasing of the reflected energy is reached by their muffling near absorber surface because of interference, which are manufactured as the package of thin plate like absorber films $[8,9]$.

2. Second group includes the materials, in which EMW energy translates to thermal energy on the basis of dielectric loses and in result of muffling of waves in absorber with structural inhomogeneity. This group includes also the absorbing coatings made from all organic and inorganic material, by which the reflecting materials (usually metallic) are coated. In this case the coatings absorb the amount of the EMW energy more than they rich to the reflective surface. The absorbers of these groups have very high design, which is presented as the tubular cells, usual cells, conical, pyramidal, honeycomb, wedge-shape, porous rubber carpets et al, the fundamental principle of which is the monotonic increasing of the amount of included particles, which are responsible in the absorbing-reflecting processes [10].

3. This group includes the ferromagnetic materials interacting with $\mathrm{E}$ and $\mathrm{H}$ fields of EMW 
and have the electric and magnetic losses. They are produced mainly as ferrite plates or the rubber carpets. The creation of the absorbers on their basis now is very perspective and actual [11].

Classification of the radio-wave absorbing materials (RAM) has a conditional character. The resonance or frequently tuned RAM provides the partial or full neutralization of the emitted energy. The neutralization effect is importable for absorber, the thickness of which is equal to $1 / 4$ of the wave length. In this case the waves reflected from absorber surface are in anti-phase to incident wave and consequently in result of interference of these waves it takes place neutralization of the waves (Yauman effect). Consequently, the considered phenomenon realized only in the case of fixed frequency of EMW.

The absorbers on the basis of ferromagnetic fillers mainly included the fine dispersed magnetic particles (the nanoparticles among them) are characterized with wide range of absorbed waves (in result of absorption and reflection of the EMW). This absorption is based on increasing of both dielectric and magnetic loses [12-14].

The RAM filled with different type ferromagnetic (shortly magnetic) high dispersive particles at irradiation by EMW under effect of alternative magnetic fields take participation in the oscillatory motion and the high frequency energy translates to the heat one. The heat energy dissipates and disappeared. There are the RAM consisting electric conducting material and dielectric layers. Weakening of the reflected wave is due to summation of the opposite phases of the waves (interference) reflected from the object metal surface, dielectric and electric conducting layer $[15,16]$. Besides of today the works on the noted problem are sufficiently large, many unresolved issues are stay, among which the mechanism of the absorption of the EMW in the heterogeneous organic or inorganic systems is very essential. One of the main embarrassing inhibitory reasons of widening the investigations on the given theme is the strong dependence of the absorption energy on the frequency of EMW.

The aim of the presented work is creation of the materials effectively absorbing RW on the basis of new polymer composites containing electric conducting and magnetic fine dispersed powders.

\section{Experimental}

Experimental manipulations were provided in two directions: 1) Obtaining of the polymer composites and 2) measurements for establishment of absorption of the EMW energy by composite materials.

\subsection{Research objects and methods of obtaining}

The samples on the basis of diane epoxy resin and some inorganic (graphite, carbon black, ferrite, cobalt micro-particles and nickel nano-particles) fillers were obtained. Polyethylene polyamine was used as a hardener. In the second series the polydimethylmethylvinylsiloxane resin as polymer matrix was used for obtaining the EMW absorber including the same fillers. The composites were obtained by following manipulations of the procedures: 1) Mixing of ingredients in the spatial mixer; 2) Placing of reaction blend to the cuvette with form of parallelepiped and sizes $70 \times 50 \times 5 \mathrm{~cm}$; 3 ) Solidification of blends; 4) 3-fold sandwich type absorbers preparation by gluing the plates with thickness $1 \mathrm{~mm}$.

With aim of study of the dependence of absorption properties of the composites the samples with different geometrical shapes and surfaces were obtained (Fig. 1). There were obtained the samples with following forms: a) saw type; b) rectangle sinusoidal and c) conic like. With this aim it was prepared the cuvettes with corresponded profiles from Teflon: a) rectangular sinusoidal, b) conical axial section and c) saw profile. 


\subsection{Testify of the obtained composites on EMW absorption}

The sample testing of the obtained composites on EMW absorption was provided on the installation presented on the Fig. 1.

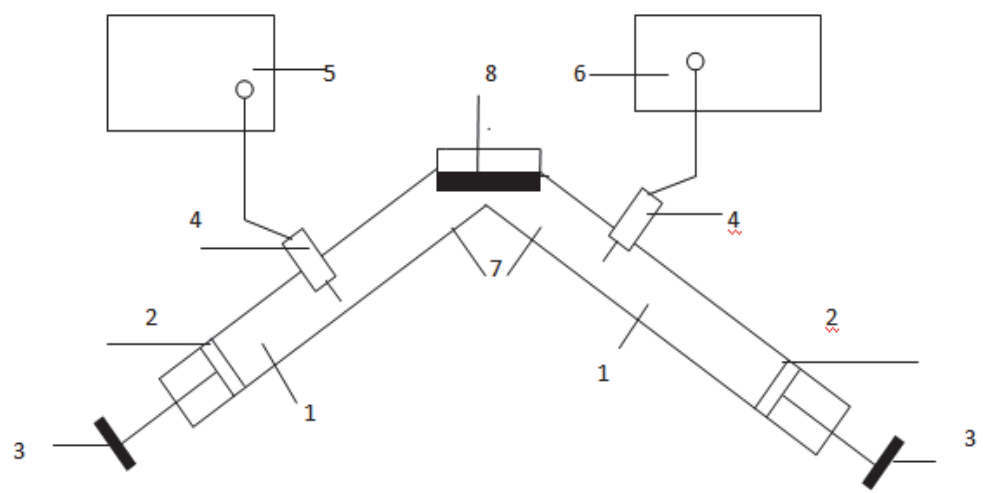

Fig. 1. Scheme of registration of the EMW reflected from samples: 1 - waveguide made from brass list,

2 - plunger - coordinator, 3 - its handle, 4 - the transmitting and receiving antennas (left contains the detector), 5 - generator of super high frequency, 6 - coordinator of the reflected EMW intensity,

7 - substrate of waveguide, 8 - reflecting plate with sample (attached from below)

The principle of working on the device is the following: EMW generated on the generator 5 with use of the transmission transfers to the waveguide and falls on the reflector plate 7 (without sample) and after reflection from it transfers to second wave guide, oriented in rectangular direction to first one, from which transmits through the receiver antenna 4 to the detector 6 and fixes as signal on the zero-indicator (is measured in the decibels). So, the device gives possibility of measuring of EMW intensity of reflected waves of any frequencies from the range 3-10 GHz. After that, they are placed on the surface of the reflector plate the sample and repeat the manipulations described above. By comparison of the intensities (in decibels) reflected from reflector without and with sample one estimates the ability of the absorption properties of the composite. Testify was provided by following manipulations: 1) switching of the device; 2) after 15-20 min the measuring of the intensity of reflected waves intensity from plate without sample; 3) displacement of the sample on the plate; 4) measuring of the wave intensity reflected from sample; 5) establishment of the absorption ability of the composite by comparison of intensities reflected from plate without and with sample.

\section{Results and discussion}

There were conducted the experiments, main aim of which was the research of the EMW absorbing ability of the obtained polymer composites taking into account of such factors, as the type and composition of the investigated composites. Besides of it was interesting to define the some features of composite structure after influence of different physical factors on the microstructure during hardening of the material (effect of high electric and magnetic fields, geometry of the samples).

\subsection{Dependence of the EMW absorption on the recipe of composites}

Experiments were conducted on the composites on the basis of diane epoxy resin and inorganic fillers - graphite, carbon black, ferrite and some metal micro-powders.

From the character of the curves presented on Fig. 2 it is clear that if at low concentrations of fillers, the curves have an ascendant character, after definite concentrations these curves become descending. It is known that in general pure polymers, as a rule are transparent to radio waves and 
after introduction to them particles effectively reactive with EMW the value of absorption or reflection processes increase. However, after definite concentration of such fillers the increasing of the EMW slows down and at future increasing of the filler concentrations the absorption of EMW decreases. The main reason of these phenomena is due to proportion of two processes absorption and reflection of the EMW after impact of them on the filler particles. If in composites containing relatively low concentrations of fillers a process of absorption prevails the process of reflection (absorption grows) at more high concentrations of the fillers the second process (reflection) gradually grows because of increasing of the integral reflecting surface (a sum of the part of filler particle surfaces located in perpendicular to waves falling on these surfaces of the fillers) and at high concentrations already reflection is prevails the absorption, in result of which the material becomes a reflector of EMW.

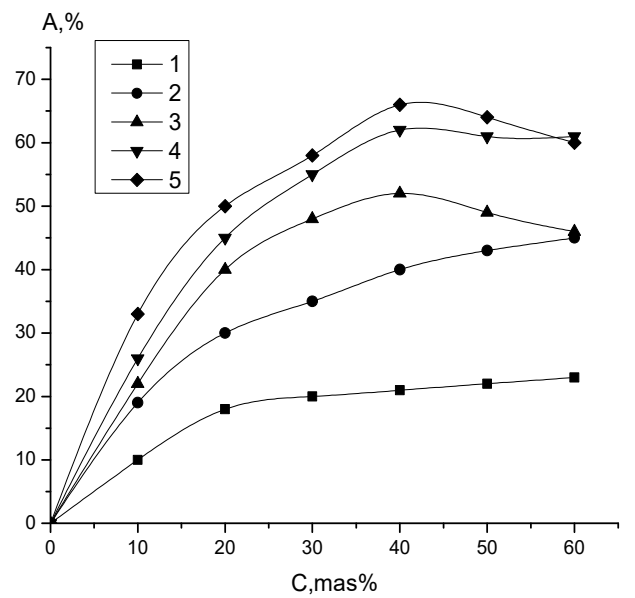

Fig. 2. Dependence of the EMW absorption of composites containing different fillers: carbon black of type P803 (1); carbon black of type P357 (2); synthetic graphite (3); natural graphite (4);

blend of synthetic and natural graphite (content 50/50\%) (5)

The analysis of the curves presented on the Fig. 2 shows that best result gives the composite containing two types of fillers - the blend of synthetic and natural graphite. This fact leads to opinion that the falling on the sample the EMW undergoes to diffraction and interference on the two types filler particles in different ways with enhancing of these processes. The composites containing natural graphite show also good absorption properties at definite filler concentrations.

\subsection{Dependence of the absorption properties of composites on the homogeneity of filler particles distribution in the polymer matrix}

It was interesting to define the dependence of EMW absorption properties of composites on the homogeneity of filler particles distribution in the polymeric matrixes. The experiment was conducted on the composites based on epoxy resin and graphite high dispersive fillers. The homogeneity of the composite plate was studied with estimation of the local elemental square $(5 \times 5 \mathrm{~mm})$ average electric resistivity on the plate. Definition of the ratio of average resistance of the elemental squares on the average resistivity of the full plate gave the degree of homogeneity of the material. From the Fig. 3 it is shown that the increasing of the structural homogeneity of the polymer composite correspondingly stipulates EMW absorption. The explanation of this phenomenon may be connected with existing of different resonance structures the polymer matrix, in result of which the absorption process is conducted with gradual connecting of this structures at different frequencies EMW. 


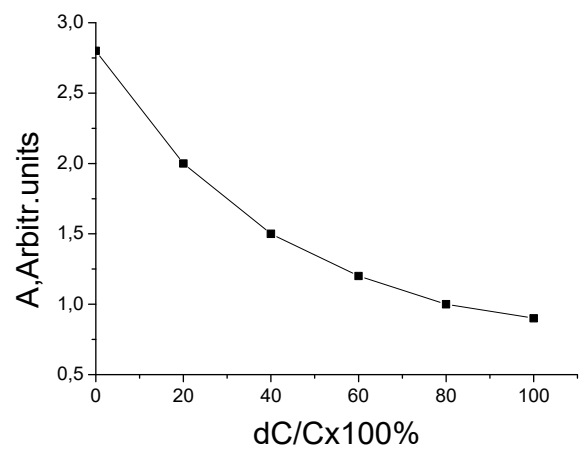

Fig. 3. Dependence of EMW absorption on the degree of homogeneity of the filler particles distribution in the polymer matrix, $\mathrm{C}$ is filler concentration and $\mathrm{dC} / \mathrm{C}$ the relative derivation of the filler concentration from it average value

\subsection{Dependence of EMW absorption of the composites on the EMW frequency}

The curves on the Figs 4-7 show that the value of the RW absorption depends both on composite composition and on the frequency of EMW. Here one can see the different extremes on the curves. The location of the minimums on the curves and their depths are dependent on the type and concentration of the fillers. Namely, the composite containing $20 \mathrm{wt} \%$ graphite is characterized with one minimum near $6,2 \mathrm{GHz}$ and 7.2 GHZ frequencies, which are correspond to 5 and $3 \mathrm{Db}$, respectively. The composite with $30 \mathrm{wt} \%$ of graphite is characterized with analogical minimum near $6.3 \mathrm{GHz}(8 \mathrm{Db})$, while for composites with 40 and $50 \mathrm{wt} \%$ such minimums (10.5 and 9.5 respectively) appear at one and same frequency $(6.2 \mathrm{GHz})$. The sample containing $50 \%$ graphite shows the similar minimums (9.5 Db at 5.5 and $6.2 \mathrm{GHz}$ respectively). Composite with $40 \mathrm{wt} \%$ graphite appears the maximum of the EMW absorption (10.5 Db).

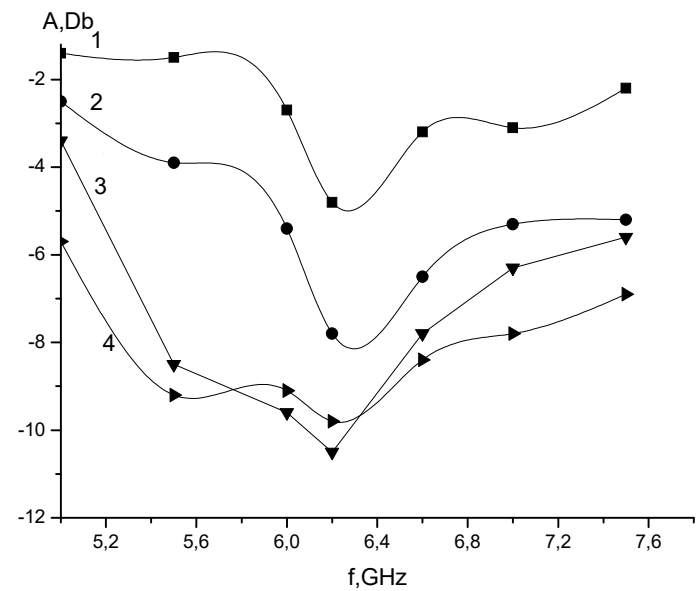

Fig. 4. EMW absorption ability of the composites based on epoxy resin and graphite for following concentrations of the filler (in wt\%): 20 (1), 30 (2), 40 (3), 50 (4)

The character of considered dependences mainly is based on the well-known phenomenon, which is responsible for the extremes of the properties at fixed concentration of the filler - on two competing processes (absorption and reflection of EMW). One from them increases at relatively low concentrations of the filler and second one - at relatively high concentrations, which is due to gradation of the filler absorption - reflection processes: at high concentrations the proportion of reflection in the reflected intensity increases, which leads to decreasing of the global absorbed intensity EMW. 
The main reason of the absorption of the EMW can be ascribed to interference-diffraction phenomena, which usually take place in the heterogeneous composites. In this case it must be foreseen a high heterogeneity of the investigated materials and based on it the heterogeneous character of the composite structure. Therefore, it is admissible that the separate region of the composite can be differed structurally from the neighbor one. Respectively the physical properties of lasts must be depended on the region coordinates. In this case it will be admissible that the EMW absorption properties of separate regions will be differ one from another in result of realization of the individual diffraction-interference processes in these regions. Therefore, the absorption properties of one and same composition can be changed by monitoring of the material microstructure, or, in other words by technological manipulations. The dependences of the absorption properties on the frequency of EMW presented on the Figs. 4-7. This phenomenon probably may be described in terms of difference in local microstructure, partially by the local resonances of diffraction-interference phenomena.

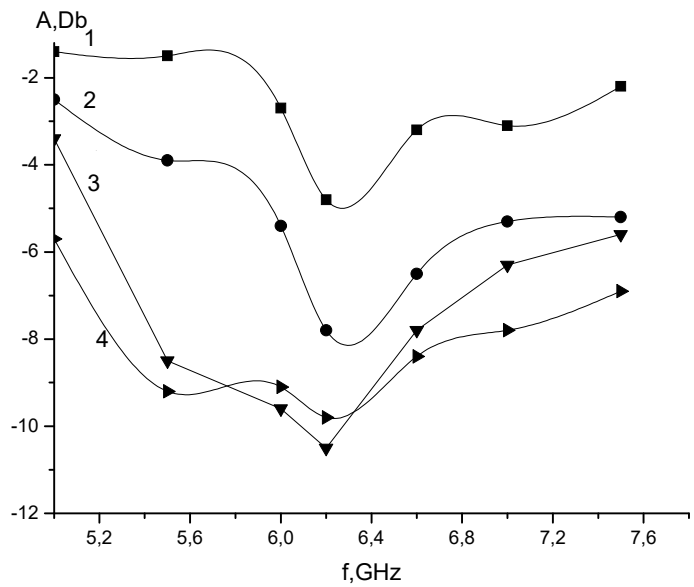

Fig. 5. EMW absorption ability of the composites based on epoxy resin and graphite for following concentrations of the filler (in wt \%): 20 (1), 30 (2), 40 (3), 50 (4)

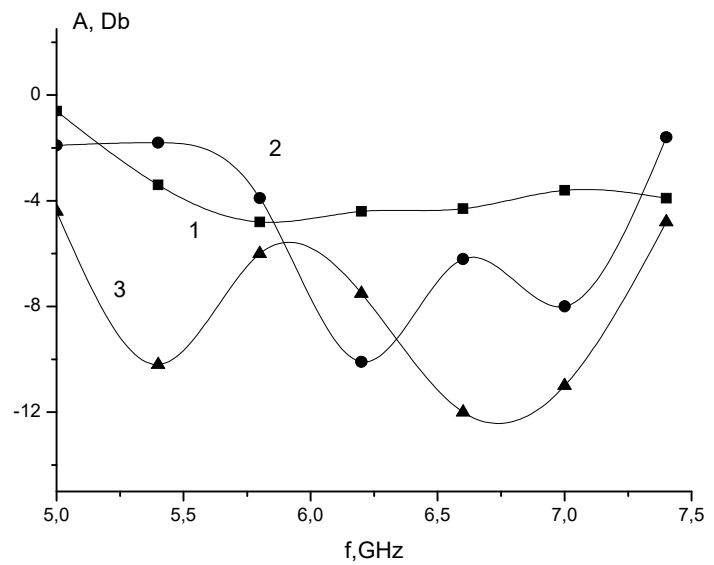

Fig. 6. EMW absorption ability of the composites based on epoxy resin and ferrite for following concentrations of the filler (in wt\%): 20 (1), 30 (2), 40 (3)

The data obtained from Figs 5-8 allow us to express the opinion that the EMW absorption ability of the composites with binary filler is essentially depends not so much on filler concentration but on the proportion of the components included to this combined filler. Here it appears so called synergistic effect. The best result (maximal absorption EMW) is reached for 
composite with $25-25 \mathrm{wt} \%$ of the graphite and ferrite powders blend.

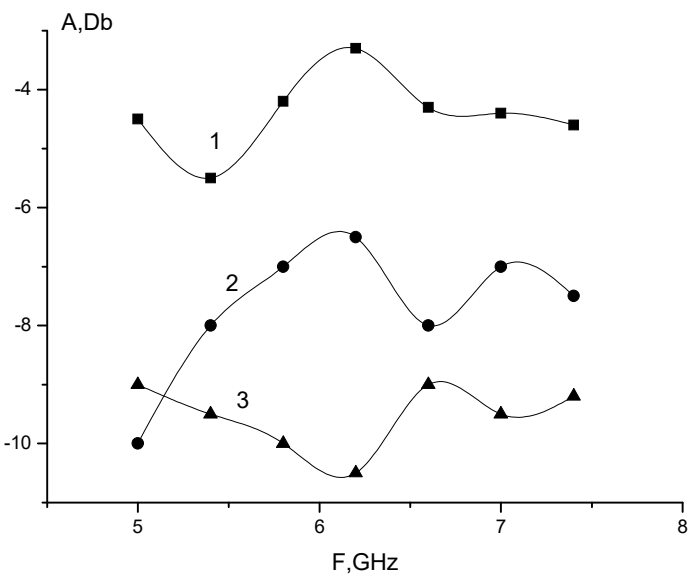

Fig. 7. EMW absorption ability of the composites based on silicon rubber and graphite for following concentrations of the filler (in wt\%): 30 (1), 50 (2), 40 (3)

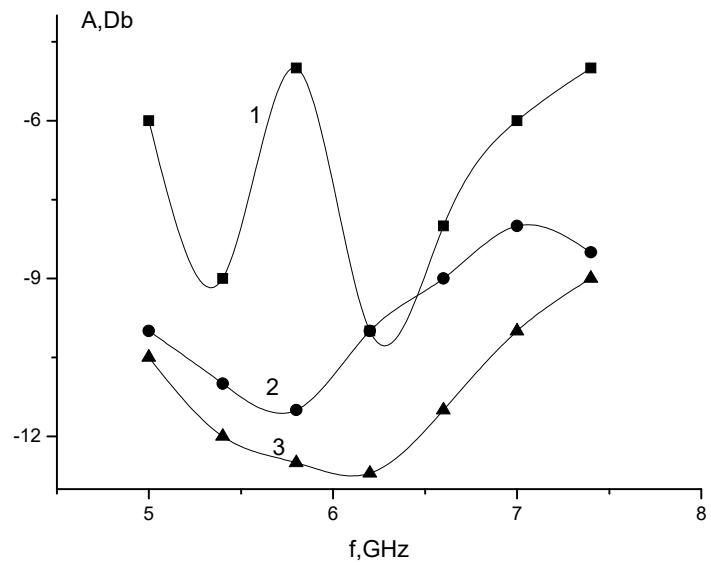

Fig. 8. EMW absorption ability of the composites based on epoxy resin and binary fillers for following proportions graphite/ferrite of the filler concentrations (in wt\%): 15/35 (1); 30/20 (2); 25/25 (3)

\subsection{Absorption of EMW by the package of the absorbing films}

From a practical point of view, it is important a definition of the EMW absorbing properties of the sandwich type packets constructed from the absorbing films with different types of the fillers and their concentrations. The testing of such sandwiches was conducted on the films packets contained three different films (both different concentrations of one type filler and different concentration of these fillers) with one and same thickness.

On the Fig. 9 it is shown the schematic picture of different sandwiches, each of which contains three different films (thickness $1 \mathrm{~mm}$ ) located differently relative to each other. experiment on revealing of the EMW absorption properties shows that the best result achieves for sandwich, in which the films are located in order of ladder like increasing of the filler concentration if the EMW is falling from film with lowest concentration of filler and vice versa at falling of EMW from reverse side of the same sandwich the result is worse than all. These results may be described as follows.

It is known [4] that protecting from EMW coatings are characterized with good absorbing properties if it microstructure contains the absorbing fillers displaced in polymer matrix with 
gradient of particles distribution in perpendicular to the absorber surface direction. It must be noted that the obtaining of such films is practically difficult for manufacture and consequently expensive. However, there are some methods, by use of which it would be simplify the design of such materials. With this aim it is possibility of obtaining of sandwich type material, made from films with gradation of distribution of filler particles. The idea, which was realized by us partially coincides with one expressed by German scientist Yauman in the middle years of XX century. for the submarines. The thickness of experimental installation of this scientist was about $10 \mathrm{~cm}$. Such construction could be used on submarines, but not avia-cosmic fly apparatus. Recently it was obtained by us the absorbers as sandwich made from thin absorbers as package of the thin films composites with different type and distribution of absorbing filler. On the scheme presented in the Fig. 9 there are presented the packages, each of which contains 3 films with different types and concentrations of the filler. Thickness of full package was about $1 \mathrm{~mm}$. The testify shows that from these packages the one with gradual increase of filler concentration in the row of the films possesses higher coefficient of EMW absorption if the wave falls from the film with less contenting of the filler. Such distribution of the films in the package is right on the basis of following considerations: EMW after falling on the film with low concentration of the filler particles will pass all the film with minimal losses of energy and with some derivation from start direction. After the entrance to the second film contenting the filler with more high concentration the EMW will pass all this film with energy losses higher than in first film and more derivation from initial direction. This tendency of the EMW will by increased at passing through the third film, after which it will reach to metal surface of the aircraft, reflected from which it will pass the package in reverse direction, although this EMW will be very week in comparison with initial one and deviated from start direction. The packages with other distribution of the films are less effective than that described above.

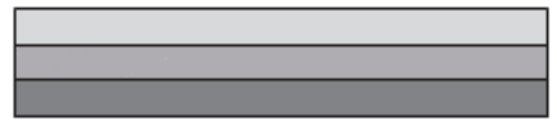

a)

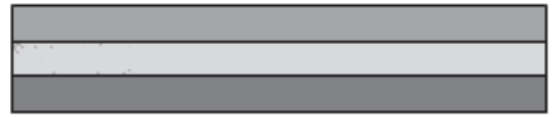

c)

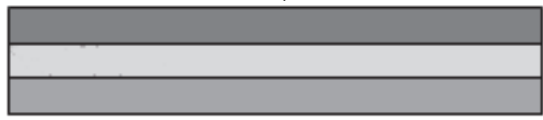

e)

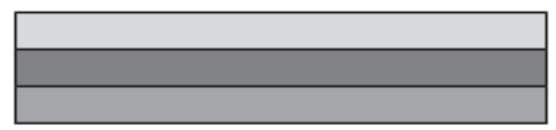

b)

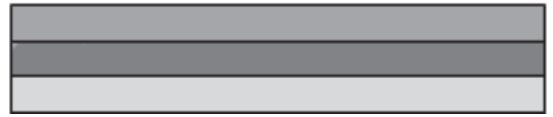

d)

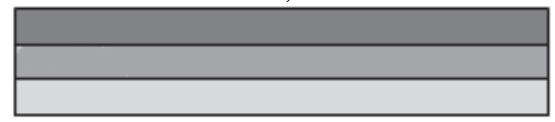

f)

Fig. 9. Schema of sandwiches with variation of dislocation of the absorber films.

Rectangle with light color corresponds to films with relatively low filler concentration, semi dark to middle and dark - high concentration of filler

On the Table 1 there are given the numerical data of the EMW absorption (in \%) by packages with three different distributions of the films.

Analogical dependences at frequencies $5.6 \mathrm{GHz}$ are presented on the Table 2.

In accordance with the Table 2 data the maximal absorption shows the package with index A, on which the wave falls from left side. The gradation of the absorption value presented in the Table 1 is determined by following terms. In the first sample (package A) the wave will pass relatively "easy" the package firstly through the plate with less content of the filler because of low concentration of one (the processes of the wave absorption-reflection processes are relatively weak). The passing of the waves through the second plate, containing more (in comparison with first plate) concentration of the graphite will absorb more amount of the EMW energy. The passing of EMW through the third plate will be characterized with more intensive absorption-reflection 
properties than previous plates. The waves falling on the metal surface (after passing through the third plate) reflects with weakened energy from last and undergoes to repeated absorption-reflection processes and therefore the energy of the waves exited from package will be essentially lower than that falling initially on the package. In case of wave falling on the package from the reverse side the energy of waves exited from the package is more than in first case because in the second case the wave falling on the third plate (with relatively high concentration of the filler) reflects from it more intensively and therefore the part of absorbed energy is much less than in first case.

Table 1. EMW absorption by sandwiches designed from films on the basis of epoxy resin and graphite with different concentration of latter $(f=8.0 \mathrm{GHz})$

\begin{tabular}{|c|c|c|}
\hline$\#$ & Position of films & Absorption of EMW, $\%$ \\
\hline 1 & $1-2-3$ & 84.4 \\
\hline 2 & $1-3-2$ & 67.7 \\
\hline 3 & $2-1-3$ & 53.3 \\
\hline 4 & $2-3-1$ & 55.2 \\
\hline 5 & $3-1-2$ & 65.6 \\
\hline 6 & $3-2-1$ & 61.3 \\
\hline
\end{tabular}

Film 1: Epoxy resin $(80 \mathrm{Wt} . \%)+$ graphite $(20 \mathrm{Wt} . \%)$ Film 2: Epoxy resin $(70 \mathrm{Wt} . \%)+$ graphite $(30 \mathrm{Wt} . \%)$ Film 3: Epoxy resin $(60 \mathrm{Wt} . \%)+$ graphite $(40 \mathrm{Wt} . \%)$

Table 2. EMW absorption ability of the sandwich type absorbers $(f=5.6 \mathrm{GHz})$. The sequence of the plates in the package (concentration of graphite in the plates) (the wave falls on the package from left side-plate with less content of the filler)

\begin{tabular}{|c|c|c|}
\hline Packing index & Position of films & Absorption of EMW, \% \\
\hline A & $30-40-50$ & 54.6 \\
\hline B & $30-50-40$ & 49.3 \\
\hline C & $40-30-50$ & 44.5 \\
\hline D & $40-50-30$ & 41.8 \\
\hline E & $50-30-40$ & 38.4 \\
\hline F & $50-40-30$ & 37.5 \\
\hline
\end{tabular}

\subsection{Effect of samples geometrical sizes on the absorption of EMW}

There were conducted the experiments on the samples obtained with use the following way: from the fluoroplast sheet (thickness $3 \mathrm{~mm}$ ) it was obtained the plates with sizes $70 \times 50 \mathrm{~mm}$. In these plates the holes with different profiles were made, which after were filled with composition material (epoxy glue $+50 \mathrm{wt} \%$ graphite) for hardening. Vertical section of this sample is shown on the Fig. 10 (top scheme corresponds to cylindrical holes, middle-conical and bottom-ladder like).

The experimental data of Table 3 allow make the conclusion that the absorption capability of the cover-protector can be regulated by change of configuration of the composite materials placed in the dielectric cover.

Table 3. The absorption capability of the composites displaced in the holes with different profiles

\begin{tabular}{|c|c|c|}
\hline$\#$ & Vertical section profile of the sample & Absorption of EMW, \% \\
\hline 1 & Cylindrical & 84 \\
\hline 2 & Conical & 91 \\
\hline 3 & Ladder like & 78 \\
\hline
\end{tabular}




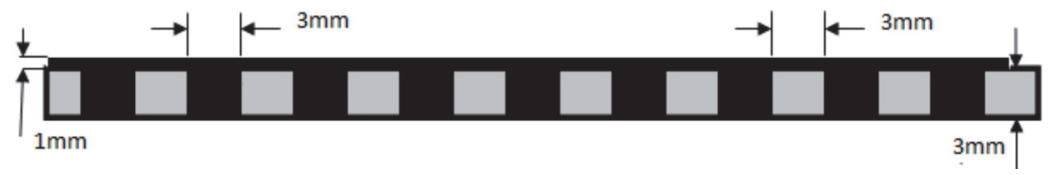

a)

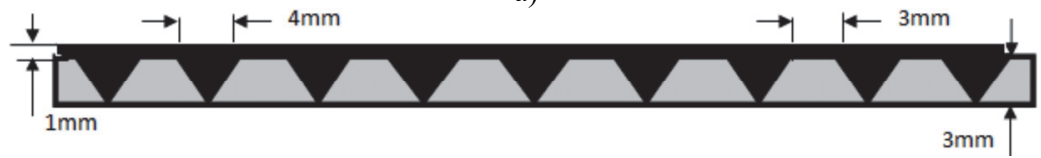

b)

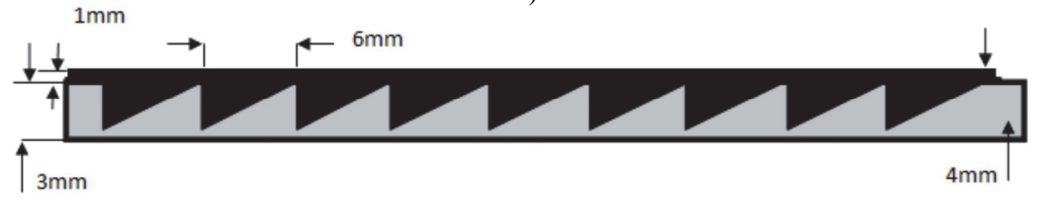

c)

Fig. 10. Vertical sections of the forms with different shapes: cylindrical; b) conical; c) ladder like. Black area - absorbent material, light color area - dielectric substrate (Teflon)

\subsection{Effect of binary fillers in the EMW absorbing properties of polymer composites}

Investigation of the EMW absorption properties of the polymer composites containing of binary fillers present a definite interest because of here is the possibility of appearing of so called synergistic effect of fillers - non-additive enhancing of some physical/chemical properties at definite proportion of the ingredients in the fillers. The composites based on epoxy glue containing of binary fillers (graphite + carbon black; graphite + ferrite; ferrite + carbon black) were used for investigation of their EMW absorbing properties. On the Figs. 11-13 it is shown the dependence of the EMW (10 GHz) absorption on the proportion of the components in binary fillers. From these dependences it is clear that the absorption of EMW in binary systems one of the important meaning has the proportion of the ingredients in composite materials. It may by proposed that binary filler promotes to creation of resonance microstructures with different volumes, which will be the basis of formation of interference-diffraction phenomena and enhancing of EMW respectively. These proportions promote to synergistic effect noted above, in this case - maximal absorption of EMW, which is unattainable at using of only one filler with any concentrations.

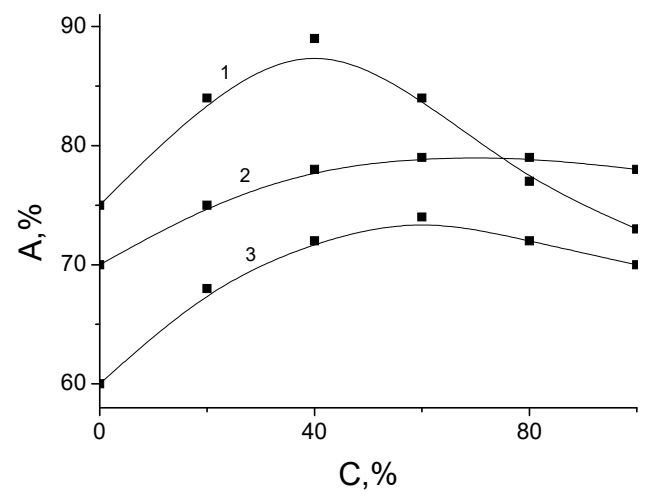

Fig. 11. Dependence of the EMW absorbing level on the proportion of fillers graphite/ ferrite in the composite based on epoxy glue. On the $x$-axes - the concentration of graphite in binary fillers for total concentration of fillers 50 (1), 40 (2) and $30 \mathrm{wt} \%$ (3) 


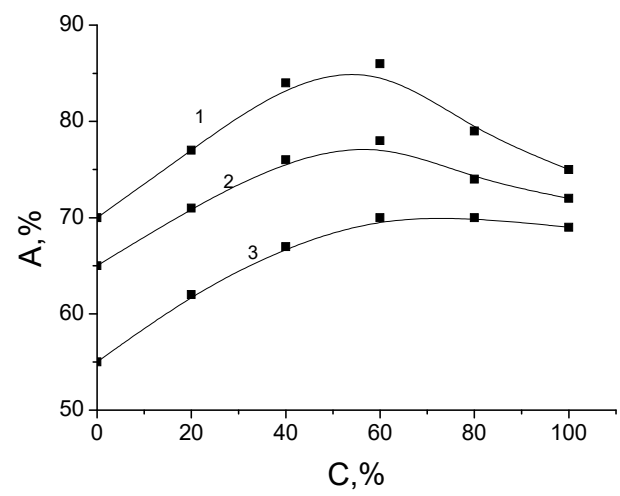

Fig. 12. Dependence of the EMW absorption on the proportion of ingredients of binary fillers in the composite based on epoxy glue and binary filler (graphite/ carbon black) for total concentrations of filler in the composites 40 (1), 50 (2) and $30 \mathrm{wt} \%$ (3); on the $x$-axis - the concentration of the graphite in binary filler

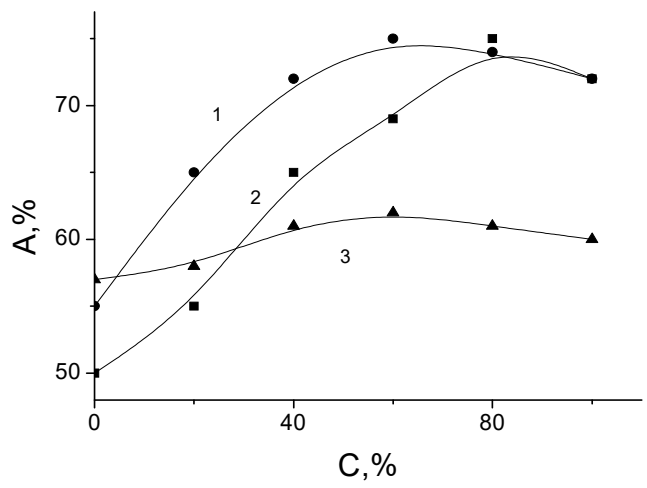

Fig. 13. Dependence of EMW absorbing processes in composites based on silicon rubber with binary filler (ferrite/carbon black) on the content of fillers at their total concentrations 30 (1), 40 (2) and $50 \mathrm{wt} \%$ (3); on the $x$-axis - the concentration of the ferrite in binary filler

\subsection{EMW absorption capability of the composites modified with constant electric and magnetic fields}

The composites with two types of the filler were selected as the research objects: natural graphite and ferrite. These materials were tested on the EMW absorption capability for study of the electric and magnetic field effect during hardening of the composites. The results of testify are presented on the Fig. 14.

From the Fig. 14 one can see that the absorption capability of the both composites increases at increasing of the electric field during process of composite hardening up to definite level, after which stopped and transacted to the saturation state. The composite in labile state creates the favorable medium for the molecular polarization, which leads to orientation of molecules. The filler particles, which are interacted with lasts receive the orderly state respectively, decrease the entropy of the system and increase the order of the particles in polymer matrix. It is one from suitable conditions for increasing of EMW absorption. It is difficult to say that at higher electric field absorption would be high, because saturation of the corresponding curve gives an opposite result. At very high fields the dielectric it will be expected a breakthrough in the dielectric, in result of which the irreversible structural transformations can be occurred.

Experiments on definition of the effect of the magnetic field were conducted on the composites based on epoxy resin and synthetic graphite and cobalt. To testify the following samples were 
underwent: 1$)$ epoxy resin + graphite $(35 \mathrm{wt} \%)$, epoxy resin + cobalt $(35 \mathrm{wt} \%)$. These composites were obtained at hardening of composites in the constant magnetic fields from the range 0.1-0.6 Tesla. On the Fig. 15 there are presented the dependences of EMW absorption of the level of "technological" magnetic field for both composites.

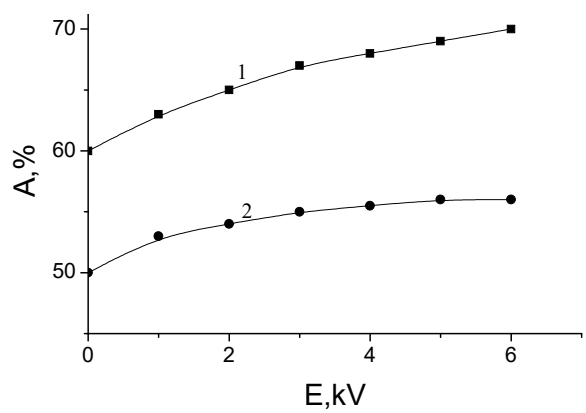

Fig. 14. Dependence of the EMW absorption on the "technological" electric field for composites based on epoxy glue with natural graphite (1) and ferrite (2) at filler concentration in samples $60 \mathrm{wt} \%$

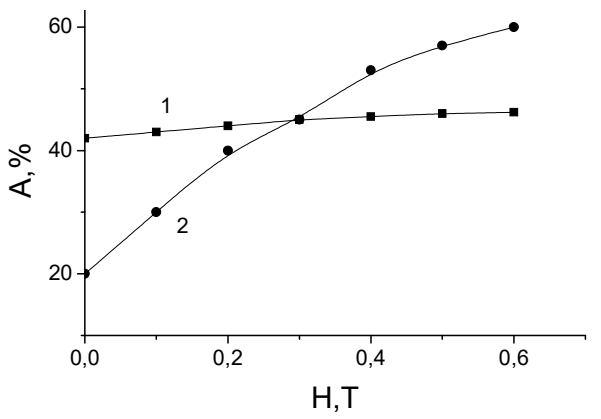

Fig. 15. Dependence of EMW absorption level on the "technological" magnetic field for the epoxy-composites containing a graphite (40 wt $\%)(1)$ and cobalt $(40 \mathrm{wt} \%)$

As in case of composites underwent to electric field, it must be foreseen the orientation role of the magnetic field. It is clear that this effect will be effective for system including the magnetic particles. From the curves presented on the Fig. 15 one can see that the magnetic field effect is more significant for the system including ferromagnetic particles than for the system including diamagnetic ones.

\section{Conclusions}

It is shown experimentally that the reception of composites and their obtaining methods are the main factors, variation of which gives the possibility of regulation of their character of EMW absorption. The dependence of the absorption of EMW (3 GHz) energy by polymer composites on the concentration of the fillers (electric conducting or ferromagnetic particles) is extreme - has a maximums at definite concentrations and type of lasts. Composites containing graphite and ferrite filler with definite proportion are characterized with synergistic effect. It is established also that the higher is the homogeneity of the degree of particles distribution in the polymer matrix the higher is the EMW absorption level, which is due to exist of resonance structure of the noted particles in the polymer medium. The composites EMW absorption may be regulated also by their hardening under conditions of the strong constant electric and magnetic fields. Increasing of the absorption of EMW by polymer composites can be reached with use of package of thin layers, in which the type, composition and order of distribution of lasts change in perpendicular to the film plane. One of the ways of change of the absorption of EMW by composites is also the regulation 
of the layers surface form. It is established that the absorption of the plate covered with low height conical holes and filled with investigated composite is essentially higher than the analogical material covered with holes with other (cylindrical or ladder-like) profiles.

\section{References}

[1] Lagarkov A. N., Pogosian M. A. Fundamental and applied problems of stels-technologies. Proceedings of Russian Academy of Sciences, Vol. 73, Issue 9, 2003, p. 848, (in Russian).

[2] Bochkarev A. M., Dolgov M. N. Radiolocation of the fly apparatus. Foreign Radioelectronics, Vol. 2, 1989, p. 3-17, (in Russian).

[3] Finkelstein M. I. Principles of Radiolocation. Guid Book for High Schools. 2nd Edition. Radio and Communications, 1983, (in Russian).

[4] Aneli J. N., Khananashvili L. M., Zaikov G. E. Structuring and Conductivity of Polymer Composites. Nova Science Publishers, New York, 1998, p. 326.

[5] Schnabel W. Polymers and Electromagnetic Radiation: Fundamentals and Practical Applications, Wiley, 2014, p. 360.

[6] Volkov V. P., Zelenetsky A., et al. Synthesis and properties of electromagnetic wave shielding polymer materials with low flammability. Journal of Applied Polymer Science, Vol. 116, Issue 5, 2010, p. 2775-2782.

[7] Batrakov K., Kuzhir P., Maksimenko S., et al. Flexible Transparent Graphene/Polymer Multilayers for Efficient Electromagnetic Field Absorption. Scientific Reports, Vol. 4, 2014, p. 231.

[8] Roberts J. A., Imholt T., Ye Z., et al. Electromagnetic wave properties of polymer blends of single wall carbon nanotubes using a resonant microwave cavity as a probe. Journal of Applied Physics, Vol. 95, Issue 8, 2004, p. 4352.

[9] Dosoudil R., Franek J., Slama J. Electromagnetic wave absorption performances of metal alloy/spinel. IEEE Transactions on Magnetics, Vol. 48, Issue 4, 2012, p. 1524-1527.

[10] Folgueras L. de Castro, Rezende Mirabel C. Multilayer radar absorbing material processing by using polymeric nonwoven and conducting polymer. Materials Research, Vol. 11, Issue 3, 2008, p. 245-249.

[11] Bregar V. B. Advantages of ferromagnetic nanoparticle composites in microwave absorbers. IEEE Transactions on Magnetic, Vol. 40, Issue 3, 2004, p. 1679-1684.

[12] Bhavsar V., Tripathi D. Advance in electronic and electronic engineering. Research India Publications, Vol. 4, Issue 4, 2014, p. 417-424.

[13] Huang J. C. Carbon black filled conducting polymers and polymer. Advances in Polymer Technology, Vol. 21, 2002, p. 299.

[14] Clingerman M. L., Weber, King J. A. Synergistic effect of carbon fillers in electrically conductive nylon. Polymer Composites, Vol. 23, Issue 5, 2002, p. 911-924.

[15] Rohde Ulrich L., Newkirk David P. EMW Circuit Design for Wireless Applications. Wiley, 2004.

[16] Keith M., Janda B., King J., Perger F., Oxby T. Polymer matrix composites. for EMI shielding applications. Polymer Composites, Vol. 26, 2005, p. 671-678.

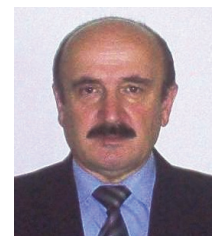

Tamaz Natriashvili received Ph.D. degree in machine technology from Georgian Technical University, Tbilisi, Georgia in 2003. Now he works as director of the Institute of Machine Mechanics. His current research interests include technical novelties in the field of automobile engines.

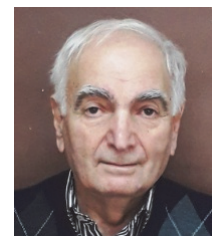

Jimsher Aneli received Ph.D. degree in chemical technology from Georgian Technical University, Tbilisi, Georgia in 1995. Now he works at the Institute of Machine Mechanics in Tbilisi, Georgia. His current research interests include technical novelties in the field of technical application of polymer composites. 
4.3. EFFECT OF TECHNOLOGICAL FACTORS ON ELECTROMAGNETIC WAVE ABSORPTION PROPERTIES OF THE POLYMER COMPOSITES.

TAMAZ NATRIASHVILI, JiMSHER ANELI, LANA SHAMANAURI

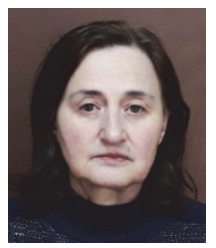

Lana Shamanauri received Ph.D. degree in chemical technology from Georgian Technical University, Tbilisi, Georgia in 2009. Now she works at the Institute of Machine Mechanics in Tbilisi, Georgia. His current research interests include Technical application of polymer composite materials. 


\title{
4.4. Studying of the seawater drops properties on superhydrophobic surface
}

\author{
Tatiana Popova ${ }^{1}$, Aleksei Ukolov ${ }^{2}$ \\ Kerch State Marine Technological University, Kerch, Crimea \\ E-mail: ${ }^{1}$ tanap178@gmail.com, ${ }^{2}$ ukolov_aleksei@mail.ru
}

\begin{abstract}
Superhydrophobic properties of the surface lead to the effect of non-wetting the liquid of a solid and the formation of a contact angle close to $180^{\circ}$. The nature of this phenomenon is explained by the features of the surface microrelief having an ordered texture consisting of microprotrusions of the cavity between which they are filled with gas. This phenomenon is widely studied and has already found its use in various practical applications where it is important that the liquid does not interact completely with a solid surface.

In that work we give a brief review of the properties of superhydrophobic surfaces and the directions of their use. The well-known methods are being analyzed for creating a superhydrophobic layer in order to select the optimal method of coating deposition, on materials in contact with the sea environment.
\end{abstract}

The results of a study of the drops of natural sea water in the Kerch Strait on the superhydrophobic surface of A40S shipbuilding steel are considered. An experimental setup and a technique for determining the droplet angle in the process of evaporation from a superhydrophobic surface are described. An analysis of the stability of the superhydrophobic state was performed with a droplet volume decrease and increase in the concentration of salt in the liquid. The influence of the force of gravity on the drops shape is estimated.

It is shown that in the evaporation process the contact angle decreases, but before the crystallization process begins, it exceeds $150^{\circ}$, thereby maintaining superhydrophobic properties of the surface. Completion of evaporation of the droplet is accompanied by the formation of a solid $\mathrm{NaCl}$ crystal, which is certainly an advantage when removing such impurities from the steel surface. In addition, such an effect will not cause degradation of the microstructure of the surface, its contamination with fine crystals, and block the gas channels providing superhydrophobic properties.

The behavior of seawater drops on the superhydrophobic surface differs from the properties of distilled water. While evaporating, the salt concentration enlarges, increasing the specific gravity of the solution. Thus, there is no significant advantage of the surface tension force with respect to the gravitational pull. This phenomenon involves the deviation of the drop's shape from the spherical in the process of evaporation, up to the point of time corresponding to the onset of crystallization of the salt and further distortion of the droplet surface.

The obtained results contribute to the further research of the properties of superhydrophobic coatings and expand the possibilities of their use in shipbuilding.

Keywords: wetting, superhydrophobic surface, surface tension, shipbuilding steel, contact angle, seawater drop.

\section{Introduction}

In the 90s of the last century, the German botanist Wilhelm Barthlott explained the appearance of the "lotus effect" - the phenomenon of low water wetting of the surface of leaves and petals of plants (Fig. 1), insect claws (Fig. 2), etc. The botanist associated this effect with the features of microstructure of surfaces interacting with water.

The microstructure of non-wetting surfaces has many microprotrusions or microvilli that reduce the interaction forces between the molecules of the liquid and the surface. This feature leads to the fact that on the surface the liquid forms globular droplets spontaneously rolling down from it (Fig. 1), collecting dust and some contaminants, thereby creating a self-cleaning effect. Thus, the "lotus effect" in nature acts as a "protector" of plants against microorganisms, fungal 
diseases, algae fouling, etc. In the case of a Gerridae, microvilli, creating non-wetting between the legs of the insect and the surface of the water (Fig. 2), facilitate smooth sliding along its surface. It should be noted that the surface of the wings of many other insects, butterflies have a similar structure. Not getting wet, insects do not lose the ability to fly. Subsequently, water-repellent surfaces were called superhydrophobic.

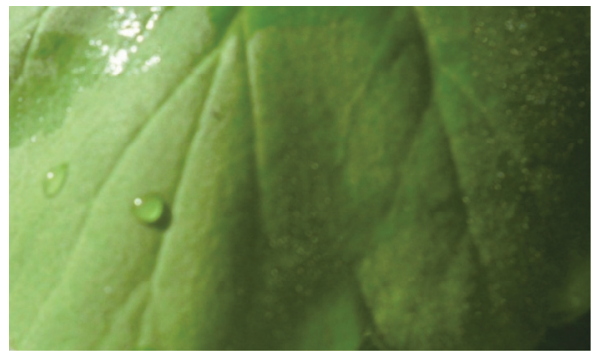

Fig. 1. Drops of water on top of the Geranium leaves

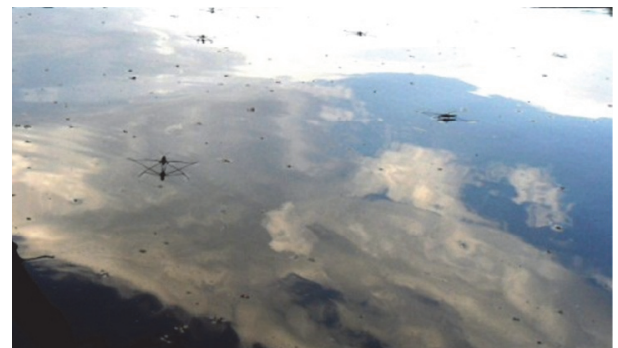

Fig. 2. Insect Gerridae on top of the water surface

The examined properties of plant and insect surfaces led to the search for functionally different superhydrophobic materials [1-3], which can be used in technology: to prevent fouling and sedimentation [4], to obtain water repellent, self-cleaning surfaces [5], reduce friction of fluid flow and increase thermal conductivity [6], in the electric power industry [7], and etc. Teflon surfaces have already become famous for having superhydrophobic properties and are being used in everyday life and production [8].

Superhydrophobic properties of surfaces depend on the characteristics, micro/nanorelief, surface energy and chemical composition of the surface substance.

To key factors affecting superhydrophobicity of the surface, X. Yao, Y. L. Song, L. Jiang include micro/nanostructures, hierarchical structures, low surface energy coatings that provide a large surface-to-volume ratio of the microfluidic device, which affects behavior and significantly increases the pressure loss of the flow to the superhydrophobic surface.

These factors are used in the manufacture of superhydrophobic surfaces on various substrates of metal, which are gradually applied in industry and everyday life [9]. So, N. Rungraeng, Y.-C. Cho, S. H. Yoon, and S. Jun have shown that plate heat exchangers with superhydrophobic surfaces can effectively prevent contamination and reduce heat transfer during the pasteurization process [10].

It is known that the effectiveness of solar panels and wind generators working in severe weather conditions is drastically reduced by the accumulation of ice and snow. Methods of removing ice from surfaces can be divided into two categories: active and passive. The active method includes thermal procedures, mechanical scraping and the use of anti-ice chemical reagents. R. Carriveau, F. Cucchiella, A. Edrisy and others consider a passive method that makes it easy to remove ice by reducing the surface energy and low adhesion strength $[11,12]$.

The passive method has a huge potential for use - airplanes, wind turbines, photoelectric devices, power lines. Using a metal surface with a superhydrophobic coating will make it possible to detach the condensed droplets from the cooled surface even under the action of gravity.

Superhydrophobic surfaces show excellent characteristics that can be used in hydrodynamics, heat engineering, power engineering, electrical and telecommunications. They have a huge potential for use, their efficiency significantly exceeds their production costs as the price of chemical reagents is not high and the methods of manufacturing such surfaces are relatively simple. After the organization of a large-scale production of water-repellent surfaces, an economic effect can be obtained.

Despite the fact that the study and production of superhydrophobic surfaces over the past fifteen years have achieved great success, many problems associated with achieving real technological applications are waiting to be solved. 
Studying the properties of superhydrophobic surfaces, we came to the possibility of their application in shipbuilding, as well as in the real operating conditions of vessels in the waters of the Black Sea [13]. We associate our research with this:

- properties of superhydrophobic states,

- interaction of such surfaces with the waters of the Black Sea,

- the development of more simple methods for creating superhydrophobic surfaces.

Our further plans lie in the direction of checking the reliability of surfaces under pressure, wear, friction, immersion, pollution and under other conditions.

\section{Main text}

The liquid practically does not wet superhydrophobic surfaces because of the special relief of these coatings. On a hard surface, there are irregularities (cavities, microprotrusions, villi) filled with air. A drop of liquid, falling on such a surface, does not penetrate into the irregularities as a result of air entrapment by microscopic irregularities.

\subsection{Contact angle}

The phenomena of wetting and non-wetting by liquid surfaces are characterized by the angle of contact (the contact angle), determining its hydrophilic or hydrophobic properties. A liquid with a hydrophilic surface forms a contact angle of less than $90^{\circ}$ (Fig. 3), with a hydrophobic angle of more than $90^{\circ}$ (Fig. 4). Surfaces having a contact angle with a fluid angle of more than $150^{\circ}$ are called superhydrophobic (Fig. 5).

In 1805, Thomas Young was the first who described the concepts of contact angle and wetting. During the next two centuries, many researchers conducted theoretical studies and developed analytical methods for studying the properties of this phenomenon.

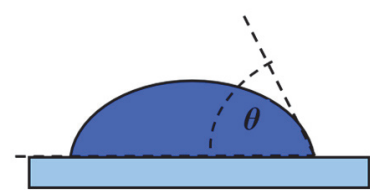

Fig. 3. Hydrophilic surface, $\theta<90^{\circ}$

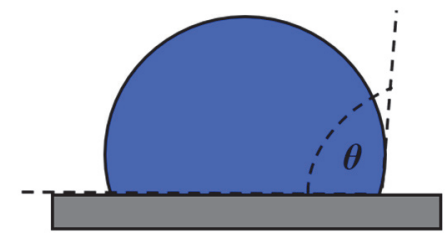

Fig. 4. Hydrophobic Surface, $\theta>90^{\circ}$

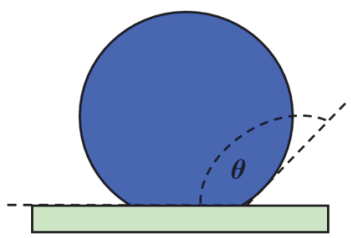

Fig. 5. Superhydrophobic surface, $\theta>150^{\circ}$

Scientists have demonstrated the various intermolecular interactions between a liquid and a solid material lead to different wetting properties [14]. Significant progress was made in studying the relationship between roughness and hydrophobicity, observing the hysteresis of the contact angle (the difference between the contact angle on the horizontal surface and the slanting), the discovery of superhydrophobicity induced by the micro- and nanoscale hierarchy [1].

The contact angle is the main index used to estimate the wetting of a solid surface. It usually characterizes the behavior of a liquid drop on a solid surface in the air and is determined by the angle between the tangent at the three-phase point and the solid surface. The relationship between the contact angle and the surface tension coefficients of interface boundaries can be described by the Young's equation:

$\gamma_{S G}-\gamma_{S L}-\gamma_{L G} \cos \theta_{c}=0$,

where $\theta_{c}$ - contact angle, $\gamma_{S G}, \gamma_{S L}$ and $\gamma_{L G}$ - surface tension coefficients of the solid/gas boundary, solid/liquid, and liquid/gas, respectively.

Methods for measuring the contact angle, can be grouped into methods of tensiometry and goniometry. 
Tensiometry methods determine the value of the contact angle using the Young Eq. (1). If the coefficients of the surface tension of the interphase boundaries do not change, the contact angle is constant and can be found from Eq. (1). Problems in measuring the edge by this method appear: first, if the values of the surface tension coefficients are unknown, secondly, impurities, the adsorption of the vapor on the solid phase, the electrostatic potential, the surface roughness, heterogeneity and external forces lead to a change in the apparent contact angle $\theta_{c}$.

In the methods of goniometry, the contact angle is observed and measured from the two-dimensional image of drops on a solid surface (Figs. 3-5).

Modern methods for measuring the contact angle of droplets, described in the literature, can be divided into two groups: analytical and exclusively image processing methods. Analytical methods require some fluid properties (for example, the surface tension coefficient) to determine the contact angle and usually cannot measure the contact angle of asymmetric drops (for example, drops on inclined surfaces). Image processing methods do not have this limitation, so they are related to common methods. But these and other methods contribute to a deeper study of the properties of superhydrophobic surfaces.

\subsection{Wentzel's and Cassie-Baxter's states}

Unevenness on superhydrophobic surfaces can both be filled with liquid partially, and not penetrate into the irregularities at all. In these cases, two states of water droplets on a solid surface are distinguished: the Wentzel's state (Fig. 6) and the Cassie-Baxter's state (Fig. 7), as it is detailed in work [7].

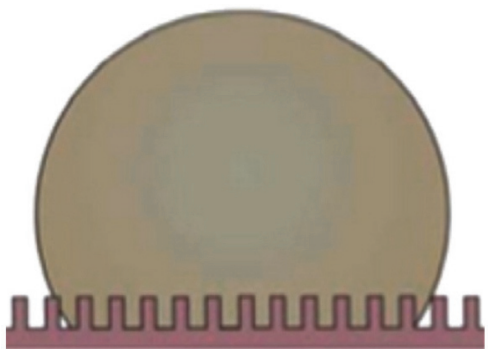

Fig. 6. Wentzel's state [7]

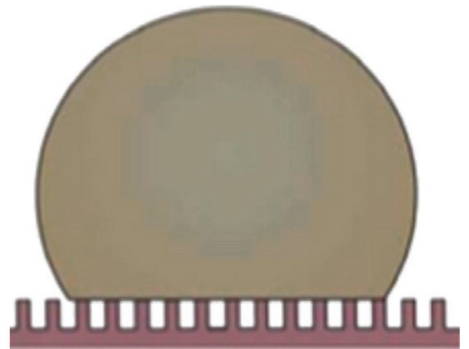

Fig. 7. Cassie-Baxter's state [7]

In the Wentzel's state, water penetrates into the structured surface, the droplets become spiked, which prevents them from slipping easily. In the Cassie-Baxter state, the liquid lies on top of a structured surface with a gas layer (as in the case of Insect Gerridae). This gas gap is an effective boundary that ensures the glide of the droplet at the liquid-gas interface.

The nature of a solid superhydrophobic surface is explained by the influence of its roughness on a drop of liquid. The wettability phenomenon of a solid surface can be interpreted using the Wenzel's and Cassie-Baxter's models (Figs. 6, 7).

Assuming that the liquid fills the space between the protrusions on the surface (Fig. 6), the Wenzel approach correlates with the apparent contact angle $\theta^{\prime}$ and the thermodynamic contact angle $\theta$ as:

$\cos \theta^{\prime}=r \cos \theta$

where $r$ is the coefficient of roughness, which determines the ratio between the true surface area and its horizontal projection. When one of the values $\left(\theta^{\prime}, \theta, r\right)$ changes, a regular change (decrease or increase) of the others occurs.

Cassie and Baxter put forward the idea that the surface captures air in hollow spaces of roughness (Fig. 7), identifying $\cos \theta^{\prime}$ of the apparent angle's contact $\theta^{\prime}$ on the superhydrophobic 
surface as follows:

$\cos \theta^{\prime}=f_{L S} \cos \theta-f_{L V}$,

where $f_{L S}$ - the proportion of the area of the liquid which is in contact with the solid, $f_{L V}-$ the proportion of the area of the liquid which is in contact with trapped air. Wherein:

$f_{L S}+f_{L V}=1$.

Thus, the hysteresis of the contact angle can distinguish two states. With greater hysteresis, the droplets will be considered to belong to the Wenzel's solution entering the surface grooves. Whereas with lower hysteresis values, the surface can be considered as a columnar structure supporting the fluid and decreasing the available area.

Since superhydrophobicity mainly arises from the air trapped in the grooves by a solid surface or coating, the presence of its volumes of very small nanoscopic scales (nanobubbles) is the subject of a wide range of interpretations and observations. Despite the existence of nanobubbles on hydrophobic surfaces, discussions still occur, where the authors compare conflicting results, and also study the properties of nanobubbles (V. Graig, X. Zhang, J. Hu [15]). Theoretically, such bubbles should dissolve under the action of their internal pressure for a short time, but experimentally with the help of a highly sensitive surface method of atomic force microscopy, unusual peculiar properties and forms of nanobubbles are found.

We have developed a technique for performing experimental studies to determine the contact angle of water droplets on the natural, polished and superhydrophobic surface of A40S $(8 \times 90 \times 50 \mathrm{~mm})$ ship steel using an installation that will be described later.

\subsection{Mechanical properties of steel grades A40S}

Steel grade A40S has high strength and ductility. It is intended for the manufacture of hulls or other welding constructions of ships and floating structures. The chemical composition of steel grade A40S is presented in Table 1.

Mechanical properties of steel grade A40S at $t=20^{\circ} \mathrm{C}$ : short-term strength limit 530-690 MPa, yield stress for permanent deformation $390 \mathrm{MPa}$, elongation at break $19 \%$.

Table 1. Chemical composition of steel A40S in shares

\begin{tabular}{|c|c|c|c|c|c|c|c|c|}
\hline Grade & $\begin{array}{c}C, \\
\text { not more than }\end{array}$ & $M_{n}$ & $S_{i}$ & $\begin{array}{c}P \text { and } S, \\
\text { not more than }\end{array}$ & $C_{r}$ & $N_{i}$ & $C_{u}$ & $A_{l}$ \\
\hline A40S & 0.12 & $0.5-0.8$ & $0.8-1.1$ & 0.035 & $0.6-0.9$ & $0.5-0.8$ & $0.4-0.6$ & $0.015-0.06$ \\
\hline
\end{tabular}

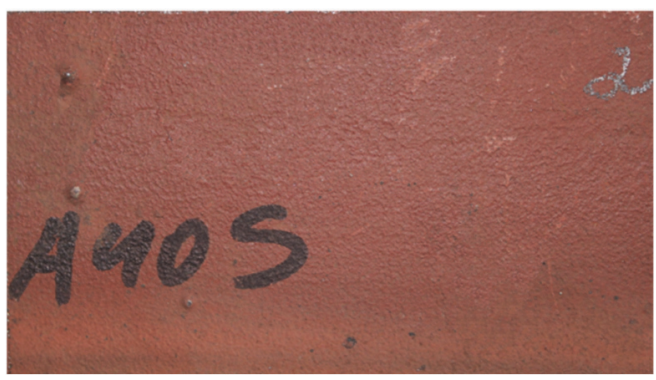

Fig. 8. Surface of experimental samples after rolling

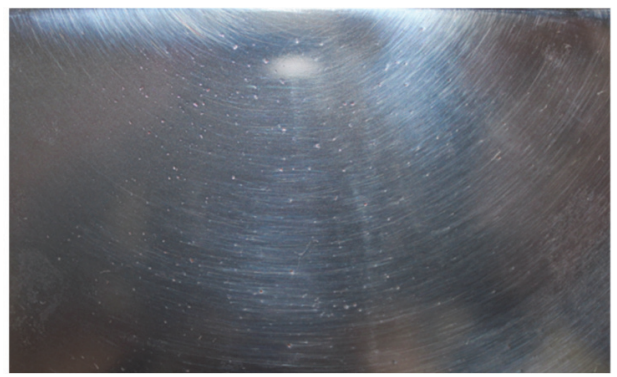

Fig. 9. Surface of experimental samples after polishing

The surface of the samples (Fig. 8) was previously ground with a decrease in the grain size of the abrasive. Final mechanical polishing was carried out with a solution of chromium dioxide $\mathrm{Cr}_{2} \mathrm{O}_{3}$ in pure kerosene, until reaching a mirror surface (Fig. 9). 
The quality of the prepared surface is also confirmed by microscopic observations (Figs. 10, 11) using an MBR-1 optical microscope with an OI-18A luminescent illuminator and a Canon EOS550D digital camera conjugated with a microscope.

Despite the presence of processing defects (Fig. 10), the polished surface has a uniform relief compared to the untreated sample (Fig. 11).

Drops of distilled water were applied to a natural (after rolled), polished and superhydrophobic (water repellent) surface.

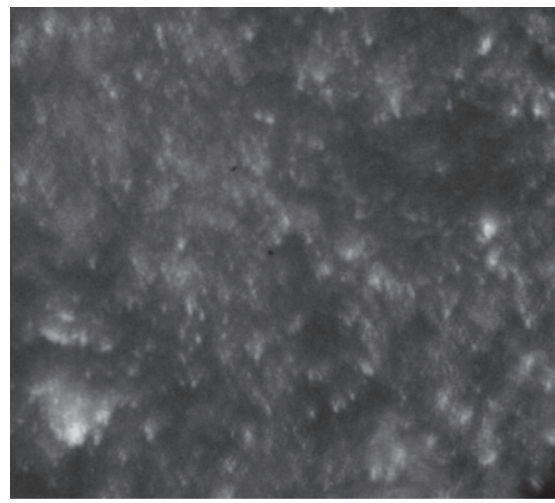

Fig. 10. Microstructure of untreated surface (steel A40S)

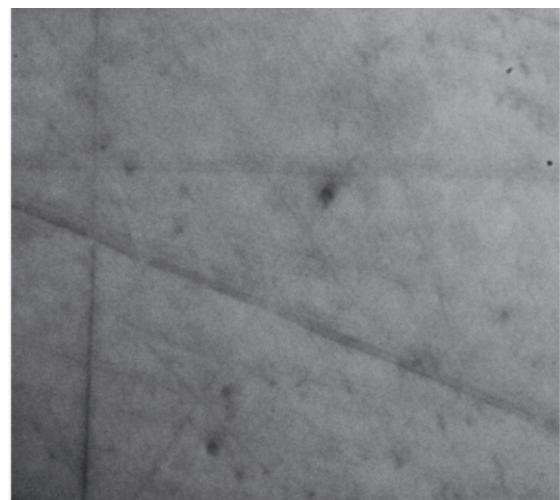

Fig. 11. Microstructure of polished surface (steel A40S)

\subsection{Methods for creating superhydrophobic surfaces and choosing the optimal path for this research}

Methods for creating superhydrophobic surfaces by processing a material with a low surface energy are mainly one-step processes and have the advantage of ease of implementation. There are many ways to make textured surfaces: mechanical pulling, laser processing and plasma processing, chemical etching, lithography, sol-gel method, layer by layer deposition and colloidal mounting, electrical or chemical reactions.

Chemical precipitation through a surface chemical reaction forms a layer with nanoscale topography, including nano-pins and nano-rods. It is convenient and quite easy to use various methods, including chemical vapor deposition (CVD), which uses a gaseous base to deposit particles or films. One can use chemical bottom desolutions (CBD) and electrochemical deposition (ECD) that use wet chemical solutions or electrochemically conductive substrates to precipitate hard metals and oxides.

We choose chemical vapor deposition (CVD) as a simple way to produce a superhydrophobic surface on a sample of A40S steel.

Superhydrophobicity of the steel surface was created with the help of the easily accessible superhydrophobic system NeverWet Base Coat. This is a new class of coatings, which causes water droplets to form almost ideal spheres, while keeping objects dry and clean.

The water-repellent properties were imparted to the surface by the precipitation of a hydrophobic solution by applying a two-layer coating. The first layer - the base layer - contains methyl isobutyl ketone, butyl acetate and mineral alcohols applied by uniform spraying in 2-3 passes from a distance of about $15 \mathrm{~cm}$ for 3-4 seconds. The sample is then left to stand for 30 minutes under normal ambient conditions maintained in the laboratory. The second layer is applied by depositing an upper coating containing silica nanoparticles. For approximately 12 hours, the surface is dried under normal laboratory conditions prior to the experiment and measurement. 


\subsection{Experimental setting}

Observation and photoshooting of the drops were carried out on a specially designed apparatus for determining the contact angle of the droplet (Fig. 12). On the optical bench (1), a specimen holder (2), a light source (3), a collecting lens (4), a digital camera Canon EOS 550D (5), linked to an ACER computer (6) are mounted to receive the image coaxiality. A drop of water after deposition on the surface of a steel sample and optical magnification is fixed by means of a viewfinder in real time by a computer.

The received images were analyzed using the sPlan 7.0 program and the built-in electronic protractor.

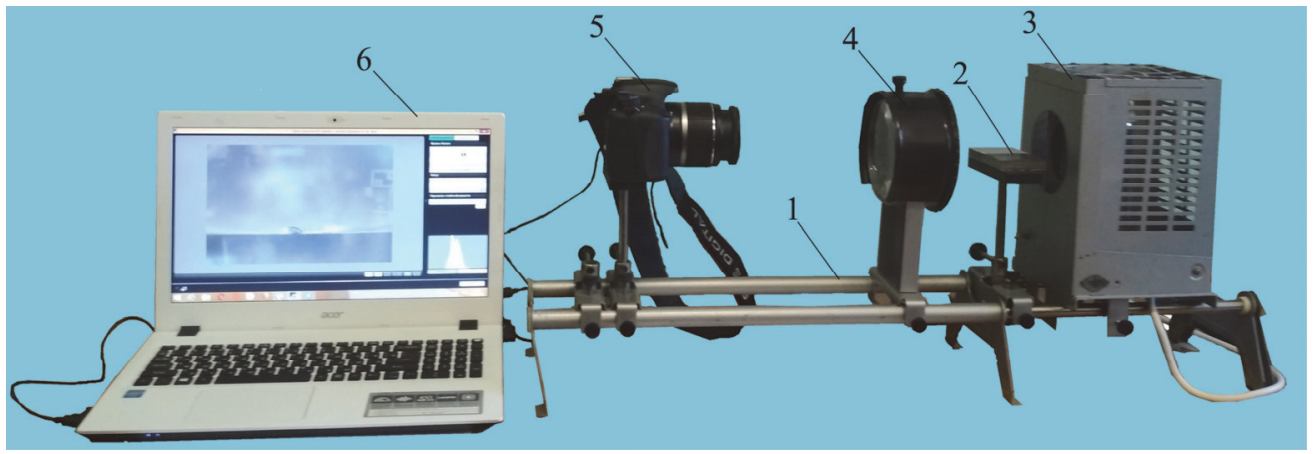

Fig. 12. Experimental setting for determining of the contact angle of the drop

\subsection{Results and discussion}

The image of water droplets on a natural (after rolled), polished and superhydrophobic surface of A40S steel is shown in the figures below (Figs. 13-15). The determination of the droplet angle by means of a graphical analysis and an electronic protractor showed that its value depends on the quality of the surface treatment. In the presence of a rough relief of the sample after steel rolling, the drop passes into the Wentzel state, which enhances the wetting effect and reduces the contact angle (Fig. 12).

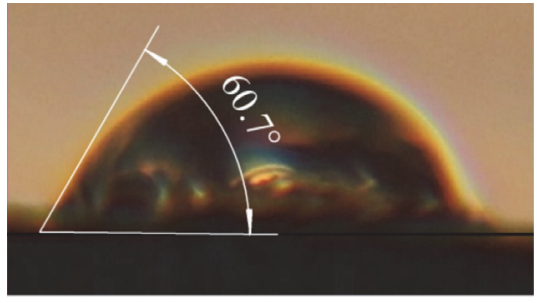

Fig. 13. A drop of water on a natural (after rolled) surface

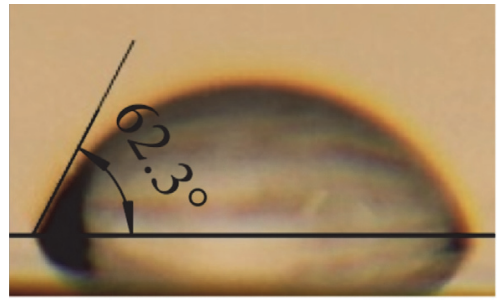

Fig. 14. A drop of water on a polished surface

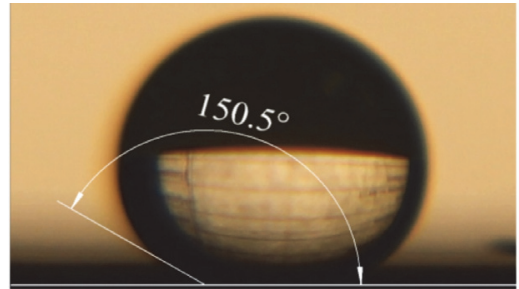

Fig. 15. A drop of water on a superhydrophobic surface 
Polishing eliminates the influence of unevenness and leads to an increase in the contact angle (Fig. 14).

The deposition of a microstructured (superhydrophobic) coating causes a transition to the Cassie-Baxter state (Fig. 15). In this state, the liquid lies on top of the structured surface above the gas layer. This gas gap is an effective medium that ensures the glide of the droplet at the liquid-gas interface. The contact angle of the surface obtained exceeds $150^{\circ}$, which is confirmed by its superhydrophobic properties.

The approbation of the method for determining the contact angle on samples of A40S shipbuilding steel using the described installation showed good agreement with the previously used scientific methods.

The superhydrophobic surfaces obtained, the images of drops on the natural (after rolling), polished and superhydrophobic surfaces do not contradict the wetting theory, which makes it possible to find ways to further extend the application of superhydrophobic coatings, including marine technologies.

The change in the angle of contact between the drop and the surfaces is due to their different processing and the presence of a superhydrophobic layer. The transition from the acute contact angle to the blunt angle on the surface of the deposited layer indicates its hydrophobic properties and the presence of the non-wetting effect, in contrast to the untreated and polished samples.

In the course of the subsequent experiment, drops of natural sea water from the Kerch Strait were used, the salinity of which was determined by the salt meter and was $17 \mathrm{ppm}$ (17 g salt $/ 1 \mathrm{~kg}$ water).

Precipitation of the drop onto the superhydrophobic surface was carried out by flowing from the tip, which provided a drop of $10^{-8} \mathrm{~m}^{3}$. Next, there was a photoshooting of the droplets evaporating (Fig. 16) in the laboratory. The interval between shots was reduced from 20 minutes at the beginning of the evaporation process up to 2 minutes at the end of the measurements. The total test time was about 4 hours (Table 2).

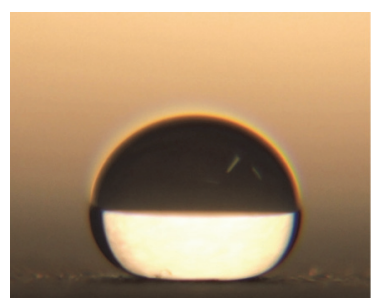

a)

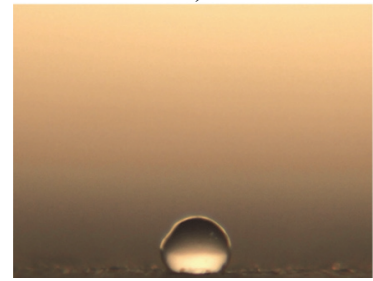

d)

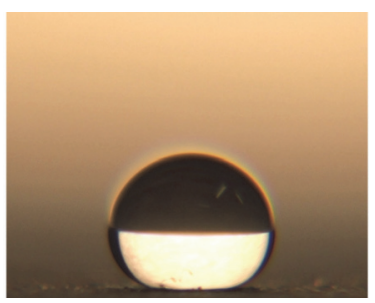

b)

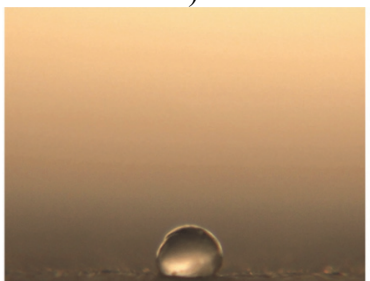

e)

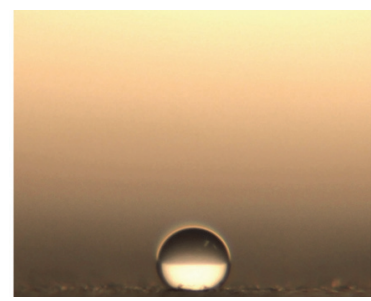

c)

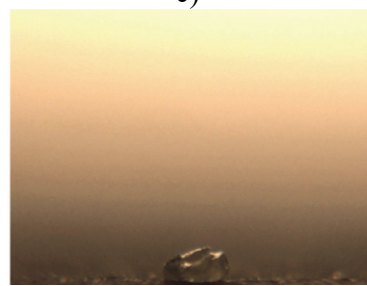

f)

Fig. 16. A sea water drop on a superhydrophobic surface during evaporation: a) $t=0 \mathrm{~min}$, b) $t=70 \mathrm{~min}$, c) $t=161 \mathrm{~min}, \mathrm{~d}) t=189 \mathrm{~min}, \mathrm{e}) t=198 \mathrm{~min}, \mathrm{f}) t=220 \mathrm{~min}$; image magnification: $\times 23$

Fig. 16 selectively shows the stages of evaporation of a drop of seawater on the superhydrophobic surface of A40S steel. The data of some properties of these droplets are shown in Table 2. Time after the droplet formation (Fig. 16(a)), after 1 hour of evaporation (Fig. 16(b)) and after 3 hours of evaporation (Fig. 15(c)), the drop gradually reduced the linear dimensions, while remaining in this form. After more than 3 hours (Figs. 16(d), 16(e)), a precipitate appears in the droplet, and the shape of the droplet is distorted, especially at the upper part. The process 
of evaporation of the salt crystal formation is completed (Fig. 16(f)). Attention is drawn to the fact that even with multiple the repetition of such an experiment as a result of the crystallization of the salt from the solution always produces a single, solid fragment of the solid phase. This phenomenon is significantly different from the results of evaporation of a drop of $\mathrm{NaCl}$ solution on hydrophilic surfaces, including shipbuilding steels. Under such conditions, a number of individual crystals arise, the size and concentration, which increases closer to the perimeter of the contour of the precipitated drop. On the superhydrophobic surface, the resultant force acting on the impurity will be directed into the droplet during evaporation, which leads to a uniform growth of the crystal during evaporation.

Table 2. Some a sea waters drops properties dependence on the evaporation time

\begin{tabular}{|c|c|c|c|c|c|c|c|c|c|c|c|}
\hline 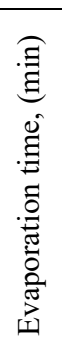 & 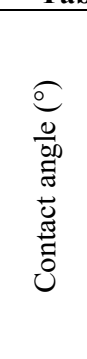 & 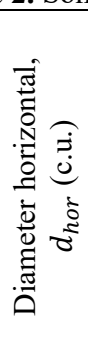 & 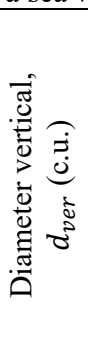 & ఫे & 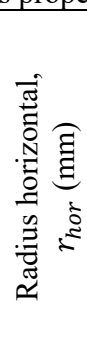 & 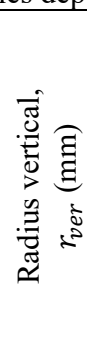 & 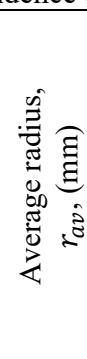 & 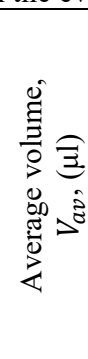 & 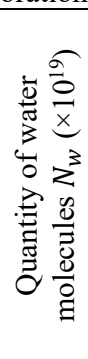 & 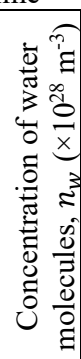 & 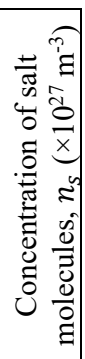 \\
\hline 0 & 168.0 & 69.0 & 54.0 & 1.278 & 1.500 & 1.174 & 1.337 & 10.00 & 33.30 & 3.32 & 0.17 \\
\hline 28 & 165.6 & 63.5 & 50.7 & 1.252 & 1.380 & 1.102 & 1.242 & 8.01 & 26.60 & 3.32 & 0.22 \\
\hline 52 & 165.5 & 58.4 & 47.3 & 1.235 & 1.270 & 1.028 & 1.145 & 6.35 & 21.10 & 3.32 & 0.28 \\
\hline 70 & 163.4 & 54.6 & 44.5 & 1.270 & 1.187 & 0.967 & 1.077 & 5.23 & 17.30 & 3.31 & 0.33 \\
\hline 94 & 167.9 & 49.1 & 41.1 & 1.195 & 1.067 & 0.893 & 0.980 & 3.95 & 13.00 & 3.30 & 0.44 \\
\hline 108 & 162.8 & 47.5 & 38.7 & 1.228 & 1.033 & 0.841 & 0.937 & 3.44 & 11.30 & 3.29 & 0.51 \\
\hline 127 & 158.9 & 40.7 & 35.5 & 1.146 & 0.885 & 0.772 & 0.828 & 2.38 & 7.78 & 3.27 & 0.74 \\
\hline 150 & 161.6 & 35 & 30.8 & 1.136 & 0.760 & 0.670 & 0.715 & 1.53 & 4.95 & 3.23 & 1.14 \\
\hline 161 & 155.1 & 31.8 & 28.2 & 1.128 & 0.691 & 0.613 & 0.652 & 1.16 & 3.71 & 3.19 & 1.51 \\
\hline 188 & 152.7 & 24.7 & 21.2 & 1.165 & 0.537 & 0.461 & 0.499 & 0.52 & 1.56 & 3.01 & 3.37 \\
\hline 189 & 154.1 & 24.1 & 20.6 & 1.170 & 0.524 & 0.448 & 0.486 & 0.4 & 1.43 & 2.98 & 3.64 \\
\hline 195 & 142.9 & 24.3 & 20.8 & 1.168 & 0.528 & 0.452 & 0.491 & 0.4 & 1.48 & 2.99 & 3.55 \\
\hline 198 & 130.4 & 23.0 & 18.8 & 1.223 & 0.500 & 0.409 & 0.454 & 0.39 & 1.14 & 2.90 & 4.46 \\
\hline 200 & 135.0 & 22.8 & 18.2 & 1.253 & 0.496 & 0.396 & 0.446 & 0.37 & 1.06 & 2.87 & 4.72 \\
\hline 202 & 135.0 & 21.7 & 17.2 & 1.262 & 0.472 & 0.374 & 0.423 & 0.32 & 0.88 & 2.79 & 5.53 \\
\hline 204 & 137.5 & 21.6 & 16.5 & 1.309 & 0.470 & 0.359 & 0.414 & 0.30 & 0.82 & 2.76 & 5.88 \\
\hline 206 & 136.0 & 21.1 & 15.8 & 1.335 & 0.459 & 0.343 & 0.401 & 0.27 & 0.73 & 2.70 & 6.48 \\
\hline 208 & 142.9 & 20.7 & 14.7 & 1.408 & 0.450 & 0.320 & 0.385 & 0.24 & 0.62 & 2.61 & 7.34 \\
\hline 210 & 142.6 & 20.8 & 14.2 & 1.465 & 0.452 & 0.309 & 0.380 & 0.23 & 0.60 & 2.59 & 7.60 \\
\hline 213 & 135.0 & 20.7 & 13.2 & 1.568 & 0.450 & 0.287 & 0.368 & 0.21 & 0.53 & 2.51 & 8.35 \\
\hline 216 & 145.0 & 21.2 & 11.5 & 1.843 & 0.461 & 0.250 & 0.355 & 0.191 & 0.45 & 2.41 & 9.31 \\
\hline 220 & 137.5 & 21.2 & 10.9 & 1.945 & 0.461 & 0.237 & 0.349 & 0.18 & 0.42 & 2.36 & 9.84 \\
\hline 223 & 135.0 & 21.2 & 11.1 & 1.910 & 0.461 & 0.241 & 0.351 & 0.18 & 0.43 & 2.38 & 9.66 \\
\hline 226 & 139.5 & 21.4 & 11.1 & 1.928 & 0.465 & 0.241 & 0.353 & 0.18 & 0.44 & 2.40 & 9.48 \\
\hline
\end{tabular}

The stability of the hydrophobic state of a drop of seawater on a structured surface was studied by measuring the contact angle (Table 2) for each observation stage. The graph of the variation of the contact angle versus the evaporation time is shown in Fig. 17.

Contact angle is defined by equation:

$\cos \theta_{C}=\frac{\gamma_{S G}-\gamma_{S L}}{\gamma_{L G}}$

where $\theta_{C}$ - contact angle, $\gamma_{S G}, \gamma_{S L}$ and $\gamma_{L G}$ - surface tension coefficients of the solid/gas boundary, 
solid/liquid, and liquid/gas, respectively.

Despite the increase in salt concentration due to evaporation of water, the contact angle at the initial stage varies slightly $\left(150^{\circ}<\theta_{C}<168^{\circ}\right)$, thus maintaining the superhydrophobic state of the drop.

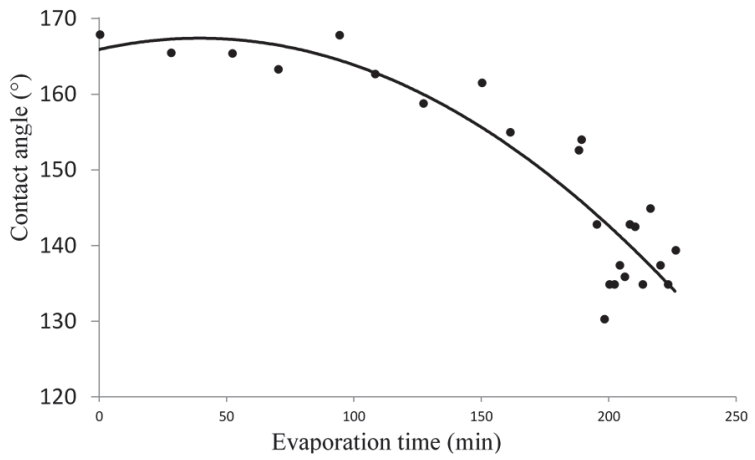

Fig. 17. The graph of the contact angles and evaporation times dependence

After 3 hours of testing, the value of the contact angle decreases. However, this evaporation stage already corresponds to the distortion of the drop-shaped form (Figs. 16(d), 16(e)), which indicates the onset of crystallization of the $\mathrm{NaCl}$-salt, which undoubtedly introduces a change in Eq. (5).

Approximately, the dependence of the surface tension $\gamma_{L G}$ on the temperature $T$, the fluid density $\rho$, and the molar mass $\mu$ of the substance is expressed by the equality:

$\gamma_{L G}=B\left(T_{k}-T-\tau\right) \cdot\left(\frac{\rho}{\mu}\right)^{\frac{2}{3}}$,

where $B$ - a constant coefficient, $T_{k}$ - the critical temperature, and $\tau$ - the magnitude of the temperature dimension. Under the isothermal conditions of the experiment, the main contribution to the change in $\gamma_{L G}$ will be made by the factor $\rho / \mu$ Eq. (6), which in turn can be represented for a mixture of substances:

$\frac{\rho}{\mu}=\frac{m\left(v_{1}+v_{2}\right)}{V m}=\frac{n_{1}+n_{2}}{N_{A}}$,

where $m$ - the mixture weight, $V$ - the drop volume, $v$ - the amount of the substance, $n-$ the concentration of the components, and $N_{A}-$ the Avogadro number. At the initial stage of the experiment, the ratio $\left(n_{1}\right) /\left(n_{2}\right) \approx 10^{2}$ and the value of the ratio Eq. (7) is mainly determined by the number of water molecules in the droplet volume. Evaporation of water leads to a decrease in its concentration and an increase in the concentration of salt in the drop. Decay $n_{1}$ occurs faster than $n_{2}$, which in time leads to an increase in their sum, and consequently to a decrease in $\theta_{C}$ Eq. (1). At the end of evaporation, $n_{1} \approx 0$, while the calculated value of $n_{2}=2 \cdot 10^{28} \mathrm{~m}^{-3}$ coincides with the concentration of molecules in the $\mathrm{NaCl}$ crystal.

In most cases, in the studies of droplets on a superhydrophobic surface, accurate analysis of the contact angle is made difficult by distortion of their shape due to gravity. According to the research [16], the droplet volume should be within 0.5-10 $\mu$ l. In general, the use of small droplets avoids the deformation of water droplets caused by gravity. However, due to the lack of proper wetting of the superhydrophobic surface, it is very difficult to create drops of water with a volume of less than $4 \mu \mathrm{l}$ on them.

In the general case, while studying liquid droplets, the actions of capillary $(\sigma L)$ and 
gravitational $\left(g \rho L^{3}\right)$ forces are compared, calculating the Bond's number (Eötvös number) by the formula:

$B_{0}=\frac{\rho g L^{2}}{\gamma}$

where $L$ - the characteristic size of the liquid drop, for example, the height at its center, $\gamma-$ the surface tension of the liquid, $g$ - the acceleration due to gravity, $\rho$ - the density of the liquid.

However, the process of evaporation of seawater drop causes variability in the density and surface tension of the liquid, which disrupts the dependence Eq. (8). Therefore, in the given work, to estimate the effect of gravity on the shape of a drop of seawater during evaporation on a superhydrophobic surface, the ratio of the horizontal radius $r_{\text {hor }}$ of the droplet to the vertical $r_{v e r}$ in the middle section of the drop was calculated. The graph of the relationship $\left(r_{\text {hor }} / r_{\text {ver }}\right)$ versus the evaporation time is shown in Fig. 18.

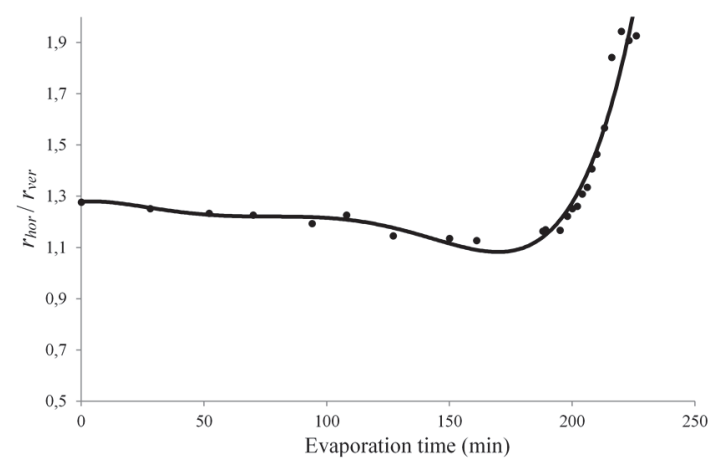

Fig. 18. The graph of the contact angles and $r_{\text {hor }} / r_{\text {ver }}$ ratios dependence

A new method for measuring the contact angles of superhydrophobic surfaces was proposed in [17]. A $5 \mu 1$ drop of water was placed on the sample, and the contact angle was measured after the droplet had decreased in volume to $0.3 \mu \mathrm{l}$ after evaporation for 40 min under ambient conditions.

The initial volume of droplets was $V=10 \mu \mathrm{l}$, and $r_{\text {hor }} / r_{\text {ver }} \approx 1.3$. In the process of evaporation, the difference in the radii of the droplet decreases, reaching its minimum at the level of $r_{\text {hor }} / r_{\text {ver }} \approx 1.16$; volume $V=0.5 \mu$ l. The minimum deviation from the spherical shape coincides with the onset of the active crystallization of salt in the droplet volume, which leads to an increase in the $r_{\text {hor }} / r_{\text {ver }}$ ratio and further coarsening of the contour. Further nucleation of the solid phase leads to an even greater discrepancy between the horizontal and vertical radii, but the predominance of salt crystals in the volume no longer makes it possible to determine the resulting formation as a drop.

In the general case, the change in the volume of the droplet from time (Fig. 19), before the onset of the phase transformation, is inversely proportional, the character of which is related to the external conditions of evaporation (humidity, saturated vapor pressure). The onset of the crystallization process is indicated by a decrease in the rate of evaporation and an equalization of the graph of $V=f(t)$ after 190 minutes of testing. At the same time, the concentration of salt in the droplet volume increases sharply (Fig. 20).

The process of phase transition takes about 30 minutes. In this case, the main contribution to volume reduction is made by the vertical component of the linear dimensions of the droplet cross section. The horizontal radius after 190 minutes of evaporation and until the time of full hardening decreases no more than $13 \%$.

The saturation concentration of $\mathrm{NaCl}$ is $35 \%$ and will be achieved when the volume is reduced 
to $0.2 \mu \mathrm{l}$. After that, the horizontal size of the droplet practically remains constant (Table 2), which causes a sharp increase in the $r_{h o r} / r_{v e r}$ ratio (Fig. 18) and the formation of an elongated crystal on the surface after evaporation of the droplet (Fig. 16(f)).

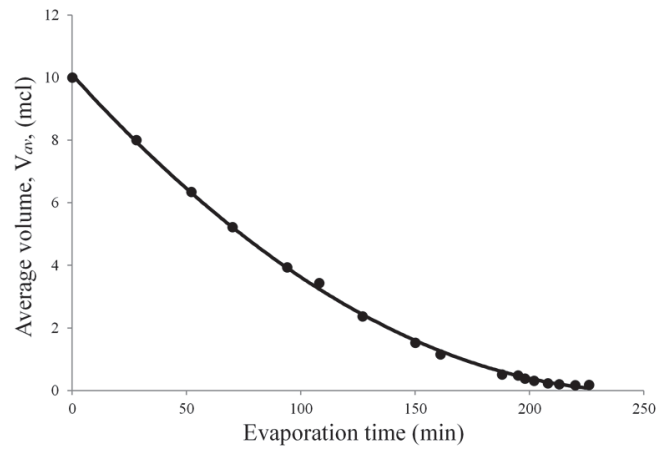

Fig. 19. The graph of the average volume and evaporation time dependence

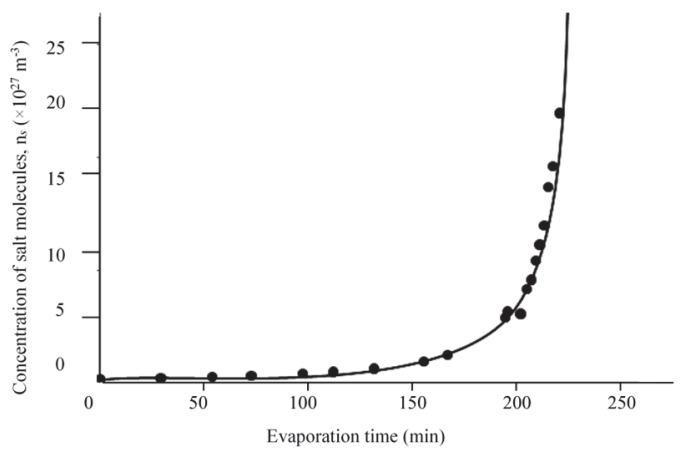

Fig. 20. The graph of the concentration of $\mathrm{NaCl}$ molecules and evaporation time dependence

Using the data of Table 2, the concentration of salt in the evaporating drop of sea water was determined by the following method. If we take the salinity of 17 pro mil (the figure characteristic of the Kerch Strait), i.e. in $1 \mathrm{~kg}$ of water contains $17 \mathrm{~g}$ of $\mathrm{NaCl}$-salt, then the salt concentration is determined by the formula:

$n_{0}=\frac{N}{V}=\frac{m}{m_{0} V}$

where $m=17 \mathrm{~g}-$ mass of salt, $m_{0}=58.44$ amu molecules $\mathrm{NaCl}, V=1, l=10^{-3} \mathrm{~m}^{3}$ (it is kilo of the substance); and will be equal to:

$n_{0}=\frac{17 \cdot 10^{-3}}{58.44 \cdot 1.66 \cdot 10^{-27} \cdot 10^{-3}}=17.5 \cdot 10^{25} \mathrm{~m}^{-3}$.

The number of salt molecules in the drop:

$N_{0}=n_{0} \cdot V_{0}=17.5 \cdot 10^{25} \cdot 10^{-8}=17.5 \cdot 10^{17}$,

where $V_{0}=10^{-8} \mathrm{~m}^{3}$ - the initial volume of the drop (experimentally determined).

To calculate the water concentration in the drop, the number of molecules in the volume was first determined without the presence of an impurity: 
$N_{0 B}=\frac{m N_{A}}{\mu}=\frac{\rho V N_{A}}{\mu}$,

and subtracted the number of salt molecules:

$N_{B}=\frac{\rho V N_{A}}{\mu}-N_{0}$

Water molecules concentration:

$n_{B}=\frac{N_{B}}{V}$

$\mathrm{NaCl}$ concentration in the crystal was determined theoretically:

$n^{\prime}=\frac{\rho_{\mathrm{NaCl}}}{m_{0}}=\frac{2165}{58.44 \cdot 1.66 \cdot 10^{-27}}=2,23 \cdot 10^{28} \mathrm{~m}^{-3}$.

The obtained theoretical data are conformed with our calculations on the experimentally obtained data.

Of course, it is difficult to affirm quantitatively good accuracy on such a scale. However, some qualitative conclusions can be made.

\section{Conclusions}

The review of the properties and methods of creating superhydrophobic surfaces is aimed at finding possible ways of implementing their various applications in the shipbuilding industry.

The work deals with the conditions of non-wetting by a liquid of hard surfaces, the dependence of the contact angle on the surface type, methods of its calculation or determination. Attention is paid to the physical mechanism of the stable state of Cassie-Baxter with air trapped by the superhydrophobic surface between the drop and its roughness.

A technique for performing experimental studies to determine the contact angle of water droplets on the natural, polished and superhydrophobic surface of A40S steel is developed. The choice of steel-type was since this steel is widely used in the world shipbuilding industry.

Superhydrophobicity of the surface on the steel was achieved by chemical vapor deposition (CVD) using the NeverWet Base Coat superhydrophobic system. The adequacy and correctness of the described and implemented technique for surface preparation and application of the superhydrophobic layer are confirmed by optical observations of water droplets and the drop contact angle analysis.

The scheme of the installation for graphical determination of the contact angle of a liquid drop on the surface of a solid is described. The images were analyzed using sPlan 7.0. The contact angle was measured by the electronic protractor built into the program. The results gave good agreement with previously used scientific methods.

In the experiment, drops of natural sea water (the Kerch Strait) were used. The determination of the contact angle of the drops showed that its value depends on the quality of the surface treatment. In the presence of a rough relief of the sample after steel rolling, the drop passes into the Wentzel's state, which enhances the wetting effect and reduces the contact angle. The change in the angle of contact between the drop and the surfaces is due to their different processing and the presence of a superhydrophobic layer. The transition from the acute contact angle to the blunt angle on the surface of the deposited layer indicates its hydrophobic properties and the presence of the non-wetting effect, in contrast to the untreated and polished samples.

The obtained superhydrophobic surfaces, the images of drops on the natural (after rolling), 
polished and superhydrophobic surfaces do not contradict the wetting theory, which makes it possible to search for ways to further extend the use of superhydrophobic coatings, including in marine technologies.

The phenomenon of evaporation of seawater drops was studied. The research of the behavior of natural sea water droplets on the created superhydrophobic surfaces on samples of A40S shipbuilding steel showed that in the process of evaporation the contact angle decreases, but before the crystallization process remains $\theta_{C}>150^{\circ}$, thereby retaining the superhydrophobic properties of the surface. Completion of evaporation of the drop is accompanied by the formation of a solid $\mathrm{NaCl}$ crystal, which, of course, facilitates the removal of such impurities from the steel surface. In addition, such an effect will not cause degradation of the microstructure of the surface, its contamination with fine crystals, and block the gas channels providing superhydrophobic properties.

Reducing the volume during evaporation does not completely eliminate the effect of gravity on the shape of the droplet, but reaches its minimum for volumes of $0.5 \mu 1$.

Thus, the obtained results contribute to the evaluation of the capabilities of superhydrophobic coatings in interaction with seawater, expand their application for self-cleaning and protection from various natural formations of surfaces of materials used by the marine industry.

The results provide an opportunity to obtain and further study protective coatings for structures and mechanisms used in the marine environment, the performance of which depends on the phenomenon of absolute non-wetting of a solid surface, including shipbuilding steel. It is necessary to conduct a thorough characterization of the mechanical and thermodynamic stability of the superhydrophobic state in real operating conditions. It is important to develop simpler and more standardized methods for creating and verifying the reliability of surfaces under pressure, wear, friction, immersion, pollution and other conditions.

\section{Acknowledgements}

This research is supported by the Kerch State Maritime Technological University under the Contract No. AAAA-A18-118021990017-5 with third-party organizations.

\section{References}

[1] Feng Li L. S. H., Li Y. S., et al. Super-hydrophobic surfaces: from natural to artificial. Advanced Materials, Vol. 14, Issue 24, 2002, p. 1857-1860.

[2] Zhang Y.-L., Xia H., Kim E., Sun H.-B. Recent developments in superhydrophobic surfaces with unique structural and functional properties. Soft Matter, Vol. 8, Issue 2, 2012, p. 11217-11231.

[3] Koch K., Bhushan B., Barthlott W. Multifunctional surface structures of plants: an inspiration for biomimetics. Progress in Materials Science, Vol. 54, Issue 2, 2009, p. 137-178.

[4] Barthlott W., Neinhuis C. Purity of the sacred lotus, or escape from contamination in biological surfaces. Planta, Vol. 202, Issue 1, 1997, p. 1-8.Neinhuis C., Barthlott W. Characterization and distribution of waterrepellent, self-cleaning plant surfaces. Annals of Botany, Vol. 79, Issue 6, 1997, p. 667-677.

[6] Yan Y. Y., Gao N., Barthlott W. Mimicking natural superhydrophobic surfaces and grasping the wetting process: a review on recent progress in preparing superhydrophobic surfaces. Advances in Colloid and Interface Science, Vol. 169, Issue 2, 2011, p. 80-105.

[7] Zhang P., Lv F.Y. A review of the recent advances in superhydrophobic surfaces and the emerging energy-related applications. Energy, Vol. 18, Issue 15, 2015, p. 1068-1087.

[8] Van der Wal P., Steiner U. Super-hydrophobic surfaces made from Teflon. Soft Matter, Vol. 3, Issue 4, 2007, p. 426-429.

[9] Yao X., Song Y. L., Jiang L. Applications of bio-inspired special wettable surfaces. Advanced Materials, Vol. 23, 2011, p. 719-734.

[10] Rungraeng N., Cho Y-C., Yoon SH., Jun S. Carbon nanotubepolytetrafluoroethylene nanocomposite coating for milk fouling reduction in plate heat exchanger. Journal of Food Engineering, Vol. 111, Issue 2, 2012, p. 218-224. 
[11] Carriveau R., Edrisy A., Cadieux P., Mailloux R. Ice adhesion issues in renewable energy infrastructure. Journal of Adhesion Science and Technology, Vol. 26, Issues 4-5, 2012, p. 447-467.Cucchiella F., D'Adamo I. Estimation of the energetic and environmental impacts of a roof-mounted building-integrated photovoltaic systems. Renewable and Sustainable Energy Reviews, Vol. 16, Issue 7, 2012, p. 5245-5259.

[13] Pridvorov B., Popova T., Ukolov A. Superhydrophobic surface. Review. Education, Science and Youth. Vol. 2017, Issue 2, 2017, p. 107-120.

[14] Bin Su, Tian Ye, Jiang Lei Bioinspired interfaces with superwettability: from materials to chemistry. Journal American Chemical Society, Vol. 138, Issue 6, 2016, p. 1727-1748.

[15] Craig V., Zhang X., Hu J. Nanobubbles at Hydrophobic Surfaces. Drops and Bubbles in Contact with Solid Surfaces. CRC Press, United States, 2012, p. 159-174.

[16] Zhao T., Jiang L. Contact angle measurement of natural materials Colloids and Surfaces B. Biointerfaces. Vol. 161, 2018, p. 324-330.

[17] Zhang X., Shi F., Yu X., Liu H., Fu Y., Wang Z., Jiang L., Li X. Polyelectrolytemultilayer as matrix for electrochemical deposition of gold clusters: towardsuper-hydrophobic surface. Journal American Chemical Society, Vol. 126, 2004, p. 3064-3065.

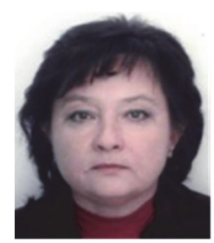

Tatyana Popova, Professor, Doctor of pedagogical sciences. Received the Doctor in pedagogy in 2011 at the National Pedagogical University after M. P. Dragomanov, Kiev, Ukraine. Now she is the Head of the Department of Mathematics, Physics and Informatics in the Kerch State Maritime Technological University. Current interests include studying the properties of superhydrophobic surfaces in seawater.

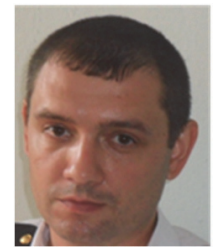

Aleksey Ukolov, Candidate of Physical and Mathematical Sciences. Received Ph.D. degree in solid state physics in 2014 at Kharkiv National University after V. N. Karazin, Kharkov, Ukraine. Currently, docent of the Department of Mathematics, Physics and Informatics of the Kerch State Maritime Technological University. Current interests include the study of low-dimensional structures in near-surface layers of solids. 


\section{Chapter 5}

Improving the quality and reliability of electronic equipment and power systems 


\title{
5.1. Acoustic emission diagnostics of solder joints on printed circuit boards
}

\author{
Igor Kovtun ${ }^{1}$, Vilen Royzman ${ }^{2}$, Andriy Voznyak ${ }^{3}$ \\ Khmelnytsky National University, Khmelnytsky, Ukraine \\ E-mail: ${ }^{1}$ dr.igorkovtun@gmail.com, ${ }^{2}$ royzman@ukr.net, ${ }^{3}$ andreyvoznyak27@gmail.com
}

\begin{abstract}
The paper represents technical diagnostics of printed circuit boards in particular the solder joints performed by commonly used through-hole and surface mount technologies. The research methods involved mechanical tensile and bend tests and acoustic emission method application with simultaneous monitoring both mechanical characteristics and acoustic emission parameters in order to find their correlation. On the base of conducted experiments method for solder joint technical diagnostics by using method of acoustic emission in pure bending cycling tests has been designed.
\end{abstract}

Keywords: solder joint, printed circuit board, technical diagnostics, electronic component, acoustic emission, tensile test, pure bending.

\section{Introduction}

Complexity and micro-miniaturization in electronic and computer production technologies constantly increase quality and reliability standards for their components. Printed circuit boards (PCB) are considered the main building blocks of modern electronics, which represent assemblies purposed to electrically connect and, what is also important, mechanically support electronic components. However, operation and even technology cause mechanical interactions and forces, acting between PCB substrate and electronic components, and spreading through their links. Such tensile, shear, bending or torque forces cause strain and stress in substrates, which are subsequently transmitted to bodies of components through the contact joints that appear to be the weakest links in the assembly. The damages of the joints cause failures of the whole electronic units and therefore require detailed studying.

The general technology for components installation (e.g. capacitors, resistors or active devices) on PCBs remains soldering although quality of soldered joints (SJ) is not always achievable due to numerous defects. Therefore, one of the primary tasks to provide reliability to SJs is to apply and improve their testing methods. The range of methods for non-destructive or destructive SJ tests provided by the state standard $[1,2]$ does not always provide proper quality selection. Since soldered joint strength is one of the indicators applied to assess its quality, development of methods for strength diagnostics of soldered joints on PCBs is the objective for the current research.

The diagnostics of SJs wad performed for two commonly used technologies: through-hole technology (THT) and surface mount technology (SMT). The research methods involved static mechanical tensile and bend tests and acoustic emission (AE) method application with simultaneous monitoring both mechanical characteristics and acoustic emission parameters.

\section{Nondestructive evaluation methods}

Application of electrical and physical methods for SJ quality control attracts close attention in nowadays. In accordance to [2] these methods include: electro-parametric, noise, acoustic emission, exoelectronic emission, photovoltaic and recombinant radiation methods. Among these methods, the most of which have range of intrinsic disadvantages such as high cost and complexity of measuring instrumentation, the method of acoustic emission has recently hastened its growth due to its high applicability for nondestructive diagnostics and prediction of strength of technical objects in general and of solder joints in particular. Table 1 shows the list of characteristics represented for comparison of acoustic emission methods with another nondestructive evaluation (NDE) methods. 
Table 1. Comparative characteristics of acoustic emission methods against other NDE methods

\begin{tabular}{|c|c|}
\hline Acoustic emission method & Other NDE methods \\
\hline Detects defects in progression & Detect geometry of defects \\
\hline Requires load testing & Does not require load testing \\
\hline Each test is unique & Tests are replicable \\
\hline Sensitive to material consistency & Less sensitive to material consistency \\
\hline Less sensitive to geometry of tested object & $\begin{array}{l}\text { More sensitive to geometry of tested } \\
\text { object }\end{array}$ \\
\hline Simplicity of application & Complexity of application \\
\hline $\begin{array}{l}\text { Transducers require minor contact area for attachment on the } \\
\text { tested object }\end{array}$ & $\begin{array}{l}\text { Require reach to the full surface of } \\
\text { tested object }\end{array}$ \\
\hline The object is completely monitored by the single test & $\begin{array}{l}\text { Subsequent monitoring parts of the } \\
\text { object }\end{array}$ \\
\hline Difficulties: sensitive to noise & Difficulties: object geometry dependent \\
\hline
\end{tabular}

Comparative analysis of NDE indicates in favor of acoustic emission method as versatile, simple and inexpensive method applicable to assessment of health conditions of the structures.

By the definition, acoustic emissions are pressure waves generated due to transient release of energy when a material is subjected to mechanical (in this case), thermal or chemical changes causing irreversible deformations or changes in atomic arrangement. The energy released travels as a spherical wavefront and is converted to electrical signal by transducers placed on the surface of the material (Fig. 1). The transducer output is filtered and amplified to eliminate ambient noise and increase the signal-to-noise ratio.

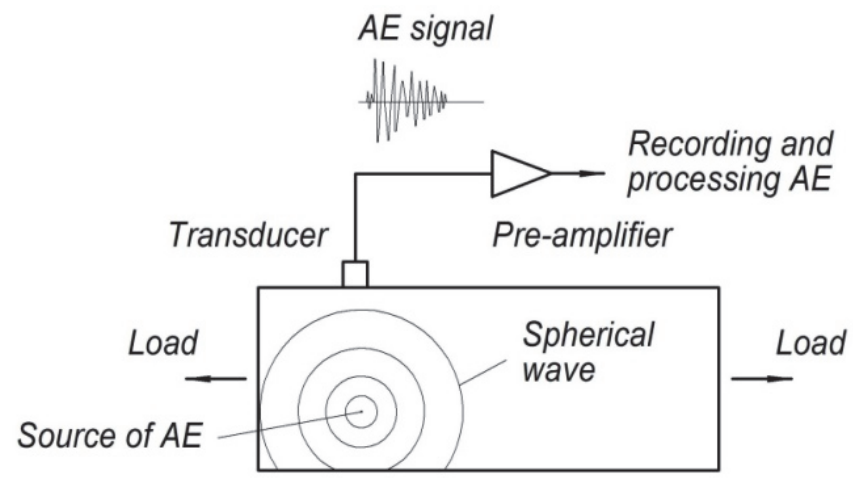

Fig. 1. The basic principle of acoustic emission generation and recording

The output signal of transducer represents high frequency oscillation (Fig. 2). The single and unique signal is named as the event of acoustic emission and assumed as the total amount of all oscillations received within time interval of $1 \mathrm{~ms}$.

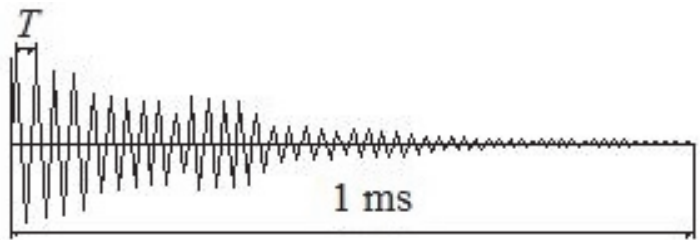

Fig. 2. Acoustic emission event image

Every event is approximated by the expression:

$U=A e^{-n t} \sin \omega t$ 
where $A$ - amplitude; $n$ - attenuation factor (factor which is determined by damping characteristics of the structure); $\omega$ - cycling frequency; $t$ - time:

$\varpi=\sqrt{p^{2}-n^{2}}$

where:

$p=c / m$,

where $c$ - rigidity (static force that causes single displacement); $m$ - reduced mass. Normally $n \ll m$ and $\omega=p$.

Oscillation is characterized by its period:

$T=\frac{2 \pi}{\sqrt{p^{2}-n^{2}}} \approx \frac{2 \pi}{p}$.

For the analysis high frequency signal (Fig. 3(a)) received from the transducer is then processed to select oscillations (Fig. 3(b)), whose amplitudes exceed discrimination threshold of the amplifier, and then events. An event is considered as collection of oscillations, received within time period of $1 \mathrm{~ms}$, and then converted into one impulse (Fig. 3(c)) of amplitude equal to maximal amplitude of oscillations collected.

The common parameters obtained by processing AE are: cumulative count of oscillations; time rate of oscillation count (count rate); cumulative count of events (total count); time rate of events count (activity) Eq. (5); amplitude of events; linear coordinate of events, which is calculated by using Doppler effect of time difference in signal arrivals to two transducers $\Delta t=t_{2}-t_{1}$ (Fig. 3(d)); power of events Eq. (7) and energy of events Eq. (8).

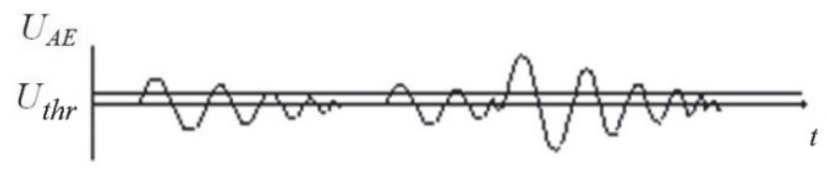

a)

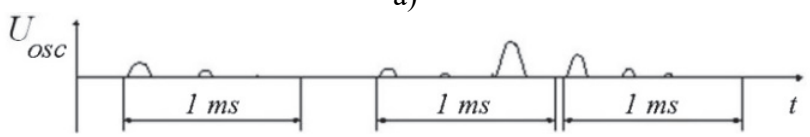

b)

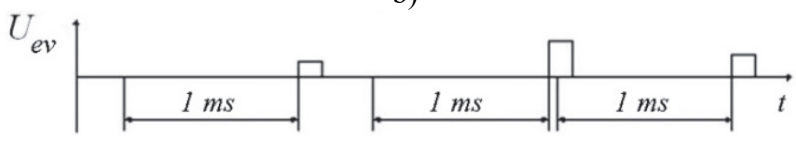

c)

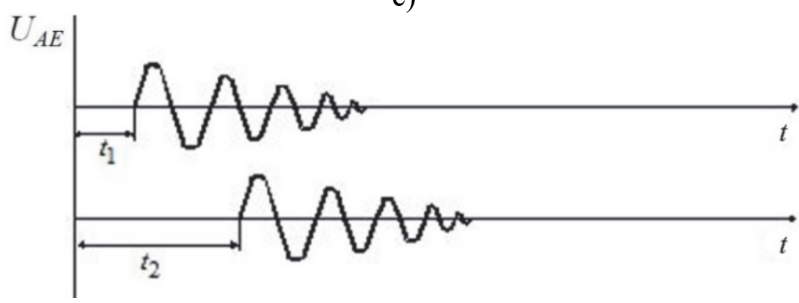

d)

Fig. 3. Acoustic emission processing: a) high frequency oscillation, b) oscillations selected,

c) events converted, d) using Doppler effect for linear location

Acoustic emission activity represents time rate of signals count: 
$N=\frac{N_{c}}{T}$

where $N_{c}$ - signals count; $T$ - time.

Power or electric power of events transduced by the piezoelectric sensor is expressed by formula:

$W=k \lim _{T \rightarrow \infty} \frac{1}{T} \int_{0}^{T} U^{2}(t) d t$,

where $k$ - proportionality factor; $T$ - time interval; $U(t)$ - amplitude of event.

Power of event is processed by measuring instrument as:

$W=U^{2} N_{o}$,

where $U$ - amplitude of event, $N_{o}$ - oscillation count per event, which is directly proportional to event duration.

Acoustic emission energy is defined by the formula:

$E=k \frac{\sum_{i=1}^{N_{c}} W_{i}}{T}$

where $k$ - proportionality factor; $W_{i}$ - power of event.

\section{Acoustic emission measuring station}

Acoustic emission station was designed in experimental laboratory of the static and dynamic strength in electronics in Khmelnytsky National University [3]. The station structure is shown in Fig. 4.

The station is created to perform online reading, saving and processing of acoustic emission parameters, received due to static and dynamic mechanical tests of the structures.

In the represented research the station was used for experimental and applied diagnostics and predicting strength of solder joints on printed circuit boards subjected to the following tests:

- Static tests conducted by tension test machine IR 5057-50;

- Dynamic tests conducted by the shaker VEDS 200;

- Thermal tests conducted in climatic chamber Feitron 3526/51.

Reading and initial processing of acoustic emission signals is performed by acoustic emission measuring instrument AF-15. Signals are transduced by the piezoelectric sensors. Final processing of the data is performed by personal computer connected to AF-15 via the designed unit of parallel input/output interface.

The following improvements have been made to create AE measuring station:

- Modification of sub-units of linear coordinates, amplitude and adder in measuring instrument AF-15;

- Design of parallel input/output interface;

- Development of the software for processing acoustic emission data.

\section{Solder joint defects}

Soldering, in which two or more items are joined together by melting and putting a filler metal (solder) into the joint, is a complex technology involving physical and chemical processes, which pass through solid, liquid and gaseous phases [1,2]. The objectives of represented research are 
two commonly used technologies: through-hole technology (THT) and surface mount technology (SMT).

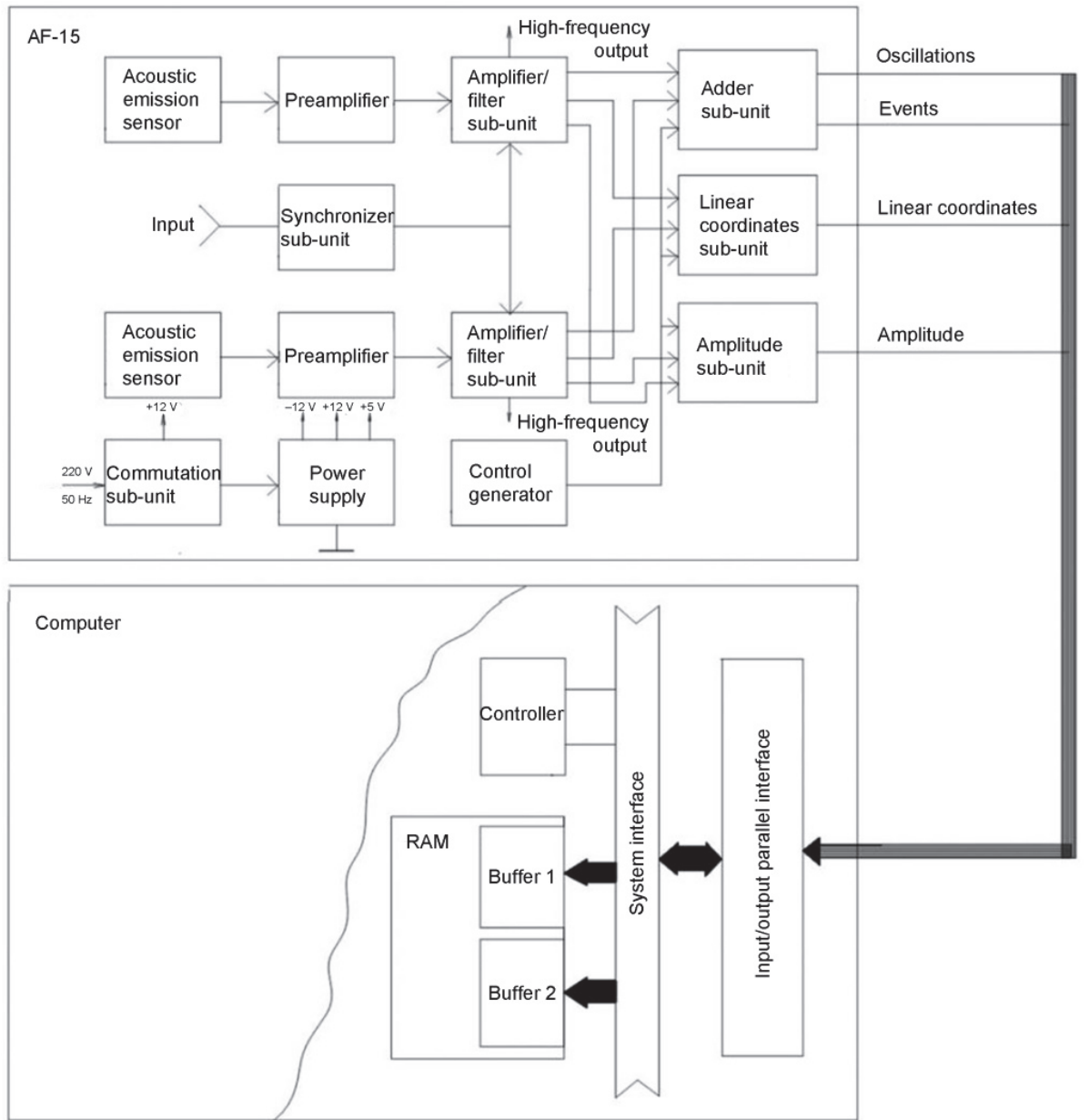

Fig. 4. Acoustic emission measuring station structure

Through-hole technology consists in mounting electronic components by leads inserted through holes on one side of the board and soldered onto copper traces on the other side. Boards may be single-sided, with an unplated component side, or more compact double-sided boards, with components soldered on both sides. Horizontal installation of through-hole parts with two axial leads (such as resistors, capacitors, and diodes) is done by bending the leads 90 degrees in the same direction, inserting the part in the board (often bending leads located on the back of the board in opposite directions to improve the part's mechanical strength), soldering the leads, and trimming off the ends. Leads may be soldered either manually or by a wave soldering machine.

In surface-mount technology components are mechanically redesigned to have small metal tabs or end caps that could be soldered directly onto the PCB surface, instead of wire leads to pass through holes. Components are much smaller and component placement on both sides of the board is more common than with through-hole mounting, allowing much smaller PCB assemblies with much higher circuit densities.

Solder joints remain the general technology for components installation, although their quality is not always achievable due to numerous defects.

One of the main parameters used to assess quality of solder joints is the mechanical 
characteristics - strength, in particular tensile and shear ultimate strength. The strength of solder joints depends on large amount of design and technology factors and thus is determined by ability of the weakest link in the structure to resist destruction. The majority of solder joint defects are represented in Table 2 .

Table 2. Types of solder joint defects

\begin{tabular}{|c|l|}
\hline Defect & \\
\hline Absence of solder & Solder is absent in a joint \\
\hline Underheated solder & Underheating solder while joining \\
\hline Cold joint & Leads mechanical displacement while solidifying \\
\hline Grainy joint & Solder contamination causing grains \\
\hline Excessive solder & Excessive amount of solder what complicates applying SJ tests \\
\hline Solder starved & Solder amount is insufficient for reliable joint \\
\hline Low wetting pad & Solder fail to wet to the contact pad \\
\hline Low wetting pin & Solder fail to wet to the lead \\
\hline Solder icicle & Sharp peaks on the solder surface \\
\hline Piercing & Small holes on the solder concave surface \\
\hline Hollows & Large holes in solder to expose on the surface \\
\hline Recess & Holes or other solder damages penetrating down to joint bottom \\
\hline Oil inclusions & Oil drops in the joint \\
\hline Solder bridge & Unintended connection between pins \\
\hline Solder beads & Solder beads of various diameter on PCB surface \\
\hline Solder spatters & Solder spatters on PCB surface \\
\hline Internal hollows & Hollows inside a solder not exposed to surface \\
\hline $\begin{array}{c}\text { Contamination and } \\
\text { foreign particles }\end{array}$ & Solder surface is contaminated what limits its control \\
\hline Flux remainders & Flux remainders on the solder surface what worsens connection \\
\hline Low-quality pins & Leads are too short or long, improperly bent (deformed), not coplanar \\
\hline Low filling & The contact hole is completely or partially unfilled with the solder \\
\hline Low adhesion & The solder is completely or partially not adhered to the basic material \\
\hline Porousness & Residual, soldering, diffusive porousness \\
\hline Solder erosion & Dissolving basic material by contacts with liquid solder \\
\hline Solder notch & Local erosion \\
\hline Solder tear & The foil is detached from PCB substrate as overheated; contact is torn a part \\
\hline Solder jumpers & Short connection between elements as soldered \\
\hline Solder contamination & Accumulation of foreign inclusions in soldering tab \\
\hline Low solder area & $\begin{array}{l}\text { Area to be soldered is insufficiently covered with solder (depends on surface } \\
\text { preparation) }\end{array}$ \\
\hline $\begin{array}{c}\text { Solder joint } \\
\text { exfoliation }\end{array}$ & Solder and contact pad are detached from PCB substrate as overheated \\
\hline & \\
\hline
\end{tabular}

\section{Through-hole technology}

Solder joints of MLT2 resistors produced by THT technology were the objectives for the research. The research was conducted in progress of strain analysis presented in [4] and aimed at assessing SJs strength by using tensile tests and acoustic emission method.

In order to assess acoustic emission signals against types of defects and find their possible correlation tests were performed for solder joints, part of which was soldered with implemented defects.

Static mechanical tensile tests were conducted for SJs installed on the PCBs into $2 \mathrm{~mm}$ contact pads. For experiments the tensile machine IP-5057-50 with $500 \mathrm{~N}$ ultimate load was used. The load measurement tolerance was under $1 \%$ of maximal load. To perform the test one lead of a resistor was soldered to metallized hole in PCB with tin-lead alloy and fixed in the upper clamps of tensile machine. PCB was firmly fixed in custom lower clamps of the machine. The load scheme 
is shown in Fig. 5.

60 solder joints were tested: 20 SJs had no defects; 20 SJs had "cold solder" defect; 20 SJs had "low solder adhesion" defect. The load was applied with the constant speed at $0.1 \mathrm{~mm}$ per minute. During the test the following AE parameters were recorded: amplitude; activity; total count.

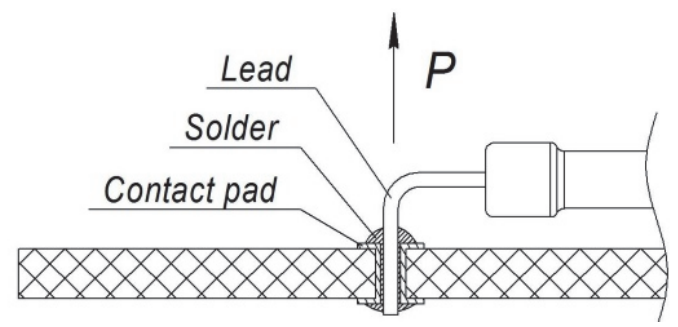

Fig. 5. Load application in solder joint tensile test

First experiments indicated the lowest tensile strength for SJs having "low solder adhesion" defect. The ultimate load for the joints with such defects was 30-40 N. Fatal damages of these joints occurred when the leads were pulled out of the solder with no acoustic emission detected. Since the case when leads are not wetted completely is very unlikely, further studying SJs with partly wetted leads were considered to be more reasonable.

The averaged results of the experiments are given in Table 3. Statistical analysis of experimental data testified of significant decreasing ultimate load for defective SJs with $95 \%$ confidence probability.

Table 3. Tensile test results for soldered joints of 60 resistors MLT 2

\begin{tabular}{|c|c|c|c|}
\hline Group & Solder joint condition & Average ultimate load, N & Average strength decrease, $\%$ \\
\hline 1 & No defect joint & 116.2 & - \\
\hline 2 & Cold joint & 44.5 & 62 \\
\hline 3 & Low adhesion joint & 75.9 & 35 \\
\hline
\end{tabular}

During the tests AE parameters were recorded along with the load progression. AE data analysis resulted in total count $\mathrm{N}$ (impulses, imp) and maximal activity Amax (impulses per second, imp/s) measured for different types of SJs during their tensile tests. Acoustic emission parameters were recorded in the load range from 0 to $44 \mathrm{~N}$ what corresponds to average ultimate load for the cold joints and makes $40 \%$ of ultimate load for no defect joints (Table 4, Fig. 6).

Table 4. Acoustic emission parameters under $40 \%$ of solder joint ultimate tensile load

\begin{tabular}{|c|c|c|c|}
\hline Group & Solder joint condition & Total count, imp & Maximal activity, imp/s \\
\hline 1 & No defect joint & 34 & 51 \\
\hline 2 & Cold joint & 70 & 210 \\
\hline 3 & Low adhesion joint & 22 & 40 \\
\hline
\end{tabular}

As foreseen, the AE character differed for different defects. Fig. 6 demonstrates acoustic emission diagrams produced by the load progression applied during tensile tests for three groups of SJs with mentioned types of defects.

Analysis of acoustic emission detected along the tests shows sensitivity and applicability of this method to detect defects in SJs and even assess their strength. Besides, it holds potential for nondestructive diagnostics of solder joints long before the final destruction.

\section{Surface mount technology}

The metallic ceramic capacitors K10-17B and K10-50B, which are surface mount devices (SMD), were objectives for the next research. Nowadays SMD technology gains popularity due 
to range of advantages before THT. Exploring, assembling and testing these capacitors are likely to cause damages in form of contact pads defoliation and detachments or even capacitor destruction [5]. Preliminary shear strength tests revealed that ultimate shear loads 2-4 times exceed tensile ones. Therefore, capacitors K10-17B and K10-50B were subjected to the tensile tests aimed at defining: ultimate loads that cause mentioned damages; tensile strain of capacitors; difference in ultimate loads for overheated solder and cold solder joints of K10-50B capacitors; correlation between AE signals and solder joint strength for varied technological conditions.

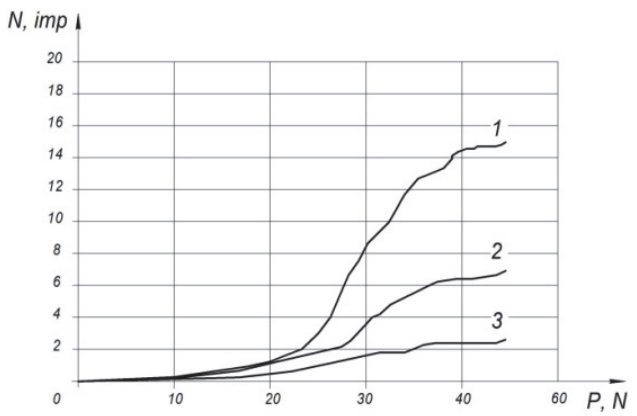

a)

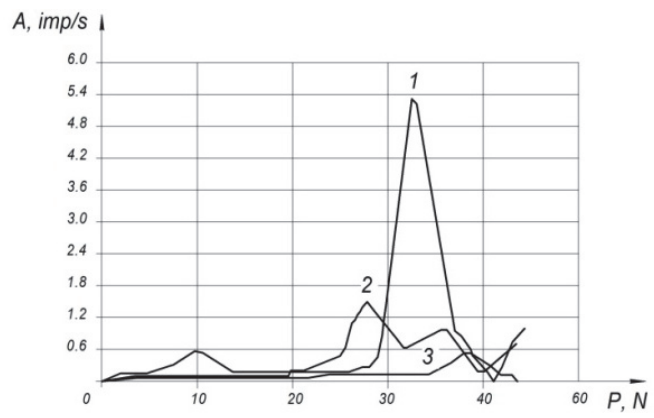

b)

Fig. 6. Acoustic emission - load diagrams during tensile tests of three types of solder joints: 1 - cold joints, 2 - low solder adhesion joints, 3 - solder joints without defects

In order to minimize errors in measuring small loads for tensile tests the special appliance was designed (Fig. 7).

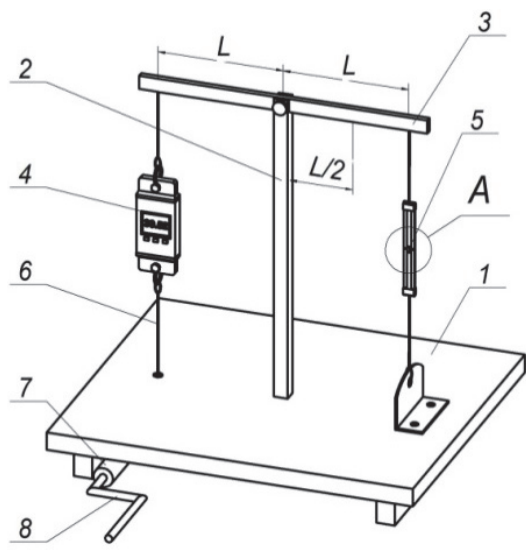

a)

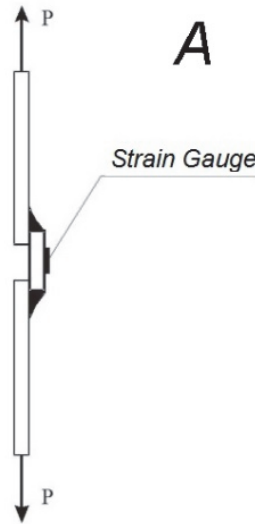

b)

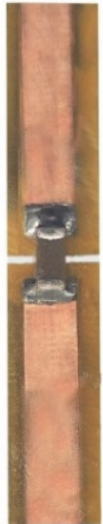

c)

Fig. 7. Manual tensile test appliance: a) structure, b) strain gauge attachment, c) capacitor installation: 1 - base; 2 - support, 3 - lever, 4 - dynamometer, 5 - capacitor, 6 - traction, 7 -drum; 8 - handle

The measuring train used in the test consisted of electro-tensometry and acoustic emission instruments. Two batches of 100 capacitors K10-17B and K10-50B were tested. Capacitors 5 (Fig. 7) were soldered by their termination caps to the ends of two strips, cut out of the PCB substrate, which were then clamped in between the lever 3 and the base 1. Turning handle 8 revolved the drum 7 and through the traction 6 pulled the left side of the lever 3 so as to apply load to capacitors; load was indicated by dynamometer 4 . To increase measurement accuracy for smaller loads the testing appliance is designed so that lever shoulders can be varied if needed.

Tests were conducted with simultaneous records of acoustic emission and loads. In all the cases destruction occurred as defoliation of a contact pad. Strain gauges were attached to capacitors (Fig. 7) with purpose to define strain versus load dependences (Fig. 8). The average 
ultimate loads measured for batches of capacitors K10-17B and K10-50B made $P_{1}=23.7 \mathrm{~N}$ and $P_{2}=31.8 \mathrm{~N}$ correspondently.

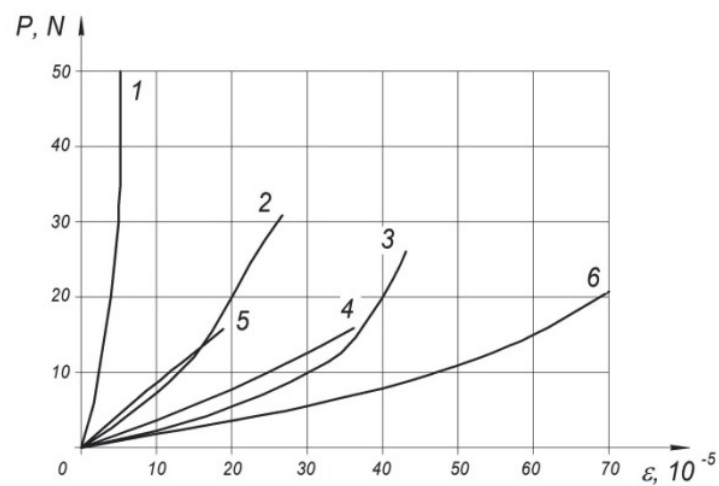

Fig. 8. Strain versus tensile load dependences for capacitors K10-17B (1) and K10-50B (2, 3-6)

The next experiment was conducted to verify the overheating effect to ultimate load of solder joints. Two groups of capacitors K10-50B that differed in soldering technologies were subjected to tensile tests. In the first group soldering was conducted at $220-270{ }^{\circ} \mathrm{C}$ temperature during $10 \mathrm{~s}$ with standard alloy POS-61, in the second group - at $94-120^{\circ} \mathrm{C}$ temperature with rosin alloy.

Data analysis (table 1) testified that overheating of solder joints improves their adherence to the contact pads and increases their tensile strength.

Significant difference between cold solder and overheated joints was indicated by acoustic emission recorded (Fig. 9) during experiment. For the cold solder AE signals appeared earlier; their total count was 1.5 times and activity -2.5 times higher than for overheated ones. Average strains recorded for cold solder and overheated joints were $\varepsilon_{1}=70 \times 10^{-5}$ and $\varepsilon_{2}=45 \times 10^{-5}$ correspondently.

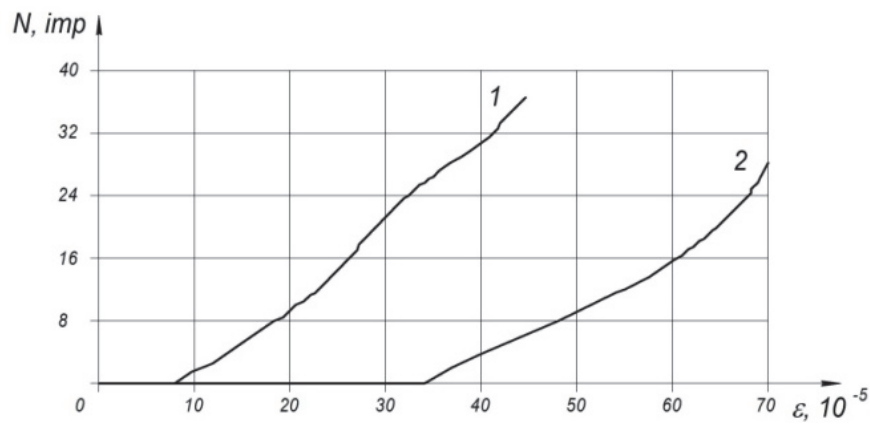

Fig. 9. Acoustic emission total count versus strain diagrams: 1 - cold solder joints with ROSE alloy, 2 - overheated joints with POS-61 alloy

The next experiment was made with conjecture that contact joint strength depends on soldering temperature. The experiment was arranged with account for limitations posed by complexity to measure solder temperature either theoretically or practically. The decision was made to remain soldering technology unchanged with an exception for contact joint temperature, which was considered to increase by preliminary heating the capacitors.

The experiment was conducted for two groups of capacitors. 1st group was soldered at the standard temperature. 2nd group was preheated before soldering in thermostat within 1 hour at the discrete temperatures: $150{ }^{\circ} \mathrm{C}, 180{ }^{\circ} \mathrm{C}, 200{ }^{\circ} \mathrm{C}, 215{ }^{\circ} \mathrm{C}, 230{ }^{\circ} \mathrm{C}, 250{ }^{\circ} \mathrm{C}$. After preheating capacitors were soldered and subjected to tensile tests by the method explained above.

Data analysis (Table 5) indicated that preheating of solder joint at $200^{\circ} \mathrm{C}$ increased its strength 
by $50 \%$. Moreover, optimal preheating temperature, producing maximal strengthening effect was defined in interval of $180-200{ }^{\circ} \mathrm{C}$.

Table 5. Tensile ultimate loads of K10-50B capacitor solder joints for different temperature modes

\begin{tabular}{|c|c|c|c|c|c|}
\hline \multicolumn{6}{|c|}{ Soldering technology modes } \\
\hline \multicolumn{2}{|c|}{ Duration: $10 \mathrm{~s}$} & \multicolumn{4}{|c|}{ Duration: $3 \mathrm{~s}$} \\
\hline \multirow{2}{*}{$t=(220-270)^{\circ} \mathrm{C}$} & \multirow{2}{*}{$\begin{array}{c}\text { Rosin alloy } \\
t=(94-120)^{\circ} \mathrm{C}\end{array}$} & \multirow[t]{2}{*}{ Standard } & \multicolumn{3}{|c|}{$\begin{array}{c}\text { Preheated in thermostat within } 1 \text { hour at the } \\
\text { temperatures, }{ }^{\circ} \mathrm{C}\end{array}$} \\
\hline & & & 150 & 200 & 250 \\
\hline \multicolumn{6}{|c|}{ Average tensile ultimate loads, $N$} \\
\hline 59.7 & 36.2 & 30.6 & 40.3 & 47.6 & 22.9 \\
\hline
\end{tabular}

\section{Pure bending theoretical model}

Conducting tensile tests with simultaneous recording acoustic emission showed perspectives for nondestructive strength diagnostics of components solder joints installed on PCB, although practical application of the method was limited by monitoring only the single component joints at the time. In order to overcome this limitation and conduct tests for entire PCB with all components installed the pure bending technique was applied (Fig. 10). The pure bending was designed to provide equal testing stress over the PCB area as applied in between two supports. Thus, all SJs installed within the tested area can be monitored simultaneously.

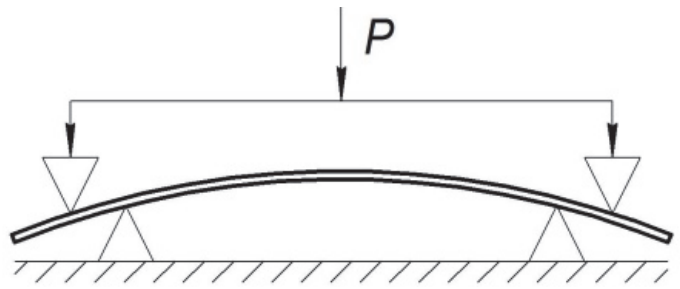

Fig. 10. Pure bending load scheme

PCBs populated with components installed by SMD technology were now the research objectives. SJ strength assessment considers solder mechanical characteristics, which are specified with consideration of design and technology of the joint. The strength of low-melting solders in cast phase is less than that of SJ. The lowest level of ultimate strength for solder POS40 in the cast phase is $\sigma_{u}=40 \mathrm{MPa}[6]$.

The ultimate strength for fiberglass substrate is $\sigma_{u}=45-100 \mathrm{MPa}$.

Assuming ultimate strength of the solder as the reference value the test stress is calculated by the formula:

$[\sigma]=\frac{\sigma_{u}}{n}=\frac{40}{2.5}=16 \mathrm{MPa}$.

where $\sigma_{u}$ - ultimate strength, $n=2.5$ - safety factor.

The possible inaccuracy entailed by no account for design and technology of the joint will deposit into the safety factor. Obtained safety factor is sufficient to provide safe testing stress $\sigma$ test within proportionality strain area of solder material, so that solder is subjected to only elastic deformations, which do not reduce its strength.

Then the load $P$ applied to PCB is specified for the nondestructive tests. Load application scheme for PCB test is shown in Fig. 11.

Maximal stress [6] is produced in dangerous part of PCB cross section - in peripheral layer, where bending moments are maximal $M=M_{\max }$. In pure bending area with length $L$ which is created by and lays in between two supports A and B, maximal stress is found by formula: 
$\sigma_{\max }=\frac{M_{\max }}{I_{a x}} y_{\max }$

where $M_{\max }$ - maximal bending moment produced by load $P ; I_{a x}$ - axial moment of inertia; $y_{\max }-$ maximal distance from the neutral line of the cross-section to the peripheral layer (fibers); $y_{\max }=h / 2$, where $h-$ thickness of PCB substrate.
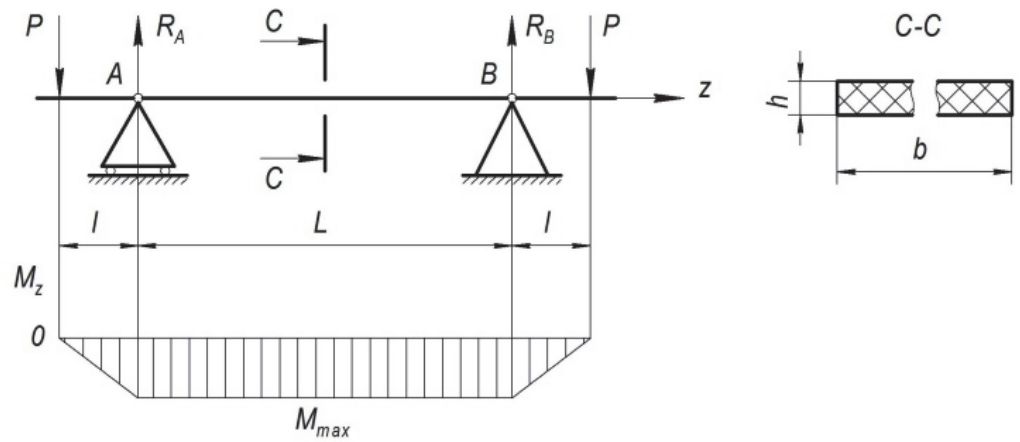

Fig. 11. Load application and diagram of internal bending moments in PCB

Since ratio $I_{a x} / y_{\max } \frac{\mathrm{I}_{\mathrm{oc}}}{\mathrm{y}_{\max }}$ represents axial moment of resistance $W_{a x}$, Eq. (2) will be shortened to:

$\sigma_{\max }=\frac{M_{\max }}{W_{a x}}$

As required by strength condition of normal stress under bending load - maximal stress produced by the applied load should comply with acceptable standard $[\sigma]$ :

$\sigma_{\max }[\sigma]$.

Performing nondestructive tests to PCBs requires maximal acceptable load estimation.

Using Eqs. (3) and (4) for which in accordance to load application scheme (Fig. 11) maximal bending moment equals:

$M_{\max }=P \cdot l$,

and axial moment of resistance of rectangular section [6] is expressed as:

$W_{a x}=\frac{b \cdot h^{2}}{6}$

Maximal acceptable load for nondestructive bending tests of PCBs should comply with condition:

$[P] \leq \frac{b \cdot h^{2}}{6 \cdot l}[\sigma]$

\section{Pure bending static tests}

Pure bending tests conducted by tensile machine IP-5057-50 indicated that the activity of acoustic emission responds to the loading speed. Since AE data analysis requires its essential 
amount the decision was made to conduct tests at maximal acceptable loading speed 100 millimeters per minute. The load was applied under acceptable limit calculated by Eq. (3) $P_{\text {test }}=36 \mathrm{~N}$.

However single load did not produce considerable activity of AE signals yet and testing was considered to conduct in multiple load and unload cycles. Each PCB was tested in 5 cycles. Minimal and maximal stresses in cycles equaled $\sigma_{\min }=0 \mathrm{MPa}$ and $\sigma_{\max }=16 \mathrm{MPa}$ correspondently. Acoustic emission was recorded during all the tests by piezoelectric gauges and via pre-amplifiers at frequency band of $0.02-0.2 \mathrm{MHz}$. The gauges were attached to PCB surface through the layer of acoustic paste.

The tests were conducted for 60 double sided fiberglass foil laminated PCBs $(320 \times 120 \times 1.5 \times 0.1 \mathrm{~mm}$ size $)$. For the experiment PCBs were prepared and sorted into three equal groups by defects embedded into their solder joints: 1) PCBs with no defects; 2) PCBs with cold joint defects; 3) PCBs with low solder adhesion defects. Each PCB was populated with one component - resistor MLT-2. In group 1 resistors were installed in compliance with technological standard [7]. In group 2 one of the resistors' leads was mounted to PCB as a cold joint, in particular as "circle crack" joint which is likely for soldering technology. In the group 3 low solder adhesion defects were embedded into solder joints for what resistors' leads had not been wetted before soldering.

During the tests the following AE parameters [8] were recorded: amplitude; activity; total count. The test results indicated that PCBs with no defect solder joints do not radiate acoustic emission during all cycles. The total count of SJs with low solder adhesion defects made 10-15 imp with amplitude $1 \mathrm{mV}$. Normally such signals are radiated at load progression and in the moments of maximal load. Signals radiated by cold joint defects are characterized by considerably higher total count (30-40 imp) with amplitude up to $3 \mathrm{mV}$. The specificity of cold joints is that $\mathrm{AE}$ appears on load discharging phase of cycles, what can be explained by cracks converging their edges. Figs. 12, 13 demonstrate acoustic emission - cycling load diagrams recorded during pure bending tests of PCBs with embedded solder joint defects.

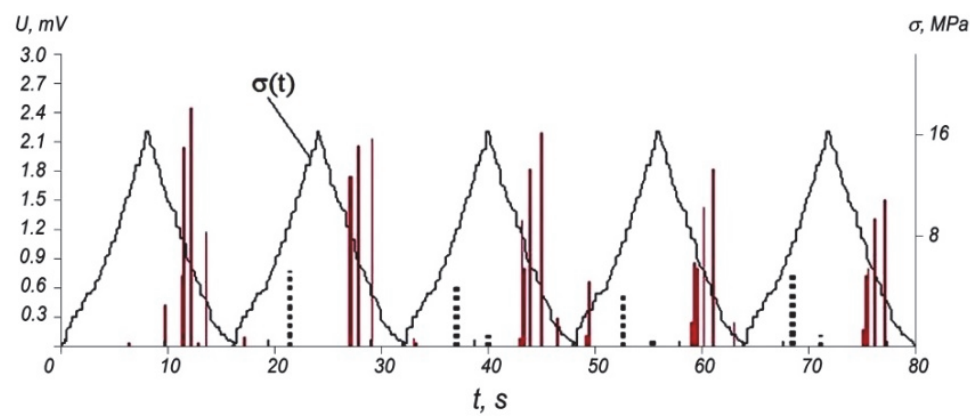

Fig. 12. Acoustic emission amplitude - cycling load diagram: solid line - low solder adhesion, dash line - cold joint

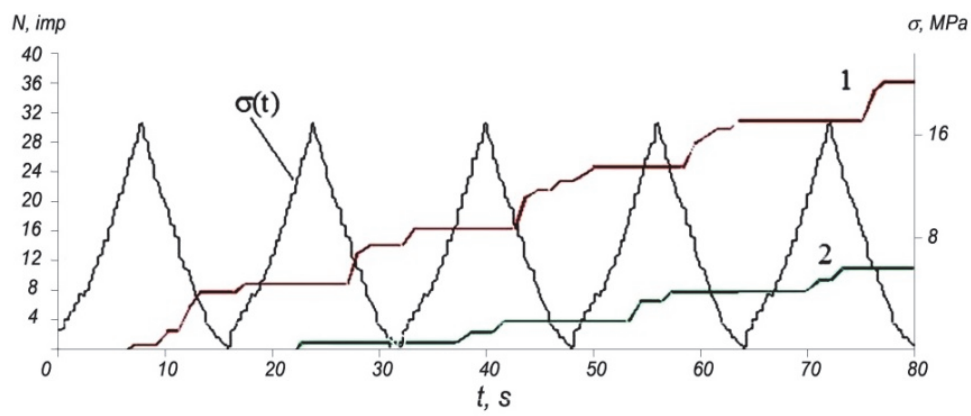

Fig. 13. Acoustic emission total count - cycling load diagram: 1 - cold joint, 2 - low solder adhesion 
AE data analysis indicated total count as the most informative parameter to correlate with various types of solder joint defects. Conducted experiments give the reason to use identified character of acoustic emission to develop methods for strength diagnostics of solder joints on printed circuit boards.

\section{Theoretical model of planar defect location}

The location accuracy of acoustic emission is strongly effected by acoustic characteristics of the transmitting medium and becomes complicated problem for a medium which is heterogeneous. So, AE spreading and location in PCBs is strongly complicated due to structural complexity of PCBs, that consist of THT or SMD components, conductive tracks, pads and other features etched from copper sheets laminated onto a non-conductive substrate, whose acoustic characteristics (Table 6) are different. The task of the current research was to develop method for planar location of AE whose sources are defects progressing in stressed PCBs when exposed to external forces.

Table 6. Acoustic characteristics of PCB structural materials

\begin{tabular}{|c|c|c|c|c|c|}
\hline \multirow{2}{*}{ Material } & \multirow{2}{*}{ Density $\rho \cdot 10^{3}, \mathrm{~kg} / \mathrm{m}^{3}$} & \multicolumn{3}{|c|}{ Wave speed $v \cdot 10^{3}, \mathrm{~m} / \mathrm{s}$} & \multirow{2}{*}{ Attenuation constant $\delta, \mathrm{m}^{-1}$} \\
\cline { 3 - 5 } & & Longitudinal & Cross & Surface & \\
\hline Textolite & $1.2-1.3$ & 2.63 & - & - & $>100$ \\
\hline Lead & 11.4 & 2.16 & 0.70 & 0.63 & $1-10$ \\
\hline Copper & 8.9 & 4.66 & 2.26 & 2.12 & $10-100$ \\
\hline
\end{tabular}

The idea of the method developed for planar location consisted in remote detection of AE by piezoelectric transducers through the volume of homogeneous medium $[10,11]$ such as water, unlike detecting AE on the PCB surface where AE signal is likely to be distorted or even lost. Water retains the most acceptable attenuation constant $\delta=2.5$ as compared to other homogeneous mediums.

The testing installation represents load appliance for PCBs and 4 piezoelectric sensors installed inside the water chamber. PESs were installed on the plane parallel to PCB at the specified distance, so that their receiving side faced the tested PCB. Acoustic waves generated by defects in PCB spread through the volume of water and reach the sensors with negligible loss or distortion.

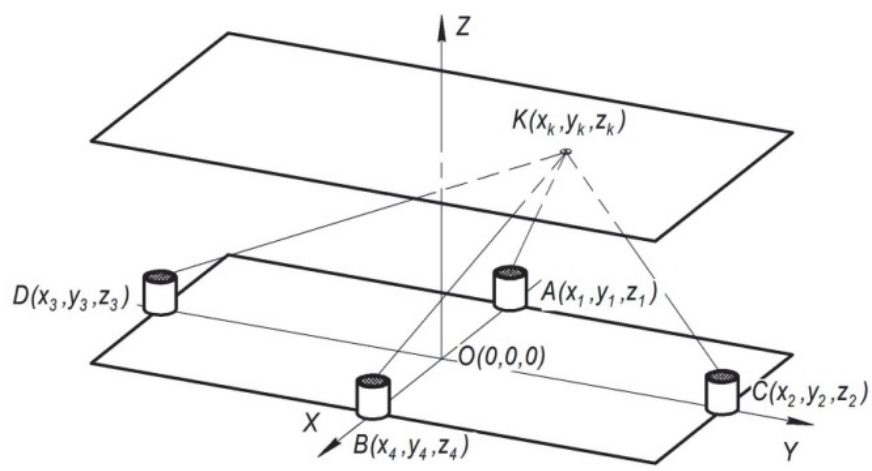

Fig. 14. Planar location scheme in acoustically transparent medium: $K$ - source of AE; A, B, C, D - piezoelectric sensors

The planar location in Cartesian coordinates is calculated in the space coordinate system $X Y Z$. 4 PESs are installed on the axes $X$ and $Y$ in $z=0$ plane (Fig. 14).

The monitored PCB lays in the plane $z=z K$, where $z K$ is distance between the PCB and PESs. The 4 channel acoustic emission system detects two time differences $\tau_{1}$ and $\tau_{2}$ of signal arrivals to two pairs of opposite sensors in two orthogonal axes. Time differences $\tau_{1}$ and $\tau_{2}$ is related to distance differences from AE source $K$ to PESs in correspondent pair: 
$\left\{B K-A K=\tau_{1} \vartheta\right.$

$\left\{C K-D K=\tau_{2} \vartheta\right.$.

where $\tau_{1}$ - time difference of signal arrivals to PESs in points $\mathrm{A}$ and $\mathrm{B} ; \tau_{2}$ - time difference of signal arrivals to PESs in points $\mathrm{C}$ and $\mathrm{D} ; v-$ ultrasonic speed in water.

The final expressions for $\mathrm{AE}$ coordinate calculation are represented as:

$$
\left\{\begin{array}{l}
x_{k}= \pm \frac{1}{2} \sqrt{\frac{\tau_{1}^{2} \vartheta^{2} \tau_{2}^{2} \vartheta^{2}\left(\tau_{2}^{2} \vartheta^{2}-\tau_{1}^{2} \vartheta^{2}+4 x_{4}^{2}-4 y_{2}^{2}\right)+4 \tau_{1}^{2} \vartheta^{2} y_{2}^{2}\left(\tau_{1}^{2} \vartheta^{2}-4 x_{4}^{2}-4 z_{k}^{2}\right)}{4 \tau_{1}^{2} \vartheta^{2} y_{2}^{2}+4 \tau_{1}^{2} \vartheta^{2} x_{4}^{2}-16 x_{4}^{2} y_{2}^{2}}}, \\
y_{k}= \pm \frac{1}{2} \sqrt{\frac{\tau_{1}^{2} \vartheta^{2} \tau_{2}^{2} \vartheta^{2}\left(\tau_{1}^{2} \vartheta^{2}-\tau_{2}^{2} \vartheta^{2}+4 x_{4}^{2}-4 y_{2}^{2}\right)+4 \tau_{2}^{2} \vartheta^{2} x_{4}^{2}\left(\tau_{2}^{2} \vartheta^{2}-4 y_{2}^{2}-4 z_{k}^{2}\right)}{4 \tau_{1}^{2} \vartheta^{2} y_{2}^{2}+4 \tau_{2}^{2} \vartheta^{2} x_{4}^{2}-16 x_{4}^{2} y_{2}^{2}}} .
\end{array}\right.
$$

In Eq. (9) positive or negative signs are selected regarding sings of $\tau_{1}$ and $\tau_{2}$.

The experimental verification of the offered method was conducted in testing installation with PESs installed at $70 \mathrm{~mm}$ distance from the tested PCB. The AE planar location diagrams obtained by regular surface location performed on the PCB surface and by the developed method are shown in Fig. 15(a) and Fig. 15(b) correspondently.
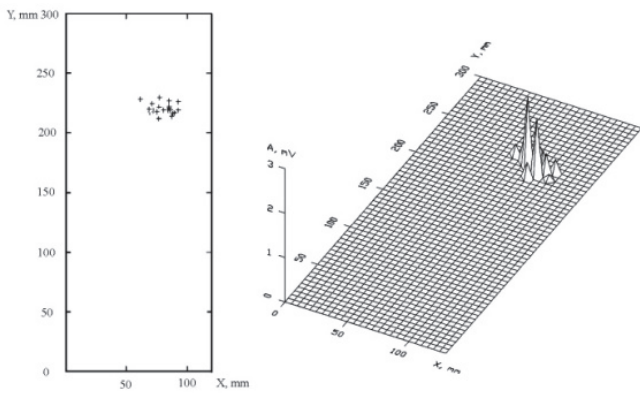

a)

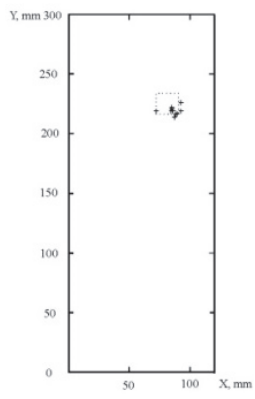

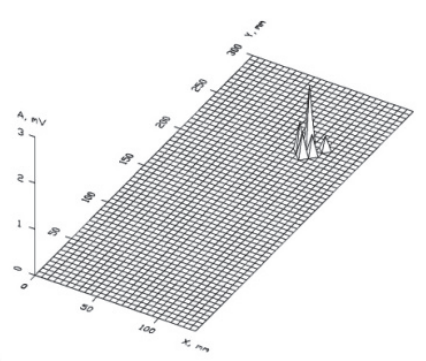

b)

Fig. 15. Acoustic emission planar diagrams recorder during PCB bending tests:

a) location on PCB surface, b) location in acoustically transparent medium

The application of developed method in acoustically transparent medium indicated that located area of the defect makes about $1 \%$ of the total PCB area, what provides accuracy 2-4 time higher than by the regular surface location.

\section{Method for technical diagnostics}

1. Printed circuit boards are tested by pure bending load under acceptable limit specified by the safety factor for solder material $n=2.5$.

2. In case acoustic emission is detected, test is repeated in 5 load/unload cycles. Acoustic emission progression during cycle test indicates of solder joint defect and such PCB is rejected and classified as joint with growing defect.

3. When required defect location is performed in order to repair it.

The developed method has been tested out on the batch of industrial printed circuit boards. The tests were conducted for 32 single sided fiberglass foil laminated PCBs $(120 \times 140 \mathrm{~mm}$ size $)$. During tests acoustic emission was detected for three PCBs what indicated progressing defects. Analysis of AE character identified type of the detected defects - low solder adhesion, which was then proved by detailed optical 10x zoom revision of rejected PCBs in places where AE was located. Defects were also tested by passing electric current through the defected solder joints and 
measuring their electric resistance. Since PCBs had been used for a long time before the tests and identified defects had remained hidden so they can have developed during their further operation and would have caused a failure of the whole electronic unit.

\section{Conclusions}

Strength diagnostics conducted for solder joints performed by through-hole and surface mount technologies on printed circuit boards by using methods of mechanical tensile and pure bending tests and method of acoustic emission with simultaneous monitoring both mechanical characteristics and acoustic emission parameters allowed to find the relationship between parameters of acoustic emission and such defects of solder joints as "cold joint" and "low solder adhesion".

Surface mount technology has been optimized by introducing solder joints preheating that results in $50 \%$ strengthening effect in the temperature interval of $180-200{ }^{\circ} \mathrm{C}$.

The theoretical model of pure bending condition for printed circuit boards has been developed in order to provide equal testing stress condition over the printed circuit board and calculate maximal acceptable load for nondestructive bending tests.

Theoretical model for planar location has been developed for defect location in printed circuit boards. The application of developed method in acoustically transparent medium indicated that located area of the defect makes about $1 \%$ of the total PCB area, what provides accuracy 2-4 time higher than that by the regular surface location.

Method for nondestructive strength diagnostics of solder joints on printed circuit boards based on pure bending cycling tests with method of acoustic emission has been developed.

\section{References}

[1] State Industrial Standard GOST 53251-2014. Printed Circuit Boards. Classification of Defects, 2014.

[2] State Industrial Standard GOST 18353-79. Nondestructive Tests. Types Classification, 1980.

[3] Royzman V. P., Kovtun I. I., Goroshko A. V. Location of defects by acoustic emission method. Visnyk of Technological University of Podillia, Vol. 1, Issue 2002, 1, p. 195-200.

[4] Kovtun I., Boiko J., Petrashchuk S., Baurienè G., Pilkauskas K. Effects of the strain transmission from the main board to the installed electronic components. Mechanika, Vol. 22, Issue 6, 2016, p. 489-494.

[5] Kühl Reiner W. Mechanical stress and deformation of SMT components during temperature cycling and PCB bending. Soldering and Surface Mount Technology, Vol. 11, Issue 2, 1999, p. 35-41.

[6] Pisarenko G. S., Kvitka O. L., Umanski E. S. Strength of Materials, 2nd Edition. High School, Kyiv, 2004, p. 655.

[7] OST4 GO.054.089. Electronic Units and Blocks. Soldering Contact Joints. Sample Technological Processes, 1982.

[8] Rao A. K. Acoustic emission and signal analysis. Defence Science Journal, Vol. 40, 1990, p. 55-70.

[9] Kuznetsov V. A., Dolgov V. A., Konevskikh V. M. Measurements in Electronics: Handbook. Energoatomisdat, Moscow, 1987, p. 512.

[10] Royzman V. P., Kovtun I. I., Goroshko A. V., Prokhorenko S. V. Device for Coordinates Determination of Acoustic Emission Source, Patent 41138, 2001.

[11] Royzman V. P., Kovtun I. I., Goroshko A. V., Prokhorenko S. V. Method for Defect Location Using Acoustic Emission Method. Patent 43125, 2002.

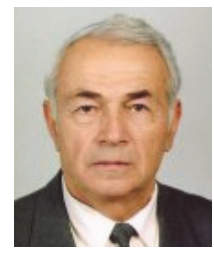

Vilen Royzman received Doctoral degree in Machine Engineering Institute of Science Academy of USSR, Moscow, Russia, in 1979. In 1980 he received title of Professor. Now he works at Khmelnytsky National University as Head of Department of Telecommunication and Radio Engineering. His current research interests include vibrations, rotor balancing, inverse problems and strength in electronics. 


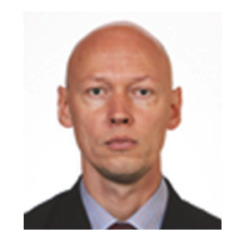

Igor Kovtun received Ph.D. degree in Khmelnytsky National University, Khmelnytsky, Ukraine, in 1998. In 2000 he received title of Associate Professor. Now he works at Khmelnytsky National University as Head of Art and Technical Graphics Department. His current research interests include strength in electronics, nondestructive control, dynamics and fault diagnostics.

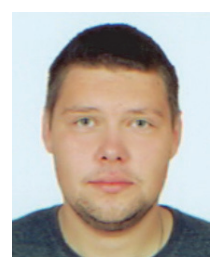

Andriy Voznyak obtained Master's degree in restoration and enhancement of wear resistance of parts and constructions specialty in Khmelnytsky National University, Khmelnytsky, Ukraine, in 2014. Now he works at Khmelnytsky National University as Research Fellow of Science and Research Department. His current research interests include strength in electronics, nondestructive control, dynamics and fault diagnostics. 


\title{
5.2. Modified method of spectral analysis of the reflection signal for damage detection systems in power lines
}

\author{
Kostyantyn Horiaschenko ${ }^{1}$, Victor Stetcuk ${ }^{2}$ \\ Khmelnitsky National University, Khmelnitsky, Ukraine \\ E-mail: ${ }^{1}$ kostyakst@ukr.net, ${ }^{2}$ sv_rt@i.ua
}

\begin{abstract}
The work is devoted to the aspects of using phase methods for measuring distances to faults in a power line. The existing phase methods are shown and discussed. It is established that spectral leakage is one of the problems of spectral analysis. An algorithm for spectral analysis based on a change in the width of the analysis window is proposed. It is shown that the proposed method provides an increase in the accuracy of determining the amplitudes of the total signal components.
\end{abstract}

Keywords: power line, phase angle shift, spectral analysis, spectral leakage.

\section{Introduction}

\subsection{The problem of measuring of parameters of power lines}

Controlling of power lines state is a well-known problem. Usually measuring of parameters of such lines requires two categories of actions [12]:

1) The first category is a global analysis. Global analysis consists in determination of the state of the whole power line. As a result, we have information about state of whole line. In compare with previously measured data, detailed measuring can be required.

2) The second category is a local analysis. Analysis consists in measuring of parameters from point to point. Many methods used to do such measuring. However, each of them has advantages and disadvantages.

The presented categories connected to each other, but they do not interchangeable. The methods in the first category put on an aim verification of readiness to work or accordance of the already on-the-road system to the further use. Therefore, we have information about state of all power line. The methods in the second category used not only with the aim of measuring of parameters of power lines. A principal difference is ability for determination of current state of each part of power line. As a result, we can control local damages at any distance in line.

Measuring of parameters of the power line or system of power lines is a difficult process which require the use of one or a few simultaneously technologies.

A lot modern methodologies used for implementation of measuring [3, 6, 12]:

1. Time Domain Reflectometry, TDR.

2. Partial Discharge, $\mathrm{PD}$ (working frequencies varies from $50 / 60 \mathrm{~Hz}$ down-to $0.02 \mathrm{~Hz}$ (VLF)).

3. $\operatorname{tg} \delta(\tan \delta)$, analysis of the dielectric penetrating on different frequencies.

4. Measuring of recovery Voltage.

5. DC Leakage Current.

6. Application of combinations of methods.

Many from these measuring methods already used in measuring devices, others are not yet accepted [10]. The use of various methods of measuring in power lines in the USA [12] are shown on Fig. 1.

For practical use only VLF, $\operatorname{tg} \delta$ and TDR accepted. Other methods are on the stage of research or do not have commercial use. We have two fundamental groups:

a) The destructive measuring methods based on use of over-voltages. Such methods not only detect defects, they speed-up destruction of power lines.

b) The non-destructive measuring methods based on use of low and nominal voltages. Those 
methods can be used without interrupting normal work of power lines.
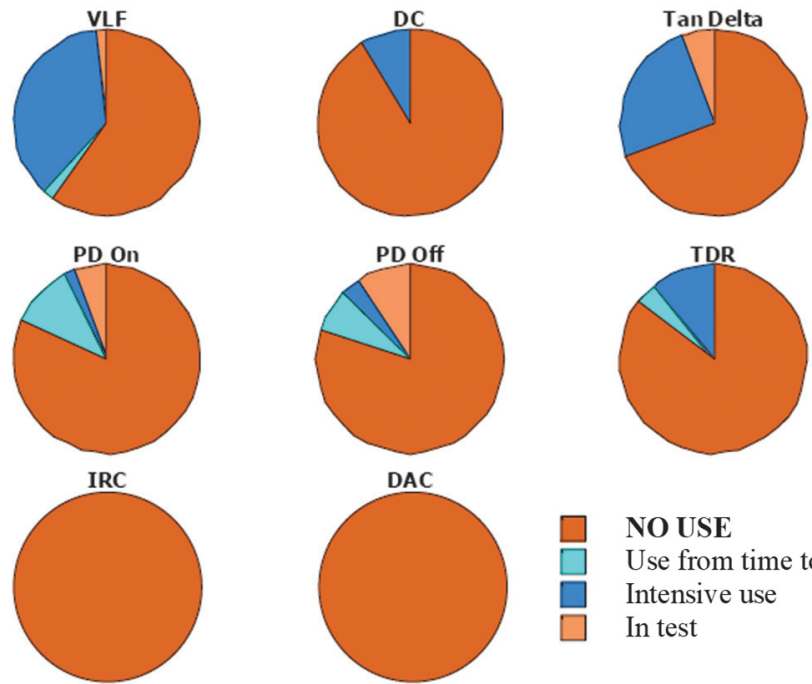

NO USE

Use from time to time

Intensive use

In test

Fig. 1. The methods of measuring: VLF - very low frequency, DC - direct current,

Tan Delta $(\operatorname{tg} \delta)$ - measuring of dielectric losses, PD On and PD - partial discharge methods,

TDR - time domain reflectometry, IRC - Isothermal Relaxation Current, DAC - Damped AC

\subsection{Known phase measuring methods for distance measuring}

\subsubsection{Classic phase measuring method}

Phase measuring methods based on measuring of phase shift [19-21] between applied test signal $\omega_{0}=U_{m 1} \cos \varphi_{1}$ and returned signal from reflection:

$\omega_{01}=U_{m 1} \cos \left(\Omega_{m} t+\varphi_{01}\right)$,

where $U_{m 1}$ - an amplitude of test signal; $\Omega_{m}$ - a frequency of scale frequency, in simplest case $\Omega_{m}=\omega_{0} ; \varphi_{01}-$ a phase angle shift on frequency $\omega_{0}$ for the same object in line.

The returned signal looks like:

$s_{2}(t)=U_{m 2} \cos \varphi_{2}=U_{m 2} \cos \left(\Omega_{m}\left(t-t_{R}\right)+\varphi_{01}+\varphi_{a p}+\varphi_{\text {ret }}\right)$

where $U_{m 2}-$ amplitude of returned signal; $\varphi_{01}-$ an initial angle; $\varphi_{a p}-$ a angle shift in the apparatus; $\varphi_{\text {ret }}-$ a phase angle shift at the reflection.

Distance to reflection in air determined as [19, 20, 22]:

$R=\frac{c\left(\varphi_{\Delta}+\varphi_{\mathrm{ap}}+\varphi_{r e t}\right)}{2 \Omega_{m}}, \quad R=\frac{c\left(\varphi_{\Delta}+\varphi_{a p}+\varphi_{r e t}\right)}{2 \omega_{0}}$,

where $c$ - speed of signal in air.

For a physical environment with speed of distribution $v$ distance will be determined as:

$R=\frac{v\left(\varphi_{\Delta}+\varphi_{a p}+\varphi_{r e t}\right)}{2 \omega_{0}}$

A value of phase angle shift $\varphi_{a p}$ can be eliminated by calibration. The phase angle shift at the 
reflection of signal $\varphi_{\text {ret }}$ strongly influence with result - distance. That is why for measuring distance by phase angle shift method value of $\varphi_{\text {ret }}$ is only $\pm 180^{\circ}$ (Fig. 2).

Use of classical phase measuring method based on the following principles in case of two or more reflections in any electrical line [18]:

- All signals pass through environments without fading;

- Speeds of wave distribution in all environments are identical;

- A signal is reflected in accordance with to the reflection coefficient of this object;

- Size of damage as small as possible, a zero-size of damage used as a rule;

- Signals passes power line pass without distortions.

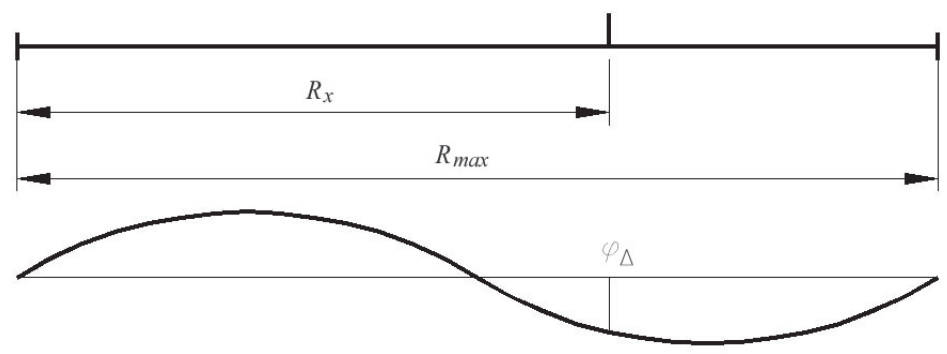

Fig. 2. Phase angle shift method

Simple case of two objects is shown on Fig. 3. Objects are located at distances $l_{1}$ and $l_{2}$ from the start of line. Accord to shown principles, a signal from the first object will be reflected with a phase angle shift $\varphi_{1}$. A phase angle shift can be expected after a formula:

$\varphi_{1}=2 \cdot 2 \pi \frac{l_{1}}{L_{\max }}$.

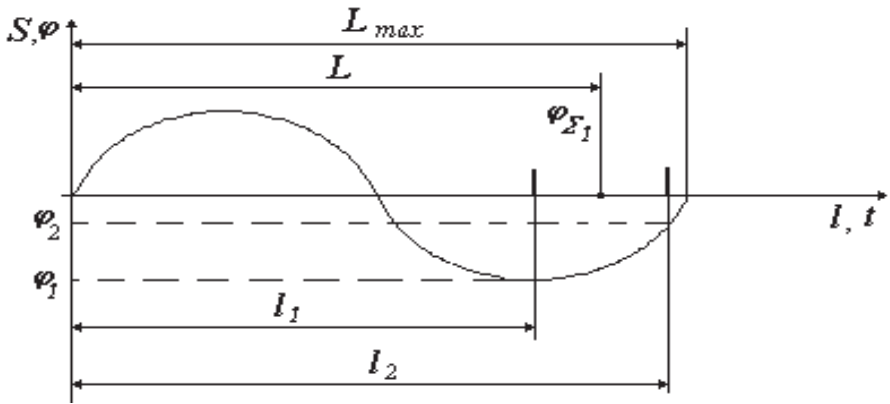

Fig. 3. Phase angle sift in case of existence of two objects

For the second object a phase angle shift $\varphi_{2}$ will be:

$\varphi_{2}=2 \cdot 2 \pi \frac{l_{2}}{L_{\max }}$

Amplitudes of signals from every object depend on reflection coefficients $\Gamma_{1}, \Gamma_{2}$ and amplitudes of input signal $a$. Thus, we have:

$a_{1}=\Gamma_{1} a, a_{2}=\Gamma_{2} a$.

All signals are harmonious with one frequency and different phase changes. Thus:

$s_{\text {refl } \Sigma}(t)=a_{1} \cos \left(\omega t+\varphi_{1}\right)+a_{2} \cos \left(\omega t+\varphi_{2}\right)$. 
In this case, from Eq. (10) with use of Eq. (4) we will receive signal like $s_{\text {refl }}(t)=A_{\Sigma} \cos \left(\omega t+\varphi_{\Sigma}\right)$ - signal from imaginary one object. And this object not at distance $l_{1}$ or $l_{2}$. This expression shows the most problem of classic phase distance measuring method no possibility to separate objects in the process of measuring.

Other problem we have is a case of vagueness of distance measuring when a wave-length $\lambda$ less than length of line $L$. Distance:

$l=\frac{\lambda(2 \pi \cdot n+\varphi)}{2 \pi}$,

where $n$ is integer from 0 to $[L / \lambda]$.

For the measuring with higher accuracy used so-called multiscale method $[16,17]$. This method based on forming of set of frequencies from the lowest frequency, wave-length of which will be greater than length of line which is tested.

Advantages of that method:

- High exactness of determination of change of phases allows to get high exactness of distance-finding to the damage;

- Using of low frequencies allows to simplify construction of measuring device and diminishes a sensitivity to the external factors;

- Measuring phase angle shift is possibly during long time which improves overall accuracy.

\subsection{Modern phase angle shift measuring methods and devices}

With grow of electronic devices with large memories and high speeds new methods of distance measuring were developed. Those methods are based on use of sine wave signals and measuring of frequency of beating, as functions of distances [4, 8, 14]. Unlike TDR, new methods belong to frequency dimension reflectometry (FDR).

There are three types of frequency dimension reflectometry that use for measuring distances to damages. To such methods belong:

1) Frequency Modulated Continuous Wave (FMCW) $[5,9,15]$;

2) Phase Detection Frequency Domain Reflectometry (PD-FDR) [9, 15];

3) Standing Wave Reflectometry (SWR).

Let consider shortly each of them.

\subsubsection{Frequency modulated continuous wave (FMCW)}

FMCW change frequency of sine wave signal very quickly. As a result, frequency shift measured between test and reflection signals converted to a corresponding delay equivalent to distance to the object. However, this method did not find application in power lines through limitation on speed in which a signal can spread in a line. Insufficient accuracy in which the frequency shift can be determined [8] is also determined.

\subsubsection{Phase detection frequency domain reflectometry (PD-FDR)}

System of measuring of distance which is based on measuring of phase angle shift shown in Fig. 4 [5-7], measures a phase angle shift between entrance and initial signals.

A voltage-controlled generator (VCO) provides a sine wave signal which steps in a line through the signal divider with a separation weak by $-10 \mathrm{~dB}$ signal of the so-called "copy" of test signal (first mixer), and returns back in a device through the second mixer.

A mixer mixes together two sine wave that gives a sum and difference of these two frequencies. After that, analogue signals transformed to digital ones for fast Fourier transformation. This is a first method offered the use of accumulation of measuring information and treatment of them in a 
single informative block.

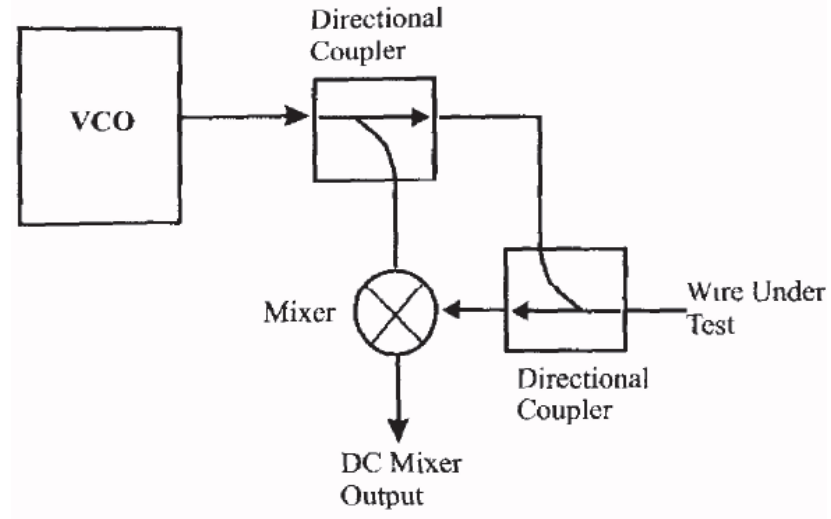

Fig. 4. Flow-chart of PD - FDR [7]
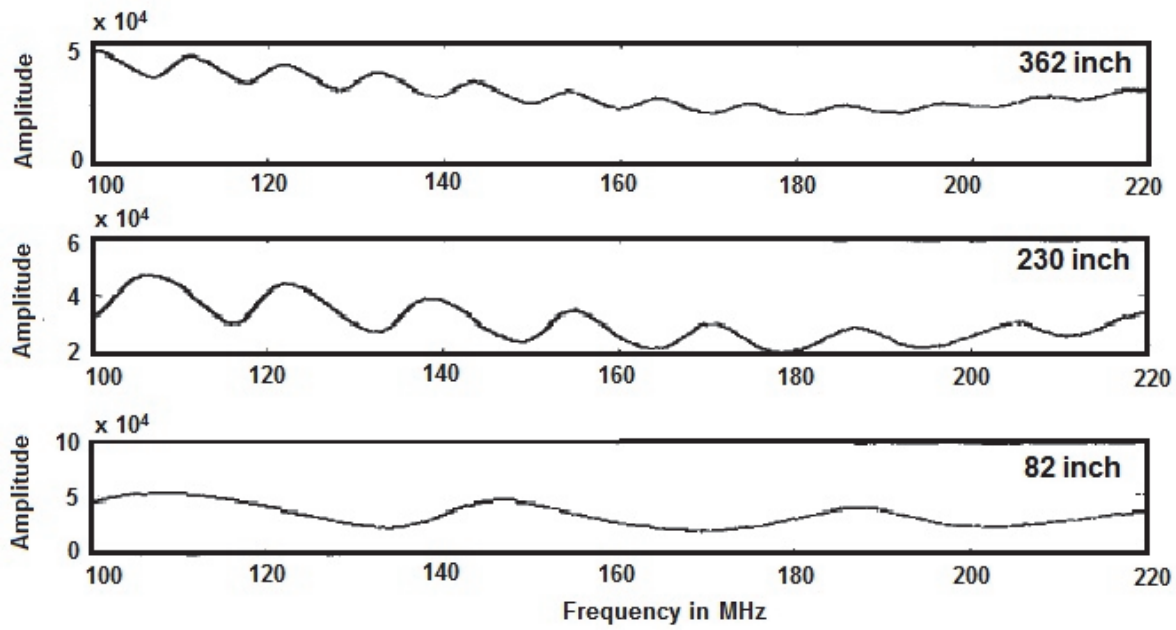

a)

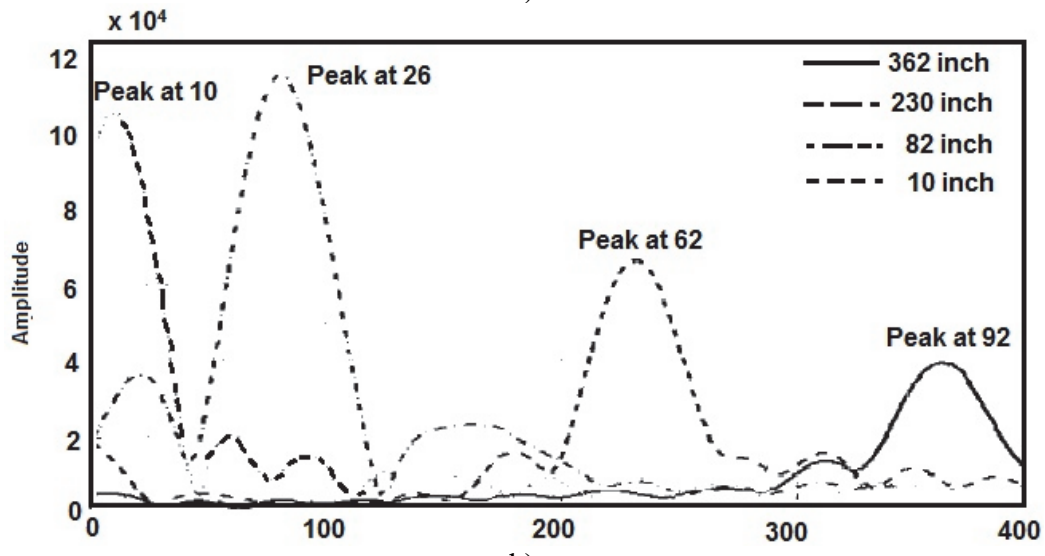

b)

Fig. 5. Result of PD-FDR for the coaxial cable of RG-58 $(50 \mathrm{ohm})$ : a) result on the exit of mixer; b) result of FFT [7]

FFT of the accumulated signal give a peak signals. Places of peaks in spectrum of FFT will 
give distances to each of them. Distance can be found as [7]:

$L=2 L_{\max }\left(\frac{\operatorname{Peak}-\operatorname{Peak}(0)}{N_{F F T}-1}\right)=\frac{1}{2}\left(\frac{\operatorname{Peak}-\operatorname{Peak}(0)}{N_{F F T}-1}\right)\left(\frac{N_{F}-1}{f_{2}-f_{1}}\right) v_{p}$,

where Peak - location of peak of delta function in FFT (integer value): $v_{p}$ - speed of distribution in a cable $(\mathrm{m} / \mathrm{s}) ; f_{1}-$ initial frequency of FDR $(\mathrm{Hz}) ; f_{2}$ - eventual frequency of FDR $(\mathrm{Hz}) ; N_{F}-$ number of frequencies in FDR, usually unit from $\operatorname{Int}\left(\frac{f_{2}-f_{1}}{\Delta f}\right) ; \Delta f$ - size of step of frequency for FDR (Hz); $L_{\text {max }}-$ maximal length, shown below; $N_{F F T}$ - number of points in FFT (integer value, on the whole $1024,2048,4096$ or 8192).

The fundamental problem of PD-FDR is very high count of points for FFT, low separation ability of objects and large range of frequencies. The typical set of answers of different lengths (on the example of wire as M27500-24SE2S23, [7]) is shown on Fig. 5.

PD-FDR has lower cost and uses simpler electronic base in compare with TDR. Components for PD-FDR could be integrated in a single chip. In addition, PD-FDR is able to work with re-reflections.

Like to the method of TDR of diminishing to distance to the object from the beginning of line requires minimisation of test signal frequency. But this is not possible to very low frequencies $[2,5,13]$.

\subsubsection{Standing wave reflectometry (SWR)}

Standing Wave Reflectometry measure the size of standing wave, created by imposition of test and reflected signals from line. A sum of these signals is two sinewave signals (see Eq. (10)) which usually identical amplitude. However, the problem of SWR is a detection of signals with low level of reflection. Therefore, the method of SWR used in case of short circuit or break.

Advantages of SWF are exactness, similar to PD-FDR in case of short circuit or break, where test and reflected signals approximately the same size. The reflected wave will be partly less, depending on distance, but require frequencies in $\mathrm{kHz}$ for measure.

\subsubsection{Mixed signal reflectometry (MSR)}

Mixed signal reflectometry (Fig. 6) is similar to PD-FDR without direction couplers or to SWR, which measures the size of standing wave. Like PD-FDR, voltage-controlled oscillator (VCO) provides a sine wave signal which steps above the given size of step. Combination of test and reflected signals passes through attenuator, which prevent the overload of mixer.

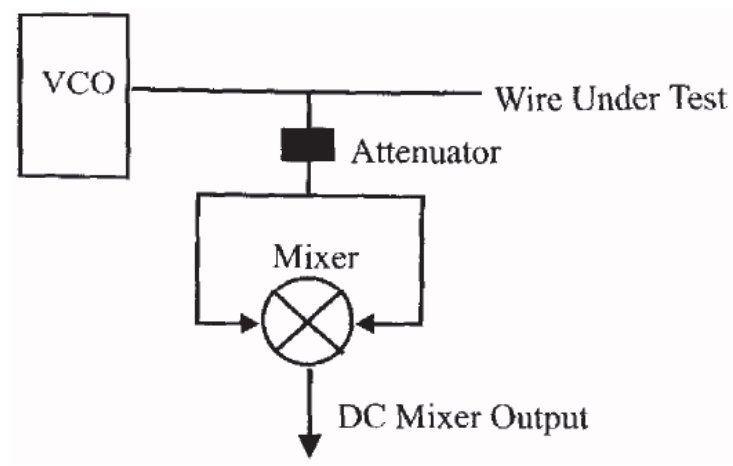

Fig. 6. Scheme diagram of MSR [7]

The method of MSR is more exact, than SWR for small reflections. However, this advantage 
does not have practical application, because it does not allow analysing very little anomalies. MSR is less expensive and less cost than PD-FDR. For the lines with interconnections, reflected signal of MSR includes reflections plus their sums and differences, which does the answer more difficult for a calculation than PD-FDR. MSR have the same limitation on the use on live wires identically to PD-FDR.

\subsection{Phase method for measuring distances of simultaneous objects in power line}

Classic phase measuring method based on determination of phase angle shift $\varphi$ between test input signal and reflection of the known frequency $f_{s}[19,20,22]$.

A vector diagram (Fig. 7) serves for explanation of problem of phase methods. Diagram shows forming of summary signal $U_{\Sigma} \cos \varphi_{\Sigma}$ [18]. An analogical diagram can be created for 3rd, 4th or other amount of damages. A result is an signal of reflection with the corresponding summary phase angle shift $\varphi_{\Sigma}$.

Multiscale method of measuring of distances to the damages leading and optical to the flow line it is known from works of such scientists as Maevsky S. M., Bajenov V. H., Baturevich E. K. The multifrequency phase measuring method is used practically for measuring.

On Fig. 8 presented reflecting in the case of two objects with zero-size damages. Distances to the damages accordingly $l_{1}=1000 \mathrm{~m}, l_{2}=2000 \mathrm{~m}$. Amplitude of the first reflection has a level a 1.0 units (vector $\mathrm{A}$ ), second is 0.5 units (vector $\mathrm{B}$ ).

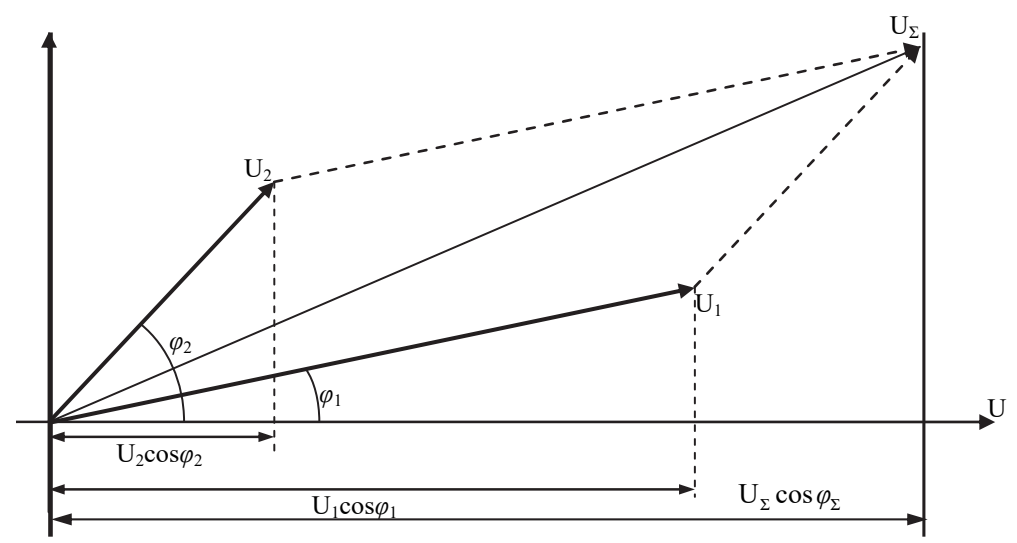

Fig. 7. Forming of reflection signal. Here: $U_{1}, U_{2}$ are amplitudes of reflections from 1st and 2nd objects, $\varphi_{1}, \varphi_{2}$ are corners of change of phase; $U_{1} \cos \varphi_{1}, U_{2} \cos \varphi_{2}$ are corresponding reflections;

$U_{\Sigma}$ it is summary amplitude and $U_{\Sigma} \cos \varphi_{\Sigma}$ is a summary signal

As shown on Fig. 8, at the change of test signal frequency in 2, 4 or 20 times, the angle shift changes gradually too. A change of the phase angle shift is proportional to frequency change.

Will pay attention to dynamics of change of vector A and vector B separately (Table 1). Especially interesting situation for understanding processes with frequencies $12 \omega$ and $20 \omega$. On these frequencies is observed transition through $2 \pi$ and $4 \pi$.

As seen from the Table 1, the increase of frequency and phase angle shift characterize every reflection. Let assume that measuring device consist of the generator of variable frequency $\omega\left(t_{i}\right)$. $\omega\left(t_{i}\right)$ is a frequency of signal during time $t_{i}, t_{i} \in\left[t_{i 1}, t_{i 2}\right]$. During time $t_{i}$ from $t_{i 1}$ to $t_{i 2}$ frequency $\omega\left(t_{i}\right)$ held unchanged and duration of interval $\left[t_{i 1}, t_{i 2}\right]$ is enough for stabilizing of transients in a power line. As a result, we have change of phase angle shift for $i$ reflection:

$d \varphi_{i}=\varphi_{i}\left(\omega\left(t_{2}\right)\right)-\varphi_{i}\left(\omega\left(t_{1}\right)\right)$,

what takes place at the change of test frequency $d \omega_{i}=\omega\left(t_{2}\right)-\omega\left(t_{1}\right)$. 


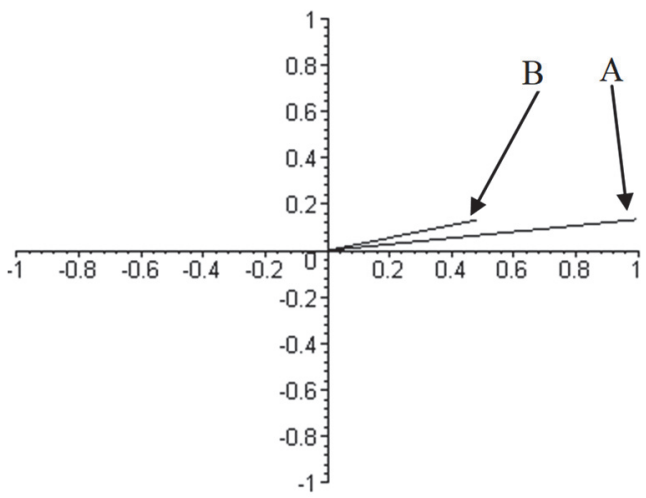

a) $\omega_{1}=1 \omega_{0}$

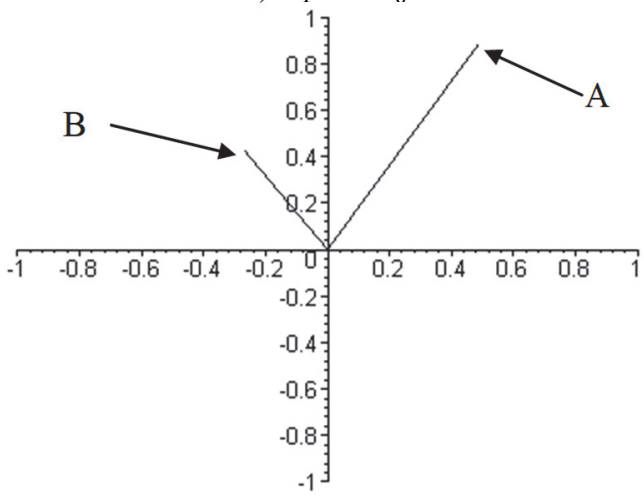

c) $\omega_{4}=4 \omega_{0}$

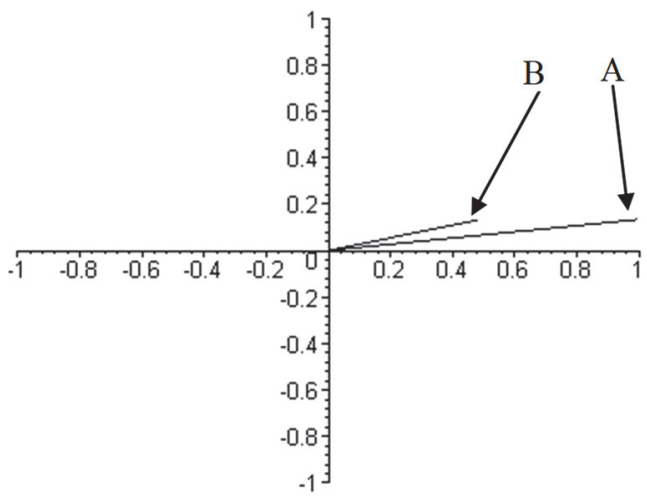

b) $\omega_{2}=2 \omega_{0}$

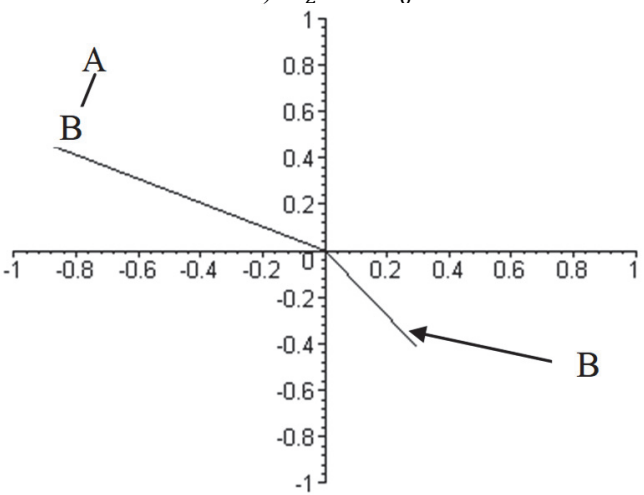

d) $\omega_{4}=8 \omega_{0}$

Fig. 8. To explain process of phase angle shift changes for two reflections on distances $l_{1}, l_{2}$ at the increases of frequency $\omega, \omega_{0}=4 \cdot 10^{3} \mathrm{~Hz}$

Table 1. Values of phase shift angles

\begin{tabular}{|c|c|c|}
\hline Frequency, $\omega$ & $\varphi_{1}, \mathrm{grad}$ & $\varphi_{2}, \mathrm{grad}$ \\
\hline $1 \omega$ & 19.2 & 38.4 \\
\hline $2 \omega$ & 38.4 & 76.8 \\
\hline $4 \omega$ & 76.8 & 153.6 \\
\hline $8 \omega$ & 153.6 & 307.2 \\
\hline $12 \omega$ & 230.4 & $\begin{array}{c}460.8 \\
(360+100.8)\end{array}$ \\
\hline $20 \omega$ & $\begin{array}{c}384.0 \\
(360+24.0)\end{array}$ & $\begin{array}{c}768.0 \\
(2 \times 360+48)\end{array}$ \\
\hline
\end{tabular}

Thus will enter a term "rotation of phase angle shift" $\Omega_{i,\left[\omega\left(t_{1}\right), \omega\left(t_{2}\right)\right]}$ :

$\Omega_{i,\left[\omega\left(t_{1}\right), \omega\left(t_{2}\right)\right]}=\frac{\varphi_{i}\left(\omega\left(t_{2}\right)\right)-\varphi_{i}\left(\omega\left(t_{1}\right)\right)}{\omega\left(t_{2}\right)-\omega\left(t_{1}\right)}=\frac{d \varphi_{i}}{d \omega_{t_{2}-t_{1}}}$,

where $t_{1}, t_{2}$ are certain physical time domains at which a value of frequency of measuring signal is became.

A value of $\Omega_{i,\left[\omega\left(t_{1}\right), \omega\left(t_{2}\right)\right]}$ is a function of only frequencies and differences between the phase shift and frequencies.

In comparing with PD-FDR, value of $\Omega_{i,\left[\omega\left(t_{1}\right), \omega\left(t_{2}\right)\right]}$ is more prediction and give ability to work with low and high frequencies. It allows utilizing several intervals of frequencies in comparing 
with PD-FDR where wide range of frequencies required.

Let assume phase angle shift for $i$ th damage is determined from the known expression as:

$\varphi_{i}=\frac{2 \pi \cdot 2 l_{i}}{\lambda}$

or in more comfortable form circular frequency $\omega_{j}$ of test signal:

$\varphi_{i}=\frac{2 \pi \cdot 2 l_{i}}{v} \cdot f_{j}=2 \frac{l_{i}}{v} \cdot\left(2 \pi \cdot f_{j}\right)=2 \omega_{j} \frac{l_{i}}{v}$

where $l_{i}-$ a distance to $i$ th object which a reflection is from; $v-$ a speed of distribution of signal in an environment; $\omega_{j}-\mathrm{a} j$ th frequency of testing signal.

In addition, expression what connect "rotation" $\Omega_{i,\left[\omega\left(t_{1}\right), \omega\left(t_{2}\right)\right]}$ of reflection vector with distance to the damage will be:

$\Omega_{i}=\frac{\left(2 \omega_{j+1} \frac{l_{i}}{v}\right)-\left(2 \omega_{j} \frac{l_{i}}{v}\right)}{\omega_{j+1}-\omega_{j}}=2 \frac{l_{i}}{v} \cdot \frac{\omega_{j+1}-\omega_{j}}{\omega_{j+1}-\omega_{j}}=2 \frac{l_{i}}{v}$

By limitation of frequencies from $\omega_{\min }$ to $\omega_{\max }$ the turn-down of phases will lay down:

$\varphi_{i \min }=2 \omega_{\min } \frac{l_{i}}{v}, \quad \varphi_{i \max }=2 \omega_{\max } \frac{l_{i}}{v}$.

From (15) there is fundamental possibility not only to define frequency of reflections vectors rotation, and it is possible to define and amount of turns of vectors of phase angle shift for the ith reflection in this range:

$N_{\text {iturn }}=\frac{\varphi_{\text {imax }}-\varphi_{\text {imin }}}{2 \pi}$,

or:

$N_{\text {i turn }}=\frac{\frac{2 l_{i}}{v}\left[\omega_{\max }-\omega_{\min }\right]}{2 \pi}=\frac{\frac{2 l_{i}}{v}\left[2 \pi f_{\max }-2 \pi f_{\min }\right]}{2 \pi}=\frac{2 \pi \frac{2 l_{i}}{v}\left[f_{\max }-f_{\min }\right]}{2 \pi}$
$=\frac{2 l_{i}}{v}\left[f_{\text {max }}-f_{\min }\right]$.

The analysis of the expression Eq. (14) will give an expression for recognition Eq. (15). As a result, we receive the following: value of Eq. (15) gave to us ability to work practically with any frequency range Eq. (15). The second feature of Eq. (15) is that $N_{i \text { turn }}$ will not be always an integer value only.

In addition, limitation of working range of frequencies is also equivalent to additional value of phase angle shift for each of signals. At $f_{\min }=0$, an initial phase angle is always $\varphi_{i} \rightarrow 0$. In common case of $f_{\min }>0$ we will also have $\varphi_{i}>0$ (clearly, that except those cases, when $\left.\varphi_{i}=2 \pi \cdot n\right)$.

In real power cables, frequencies lower then $10-20 \mathrm{kHz}$ does not allow to archive acceptable qualify of measuring due to variable parameters of power line on low frequencies.

For example, on Fig. 9 shown code in Wolfram Mathematica for modelling power line from $0.001 \mathrm{~Hz}$ to $600 \mathrm{kHz}$.

After that will be received figures of phase shift $\beta[\omega]$ (Fig. 10), real and imaging parts of wave 
impedance $\dot{Z}_{i m p}[\omega]$ (Fig. 11). Parameters of power line are stable for measuring only at high frequencies.

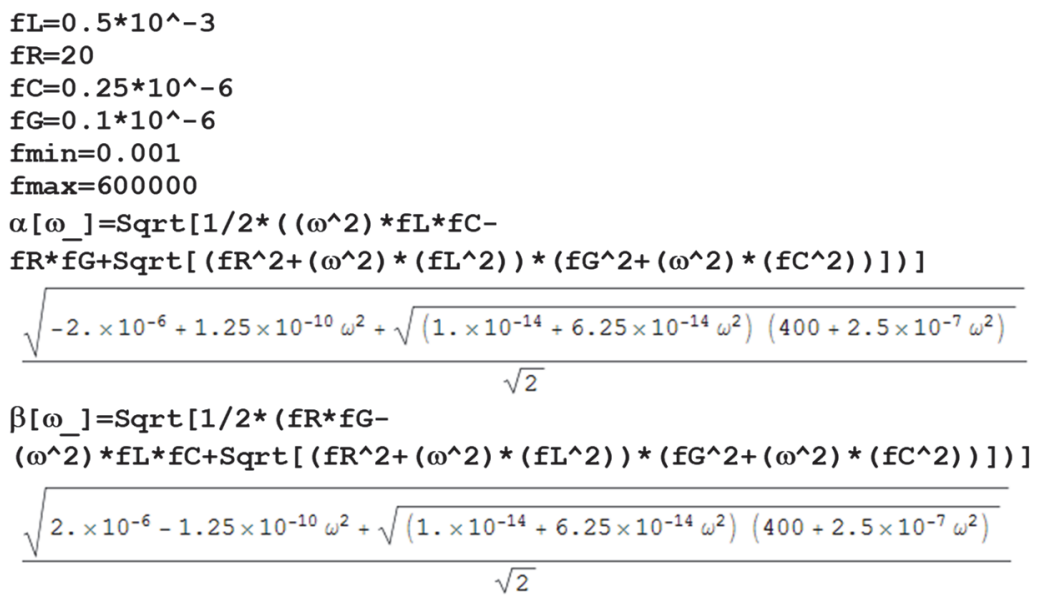

$Z[\omega]=\operatorname{Sqrt}[(f R+I * \omega * f L) /(f G+I * \omega * f C)]$

$\sqrt{\frac{20+(0 .+0.0005 i) \omega}{1 . \times 10^{-7}+\left(0 .+2.5 \times 10^{-7} i\right) \omega}}$

$\gamma\left[\omega_{-}\right]=\beta[\omega]+I \star \alpha[\omega]$

$\frac{\sqrt{2 . \times 10^{-6}-1.25 \times 10^{-10} \omega^{2}+\sqrt{\left(1 . \times 10^{-14}+6.25 \times 10^{-14} \omega^{2}\right)\left(400+2.5 \times 10^{-7} \omega^{2}\right)}}}{\sqrt{2}}+$

$\frac{i \sqrt{-2 . \times 10^{-6}+1.25 \times 10^{-10} \omega^{2}+\sqrt{\left(1 . \times 10^{-14}+6.25 \times 10^{-14} \omega^{2}\right)\left(400+2.5 \times 10^{-7} \omega^{2}\right)}}}{\sqrt{2}}$

Plot $[\alpha[w],\{w, f m i n, f \max \}$, PlotLegends $\rightarrow$ "Expressions"]

Plot $[\beta[w],\{w, f \min , \operatorname{fmax}\}$, PlotIegends $\rightarrow$ "Expressions "]

Plot $[\{\operatorname{Re}[z[w]], \operatorname{Im}[z[w]]\},\{w, f \min , \operatorname{fmax}\}$, PlotIegends $\rightarrow$ "Expressions "]

Fig. 9. Code for modelling power line from $0.001 \mathrm{~Hz}$ to $600 \mathrm{kHz}$ in Wolfram Mathematica

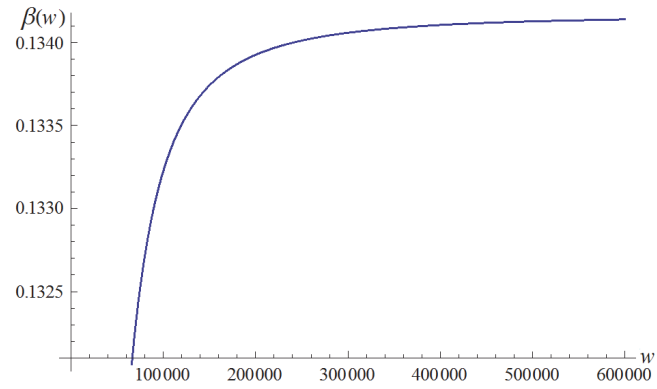

Fig. 10. Phase shift $\beta[\omega]$

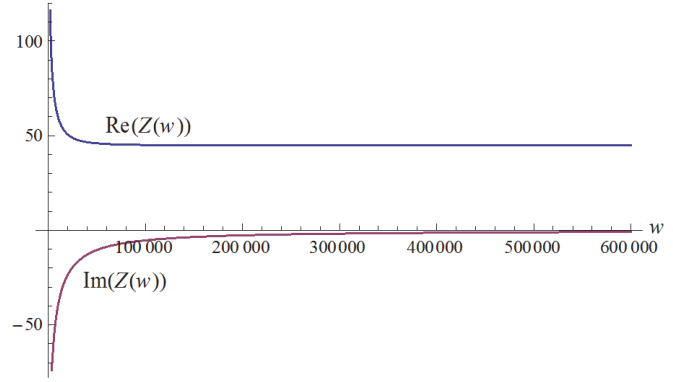

Fig. 11. real and imaging parts of wave impedance $\dot{Z}_{i m p}[\omega]$

\section{Signal modelling}

On Fig. 12 it is shown distribution of the reflected signals $0.5 \cos (6 \varphi)+0.7 \cos (8 \varphi)+0.9 \cos (10 \varphi)$ for the range $\varphi=0.2 \pi$. That signal for modelling power line with 3 objects in it with distance 6:8:10 respectively. As were shown on Fig. 5(a) high frequency belong to far object. Limitation in a frequency range taken from Fig. 10 and Fig. 11. As 
evidently, the initial phase of signals in allowed zone are different on such condition.

A spectral analysis of reflection signal with use of Fourier transformation with variable window size.

As were specified early, signal in phase measuring methods is sum of harmonics signals of the same frequency but with different phase angle shifts (see (10)). Due to different values of $\varphi_{i}$ values of $\Omega_{i,\left[\omega\left(t_{1}\right), \omega\left(t_{2}\right)\right]}$ are different too. So, the object of analysis is a set of $\Omega_{i,\left[\omega\left(t_{1}\right), \omega\left(t_{2}\right)\right]}$.

Among the existent methods used for signal analysis, the most used methods are [1, 22, 23]:

- Fourier transformation;

- Wavelet analysis;

- Other methods of analysis.

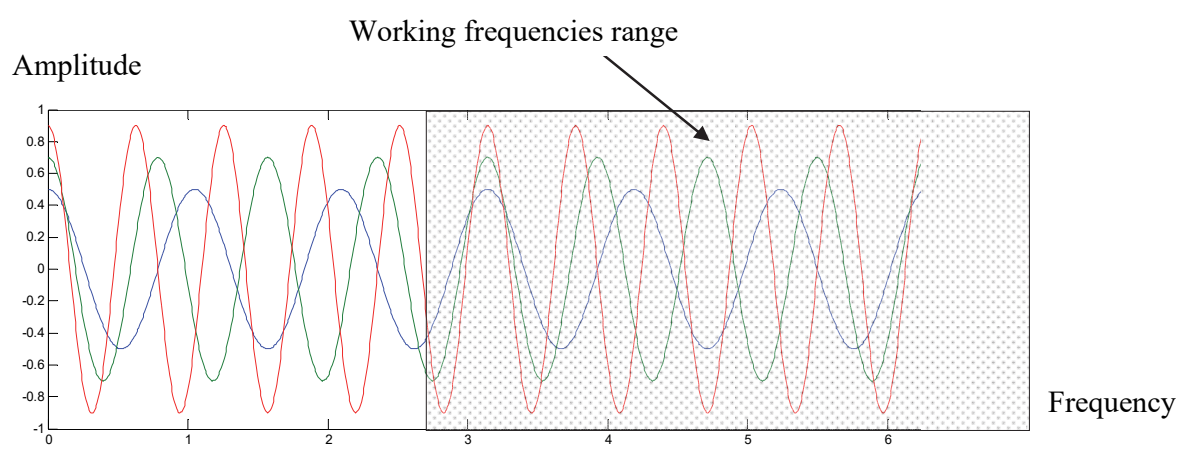

Fig. 12. Distribution of projections of vectors from the signals of reflections $($ signal $0.5 \cos (6 \varphi)+0.7 \cos (8 \varphi)+0.9 \cos (10 \varphi)$ )

Fourier transformation is the well-known basic instrument of signals analysis in a frequency area. It is known that the periodic signals of kind $x(t)=x\left(t+m T_{O}\right)$ appear as a row of a sum of harmonic constituents.

In practice of spectral analysis of the real signals does not operate a continuous signal $x(t)$ and only by the eventual sequence of their selections $x(n)$. For this reason, in the technique of digital analysis of signals, discrete Fourier transformation is used.

The one of the main problem of FFT is analysing periodical signal limited in time. There are signals with period match analysis window size. And others are not equal to analysis window. Thus, if a periodical signal does not match with FFT window size additional components appear in a spectrum. This phenomenon named spectrum leakage. Spectrum leakage is possible to illustrate with the calculation of spectrum of discrete harmonic signal (Fig. 13) on an example. Discrete signal contains 16 points of harmonic signal with own period even to a 4 point (Fig. 13(a)) the periodically continued signal is periodic to FFT window.

If signal does not periodic to FFT window spectrum leakage will be observed (Fig. 13(b)). Spectrum of signal consists of additional harmonics. For PD-FDR or any equivalent method this mean additional objects in line.

To determine the methods for controlling the spread of the spectrum of the input signal, it is necessary to establish the factors that lead to the occurrence of this phenomenon.

Understanding the causes of the phenomenon in this case will allow taking a number of measures to eliminate the spread of the spectrum.

As were shown above, the phenomenon of the spectrum leakage is the appearance of additional harmonics in the spectrum. Moreover, these additional harmonics complicate the process of identifying harmonics from the real components of the signal. That is, there is a process of "masking" the signals. In order to deal with the spectrum leakage in the practice of digital analysis, it is known that window functions are used, but in this case, they do not give us the result we need because they reduce the phenomenon associated with the non-periodicity of the signal for analysis.

Window functions are used to reduce signal at beginning and ending of FFT window. For 
example, Fig. 13 show use of Hann window function and result spectrum.

Given the complexity of the introduction of wavelet analysis and the need to create application software that would allow simple mathematical transformations, it would be able to transfer to 32-bit or 8-bit computing environment of small and medium computing power, proposed the creation of an algorithm for spectral analysis on based on the modified Fourier transform algorithm. Software implementations of the Fourier transformation are known for a large number of hardware and software environments.
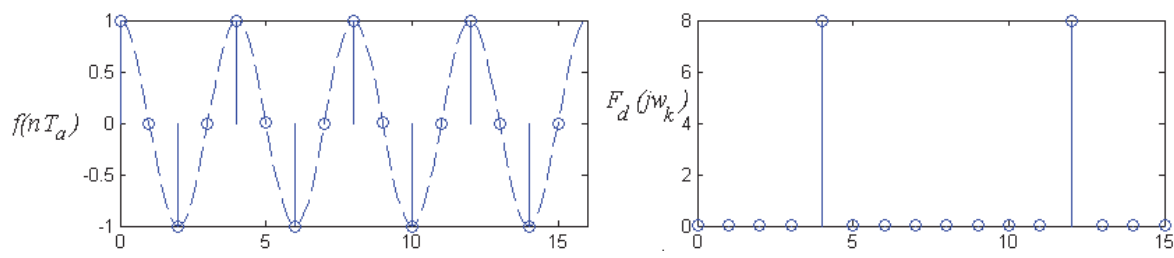

a)
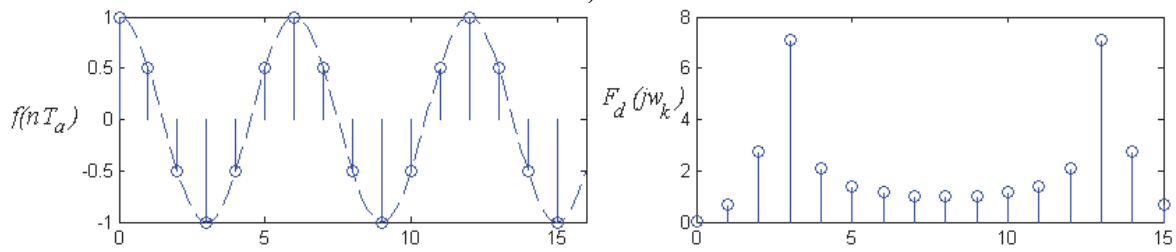

b)
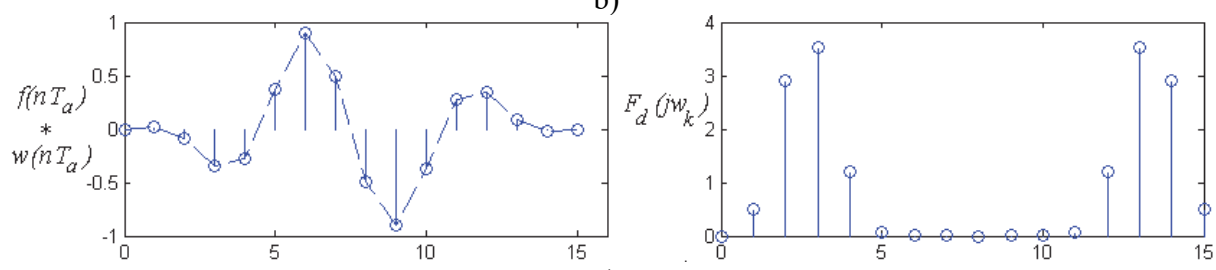

c)

Fig. 13. FFT for periodical signal: a) unperiodical signal and spectrum leakage, b) reducing spectrum leakage with Hann window function

\subsection{A flow-chart of spectral analysis algorithm}

However, existing implementations does not allow detect signals with such incomplete period, since according to the FFT, the total signal represents a series of whole-periodic functions. Thus, if we change the number of points for FFT, it is quite clear that a signal with a non-integer period can transform into a signal with an integer number of periods, at the same time. But at the same time signals with integer number of periods will become signals with non-integer periods.

As a result of this, it is suggested to produce multiple analyses of the same signal by FFT. However, every next time of analysis the same signal point for FFT will be reduced. Since our signal represents a sum of harmonic signals, changing the window width will be in the coincidence of the whole number of periods. Accordingly, such signals in the output of FFT will have the highest amplitudes at a certain value of the window width. Consequently, the gradual decrease in the width of the window will lead to the discovery of various spectral components in the total signal.

Since there will be a rise and fall in the spectrograph, then it is proposed to highlight the largest and smallest values for each spectrogram in a single array. The aim of this is to determine the harmonics that have reached the highest value in the process of spectral analysis of our signal.

The algorithm for determining the spectral components of the total signal by manipulating the width of the window FFT is as follows:

1) Measuring of the input signal with the definition of $N$ points. 
2) A FFT cycle is performed. Result stored in arrays of maximal and minimal values.

3) Determine the spectral components with the greatest and smallest amplitudes. The result is remembered by adding to the result to the results of the previous cycles of the FFT.

4) Reduce the width of the FFT window by dropping one point from the end.

5) If the number of readings is greater than the minimum value of $N_{\min }$, go to paragraph 2.

Conditionally, the flow-chart of algorithm of spectral analysis of signal with the change of width of window is shown on Fig. 14.

Software was created in the MatLab using the proposed algorithm.

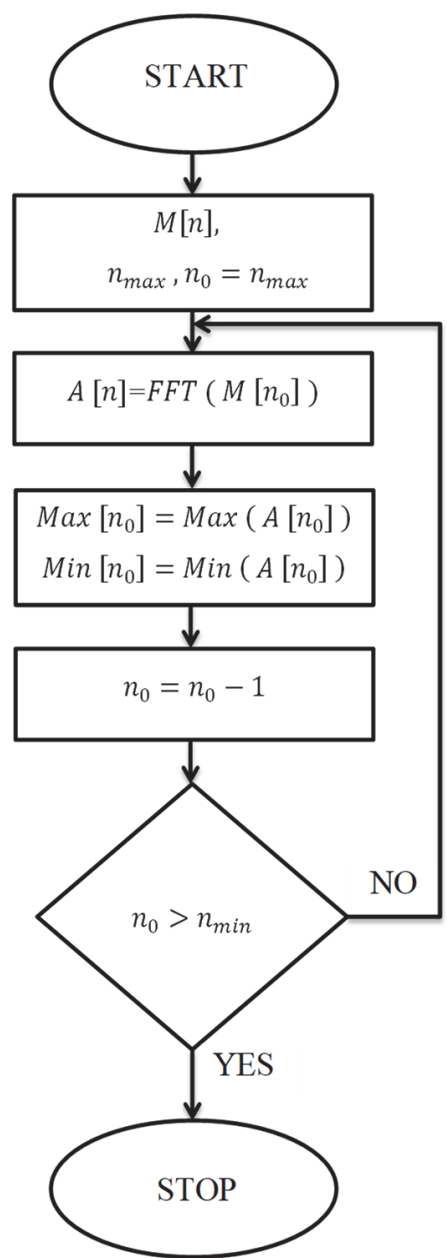

Fig. 14. Flow-chart of algorithm of spectral analysis with the change of width of window. Here $M[n]$ is an array of input data from measuring; $n_{\max }$ it is a number of the last element in an array $M[n]$; $F F T$ it is a function of transformation of Fourier; $A[n]$ - the array of accordions is got on the $i$ th step of transformation; $\operatorname{Max}\left[n_{o}\right]$ and $\operatorname{Min}\left[n_{o}\right]$ are arrays of most and the least amplitudes of accordions

\subsection{Result of proposed algorithm}

Consider the work of the proposed algorithm for spectral analysis of the signal with the change in the width of the FFT window. A signal is taken as a test signal (Fig. 15(a)) $-20 \cos (64 t+0.7 \pi)-32 \cos (74.3 t+1.2 \pi)+49 \cos (106.2 t+3.2 \pi)$. 


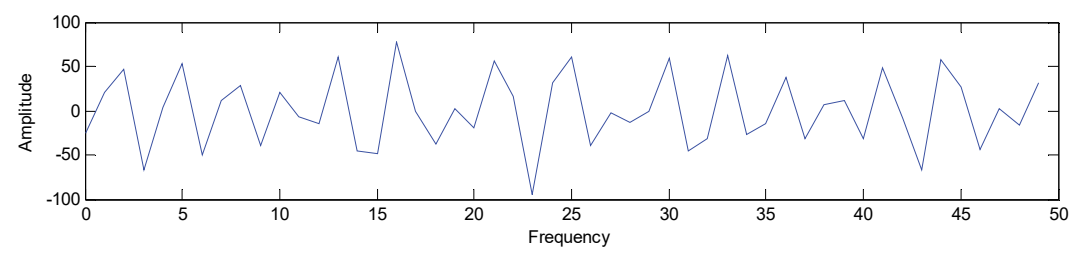

a)

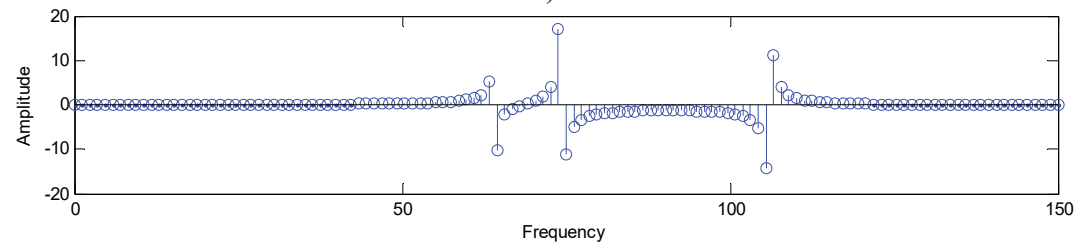

b)

Fig. 15. a) Signal, b) spectral transformation for the signal of kind $S(t)=-20 \cos (64 t+0.7 \pi)-32 \cos (74.3 t+1.2 \pi)+49 \cos (106.2 t+3.2 \pi)$

Fig. 16 shows the dynamics of changes of spectral components at the terms of FFT window width changed. It stipulates the necessity of setting of norms of the got result for a coercion to the only dimension to the landmark of frequency. At the same time, such setting of norms introduces error setting of norms, as spectral components, which have a whole number $N_{i, F F T}$ led to the corresponding rationed whole numbers:

$N_{j, F F T}=\operatorname{round}\left[\frac{F s}{2} \cdot \frac{1}{N F F T^{\prime}} \cdot k\right], \quad k=1, \ldots, N F F T^{\prime}, N F F T^{\prime}=\frac{N F F T}{2}, \ldots, N F F T$,

where $F S$ is frequency of input signal discretization; NFFT it is an amount of points of FFT.

For comfort of analysis, the result after normalization is shown on Fig. 17.

After FFT as shown on Fig. 15, amplitudes are only 12.1, 19.3, 16,1. After comparing result of FFT from Fig. 15 and from Fig. 17 can be noted following: the presence of three signals with amplitudes by modules 7.2, 13.1 and 17,4. Correlation between those amplitudes are closer to original ones in test signal.

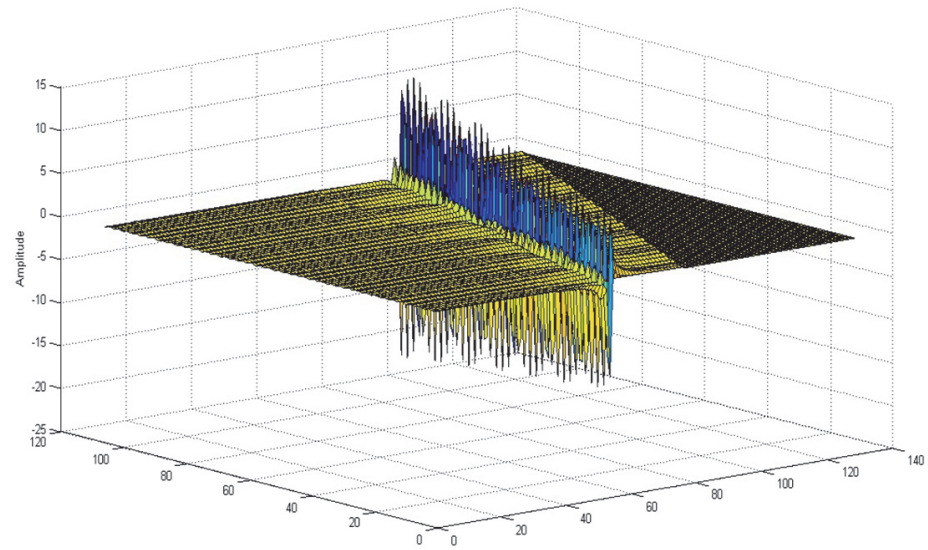

Fig. 16. Result in a 3-dimension view

Thus, offered method of determination of spectral component signals, which consist of harmonious signals with variables periods in compare to FFT window width allows to set both the 
presence of spectral components and pick up correct amplitudes of these components. After FFT as shown on Fig. 15, amplitudes are only 12.1, 19.3, 16,1.

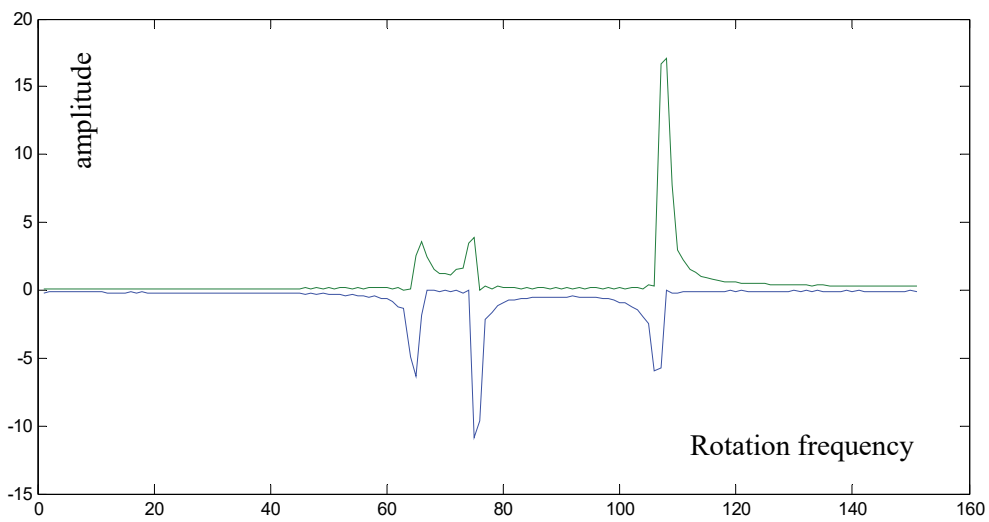

Fig. 17. Result of selection of spectral components by

determination of maximal and minimum values of amplitudes of spectrums

\section{Conclusions}

There are a huge number of methods of spectral analysis, but they have properties that complicate their use in practice. Fast Fourier transform is a simple analysis method. However, the Fourier transform has errors in determining the frequencies and amplitudes of the components when the width of the analysis window and the period of the input signal are not identical. Spectral analysis based on wavelet analysis is a powerful tool, but complex and not sufficiently convenient for transfer to automated measuring systems.

The algorithm of the spectral analysis of the signal with the change in the width of the window is proposed. A simulation has been performed. Application of a variable width of a window allows reduce the influence of the spectrum leakage.

It is established that existing phase measuring methods have fundamental frequency constraints, especially at low frequencies below $100 \mathrm{kHz}$. Therefore, phase measuring methods have additional difficulties in practical application, since one of the key properties is considered to be the measurement at frequencies close to zero. At the same time, the frequency limitation is not principle for the presented phase-frequency method.

\section{References}

[1] Amara Graps An introduction to wavelets. IEEE Computational Science and Engineering, Vol. 2, Issue 2, 1995, p. 50-61.

[2] Basava Santi B. Detection and Location of Cable Faults Using Reflectometry Methods. M.S. Thesis, Ulah State University, 2004.

[3] Filomena Andre D., Resener Mariana, Salim Rodrigo H., Bretas Arturo S. Fault location for underground systems with capacitive current compensation. 16th PSCC, Glasgow, Scotland, 2008.

[4] Chen C. S., Rocmer L. E., Grumbach R. S. Cable diagnostics for power cables. IEEE Annual Conference of Electrical Engineering Problems in Rubber and Plastic Industries, 1978.

[5] Chung You Chung, et al. Non-destructive fault location on aging aircraft wiring networks Part 1 Cost-optimized solutions. IEEE AS and USNC/URSI National Radio Science Digest, Columbus, Ohio, 2003.

[6] Hampton R. N., Perkel J., Hernandez J. C., Begovic M., Hans J., Riley R., Tyschenko P., Doherty F., Murray G., Hong L., Pearman M. G., Fletcher C. L., Linte G. C. Experience of Withstand Testing of Cable Systems in the USA. CIGRE 2010, p. B1-303. 
[7] Chung You Chung, Turse Cynthia, Pruitt Jeremy Application of phase detection frequency domain reflectometry for locating faults in an F-I8 flight control harness. IEEE Transactions on Electromagnetic Compatibility, Vol. 47, Issue 2, 2005, p. 127-114.

[8] Furse C., Kamdar N. An inexpensive distance measuring system for navigation of robotic vehicle. Microwave and Optical Technology Letters, Vol. 33, Issue 2, 2002, p. 84-87.

[9] Furse Cynthia, Chung You Chung, Dangol Rakesh, Nielsen Marc, Mabey Glen, Woodward Raymond Frequency domain reflectometry for on board testing of aging aircraft wiring. IEEE Transactions on Electromagnetic Compatibility, 2003, p. 306-315.

[10] Hartlein R. A., Hampton R. N., Perkel J. Some considerations on the selection of optimum location, timing, and technique, for diagnostic tests. IEEE Power Engineering Society (PES) General Meeting Panel Session, Pittsburg, PA, 2008.

[11] Oppenheim A. V. Digital Signal Processing. Prentice-Hall, Englewood Cliffs, N.J., 1975.

[12] Hartlein R., Hampton N., Hernández J. C., Perkel J. Overview of Cable System Diagnostic Technologies and Application: Cable Diagnostic Focus Initiative Project (CDFI): 04-211/04-212/09166. The National Electric Energy Testing Research and Applications Center (NEETRAC), Georgia, 2010, p. 323.

[13] Schmidt Mark Use of TDR for Cable Testing. M.S. Thesis, Utah State University, Logan, Utah, 2002.

[14] Smith Paul Spread Spectrum Lime, Domain Reflectometry. Ph.D. Dissertation, Utah Slate University, 2003.

[15] Tsai P., Lo C., Chung Y. C., Furse C. M. Mixed signal reflectometer for location of faults on aging wiring. IEEE Sensor Journal, Vol. 5, Issue 6, 2005, p. 1479-1482.

[16] Abramov K. K. Simulation and Calculation of Communication Cables on the Computer. Communication, Moscow, 1979, p. 79.

[17] Gillmanov Eduard Akhnafovich. Improvement of efficiency of cable transmission lines operation on the basis of their diagnostics by pulsed reflectometry. Ufa, 2009, p. 20.

[18] Horiaschenko K. L. Pulse-phase measurements for a line with two inhomogeneities. Measuring and computing engineering in technological processes. Khmelnytskyi. Vol. 1, 2003, p. 80-82.

[19] Cable Products. Terms and Definitions. GOST 15845-80, 1981, p. 18.

[20] Glebovich G. V., Andriyanov A. V., Vvedensky Yu. V. Investigation of Objects with Picosecond Pulses. Radio and Communications, Moscow, 1984, p. 256.

[21] Lyubchik V. R. Development of the phase method for measuring distances to two objects. Bulletin of the Technological University of Podillya, Vol. 2, 2004, p. 108-114.

[22] Maevsky S. M., Bazhenov V. G., Baturevich E. K., Kuts Yu V. Application of Methods of Phase Measurement for Precision Measurement of Distances. Higher School, Kiev, 1983, p. 84.

[23] Otnes R., Enoxon L. An Applied Analysis of Time Series: Basic Methods. Mir, Moscow, 1982, p. 430.

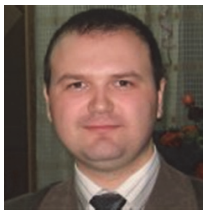

Kostyantyn Horiaschenko received Ph.D. degree in Kyiv National University of Technology and Design in 2005. Khmelnitsky National University. His current research interests include control and fault diagnosis in power lines.

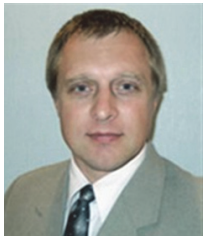

Victor Stetcuk received Ph.D. degree in Khmelnitsky National University in 2012. Now he works at Khmelnitsky National University. His current research interests include telecommunications and power electronics. 


\title{
5.3. Rank analysis in the problems of optimization of local power engineering
}

\author{
Serhii Denysiuk ${ }^{1}$, Vasylenko Vira ${ }^{2}$ \\ National Technical University of Ukraine, Igor Sikorsky Kyiv Polytechnic Institute, Kyiv, Ukraine \\ E-mail: ${ }^{1}$ spdens@ukr.net, ${ }^{2}$ veravasylenko@gmail.com
}

\begin{abstract}
The monograph considers the problems of efficient use of energy resources. The question of application of the technocenological approach for optimal management of energy consumption with the use of rank analysis, which includes procedures of interval estimation, parametric rationing, forecasting and standardization of consumption of resources is considered. The paper presents an algorithm for conducting rank analysis for forecasting power consumption and technocenose optimization. The practical example of technocenose optimization is presented and the rank-parametric distribution of technocenosis is shown on the parameter of electric consumption.
\end{abstract}

Keywords: energy efficiency, technocenosis, rank analysis, optimization of technocenosis.

\section{Introduction}

At present, energy consumption in the world has been increasing and fossil fuels comprise large proportion to the overall energy use. In addition to the concerned issue on future exhaustion of resources, global warming is also becoming a serious concern due to higher concentration of $\mathrm{CO} 2$ emissions in the air through the use of these resources. In regard to effective countermeasures against these global issues, the international community has actively made efforts on the promotion of energy conservation such as building the international framework in accordance with the Kyoto Protocol. On the other hand, countries still tend to focus on economic development rather than environmental countermeasures. Especially in developing countries, demand for energy has continuously increased due to the population growth and industrialization. It is expected that the world energy consumption will significantly increase, mainly in developing countries in the 21 st century. It is necessary for both developed and developing countries to promote energy conservation in order to resolve the global issues for the future.

The basis of energy conservation in power systems is the planned implementation of a complex of technical and technological measures and optimization of energy consumption of their individual elements at the system level. The purpose of optimization is to streamline the energy consumption of the power system objects, to save energy resources received from the consumed primarily through organizational measures, as well as to create scientifically grounded assumptions for conducting targeted energy surveys, followed by the implementation of technical and technological measures for energy conservation [1].

Describing processes in the energy system, we use the following definitions:

Technical power system (TPS) - the summation of numbers of equipment and enterprises that interact with each other for the production, consumption or transformation, storage, transportation or processing of energy products.

Energy product - finished product, which is used mainly for the production of mechanical work.

Natural resources are used as inputs to TPS, in other words the summation of equipment and enterprises, the main outputs of which are products and services, but which also produce by-products and emissions. Natural resources of other energy sources such as solar radiation, tidal energy, geothermal energy, wind and heat are also used for direct conversion into mechanical, thermal or electrical energy.

Products that are outputs of the technical power system are inputs to other technical power systems or are used to provide services. At the end of their exploitation, they are reused within the technosphere or returned to the environment as emissions. Thus, inputs to the technosphere are 
natural resources, and the outputs of services provided to society, emissions and operational influences [2, 3].

Energy resources directly consumed

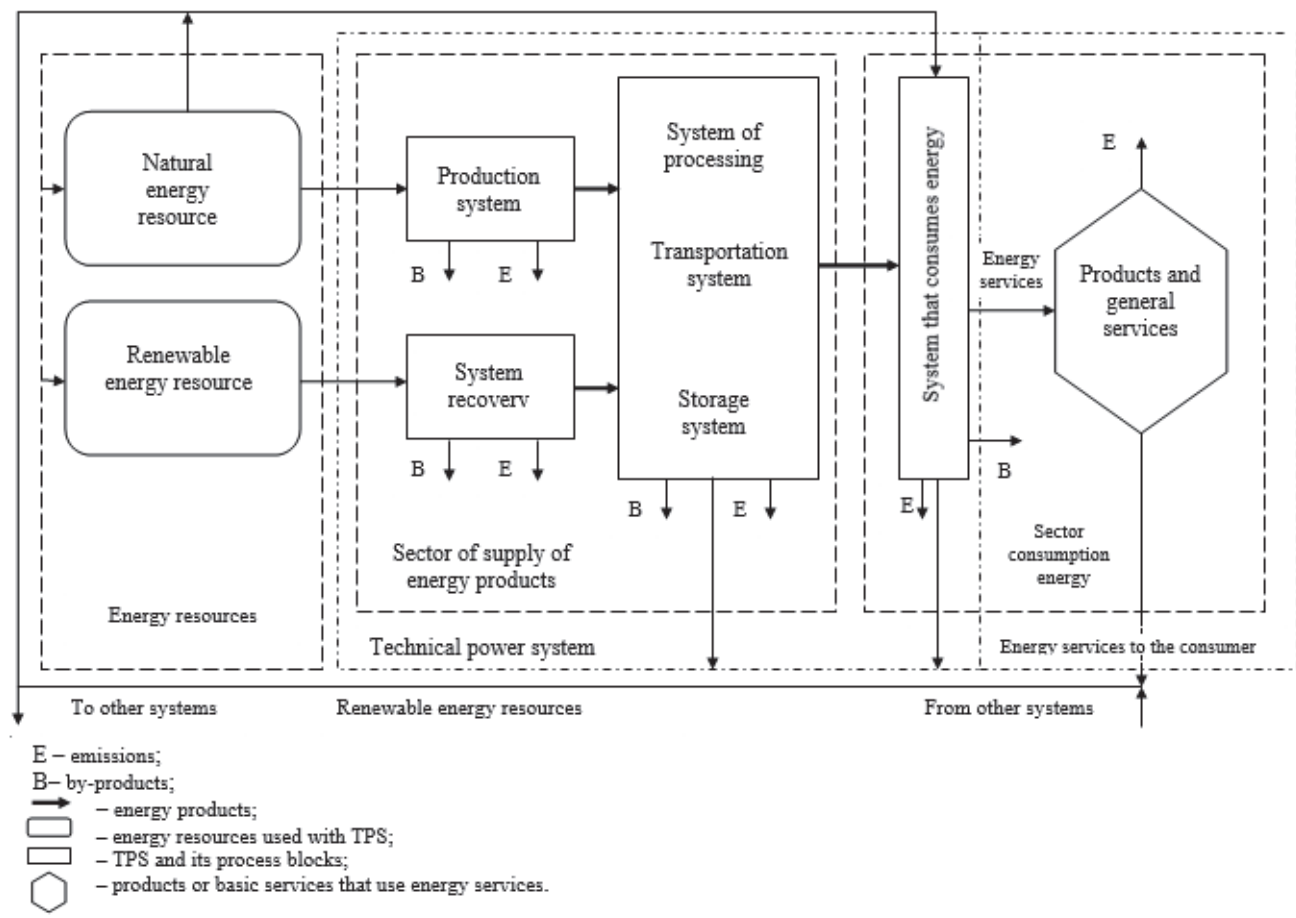

Fig. 1. The general model of the technical power system

\section{The theoretical part}

The general methodology of research and implementation in the field of energy conservation, according to the classification introduced in $[4,5]$, can be conditionally divided into three levels (Fig. 2):

1) The first level corresponds to activities aimed at specific technical and technological developments that contribute to reducing energy consumption (improvement of heat supply systems, replacement of outdated lighting devices, introduction of energy-efficient frequency controlled electric drives, modernization of internal equipment of buildings and structures, etc.).

2) At the second level, optimization of energy consumption of TPS as a whole is carried out. As a methodological basis at this level, a rank analysis based on the technocenological approach, Zipf mathematical statistics and the theory of hyperbolic infinitely divisive distributions is widely used. Given the conceptual and methodological differences that underlie research at the second level, it is considered to be systemic in relation to the level of research relating to specific technical and technological solutions in the field of energy conservation [6].

3) Strategic planning and forecasting of the TPS is carried out at the third level of activity (maneuvering of the maximum load, reduction of losses in the lines, effective control, etc.).

Real management of technocenosis is possible only in the conditions of correct understanding the object of management, as well as the introduction of correct methods for optimal management of this object.

Biological terminology is used to describe the technocenosis [7]:

- individual - an integral part of the cenosis - are personified TPS, which carry out unique activities, have an individual name, are taxpayers, independently distribute and consume resources; 
- view - a group of elements, systematized by the nature of the activity, size;

- population - a group of individuals of the same species;

- caste - a group in which each species is represented by an equal number of individuals.

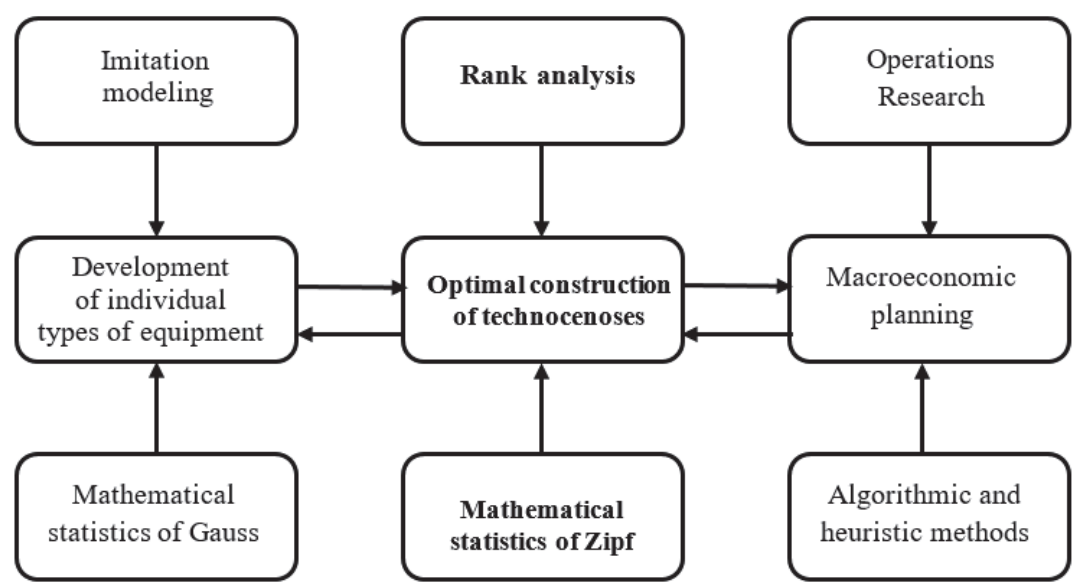

Fig. 2. Levels of research in the field of energy saving TPS

The main properties of technocenosis [8]:

1) The name and number of all available (winding transformer wire, fasteners, tools, instruments, etc.) cannot be listed. Each of the objects is discretely allocated as an individual, but at the same time it can be described by a variable parameter (costs, electricity consumption) from a continuous series of positive integers. The discreteness and finiteness of the elements forming the technocenose, as well as the continuity and possible change of the cenosis parameter in general, allow it to be formalized to describe it for evaluation of the past, management of the current state and forecast of development;

2) Uncertainty of the boundaries of electricity supply. They are not limited only to the master plan: own electric networks go beyond its limits, which feed on third-party consumers (the same thing - inside).

Speaking about the indicators that characterize the technocenosis (regardless of their verbal or formal representation), one should bear in mind:

1) The price cannot be adequately described by a system of indicators, any system is fuzzy and incomplete, an increase in the number of indicators and an increase in accuracy (reliability) does not approach to the selection of the technocenosis;

2) Two cenoses, described by one system of indicators, which coincide within the limits of accuracy adopted for this class, may differ in essence (characteristics, parameters, representations) [8].

Technocenosis has the following pronounced specific properties $[4,5,9]$ :

1) interconnectedness - the individual objects of technocenose are statistically significantly related to a single system, but the type of connections in this case is special - these are so-called "weak" connections that are not reduced to the Newton's law, Ohm's law, Kirchhoff's law et al.;

2) "Gaussless" - statistical samples of the parameters of individuals (objects) have Zipf properties (the dependence of the mean and variance on the sample size is significant), that is, the law of large numbers is not executed, which consists in the fact that the average statistical value should converge on the probability of mathematical expectation. This suggests that, before starting to manage the infrastructure object, it is necessary to find out whether it is a technocenosis and, consequently, in general, whether it is possible to apply it to the technocenological methodology.

The main instrument of the technocenological approach in the study of complex technical systems is the rank analysis - the method of research of large technical systems (infrastructure), 
which aims at their statistical analysis, as well as optimization, and uses the form of generic and rank distributions as the main criterion. Hyperbolic $H$-distributions are used to describe technocenosis.

Ranked distribution refers to the downward sequence of parameter values, arranged in such a way that each subsequent number is smaller than the previous one, and is brought into line with the rank (number in order, number of integers, arranged in ascending order) [3].

The structure of the cenosis is described by species distribution - the dependence of the number of species on the number of individuals in the form, ranked species distribution and rank distribution by parameter [7].

The mathematical apparatus of the cenological method is represented by three models of hyperbolic $H$-distribution (Fig. 3) [1, 8]:

1) Species:

$\Omega(x)=\frac{W_{0}}{x^{1+\alpha}}$

where $x \in(1 ; \infty]$ - a continuous analogue of population numbers $i,(i$ - always a discrete value, $i=[x]) ; a>0$ - characteristic index; constant distribution $\gamma=1+a ; W_{0}=A \cdot S$, $W_{1}=\left[W_{0}\right]$, where $W_{0}-$ the theoretical, not necessarily discrete value, and $W_{1}-$ actual (experimental) value of the first point; $A$ - constant distribution, which is found from the conditions of valuation.

2) Ranked:

$\Lambda(x)=\frac{B}{r^{\beta}}, \quad \omega(r)=\frac{u_{r}}{U}, \quad U=\sum_{r=1}^{S} u_{r}$,

where $u_{r}$ - the number of species of species (the population of the $s_{r}$ species), which corresponds to rank $r$ in the total number of individuals $U$. Rank of the species $s=1,2, \ldots, s_{r}, \ldots, S-$ is a serial number (line number). $1>B>0, \beta>0$ - constants of a rank-like $H$-distribution.

The species and rank distribution are used to study and manage the structure of installed equipment and equipment that is being repaired (electrical installations), that is, for discrete quantities. But the power supply system is characterized by continuous quantities (electricity consumption, heat consumption, capacity, cost, costs). In this case, the hyperbolic rank $H$-distribution by parameter [10] is used.

3) Ranked by parameter.

Let set of objects that form a certain integrity (all units of a single enterprise) and which correspond to cenological criteria. Each object is characterized by one or more parameters that are expressed numerically. After defining the parameter, you can arrange the set, placing all objects in order of decreasing the parameter, and obtain a hyperbolic rank $H$-distribution by parameter. The area under the curve of the rank $H$-distribution by parameter characterizes the energy consumption of the enterprise as a whole:

$W(x)=\frac{W_{1}}{r^{\beta}}$

where $r$ - the rank of the object; $\beta$ - an indicator that determines the degree of steepness of the distribution curve; $W_{1}=W_{\max }(x)$ - the constant for which the maximum value of the energy consumption of the largest consumer is taken:

$\beta=\log _{r} \frac{W_{1}}{W(x)}$ 
The ranking by parameter allows us to talk about the optimality, the effectiveness of the cenosa as a whole. Next it is necessary to investigate the structure of the cenosis. The best is the state of technocenosis, in which the parameter is within range $0.5 \leq \beta \leq 1.5$.

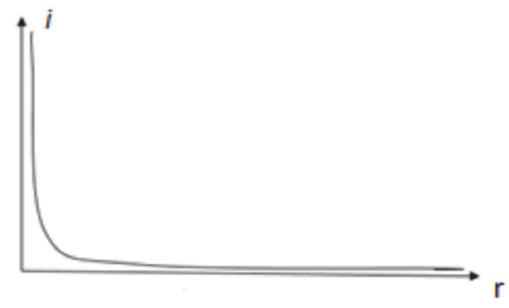

a)

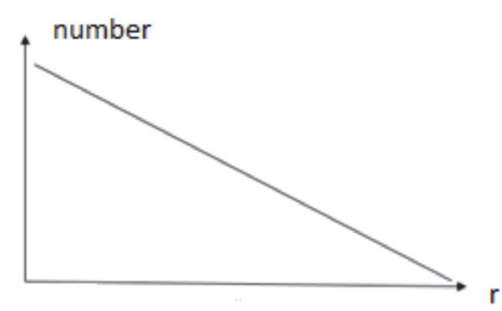

c)

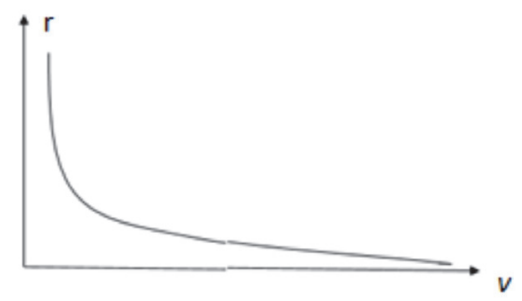

b)

Fig. 3. Types of $H$-distributions: a) species distribution, b) rank distribution, c) ranked by parameter distribution

Removal of abnormal variations in the species distribution is based on the analysis of deviations from the approximating curve: upwards - indicate a lack of unification of species, and downward - on the contrary, excessive. Parametric optimization determines the detailed mechanism of the process of improving the structure of the cenosis on the basis of characteristic indicators.

The energy consumption of a separate TPS using a technosenological approach is considered not in isolation but correlated with other consumers, hierarchically systematized. Detection of the tendency of the electricity consumption of the power system of the region, analysis of the parameters of rank distributions creates the basis for optimizing the management of the electricity consumption of the infrastructure region, enabling more accurately predict the further development of the energy complex, assess its stability [11].

\section{Materials and research results}

Investigating cenosis as an integrity reduces to their systematic description of the hierarchical system of indicators (which is necessary for the identification of the cenosis) and to the structural cenological description. Consider the technocenosis of the TPS on the example of the specific energy consumption by technoenese individuals. This cenosis is limited in space - the total number includes many functionally isolated individuals, not connected with each other by strong ties. There is also a single infrastructure that includes a power supply system, as well as a control system for operation and operation.

Energy consumption of the power system is described by analytical dependence:

$W(x)=\frac{W_{1}}{r^{\beta}}=\frac{501316.2}{r^{0.725}} \Rightarrow \beta=0.725$.

According to the above expression, one can determine some range of optimal states of the system and graphically display it as a certain band on the rank distribution graph (Fig. 4). 


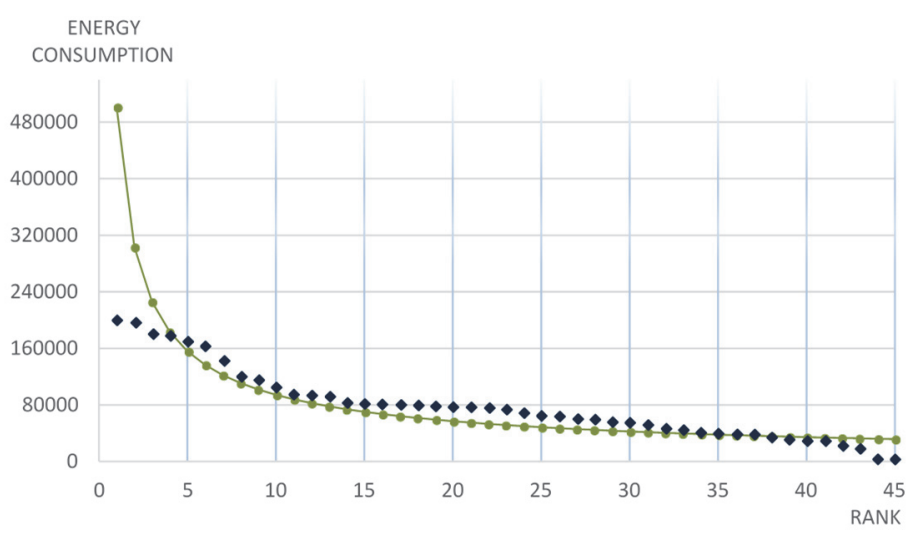

a)

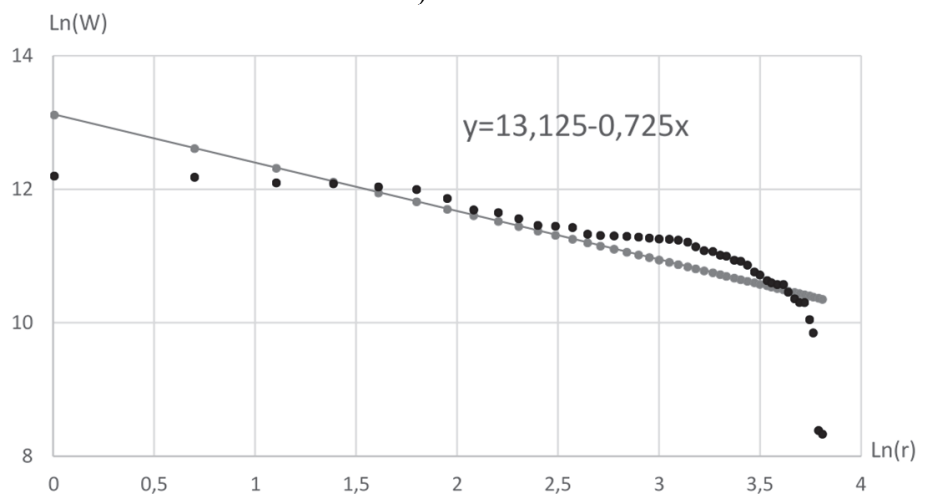

b)

Fig. 4. The parametric distribution of the technocenosis of the TPS:

a) points - empirical data, b) solid line - approximation curve

Fig. 4 shows that the specific power consumption is not optimal enough and needs to be significantly improved. The parameter of the $H$-distribution is not within the range, the energy consumption curve is far removed from the optimal approximation curve, which indicates the inadequate performance of the system as a whole. Needed additional work with technocenose: nomenclature optimization; purposeful removal of abnormal individuals; parametric optimization; improvement of parameters of abnormal individuals, etc.

Rank analysis never ends with the approximation of the relevant technocenose distributions. It always follows optimization, because our main task is to determine the directions and criteria for improving the existing technocenose. The procedure for optimizing any cenosis is aimed at eliminating abnormal deviations in rank distribution. After the detection of anomalies in the graphical distribution of the tabulated distribution, individuals are identified, "responsible" for the anomaly, and identified the priority measures to eliminate them.

The algorithm for conducting rank analysis for optimizing technocenosis includes the following steps:

Step 1. Selection of the cenosis.

Selection of technocenosis is accompanied by its description. For this purpose, a special database is provided, which includes systematized and standardized, sufficiently complete and at the same time, without unnecessary details, information on the types and individuals of technocenosis.

Step 2. Setting of the shaping parameters.

Elements technocenose are allocated on the basis of the database. For each item there must be some documentation in the database. If we consider the case with TPS, then the database should 
contain data on monthly energy consumption.

Step 3. Ranking - parametric description of the cenosis.

The first rank is assigned to an object with the highest energy consumption, further decreasing.

Step 4. Construction of the tabulated rank distribution and the graphical rank parametric distribution of the existing technocenosis.

Step 5. Calculation of the degree of steepness of the hyperbolic $H$-distribution curve. Approximation of distributions.

Step 6: Optimize the technocenosis.

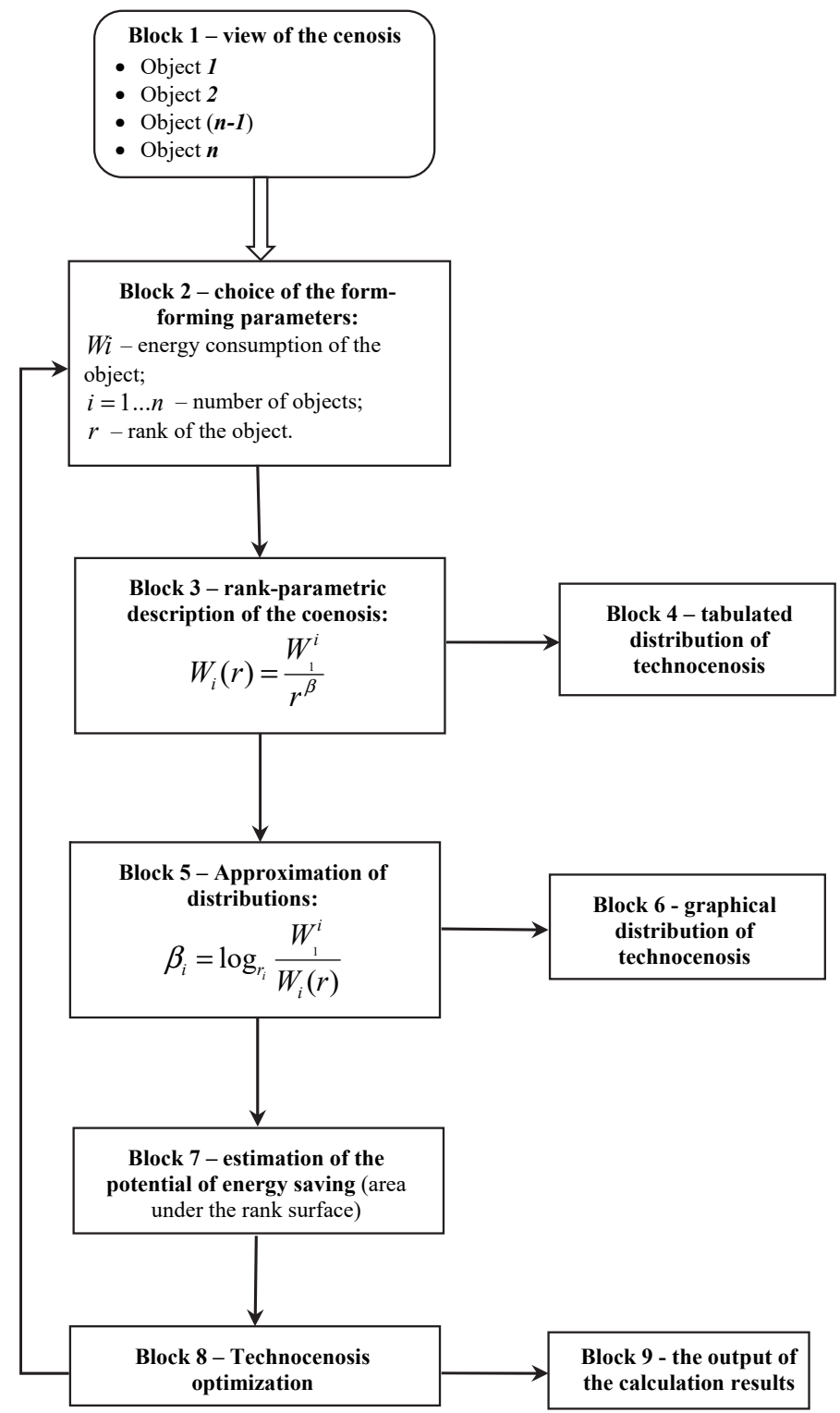

Fig. 5. Block-diagram of algorithm for conducting rank analysis for optimization of technocenosis

The best known technocenosis, which is a set of technical products, individuals who, on the one hand, their combined functional parameters provides performance tasks, and on the other - 
has a maximum entropy, so the total energy resources embodied in technical products for their manufacturing, distributed evenly according to the populations types of equipment. Optimal improvement of the structure proposed tehnotsenozu carried out in two phases: the first phase to determine and optimize the basic types, and the second - to form a species diversity of species and parameters of technical products that meet the requirements dictated by law optimal construction technocenosis.

After this analysis it is possible to proceed with the forecasting of energy consumption on the basis of technocenological approach. The block diagram of the algorithm for conducting rank analysis for the optimization of technocenosis is presented in the Fig. 5.

\section{Conclusions}

The advantage of technocenological method and rank analysis is the optimal reflection of the process of functioning of technocenous objects in the future, taking into account possible changes in technology, infrastructure, and the use of resources. It is a disadvantage to note that the method based on a statistical model, as well as similar methods, with high accuracy calculates the value of short-term forecasting (according to studies, the exact forecast can be obtained for 1 to 2 years, after which the error is sharply increasing). Another disadvantage is the inability to implement criteria based on comparing power management options. These disadvantages can be eliminated. This requires the creation of a dynamic adaptive model that reflects the process of power consumption at a depth of 5 to 7 years or more.

This algorithm can be widely used for solving actual tasks of design, construction and operation of power systems, as well as for improving the quality and reliability of energy supply and implementation of energy saving measures, as well as new projects in the field of alternative energy.

\section{References}

[1] Vasylenko V. System efficiency of functioning of the power system with controlled loads. Power Engineering: Economics, Technology, Ecology. Vol. 1, 2015, p. 70-81.

[2] DSTU ISO 13600-2001 Power Engineering Systems. Basic Provisions, IDT, 1997.

[3] DSTU ISO 13601-2001. Power Engineering Systems. Structure for Analysis. Energy Supply and Consumption Sectors, IDT, 1998.

[4] Gnatyuk V. Technique, Technosphere, Energy Saving. Internet Moscow, 2012.

[5] Gnatyuk V. The Law of Optimal Construction of Technocenosis. Kaliningrad, 2014.

[6] Prokopchik V. Improvement the Quality of Power Supply and Efficiency of Electrical Equipment of Enterprises with Continuous Technological Processes. Gomel, 2002.

[7] Kudrin B. Power Supply for Industrial Enterprises: a Textbook for Students of Higher Educational Institutions. Moscow, 2005.

[8] Kudrin B. Tsenological bases of management by an electric economy of the consumer. Industrial Power, Vol. 9, 2015, p. 38-46.

[9] Denisyuk S., Vasilenko V. Optimal management of energy consumption of budget organizations and institutions as objects of technocenosis. Bulletin of the Kiev National University of Technology and Design, Series Technical Sciences, Vol. 5, Issue 114, 2017, p. 97-105.

[10] Kosharnaya Y. Use of methods of cenological and cluster analysis in energy audits of industrial enterprises. Proceedings of the 7th International Scientific and Practical Internet Conference on Energy and Resource Saving 21 st Century, Orel, 2009.

[11] Fedorova S., Tretyakov A. Application of the technocenologycal approach to the analysis of power consumption and energy saving of enterprises of the Sverdlovsk Region. Bulletin of the South Ural State University, Vol. 16, 2012, p. 92-97. 


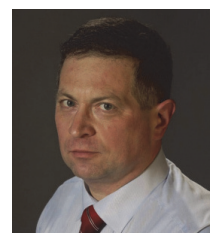

Serhii Denysiuk Doctor of technical sciences, Professor, Director of the Institute of Energy Saving and Energy Management, National Technical University of Ukraine "Igor Sikorsky Kyiv Polytechnic Institute", Kyiv, Ukraine. He is specialist in energy security and energy conservation.

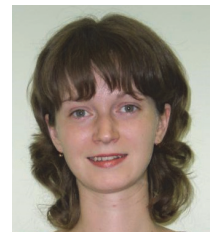

Vira Vasylenko, Assistant of the Institute of Energy Saving and Energy Management, National Technical University of Ukraine "Igor Sikorsky Kyiv Polytechnic Institute", Kyiv, Ukraine. Her current research interests include development of methods for increasing the efficiency of functioning of power supply systems with controlled loads through the implementation of the principles and laws of multi-agent management. 


\title{
5.4. Principles of constructing invariant piezoresonance devices based on adaptive multifrequency systems with a predictive standard
}

\author{
Sergey Pidchenko ${ }^{1}$, Alla Taranchuk ${ }^{2}$ \\ Khmelnitsky National University, Khmelnitsky, Ukraine \\ E-mail: ${ }^{1}$ sergpchn@gmail.com, ${ }^{2}$ allatr@ukr.net
}

\begin{abstract}
The paper represents principles of developing invariant to disturbing factors piezoresonance units with controlled dynamics, which are assumed to be the adaptive control system with predictive reference model. There are formulated the objectives and criteria for terminal control and given results of research of piezoresonance three-frequency oscillation system dynamics on the base of developed mathematical model, containing generalized reduced differential equations, describing character of oscillations' amplitude, phases and voltages of auto-bias in excitation channels. On the base of modified method of simplexes the oscillation system parameter optimization is performed by minimum time criterion for three-frequency oscillation mode setup.
\end{abstract}

Keywords: quartz resonator, quartz crystal oscillator, multi-frequency oscillation system, frequency instability, destabilizing impact factors.

\section{Introduction}

The intensive progress in radio-electronics and measuring technology based on implementing digital and microprocessor technology increases the role of electronic components providing generation of high-stability clock frequency oscillations. The most common sources of reference oscillations are crystal oscillator (XO). Due to high stability of oscillation frequency, minor mass and size parameters and relatively low cost XOs are widely applied to systems for data transmission and radio communication, radio location and radio navigation, radio engineering measurements and telemetry, in computer technology and domestic radio devices. Also, piezoresonance (quartz) auto-generating sensors of physical parameters such as pressure, strain, acceleration, temperature, humidity and others are traditionally applied.

At the same time the further development in functionality of piezorasonance devices (PRD), quartz resonators $(\mathrm{QR})$ and crystal oscillators, filters, piezoresonance transducers of physical parameters, which are undetectable parts of telecommunication and measuring systems, requires improving accuracy of their characteristics: stability of resonance frequency, bandwidth, parameters of generated oscillations and characteristics of physical measure transformations. Design, technological and functional methods (concerning compensation of destabilizing factors) are likely to meet difficult contradiction with modern requirements to microminiaturization, reliability, energy consumption, readiness time and costs.

One of the perspective ways along this line represent functional and algorithmic approaches to provide invariance of PRD to destabilizing factors. It refers to multi-frequency excitation mode of quartz resonator, what allows combining its basic function - frequency determining (stabilizing) function and additional (measuring) function to improve accuracy of the current QR condition identification.

However, proceeding to multi-frequency oscillation mode (MOM) of $\mathrm{QR}$, because of competition of oscillations in excitation channels of QR, significantly complicates PRD dynamics, what may cause a loss of oscillation stability and essential increasing of transition processes in comparison with single-frequency mode. This fact significantly increases requirements to multi-frequency PRD parameter optimization to provide high sustainability and stability of oscillations and also decreasing its readiness time [1-6]. 


\section{The multi-frequency functional-algorithmic methods to ensure invariant piezoresonance devices}

It is known [7], that the absolute coordinates $y_{i}$ of the invariance of influence $x_{j}$ :

$y_{i}(p)=\boldsymbol{\Phi}(p) x_{j}(p), \quad i=\overline{1, n}, j=\overline{1, m}$.

Necessary that:

$\Phi_{i j}(p)=\left(A_{i j_{0}} p^{r}+A_{i j_{1}} p^{r-1}+\cdots+A_{i j_{r}}\right) B_{i j}^{-1}\left(p^{s}, p^{s-1} \ldots p\right) \equiv 0$, $A_{i j_{l}} \equiv 0, \quad \forall l=\overline{0, r}, \quad r \leq s$.

Having presented $A_{i j_{l}}=A_{l}^{(1)}+A_{l}^{(2)}$ (principle of dual channel), we get invariance condition Eq. (2) for passive differential (without identification) system:

$A_{l}^{(1)}=-A_{l}^{(2)}$,

which is satisfied by the selection of parameters of channel, for example, using two piezoresonance oscillation system (POS), which opposite in sign to the temperature coefficient of frequency. In the ideal case $y_{i}(t) \equiv g_{i}(t)$, that the real movement of system is corresponds exactly to the desired.

In practice, however $y_{i}(t)-g_{i}(t)=\varepsilon$, so further considered invariance PRD with accuracy to $\varepsilon$ (the technical invariance). Beside this, because $j>1$, is introduced the concept of multivariate.

In accordance with Eq. (1), PRD represented as multilateral multi-frequency transducer (MFT), which includes $m$ destabilizing channels and $n$ control channels.

It should be noted that the separation of all control channels to control and destabilizing it is a conditional. For example, the invariance of quartz oscillator requires fulfillment of Eq. (2) for all $m+n$ channels, but also the invariance of piezoresonance sensor the one physical quantity requires fulfillment of conditions for $m+n-1$ channels. This choice allows the model with the same position is solve the problem of invariance of all existing PRD [7, 8].

\section{Generalized formalized model of invariant PRD with current identification of impacts}

Generalized invariant structure PRD with current identification impacts is developed on the basis of multiply system with combined management $[5,6]$, there are two regulation contours by the disturbance and the deflection (open-circuited and closed) can be synthesized separately, which is so important for solving the problem of invariance of piezoresonance devices. Given that the question of synthesis of closed circuit is well-researched of modern theory's automatic control, must pay attention to the synthesis of open circuit and solving insufficiently known its main tasks for formation of multi-frequency (multidimensional) information signal and for to control POS and identification of destabilizing impact factors (DIF). In the methodical plan is also important that the system is controlled with a combined basis for synthesis adaptive of systems without search, so that is the proposed approach has the possibility for further development [6].

The principal difference between the proposed formal model (Fig. 1) and traditional method is to measure the vector DIF $\mathbf{X}$. The sensor unit $\mathbf{S}$ is connected directly to the input of controlled perturbations MFT (multi-dimensional object to control) (point a) in the traditional system.

In the proposed system are measurements DIF of carried by identifier I for directly observed vector $\mathbf{Y}_{C 0}$ and a priori known characteristics $\mathbf{A}$ of the converter CCP (converter controlled perturbations). Without considering the vector's uncontrolled effects $\Theta$ is actually observed vector $\Delta \mathbf{Y}_{C}=\Delta \mathbf{Y}_{C 0}+\Delta \mathbf{Y}_{U N C}$, that is leading to identification errors DIF. 


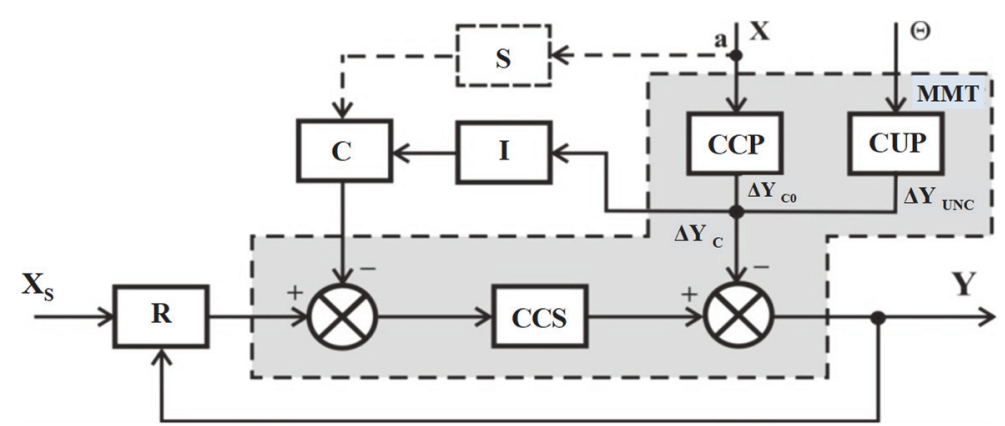

Fig. 1. The formalized model of invariant PRD: $\mathrm{CCP}-$ converter controlled perturbations, CCS - converter control signal, CUP - converter uncontrolled perturbation, $\mathrm{I}$ - identifier, $\mathrm{C}$ - compensator, $\mathrm{R}$ - regulator, $\mathrm{S}$ - sensor unit

We write the condition of invariance of the traditional system to:

$\mathbf{K}_{C 0}=\mathbf{W}^{-1} \mathbf{A} \mathbf{S}_{0}^{-1}$,

where are $\mathbf{K}_{C 0}, \mathbf{S}_{0}$ - the matrixes of the coefficient of transfer compensator (C) and the ideal sensor (S); $\mathbf{W}$ - the matrixes of the coefficient of signal for controlling.

In fact, there is always uncertainty $\mathbf{S}=\mathbf{S}_{0}+\Delta \mathbf{S}$, which is equivalent to the uncertainty compensator $\Delta \mathbf{K}_{S}$. Then:

$\mathbf{K}_{S 0}+\Delta \mathbf{K}_{S}=\mathbf{W}^{-1} \mathbf{A}\left(\mathbf{S}_{0}+\Delta \mathbf{S}\right)^{-1}$.

As $\left(\mathbf{S}_{0}+\Delta \mathbf{S}\right)^{-1}=\left[\mathbf{S}_{0}\left(\mathbf{E}+\mathbf{S}_{0}^{-1} \Delta \mathbf{S}\right)\right]^{-1}=\left(\mathbf{E}+\mathbf{S}_{0}^{-1} \Delta \mathbf{S}\right)^{-1} \mathbf{S}_{0}^{-1}$, where $\mathbf{E}$ - identity matrix, if $\left\|\mathbf{S}_{0}^{-1} \Delta \mathbf{S}\right\| \leq\|\Delta \mathbf{S}\| \cdot\left\|\mathbf{S}_{0}^{-1}\right\|<1$, than $\left(\mathbf{E}+\mathbf{S}_{0}^{-1} \Delta \mathbf{S}\right)^{-1}=\mathbf{E}+\sum_{k=1}^{\infty}\left(-\mathbf{S}_{0}^{-1} \Delta \mathbf{S}\right)^{k}$. Confining the first order of smallness in $\Delta \mathbf{S}$, we get:

$\Delta \mathbf{K}_{\mathrm{S}}=-\mathbf{W A S}_{0}^{-1} \Delta \mathbf{S S}_{0}^{-1}$.

The condition of invariance of the proposed system by $\mathbf{X}$ is written as:

$\mathbf{K}_{I 0}=\mathbf{W}^{-1} \mathbf{I}_{0}^{-1}$.

Acting similarly, we get

$\Delta \mathbf{K}_{I}=-\mathbf{W}^{-1} \mathbf{I}_{0}^{-1} \Delta \mathbf{I I}_{0}^{-1}$,

where $\Delta \mathbf{I}$ characterizes the uncertainty identification of impact factor.

Introducing the Euclidean norm and relative compensation uncertainty $\delta_{K_{I}}=\left\|\Delta \mathbf{K}_{I}\right\| /\left\|\mathbf{K}_{I 0}\right\|$, $\delta_{K_{S}}=\left\|\Delta \mathbf{K}_{S}\right\| /\left\|\mathbf{K}_{S 0}\right\|$, we get:

$\delta_{K_{S}}=\left\|\mathbf{S}_{0}^{-1}\right\| \cdot\|\Delta \mathbf{S}\|, \quad \delta_{K_{I}}=\left\|\mathbf{I}_{0}^{-1}\right\| \cdot\|\Delta \mathbf{I}\|$.

Given that fact, as $\mathbf{I}_{0}=\mathbf{E}$ and $\mathbf{S}_{0}=\mathbf{E}$, we get from Eqs. (6), (8) for the actual value of the system $\|\Delta \mathbf{S}\| \gg\|\Delta \mathbf{I}\|$, and therefore $\delta_{K_{S}} \gg \delta_{K_{I}}[6]$.

\section{Developing invariant piezoresonance units with controlled dynamics}

The architecture of invariant multi-frequency piezoresonance units (PRU) with controlled dynamics (IMPRU/CD) represented as adaptive controlling system with predictive reference model (Fig. 2) is considered. Its main component is the PRU core - multi-frequency 
piezoresonance oscillatory system (MPOS) with supporting control circuits, interfacing, thermal and vibrational stabilization which is exposed to destabilizing disturbing factors. On the base of monitoring signals vector $\mathbf{Y}$ the system of optimal (suboptimal) assessment and identification accomplishes assessment of condition vector $\widehat{\mathbf{X}}_{c}$ and assessment of parameters' vector $\widehat{\mathbf{X}}_{P}$ of PRU mathematical model (parametric identification). The optimal control system, basing on reference model and current assessment of PRU condition vector, forms the vector of control signals $\mathbf{u}$, thereby providing the system to move in accordance to specified optimization criteria and limits $\mathbf{L}$ related to distinguished physical implementation of MPOS. The control signal $\mathbf{u}^{\prime}$ is also used by the optimal assessment and identification system (Fig. 2).

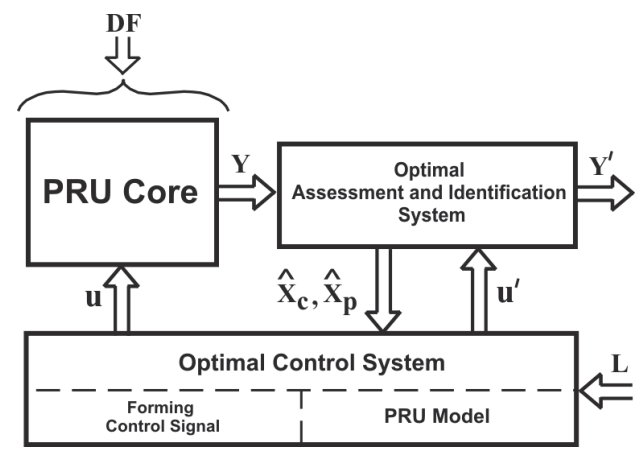

Fig. 2. The generalized architecture of IMPRU/CD

To identify optimal control criteria in accordance to the offered concept for creating IMPRU/CD the control process will be divided into two stages. At the first stage of the setting oscillation the control signal is formed in each excitation channel, which provides coming onto stationary mode for the shortest reachable time to set stable multi-frequency oscillation mode. At the second stage of the stabilizing oscillation the control signal is formed to support stability of generated oscillations in condition of destabilizing factors (providing technical invariance) [9-11].

The problem of control is defined as followed. The mathematical model of the system is specified as the differential equation system:

$\dot{\mathbf{x}}=\Phi(\mathbf{x}, \mathbf{u})$,

at initial condition:

$\mathbf{x}(0)=\mathbf{x}_{0}$,

where $\Phi=\left(\phi_{1}, \ldots, \phi_{n}\right)^{T}$ - vector function of the right part of the Eq. (10) of $\mathrm{n}$ dimension; $\mathbf{x}=\left(x_{1}, \ldots, x_{n}\right)-$ vector of phase coordinate of $\mathrm{n}$ dimension; $\mathbf{u}=\left(u_{1}, \ldots, u_{k}\right)$ - vector of control signals of $\mathrm{k}$ dimension.

The optimal control signal $\mathbf{u}(t)$ is to be found within $t \in[0, T]$ time period for system Eq. (10) at initial condition Eq. (11), providing for each excited oscillation mode the movement $x_{i}(t)$ at needed accuracy on the pass $x_{i}^{*}(t)$ :

$\left|x_{i}^{*}(t)-x_{i}(t)\right| \leq \varepsilon_{i}, \quad i=\overline{1, n}$,

where $\varepsilon_{i}$-specified tracking accuracy.

The movement pass $x_{i}^{*}(t)$ is defined by PRU reference model of the optimal control system (Fig. 2), which allows, on the basis of priori and current information of IMPRU/CD condition, defining the final time $T^{*}$ and optimal pass for coming onto stationary mode of multi-frequency oscillations in accordance to criteria of time setting minimum $[10,11]$ : 
$\tau_{\text {stat }}^{\text {opt }}=\min _{\mathbf{P} \in \mathbf{D} 1 \leq i \leq n} \max _{\text {stat }_{i}}$

$\mathbf{D}=\left\{\mathbf{P} \in \mathfrak{R}^{N}: p_{\text {jmin }} \leq p_{j} \leq p_{\text {jmax }}, 1 \leq j \leq N\right\}$,

where $\tau_{\text {stat }_{i}}$ - time for setting stationary oscillation of mode $\mathrm{i}$, defined by the specified longtime stability of oscillations $\delta_{i}$, assuming structural specificity of PRU and its functionality; $\mathbf{P}_{\min }=\left\{p_{1 \min }, \ldots, p_{N \min }\right\}, \mathbf{P}_{\max }=\left\{p_{1 \max }, \ldots, p_{N \max }\right\}-$ specified vectors of minimal and maximal acceptable values of IMPRU/CD core parameters.

The problem Eqs. (10)-(13) may be considered as a problem of the terminal control according to which within the time $T^{*}$ the system is to transit from initial condition $\mathbf{x}_{0}$ to terminal one:

$\mathbf{x}\left(T^{*}\right)=\mathbf{x}_{T^{*}}: \mathbf{x}_{0} \rightarrow \mathbf{x}_{T^{*}}$

In this case the optimal control algorithm is defined on the basis of minimizing the functional, which represents energy consumption of acceleration:

$G(\mathbf{u})=\frac{1}{2} \sum_{i, j}^{n}\left(\ddot{x}_{i}^{*}(t)-\ddot{x}_{i}(t, \mathbf{u})\right)\left(\ddot{x}_{j}^{*}(t)-\ddot{x}_{j}(t, \mathbf{u})\right)$.

From condition of reaching proximity to absolute minimum:

$\min _{G} G(\mathbf{u})=G\left(\mathbf{u}^{*}\right)=0$.

On control signals $\mathbf{u}[6]$.

Solving problems Eq. (13)-(16) in accordance to characteristics (physical properties) of PRU and its functionality is followed by limitations to oscillation amplitude $U_{i}$, initial frequency run-out $\Delta \omega_{i}$, maximal power of quartz resonator excitation $P_{\text {excit }}$ and its increasing rate on the stage of setting multi-frequency mode for reducing thermal and dynamical instability of oscillation frequency:

$U_{\min } \leq U_{i} \leq U_{\max }$

$\Delta \omega_{i} \leq \Delta \omega_{\text {ultim }}, \quad i=\overline{1, n}$,

$P_{\text {excit }} \leq P_{\text {ultim }}, \quad \frac{d P_{\text {excit }}}{d t} \leq K_{d}^{(P)}$.

Upon reaching terminal condition Eq. (14) the system location relative to set condition $\mathbf{x}_{T^{*}}$ is stabilized. At this stage the minimizing functional Eq. (15) becomes:

$G(\mathbf{u})=\frac{1}{2} \sum_{i, j}^{n}\left(x_{T_{i}^{*}}-x_{i}(t, \mathbf{u})\right)\left(x_{T_{j}^{*}}-x_{j}(t, \mathbf{u})\right)$.

Providing minimizing deviation of the system $\Delta x_{i}=\left|x_{T_{i}^{*}}-x_{i}(t, \mathbf{u})\right|, i=\overline{1, n}$.

\section{Strategy of optimal control for software trajectory motion of PRD with predictive model}

The designed concept for invariant PRD like adaptive system with predictive standard requires solving inverse dynamics problem. It requires determination of the dynamic system motion and its parameters under a condition of performing of the motion corresponding to given trajectory. In accordance to specificity of development and exploiting IMPRU/CD control process is performed by two stages. 
The first stage is the oscillation forming. Control impacts are formed in each exciting channels. The impacts provide the output to the stationary mode under a minimum time of set of stable multi-frequency oscillatory mode.

Second stage is the oscillation stabilization. Control of IMPRU/CD is directed to support of generated oscillations stability under influence of destabilizing factors, i.e. providing technical invariance [6].

We will carry out the construction of optimal or sub-optimal control law according to generalized work criterion that has good results at the stage of analytical construction during a designing period. Using this approach allows not only simplifier the procedure of obtaining the optimal control laws, but often do not obtain the functional dependences due to the cumbersomeness and complexity related to control laws. In such case, solving a problem comes to an end with an algorithm which performs optimization during system functioning and it is convenient from the point of view of a realization of microprocessor control.

Let us consider controlled process in the form of:

$\dot{y}_{i}+f_{i}\left(x_{1}, \ldots, x_{n}, y_{1}, \ldots, y_{m}, t\right)=0, \dot{x}_{j}=u_{j}$,

where $f_{i}$ is differentiable or piecewise differentiable functions; $\mathbf{x}=\left(x_{1}, \ldots, x_{n}\right)$ is the state vector of the control elements; $\mathbf{y}=\left(y_{1}, \ldots, y_{m}\right)$ is the state vector of the control object; $\mathbf{u}=\left(u_{1}, \ldots, u_{m}\right)$ is the control vector, $i=\overline{1, n}, j=\overline{1, m}$.

In considered case, the control of the rates related to the variations of controlled elements is performed. Object non-stationarity can be taken into account by extension its state vector.

Equation of system free movement Eq. (16) can be written as:

$\dot{\mathbf{y}}+f(\mathbf{x}, \mathbf{y}, t)=0, \quad \dot{\mathbf{x}}=0$.

E.g. free system movement is the movement under fixed locations of the control elements.

For the task formulated by using an Eq. (19), we have expanded state vector $(\mathbf{x}, \mathbf{y})$. According to optimal controlling the minimum of the functional [11]:

$I=V_{\text {set }}\left(\mathbf{x}\left(t_{2}\right), \mathbf{y}\left(t_{2}\right)\right)+\int_{t}^{t_{2}} Q(\mathbf{x}, \mathbf{y}, t) d t+\frac{1}{2} \int_{t}^{t_{2}} \sum_{j=1}^{m} \frac{u_{j}^{2}+u_{j o p t}^{2}}{k_{j}^{2}} d t$.

Provides the control of the form of:

$u_{j}=u_{j_{o p t}}=-k_{j}^{2} \frac{\partial V}{\partial x_{j}}$

where $V=V(y, t)$, is the solving the following equation:

$\frac{d V}{d t}-\sum_{i=1}^{n} f_{i} \frac{d V}{d y_{i}}=-Q$

Under boundary condition of $V_{t=t_{2}}=V_{\text {set }} ; f_{i}, Q$, where $V_{\text {set }}$ are given uninterrupted functions; $k_{j}^{2}>0$ are given coefficients.

In order to provide prediction of object behavior, model of free movement must operate in faster mode as $t^{\prime}=t / \chi$, where $\chi=$ const $\gg 1$. Then, the equation of prediction model can be written as: 
$\frac{d \mathbf{y}_{M}}{d t^{\prime}}+\chi \cdot f\left(\mathbf{x}_{M}, \mathbf{y}_{M}, \chi t^{\prime}\right)=0, \frac{d \mathbf{x}_{M}}{d t^{\prime}}=0$

A prediction model has to provide the integration of free movement during total optimization interval from $t=t_{1}$ till to $t_{2}$ by using faster rate under initial conditions given by control (estimation) system. Integration rate that defined by $\chi$ value is selected in such a manner that for each cycle of $\Delta t_{c}$ (in many times less than $t_{2}-t$ ) several runs of a free movement were performed during the interval of $t_{2}-t$. The latter procedures are necessary for numerical evaluation the partial derivations $\partial V / \partial y_{i}$. Because of this, the values $\chi$ in real-life control of a process must be of the value equal to dozens, hundreds and even thousands units.

The beginning of each cycle coincides with current time moment $t$ with accuracy equal to $\Delta t_{c}$. At the beginning of each cycle, control and estimation system operating with real-life controlled process defines the state vector $\mathbf{x}(t)$ and gives initial conditions for free movement model Eq. (22). As a result, the following equality is provided for beginning of each cycle:

$\mathbf{y}_{M}(t)=\mathbf{y}(t)$.

Under free movement of the system, the left part of Eq. (23) transforms to the total derivative under time as:

$\dot{V}=-Q$.

Therefore, we obtain:

$V\left(\mathbf{y}_{M}\left(t_{2}\right)\right)-V\left(\mathbf{y}_{M}\left(t_{1}\right)\right)=-\int_{t_{1}}^{t_{2}} Q\left(\mathbf{y}_{M}, t\right) d t$,

and for a terminal task:

$V\left(\mathbf{y}_{M}\left(t_{2}\right)\right)=V_{\text {set }}\left(\mathbf{y}_{M}\left(t_{2}\right)\right)$.

Thus, for free movement system mode under predictive model, the following computations must be performed:

$V=V_{\text {set }}\left(\mathbf{x}_{M}\left(t^{\prime}{ }_{2}\right), \mathbf{y}_{M}\left(t^{\prime}{ }_{2}\right)\right)+\chi \int_{t^{\prime}=t / \chi}^{t_{2}^{\prime}=t_{2} / \chi} Q\left(\mathbf{x}_{M}, \mathbf{y}_{M}, \chi t^{\prime}\right) d t^{\prime}$.

In order to evaluate by using numerical technique the partial derivations $\partial V / \partial x_{j}$, the variability of initial requirement related to $\mathbf{x}_{M}$ is performed for each run of the model Eq. (24).

Let us consider the features necessary for selection of integrand for minimizing functional $Q(\bullet)$ given in the form Eq. (21). The features depend on the difference between output vector $\mathbf{y}_{M}$ contained in the model Eq. (24) and the estimate of the control process state $\hat{\mathbf{y}}$ :

$Q=Q\left(\mathbf{y}_{M}-\hat{\mathbf{y}}\right)$.

Let us introduce the square functional as:

$Q=\left(\mathbf{y}_{M}-\hat{\mathbf{y}}\right)^{T} \beta\left(\mathbf{y}_{M}-\hat{\mathbf{y}}\right)$,

where $\beta$ is the diagonal matrix contained the elements that are proportional to the maximum errors 
related to the corresponding coordinates (principle of the contribution of the errors).

By exploiting the optimal Kalman filter or suboptimal estimation procedure, it is necessary to define the following:

$\beta=\widehat{\mathbf{R}}^{-1}$,

where $\mathbf{R}$ is the error covariance matrix or their estimates $\widehat{\mathbf{R}}$.

It is also interesting an approach to forming the sequence of quality functional which describes the energy of system movement, for example, the minimum of acceleration energy as:

$Q=\left(\mathbf{y}_{M}-\hat{\mathbf{y}}\right)^{T} \beta\left(\mathbf{y}_{M}-\hat{\mathbf{y}}\right)$.

This approach allows improving the dynamic accuracy for control of transmission processes in IMPRU/CD on the stage of oscillation reconstruction [6].

Several limitations exist for solving the task Eqs. (19)-(24) according to physical peculiarities and functional assignment of PRD (Eq. (17)).

In order to give limitations, expanded state vector $(\mathbf{x}, \mathbf{y})$ can be represented in the form of some space state domain $G$, which we will consider as enclosed and simply-connected. Let us give the penalty function $Q_{f}(\mathbf{x}, \mathbf{y})$, which is equal to zero inside and on the borders of the domain $G$ and rather fast accrue in given domain. Assuming that the domain $G$ is described by the equation $Q_{f}(\mathbf{x}, \mathbf{y})=0$, we represent the integrand function of the functional of quality related to generalized work Eq. (21) as:

$Q=Q_{o p t}+Q_{f}$

For what inside the domain of limitations $Q=Q_{\text {opt }}$. The function $Q_{\text {opt }}$ is determined by the limitations of Eqs. (31)-(33) given inside the domain $G$, and the limitations of Eq. (34) are given for the minimized functional (21) with help of the penalty function $Q_{f}$ Eq. (34).

It should be noted that described above algorithm corresponds to the terminal (quasi-terminal) control state $V_{t=t_{\text {set }}}=V_{\text {set }}$ which is related to the first control stage, e.g. forming stable and multi-frequency oscillating mode in IMPRU/CD. However, it can be simply transformed to the non-terminal algorithm of control by using transition to "sliding" optimization interval for which optimization interval is given as $t_{2}=t+T_{\text {set }}$, where $T_{\text {set }}$ is the given optimization interval. According to that, predictive model performs integration of the Eq. (24) within the interval from $t / \chi$ till to $\left(t+T_{\text {set }}\right) / \chi$. As the function of $V_{\text {set }}$ can be selected, an arbitrary function, including $V_{\text {set }} \equiv 0$.

After achievement the terminal state Eq. (28), stabilization of location of the system relatively given state is performed $V_{0} \equiv V_{S T}$. For this reason, transition to the "sliding" optimization interval $T_{S T}$ is executed.

Let us define productivity of control microprocessor system necessary for realization of technical invariance principle in PRD using adaptive system with predictive standard. If under condition of single-entry numerical integration for optimization interval equal to $T_{S T}$ necessary $N$ operations, then performance of microprocessor device can be approximately estimated as follows:

$n_{M}=\frac{m+1}{2 \Delta t_{c}} N$

It was assumed here that number of components of influence vector which give control process is equal to $\mathrm{m}$ and binary system of numerical differentiation is exploited. Value of $\Delta t_{c}$ is defined by a necessary velocity of the update for given influences. For example, for $m=3, \Delta t_{c}=0.5 \mathrm{~s}$, $N=2 \cdot 10^{5}$ microprocessor device must provide productivity in the level of $n_{M}=8 \cdot 10^{5}$ operations 
per one second $[6,11]$.

\section{Mathematical model of multi-frequency piezoresonance oscillation system}

High effectiveness of multi-frequency oscillating mode is provided by condition of high stability in generated oscillations. This is possible only with using filtering schemes in which QR is embedded in feedback circuit (FC) and is excited in the frequencies close to those of its own consequent resonances. At limited quantity of generated oscillations the other oscillator schemes can be used, for example those of oscillating type [12, 13].

The basic IMPRU/CD core architecture represents MPOS to have principles of creating filtering schemes implemented (Fig. 3). It incorporates passive multi-frequency quartz quadripole unit (MQU) on the base of quartz resonator with $\mathrm{m}$-frequencies generating $z_{q_{j}}$ and $\mathrm{n}$ excitation channels embedding the generalized non-linear component (NLC) and phasing selective feedback circuit (FBC). The automatic bias circuits with complex equivalent resistance $z_{b_{i}}$ are used for stabilizing NLCi operational mode. The selective non-linear circuits $\mathrm{FBC}$ with gain $K_{j i}\left(j w, u_{\Sigma}, \tau\right)$, $j=\overline{1, m}, i=\overline{1, n}$, except for their function to set required amplitude-phase ratio in excitation channels, provide significant reducing competition in oscillations due to their own selective properties $K_{j i}(j w)$ and also automatic adjustment $K_{j i}\left(u_{\Sigma}\right)$ of oscillation amplitudes for fixing the specified (ultimately acceptable) power dissipation on QR.

Fig. 3 has the following symbols' identifications:

- $z_{i n_{\Sigma}}=R_{i n_{\Sigma}} /\left(1+j w \tau_{i n_{\Sigma}}\right), R_{i n_{\Sigma}}^{-1}=\sum_{i} R_{i n_{i}}^{-1}, C_{i n_{\Sigma}}=\sum_{i} C_{i n_{i}}-$ the complex equivalent total resistance of partial FC input circuits;

- $z_{\text {out }_{\Sigma}}=R_{\text {out }_{\Sigma}} /\left(1+j w \tau_{\text {out }_{\Sigma}}\right), R_{\text {out }_{\Sigma}}^{-1}=\sum_{i} R_{\text {out }_{i}}^{-1}, C_{\text {out }_{\Sigma}}=\sum_{i} C_{\text {out }_{i}}$ - the complex equivalent total resistance of output $\mathrm{NLC}_{\mathrm{i}}$ circuits;

- $z_{a b_{i}}=R_{a b_{i}} /\left(1+j w \tau_{a b_{i}}\right)$ - complex resistance of auto-bias circuit $i$

- $\tau_{a b_{i}}=R_{a b_{i}} C_{a b_{i}}, \tau_{i n_{\Sigma}}=R_{i n_{\Sigma}} C_{i n_{\Sigma}}, \tau_{o u t_{\Sigma}}=R_{o u t_{\Sigma}} C_{o u t_{\Sigma}}$ - time constants;

- $i_{\Sigma}=\sum_{i} i_{\text {out }}\left(e_{i}\right)$ - total current of $\mathrm{NLC}_{\mathrm{i}} ; e_{i}(\tau)=\sum_{j} v_{i}(\tau)+E_{i}+\tilde{E}_{i}(\tau)$ - control voltage in NLCi input, where $E_{i}, \tilde{E}_{i}(\tau)$ - constant and variable components of auto-bias voltage;

- $u_{\Sigma}(\tau)=U_{0}(\tau)+\sum_{j} U_{j}(\tau) \cdot \cos \left[w_{j} t+\varphi_{j}(\tau)\right]-$ the total voltage in FC circuit input, where $U_{j}(\tau), w_{j}$ and $\varphi_{j}(\tau)$ - envelope, frequency and phase of oscillation $j$ correspondently, $\tau=t-t_{0}-$ time interval from initial moment $t_{0}$ (MPOS startup moment) [6].

MPOS operation (Fig. 3) is described by the system of differential equations:

$$
\begin{aligned}
& u_{\Sigma}=Z(p) \cdot \sum_{i} i_{\text {out }_{i}}\left(\sum_{j} K_{j i} \cdot u_{\Sigma}, \tilde{E}_{i}, E_{i}\right), \\
& E_{i}=-z_{\text {ab }_{i}}(p) \cdot i_{\text {out }_{i}}\left(\sum_{j} K_{j i} \cdot u_{\Sigma}, \tilde{E}_{i}, E_{i}\right),
\end{aligned}
$$

where:

$Z(p)=\frac{z_{\text {in }_{\Sigma}}(p) z_{\text {out }}(p)}{z_{\text {in }}(p)+z_{\text {out }}(p)+z_{q}(p)^{\prime}}$

Is symbolic gain of MQU, representing control resistance of MPOS; $p \equiv d / d t$.

Representing control resistance $Z(p)$ Eq. (12) as the ratio: 
$Z(p)=\frac{\delta \cdot P(p, \delta)}{Q(p, \delta)}$

where $P(p, \delta), Q(p, \delta)$ - polynomials of $p ; \delta$ - low parameter, physically determined for resonance systems as damping ratio, and taking into account that in the general case solving system Eq. (36) is as sum of oscillations with frequencies close to resonance ones of QR $\omega_{q_{j}}$ :

$u_{\Sigma}=\sum_{j=1}^{m} U_{j}(\tau) \cos \left(\omega_{q_{j}} t+\varphi_{j}(\tau)\right)$

where $U_{j}(\tau)$ and $\varphi_{j}(\tau)$ - slowly changing parameters, the output current of NLCi with low parameter $\delta$ accuracy can be written as:

$i_{\text {out }_{i}}\left(e_{i}\right)=I_{o_{i}}\left(V_{j i}, E_{i}, \tilde{E}_{i}\right)+\sum_{j=1}^{m} I_{j i}\left(V_{j i}, E_{i}, \tilde{E}_{i}\right) \cos \left[\omega_{q_{j}} t+\psi_{j i}(t)\right]$,

where $I_{o i}-$ constant component; $I_{j i}=\bar{S}_{j i}\left(V_{j i}, E_{i}, \tilde{E}_{i}\right) V_{j i}=\bar{S}_{j i}\left(V_{j i}, E_{i}, \tilde{E}_{i}\right) U_{j} K_{j i} ; \psi_{j i}=\varphi_{j}+\Delta \varphi_{j i}$; $K_{j i}, \Delta \varphi_{j i}$ - gain and phase shift of circuit $i$ of $\mathrm{FC}$ for resonance frequency $j$ of $\mathrm{QR}$; $\bar{S}_{j i}\left(V_{j i}, E_{i}, \widetilde{E}_{i}\right)=\frac{1}{V_{j i}} L\left[i_{\text {out }}\left(V_{j i}, E_{i}, \widetilde{E}_{i}\right)\right]$ - mean slope for oscillation $j ; \underset{j}{L}[\bullet]-$ mean operator.

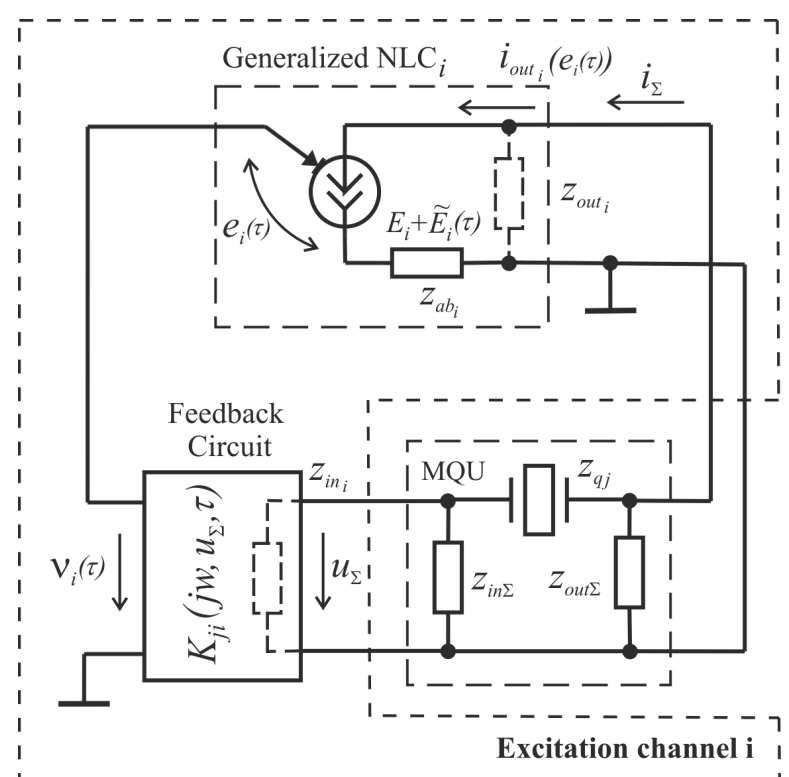

Fig. 3. The basic $\overline{\mathrm{IM}} \overline{\mathrm{PR}} \overline{\mathrm{U}} / \overline{\mathrm{C}} \overline{\mathrm{D}}$ core architecture

Having entered complex amplitudes $\dot{U}_{j}=U_{j} \exp \left(j \varphi_{j}\right), \dot{I}_{j i}=I_{j i} \exp \left(j \psi_{j i}\right)$ and complex $\mathrm{FC}$ gain $\dot{K}_{j i}=K_{j i} \exp \left(j \Delta \varphi_{j i}\right)$, and split real and imaginary units the reduced equations for amplitudes, MPOS oscillation phases and auto-bias voltages become $[6,12,13]$ :

$T_{j} \frac{d U_{j}}{d t}=\left[R_{e_{j}} K_{\phi_{j}} \sum_{i=1}^{n} \bar{S}_{j i}\left(V_{j i}, \tilde{E}_{i}, E_{i}\right) \cdot K_{j i} \cos \left(\Delta \varphi_{j i}+\Delta_{\phi_{j}}\right)-1\right] \cdot U_{j}$, 


$$
\begin{aligned}
& T_{j} \frac{d \varphi_{j}}{d t}=R_{e_{j}} K_{\phi_{j}} \sum_{i=1}^{n} \bar{S}_{j i}\left(V_{j i}, \tilde{E}_{i}, E_{i}\right) \cdot K_{j i} \sin \left(\Delta \varphi_{j i}+\Delta_{\phi_{j}}\right)-\Delta \omega_{j} T_{j}, \\
& T_{a b_{i}} \frac{d \tilde{E}}{d t}=-\left[R_{a b_{i}} I_{0 i}\left(V_{j i}, \tilde{E}_{i}, E_{i}\right)+\tilde{E}_{i}\right],
\end{aligned}
$$

or as generalized matrix:

$$
\begin{aligned}
& \mathbf{T} \frac{d \mathbf{U}}{d t}=\left[\mathbf{G}_{R} \cdot \mathbf{R}-\mathbf{E}_{(m m)}\right] \cdot \mathbf{U}, \\
& \mathbf{T} \frac{d \Phi}{d t}=\left[\mathbf{G}_{I} \cdot \mathbf{R}-\Delta\right] \cdot \mathbf{E}_{(m 1)}, \\
& \mathbf{T}_{a b} \frac{d \tilde{\mathbf{E}}}{d t}=-\left(\mathbf{R}_{a b} \cdot \mathbf{I}_{0}+\tilde{\mathbf{E}}\right),
\end{aligned}
$$

where $\mathbf{T}=\operatorname{diag}\left(T_{1}, \ldots, T_{m}\right), \mathbf{T}_{a b}=\operatorname{diag}\left(\tau_{a b_{1}}, \ldots, \tau_{a b_{n}}\right)-$ time constant matrixes of partial oscillation system $T_{j}=2 / \omega_{q_{j}} \delta_{j}$ of $m \times m$ dimensions and auto-bias circuits NLCi of $n \times n$ dimensions; $\mathbf{U}=\left(U_{1}, \ldots, U_{m}\right)^{T}, \Phi=\left(\varphi_{1}, \ldots, \varphi_{m}\right)^{T}$ and $\tilde{\mathbf{E}}=\left(\tilde{E}_{1}, \ldots, \tilde{E}_{n}\right)^{T}-$ vectors of oscillation amplitudes and phases of $m$ dimension and vector of auto-bias voltage of $n$ dimension; $\mathbf{G}_{R}=\operatorname{Re} \dot{\mathbf{G}}=|\dot{\mathbf{G}}| \cos \Delta \varphi^{T}, \mathbf{G}_{I}=\operatorname{Im} \dot{\mathbf{G}}=|\dot{\mathbf{G}}| \sin \Delta \varphi^{T}$ - matrixes of real and imaginary units of equivalent complex conductance of active part of $\operatorname{MPOS}|\dot{\mathbf{G}}|=\left(\bar{S}_{j i} K_{j i}\right)_{j=1, i=1}^{m, n}$ of $m \times n$ dimensions; $\mathbf{R}=\mathbf{R}_{e} \cdot \mathbf{K}_{\phi}$ - matrix of reduced resistances; $\mathbf{R}_{e}=\operatorname{diag}\left(R_{e_{1}}, \ldots, R_{e_{m}}\right)$ - matrix of equivalent resistances $R_{e_{j}}=R_{i n_{\Sigma}} \cdot R_{o u t_{\Sigma}} /\left(R_{i n_{\Sigma}}+R_{o u t_{\Sigma}}+R_{q_{j}}\right)$ of $m \times m$ dimensions, $\mathbf{K}_{\phi}=\operatorname{diag}\left(K_{\phi_{1}}, \ldots, K_{\phi_{m}}\right)-$ matrix of equivalent phase link gains $K_{\phi_{j}}=1 / \sqrt{1+\left(\omega_{q_{j}} \cdot T_{\phi_{j}}\right)^{2}}$, $T_{\phi_{j}} \approx R_{i n_{\Sigma}}\left(C_{0}+C_{i n_{\Sigma}}\right)+R_{\text {out }}\left(C_{0}+C_{\text {out }}\right)$ - time constant of equivalent phase link, providing phase shift $\Delta \varphi_{\phi_{j}}=-\operatorname{arctg}\left(\omega_{q_{j}} \cdot T_{\phi_{j}}\right)$ between immediate values of voltage $u(t)$ and current $i(t) ; \omega_{q_{j}}, C_{0}$ - resonance frequency and parallel capacity of QR; $\Delta \varphi=\left(\Delta \varphi_{j i}+\Delta \varphi_{\phi_{j}}\right)_{j=1, i=1}^{m, n}-$ matrix of $m \times n$ dimensions, which provides phase relations in excitation channels of MPOS; $\Delta=\operatorname{diag}\left(\Delta \omega_{1} T_{1}, \ldots, \Delta \omega_{m} T_{m}\right) \quad-\quad$ matrix of generalized detuning, $\Delta \omega_{j}=\omega_{q_{j}}^{2}\left(R_{\text {in }_{\Sigma}} \tau_{\text {in }_{\Sigma}}+R_{\text {out }} \tau_{\text {out }}\right) / 2 Q_{q_{j}} R_{q_{j}}$ - frequency adjustment to oscillation $j$ explained by the fact that reduction was produced relative to natural frequencies of $\mathrm{QR} \omega_{q_{j}}$ with no respect to phase-shift in FC circuits; $Q_{q_{j}}, R_{q_{j}}$ - quality factor and dynamic resistance of QR for oscillation frequency $j ; \mathbf{R}_{a b}=\operatorname{diag}\left(R_{a b_{1}}, \ldots, R_{a b_{n}}\right)$ - matrix of auto-bias resistance of $n \times n$ dimensions; $\mathbf{I}_{0}=\left(I_{0_{1}}, \ldots, I_{0_{n}}\right)^{T}-$ vector of constant components of output currents $i_{\text {out }_{i}}\left(e_{i}\right) ; \mathbf{E}_{(m m)}$, $\mathbf{E}_{(m 1)}$ - unit matrix of $m \times m$ dimensions and unit column vector $m \times 1 ; m$ - quantity of generating frequencies, $n$ - quantity of excitation channels.

\section{Dynamics of multi-frequency piezoresonance oscillation system}

The case considers three-frequency excitation of the main mode of oscillations of multi-frequency QR and two additional ones - informative: temperature and vibration $(m=n=3)[6,14]$. At polynomial approximation the output current of NLCi (Fig. 2) can be represented as:

$i_{\text {out }_{i}}\left(e_{i}\right)=I_{s}\left[\hat{a}_{0}+\sum_{r=1}^{k} \hat{a}_{r}\left(\hat{v}_{i}+X_{b_{i}}\right)^{r}\right], i=\overline{1, n}, k=3,5$, 
where $\hat{v}_{i}=v_{i} \cdot S_{0} / I_{s}-$ normalized control voltage; $X_{b_{i}}=\bar{x}_{b_{i}}+\tilde{x}_{b_{i}}, \bar{x}_{b_{i}}=\left(E_{i}-E_{s}\right) \cdot S_{0} / I_{s}$, $\tilde{x}_{b_{i}}=\tilde{E}_{i} \cdot S_{0} / I_{s}-$ constant and variable components of normalized bias; $\hat{a}_{0}=a_{0} / I_{s}$, $\hat{a}_{r}=a_{r} \cdot I_{s}^{(r-1)} / S_{0}^{r}-$ normalized coefficients of approximating polynomial; $I_{s}, E_{s}-$ the coordinates of the point in the center of volt-amps diagram (VAD) with maximal gain slope $S_{0}$.

Having used trigonometry formulas of multiple argument and averaging accordantly to Eq. (41), the spectral components in NLCi input at approximation by polynomial of the third degree will be written as:

$I_{j i}=V_{j i} \cdot \bar{S}_{j i}=V_{j i} \cdot S_{0}\left[A_{1 i}+0,75 \cdot \hat{a}_{3} B_{1 i}\right]$,

$I_{0_{i}}=I_{s}\left[A_{2 i}+0,5\left(\hat{a}_{2}+3 \hat{a}_{3} X_{\mathrm{b}_{i}}\right) B_{2_{i}}\right]$.

Having substituted the expressions (49), (50) into reduced Eqs. (42)-(44) and assuming $K_{\phi_{j}} \approx 1$ and $\Delta \varphi_{\phi_{j}} \approx 0$, the three-frequency MPOS $(m=3)$ motion equation with three non-linear components $(n=3)$ will be obtained:

$\xi_{j} \frac{d \widehat{U}_{j}}{d \hat{t}}=S_{0} R_{e_{j}} K_{j j} \cdot \widehat{U}_{j}\left(\sum_{i=1}^{3}\left(A_{1_{i}}+0,75 \hat{a}_{3} B_{1_{i}}\right) \cdot \gamma_{j i}^{2} \frac{K_{i i}}{K_{j j}}-\frac{1}{S_{0} R_{e_{j}} K_{j j}}\right)$,

$\xi_{j} \frac{d \varphi_{j}}{d \hat{t}}=S_{0} R_{e_{j}} K_{j j} \cdot \sum_{i=1}^{3}\left(A_{1_{i}}+0,75 \hat{a}_{3} B_{1_{i}}\right) \cdot \gamma_{j i} \sqrt{1-\gamma_{j i}^{2}} \frac{K_{i i}}{K_{j j}}-\xi_{j} \Delta \omega_{j}$,

$\mu_{i} \frac{d X_{b_{i}}}{d \hat{t}}=-S_{0} R_{a b_{i}} \cdot\left(A_{2_{i}}+\frac{X_{b_{i}}}{S_{0} R_{a b_{i}}}+0,5\left(\underline{a}_{2}+3 \underline{a}_{3} X_{b_{i}}\right) B_{2_{i}}\right)+\bar{x}_{b_{i}}$.

The correspondent Eqs. (51)-(53) at approximating transfer characteristics by polynomial of the fifth degree (48) are given in [6].

The following symbols used in Eqs. (51)-(53) stand for:

$A_{1_{i}}=\sum_{r=1}^{3} r \hat{a}_{r} \cdot X_{b_{i}}^{(r-1)}, \quad A_{2 i}=\hat{a}_{0}+\sum_{r=1}^{3} \hat{a}_{r} \cdot X_{b_{i}}^{r}$

$B_{1_{i}}=K_{i i}^{2} \sum_{k=1}^{3} b_{k i j} \cdot \widehat{U}_{k}^{2}, \quad B_{2 i}=K_{i i}^{2} \sum_{j=1}^{3} \gamma_{j i}^{2} \cdot \widehat{U}_{j}^{2}$.

$b_{k i j}=\gamma_{k i}^{2}$, for $k=j$ and $b_{k i j}=2 \gamma_{k i}^{2}$, for $k \neq j ; \gamma_{j i}=\frac{K_{j i}}{K_{i i}}=\cos \Delta \varphi_{j i}-$ coefficients of suppressing inter-channel interference (parasite) oscillations $(j \neq i) ; \widehat{U}_{j}=S_{0} U_{j} / I_{s}$ - normalized voltage in MQU output; $\xi_{j}=\frac{T_{j}}{\max _{1 \leq j \leq 3} T_{j}}, \mu_{i}=\frac{T_{a b_{i}}}{\max _{1 \leq j \leq 3} T_{j}}-$ normalized time constants of partial oscillation system $j$ and bias circuit $i ; \hat{t}=\frac{t}{\max _{1 \leq j \leq 3} T_{j}}-$ normalized time; $j=\overline{1,3}, i=\overline{1,3}$.

Activity of excited oscillations is determined by regeneration parameter to be found from (51) when $\widehat{U}_{j}=0$ :

$K_{j}^{r e g}=R_{e_{j}} K_{j j} S_{s_{j}}=R_{e_{j}} K_{j j} S_{0} \times \sum_{i=1}^{3}\left(\sum_{r=1}^{3,5} r \hat{a}_{r} X_{s_{j}}^{(r-1)}\right) \gamma_{j i} \frac{K_{i i}}{K_{j j}}$,

where $S_{s_{j}}$ - gain slope of volt-amps diagram in still point for oscillation $j ; X_{s_{j}}$ - bias in the still 
point for NLCi, being the solution for non-linear equation:

$S_{0} \cdot R_{\mathrm{a} b_{i}}\left(\hat{a}_{0}+\frac{X_{b_{i}}}{S_{0} \cdot R_{\mathrm{a} b_{i}}}+\sum_{r=1}^{3} \hat{a}_{r} \cdot X_{b_{i}}^{r}\right)-\bar{x}_{b_{i}}=0$.

Figs. 4, 5 shows distinguished case of stabilizing oscillations in three-frequency MPOS, as result of numerical integrating Eqs. (51)-(53). The bipolar transistors KT368 (2SC9018) are used as active components (NLCi), which have average statistical values of VAD parameters: $S_{0}=0.1 \mathrm{~A} / \mathrm{V} ; \hat{a}_{0}=0.95 ; \hat{a}_{1}=0.55 ; \hat{a}_{2}=0.051 ; \hat{a}_{3}=-0.054$, when approximation error $\varepsilon \leq 5 \%$. Initial values of low-varying parameters made: $T_{j}=0.1 \mathrm{~s} ; T_{a b_{i}}=1 \cdot 10^{-4} \mathrm{~s} ; R_{a b_{i}}=1 \mathrm{k} \Omega$; $K_{j j}=-20 \mathrm{~dB} ; \bar{x}_{b_{i}}=30$.

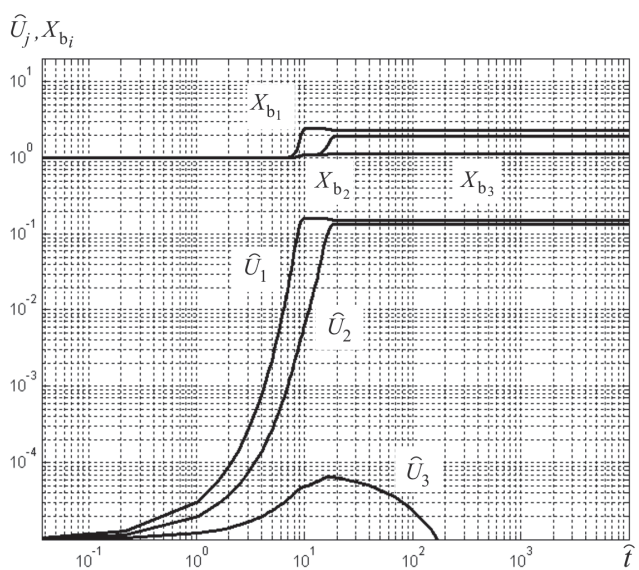

a)

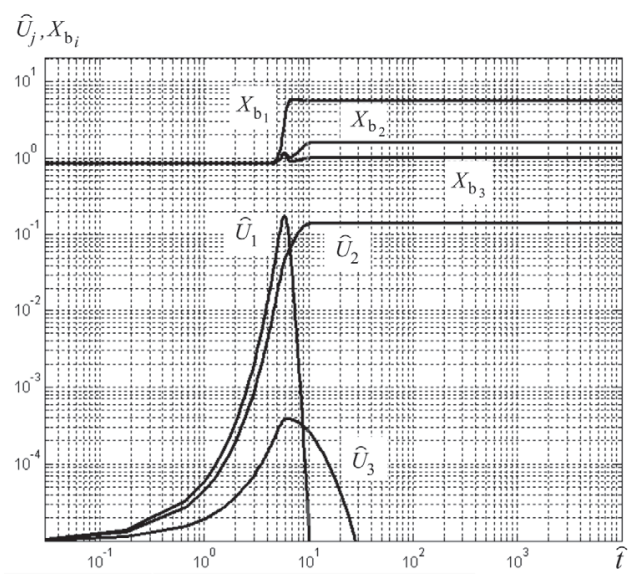

b)

Fig. 4. Distinguished case of appearing competition of oscillations in excitation channels of MPOS: a) suppressing energetically less active oscillations,

b) suppressing energetically more active oscillation by less active one

It's obvious that high competition in excitation channels $\left(\gamma_{j i}=-6 \mathrm{~dB}\right)$ can bring to situations when oscillations with low energetic activity (oscillation $\widehat{U}_{3}$, Fig. 4(a), (b)) can be suppressed by more active oscillations (with high regeneration coefficient $K_{j}^{r e g}$ ). At the same time the overly high increasing regeneration parameter $K_{j}^{\text {reg }}$ can cause a breakdown of more energetically active oscillations (oscillation $\widehat{U}_{1}$, Fig. 4(b)) in excitation channels of MPOS. Reducing competition of oscillations due to increasing selective properties of FC circuits down to $\gamma_{j i}=-12 \mathrm{~dB}$ provides stable three-frequency mode of oscillation (Fig. 5 (a)) even when regeneration coefficient values $K_{1}^{r e g}=1.25, K_{2}^{r e g}=1.5 K_{3}^{r e g}$ are growing significantly $[6,14,15]$.

In this case it assumes using the term of low or high active oscillation (oscillations) in relation to the other oscillations of MPOS, which, being energetically all-sufficient in one-frequency mode, may fade on transiting to multi-frequency mode of oscillations in the certain conditions.

Fig. 6(a) represents approximated relationships of oscillation stabilizing time $\tau_{\text {stat }}=\max _{1 \leq j \leq m} \tau_{\text {stat }_{j}}$ at various partial coefficients of FC. It also has bifurcation curve that determines a range of stable MOM. Evidently, at high values of variations $\chi=\max _{1 \leq j \leq m} K_{j}^{r e g} / \min _{1 \leq j \leq m} K_{j}^{r e g}=\max _{1 \leq j \leq m} R_{e_{j}} / \min _{1 \leq j \leq m} R_{e_{j}}$ of regeneration coefficients the oscillation stabilizing time $\tau_{\text {stat }}$ is significantly increasing (by an order and more!). Compensation of this events when having the great scatter of equivalent resistances $R_{e_{j}}$ is possible by selecting appropriate coefficients $K_{j j}$ of partial FC (increasing energy of oscillations), what it's not only 
improving dynamic properties of MPOS, but also brings to extending range of stable MOM (Fig. 6(a)).

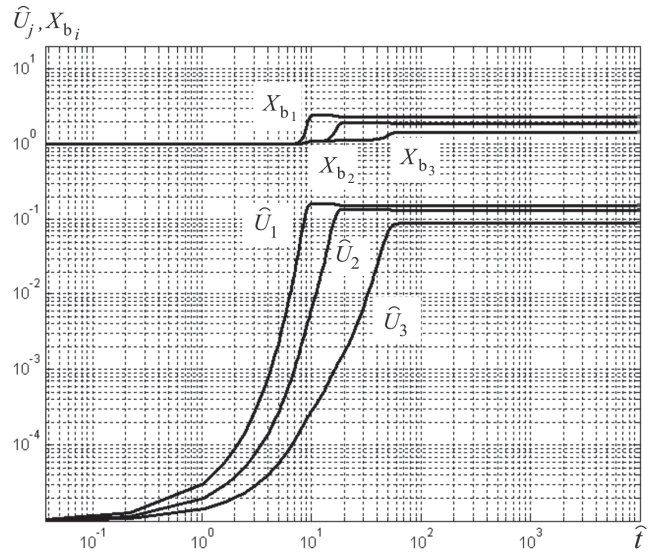

a)

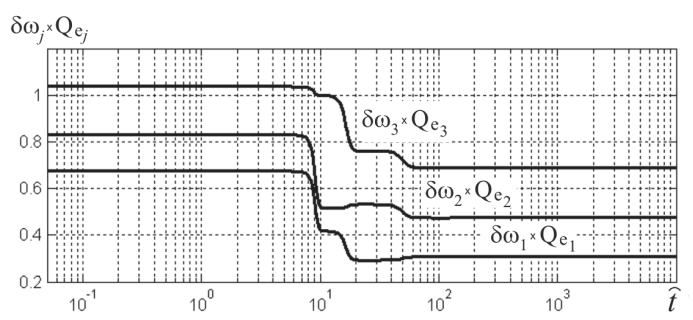

b)

Fig. 5. Stable three-frequency oscillation mode: a) setting oscillation amplitudes and auto-bias voltages, b) initial run-out of oscillation frequencies, caused by amplitude and phase conversion in excitation channels

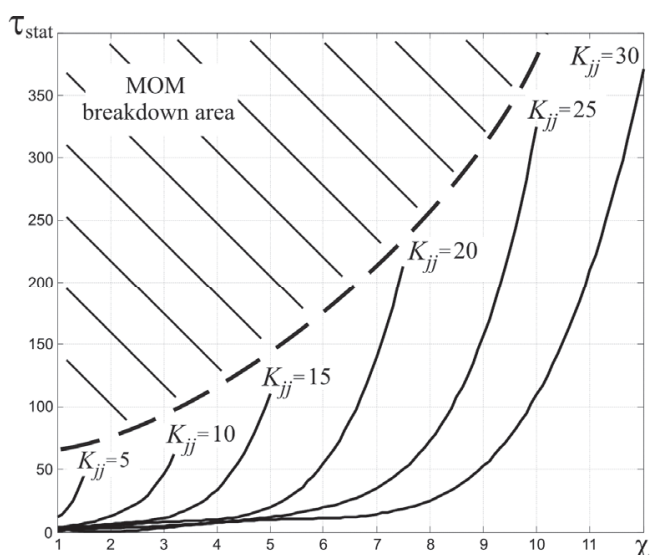

a)

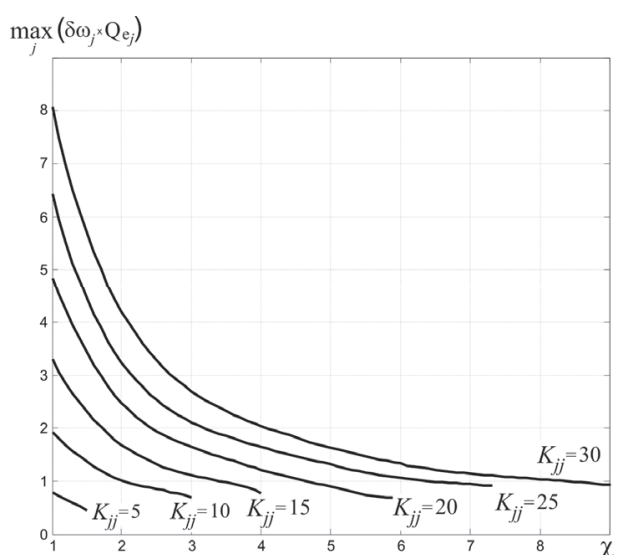

b)

Fig. 6. a) Dependences of normalized oscillation stabilizing time and b) initial frequency run-out on MPOS parameters

The quite important characteristic of MPOS dynamics is group run-out of oscillation frequencies $\delta \omega_{j} Q_{e_{j}}$, which is defined according to Eq. (52). The modeling results indicate that amplitude-phase conversion slightly exhibits for energetically "weak" oscillations (Fig. 5(b)). At joint control of oscillation amplitude and voltage of auto-bias the relative run-out of frequencies $\delta \omega_{j}=\left(\omega_{q_{j}}\right)^{-1} \cdot \Delta \omega_{j}(\hat{t})=\left(\omega_{q_{j}}\right)^{-1} \cdot \frac{d \varphi_{j}}{d \hat{t}}$ may reach values from $10^{-8}$ to $10^{-6}$, and significantly reduce on increasing coefficients $K_{j j}$ of partial circuits of FC (Fig. 6(b)).

\section{Strategy ground for optimal parameters search in MPOS}

A. General statement of PRD parametric synthesis.

In the general statement of MPOS parametric synthesis the required solution will be found by criterion Eq. (13): 
$\tau_{\text {set }}^{\text {opt }}=\min _{\lambda \in D} \tau_{\text {set }}(\lambda)$

$D=\left\{\lambda \in R^{N}: \lambda_{i_{\min }} \leq \lambda_{i} \leq \lambda_{i_{\text {max }}}, 1 \leq i \leq N\right\}$

where duration of transient process $\tau_{\text {set }}$ is determined by solving Eqs. (51)-(53); $\lambda_{\min }=\left(\lambda_{1_{\min }}, \ldots, \lambda_{N_{\min }}\right), \lambda_{\max }=\left(\lambda_{1_{\max }}, \ldots, \lambda_{N_{\max }}\right)-$ vectors set for minimally and maximally acceptable values of parameters of MPOS circuits.

Taking into account that the requirements to expanding stable oscillation range and shortening transient process duration become in contradiction with requirements to stability of generated oscillations poses additional limitations to values of oscillation amplitudes and initial frequency deflections $\widehat{U}_{j}$ and $\delta \omega_{j}$ Eq. (17):

$$
\begin{aligned}
& \max _{1 \leq j \leq m} \widehat{U}_{j} \leq \widehat{U}_{\text {lim }}, \\
& \max _{1 \leq j \leq m} \widehat{U}_{j} / \min _{1 \leq j \leq m} \widehat{U}_{j} \leq l, \\
& \max _{1 \leq j \leq m} \delta \omega_{j} \leq \delta \omega_{\text {lim }},
\end{aligned}
$$

where $\max _{1 \leq j \leq m} \widehat{U}_{j}, \min _{1 \leq j \leq m} \widehat{U}_{j}$ - maximal, minimal values of MPOS oscillation amplitudes; $\widehat{U}_{\text {lim }}-$ maximal limit of oscillation amplitudes, which is determined by maximally acceptable dissipation power in QR; $l$ - coefficient that defines maximally acceptable amplitude difference $\widehat{U}_{j}$, normally $l=(1.5, \ldots, 3)[13-15]$.

To solve synthesis problem (56), (57) the following circumstances are considered:

- high complexity of the optimization object, that often prevents analytical finding interconnection between oscillation setup time $\tau_{\text {set }}$ and MPOS parameters vector $\lambda$;

- complex, non-linear and possibly multi-extreme character of the objective function at the large number of limitations;

- large number of possible vector $\lambda$ parameters, wide diapason of their vibration, therewith initial values of parameters can be outlying the optimal range;

- necessity of considering parameter scatter for real elements and calculation errors, and providing physical realization of MPOS.

B. Method for optimal parameter search in MPOS.

All existing methods for solving optimization problems can actually be divided into two groups: methods based on using as values of function to be minimized so its derivatives (gradient, first or second order methods); and algorithms, which use only values of function to be minimized (non-gradient or direct search methods).

Considering specificity of MPOS (51)-(53) dynamics equations for solving parametric optimization problem the most acceptable methods are direct search methods (non-gradient), which are based on calculation of only values of objective function. Ones of the most effective direct search methods are simplex methods, which can be easily modified for solution of conditional optimization problems (for example, $S^{2}$ - method or Hooke-Jeeves method) [16].

For parametric synthesis of PRD in the mode of quartz resonator multi-frequency excitation the modified method of $S^{2}$ or method of complexes is used. The basis of this method is building regular simplex, which is represented in $N$-dimensional space as polyhedron built by $N+1$ points - vertexes equally distanced from one another. For example, in case of two variables the correct simplex is equilateral triangle, and in three-dimensional space - tetrahedron.

To build new simplex on the plane the vertex with highest value of the objective function is moved to the needed distance along the line, which is drawn through the gravity center of other vertexes of simplex. The point obtained in this way is the vertex of new simplex, and the vertex selected in initial simplex is excluded. Therewith only one additional value of objective function is to be calculated. The search is finalized when simplex dimensions or differences in function 
values in vertexes become quite small values, which are determined by maximally acceptable error tolerance.

Realization of complex method requires the following operations. The rigid acceptable point $\lambda_{0}$, reflection parameter $\beta$ (recommended value $\beta=1.3$ ) and calculation errors $\varepsilon$ and $\delta$ are set in advance.

C. Building initial complex.

1) Finding coordinate $\lambda_{i}^{N}$ :

$\lambda_{i}^{N}=\lambda_{0}^{N}+\Xi_{i}^{N}, \quad i=\overline{1, S}$,

where $\Xi^{N}$ - vector-row of evenly distributed numbers of $N$ dimension, for each the following is true $\left\|\Xi^{S}\right\| \ll R ; R$ - radius of gravity hyperbole of the objective function minimum; $S=N+1$.

2) Finding center of gravity: if $\lambda_{i}^{N}$ - unacceptable point, then center of gravity is found for previously found points:

$\hat{\lambda}=\frac{1}{i} \sum_{i} \lambda_{i}^{N}$,

and assumption is made that:

$\lambda_{i_{\text {new }}}^{N}=\lambda_{i}^{N}+0.5\left(\hat{\lambda}-\lambda_{i}^{N}\right)$.

Procedure is repeated until $\lambda_{i_{\text {new }}}^{N}$ becomes acceptable.

The operations $(\mathrm{C} 1, \mathrm{C} 2)$ are repeated until sufficient number of points is obtained $(i=S)$, then the setup time is defined as $\tau_{\text {set }_{i}}\left(\lambda_{i}^{N}\right)$.

D. Complex reflection.

1) Point selection $\lambda_{\max }^{N}$ :

$\tau_{\max }=\tau_{\text {set }}\left(\lambda_{\max }^{N}\right)=\max _{i} \tau_{\text {set }_{i}}\left(\lambda_{i}^{N}\right)$.

2) Finding gravity center $\hat{\lambda}$ and next point:

$\lambda_{\text {repl }}^{N}=\hat{\lambda}+\beta\left(\hat{\lambda}-\lambda_{\text {max }}^{N}\right)$.

3) Verification of the conditions: if $\lambda_{\text {repl }}^{N}$ - acceptable point and $\tau_{\text {set }}\left(\lambda_{\text {repl }}^{N}\right)<\tau_{\text {max }}$, the verification of conditions $(\mathrm{E})$ is proceeded;

4) Verification of the conditions: if $\lambda_{\text {repl }}^{N}$ - unacceptable point or $\tau_{\text {set }}\left(\lambda_{\text {repl }}^{N}\right) \geq \tau_{\text {max }}$, the distance between $\lambda_{\text {repl }}^{N}$ and gravity center $\hat{\lambda}$ is two times reduced, the search is continued until condition (D3) is fulfilled.

E. Verification of the conditions of finalizing calculations.

1) Definitions:

$\left\langle\tau_{\text {set }}\right\rangle=\frac{1}{S} \sum_{i=1}^{S} \tau_{\text {set }_{i}},\left\langle\lambda^{N}\right\rangle=\frac{1}{S} \sum_{i=1}^{S} \lambda_{i}^{N}$.

2) Verification of the conditions; if:

$\sum_{i=1}^{S}\left(\tau_{\text {set }_{i}}-\left\langle\tau_{\text {set }}\right\rangle\right)^{2} \leq \varepsilon,\left\|\lambda_{i}^{N}-\left\langle\lambda^{N}\right\rangle\right\| \leq \delta$, 
then calculation is completed; otherwise transition to (D1) is proceeded [6].

\section{Finding optimal parameter vector of MPOS}

The parametric optimization of MPOS will be performed, so that three-frequency quartz resonator is realized as AT-cut of resonator with basic resonance frequency $f_{q_{1}}=10009987 \mathrm{~Hz}$, quality factor $Q_{q_{1}}=103000$, dynamic resistance $R_{q_{1}}=24 \Omega$, frequencies of two anharmonics $f_{q_{2}}=10197018 \mathrm{~Hz}$ and $f_{q_{3}}=10268125 \mathrm{~Hz}$, quality factors $Q_{q_{2}}=84000, Q_{q_{3}}=61000$ and dynamic resistances $R_{q_{2}}=60 \Omega$ and $R_{q_{3}}=120 \Omega$ correspondently.

The optimization parameter vector $\lambda=\left(X_{s_{i}}, K_{j j}\right)$ will be embedded by values, which determine direct current operation mode for non-linear elements and amplifying partial circuits of the feedback (FB). The selected features of FB circuits are fixed at level $\gamma_{j i}=-10 \mathrm{~dB}$, what provides acceptable stability for multi-frequency generation mode. The variation diapason for value $X_{S_{i}} \in[-0.6 ;-1.5]$ is selected by design considerations, what is equivalent to changes of $R_{a b_{i}}$ in diapason $(650, \ldots, 2500) \Omega$.

The initial approach point (Fig. 7) is selected to the point which corresponds to generator nominal parameters: $\lambda_{0}=(-1 ; 20) ; \tau_{\text {set }_{0}}\left(\lambda_{0}\right)=34.31$. According to optimization algorithm the problem of dimension $N=2$ requires building three points of initial simplex, each of which $\lambda_{i}=\left(\lambda_{1}^{(i)}, \lambda_{2}^{(i)}\right)$ can be obtained from the following relations:

$\lambda_{1}^{(i)}=-1.5+0.9 \cdot \xi_{1}^{(i)}, \lambda_{2}^{(i)}=5+25 \cdot \xi_{2}^{(i)}$,

where $\xi_{1}^{(i)}$ and $\xi_{2}^{(i)}$ - random numbers, which are evenly distributed in the interval [0;1].

The assumption is made that random numbers generator generates $\xi_{1}^{(1)}=0.9$ and $\xi_{2}^{(1)}=0.5$. Then $\lambda_{1}=(-0.69 ; 17.5)$. This point is acceptable and memorized as the first point.

The next assumption is made that sensor generates $\xi_{1}^{(2)}=0.27$ and $\xi_{2}^{(2)}=0.2$. Then $\lambda_{2}=(-1.26 ; 10)$. This point is unacceptable, so moving towards to gravity center of already found vertexes gives:

$\hat{\lambda}_{0}=0.5\left(\lambda_{0}+\lambda_{1}\right)=(-0.845 ; 18.75)$.

New point $\lambda_{2_{\text {new }}}$ is acceptable and memorized.

Final assumption is made that $\xi_{1}^{(3)}=0.33, \xi_{2}^{(3)}=0.7$. Then $\lambda_{3}=(-1.2 ; 22.5)$. Next point is found by reflection:

$\lambda_{4}=\hat{\lambda}_{1}+1.3\left(\hat{\lambda}_{1}-\lambda_{2_{\text {new }}}\right)=(-0.843 ; 27.31)$.

This point is unacceptable. Therefore, consequently moving towards to gravity center $\hat{\lambda}_{1}$ new point is found as $\lambda_{4_{\text {new }}}=(-0.93 ; 21.82)$, which is acceptable and memorized (Fig. 7).

Next operations return optimal parameter vector of generator $\lambda^{*}$. Continuing iteration processes does not result in changes of gravity center location (Fig. 7). Stopping calculations for the value $\varepsilon=0.01$ results in vector of optimal values for MPOS $\lambda^{*}=\left(X_{S_{i}}^{*}, K_{j j}^{*}\right)=(-1.157 ; 26.61)$ and values $\tau_{\text {set }}^{*}\left(\lambda^{*}\right)=11.27$.

Thus, as result of two-directional optimization of MPOS parameters the multi-frequency oscillations mode setup process duration reduced from $0.082 \mathrm{~s}$ to $0.0225 \mathrm{~s}$ (more than by three times in comparison with input value). At the same time changing only one parameter (increasing $K_{j j}$ to value 23.7 or $X_{S_{i}}$ to -0.6 ) the gain is obtained only by two times or at $95 \%$ correspondently.

The gravity center movement trajectory and also rapid convergence of the iterative process to the point of MPOS parameter optimal values approves high efficiency of applied optimization 
method. Noteworthy is that efficiency of parametric optimization significantly increases if the preliminary study of objective function is performed, for example, by brute-force search method $[6,12-15]$.

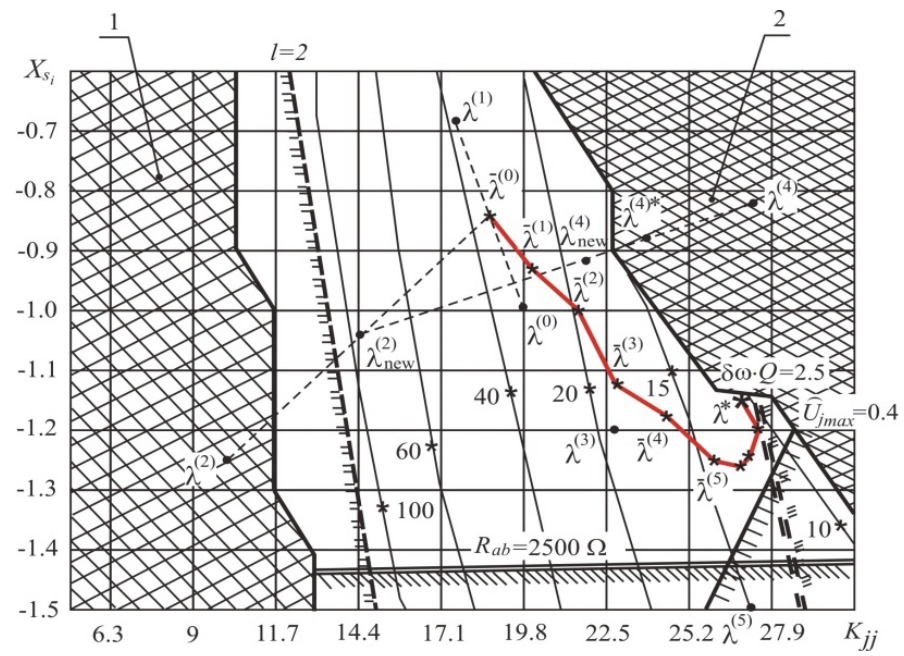

Fig. 7. Finding optimal parameter vector of MPOS: 1,2 - break areas of multi-frequency oscillation mode

\section{Conclusions}

The offered approach to developing piezoresonance units with controlled dynamics, which are represented as the adaptive control system with predictive reference model, allows creating the new class of PRU to be invariant to disturbing destabilizing factors. This approach grounds on the principle of using natural redundancy in multi-frequency basis of PRU core - multi-frequency piezoresonance oscillation system what allows on the base of invariance theory not only synthesizing system with current identification of disturbing factors, but also adapting PRU relative to their effect.

The presented model invariant formalized PRD involves review in linear approximation. Accounting anisochronism and parametric phenomena POS calls for the use of non-linear approach, which is in the part of invariance has a number of significant features.

In particular, the terms of compensation for nonlinear combined system must perform more complex functions than linear. You need to take into account not only the influence of perturbing factors, and some coordinate system. This requirement cannot be nominated if in the invariant device the linear object only in relation to perturbation. In this case compensator must contain the same nonlinearity, as direct circle of influence disturbance, that circle of the direct influence of perturbation. Conditions invariance is to provide symmetry impact perturbation $X$ on vector output $\mathrm{Y}$, and it is determined almost the same as in linear systems.

Using of suggested conceptual states related to design invariant multi-frequency piezo-resonance devices with controlled dynamics in the form of adaptive-self-tuning systems with the fast operating predictive standard provided creation of novel class invariant PRD which accuracy performance is of maximum close to potentially possible level. Control the trajectory operating in a real-time environment is performed on the basis of predictive numerical analysis of the core dynamics in IMPRU. Model parameters are given corresponding to the results of identification of current state QR under multi-mode excitation and taking into account the constructive peculiarities of PRD concrete type.

In order to provide given system operation mode under its incomplete parametrical distinctness (robustness), two-stage interval-approximation control law has been developed. The law provides dividing the process into the sequence interval-local approximation tasks within the limits of two 
stages for restoration and stabilization of multi-mode oscillation mode. During the first stage, adaptive control task is solved for each separate mode of IMPRU in order to provide operating the system under stationary oscillation mode in minimum time. During the second stage, control influences are formed. The latter ones are directed to the system stabilization under influence of destabilizing factors, i.e. providing technical invariance. Criteria for an optimal control in IMPRU/CD are analytically designed. That provides for each stage the minimization of energy expenses for optimal system operating trajectory. Local and global stability of interval-approximation control technique IMPRU/CD is demonstrated. Estimate of productivity of digital control system in IMPRU/CD confirms opportunity of physical realization of given conception by using wide spread ARM processes.

Using the developed mathematical model MPOS having in its base reduced differential equations for amplitudes, phases and voltages of auto-bias as predictive reference model of PRU core allows accomplishing effective controlling IMPRU/CD in accordance to the criteria of minimum of energy consumption as at the stage of setting multi-frequency oscillation mode so at the stage of stabilizing multi-frequency oscillations.

Method of complexes, which is related to direct search methods can be effectively used for parametric optimization of MPOS as in the project stage so in the stage of system functioning with purpose of its adaptation to external destabilizing factors. This method has certain advantages:

- logical structure of the method and necessary calculations are distinguished by simplicity and therefore its software realization is not complicated;

- low level of requirements to memory size of microcomputer and minor quantity of preliminary parameters to be installed;

- efficiency of the method is provided even when objective function calculation error is high, because while reflecting of the complex the highest value of objective function and gravity center $\hat{\lambda}$, which is obtained by averaging, are used.

Nevertheless, application of method of complexes is to consider some of its features:

- because all coordinates of complex vertexes depend on the same scale factor - reflection parameter $\beta$, the variable scaling is reasonable for practical problems in order to obtain comparable values;

- although the method of complexes does not require objective function continuity since it does not use derivative values, the function definition area needs to be convex, otherwise in some cases converging of the method is not provided. Such cases are unlikely in practice and using method normally gives good results.

\section{References}

[1] John Vig R. Quartz crystal resonators and oscillators for frequency control and timing applications a tutorial. IEEE International Frequency Control Symposium Tutorials, 2004.

[2] Lam C. A review of the recent development of MEMS and crystal oscillators and their impacts on the frequency control products industry. IEEE Ultrasonics Symposium, Beijing, China, 2008, p. 694-704.

[3] Taranchuk A., Pidchenko S., Skovryha O. Pressure transducer based on the dual-mode piezoresonant sensors with modulated interelectrode gap. Proceedings of IEEE 36th International Conference on Electronics and Nanotechnology, Kiev, Ukraine, 2016, p. 261-263.

[4] Pidchenko S. Dual-frequency temperature-compensated Quartz Crystal Oscillator. Proceedings of IEEE 23th International Crimean Conference on Microwave and Telecommunication Technology, Sevastopol, Crimea, Ukraine, 2013, p. 669-670.

[5] Bargin V., Zelensky A., Kolpakov F., et al. multi-mode quartz resonator-thermosensor. Electronic Engineering. Radio-Components, Vol. 10, Issue 2, 1972, p. 54-57, (in Russian).

[6] Pidchenko S. Theory and Fundamentals Implementation of Invariant Piezoresonance Devices and Systems. KNU, Khmelnytskyi, 2014, p. 400.

[7] Mensky B. The Principle of Invariance in Automatic Regulation and Control. Mashinostroenie, 1972, p. 248, (in Russian).

[8] Kharkevich A. Favourites Proceedings, Vol. 1, Nauka, 1973, p. 400, (in Russian). 
[9] Pidchenko S., Taranchuk A., Spivak A., Akulynichev A. The technical invariance of piezoresonance devices of the info communication systems. Proceedings of IEEE 3rd International Scientific-Practical Conference Problems of Info Communications Science and Technology, Kharkiv, Ukraine, 2016, p. 71-72.

[10] Pidchenko S., Taranchuk A., Totsky A., Akulynichev A. Providing of invariance property for piezo resonance devices on the basis of adaptive systems contained predictive standard. Proceedings of IEEE 4rd International Scientific-Practical Conference Problems of Info Communications Science and Technology, Kharkiv, Ukraine, 2016, p. 572-575.

[11] Krasovskii A., Bukov V., Shendrik V. Universal Algorithms for Optimal Control of Continuous Processes. a Series of Theoretical Foundations of Technical Cybernetics. Nauka, Moscow, 1977, p. 272.

[12] Pidchenko S., Taranchuk A. Principles of quartz multi-frequency oscillatory systems with digital compensation of temperature and vibrational instability frequency. Proceedings of IEEE International Conference Radio Electronics and Info Communications, Kiev, Ukraine, 2016, p. 281-284.

[13] Zelenskiy A., Pidchenko S., Taranchuk A. Multifrequency core structure of an invariant quartz oscillatory system. Proceedings of IEEE 11th International Conference on Modern Problems of Radio Engineering, Telecommunication and Computer Science, Lviv-Slavske, Ukraine, 2012, p. 125.

[14] Taranchuk A., Pidchenko S., Khoptinskiy R. Dynamics of temperature-frequency processes in multifrequency crystal oscillators with digital compensations of resonator performance instability. Radioelectronics and Communications Systems, Allerton Press Inc. USA, Vol. 58, Issue 6, 2015, p. 250-257.

[15] Pidchenko S., Taranchuk A., Totsky A. Multi-frequency quartz oscillating systems using digital compensation of frequency instability caused by variations of temperature and vibrations. Begell House Inc., USA, Vol. 76, Issue 13, 2017, p. 1193-1200.

[16] Reklaitis G., Ravindrank A., Ragsdell M. Engineering Optimization: Methods and Applications. John Wiley and Sons, 1983.

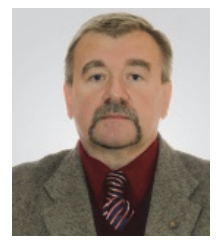

Sergey Pidchenko, D.Sc., Associate Professor in Department of Telecommunication and Computer-Integrated Technologies at Khmelnytsky National University. The field of scientific interests includes the problems of generating, forming and processing highly stable multi-frequency signals in info communication systems. Author of more than 120 publications in this field of research.

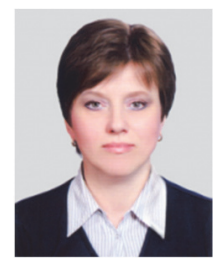

Alla Taranchuk, Ph.D., Associate Professor in Department of Telecommunication and Computer-Integrated Technologies at Khmelnytsky National University. The field of scientific interests includes the problems of generating, forming and processing highly stable multi-frequency signals in telecommunication systems. Author of more than 80 publications in this field of research. 


\section{Chapter 6}

\section{Increasing the reliability and durability of building}

materials 


\title{
6.1. The industry of building materials of Ukraine in the context of globalization
}

\author{
Petro V. Zakharchenko ${ }^{1}$, Oleksandr M. Gavrysh ${ }^{2}$, Roman D. Zakharienkov ${ }^{3}$ \\ ${ }^{1,2}$ Kyiv National University of Building and Architecture, Kyiv, Ukraine \\ ${ }^{3}$ Ukrainian Association of Styrofoam Manufacturers, Kyiv, Ukraine \\ E-mail: ${ }^{1} t k d 362 @ u k r . n e t,{ }^{2} t k d 362 @ u k r . n e t,{ }^{3}$ office@aspp.com.ua
}

\begin{abstract}
The condition of the building industry and the building materials industry in Ukraine is considered, production and sale of building materials are shown in dynamics. Modern building materials displace from the market obsolete inefficient products.
\end{abstract}

Keywords: brick (ceramic, silicate), autoclaved aerated concrete, dry building systems, thermal insulation products.

The building industry of Ukraine has evolved considerably during the years of independence. The large state-owned monopolies (ministries, republican and regional associations, etc.) have disintegrated or corporatized, state enterprises turned into LLCs, JSCs and others, there is practically no state order for housing construction, requirements for thermal protective characteristics of buildings and structures have significantly increased. All this, as well as the appearance of world-famous companies and technologies in Ukraine, has changed the vector of the development of the building materials industry. In this paper, we analyze the dynamics of production of the main types of building materials over the past 27 years.

Previous studies [1] found that builders during the crisis dramatically reduce the volume of construction and increase them just before the next economic downturn. The indicated dependence is particularly clearly observed in housing construction. If we recall that the vast majority of residential multi-apartment construction projects last about 3 years, it becomes clear that the implementation of the vast majority of such projects begins at the peak of demand during economic growth, and the time of their completion falls at the bottom of demand during the economic crisis, and forces organization to stop construction and threatens them with bankruptcy. So, during the economic downturns of 2002 and 2008, the rate of commissioning of housing has fallen sharply (because during a crisis it is possible to realize apartments only in completed facilities). In 2016, the volume of housing commissioning fell to 9367 ths sq. meters (Fig. 1).

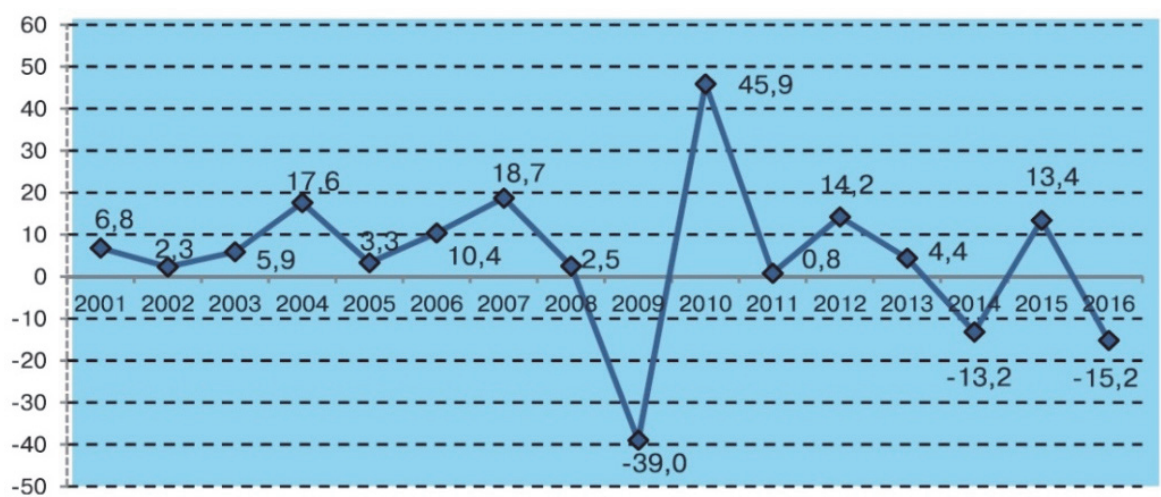

Fig. 1. Commissioning of housing in Ukraine in 2001-2016, in \% to the previous year

The paper examines three markets for building materials, which determine the current level of construction.

1. Structural materials for the building of outer walls and inter-apartment/interior partitions, which include: brick (ceramic and silicate), ceramic blocks, blocks from autoclaved aerated concrete and products of house-building combines wall panels. 
2. Dry building mixtures and plate materials for interior decoration of premises. This market includes building mortars, dry building systems (plasterboard), other plate materials (chipboard, fiberboard, magnesite).

3. Heat and sound insulation materials and products. This market includes the following groups of products: products based on mineral, silicate and glass wool, expanded polystyrene, foam plastics, insulating products based on expanded perlite.

Brick is one of the most common building materials used in the construction of buildings and structures, laying foundations, facing facades and when building internal partitions. The material successfully competes with cheaper for today concrete products, cinder blocks and wooden structures.

By composition and method of production, the brick is divided into ceramic and silicate.

In 1990, the volume of production of ceramic bricks was 7 billion pieces conv. bricks/year, about half of which was produced in factories with outdated seasonal technology and provided the production of M75 bricks (of rather poor quality). In the $90 \mathrm{~s}$, production at almost all these plants was stopped. In the new millennium, modern technologies for the production of ceramic bricks have come to Ukraine, plants have been built and reconstructed: Slobozhanska Budivelna Keramika (SBK., Romny, Sumy region), Kerameya (Sumy), Euroton (Yavoriv, Lviv region), Zagvizdiansky brick factory (Ivano-Frankivsk), "Rusinia" (Mukachevo, Zakarpattia region), a brick factory of the Kolomoyskyi plant office of building materials (Ivano-Frankivsk region), Novoaleksandrovsky brick factory (Dnepropetrovsk region), Belotserkovsky brick factory (Kyivs'ka obl.). These enterprises produce high-quality ceramic bricks: facial, clinker and ceramic blocks.

Perspective wall material - ceramic blocks are made from natural raw materials - clay. In order to obtain a porous structure in the process of production, crushed wooden sawdust or other organic components are added to the clay mass, which burn out during brick burning, forming a large number of closed micro-cavities. Ceramic blocks have high thermal insulation properties and are more economically more profitable than conventional brick. In this case, the ceramic block retains the stability and strength of a conventional brick. According to the classification of the State Statistic Service adopted in Ukraine, ceramic brick as a construction material for wall making refers to the code "Ceramic brick, non-refractory, building". The dynamics of production of ceramic non-refractory brick is shown in Fig. 2.

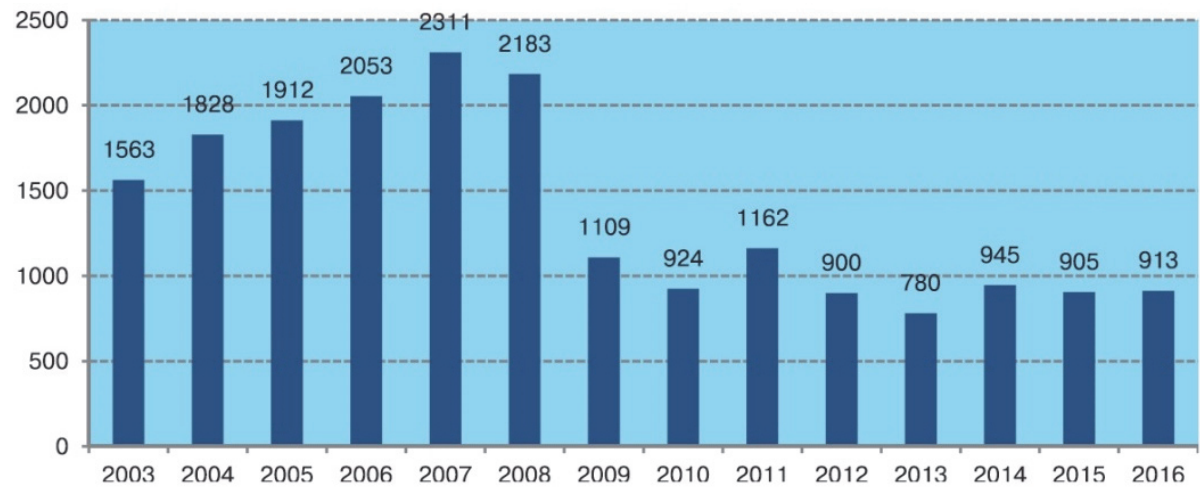

Fig. 2. Manufacture of ceramic non-refractory building brick in years 2003-2016, mln. conv. bricks

During the period 2003-2016, the production of building bricks ranged from 1.5-2.3 billion pcs. conv. bricks/year, but the last 10 years the production volumes are less than 1 billion pcs. conv. brick/year.

Thus, it can be concluded that over the years of Ukraine's independence, the volumes of ceramic bricks production have decreased 8 times, and almost half of the brick volumes are 
represented by high-tech products.

The silica brick is made of sand, lime and a small fraction of additives with the addition of water. Silica bricks are not subjected to roasting, but it is treated with steam in an autoclave. The process of production of silicate brick can be briefly described as follows: a moist mixture of $90 \%$ sand, $10 \%$ lime is poured into a press where a raw brick is formed, then it is loaded into an autoclave and exposed to saturated steam at a temperature of $170-200^{\circ} \mathrm{C}$ and pressure of $8-12$ atmospheres. If weather-resistant, alkali-resistant pigments are added to this mixture, a colored silica brick is obtained. The water resistance of the silica brick is lower than that of the ceramic brick. Silica brick is recommended for use in laying load-bearing walls and various partitions, but it is strictly forbidden to use it when laying foundations, in laying ovens, fireplaces, pipes, socles, etc. The advantage of silica brick in front of ceramic is its increased sound insulation characteristics, which is important for erection inter-apartment or interior walls.

In 1990, the volume of production of silica bricks amounted to 2 billion pcs. conv. bricks per year. Considering the quite high technological level and significantly lower energy consumption of the technology, its production has decreased significantly less than the production of ceramic up to 550 million pes conv. bricks per year.

The most popular from the point of view of energy efficiency of buildings and structures are modern products made from autoclaved aerated concrete, which provide the normative heat resistance of the walls when laying the blocks in one row (single-layer wall structure, wall width $400-500 \mathrm{~mm}$ at a material density of $300-400 \mathrm{~kg} / \mathrm{m}^{3}$ ).

Aerated concrete is an energy-saving building material of the porous structure. Its pores contain from 50 to $90 \%$ of air, which distinguishes concrete from other building products by the properties of the material. With a change in the porosity of the material, there is a logical change in its performance indicators - density, heat conductivity, strength.

Aerated concrete is an artificial stone, which is formed during the hardening of a porous mixture, including finely dispersed filler, blowing agent and water. Aerated concrete has the properties, on the one hand, of stone, on the other - of wood. The combination of these properties makes it an excellent building material.

Residential buildings, industrial and public buildings, as well as various agricultural premises, are being built from aerated concrete:

- aerated concrete with a porosity of 50-60\% (density of $1000-800 \mathrm{~kg} / \mathrm{m}^{3}$ ) has a sufficiently high strength and can be used as a structural material in low-rise buildings (buildings up to 5 floors), replacing silica and ceramic bricks, lightweight concrete;

- aerated concrete with a porosity of $75-80 \%$ (density of $600-300 \mathrm{~kg} / \mathrm{m}^{3}$ ) is approaching to wood by operational parameters, a combination of high thermal insulation and strength characteristics in the material allows it to be used in single-layer outer walls of a building up to 3 floors. Walls from aerated concrete can be used separately, as well as in combination with brick, concrete (prefabricated, monolithic) and other types of products;

- aerated concrete with a porosity of more than $80 \%$ (density $300-200 \mathrm{~kg} / \mathrm{m}^{3}$ ) belongs to the class of heat-insulating materials and can be used for erecting interior partitions. At low density, it has many advantages over other types of organic and mineral heat-insulating materials: stiffness, non-combustibility, bio-stability, environmental cleanliness, preservation of physical and technical parameters during long-term operation, durability, and availability of own raw material base for manufacturing of the products (cement, lime, sand).

The dynamics of production of this material is impressive. In 1990, Ukraine produced 400 ths. $\mathrm{m}^{3}$ of products from autoclaved aerated concrete per year (which corresponds to $400 \mathrm{mln}$. pcs. conv. bricks/year), and already in 2017 - the volume of its production exceeded $3.6 \mathrm{mln} . \mathrm{m}^{3} /$ year (corresponding to 3.6 billion pcs. conv. bricks/year) 
Table 1. Characteristics of technological lines and sales volumes of small blocks of producers of ANB

\begin{tabular}{|c|c|c|c|c|c|c|}
\hline \multirow[t]{2}{*}{ Manufacturer } & \multirow[t]{2}{*}{ Equipment } & \multirow[t]{2}{*}{ Location } & \multirow{2}{*}{$\begin{array}{l}\text { Maximum } \\
\text { production } \\
\text { line power }\end{array}$} & \multirow{2}{*}{\multicolumn{2}{|c|}{\begin{tabular}{|c|}
$\begin{array}{c}\text { Sales volume in } \\
2016, \text { ths. } \mathrm{m}^{3}\end{array}$ \\
$\mathrm{~m}^{3} /$ day ths. $\mathrm{m}^{3} /$ year \\
\end{tabular}}} & \multirow[t]{2}{*}{ Change $\%$} \\
\hline & & & & & & \\
\hline $\begin{array}{l}\text { AEROC } \\
\text { (2 plants) }\end{array}$ & $\begin{array}{c}\text { WEHRHAHN } \\
\text { (Obukhiv) Universal- } \\
60 \text { (Obukhiv) Hess } \\
\text { (Berezan) }\end{array}$ & \begin{tabular}{|} 
Obukhiv city \\
and Berezan city \\
Kyivs'ka oblast'
\end{tabular} & 3200 & 1000 & 1033 & $+12 \%$ \\
\hline $\begin{array}{l}\text { Oriyentyr- } \\
\text { Budelement }\end{array}$ & $\begin{array}{l}\text { Hetten (modernized } \\
\text { line) }\end{array}$ & $\begin{array}{c}\text { Brovary city } \\
\text { Kyivs'ka oblast' }\end{array}$ & 3650 & 1300 & 1349 & $+19 \%$ \\
\hline UDK & Masa-Henke & Dnipro city & 1500 & 430 & 412 & $+27 \%$ \\
\hline Energy Product & Durox & $\begin{array}{c}\text { Nova Kakhovka } \\
\text { city Kherson } \\
\text { Oblast }\end{array}$ & 1300 & 380 & 383 & $+16 \%$ \\
\hline Ju-Ton & WKB Systems & $\begin{array}{l}\text { Voznesensk city } \\
\text { Mykolaiv Oblast }\end{array}$ & 850 & 280 & 175 & $+17 \%$ \\
\hline TBK & Wuxi Mettle & Kherson city & 400 & 120 & 0 & - \\
\hline $\begin{array}{c}\text { PE } \\
\text { Budtehnologiya-N }\end{array}$ & $\begin{array}{l}\text { Universal-60 } \\
\text { (2 lines) }\end{array}$ & $\begin{array}{l}\text { Kupyans'k city } \\
\text { Kharkiv Oblast }\end{array}$ & 600 & 200 & 11 & $+4 \%$ \\
\hline KBM Corporation & Hetten & Kharkiv city & 600 & 200 & 150 & $+4 \%$ \\
\hline PE Autocraft & $\begin{array}{l}\text { Ukrainian equipment } \\
\text { (PE INTeRBudMa) }\end{array}$ & $\begin{array}{c}\text { Bershad town } \\
\text { Vinnytsia Oblast }\end{array}$ & 250 & 80 & 0 & - \\
\hline $\begin{array}{l}\text { Zhytomyr silicate } \\
\text { products factory }\end{array}$ & $\begin{array}{l}\text { Universal-60 } \\
\text { WEHRHAHN }\end{array}$ & Zhytomyr city & 250 & 80 & 46 & $+10 \%$ \\
\hline $\begin{array}{l}\text { Dnieper Plant of } \\
\text { Building Materials }\end{array}$ & Extra block & Dniepro city & 150 & 50 & 40 & $+100 \%$ \\
\hline Silikatobeton & China & Sumy city & 400 & 150 & 1 & - \\
\hline $\begin{array}{c}\text { Teplobud- } \\
\text { Siverschyna }\end{array}$ & USSR & Chernihiv city & 20 & 5 & 4 & - \\
\hline & & Total: & 13710 & 4275 & 3604 & $+15 \%$ \\
\hline
\end{tabular}

In 1990, more than half of the housing was built from claydite-concrete panels, which, according to the State Statistic Service of Ukraine, refer to "elements of the construction of prefabricated buildings for housing and civil construction from cement, concrete or artificial stone". In recent years, the share of these products accounted for less than $15 \%$ of the total volume of wall materials and only in 2016 reached almost $25 \%$.

The greatest development during the years of Ukraine independence has received the subsector of dry construction [2-5].

Dry construction is the installation of billets, building products and materials produced by industry, with the exception of the so-called "wet" processes (preparation of masonry, plaster solutions, concrete, etc.). Dry construction includes non-load-bearing structures that limit space (interior partitions), as well as interior finishing and covering of load-bearing walls, ceiling, and the installation of floors.

The development of the method of dry construction is closely related to the industrial production of gypsum boards. Since the late 50 -ies in construction started to use in large volumes of building parts from plasterboards for light partitions and wall cladding. However, then the construction was much easier in comparison with the complexity and capabilities of today's dry construction. At the moment, this method is rapidly spreading and has an increasing influence on all spheres of construction: high-performance combined materials, heating and air conditioning systems integrated into dry construction, systems of ceiling, walls and floors with high sound insulation properties, board materials with wooden, glass, steel and aluminum coating, plasterboards with high heat accumulation are the several examples of development when technical and design innovation potential is almost unlimited. Plate materials in composition and design can meet almost all requirements due to structural changes and special additives. 


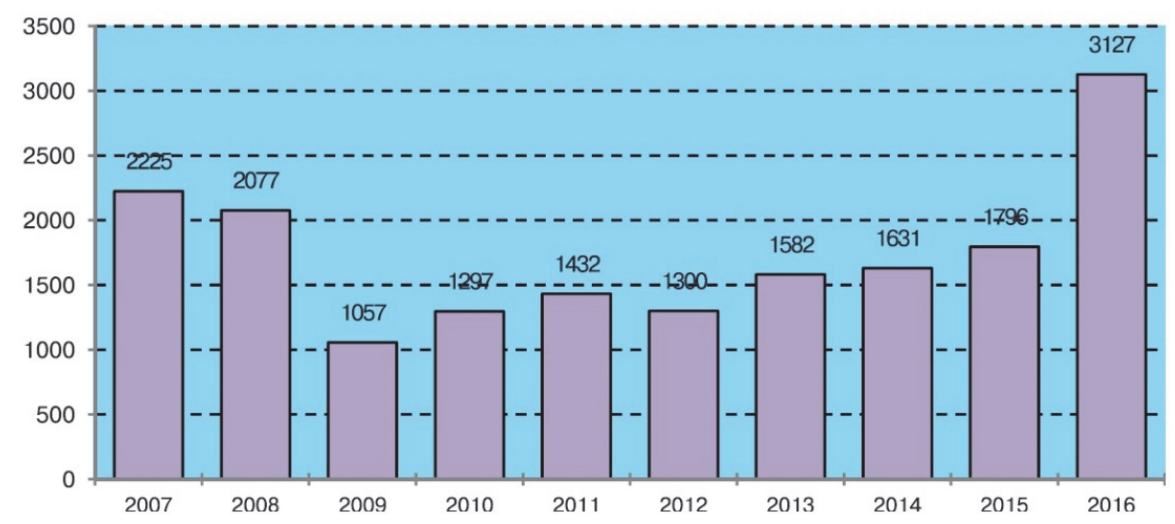

Fig. 3. Production of elements of the prefabricated structures for construction from cement, concrete or artificial stone for 2007-2016 ths. $\mathrm{m}^{3}$

The implementation of dry construction systems, allows you to abandon the "wet" processes, increases in several times labor productivity and provides an opportunity to perform interior design of premises more better. The main material of dry construction is plasterboards.

Plasterboards are sheet products, consisting of a non-combustible gypsum core, the surface of which, besides the end edges, is faced with cardboard that glued to the core.

Plates are designed for facing walls, partition making, fire retardant structures, manufacturing of decorative and sound-absorbing products.

Depending on the properties and area of application, the plasterboards are divided into the following types:

- conventional (PB),

- moisture-resistant (PBM),

- increased resistance to open flame (PBIR),

- moisture-resistant increased resistance to open flame (PBMIR).

$\mathrm{PB}$ and PBIR are used in buildings and premises with dry and normal humidity regime.

PBM and PBMIR are used in buildings and premises with an increased humidity regime.

PBIR and PBMIR it is appropriate to use for cladding of structures with the purpose of increasing their limit of fire resistance in premises with increased fire danger.

Dimensions of plates, mm: length from 2000 to 4000 in increments of $500 \mathrm{~mm}$; width 600 and 1200 ; thickness $6.5 ; 8.0 ; 9.5 ; 12.5 ; 14.0 ; 16.0 ; 18.0 ; 20.0 ; 24.0$. Plates should have a rectangular shape in the plan.

PB produce with different in form longitudinal edges, glued with cardboard, which determine the way and quality of sealing joints between neighboring PBs.

Dynamics of plasterboard production in Ukraine is shown in Fig. 4.

In 1990, Ukraine produced about $6 \mathrm{mln}$. sq. m. of plasterboards. During 2003-2008, the production of plasterboards increased from $27.0 \mathrm{mln}$. sq. $\mathrm{m}$. to $91.5 \mathrm{mln}$. sq. $\mathrm{m}$. That is, in five years the volume of production has grown 3.4 times. The greatest growth occurred in 2007, mainly due to the commissioning of new capacities by a group of KNAUF companies. In 2009 there was a drop-in production by almost a third, but in 2010-2011 production volumes gradually increased and almost reached the 2007 levels, but in 2012 the figures decreased, and in 2013-2016 the production of the PB remained at the level of 2006.

Chipboard - this is the most common material in the production of furniture and construction. It was first started to produce in the city of Bremen (Germany) in July 1941 at the factory of the TORFITWERKE G.A. HASEKE Company. The main advantages of particle board are ease of processing, economy, reliability and high practicality, in addition, it is environmentally friendly material.

Modern production technologies make it possible to achieve a sufficiently high quality and 
safety of particle board products. The particle board is produced by way of hot pressing of largedispersed chips, which obtained from technical wood of any kind and from waste of woodworking enterprises with the simultaneous introduction of thermosetting synthetic resin, as well as hydrophobizing (such increasing the water-repellent properties), antiseptic and other additives, due to which the plate acquires a special strength and durability.

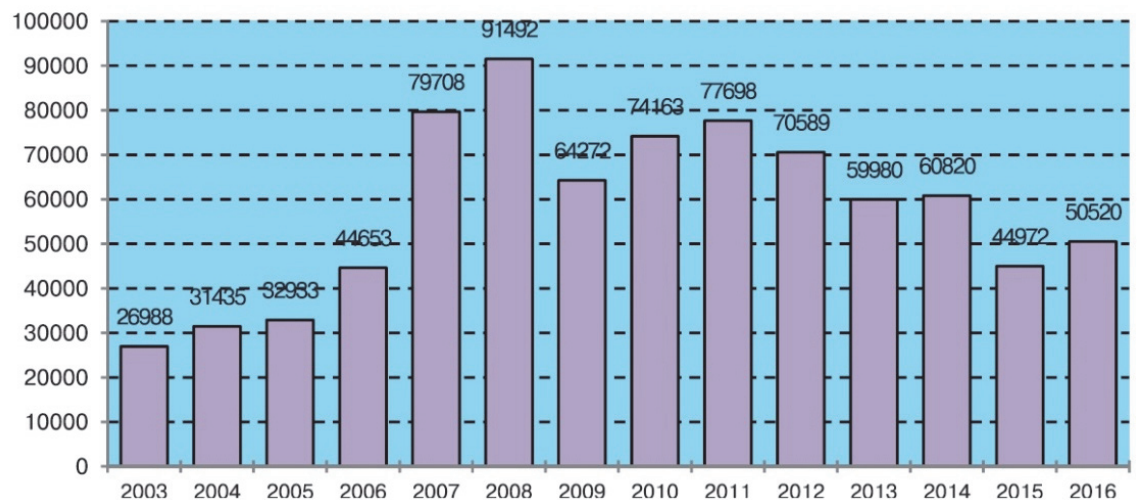

Fig. 4. Manufacture of gypsum products for construction (plasterboards) in 2003-2016 years ths. $\mathrm{m}^{2}$

The use properties of the particle board depend mostly on their density, the shape and size of the chips, and the quantity and quality of the binder component. There are plates with a very small density $\left(350-450 \mathrm{~kg} / \mathrm{m}^{3}\right)$, small $\left(450-650 \mathrm{~kg} / \mathrm{m}^{3}\right)$, medium $\left(650-750 \mathrm{~kg} / \mathrm{m}^{3}\right)$ and high $\left(700-800 \mathrm{~kg} / \mathrm{m}^{3}\right)$ density. There are single-, three- and five-layer boards.

Fiberboards are made by hot pressing of pulp, consisting of organic, mainly cellulose, fibers, water, fillers, synthetic polymers and some special additives. The raw material for the production of boards is waste of woodworking industries and logging (cod, fines), stalks of bulrush, flax hurds and other plant materials. Depending on the pressure while pressing and the type of further processing, the wood fiber boards are divided into super-hard, hard, semi-solid and soft (insulation-finishing and insulating). For interior decoration of buildings rigid boards are used, super-hard is used for covering floors.

Fiberboard is used in housing and industrial construction for heat and sound insulation of the roof, interfloor ceilings, walls, for finishing premises, etc. Fiberboard is especially widely used in low-rise, cottage and rural construction, in standard house-building, and also in the production of furniture and packaging.

OSB (Oriented Strand Board) - boards from oriented shavings of coniferous trees, are pressed under high pressure into three layers and seep with adhesive waterproof resin. OSB-plates are produced in a format up to $3.6 \times 7.2$ meters, with a thickness from $6 \mathrm{~mm}$ to $30 \mathrm{~mm}$. In Ukraine, the most common are OSB-boards measuring $1.25 \times 2.5 \mathrm{~m}$ and thickness of $10 \mathrm{~mm}$ and $12 \mathrm{~mm}$. They are used for wall covering, roofing, as supporting surfaces for floors, supporting structures, formwork for concrete construction and the like. They are used, next to plywood, for the manufacture of strong elements of building structures, containers, packaging, furniture and so on.

Their density is $600-660 \mathrm{~kg} / \mathrm{m}^{3}$. The flexural strength is $25 \mathrm{~N} / \mathrm{mm}^{2}$ along the fibers, $14 \mathrm{~N} / \mathrm{mm}^{2}$ across the fibers. The thermal conductivity is $0.13 \mathrm{~W} /(\mathrm{mK})$.

OSB on $90 \%$ consists of natural wood, has all the characteristics of the array, but does not have knots and other defects. When working with the OSB, the tools are subjected to less wear.

According to the classification of the State Statistic Service adopted in Ukraine, board materials (plasterboards, fiberboards) as a structural material for making partitions refer to the code "Particle boards unprocessed" and "Panels, plates, blocks and similar articles of vegetable fibers or wood waste, agglomerated with mineral binders".

The dynamics of production of wood-based panels is shown in Fig. 5. 


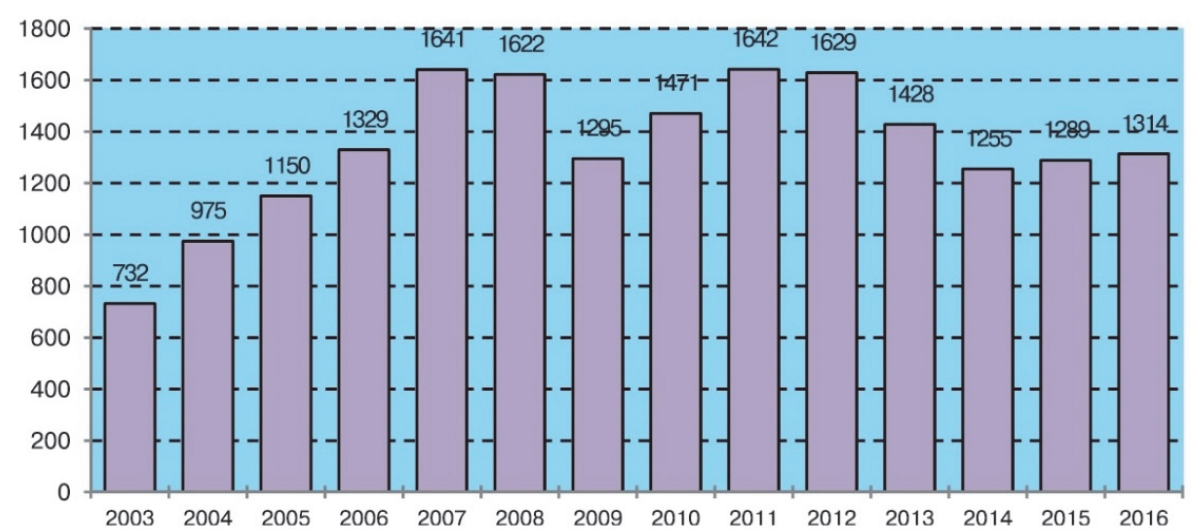

Fig. 5. Manufacture of unprocessed plasterboard in 2003-2016, ths. conv. $\mathrm{m}^{3}$

The market of dry building mixtures directly depends on the production of binders: cement, gypsum, lime. The volume of cement production in 1990 exceeded 20 million t/year. In the 90's, the volume of production and consumption of cement fell to 5 million $t / y e a r$. In the $2000 \mathrm{~s}$, cement production began to grow and reached its peak in year 2007 of 15 million t/year.

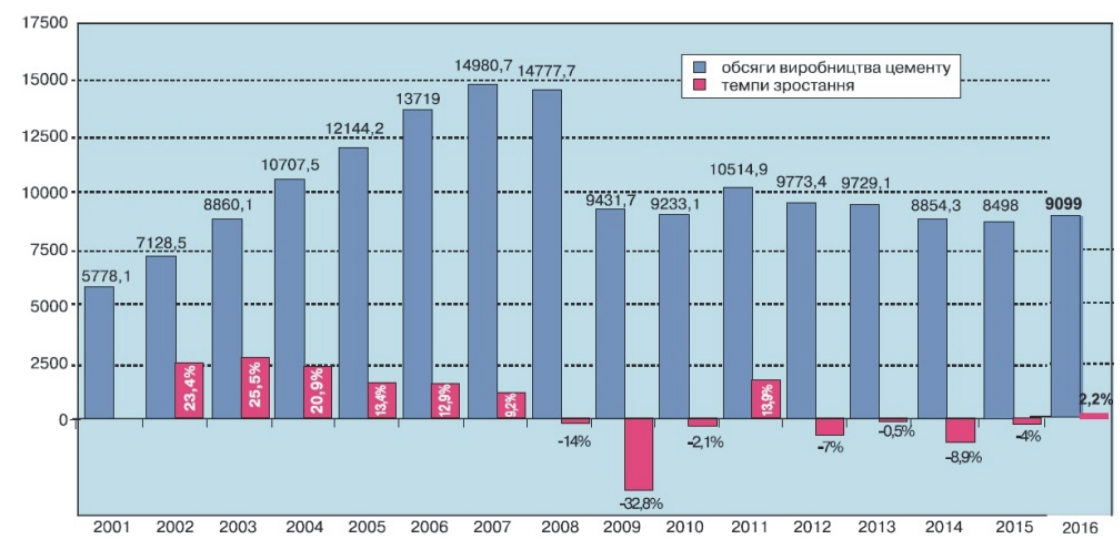

Fig. 6. Manufacture of cement by the enterprises of the association "Ukrcement", in 2001-2016 thousand tons

However, already 2008 is characterized by a decrease of $1.5 \%$. Due to the economic crisis during 2009-2010, there was a rapid decline in the volume of cement production (by about $37 \%$ ). In 2011, the cement industry was gradually emerging from the crisis, production volumes reached the level of 2004, but in 2012-2015 the production rates fell somewhat, and in 2016 they increased on 500 thousand t. (Fig. 6).

Information on the main producers and volumes of cement production in Ukraine is given in Table 2.

In 2014, 34 rotary kilns operated in the cement industry of Ukraine against 41 in 2013, 6 of them by dry method of production, 28 by wet ones. The production potential for clinker output was 15 million 133 thousand t/year, including 5 million 401 thousand t/year on dry production method or $35.7 \%$ of total production capacity. Loading of the rotary kilns in 2015-2016 is amounted to $40.1 \%$, while the use of cement production capacities was $45.2 \%$.

As can be seen from the table below, almost all Ukrainian cement plants are owned by leading foreign firms. Considering the high energy production costs and the high cost of natural gas, almost all these plants have been converted to cement clinker firing by the pulverized coal mixture. 
Table 2. Manufacturers of cement in Ukraine

\begin{tabular}{|c|c|}
\hline Owner & Enterprise \\
\hline Dyckerhoff (Germany) & "Volyn-Cement" \\
\cline { 2 - 2 } & "Yugcement" \\
\hline \multirow{2}{*}{ CRH (Ireland) } & "Mykolaivcement" \\
\cline { 2 - 2 } & "Podilsky Cement" \\
\cline { 2 - 2 } HeidelbergCement (Germany) & "Cement Odessa" \\
\cline { 2 - 2 } & "Dryvyi Rih Cement" \\
\cline { 2 - 2 } & "Dnieprocement" \\
\cline { 2 - 2 } & "Cement of Donbass" \\
\hline Eurocement (Russia) & "Pushka" Kramatorsk Cement Plant \\
\cline { 2 - 2 } & "Balcem" \\
\hline Ukrainian owners & "Bakhchisaray combine "Budindustriya" \\
\cline { 2 - 2 } & "Ivano-Frankivsk Cement" \\
\cline { 2 - 2 } & "Promcement" \\
\hline
\end{tabular}

In the second half of the $1990 \mathrm{~s}$, in Ukraine started the production of dry construction mixtures $[6,7]$.

Dry mixtures, in comparison with traditional solutions and concrete, have a number of advantages:

- a minimum of technological operations to bring dry mixtures into operation (mixing with water)

- reduction by $5-7 \%$ in solution waste as a result of batch dosing;

- saving of 10-15\% of cement due to the use of plasticizing and water-retaining additives;

- stability of the composition of dry mixtures as a result of precise dosing of components and the effectiveness of their mixing;

- increasing the productivity of workers in 1.5 to 3 times due to the achievement of high technological properties of mortar mixtures and their mechanized application to finishing surfaces;

- decreasing of 10-15\% of transportation costs and an improvement in the quality of work, while reducing the labor intensity of technological processes.

Due to above advantages and unique properties, dry mixes have a wide range of applications in construction. Dry building mixtures (hereinafter-mixtures) are classified by:

- type of binder used;

- the largest size of aggregates or fillers;

- the main purpose. Depending on the type of binder used, the mixture is divided into cement, gypsum, calcareous, polymeric and complex.

Dry building mixtures modified on a cement basis of all groups should:

- when performing external works, ensure high resistance of coatings to moisture and various climatic factors, including negative and elevated temperatures;

- when performing external works, the water absorption coefficient should not exceed $0.2 \mathrm{~kg} / \mathrm{m}^{2} \times \mathrm{h} 0.5$ (group III 3 )

- easy to apply and to level.

Dry building mixes modified on a cement basis are a traditional finishing material, which is widely used for interior and exterior decoration. According to the classification of the State Statistic Service of Ukraine "Dry mortars modified on cement basis" refer to the code "Building mortars and concretes (dry), except concrete ready mix". Below are the changes that occurred in this segment during 2004-2016.

Next, consider the dynamics of production of dry building mixtures on a cement basis (Fig. 7).

As can be seen from the chart, after the fall in 2009, in 2010-2013, this segment of the market not only recovered, but also significantly exceeded pre-crisis indicators. In 2014-2015 the production of the building mixtures on a cement basis significantly decreased, and in 2016 the 
production volumes increased by about $45 \%$.

The commodity structure of the building mixtures on a cement basis in 2016 is shown in Fig. 8 .

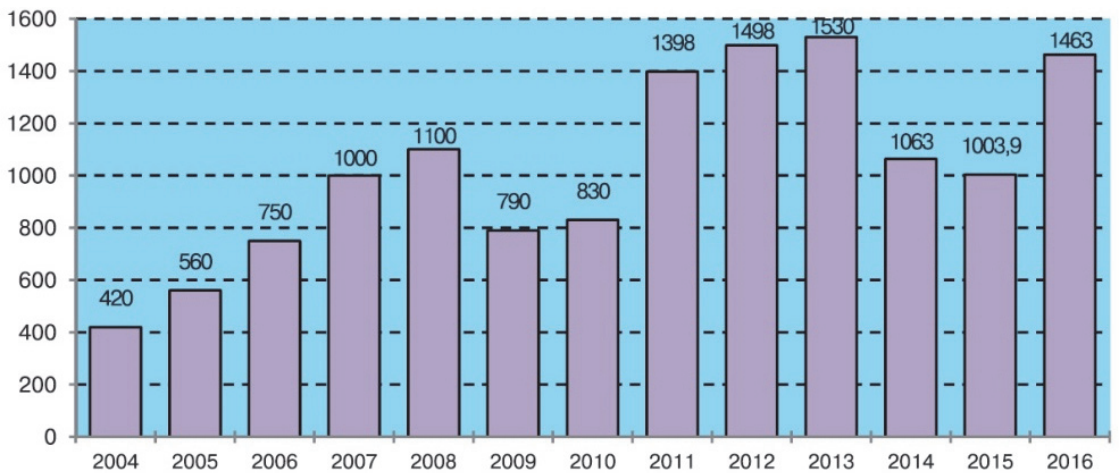

Fig. 7. Dynamics of the building mixtures on a cement basis market for 2004-2016, ths. $t$

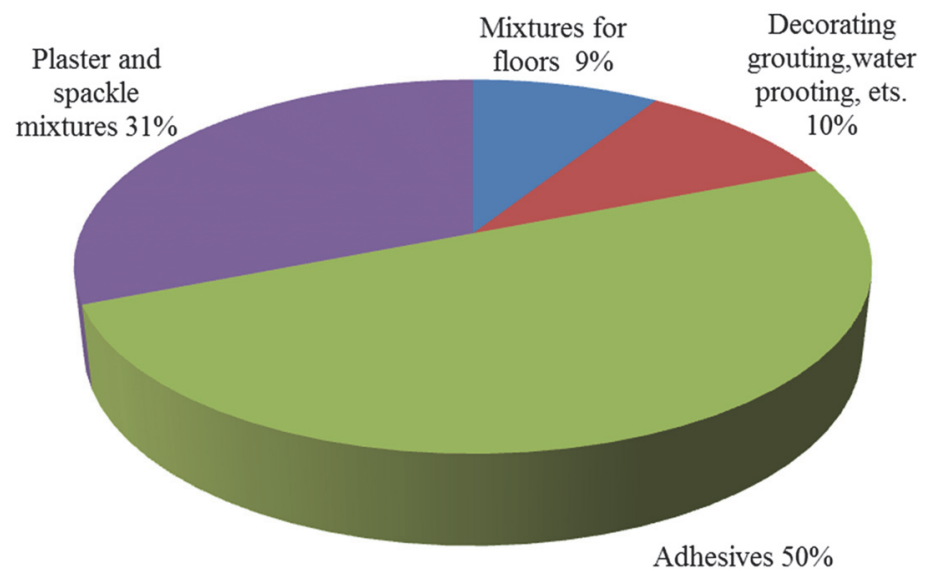

Fig. 8. Commodity structure of the building mixtures on a cement basis in $2016, \%$

Table 3. The main operators in the market of dry construction mixtures in 2016

\begin{tabular}{|c|c|c|}
\hline Trade Mark & Volume of production, ths.t. & $\%$ \\
\hline Ceresit & 360 & 39.2 \\
\hline Siltek & 80.4 & 5.7 \\
\hline Anserglob & 53.6 & 6.1 \\
\hline Majster & 91.7 & 6.5 \\
\hline Polimin & 175 & 12.4 \\
\hline Kreisel & 101.6 & 7.2 \\
\hline Ferozit & 56.5 & 4.0 \\
\hline BudMajster & 59.3 & 4.2 \\
\hline Baumit & 67.7 & 4.8 \\
\hline Artisan & 31.0 & 2.2 \\
\hline others & 108.7 & 7.7 \\
\hline
\end{tabular}

Gypsum mixes (group III2) are intended for finishing of interior surfaces of premises. They include gypsum, lime, fillers, polymer modifying components, a setting retardant and other impurity.

Gypsum mixtures must:

- not be destroyed during periodic short-term humidification;

- provide the ability to control the setting process in a wide time range (1-2 hours). 
Mixtures for surface preparing are the systems containing mineral binders, fillers and various impurities, including those that reduce adherence of the mortar mixture to the tool.

Mixtures for surface preparing for finishing should:

- have adhesion with the base not less than $0.5 \mathrm{MPa}$;

- have a vapor permeability of not less than $0.1 \mathrm{mg} /(\mathrm{m} \times \mathrm{h} \times \mathrm{Pa})$

- be frost-resistant (for outdoor work), to withstand at least 50 freeze-thaw cycles;

- easily fit on the basis, not leave strips, lumps and not follow the tool;

- have resistance to cracking and minimal shrinkage, should not exceed $0.2 \%$;

- be polished and be painted, including paints on organic solvents;

- not be dripping down from the vertical surfaces;

- be water resistant.

According to the classification of the State Statistic Service of Ukraine, production, export and import of dry building mixes on a gypsum basis fall under the codes 26.53.10 "Gypsum binders", 2520201000 "Gypsum building" and 2520209000 "Gypsum other".

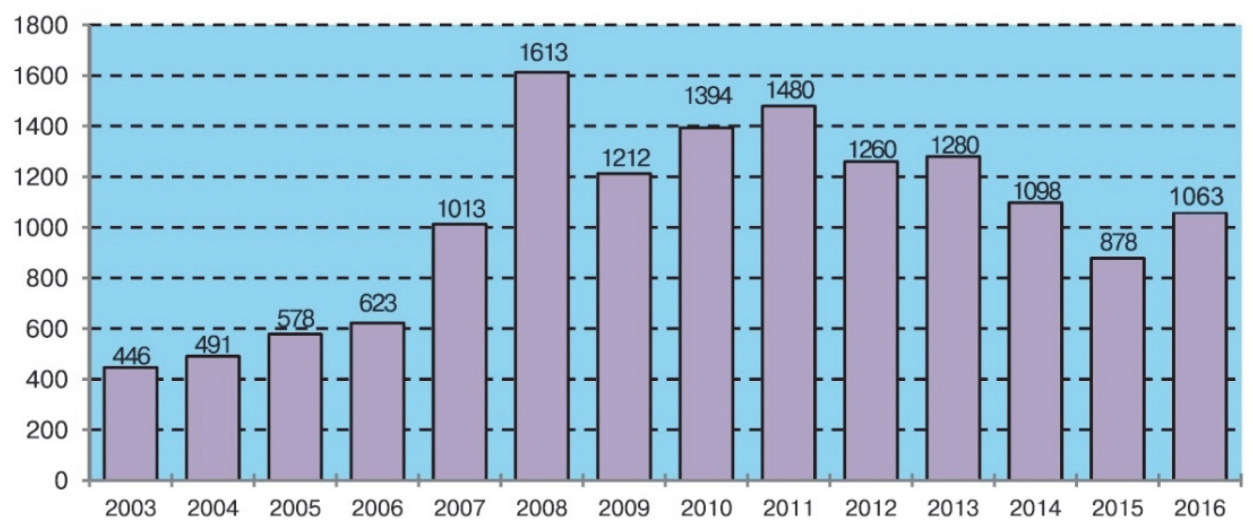

Fig. 9. Production of construction gypsum and gypsum binders for 2003-2016, ths. $t$

During the period of 2003-2008, the production of construction gypsum and gypsum binders increased in 3.6 times. The greatest growth occurred in 2007 and 2008, the increase was about $60 \%$. Mainly due to the commissioning of new capacities by a group of KNAUF companies. Due to the crisis in 2009, production volumes decreased by $25 \%$, and already in 2010-2011 production growth resumed and it almost reached the pre-crisis level, but in 2012 production volumes decreased. The production of gypsum binders in 2012-2013 was 1260-1280 ths. t, and in 2014-2016 it was 900-1100 ths. t.

The main suppliers are Knauf Marketing (TM Knauf), Helios (Lviv, TM Ferozit), Fomalhaut-Polimin (Kyiv, TM Polimin), Polirem-Center (Kiev, TM "Polirem"), "Factory of the building mixtures "BudMajster" (Pavlograd, TM "BudMajster"), "KREISEL-Building Materials" (TM “Kreisel”), “Terminal M” (TM “Siltek”), “Akvalit” (TM “Akvalit”).

The requirements for the thermal efficiency of buildings and structures in Ukraine are constantly growing. In 1960 , the standard resistance to heat transfer $R$ was $0.7 \mathrm{~m} \cdot \mathrm{K} / \mathrm{W}$. In 1995 , it increased to $1.8-2.2(\mathrm{~m} \cdot \mathrm{K}) / \mathrm{W}$, in $2006-$ up to $2.8(\mathrm{~m} \cdot \mathrm{K}) / \mathrm{W}$, the current norm from $2015 R$ is equal to $3.3(\mathrm{~m} \cdot \mathrm{K}) / \mathrm{W}$.

One of the main reserves of energy saving is the reduction of thermal and sound insulation properties of housing and civil facilities to the modern European level, which, along with saving energy resources, will solve the problem of ensuring the normative level of comfort in the residential environment, the absence of which has become a serious social problem for residents of multi-family housing.

Modern systems for home heat insulation make it possible to reduce the cost of heating, and also have optimal sound-absorbing properties, protect against vibration. For the production of 
systems are used heat and sound insulation materials such as mineral wool products, extrusion and beaded expanded polystyrene and the like [8].

Each of the materials has its advantages and their engineering justified combination makes it possible to make the construction of thermal insulation at home as efficient and durable as possible.

The main types of heat and sound insulation used in Ukraine today are polystyrene and other foam plastics, their share is more than $35 \%$, mineral wool and glass wool products occupy $35 \%$ and $25 \%$ respectively, and about $1 \%$ fall on other types of heat-insulating materials (see the following diagrams). In general, the market of thermal insulation materials in Ukraine is quite stable.

At the same time, it should be determined that the volume of the rock wool segment showed a positive trend and increased by $0.3 \%$ compared to 2012, while the segment of the expanded polystyrene foam market increased by $0.9 \%$. This trend indicates that the participants of the building materials market prefer the products of stone wool as safer and non-combustible and cheaper products made of expanded polystyrene.

The world and domestic construction industry today offers a wide choice of heat and sound insulation materials, each of which has its own technical characteristics and scope.

In the general balance of the use of heat and sound insulation in Ukraine, which has developed today, about 65 to $70 \%$ of materials are used in enclosing structures, about $15-17 \%$ are used for installation insulation, $8-10 \%$ for heat insulation of pipelines, $4-5 \%$ for sound insulation, and for the refrigeration industry - about $0.6 \%$.

Nomenclature and classification of heat-insulating and acoustic products.

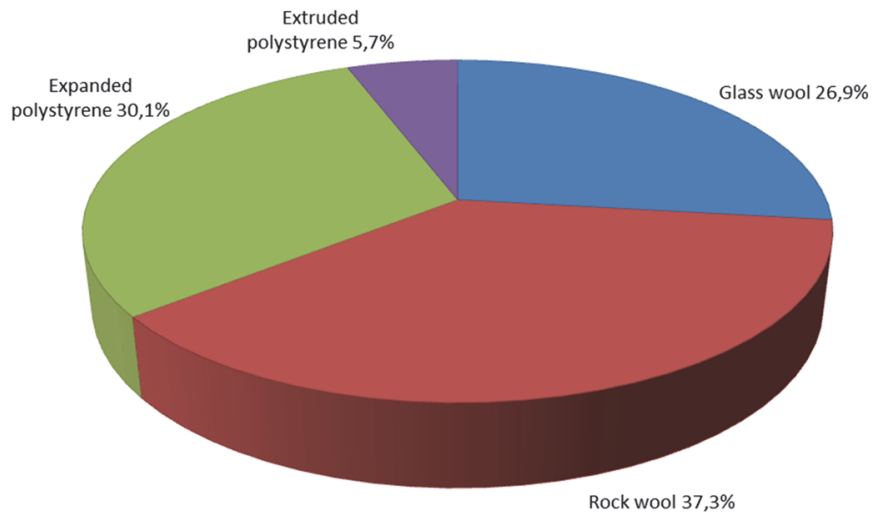

Fig. 10. Market structure of heat and sound insulation materials of Ukraine in 2016, thousand cubic meters

Heat-insulating and soundproof materials according to GOST B16381: 2011, are classified according to form, appearance, structure, type of raw material, density, stiffness (relative deformation of compression), thermal conductivity, flammability.

In terms of form and appearance, the materials are divided into:

- piece products (plates, blocks, brick, cylinders, semi-cylinders, segments);

- rolled and corded (mats, cords, tows);

- loose materials (mineral wool and glass, expanded perlite and vermiculite).

cotton wool and products based on it

One of the main types of heat and sound insulation materials in our country and abroad today is silicate wool and products based on it. This production accounts for more than $50 \%$ of the total volume of thermal insulation materials.

The use of fibrous products in construction during the warming of brick walls reduces by $50 \%$ the demand for bricks, reduces the consumption of cement and lime by 2.5-3 times, labor costs at construction sites and the estimated cost of construction with the same heat resistance of enclosing structures. 
Silicate wool (the general name of glass and mineral wool) and products made of it are the most common form of heat and sound insulation materials. Silicate wool consists of fibers of microscopic thickness, which in most cases are placed chaotically, and the particles of solidified melt that have not formed into fibers (so called "korolki"). The quality of melt processing in fiber is determined by the presence of "korolki" contained in the resulting silicate wool, and thus, by the purity and insulation characteristics of silicate wool.

The main property of silicate wool is incombustibility in combination with high heat and sound insulating ability, resistance to temperature deformation, non-hygroscopicity, chemical and biological resistance, environmental friendliness and ease of installation work.

Products made of these materials retain thermal insulation and mechanical properties at the initial level for decades.

In addition, the use of products from these materials provides a good sound insulation of enclosing structures. Mineral and glass wool significantly reduce the risk of standing sound waves inside the enclosing structures, providing an increase in the insulation index from air noise.

The advantages of mineral wool and fiberglass insulation are the ability to apply them depending on their technical characteristics not only in the form of solid and semi-solid slabs in the facade structures, but also in the form of soft slabs and mats in frame structures, for example, in panels of the type "sandwich".

Production of silicate wool and products from it shows a positive dynamics: from 370 ths. $\mathrm{m}^{3}$ in 1990 to $5.2 \mathrm{mln} . \mathrm{m}^{3}$, while the presence of basalt deposits in Ukraine allowed producing of stone wool at a higher pace in comparison with glass wool.

Table 4. Market of heat insulation materials of Ukraine

\begin{tabular}{|c|c|c|c|c|c|}
\hline \multirow[b]{2}{*}{ Thermal insulation material } & \multicolumn{2}{|c|}{2015} & \multicolumn{2}{|c|}{2016} & \multirow{2}{*}{$\begin{array}{c}\text { Growth } \\
2015 / 2016, \%\end{array}$} \\
\hline & $\begin{array}{l}\text { Volume, } \\
\text { ths. } \mathrm{m}^{3}\end{array}$ & Share, $\%$ & $\begin{array}{l}\text { Volume, } \\
\text { ths. } \mathrm{m}^{3}\end{array}$ & Share, $\%$ & \\
\hline Rock wool & 2943.4 & 37.2 & 3024.9 & 37.3 & 2.8 \\
\hline Glass wool & 2122.0 & 26.8 & 2176.1 & 26.9 & 2.5 \\
\hline Expanded polystyrene EPS & 2480.2 & 31.2 & 2442.4 & 30.1 & -1.5 \\
\hline Extruded polystyrene XPS & 376.6 & 4.8 & 461.0 & 5.7 & 22.4 \\
\hline TOTAL & 7922.2 & 100.0 & 8104.4 & 100.0 & 2.3 \\
\hline
\end{tabular}

The segment of mineral wool insulation in 2016 grew by more than $2.5 \%$ compared to 2015 . If we consider the thermal insulation markets on the basis of glass fiber and basalt separately, then the consumption of fiberglass insulation increased by $2.5 \%$, based on basalt by $2.8 \%$. According to the forecasts of some experts, in the future, these growth rates should be maintained.

More than $95 \%$ of the needs of the Ukrainian market of thermal insulation on the basis of fiberglass is satisfied by the products of four manufacturers of mineral wool insulation "TechnoNicol”, "Knauf Insulation”, URSA, ISOVER.

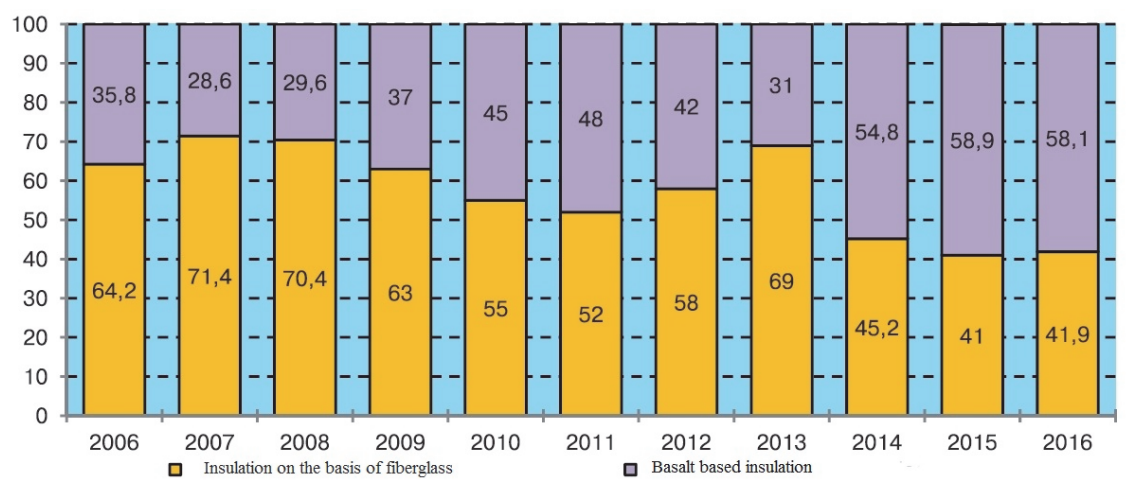

Fig. 11. Distribution of the market of fibrous thermal insulating materials of Ukraine in 2006-2016, \% 
6.1. THE INDUSTRY OF BUILDING MATERIALS OF UKRAINE IN THE CONTEXT OF GLOBALIZATION. PETRO V. ZAKHARCHENKO, OLEKSANDR M. GAVRYSH, ROMAN D. ZAKHARIENKOV

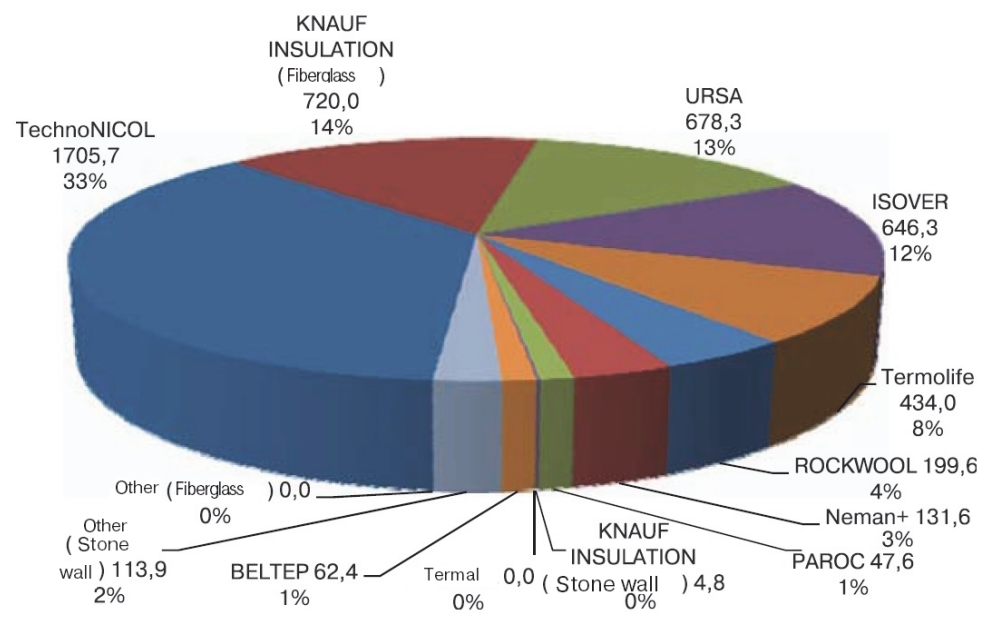

Fig. 12. The market structure of the mineral wool (stone wool + glass wool) of Ukraine 2016 ths. $\mathrm{m}^{3}, \%$

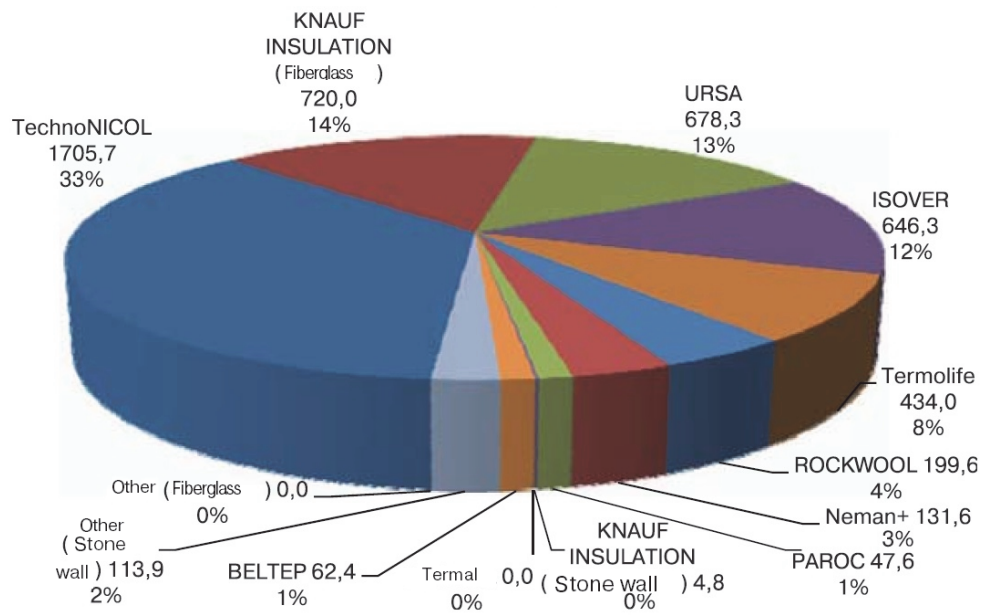

Fig. 13. The market structure of the expanded polystyrene thermal insulation EPS of Ukraine 2016, ths. $\mathrm{m}^{3}, \%$

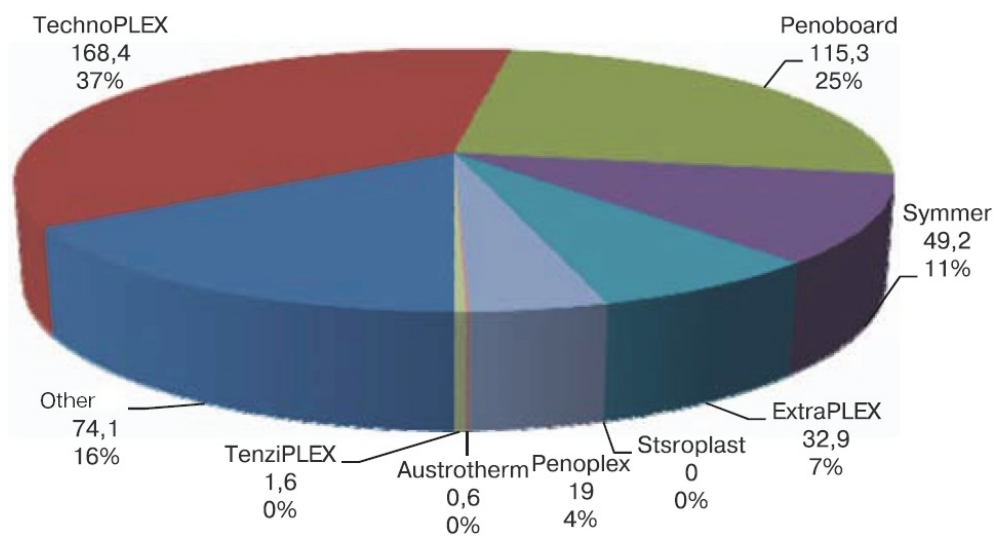

Fig. 14. The market structure of the expanded polystyrene thermal insulation XPS of Ukraine 2016, ths. $\mathrm{m}^{3}, \%$

The market structure of the expanded polystyrene thermal insulation EPS of Ukraine 2016 is 
presented in Fig. 13. The market structure of the expanded polystyrene thermal insulation XPS of Ukraine 2016 is presented in Fig. 14.

By the results of 2016, it is evident that the tightening of the requirements for energy efficiency of buildings and the introduction of a regime of increased energy savings and the economic crisis have led to changes in the market of TIM, but those changes will further activate the Ukrainian market as early as 2017. Forecasting trends for 2018, it should be noted that the segment of light positions of mineral wool will further develop.

In 2016, a new thermal insulation material produced by BASF Neopor appeared on the thermal insulation market.

Neopor ${ }^{\circledR}$ is the newest technical solution offered by BASF for effective thermal insulation in the construction of new and reconstruction of existing buildings. BASF represents Neopor ${ }^{\circledR}$ as an innovative development based on polystyrene with the trade name Styropor ${ }^{\circledR}$, has been successfully applied for thermal insulation and packaging for many years. Neopor ${ }^{\circledR}$ is a polymer (EPS) granulate of expanded polystyrene. Expanded polystyrene based on Neopor ${ }^{\circledR}$ is of silvery-gray color, because it contains graphite, which significantly increases the thermal insulation properties of the material. Expanded polystyrene based on Neopor ${ }^{\circledR}$ significantly exceeds other insulation materials, because compared to the latter it has much higher thermal insulation properties. This decisive advantage reduces the cost of heating the house and increases the value of housing, taking into account the growing environmental requirements. As a result: Neopor ${ }^{\circledR}$ is a contribution to resource saving and the opportunity to create a favorable microclimate in a residential area.

Excellent efficiency of thermal insulation made of Neopor ${ }^{\circledR}$ material is a significant advantage in practical construction for architects, engineers, builders and developers. Absorption or reflection of infrared radiation with Neopor ${ }^{\circledR}$ material significantly reduces the thermal conductivity of the building. The material to a lesser extent than conventional thermal insulation boards, allows the heat to pass through.

Absorption and reflection of infrared radiation can significantly reduce the heat outflow caused by radiation. Using of Neopor ${ }^{\circledR}$ is most advantageous where, with a low absolute density of thermal insulation, you need to achieve maximum efficiency of thermal insulation. Neopor® thermal insulation with a density of $15 \mathrm{~kg} / \mathrm{m}^{3}$ are provided, for example, with a thermal conductivity of $\leq 0.032 \mathrm{~W} /(\mathrm{m} \cdot \mathrm{K})$. For a typical EPS of comparable density, the thermal conductivity parameter is $\geq 0.037 \mathrm{~W} /(\mathrm{m} \cdot \mathrm{K})$.

The production of thermal insulating products based on expanded perlite is successfully developing in Ukraine.

Expanded perlite is the basis of effective heat and sound insulation materials. One of the significant advantages of perlite is that it reduces fire hazard and increases the fire resistance of structures. Perlite is part of flame retardant paints, pastes, fire retardant plaster, it is used as fillers for light concrete. Perlite materials are capable of improving the physical properties of existing structures. The regulation of the humidity of constructions and of the microclimate of premises is one of the peculiarities of materials based on perlite. This material is biologically stable, such that it cannot be decomposed and rotten under the action of microorganisms, is not a favorable environment for insects and rodents. Chemically inert - neutral to alkali and weak acids. Perlite is environmentally friendly and sterile material, non-toxic, does not contain heavy metals.

Expanded perlite is a porous material obtained by thermal treatment of crushed volcanic water-containing rocks. After firing, expanded perlite is divided into sand $(<5 \mathrm{~mm})$ and crushed stone $(5-20 \mathrm{~mm})$, depending on the grain size.

Expanded perlite sand DSTU BV. 2.7-157: 2011 (GOST 10832-2009). Building materials. The expanded perlite sand and crushed stone. Technical specifications. Divide into ordinary PSO - with grains of any fractions in the size from $0.16 \mathrm{~mm}$ to $0.5 \mathrm{~mm}$, large PSL - with grains of the fraction (1.25-5) $\mathrm{mm}$, the average PSA - $(0.16-2.5) \mathrm{mm}$, small PSS - $(0.16-1.25) \mathrm{mm}$, powdered PSP - less than $0.16 \mathrm{~mm}$. 
Table 5. Technical data Neopor®

\begin{tabular}{|c|c|c|c|c|c|c|}
\hline \multirow{2}{*}{ Property } & \multirow[t]{2}{*}{ Unit } & \multirow{2}{*}{$\begin{array}{l}\text { Designation } \\
\text { code acc. to } \\
\text { EN } 13163\end{array}$} & \multicolumn{3}{|c|}{$\begin{array}{l}\text { Metrics for insulation products made } \\
\text { of Neopor } \AA \text { EPS } \AA\end{array}$} & \multirow[t]{2}{*}{ Standard } \\
\hline & & & EPS 70 & EPS 100 & EPS 150 & \\
\hline $\begin{array}{c}\text { Thermal } \\
\text { conductivity, } \lambda_{D}\end{array}$ & $\mathrm{~W} /(\mathrm{m} \cdot \mathrm{K})$ & - & $\geq 0.031$ & $\geq 0.030$ & $\geq 0.030$ & EN 13163 \\
\hline $\begin{array}{c}\text { Thermal } \\
\text { conductivity, rated } \\
\text { value }\end{array}$ & $\mathrm{W} /(\mathrm{m} \cdot \mathrm{K})$ & - & $\geq 0.032$ & $\geq 0.031$ & $\geq 0.031$ & $\begin{array}{l}\text { Deutsche } \\
\text { a.b.Z }\end{array}$ \\
\hline $\begin{array}{l}\text { Compressive } \\
\text { strength at } 10 \% \\
\text { deformation }\end{array}$ & $\mathrm{kPa}$ & CS (10) & $\geq 70$ & $\geq 100$ & $\geq 150$ & EN 826 \\
\hline $\begin{array}{l}\text { Tensile strength } \\
\text { perpendicular to } \\
\text { plane of board }\end{array}$ & $\mathrm{kPa}$ & $\mathrm{TR}$ & $\geq 100$ & $\geq 150$ & $\geq 200$ & EN 1607 \\
\hline Flexural strength & $\mathrm{kPa}$ & BS & $\geq 115$ & $\geq 150$ & $\geq 200$ & EN 12089 \\
\hline Shear strength & $\mathrm{kPa}$ & $\mathrm{t}$ & $\geq 35$ & $\geq 60$ & $\geq 85$ & EN 12090 \\
\hline $\begin{array}{c}\text { Dimensional } \\
\text { stability after } 48 \mathrm{~h} \\
\text { at } 70^{\circ} \mathrm{C}\end{array}$ & $\%$ & DS $(70,-)$ & $\leq 1$ & $\leq 1$ & $\leq 1$ & EN 1604 \\
\hline $\begin{array}{c}\text { Deformation after } \\
48 \mathrm{~h} \text { at } 20 \mathrm{kPa} \text { and } \\
80^{\circ} \mathrm{C}\end{array}$ & $\%$ & DLT (1) 5 & - & $\leq 5$ & $\leq 5$ & EN 1605 \\
\hline $\begin{array}{c}\text { Deformation after } \\
168 \mathrm{~h} \text { at } 40 \mathrm{kPa} \\
\text { and } 70^{\circ} \mathrm{C}\end{array}$ & $\%$ & DLT (2) 5 & - & - & $\leq 5$ & EN 1605 \\
\hline $\begin{array}{c}\text { Water vapor } \\
\text { diffusion } \\
\text { resistance factor } \mu\end{array}$ & - & - & $20-40$ & $30-70$ & $30-70$ & EN 12086 \\
\hline $\begin{array}{l}\text { Thermal linear } \\
\text { change coefficient }\end{array}$ & $\mathrm{K}^{-1}$ & - & $60-80 \cdot 10^{-6}$ & $60-80 \cdot 10^{-6}$ & $60-80 \cdot 10^{-6}$ & $\begin{array}{c}\text { DIN } \\
53752 \\
\end{array}$ \\
\hline Reaction to fire & Euroclass & - & $\mathrm{E}$ & $\mathrm{E}$ & $\mathrm{E}$ & $\begin{array}{c}\text { EN } \\
13501-1 \\
\end{array}$ \\
\hline $\begin{array}{l}\text { Chemical } \\
\text { resistance }\end{array}$ & \multicolumn{6}{|c|}{ Insensitive to water, most acids, and lyes. Sensitive to organic solvents. } \\
\hline $\begin{array}{l}\text { Biological } \\
\text { behavior }\end{array}$ & \multicolumn{6}{|c|}{$\begin{array}{l}\text { No influence on microorganisms. Does not decay or rot. Chemically neutral, not } \\
\text { soluble in water. No known health hazards. }\end{array}$} \\
\hline
\end{tabular}

Perlite-cement dry mixtures. Used in Europe and Ukraine in the form of heat-insulating plaster mixtures, lightweight billets for the floor and solutions for masonry. They can be used both for the construction of new and for the reconstruction of old buildings that do not meet the current requirements for thermal protection.

Perlite-concrete wall blocks. Full-walled blocks with the density of $500-700 \mathrm{~kg} / \mathrm{m}^{3}$ are manufactured using the vibration-pressing method and are used in Ukraine for the construction of low-rise, multi-storey and high-rise buildings. Due to the granular structure and the presence of a glassy phase of perlite filler, perlite concrete has better thermal-technical characteristics than autoclaved cellular concrete of similar density.

Thermal-insulated plates in the shell. Promising development of NDIBMV - non-flammable thermal-insulated plates made of expanded perlite on an inorganic binder in a shell with the effect of reflective insulation.

Characteristics:

- density - 200-250 kg/m³

- thermal conductivity (piped products) - 0.05-0.06 W/(mK);

- thermal conductivity (using vacuum or modified gas medium) $-0.02-0.03 \mathrm{~W} /(\mathrm{mK})$; 
- compressive strength is $0.2-0.3 \mathrm{MPa}$.

Perlite products on liquid glass are produced of two types - perlitephosphogel and perlite calcine lightweight.

Perlitephosphogel products consist of perlite expanded sand (60-70\%) and liquid glass (40-30\%). Products are used for fire protection and thermal insulation of building structures, equipment, pipelines at temperatures up to $600^{\circ} \mathrm{C}$.

When installing a waterproofing coating, plates, on which paper is fixed with the help of bitumen, are used to insulate structures, equipment and tanks.

The products are manufactured in the form of plates, shells (semicylinders) and segments with a length of 450-1000 mm, width (for plates) - 250-500 $\mathrm{mm}$ and internal diameter (for shells and segments) - 57-426 mm, thickness 40-100 mm.

Perlite-ceramic products are made of a mixture of expanded perlite sand and plastic refractory clay.

The products are intended for thermal insulation of industrial equipment, furnaces and pipelines at a temperature of no more than $875^{\circ} \mathrm{C}$.

Perlite-ceramic products are divided according to the density into brands: 200, 220, 250, 300, 350,400 . Products are produced in the form of plates, bricks, shells and segments.

Lightweight refractory perlite-chamotte products consist of expanded perlite sand (3-13\%), refractory clay (16\%) and chamotte (71-81\%).

Based on the above, we can conclude that the building materials industry of Ukraine is a powerful player in the market of building materials and products and a leading country in the post-Soviet space in attracting of modern foreign technologies.

It can be predicted that by 2025 traditional building materials, ceramic and silicate bricks will be replaced with clinker bricks and products made from autoclaved aerated concrete. The segment of dry building mixtures will significantly increase its presence in the total production of binders. With an increase in construction volumes, dry construction systems (plasterboards), whose production capacity can reach $200 \mathrm{mln} . \mathrm{m}^{2} /$ year, will be increasingly in demand.

\section{References}

[1] Zakharchenko P. V., Gavrysh A. M., Sokha V. G., Reva V. I., et al. A Guide to the Market of Materials for Internal Arrangement and Decoration of Premises (According to 2015). Under the General Editorship of Prof. Zakharchenko P. V., KNUBA, IE Pavlenko, 2016, p. 252.

[2] Zakharchenko P. V., Lenga G., Gavrysh A. M., Piven N. M. Technology and Goods Management of Dry Construction Systems. KNUBA, Edition 2, IE Pavlenko, 2011, p. 512.

[3] Zakharchenko P. V., Gavrysh A. M., Kashchenko T. O., Piven N. M. Workshop on Technology and Goods Management of Dry Construction Systems: Tutorial. KNUBA, Edition 2, LLC Publishing Workshop, 2016, p. 384.

[4] Zakharchenko P. V., Gavrysh A. M., Karpenko O. P., Petukhova O. M. Technology and Goods Management of Dry Construction Systems: Fire Protection of Building Structures. Tutorial. KNUBA, IE Pavlenko, 2012, p. 392.

[5] Zakharchenko P. V. Recommendations on the design and installation of partitions with plasterboards KNAUF, which are required fire safety and fire protection of building structures. Annex 2 to the tutorial "Technology and goods management of dry construction systems: fire protection of building structures". KNUBA, IE Pavlenko, 2012, p. 50.

[6] Karapuzov E. K., Sokha V. G., Ostapenko T. Ye Materials and Technologies in Modern Construction: Textbook. Higher Education, 2006, p. 495.

[7] Zakharchenko P. V., Sokha V. G., Piven N. M. Waterproofing Systems. Consumer Properties. Test Methods and Devices of Arrangement. Tutorial. KNUBA, LLC Publishing Workshop "SPD Pavlenko", 2017, p. 180.

[8] Zakharchenko P. V., Gavrysh O. M., Dolgy E. M., Galagan Y. O. Thermal and Sound Insulation Materials and Products in Energy-Saving Technologies. Textbook. Masters, 2008, p. 340. 


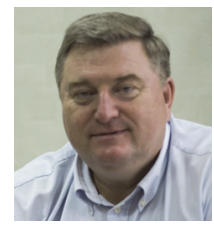

Petro V. Zakharchenko, Kyiv Nationality University of Building and Architecture. Honored master Education of Ukraine, Full Member of the Academy of Building of Ukraine. Professor, Doctor of Philosophy, Ph.D. Technical Science, head of Department of Goods Management and Commercial Activity in Building. Total number of his publications is 220 .

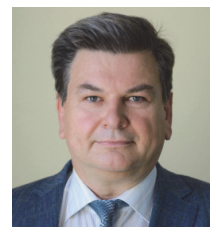

Oleksandr M. Gavrysh, Kyiv Nationality University of Building and Architecture. Honored Builder of Ukraine. Professor, Doctor of Philosophy, Ph.D. of Philological Science. Full Member of the Academy of Building of Ukraine. Total number of scientific publications is 115 .

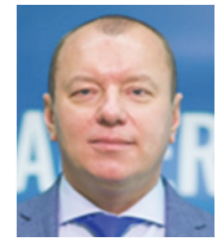

Roman D. Zakharienkov, State University of Telecommunications, Communications Engineer. President of Ukrainian Association of Styrofoam Manufacturers, Corresponding Member of the Academy of Building of Ukraine. The Association's purpose is solving common problems for certification of raw materials and products, dissemination of information about products and possible technologies for its use, support for the development and introduction of appropriate production and technologies, study and sharing the best practices of manufacturers and users of products, assistance in attracting domestic and foreign investment in the industry. 


\title{
6.2. A new method for determining an acoustical characteristic of building materials
}

\author{
Tamaz Natriashvili ${ }^{1}$, Merab Chelidze ${ }^{2}$, David Nizharadze ${ }^{3}$, Jemal Javakhishvili ${ }^{4}$ \\ LEPL R. Dvali Institute of Machine Mechanics, Tbilisi, 0112, Georgia \\ ${ }^{2}$ Corresponding author \\ E-mail: ${ }^{2}$ merabchelidze@yahoo.com
}

\begin{abstract}
The modern digital technique and software give the ability that the sound absorption coefficient was measured by the method of the sound amplitudes decay in a closed chamber, namely in the impedance tube, by means of the ratio of the subsequent attenuating reflected waves. Taking into account the influence of the length and diameter of the impedance tube on the process of decay of sound waves, a new study of sound propagation in an impedance tube is presented, on the basis of which the sound absorption coefficient is determined much easier.
\end{abstract}

Keywords: sound, wave, reflection, reverberation, decay, absorption, measurement, vibration.

\section{Introduction}

Sound, a form of energy, is caused by molecules vibrating in a gas, liquid or solid and it propagates in the air as waves by changing of atmospheric pressure i.e. by disturbance of the air pressure. Sound after exposure to solid materials is converted into vibration and thus spreads into them. The frequency or pitch of the noise, measured in hertz, is the number of sound waves emitted per second.

Noise can be described as unwanted sound, the intensity of sound depends on pressure levels which are measured in decibels $(\mathrm{dB})$. Previous studies have shown that the results for an absorption coefficient test are dependent on the testing method, that is, the absorption coefficients of the same material with the same properties will vary depending on the testing method. Sound insulation refers to the ability of the building material to resist the transmission of airborne and impact sound. Some existed typical noise levels are given in Table 1. It should be noted that all the noise levels presented in Table 1 are different for different countries, for example, the highly developed countries have more stringent requirements.

Table 1. noise levels in pascals and decibels

\begin{tabular}{|c|c|c|c|}
\hline Noise & Explanations & Pascal & $\mathrm{dB}$ \\
\hline Lower threshold of audibility & Sound is not heard & $2 \cdot 10^{-5}$ & 0 \\
\hline A quiet rustle of leaves & Is heard at a distance of $1 \mathrm{~m}$ & $6.32 \cdot 10^{-5}$ & 10 \\
\hline At night & \multirow{2}{*}{$\begin{array}{l}\text { The sound pressure level provided for by the } \\
\text { standards for living rooms }\end{array}$} & $6.3 \cdot 10^{-4}$ & 30 \\
\hline In the afternoon & & 0.002 & 40 \\
\hline $\begin{array}{l}\text { In the workplace intermittent } \\
\text { noise }\end{array}$ & The sound level should not exceed & 6.3 & 110 \\
\hline Impulse noise at the workplace & $\begin{array}{l}\text { Permissible for a short period in the form of } \\
\text { impulse noise }\end{array}$ & 35.1 & 125 \\
\hline Jet plane at the start & Feeling of pain in the ears at a distance of $1 \mathrm{~m}$ & 63 & 130 \\
\hline $\begin{array}{l}\text { The environment in which high } \\
\text { sound pressure }\end{array}$ & $\begin{array}{l}\text { Even for a short period of time it is } \\
\text { inadmissible to remain }\end{array}$ & 112.5 & 135 \\
\hline Excessive sound pressure & $\begin{array}{l}\text { Causes a rupture of the septum of the ear can } \\
\text { even lead to death }\end{array}$ & 2000 & 160 \\
\hline Epicenter of the explosion & Causes death of a person & 200000 & 200 \\
\hline
\end{tabular}

\section{The new heat and sound-insulation building materials}

To solve the problem necessary the creation of low-cost building materials based on local resources. Creation of such materials in the local area makes it possible to improve, as the sound 
insulation so heat insulating characteristics, which is connected with the decision of very important social and economic problems. It should be noted soundproofing material has very good heat insulation characteristics as well.

Sound insulation and heat proofing characteristic of building wall partitions are improved greatly when foam concrete is used in it as a layer of the sandwich. For obtaining foam concrete is used soon spoiling organic components that are very uneasy (clumsy) for little and serial enterprises in terms of storage and transportation costs. The foam material created by us on the basis of local raw materials is cheap and durable because it is based on inorganic materials and they are rosin and glue. The noted technological process is new and, possibly, promising in our market.

To develop a new production technology for relatively cheap sound-proof building materials and to determine the acoustic and heat non-conductive characteristics for the materials obtained, it is necessary to simplify the method of determining the absorption coefficient of materials, so that in practice it is widely used in production and at homes by citizens.

\section{Theory of propagation and reflection sound waves.}

Measureless and frequency dependent the sound absorption coefficient $\alpha$ presents the main acoustical characteristic of materials and in agreement of the determination is the ratio of the absorbed $E_{a b s}$ and the incident $E_{\text {inc }}$ energies [1-3].

$\alpha=\frac{E_{a b s}}{E_{\text {inc }}}$.

Though in some cases $\alpha$ is determined as a relation all not reflected energies with incident energy:

$a=1-\frac{E_{\text {ref }}}{E_{\text {inc }}}=\frac{E_{a b s}+E_{\text {tran }}}{E_{\text {inc }}}$,

where in a consent of the conservation energy $E_{\text {inc }}=E_{\text {ref }}+E_{a b s}+E_{\text {tran }}$.

Besides of the vacuum, the sound is propagated in any materials. The sound is appeared by any disturbance of the air pressure $p$; density $\rho$; environment particles speed $v$ and temperature $t$. The functional connection between air pressure and density is expressed by adiabatic equation $p=f(\rho)$. The differentiation of which gives the sound composition equation [4-6]:

$\frac{\partial p}{\partial x}=\frac{1}{c^{2}} \frac{\partial p}{\partial t}$

where $c$ - the speed of sound propagation

The solution of the equation of (1) may be presented by two periodic and harmonic propagating waves [6]:

$p=A \cos (\omega t-k x)+B \cos (\omega t+k x)$.

For periodic oscillations (4) takes view:

$p=f_{1}\left[\frac{c}{\omega}(\omega t-k x)\right]+f_{2}\left[\frac{c}{\omega}(\omega t+k x)\right]$,

where $k=\frac{\omega}{c}=\frac{2 \pi f}{c}=\frac{2 \pi}{\lambda}$ waves oscillations, $\lambda=\frac{c}{f}-$ is length of waves.

For harmonic waves, Eq. (5) takes the following form: 
$p=A \cos \frac{\omega}{c}(c t-x)+B(c t+x)=A \cos (\omega t-k x)+B \cos (\omega t+k x)$.

The Eq. (6) frequently is presented in a complex form for simplifying summing calculation operations of different frequency waves:

$p=\left(\bar{A} e^{-j k x}+\bar{B} e^{-j k x}\right) e^{-j k x}=\bar{A} e^{-j k x-j k x}+\bar{B} e^{-j k x-j k x}$.

In accordance with equations of (6) and (7) the incident and reflected flat waves are summed that is, depending on the phase of the waves, the corresponding amplitudes increase or decrease. In accordance with equations of (4) $p=X(x) T(t)$ if there will be taken two private solutions and they will be supplied in Eq. (3) then will be obtained equations which left and right parts will equal [6]. There may be such solution where they will equal to $-k^{2}$ :

$\frac{1}{C^{2}} \frac{\partial^{2} T}{\partial t^{2}}=\frac{\partial^{2} p}{\partial t^{2}}+\frac{1}{X} \frac{\partial^{2} X}{\partial x^{2}}=-k^{2}$.

From Eq. (8) are obtained two equations with following solutions, which are analogous of oscillation of material [6]:

$\frac{\partial^{2} X}{\partial x^{2}}=-k^{2} X, \quad X=A \cos \left(k x+\varphi_{x}\right), \quad \frac{\partial^{2} T}{\partial t^{2}}=-k^{2} c^{2} T=\omega^{2} T, \quad T=B \cos \left(\omega t+\varphi_{t}\right)$,

where $\omega^{2}=k^{2} c^{2}$.

The solution of wave equations is equal:

$p=X T=C \cos \left(k x+\varphi_{x}\right) \cos \left(\omega t+\varphi_{x}\right)$.

The obtained equation describes free oscillation of standing waves. So private solution of the equation $p=X(x) T(t)$ gives us possibility from endless solutions were searched the standing waves equation. The solution of plane waves can be represented as in form standing waves so moving waves. Moving plain waves are characteristic for unstable processes and standing waves for periodic and stable processes [6,7].

Usually, $\alpha$ is in a functional dependence with the sound incidence angle of $\theta_{\text {inc }}$. When sound waves are fallen on the materials by the right angle the absorption ability of the materials are denoted by $\alpha N$. So, the simplest method evaluation of materials absorption ability $\alpha N$ and surface impedances $\partial N$ are to use the impendance tube in which are generating plane waves [3-5].

When a simple wave falls at a right angle at the point $x=0$ on the body of separation of two media (Figure 1), then a part of the wave is reflected, and the other part is transmitted into the second environment. The propagation of sound in both media is described by the wave equation and they can be integrated independently of each other $[3,6]$.

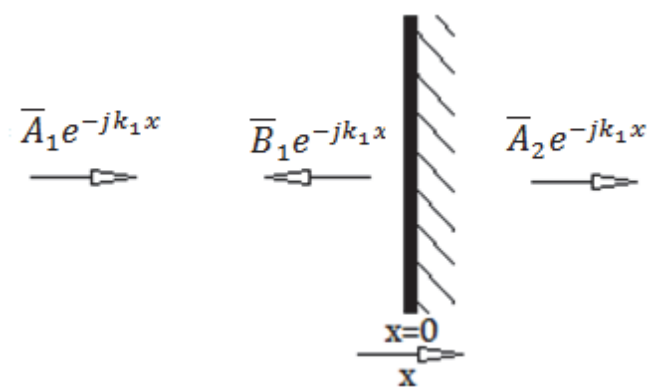

Fig. 1. Sound reflection, transition and two media separating barrier 
The solution of the incident and reflected wave equations is equal to:

$\bar{p}_{1}=\bar{A}_{1} e^{-j k_{1} x}+\bar{B}_{1} e^{-j k_{1} x}$

But the solution of the transmitted equation is equal to:

$\bar{p}_{2}=\bar{A}_{2} e^{-j k_{1} x}$.

Since the second environment is presented as endless that $\bar{B}_{2}=0$ so the sum of incident and reflected sounds pressures P1 located at left side of dividing barrier must be equal to penetrated sound pressure $\mathrm{P} 2$ located at the right side of the barrier [6]:

$\left(\bar{P}_{1}\right)_{x=0}=\left(\bar{P}_{2}\right)_{x=0}, \bar{A}_{1}+\bar{B}_{1}=\bar{A}_{2}$.

Theoretical analysis of the above-described sound equations shows that the sound wave in materials behaves similarly to the vibration wave, which makes it possible for the absorption coefficient to be determined by the decay process of the reverberating amplitudes, as in the case of the theory of oscillations. Moreover, the sound in the materials is spread in the form of vibration and vibration is the source of the creation and propagation of sound waves in the atmosphere.

The full period of propagation plane waves from one side covers of the tube to another and back, i.e. one full cycle $T_{\text {lon }}$, is a ratio of the double length of the tube to the speed of sound propagation in air. If the length of the tube is more than 4 diameters, stable flat and standing waves form in the tube in the longitudinal direction $[3,4,7]$. When the noise source is turned off, in the tube, the amplitudes of the standing and plane waves are fallen at the same speed. In accordance with formula (1), the value of decay of sound amplitudes in each cycle is equal to the transmitted and absorbed sound energy by the materials on the side covers of the tube.

White and octave waves emitted by a sound source create very complexly and unstable reverberating waves in the impedance tube when simple (coherent) sound waves of frequency $f$ create simple waves oscillating with a frequency $f$ in the tube. Sound waves, circulating in a closed impedance tube, are repeatedly reflected from the side covers of the tube. The resulting reverberating plane waves act on each other, are summed up and create stable standing waves in the tube (Fig. 2). The absorbent material, that is fixed to the side end of the tube (Fig. 2), causes a change in the amplitude of the standing waves [3, 8, 9]. Experimental investigation showed that little appreciable differences are obtained in the results from using the short and long tubes.

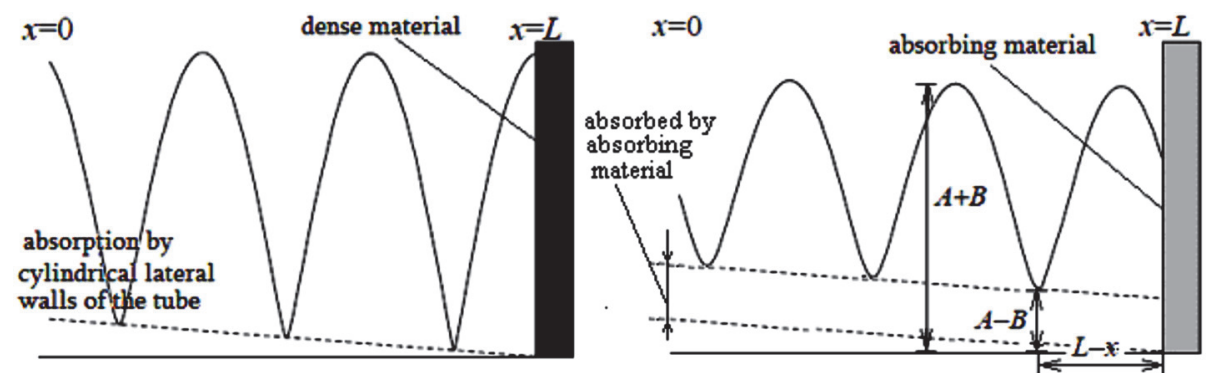

Fig. 2. Standing waves in the impedance tube

\section{Experimental investigation and obtained results}

Performed theoretical and experimental studies, by the help of modern digital technologies, have shown that the propagation of the waves of white noise in a closed tube creates and establishes a complex unstable reverberating oscillation (Fig. 3(a)). It should be noted that in the 
case of white noise and an impedance tube with a length $L=0.63 \mathrm{~m}$, along the tube side, covers clearly are visible longitudinal reverberating sound waves oscillations with the frequency of $f_{\text {lon }}=300 \mathrm{~Hz}$, whose frequency is equal to the ratio of the sound speed $s$ to the double length of the tube $2 \mathrm{~L}$, i.e. $f_{\text {lon }}=s / 2 \mathrm{~L}[3,13]$. If the sound source is coherent, for example with a frequency of $2000 \mathrm{~Hz}$, coherent waves with a frequency of $2000 \mathrm{~Hz}$ are also installed in the tube, but in this case, practically, noticeable longitudinal oscillations with frequency $f_{\text {lon }}=300 \mathrm{~Hz}$ are not detected (Fig. 3(b)).

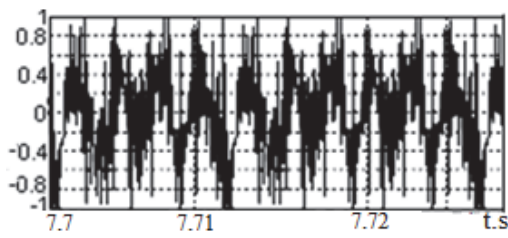

a) $f_{1}=300 \mathrm{~Hz} ; \tau=0.0033 \mathrm{sec}$

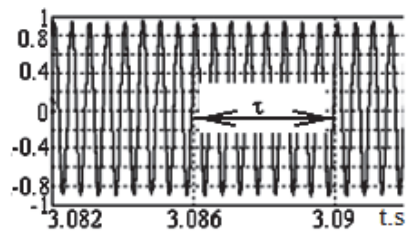

b) $f=2000 \mathrm{~Hz} ; \tau_{f}=0.0005 \mathrm{sec} ; \tau=0.0036 \mathrm{sec}$

Fig. 3. Reverberating white a) and coherent b) sound waves in the impedance tube

In the tube, since the moment the sound source is turned off, a transient process of decay of the reverberating amplitudes occurs until the sound waves completely disappear (Fig. 4(a)). Approximately the frequency of the decay of longitudinal waves with white noise is equal to $f_{\text {lon }}=300 \mathrm{~Hz}$, and for a coherent noise source Fig. $4(\mathrm{~b}), f_{\text {lon }}=285 \mathrm{~Hz}(\tau=0.0036 \mathrm{sec})$, and both are related to the length of the impedance tube.

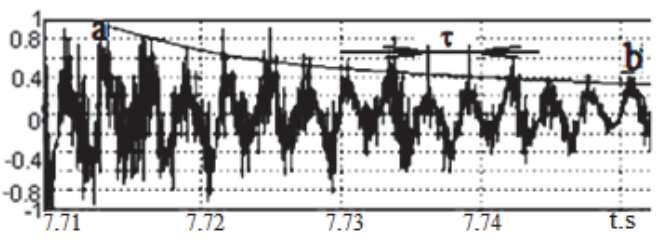

a) $f_{\text {lon }}=300 \mathrm{~Hz}, \tau=0.0033 \mathrm{sec}$

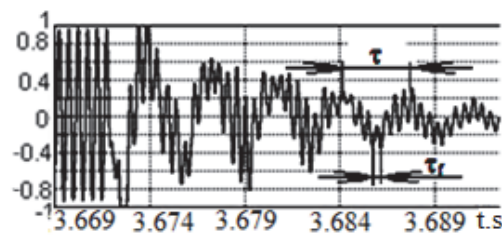

b) $f_{\text {lon }}=285 \mathrm{~Hz}, \tau_{f}=0.0005 \mathrm{sec}, f=2000 \mathrm{~Hz}$

Fig. 4. Decay processes of white a) and coherent $b$ ) reverberating sound waves

According to Fig. 4(b), the period $\tau$ and the frequency $f$ of the decay reverberant sound amplitudes can be calculated as follows. The period of coherent sound waves $\tau_{f}$ is equal to time mark interval divided by the number of amplitudes of this interval $\tau_{f}=(3.689-3.684) / 11=0.0005 \mathrm{sec}$. Thus, in accordance with the period $\tau_{f}$, the frequency of the noise source is $f=1 / \tau_{f}=1 / 0.0005=2000 \mathrm{~Hz}$. Consequently, the period and frequency of longitudinal oscillations will be $\tau=n \tau_{f}=7 \cdot 0.0005=0.0035 \mathrm{sec} . f_{\text {lon }}=1 / \tau=285 \mathrm{~Hz}$. Where $\mathrm{n}$ is the number of amplitudes in the period $\tau$. The curve ab in Fig. 4(a) shows the average rate of the decay process of group amplitudes of longitudinally reverberating waves.

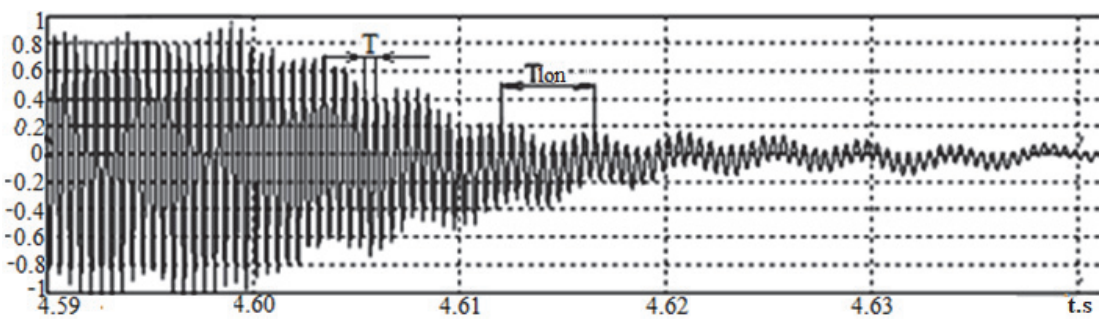

Fig. 5. Sound decay exited frequency $f=2000 \mathrm{~Hz}$, decay frequency $f_{\text {lon }}=200 \mathrm{~Hz}, L=0.8 \mathrm{~m}$

It should be noted that in any process of decaying waves, in conjunction with the frequency $f$ emitted by the sound source, frequencies of longitudinal waves are observed, which are created in 
a long tube by frequent reflections from the side covers of the tube (Figs. 5-7).

To improve the accuracy of measurement, the sound reflection coefficient of the tube wall should be close to 1 , i.e. material for the tube should be bronze, with a minimum wall thickness of $5 \mathrm{~mm}$, although the tube material may be steel $[5,10,11]$.

Thus, in an impedance tube, two types of waves are simultaneously generated: 1) waves with a frequency $f$ emitted by the sound source, and 2) longitudinally reflecting waves produced in the tube. The process of decay standing waves in an impedance tube are combinations of sums of frequencies $f$ and $f_{l o n}$, which decay at different frequencies and times.

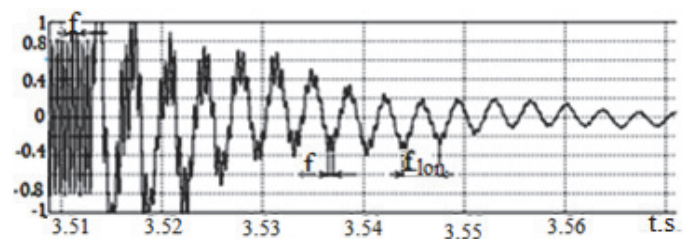

Fig. 6. Sound decay, exited frequency $f=2000 \mathrm{~Hz}$, decay frequency $f_{\text {lon }}=250 \mathrm{~Hz}, L=60 \mathrm{~cm}$

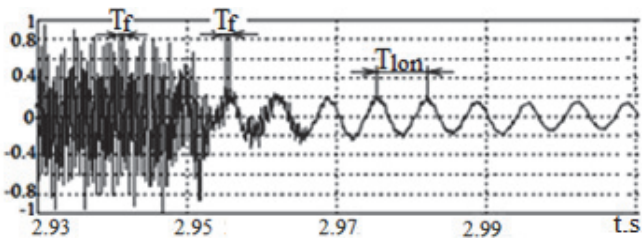

Fig. 7. Sound decay, exited frequency $f=2000 \mathrm{~Hz}$, decay frequency $f_{\text {lon }}=150 \mathrm{~Hz}, L=1 \mathrm{~m}$

The fact that the ability of a material to absorb sound energy is related to the frequency of sound is clearly visible in Figs. 6 and 7, where high-frequency $f$ waves emitted from a sound source are absorbed faster than longitudinally reverberating low-frequency sound waves $f_{\text {lon }}$ excited in an impedance tube, although at a given frequency, in the presence of another absorbent material (e.g., a cork tree), they can decay simultaneously (Fig. 5).

Each longitudinally oscillating amplitude (wave), reflected by the side covers during the decay, contains a set of white noise amplitudes emitted by the noise source (Fig. 4(a)), and in the case of coherent noise, it contains only a few amplitudes $[12,13]$. The number of coherent amplitudes contained in longitudinally oscillating waves is directly related to the tube length and is equal to $n=L \cdot 2 \cdot f / s$. Fig. 6 shows that one longitudinal wave of $2000 \mathrm{~Hz}$ contains 7 radiated waves when the tube length is $0.6 \mathrm{~m}$, but with a tube length of $1 \mathrm{~m}$, one amplitude contains 13 emitted coherent amplitudes from the noise source Fig. 7.

In accordance with formula (1), the absorption coefficient $\alpha$, in the case of amplitudes decay of complex form, also can be determined by the ratios of reflected amplitudes Figs. 5-7.

Figs. 8 and 9 show the processes of decay in the presence of coherent sounds of 100 and $2000 \mathrm{~Hz}$, occurring in an impedance tube of length $L=0.585 \mathrm{~m}$. In Fig. 8, we see the process of decay of a sound with a frequency $f=100 \mathrm{~Hz}$, whose decay frequency is $f_{w}=47 \mathrm{~Hz}$ which contains 5.7 waves, the decay frequency of which is $f_{\text {lon }}=285 \mathrm{~Hz}$. Since the length of the sound wave of 100 Hertz is $334 \cdot 0.01=3.34 \mathrm{~m}$ and 5.8 times the length of the tube $L=0.585$, then for one full period of the $100 \mathrm{~Hz}$ wave, the tube reflects 5.8 times in lengthwise direction, so $L_{w}=(1 / 285) \cdot 6 \approx 47 \mathrm{~Hz}$.

As for Fig. 9, the length of one sound wave of $2000 \mathrm{~Hz}$ is $0.167 \mathrm{~m}$ and 3.5 times less than the length of the tube $L=0.585 \mathrm{~m}$. A very interesting process takes place in an impedance tube when the source of coherent noise is cut off. In the process of sound attenuation, because of the absence of waves emitted by the sound source, the previous reflected waves assume the role of a sound source, so in this case standing waves are created by additional reflected waves, otherwise standing waves will not be created in the tube (Eqs. (6), (7)). Thus, in the decay process, in closed spaces, twice reflected two waves moving in opposite directions create standing waves which frequency is increased two times Fig. 9. Therefore, out of 3.5 longitudinally oscillating amplitudes, 7 standing oscillating amplitudes are obtained.

To achieve standing waves, it is necessary that the length of the tube is more than 3-4 diameters, and above $1700 \mathrm{~Hz}$ the diameter of the tube should not exceed $30 \mathrm{~mm}$. Since the decay of sound waves at $2000 \mathrm{~Hz}$ recorded in Figure 9 was performed by a tube $100 \mathrm{~mm}$ in diameter, so the shape of the decay differs from the decay mode shown in Fig. 10 where the decay process was made by 
means of a tube with a diameter of $25 \mathrm{~mm}$. So high-frequency sound waves emitted by a noise source during the decay process at each longitudinal reverberating amplitude (wave) do not practically change in magnitude.

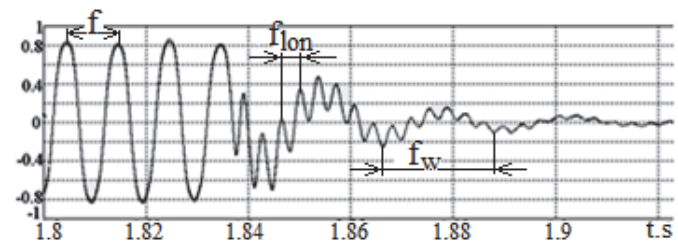

Fig. 8. Sound decay, exited frequency $f=100 \mathrm{~Hz}$, decay frequency $f_{\text {lon }}=285 \mathrm{~Hz}, f_{w}=47, L=58.5 \mathrm{~cm}$

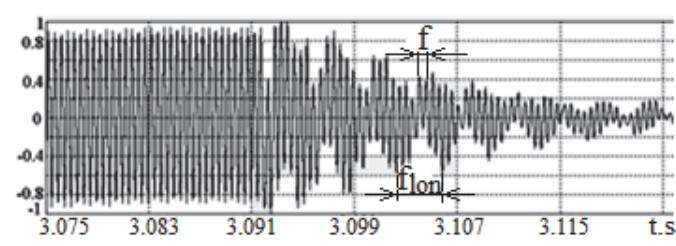

Fig. 9. Sound decay, exited frequency $f=2000 \mathrm{~Hz}$, decay frequency $f_{\text {lon }}=285 \mathrm{~Hz}, L=58.5 \mathrm{~cm}$

Fig. 11 shows the process of decay of waves in an impedance tube, when high-frequency sound waves emitted by a noise source have $f=200 \mathrm{~Hz}$. and the frequency of standing waves created in the impedance tube has $f_{\text {lon }}=285 \mathrm{~Hz}$. In this case, the amplitudes of the longitudinal oscillations, as compared with Fig. 8, increase significantly since the indicated frequency is closer to each other, i.e. a resonant phenomenon is created in the tube and when the source of noise is turned off, the decay of the amplitudes of the longitudinal oscillations occurs almost at the frequency that has a coherent noise source.

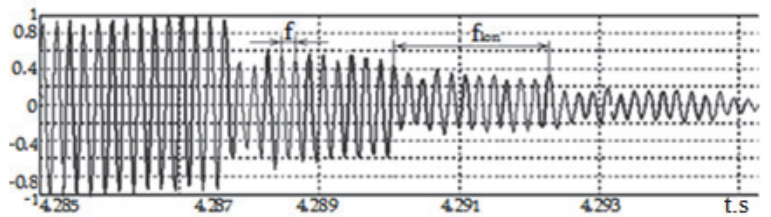

Fig. 10. Sound decay, exited frequency $f=5000 \mathrm{~Hz}$, decay frequency $f_{\text {lon }}=415 \mathrm{~Hz}, L=40 \mathrm{~cm}$

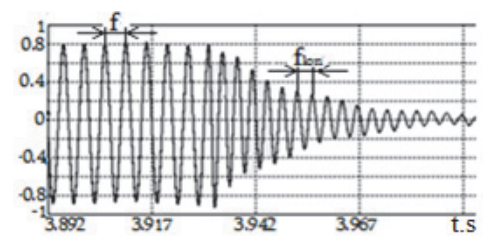

Fig. 11. Exited frequency $f=200 \mathrm{~Hz}$, decay frequency $f_{\text {lon }}=285 \mathrm{~Hz}, L=58.5 \mathrm{~cm}$

The program VT RTA-168 makes it possible to look for such a deployment scale when the longitudinal decay waves (Fig. 12(a)) will be represented in a clean longitudinal reverberating form without shown of waves emitted from the sound source (Fig. 12(b)). The curve ab on both oscillograms shows the time duration and the attenuation rate of the longitudinal oscillation amplitudes in Fig. 8(a) in the expanded form and in Fig. 8(b) in compressed form.

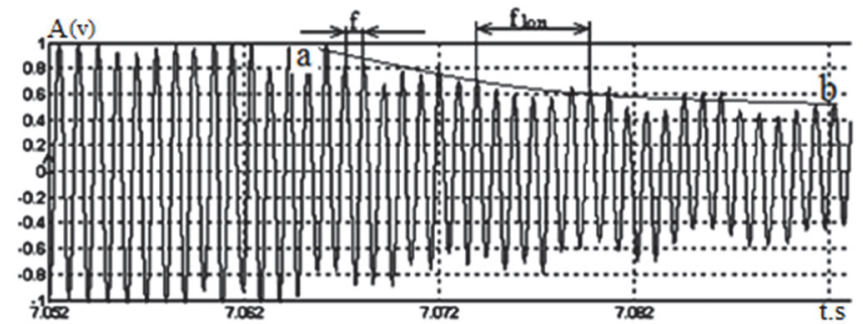

a)

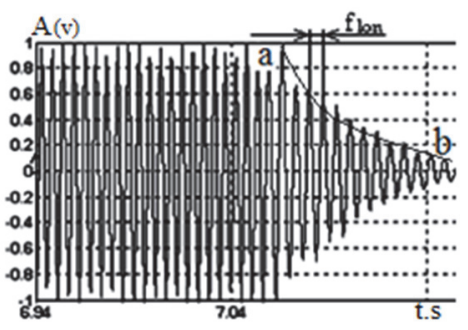

b)

Fig. 12. Sound decay processes, a) exited $f=1000 \mathrm{~Hz}$, decay $\left.f_{\text {lon }}=150 \mathrm{~Hz}, \mathrm{~b}\right)$ Waveform is compressed

The rate of decay of plane waves in an impedance tube is determined by the imperfect reflection of sound from both sides, the propagation of sound in the air, and the resistance of the side walls to the sound waves propagating along the length of the tube, as confirmed by experimental studies. If one side tube cover is replaced by a sample material that has a different absorbent capacity, the sound decay rate in the tube will be changed accordingly Fig. 13. 

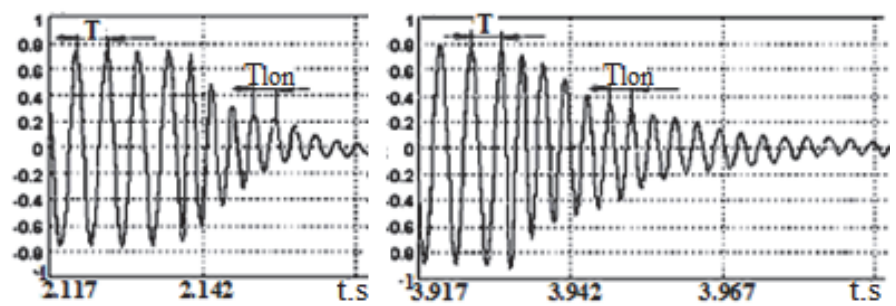

Fig. 13. Sound decay of concrete and marble, exited $f=200 \mathrm{~Hz}$, decay $f_{\text {lon }}=250 \mathrm{~Hz}, L=65 \mathrm{~cm}$

On the basis of experiments, a theoretical point of view was proved that by the selection of the tube length, the wave frequency created in the tube maybe coincided with the frequency of the coherent wave radiated from the sound source i.e., when the length of the tube coincides with a certain wavelength of sound. When the sound frequencies $f$ and $f_{\text {lon }}$ are the same, the wave attenuation is determined mainly by the absorbing capacity of the material situated from the longitudinal side of the tube.

But it should be noted that in all the cases considered, we are dealing with the absorption coefficient associated with the frequency of longitudinal oscillations arising in the tube itself, rather than the frequency emitted by the noise source. But on the basis of the conducted experiments it can be concluded that for a certain ratio of the length of the tube and the length of the exciting coherent wave, the frequencies $f$ and $f_{\text {lon }}$ can coincide with each other Fig. 14.

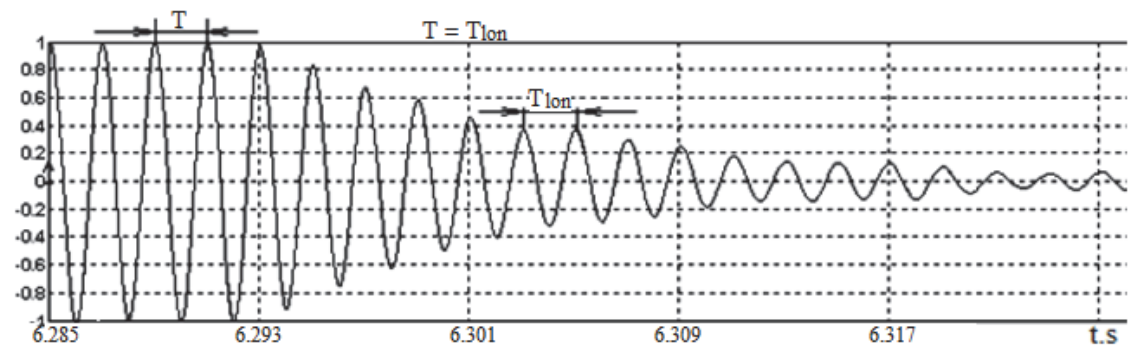

Fig. 13. Sound decay, exited frequency $f=500 \mathrm{~Hz}$, decay frequency $f_{\text {lon }}=500 \mathrm{~Hz}$

So, the equality of frequencies $f_{\text {lon }}$ and $f$ is reached by regulating of changing the tube length. To achieve this, the length of the tube should be $1 / 4$ of the length of the sound waves. In addition, to ensure flat waves, the length of the tube should be more than 3/4 tube diameters.

Fig. 15 shows the recorded waveforms in which the damped frequency processes of 200, 500, $1000,2000,5000$ and $10,000 \mathrm{~Hz}$ emitted by the sound sources and the frequency of the decay waves generated in the impedance tube are equal, and this was achieved by adjusting the length of the tube for each frequency. Using the oscillograms obtained in this way, the absorption coefficients of the materials studied for any spectrum were determined. The exception was $10,000 \mathrm{~Hz}$, where, during the decay, the frequency was $10,000 \mathrm{~Hz}$ at a frequency of $3,000 \mathrm{~Hz}$. According to the calculation, the length of the tube should be $17 \mathrm{~mm}$ in order for the decay of the amplitudes to occur at a frequency of $10,000 \mathrm{~Hz}$ Since the length of the microphone was $20 \mathrm{~mm}$, we had to use a tube $25 \mathrm{~mm}$ long. Thus, in the process of damping together $10,000 \mathrm{~Hz}$, the attenuation frequency of $3000 \mathrm{~Hz}$ is also allocated. The waveforms of decay shown in Fig. 15 are obtained for the case when the sample of the test was the cork tree installed at the side end of the tube. 
6.2. A NEW METHOD FOR DETERMINING AN ACOUSTICAL CHARACTERISTIC OF BUILDING MATERIALS.

TAMAZ NATRIASHVILI, MERAB CHELIDZE, DAVID NizHARADZE, JEMAL JAVAKHISHVILI
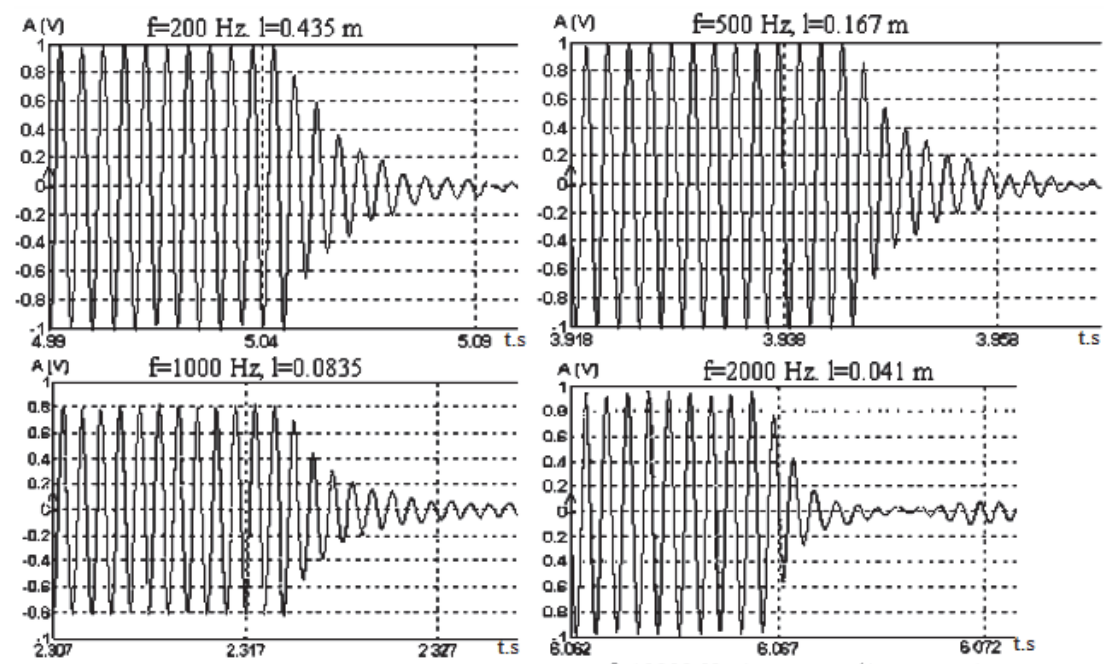

Alv $f=5000 \mathrm{~Hz}, 1=0.033 \mathrm{~m} \quad$ A.M $\quad f=10000 \mathrm{~Hz}, 1=0.025 \mathrm{~m}(1=0.017 \mathrm{~m})$

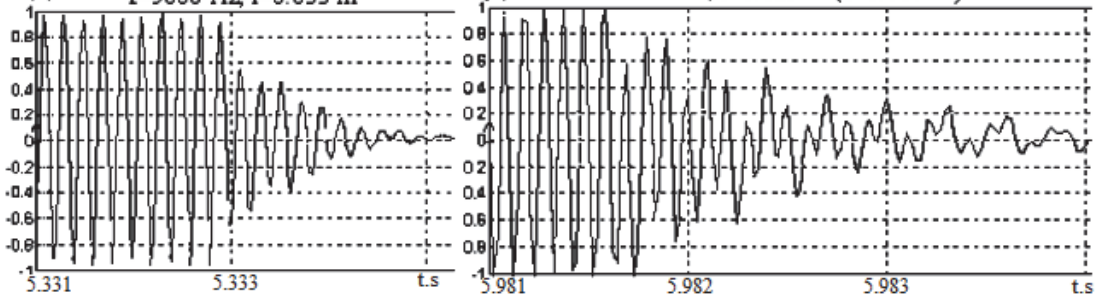

Fig. 15. Achievement of equality of excitement and fading frequencies by means of regulation of the tube length (sample material is cork)
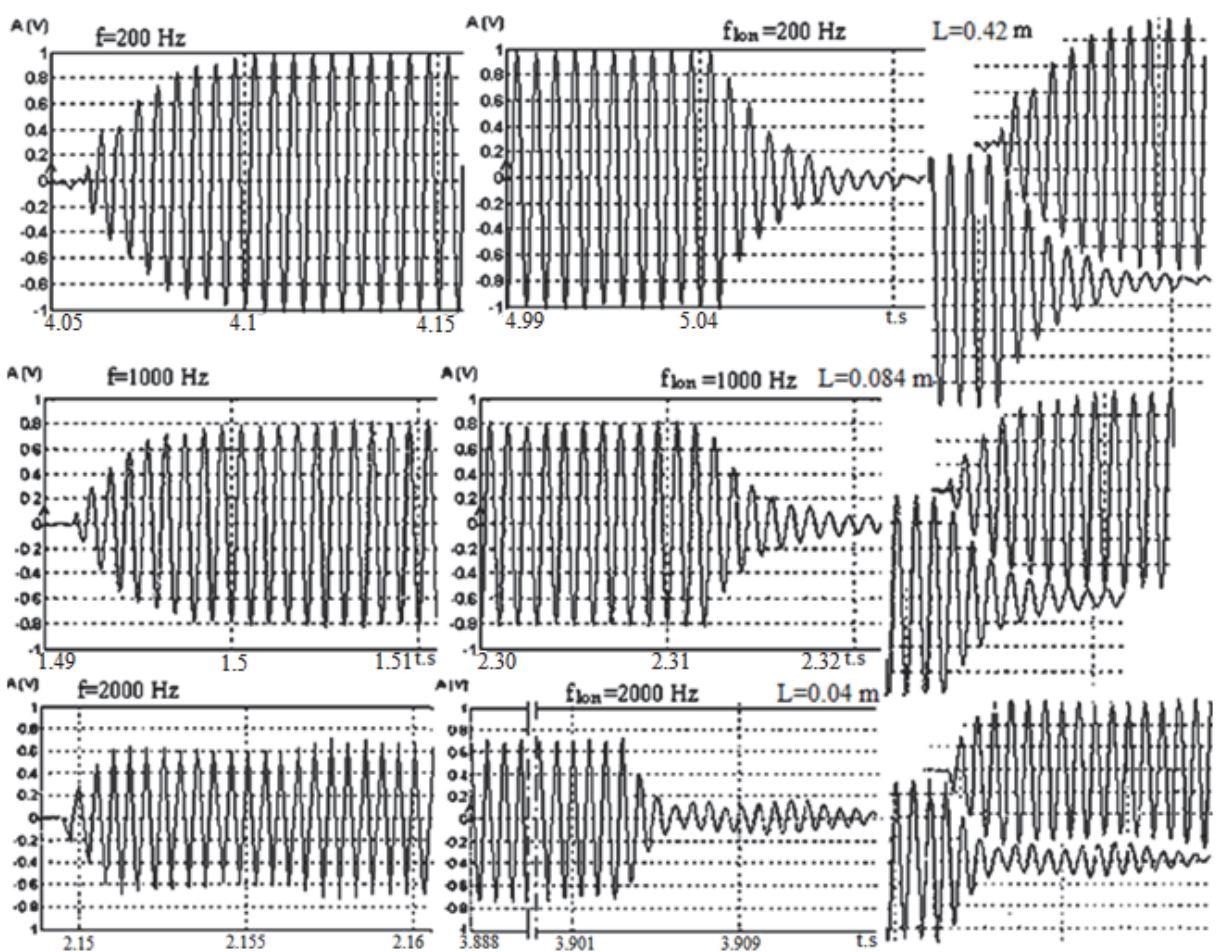

Fig. 16. Mirror images of forming and decay amplitudes at the presence of low sound energy

VOL. 2. QuAlity AND RELIABILITY OF TECHNICAL SYSTEMS: THEORY AND PRACTICE. ISBN 978-609-96036-0-5 
Theoretically, at low power, in a closed space, in particular in an impedance tube, the amplitudes of sound waves should increase gradually and also gradually decrease, i.e. have an antipolar form, which is fully proved by experimental studies. Fig. 16 shows the processes of increasing and decreasing amplitudes of standing waves in a closed space at the time of the switching on and turn off the low-level noise source. With a low-power sound source during the study, it was also found that in closed chambers (in the tube), the growth and decay of the sound amplitudes occurs at the same rate and they have the form of mirror images (Fig. 16).

The mirror images of processes of forming and attenuation of sound standing waves once more confirm that the presented method is correct, perspective and new, furthermore sound absorption coefficient can be determined also by the process of increase of reverberating sound amplitudes. Especially it should be noted that the process of decay of the reverberating sound amplitudes obtained by the method described above is very stable. In addition, the accuracy of the measurement of decay rates is practically independent of the measurement conditions, types of measuring instruments and their accuracy.

Experimental studies show that the amplitudes of reverberating sounds decay nonlinearly [4], which corresponds to the def curve shown on the oscillogram of Fig. 17. The rate of decay on the intervals de and $e_{f}$ is different and consequently different absorption coefficient will be obtained. If we intend that de and $e_{f}$ are linear intervals in conformity with linear intervals ac and $c_{b}$ than the formula for calculation sound absorption coefficient will be simplified. According to oscillograms of the decay of reverberating sound waves, the sound absorption coefficient can be calculated from the following simple Eq. (14), in a manner analogous of calculation the damping coefficient in the theory of oscillations $[10,14,15]$.

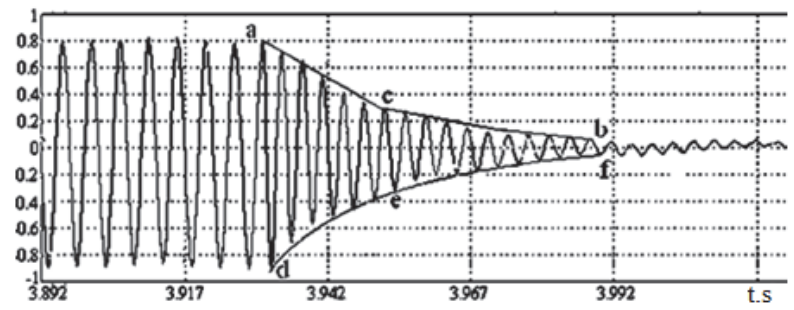

Fig. 17. Determination of sound absorption coefficient by decay of reverberation amplitudes

$\alpha_{k}=\frac{A_{a}-A_{b}}{m A_{a}}$,

where $A_{a}$ and $A_{b}$ - maximal and minimal amplitudes on the calculation interval accordingly, $m$ the number of amplitudes of decay, or increase on the calculation interval. Evidently, that average value of absorbing coefficient, entirely for def curve, will be the average mathematical sum of separated ac and $c_{b}$ linear intervals. At least from a practical point of view, it is desirable that a certain absorption coefficient indicates a high sound pressure at a level of 120-70 dB. In Fig. 14, such an interval is represented by the nonlinear curve de and linear ac. In the theory of vibration, the absorption coefficient for ac range is calculated using $[a, b]$ interval by adding 2 in the Eq. (15):

$\alpha_{k}=\frac{2}{m} \frac{A_{a}-A_{b}}{A_{a}}$

In such cases, it is desirable that the lengths of high inclination curves $a_{c}\left(d_{e}\right)$ and low inclination curves $c_{b}\left(e_{f}\right)$ were approximately equal.

Theoretically, when the length of the radiated wave is bigger than the length of the tube the length of the tube must be increased otherwise the frequency of generating of the plain waves become higher i.e. in the tube will be created high-frequency standing waves. 


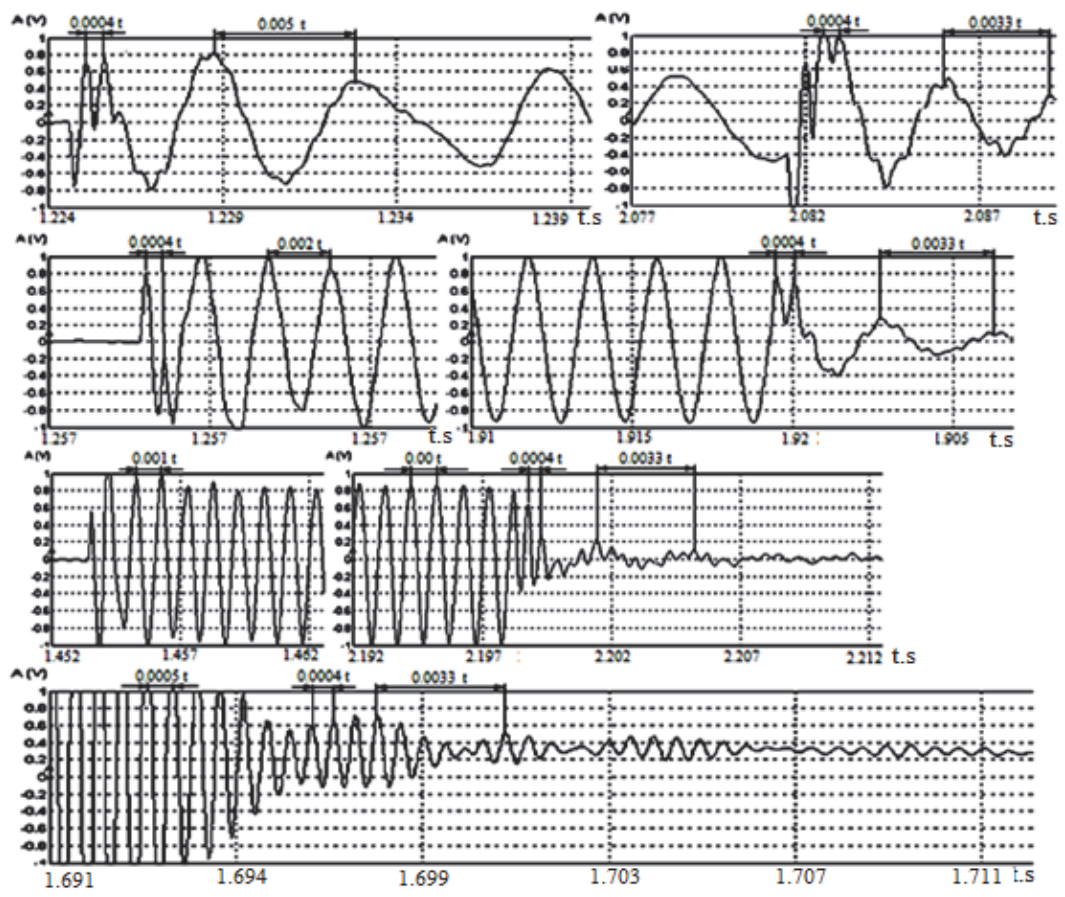

Fig. 18. The tube length and reverberation sound frequency

Figure 18 shows the processes of creation and attenuation of frequency waves of $2500 \mathrm{~Hz}$, when 200, 500, 1000 and 2000 frequency waves excited from a noise source are acted in a tube with a length of $35 \mathrm{~mm}$. At the beginning of short periods of growth and decay of amplitudes, i.e., pulses of $0.0004 \mathrm{sec}$. appear during periods of unstable processes. For all waves, the fundamental attenuation frequency is $300 \mathrm{~Hz}$, and it is accompanied by a frequency of $2500 \mathrm{~Hz}$.

Based on numerous experimental studies, a new method for measuring the sound absorption coefficient showed a greater sensitivity to the measurement of the acoustic properties of materials. Fig. 19 shows some oscillograms of the decay of the sound amplitudes, the absorption coefficients of which were calculated from Eq. (14). In addition, the method for determining the sound absorption coefficient, based on the decay of the sound amplitudes, proved to be very stable and accurate in spite of the measurement requirements, measuring devices accuracy and types were violated.
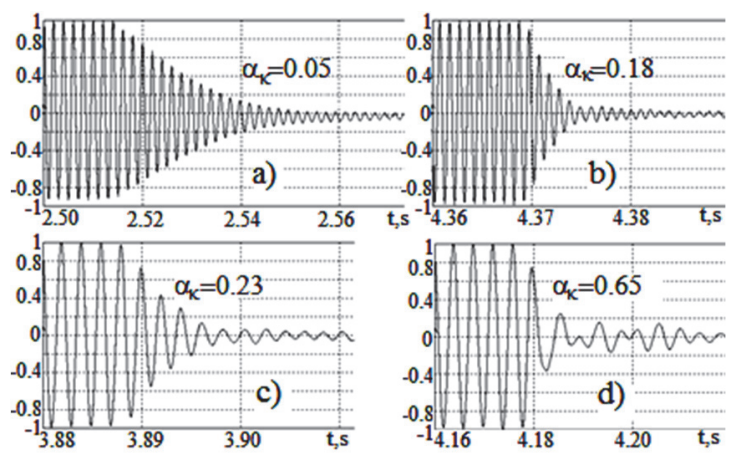

Fig. 19. Oscillograms of attenuation amplitudes at $500 \mathrm{~Hz}$ with samples of a) water, b) tiles,

c) plywood and d) cotton
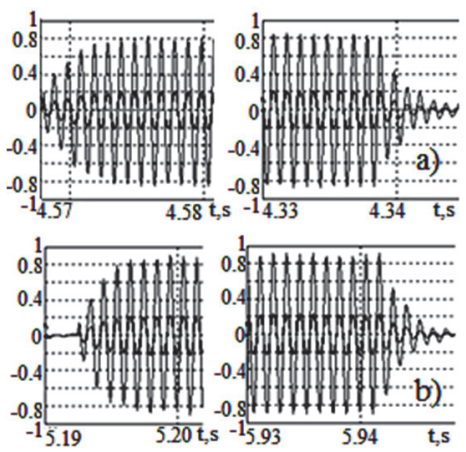

Fig. 20. Oscillograms a) a tightly closed lid, b) an incomplete lid on the side of the noise source 
For example, Fig. 20 shows the entering and decay waveforms that were obtained for a wet porcelain plate when, in one case, the tube cover was tightly closed $\alpha_{k}=0.125$ and in another case, the cover on the side of the noise source was practically open $\alpha_{k}=0.128$. Since the absorption coefficient is determined by the ratio of the subtraction of the maximum and minimum amplitudes $\left(A_{a}-A_{b}\right)$ to the maximum amplitude Aa of the given interval, therefore errors that appearing due to inaccuracies of the measuring devices, instability of measurement conditions and the environment are not great, unlike the existing methods in which they greatly affect the obtained results. If equal values appear in the numerator and denominator simultaneously, in our case the measurement errors, they will not cause the change of the fraction. Here the main thing is that during the decay of the sound amplitude (approximately $0.01 \mathrm{sec}$.), no changes occurred on the background sound.

On the basis of theoretical and experimental studies shown that the sound absorption coefficient for various materials possible to determine in the open space without an impedance tube by means of conventional measuring instruments in acceptable accuracy for wide consumers. As studies have shown for carrying out measuring operations, a test material of approximately $1 \mathrm{~m}^{2}$ area and a reflecting wall is required and the possibility to vary the distance from 0.03 to 1 $\mathrm{m}$. The microphone should be installed in the center next to the test material and the loudspeaker at the wall also in the center. Other reflective materials should be removed at least $3-5 \mathrm{~m}$ that during the attenuation of the reverberating amplitudes, sound reflected from other materials was not penetrated into the work area.

Fig. 21 shows the process of decay of reverberant sound amplitudes when the distance between the sample and the reflecting wall is $L=0.93 \mathrm{~m}$. On the basis of investigation may be said the process of decay sound waves in the open space is identical to the process that takes place in the closed tube, but in order to achieve the same sound pressure level in open space, it is necessary to increase the loudspeaker capacity by more than 70-80 units in comparison to the tube where it was 1-2 units. When the distance from other reflective materials was more than $3 \mathrm{~m}$, the effect of the reflective waves of the spectra of 500 and $5000 \mathrm{~Hz}$ was minimal, but the condition was broken for the $2000 \mathrm{~Hz}$ oscillogram.
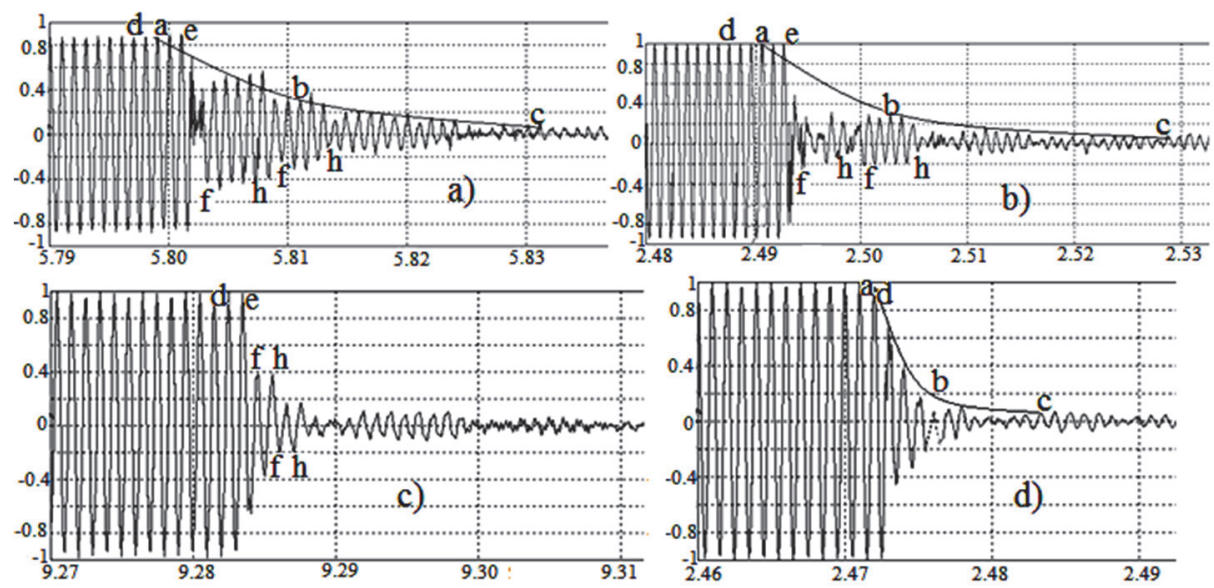

Fig. 21. Determination sound absorption coefficient in the open space

The oscillograms a) and b) of figure 21 are obtained at the condition of the distance $L=0.9 \mathrm{~m}$. At this distance, about 5 waves with a frequency of $1000 \mathrm{~Hz}$ are placed. Indeed $334 / 2 \cdot 1000=0.167 \mathrm{~m}$ and $0.9 / 0.167=5.4$ that clearly is seen on the decay regimes oscillograms. Each stage (step) of the decay contains almost five amplitudes. The oscillogram of the decay mode of the sound shown in Fig. 21(b), differs from the oscillogram Fig. 21(a) only in that the distances between plywood and the wall was changed insignificantly, which caused every second half-wave 
of the sound wave to enter the antiphase state, i.e., on the basis of Eqs. (6), (7), the amplitudes of the corresponding periods are suppressed.

Although the average decay rate of the amplitudes is the same in both cases. The oscillogram showed in Fig. 18(c) was obtained when the distance was $a=0.20 \mathrm{~m}$. In this case, we have two amplitudes at each level (stages). But oscillogram d) was obtained when the distance was $9 \mathrm{~mm}$ i.e. $1 / 4$ the part of the length of $1000 \mathrm{~Hz}$ of sound wave frequency. In this case, the decay process proceeds at the same frequency, which has a source of coherent noise i.e. the decay process of reverberating waves is analogous that happens in the impedance tube shown in Figs. 13, 15. It should be specially noted that all four oscillograms obtained by the decay processes performed on the plywood have approximately equal attenuation rate and absorption coefficient $\alpha=0.27-0.29$, calculated by the Eq. (14). Each oscillogram has three and a half steps of the amplitudes of decay.

As seen when calculating the absorption coefficient using the presented method, the time factor does not play any real role since the damping times a) and b) of the oscillogram are 2.5 times larger than the decay time of the regime c) and 5 times of the regime d) but obtained sound absorption coefficient for four cases are practically is equal.

When the sound incident on the material, it is reflected, absorbed and transmitted, as shown in Fig. 22.

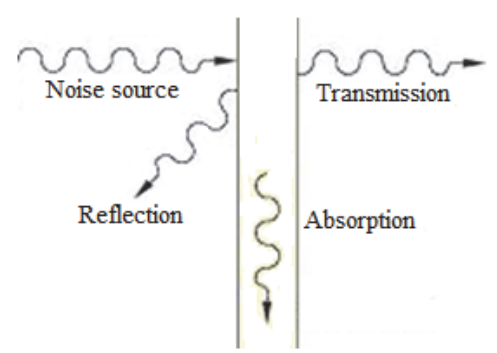

Fig. 22. Sound incident on a material

Ideally, the sound absorption coefficient defined as the fraction of the randomly incident sound power absorbed by the surface, but in the practice (experimentally) it is operationally defined as a collection of absorbed and transmitted parts of the sound. In this sense an open window is considered a "perfect" absorber, but is really a perfect transmitter. The coefficients determined are based on this conception; that is, that what is not reflected is "absorbed". The open window is taken as the standard absorber with a coefficient of 1.00, or 100 per cent absorption [4].

As practice shows, in a number of cases, it is necessary to know the absorbing capacity of materials, regardless of the determination of transmitted energy, which is still difficult to determine. It can be said that today used method for this purpose is based on the simultaneous use of four microphones in an impedance tube which requires quite complicated calculations, highprecision measuring types of equipment and so on. This method is based on the theory of transfer function method for sound propagating in a tube where the acoustic transfer function is calculated as a substitute of the spectral densities. This method requires a four-channel FFT analyzer and four spaced microphones and working with the convolution integrals and their Fourier transforms.

The obtained results of the decay process of complex and coherent reverberation sound waves performed in closed spaces showed that the determination of the sound absorption coefficient can be greatly simplified. This method allows that instead of a tube with four microphones, a tube with two microphones were used, where the sample separates the cameras. Theoretically, if the sound is not transmitted completely through the test material, the absorbed energy will be equal to the energy not reflected, that is, subtraction of the incident and reflected energies, and coefficient of absorption by material will equal $\alpha_{t}=1-\alpha$.

But in the case when a part of the sound waves is transmitted through the test material, then the energy absorbed by the material is equal to the subtraction of the energy of the incident and 
transmitted energy, ie, the damping factor will be equal to the ratio of the subtraction of the sound energy of the incident and transmitted amplitudes of the first and second cameras by a fixed corresponding amplitude of the first camera. Since the values of the incident and the transmitted sound amplitudes are measured in different chambers, it is obvious that in such cases it is necessary to increase the requirements for the accuracy of the measuring instruments and the correct installation of the test material in the measurement work.

Fig. 23 shows the processes of decay and transmission of sound waves in the presence of test materials mouse pad and cotton.

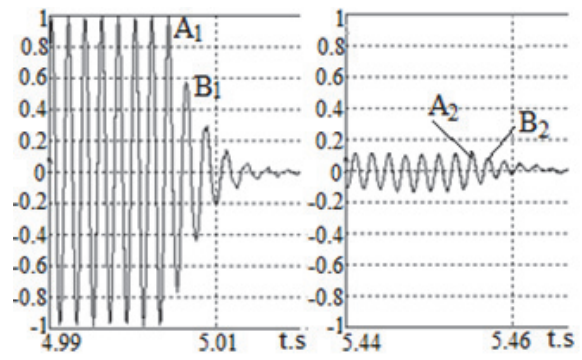

Fig. 23. Decay and transmited processes at the presence of mouse pad testing material

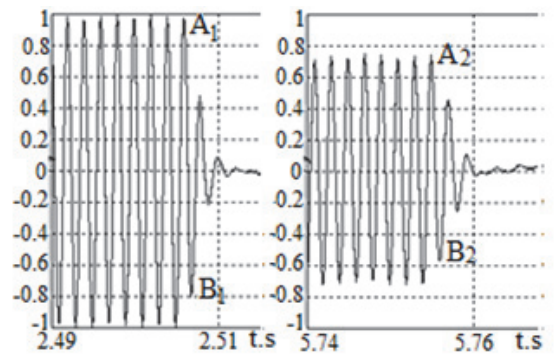

Fig. 24. Decay and transmited processes at the presence of cotton testing material

The presented oscillogram makes it possible to measure the damping coefficient of the material according to the Eq. (16):

$\alpha_{t}=\frac{A_{1}-A_{2}}{A_{1}}$.

In Figs. 23 and 24, A1 and B1 denote the sound amplitudes of the decay in the room of the sound source and A2, B2 - the sound amplitudes in the sound receiving room.

Although a clean cotton fabric has a higher sound absorption capacity than a mouse pad, a clean cotton fabric lets more sound energy into the receiving chamber than a mouse pad i.e. mouse pad directly absorbs more sound energy than cotton fabric.

It should be noted that when coherent sound passes through the materials, in some cases additional spectral components appear Fig. 25, which in principle makes it possible to investigate the structural state and damage of some materials.
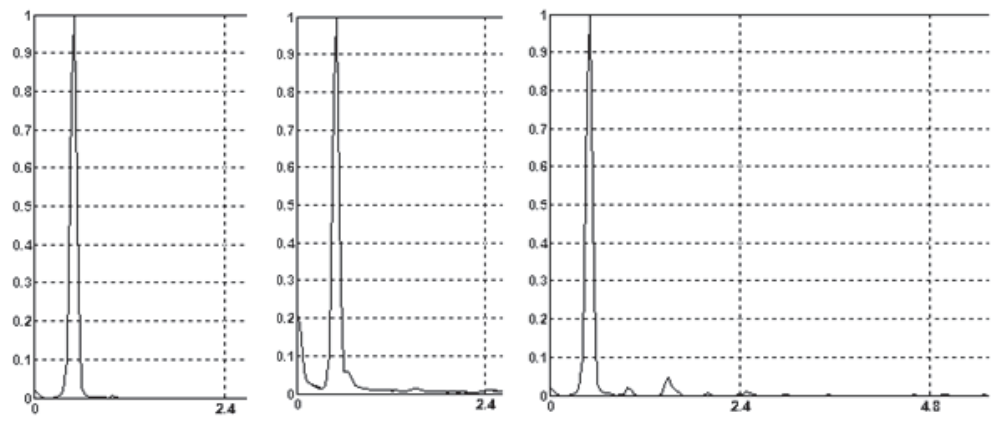

Fig. 25. Amplitude-frequency features of $500 \mathrm{~Hz}$ transmitting coherent sound source waves

The conducted studies showed that, according to the proposed method, the results of the test of the study and determination of the absorption coefficient do not depend on the test condition, i.e. the absorption coefficients of the same material with the same properties do not depend on the test condition, accuracy, and type of measuring instruments. The method of determination of the 
absorption coefficient by means of decay of the reverberating waves is sufficiently stable, demonstrates a high sensitivity to the materials acoustic characteristics and a low sensitivity to errors admitted during the measurement. Therefore, may be concluded that after some further improvement, the presented method has a good prospect of wide use.

More than 200 the scientific literature, which was considered and analyzed by us, that cover period of 1927-2014 years, among them 6 fundamental monographs, did not contain calculations of absorption coefficient by the presented method and are not shown waveforms in which clearly are shown decay and rate tempo of reverberation amplitudes in the materials. By noted waveforms, graphically, may be determined absorption coefficient in the materials that are a new method and must be perspective for further elaboration and refinement.

\section{Conclusions}

The method of determining the absorption coefficient in materials by the decay of standing waves with the help of an impedance tube allows, with the help of cheap and low-current devices, the measured value of the coefficient was practically acceptable and accurate, which gives a wide possibility of its use in practice.

The method of determining the absorption coefficient using the process of reverberating decay waves is fairly stable and shows a low sensitivity for all made errors in the measurement process.

The wide dissemination of modern digital technologies, PCs and software allows measuring the ability of sound absorption of materials and absorption coefficient with the necessary accuracy without special laboratories in conditions of wide consumptions.

The conducted studies showed that, according to the proposed method, the results of the test for the absorption coefficient do not depend on the condition of performing test, that is, the absorption coefficients of the same material with the same properties do not depend on the test condition, accuracy, and type of measuring instruments.

If we do not take into account the sound attenuation in the air, the time factor plays no real role in calculating the absorption coefficient by the presented method.

Since the absorption coefficient is determined by the ratio of the subtraction of the maximum and minimum amplitudes to the maximum amplitude of this interval, errors resulting from inaccuracies in measuring devices, instability of the measurement conditions and the environment are small, since the errors occurring in the numerator and denominator simultaneously, in our case measurement errors, they do not cause a change in the fraction, only here is necessary that the measuring devices were linear in the measuring ranges.

\section{References}

[1] Acoustics in Buildings. FESI Document A4. October, 2007.

[2] Igolkin A. A., Rodionov L. V., Chess E. V., Kox A. I. The Sound-Absorption. The Methods of the Measurements. Samarskiy State Aerospace University, Name of the Academician S.P. Koroleva, 2010.

[3] McGrory Mathew, Castro Cirac Daniel, Gaussen Olivier, Cabrera Densil Sound absorption coefficient measurement: re-examining the relationship between impedance tube and reverberant room methods. Proceedings of Acoustics, Fremantle, Australia, 2012.

[4] Watson Floyd R. The Absorption of Sound by Materials. University of Illinois Bulletin. Vol. 25, 1927.

[5] Seybert Andrew F. Notes on Absorption and Impedance Measurements Two Microfone. University of Kentucky Lexington, KY 40506-0108, 2007.

[6] Eugen Skudrzyk The Foundation of Acoustic. Basic Mathematics and Basic Accounts. SpringerVerlag, Wien, New York, Vol. 1, 1971, p. 520.

[7] Kinsler Lawrense E., Frey Austrin R., Coppens Alen B., Sanders Jeimes V. Fundamentals of Acoustics. 4th Editions. New York, Toronto, 1982, p. 550.

[8] Crendall I. B. Acoustics. 2009, p. 168.

[9] Seybert Andrew F. Notes on Absorption and Impedance Measurements. University of Kentucky Lexington, http://www.spectronics.net/Notes\%20on\%20Absorption\%20Measurements.pdf 
[10] Genkina M. D. The Vibrations in Technique. Reference Book in 6 volumes. Under Editing. Machine building, 1981.

[11] Muehleisen R. T. Measurement of the Acoustic Properties of Acoustic Absorbers. Illinois Institute of Technology, 2007, p. 30.

[12] Chelidze M. A., Nizharadze D., Javackishvili Tedoshvili J. M. Investigation of sound absorption. Modern achievements of Science and Education 7th International Conference, Jerusalem (Israel), 2016, p. 66-71.

[13] Chelidze M. A. Investigation of Sound absorption coefficient by decay of standing waives in the impedance tube. GESJ: Physics, Vol. 1, Issue 17, 2017.

[14] Chelidze M. A., Javaxishvili J., Nizharadze D., Tedoshvili M. Investigation of sound absorption coefficient by energetic method. Proceedings of 7th International Conference on Science and Education, Rome (Italy), 2017, p. 3-9.

[15] Chelidze M. A. Investigation of sound absorption characteristics in materials by help of subtraction method on the base of PC. Science and Education 8th International Conference, 2015, p. 24-27.

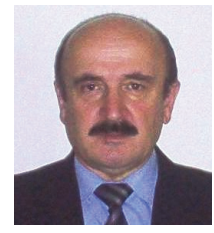

Tamaz Natriashvili received $\mathrm{Ph} . \mathrm{D}$. degree in machine technology from Georgian Technical University, Tbilisi, Georgia in 2003. Now he works as director of the Institute of Machine Mechanics, Tbilisi, Georgia. His current research interests include technical novelties in the field of automobile engines.

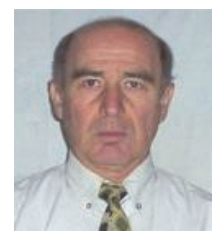

Merab Chelidze received Ph.D. degree by the decision of the Council of the Georgian Polytechnical Institute of March 29, Tbilisi, Georgia, 1982. Now he works at Institute of Machine Mechanics. His current research interests include mathematical modeling, dynamics investigation, machines calculation on the toughness and endurance, the study of problems of determining of acoustical characteristic of building.

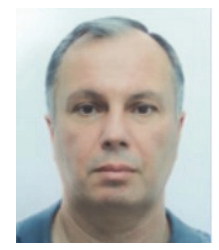

David Nizharadze received Ph.D. degree in biology science from the Institute of Plant Biochemistry and Biotechnology of the Georgian Science Academy, Tbilisi, Georgia, in 1989. Now he works at the Rafael Dvali Institute of Machine Mechanics, Tbilisi, Georgia. His current research interests are modern thermal insulating materials.

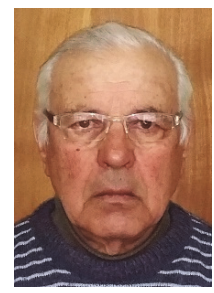

Jemal Javakhishvili received Ph.D. degree in technical science and engineering from the Polytechnic University of Baku, Baku, Azerbaijan, in 1983. Now he works at the Rafael Dvali Institute of Machine Mechanics, Tbilisi, Georgia. His current research interests include investigations in the heat engines and modern thermal insulating materials. 


\section{Index}

absorption, 184, 185, 186, 187, 188, 189, 190, 191, 192, 193, 194, 195, 283, 293, 294, 295, 296, 298, 300, 302, 303, 304, $305,306,307$

acoustic emission, 214, 215, 217, 219, 220, $221,222,223,224,225,226,227,228$

attractor, 81,86

autoclaved aerated concrete, 276, 278, 291

automatic balancing, 26, 29, 40, 42, 46, 49, $51,57,91$

brick, 276, 277, 278, 286

business process, $5,6,7,10$

computer-aided engineering, 18, 19

contact angle, 36, 69, 198, 200, 201, 202,

204, 205, 206, 207, 208, 210, 211

contact strength, 104, 106, 115, 116, 117

cutting tool, 91, 94, 163, 165, 166, 168,

$169,172,173,174,175,176,177,178$,

$179,180,181,182$

cutting tools, $136,163,165,168,174,175$, 181

dry building systems, 277

effective current density, 134, 155

energy efficiency, 81, 278, 289

experiment, 38, 49, 104, 105, 112, 114,

116, 169, 187, 190, 203, 205, 206, 207,

$210,222,225$

functional approach, 2, 8, 10

high-rate deformation, 119, 120, 123, 130

innovation, 15, 17, 24

integrated comprehensive systems and

technologies, 14

invariant, 83, 256, 257, 259, 272

measurement, 40, 60, 61, 67, 71, 72, 77, 93,

$97,108,121,122,203,219,221,244$,

298, 302, 303, 304, 306, 307

mechanical vibratory system, 63,72

microhardness, 104, 106, 109, 110, 117,

$136,149,151,163,164,165,168,169$,

$170,171,172,174,175,181,182$

nanostructures, 134, 135, 136, 139, 144,

$145,146,147,148,149,152,153,155$,

$156,160,161,163,166,167,168,169$,

$172,181,199$

numerical methods, 92, 93, 119

optimization of technocenosis, 252, 253

phase angle shift, 231, 232, 233, 236, 237, 238

polymer composite, 187 power line, 230, 232, 236, 238, 239

printed circuit board, 228

process approach, 2, 10, 11

process model, 3

protective element, 119, 125, 126

protective elements, 119, 123, 124, 125,

126,130

pure bending, 214, 223, 225, 227, 228

quality management system, 2, 11

quartz resonator, 255, 259, 263, 269, 271

rank analysis, 246, 247, 248, 251, 252, 253

reflection, 185, 186, 187, 188, 191, 231,

232, 233, 235, 236, 238, 240, 253, 270,

271, 273, 289, 294, 295, 298, 299

reverberation, 302, 303, 305, 307

rolling bearing, 61

rope block, 104, 105, 106, 111, 112, 114, $115,116,117$

rotor, $26,27,28,29,35,36,38,42,43,44$, $45,46,47,48,49,50,51,52,53,54,55$,

$57,60,74,75,76,77,78,79,81,82,84$,

$86,87,125$

rotor stabilization, 82

sandwich type absorber, 184

seawater drop, 208

self-balancing, 26, 28, 29, 52, 53, 54, 55, 57

shipbuilding steel, 198, 205, 211

shock, 61, 62, 119, 121, 123, 129, 130

solder joint, 214, 219, 220, 221, 222, 225, 226, 227

sound, 40, 277, 278, 279, 280, 281, 285 , 286, 287, 289, 293, 294, 295, 296, 297 , 298, 299, 300, 301, 302, 303, 304, 305, 306, 307

spectral analysis, 96, 100, 230, 240, 241, 242, 244

spectral leakage, 230

spindle unit, $60,61,62,64,67,72$

stability, 44, 45, 47, 74, 84, 86, 90, 91, 92,

$105,198,206,211,250,255,258,259$,

$260,263,269,271,273,277,278,283$,

290

superhydrophobic surface, 198, 199, 201, 202, 203, 204, 205, 207, 208, 210

surface plastic deformation, 104

surface tension, 41, 198, 200, 201, 206, 207, 208

synergetic control theory, 81,87 
synergistic effect, 184, 189, 193, 195

technical diagnostics, 214, 227

technocenosis, 246, 247, 248, 249, 250, 251,252

technological processes, 104, 119, 127, 130,283

temperature, $35,134,135,137,139,143$, $144,145,146,147,148,149,152,161$, $169,170,171,172,207,222,223,228$, $255,256,265,278,287,291,294$

tensile test, 220,221

thermal stress, 134, 135, 138, 144, 145, $146,147,148,152$

thermal stresses, 134, 135, 144, 145, 146, $147,148,161$

three-dimensional models, 119, 130

ventricular assist device, 74, 87 vibration, 26, 27, 29, 35, 40, 50, 51, 53, 54, $55,57,60,61,62,66,68,69,70,72,90$, $91,92,93,94,98,99,100,265,269$, 285, 290, 293, 296, 302

vibration diagnostic, 60, 94 vibroactivity, 26, 34, 35 washing-machine, 32, 34, 35

wave, $28,100,184,185,186,191,192$, $218,232,233,235,238,239,295,296$, 298, 299, 300, 302, 304, 305

wear, 36, 39, 40, 74, 75, 90, 93, 104, 105, $106,110,111,114,115,116,117,136$, $149,163,164,166,181,200,211,281$ wedge roller, 104, 111, 112, 114, 116, 117 wetting, 198, 200, 204, 205, 207, 210, 211 , 219 


\author{
JVE INTERNATIONAL
} Www.jvejournals.com 